\title{
Isolierung, Strukturaufklärung und Biosynthese von Sekundärmetaboliten endophytischer Pilze aus Algen und Pflanzen mariner Habitate
}

\author{
Dissertation \\ zur Erlangung des Doktorgrades \\ der Mathematisch-Naturwissenschaftlichen Fakultäten \\ der Georg-August-Universität zu Göttingen
}

vorgelegt von

Oliver Schlörke

aus Berlin

Göttingen 2005 
D7

Referent: Prof. Dr. A. Zeeck

Korreferent: Prof. Dr. L. F. Tietze

Tag der mündlichen Prüfung: 27. April 2005 
Für meinen Vater,

der meine Begeisterung für die Chemie geweckt hat

„Der Beginn aller Wissenschaften ist das Erstaunen, dass die Dinge so sind, wie sie sind."

Aristoteles (384-322 v. Chr.) 
Die vorliegende Arbeit wurde im Institut für Organische und Biomolekulare Chemie der Universität Göttingen in der Zeit von Januar 2002 bis März 2005 unter Anleitung von Prof. Dr. A. Zeeck durchgeführt.

Herrn Prof. Dr. A. Zeeck danke ich für die interessante Aufgabenstellung, sein stetes Interesse am Fortgang dieser Arbeit sowie für viele wertvolle Diskussionen und Anregungen 


\section{Inhaltsverzeichnis}

\section{A Theoretischer Teil}

I Einleitung

1. Marine Naturstoffe in der Wirkstoffforschung 1

2. Sind Mikroorganismen die wahren Produzenten mariner Naturstoffe? 5

3. Endophytische Pilze als Quelle neuer Naturstoffe 8

$\begin{array}{ll}\text { 4. Projektbeschreibung } & 10\end{array}$

5. Aufgabenstellung 11

II Herkunft und Screening der endophytischen Pilzen 12

1. Screening der endophytischen Pilze 12

$\begin{array}{lr}\text { 1.1. Screeningmethoden } & 12\end{array}$

1.2. Durchführung des chemisch-biologischen Screenings 13

2. Herkunft der bearbeiteten endophytischen Pilze und Stammauswahl 16

2.1. In Braunschweig isolierte endophytische Pilzstämme 16

$\begin{array}{ll}\text { 2.2. In Göttingen isolierte endophytische Pilzstämme } & 17\end{array}$

2.3. Der Stamm A6651 20

III Sekundärmetabolite in Braunschweig isolierter endophytischer Pilze 21

1. Microsphaeropsis sp. Stamm $6288 \quad 21$

1.1. Spiciferone 22

1.1.1. Spiciferon A (17) 22

1.1.2. Prospiciferon A (18) 22

1.1.3. Biosynthese von Prospiciferon A (18) und Spiciferon A (17) 25

1.2. $\alpha$-Pyrone 26

1.2.1. Taiwapyron (22) 26

$\begin{array}{ll}\text { 1.2.2. Dehydrotaiwapyron (23) } & 27\end{array}$

$\begin{array}{ll}\text { 1.2.3. 5,6-Dihydrotaiwapyron (24) } & 27\end{array}$ 
1.2.4. 7-(1-Methyl-ethyliden)-taiwapyron (25)

1.2.5. $\alpha$-Pyrone vom Typ des Taiwapyrons 31

1.2.6. Überlegungen zur Biosynthese der $\alpha$-Pyrone 32

1.2.7. Zur Biosynthese der vom Stamm 6288 gebildeten $\alpha$-Pyron 35

1.3. Betaenone 36

1.3.1. Betaenon G(39) 36

1.3.2. Betaenon $\mathrm{H}(\mathbf{4 0}) \quad 38$

1.3.3. Betaenon I (41) 39

1.3.4. Betaenon J (42) 40

1.3.5. Die Gruppe der Betaenone und Stemphyloxine 41

1.4. Solanapyrone 43

1.4.1. Solanapyron H (48) 43

1.4.2. Solanapyron I (49) 45

1.4.3. Solanapyron J (51) 46

1.4.4. Die Gruppe der Solanapyrone $\quad 47$

1.5. Überlegungen zur Bildung von Solanapyron J (51) und

7-(1-Methyl-ethyliden)-taiwapyron (25) 48

1.6. Beurteilung der Biosyntheseleistung des Stammes 50

2. Alternaria sp. Stamm 6587 und 6588

2.1. Alternariol (55) 51

2.2. Alternariol-9-methylether (56) 52

2.3. Perylenchinone 53

2.3.1. Alterperylenol (57) 53

2.3.2. 5,6-Dihydroalterperylenol (58) 54

2.3.3. Stemphyperylenol (59) 54

2.3.4. Zur Biosynthese der isolierten Perylenchinone 55

2.4. Variation des Metabolitenmusters durch Enzyminhibitoren 57

2.4.1. 1,3,8-Trihydroxynaphthalen (65) 58

2.4.2. 2-Hydroxyjuglon (69) 58

2.4.3. Sphaerolon (70) 59

2.4.4. Scytalon (64) 61

3. Weitere in Braunschweig isolierte endophytische Pilze 62

3.1. Ascotricha chartarum Stamm $6303 \quad 62$

3.2. Stamm 6325 
IV Sekundärmetabolite in Göttingen isolierter endophytischer Pilze

1. Chaetomium sp. Stamm Gö 100/2 64

1.1. Die Chaetocyclinone A, B und C (82 - 84) 65

1.2. Untersuchungen zur Biosynthese von Chaetocyclinon A (82) 66

1.3. Überlegungen zur Biosynthese von Chaetocyclinon B (83) und C (84) 67

2. Aspergillus flavus Stamm Gö 100/4 69

2.1. Kojic acid (91) 69

2.2. Miyakamide 70

2.2.1. Miyakamid $\mathrm{A}_{2}$ (92) 70

2.2.2. Miyakamide $\mathrm{B}_{1}(\mathbf{9 3}) \quad 71$

2.2.3. Miyakamide in der Literatur $\quad 72$

2.3. Arthrographol (96) 73

2.4. Arthrolacton (98) 74

2.5. Untersuchungen zur Biosynthese von Arthrographol (96) 75

3. Chaetomium sp. Stamm Gö 100/9 80

3.1. Orsellinsäure und mit Orsellinsäure acylierte Verbindungen 81

3.1.1. Orsellinsäure (100) 82

3.1.2. Orsellide A (101) und B (102) 82

3.1.3. Orsellid C (104) 87

3.1.4. Orsellid D (105) 88

3.1.5. Orsellid E (106) 89

3.1.6. (4-Hydroxy-pent-2-on-1-yl)-orsellinat (107) 89

3.1.7. (Pent-3-en-2-on-1-yl)-orsellinat (108) 90

3.2. Literaturbekannte Verbindungen mit Desoxyzuckern 91

3.3. Untersuchungen zur Biosynthese der Orsellide 92

3.4. Überlegungen zur Biosynthese der Orsellide 98

3.5. Überlegungen zur Bildung von (4-Hydroxy-pent-2-on-1-yl)-orsellinat (107) und (Pent-3-en-2-on-1-yl)-orsellinat (108) 100

$\begin{array}{ll}\text { 3.6. Thiodiketopiperazine } & 101\end{array}$

3.6.1. Chetomin (119) 101

3.6.2. Chetoseminudin A (120) 102 
3.6.4. Die Gruppe der Thiodiketopiperazine 103

$\begin{array}{ll}\text { 3.7. Fumitremorgin C (125) } & 105\end{array}$

4. Trichoderma sp. Stamm Gö 101/19 106

4.1. Sorbicillin (126) 106

5. Humicola grisea Stamm Gö 101/26 107

5.1. 6-Methoxysterigmatocystin (127) 107

$\begin{array}{ll}\text { 5.2. Fuscoatrosid (128) } & 108\end{array}$

V Sekundärmetabolite weiterer untersuchter Pilze 109

1. Der Stamm A6651 109

$\begin{array}{ll}\text { 1.1. Eremophilane } & 109\end{array}$

1.1.1. Dihydrobipolaroxin (129) 109

1.1.2. Bipolaroxin (130) 110

1.1.3. Dendryphiellin E (131) 111

1.1.4. KM-01 (132) 112

1.1.5. Metabolit A6651-E (133) 113

1.1.6. Die Gruppe der Eremophilane 114

1.1.7. Zur Biosynthese der isolierten Eremophilane 115

1.2. Dendryphiellinsäure A (139) 117

$\begin{array}{ll}\text { 1.3. Cynodontin (140) } & 117\end{array}$

1.4. Cerebrosid C (141) 119

$\begin{array}{ll}\text { 1.5. Variation der Fermentationsbedingungen } & 120\end{array}$

$\begin{array}{ll}\text { VI Zur biologischen Aktivität der isolierten Naturstoffe } & 121\end{array}$

$\begin{array}{ll}\text { VII Diskussion und Ausblick } & 123\end{array}$

VIII Zusammenfassung der Ergebnisse $\quad 126$ 


\section{B Experimenteller Teil}

I Allgemeines

1. Instrumentelle Analytik

2. Chromatographische Methoden

3. Mikrobiologische Methoden

4. Allgemeine Arbeitsvorschriften

II Sekundärmetabolite aus endophytischen Pilzstämme

1. Stammhaltung und Langzeitkonservierung

2. Durchführung des chemisch biologischen Screenings

3. Aufarbeitung von Fermentationsansätzen

3.1. Kultivierung in Schüttelkolben 138

3.2. Kultivierung als Ruhekultur 138

4. Microsphaeropsis sp. Stamm 6288

4.1. Kultivierung und Aufarbeitung

4.2. Variation der Kultivierungsbedingungen

4.3. Aufnahme einer Wachstumskurve

4.4. Durchführung der Fütterungsexperimente 139

4.5. Isolierung und Charakterisierung der Metabolite 140

5. Alternaria sp. Stamm 6587 und 6588

5.1. Kultivierung und Isolierung der Metabolite

5.2. Kultivierung unter Zusatz von Enzyminhibitoren

5.3. Charakterisierung der Metabolite

6. Aspergillus flavus Stamm Gö 100/4

6.1. Kultivierung und Isolierung der Metabolite

6.2. Aufnahme einer Wachstumskurve

6.3. Durchführung der Fütterungsexperimente

6.4. Charakterisierung der Metabolite

7. Chaetomium sp. Stamm Gö 100/9

7.1. Kultivierung und Isolierung der Metabolite

7.2. Aufnahme einer Wachstumskurve

7.3. Durchführung der Fütterungsexperimente 
\begin{tabular}{ll} 
7.4. & Charakterisierung der Metabolite \\
\hline
\end{tabular}

8. Trichoderma sp. Stamm Gö 101/19 181

8.1. Kultivierung und Isolierung der Metabolite 181

8.2. Charakterisierung der Metabolite 181

9. Humicola grisea Stamm Gö 101/26 182

9.1. Kultivierung und Isolierung der Metabolite 182

9.2. Charakterisierung der Metabolite 182

10. Der Stamm A6651 184

10.1. Kultivierung und Aufarbeitung 184

10.2. Variation der Fermentationsbedingungen 184

10.3. Isolierung und Charakterisierung der Metabolite 185

$\begin{array}{lll}\text { C } & \text { Literaturverzeichnis } & 193\end{array}$ 


\section{A. Theoretischer Teil}

\section{Einleitung}

\section{Marine Naturstoffe in der Wirkstoffforschung}

Unter den verschiedenen Quellen für die Suche nach neuen Arzneimitteln zur Behandlung von Krankheiten spielen Substanzen aus der belebten Natur, die so genannten Naturstoffe, eine herausragende Rolle. Die Tatsache, dass 61 \% der in den Jahren 1981 bis 2002 als Medikamente eingeführten Substanzen aus Naturstoffen bestehen oder auf Basis von Leitstrukturen aus der Natur entwickelt wurden, ${ }^{*}$ verdeutlicht die Bedeutung der Naturstoffe für die Pharmaforschung. ${ }^{1}$ In bestimmten therapeutischen Bereichen ist der Anteil sogar noch größer: z. B. bei den Krebstherapeutika (74 \%) und antibakteriellen Wirkstoffen (78 \%).

Während Pflanzenextrakte als Quelle von Arzneistoffen in der Vergangenheit eine besonders wichtige Rolle spielten, ${ }^{2}$ wurden Erfolge bei der Bekämpfung vieler bis dahin tödlich verlaufender Infektionskrankheiten erst durch die Entdeckung antibiotisch aktiver bakterieller oder pilzlicher Naturstoffe möglich. Mit der Entwicklung der ersten Antibiotika, wie z. B. des Penicillins $^{3}$ und des Streptomycins ${ }^{4}$ zur Mitte des letzten Jahrhunderts, rückten Mikroorganismen als Arzneistoffquelle in den Vordergrund. Dass Substanzen aus Mikroorganismen oder Pflanzen sich als Startpunkte für die Suche nach Wirkstoffen gegen pilzliche oder bakterielle Infektionen anbieten, ist aufgrund ihrer Bedeutung in den Produzenten verständlich, schützen sie diese doch unter anderem vor Konkurrenten oder Schädlingen. ${ }^{5}$

Nahezu alle bis zum heutigen Tag aus der Natur entwickelten Arzneistoffe stammen aus terrestrischen Organismen, obwohl ca. 70 \% der Erdoberfläche von Ozeanen bedeckt sind und die Weltmeere eine immense Vielfalt an Lebensformen beherbergen. Von jeher spielen die Meere eine wichtige Rolle als Nahrungslieferant für den Menschen, als Quelle für Arzneistoffe blieben sie dagegen über lange Zeit hinweg unberücksichtigt. Den Beginn der marinen Naturstoffforschung markierte Anfang der fünfziger Jahre des letzten Jahrhunderts die Isolierung der ungewöhnlichen Nucleoside Spongouridin (1) und Spongothymidin (2) aus dem Meeresschwamm Cryptotethya crypta durch Bergmann und Feeney. ${ }^{6}$

\footnotetext{
* Naturstoffe $6 \%$, Naturstoffderivate $27 \%$, synthetische Komponenten mit Naturstoff-basiertem Pharmakophor $5 \%$, synthetische Produkte, die aufgrund von Wissen aus der Naturstoffforschung erstellt wurden (Naturstoffimitationen) $23 \%$. In diesen Zahlen nicht enthalten sind Derivate oder Analoga von schon auf dem Markt befindlichen Wirkstoffen.
} 
Beide Verbindungen enthalten als Zuckerbestandteil Arabinose statt Ribose und führen in menschlichen Zellen zum Abbruch der Nucleinsäure-Synthese. Dies macht man sich gezielt für die Behandlung von Krebserkrankungen bzw. viralen Infektionen zu Nutze. So wurden basierend auf diesem Wirkprinzip antiviralen Arzneistoffe wie $\operatorname{Vidarabine}^{\circledR}(3)$, $\operatorname{Udicil}^{\circledR}$ (4) oder Aciclovir ${ }^{\circledR}$ entwickelt. Diese sind bis heute die einzigen in klinischer Anwendung befindlichen Wirkstoffe, die sich von marinen Naturstoffen ableiten.

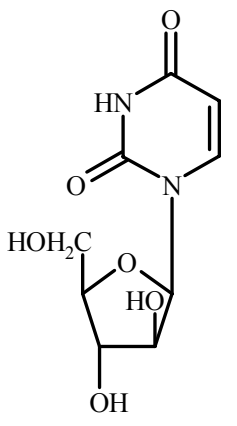

1

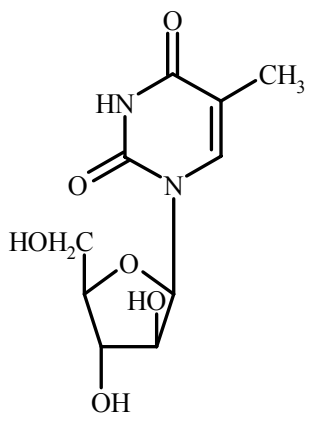

2

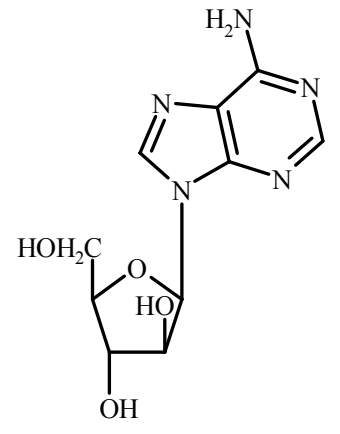

3

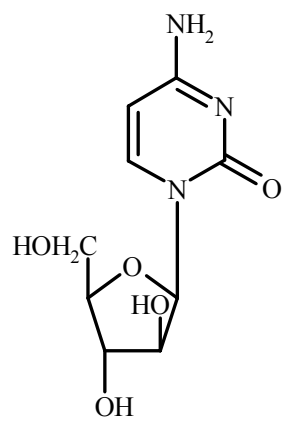

4

Inzwischen ist die Zahl bekannter Naturstoffe aus marinen Organismen auf über 7000 angewachsen. $^{7}$ Dieser Zuwachs hängt zum einen mit den Weiterentwicklungen der Tauchtechnik zusammen, die es marinen Biologen und Naturstoffforschern gestatten ihre Forschungsobjekte auch in entlegenen Ozeanregionen zu erreichen und zu beobachten. Zum anderen führten die Entwicklungen neuer Methoden in der Massenspektrometrie und NMR-Spektroskopie dazu, dass die Aufklärung auch komplexer Strukturen mit immer geringeren Substanzmengen möglich wurde.

Betrachtet man diejenigen Organismengruppen, die auf dem Land bzw. im Meer bislang die meisten biologisch aktiven Naturstoffe hervorgebracht haben, so tun sich deutliche Unterschiede auf: In der Gruppe terrestrischer Organismen sind ganz eindeutig die höheren Pflanzen an erster Stelle zu nennen. Im Meer dagegen sind Wirbellose (so genannte Invertebraten), wie z. B. Schwämme, Tunikaten oder (zumeist schalenlose) Schnecken, die reichsten Naturstoffquellen. Eine einleuchtende Erklärung für das gehäufte Auftreten bioaktiver Naturstoffe in wirbellosen Organismen liegt in der Ökologie dieser vielfältigen Organismen begründet. Marine Invertebraten, wie z. B. Schwämme oder Tunikaten, sind ähnlich wie Pflanzen sessile Organismen, die auch bei einem Angriff nicht die Flucht ergreifen können. Ferner verfügen sie nicht über einen schützenden Panzer oder andere morphologische Schutzmechanismen. 
Viele Schwämme oder andere Invertebraten leben darüber hinaus exponiert auf Felsen oder Korallenriffen und sind daher für Fraßfeinde meist leicht zu entdecken. Ferner stellen gerade Schwämme für karnivore Organismen auf Grund ihres hohen Stickstoffgehaltes eine metabolisch lohnende Nahrungsquelle dar, denn im Meer ist das Stickstoffangebot limitiert. Daher ist es kaum verwunderlich, dass diese Organismen oft Naturstoffe mit fraßhemmenden und/ oder toxischen Eigenschaften produzieren und sich auf diese Weise vor Umweltgefahren schützen.

Unter den gegenwärtig in verschiedenen Phasen der klinischen Prüfung stehenden marinen Naturstoffen machen die aus Invertebraten isolierten Substanzen einen besonders großen Teil aus: Von den etwa 14 Naturstoffen (oder Naturstoffanaloga) stammen 13 aus Invertebraten, lediglich eine Verbindung, das Squalamin-Lactat, wird aus Haien als Vertretern mariner Wirbeltiere gewonnen. ${ }^{8}$ Am weitesten in der Arzneistoffentwicklung fortgeschritten ist das Ziconotid. Beim Ziconotid handelt es sich um ein aus 25 Aminosäuren bestehendes Peptid, das aus dem Gift mariner Kegelschnecken (Conus-Arten) stammt und von den Schnecken zum Lähmen ihrer Beutetiere verwendet wird. Es wurde kürzlich von der FDA (Food and Drug Administration) zugelassen und soll Anfang 2005 unter dem Namen Prialt ${ }^{\circledR}$ als Schmerzmittel zur Behandlung von Patienten, die unter starken chronischen Schmerzen leiden, aber die Morphine in klassischen Arzneimitteln nicht vertragen, in den USA eingeführt werden. ${ }^{9}$ Das marine Alkaloid Ecteinascidin 743 (5) wird aus dem marinen Manteltierchen Ecteinascidia turbinata gewonnen ${ }^{10}$ und befindet sich momentan in der klinischen Erprobung gegen verschiedenen Tumorerkrankungen (Phase II und III). Zum Wirkmechanismus gibt es eine Vielzahl von Studien, die allerdings mit Vorsicht zu betrachten sind, da bei einigen dieser Untersuchungen Konzentrationen von 5 verwendet wurden, die um Größenordnungen über den minimalen Wirkkonzentrationen liegen. In physiologisch wirksamen Konzentrationen hemmt 5 die Zellteilung in der $\mathrm{G}_{2} / \mathrm{M}$-Phase und interagiert mit dem DNA-Reperaturprotein TC-ER. Außerdem inhibiert 5 die translationale Aktivierug des MDRl-Gens (MDR: multi drug resistance), was zu einem synergistischen Effekt mit Chemotherapeutika, die ein Substrat für MDR1 darstellen, führt. ${ }^{11}$

Ein weiteres viel versprechendes Krebstherapeutikum ist Bryostatin 1 (6), das aus dem Moostierchen Bugula neritina isoliert wurde und sich als wirksam gegen verschiedene Krebsarten erwiesen hat. Der Wirkmechanismus von Bryostatin 1 auf molekularer Ebene ist bisher nicht vollständig verstanden. Es induziert verschiedene zelluläre Antworten, einschließlich der Stimulation des Immunsystems und der Induktion von Apoptose in Tumorzellen. Einige dieser Effekte beruhen auf der Wechselwirkung des Naturstoffs mit 
verschiedenen Proteinkinase $\mathrm{C}$ Isoenzymen. ${ }^{12}$ Bryostatin 1 befindet sich zurzeit in 50 verschiedenen klinischen Studien, unter anderem zur Behandlung von Melanomen, Leukämie sowie Brust- und Lungenkarzinomen. Dabei hat sich gezeigt, dass die besten Ergebnisse bei einem kombinierten Einsatz mit anderen Krebstherapeutika erzielt werden. ${ }^{13}$

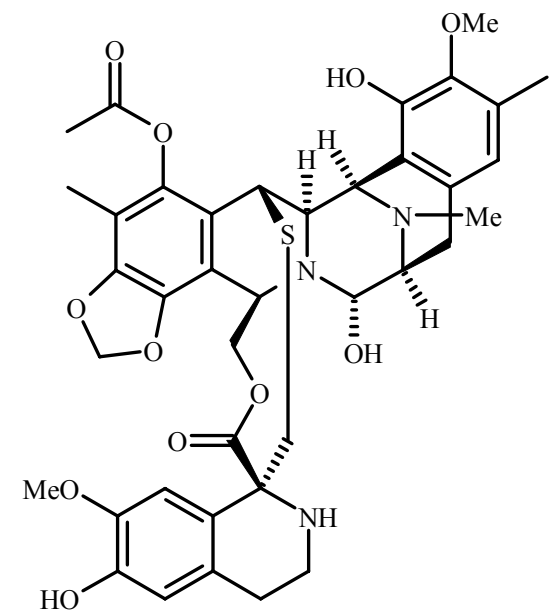

5

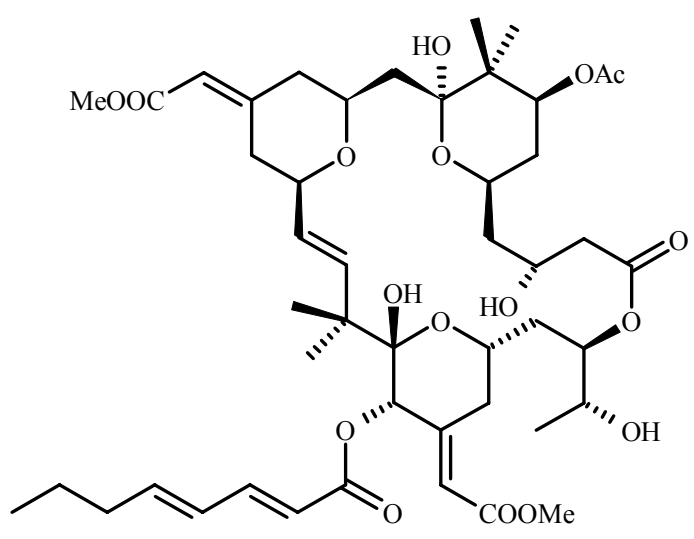

6

In der Mehrzahl der Fälle ist die Beschaffung ausreichender Substanzmengen mariner Naturstoffe mit enormen Schwierigkeiten verbunden. So werden aus $1000 \mathrm{~kg}$ Moostierchen nur $1.5 \mathrm{~g}$ Bryostatin 1 erhalten. Die komplexe Struktur des Naturstoffes verhindert einen ökonomisch vertretbaren Zugang durch Totalsynthese. Zurzeit werden Struktur-WirkungsBeziehungen an vereinfachten synthetischen Bryostatin-Analoga durchgeführt. ${ }^{14}$

Eine andere Möglichkeit um die Beschaffungsprobleme beim Bryostatin 1 (6) zu lösen, wurde von M. HAYGOOD aufgezeigt. Mit Hilfe von Gensonden konnte sie nachweisen, dass das Moostierchen Bugula neritina ein symbiotisches $\gamma$-Proteobakterium "Candidatus Endobugula sertula" enthält, welches für die Produktion von 6 verantwortlich gemacht wird. ${ }^{15}$ Tatsächlich gelang kürzlich die Isolierung eines $65 \mathrm{~kb}$ großen Genclusters, der für eine modulare Polyketidsynthase mit über dreißig katalytischen Domänen kodiert. Erste Analysen legen die Vermutung nahe, dass der Gencluster für die Bildung des Bryostatingrundgerüstes verantwortlich ist. ${ }^{16}$ Falls es gelingt, diesen Gencluster heterolog zu expremieren, könnten größere Mengen an Bryostatin 1 durch Fermentation gewonnen werden. 


\section{Sind Mikroorganismen die wahren Produzenten mariner Naturstoffe?}

Wirbellose marine Organismen, wie z. B. Schwämme, die als Quelle von pharmakologisch aktiven Naturstoffen in Frage kommen, leben in komplexen, bis heute kaum verstandenen Lebensgemeinschaften, unter Beteiligung diverser Mikroorganismen, die zum Teil einen beträchtlichen Anteil an der Biomasse ihrer Wirte ausmachen können. Daher ist die Vermutung nahe liegend, dass solche assoziiert lebenden Mikroorganismen durch eigenständige Biosyntheseleistungen zum Metabolitenspektrum des gesamten Organismus beitragen. $^{17}$

In der Tat erscheint diese Hypothese glaubhaft, wenn man die auffallende strukturelle Analogie zwischen Naturstoffen mariner Invertebraten und mikrobiellen Sekundärstoffen betrachtet. Dies führte zu der Vermutung, dass einige der aus Invertebraten isolierten Naturstoffe, wie beim Bryostatin-Produzenten Bugula neritina gezeigt, in Wahrheit von Mikroorganismen produziert werden. So besitzt das bereits im vorherigen Kapitel erwähnte Ecteinascidin 743 (5) große Ähnlichkeit zu den von Mikroorganismen gebildeten Saframycinen und Safracinen. Diese strukturelle Ähnlichkeit nutzt man für die semisynthetischen Produktion von 5 ausgehend von Safracin B (7), welches von Pseudomonas fluorescens gebildet wird, aus. Durch diese Methode konnte von 5 eine für die klinische Erprobung ausreichende Menge hergestellt werden. ${ }^{18}$ Unterstützt wird die Vermutung, dass 5 von Mikroorganismen gebildet wird, durch die Tatsache, dass mit den Renieramycinen, wie Renieramycin E (8), weitere den Safracinen ähnliche Verbindungen aus Schwämmen der Gattung Reniera isoliert werden konnten.

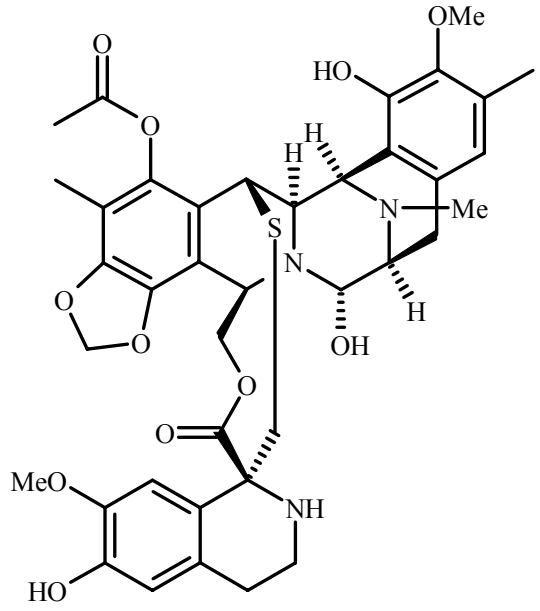

5

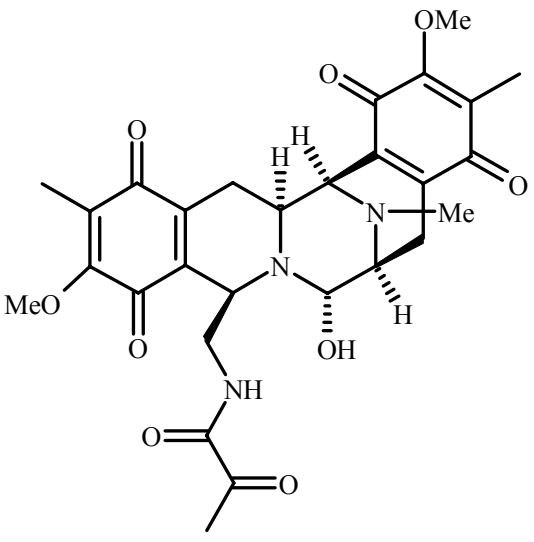

7

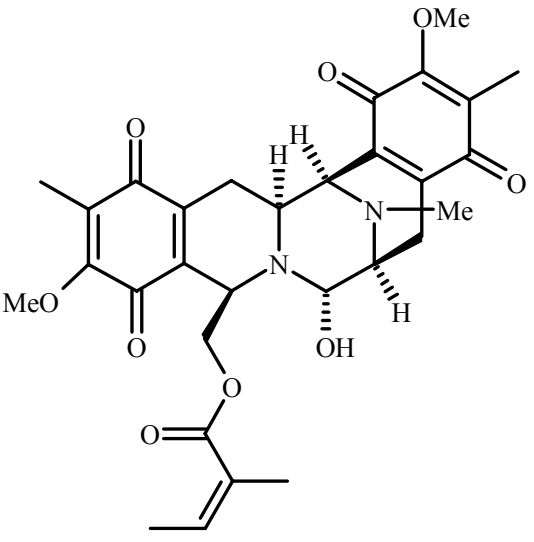

8 
Ein weiteres Beispiel, das die Bedeutung von assoziierten Mikroorganismen für die Naturstoffbiosynthese in marinen Invertebraten belegt, ist der Schwamm Theonella swinhoei, der im Pazifik und im Indopazifik vorkommt und das cyclische Peptid Theopalauamid (9) sowie das Makrolid Swinholid A (10) enthält. Während Theopalauamid das Wachstum von Pilzen inhibiert, zeichnet sich Swinholid A durch starke cytotoxische Aktivität aus. Nach mechanischem Aufschluss des Schwammgewebes konnten mittels differentieller Zentrifugation verschiedene Zellfraktionen erhalten werden, die aus schwammassoziierten Bakterien bzw. aus Schwammzellen bestanden. In einer Fraktion, die hauptsächlich filamentöse Bakterien aufwies, wurde das Theopalauamid als Hauptkomponente detektiert, während sich das Swinholid A in einer anderen Fraktion wiederfand, die vornehmlich unizelluläre Bakterien enthielt. ${ }^{19}$ Die Theopalauamid produzierenden Bakterien sind mittlerweile mittels 16S rRNA Gensequenzierung als $\delta$-Proteobakterien identifiziert worden. ${ }^{20}$ Versuche zur Kultivierung dieses Bakteriums schlugen bislang allerdings fehl.
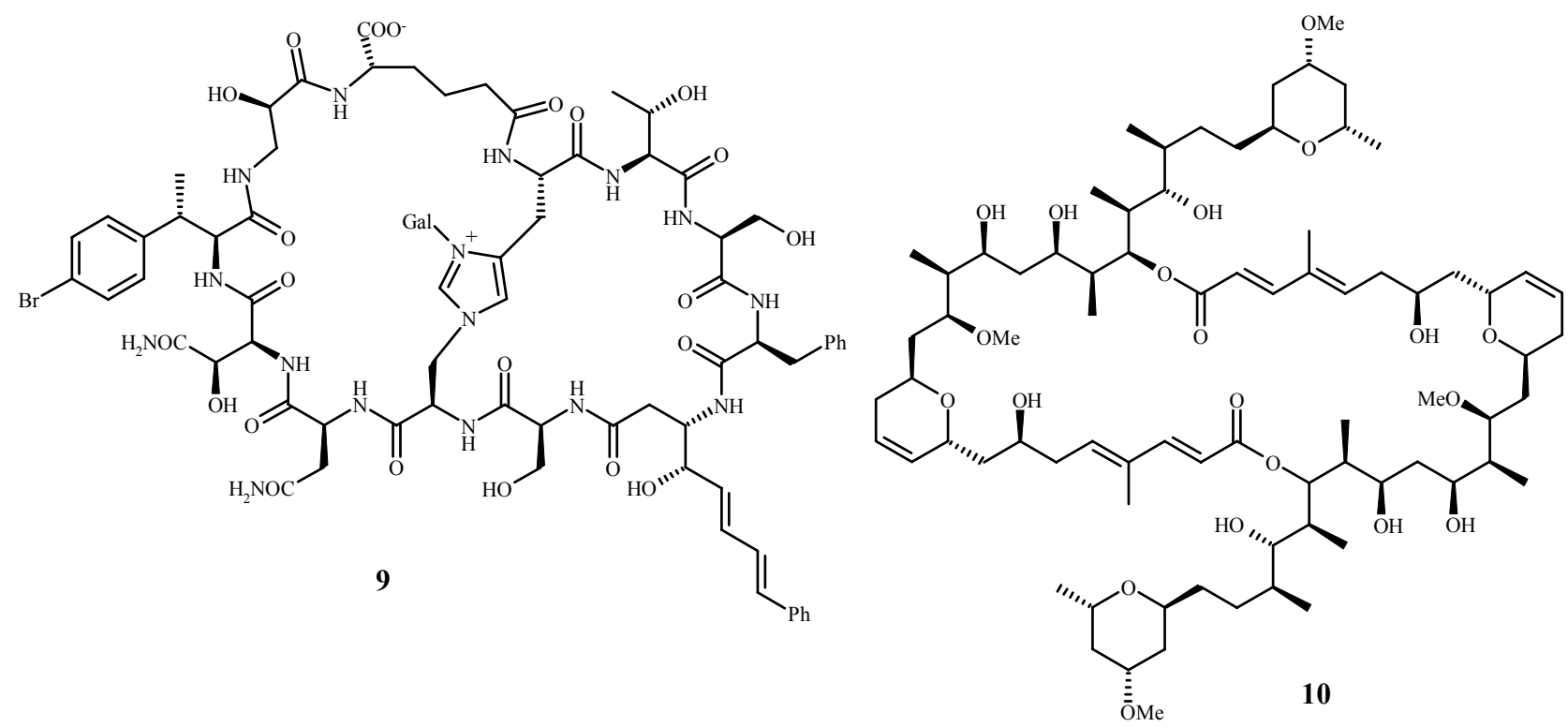

Das Polyketid Pederin (11) ist eine cytotoxische Verbindung, deren Derivate sowohl in verschiedenen Schwammgattungen zu finden sind, als auch in Käfern der Gattungen Paederus und Paederidus. Die Käfer nutzen Pederin als chemische Waffe, um sich und ihre Nachkommen vor Fraßfeinden zu schützen. ${ }^{21}$ Der Abwehrstoff wird nur in den weiblichen Käfern gebildet und bei der Eiablage auf das Gelege übertragen - was nicht nur die Eier vor Feinden schützt, sondern auch die Larven der nächsten Generation. Mittlerweile konnte nachgewiesen werden, dass Bakterien für die Pederin-Biosynthese in den Käfern verantwortlich sind. So konnte ein $100 \mathrm{~kb}$ großer Genombereich isoliert werden, der acht Gene enthält - wie man es für die Pederin-Biosynthese erwartet. 
Um Einblicke in die Biologie des Produzenten zu erhalten, wurden auch Gene, die außerhalb des für die Pederin-Biosynthese verantwortlichen Genclusters liegen, sequenziert. Die meisten dieser Gene, insbesondere solche des primären Metabolismus, besitzen hohe Homologie zu Genen des Bakteriums Pseudomonas aeruginosa. ${ }^{22} \mathrm{Ob}$ auch die zur Pederin-Klasse gehörenden Schwammmetabolite Mycalamid $A^{23}$ (12) und Onnamid $A^{24}$ (13) von Bakterien gebildet werden, kann bisher allerdings nur vermutet werden.

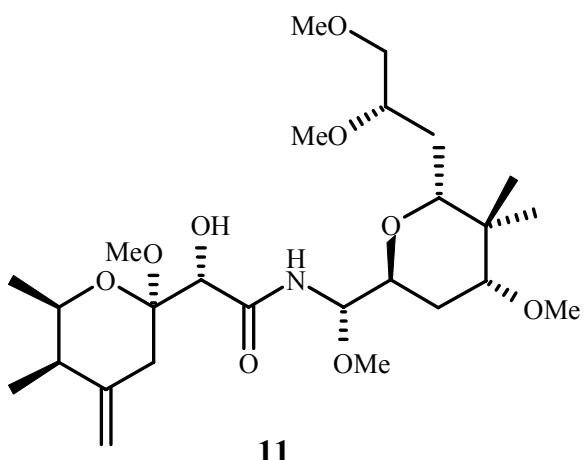<smiles>C=C1C[C@](OC)([C@H](O)C(=O)N[C@H]2OCO[C@H]3[C@@H]2O[C@H](C[C@H](O)CO)C(C)(C)[C@H]3OC)O[C@H](C)[C@@H]1C</smiles>

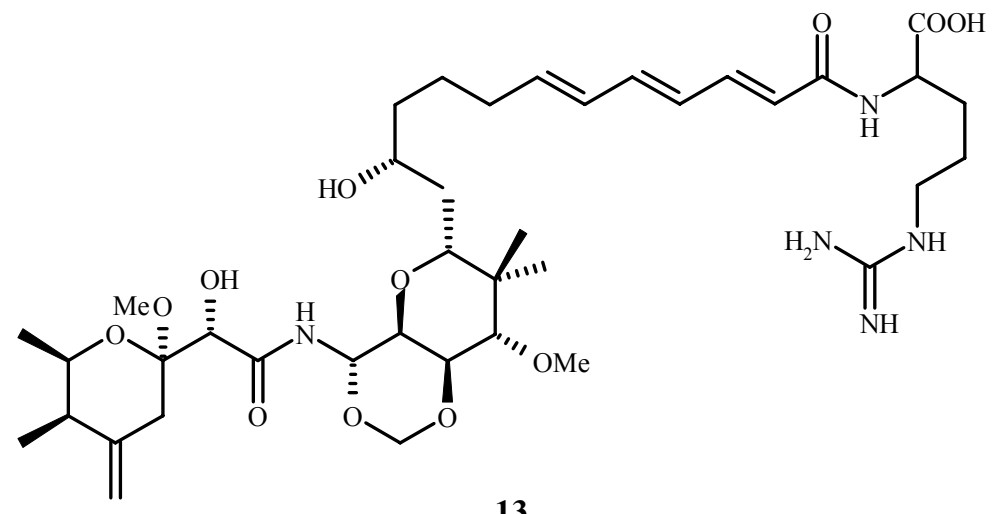

13

Wenn es gelingt, die für die Produktion mariner Naturstoffe verantwortlichen Mikroorganismen zu kultivieren oder ihr Biosynthesepotential durch genetische Methoden zu erschließen, könnten die für die präklinische und klinische Prüfung sowie im Falle einer späteren Anwendung als Medikament erforderlichen Substanzmengen durch Fermentation gewonnen werden. Außerdem würde dies einen Beitrag zum Erhalt der durch Eingriffe des Menschen bedrohten Biodiversität der Meere leisten und eine nachhaltige Nutzung biologischer Ressourcen ermöglichen. 


\section{Endophytische Pilze als Quelle neuer Naturstoffe}

Bei Pilzen ist bekannt, dass sie häufig in Symbiose mit anderen Organismen leben, sei es in Form der Lebensgemeinschaft Flechte, durch Verknüpfung mit Pflanzen über eine Mykorrhiza oder als Endosymbionten von Pflanzen und Tieren. Als endosymbiontisch werden Pilze bezeichnet, die entweder die Wirtszellen kolonialisieren oder in den Zellzwischenräumen überleben können, ohne der Pflanze dabei sichtbaren Schaden zuzufügen. ${ }^{25}$ In nahezu jeder Pflanze können endophytische Pilze gefunden werden, wobei die Wechselwirkungen vom Parasitismus bis zur Symbiose reichen können. ${ }^{26}$

Das genaue Studium dieser Wechselwirkungen wird durch die Tatsache erschwert, dass aus einer einzigen Pflanze bis $\mathrm{zu}$ mehrere hundert verschiedene endophytische Pilze isoliert werden können. ${ }^{27}$ Unter den isolierten Pilzen befinden sich immer auch ubiquitär verbreitete Arten wie Fusarium sp., Penicillium sp. oder Aspergillus sp., bei denen nicht davon ausgegangen werden kann, dass es sich um wirtspezifische Symbionten handelt.

Bei verschiedenen Gräsern konnte gezeigt werden, dass sie nach Infektion mit bestimmten Endophyten über eine gesteigerte Resistenz gegen Trockenheit verfügen. ${ }^{28}$ Auch werden von endophytischen Pilzen Substanzen gebildet, die ihren Wirt vor Fraßfeinden ${ }^{29}$ und pathogenen Pilzen und Bakterien ${ }^{30,31}$ schützen. In der Literatur sind zahlreiche Metabolite aus Endophyten beschrieben worden, die antibakteriell, antifungisch oder insektizid wirksam sind. ${ }^{32}$ Dazu gehören die antifungisch wirksame Ambuic $\operatorname{acid}^{33}$ (14), die Tetramsäure Cryptocin (15), die eine antimycotische Aktivität gegen den Reisschädling Pyricularia oryzae aufweist ${ }^{34}$ oder das immunsupressive Subglutinol $\mathrm{A}^{35}$ (16).
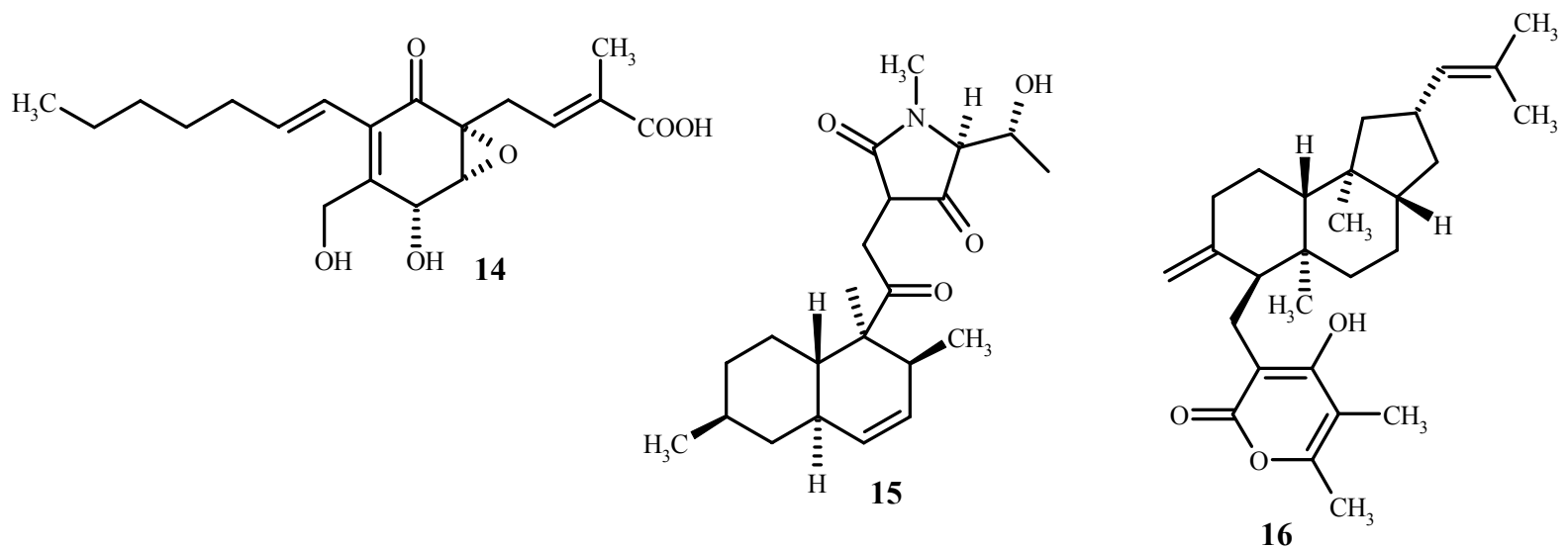

Große Beachtung fand 1993 die Isolierung von Paclitaxel (17) aus dem endophytischen Pilz Taxomyces andreanae. ${ }^{36}$ Paclitaxel, der Wirkstoff des Cytostatikums Taxol, das durch 
Stabilisierung der Mikrotubuli die Zellteilung verhindert, wurde zuerst aus der Rinde der pazifischen Eibe Taxus brevifolia isoliert und wird seit 1982 als Cytostatikum klinisch eingesetzt. ${ }^{37} \mathrm{Da}$ die Rinde der Eibe nur begrenzte Mengen Paclitaxel enthält, gewinnt man Taxol zur Zeit semisynthetisch aus der Vorstufe Baccatin III, die aus den nachwachsenden Teilen (Nadeln, Triebe) verschiedener Eibenarten stammt.

Mit der Isolierung von 17 aus T. andreanae wurde die Hoffnung verbunden, Paclitaxel durch Fermentation gewinnen zu können. Allerdings werden von dem Pilz nur geringe Mengen Paclitaxel (20 - 50 ng/L) gebildet, die auch nach Optimierung der Ausbeute auf $0.2-0.3 \mu \mathrm{g} / \mathrm{L}$ für eine kommerzielle Nutzung nicht ausreichend sind. In den folgenden Jahren konnten auch aus anderen Pflanzenarten weitere Paclitaxel produzierende Endophyten isoliert werden, ${ }^{38}$ die 17 aber auch nur in sehr geringen Ausbeuten bilden. Bei einem dieser endophytischen Pilze konnte die Produktion von 17 durch die Zugabe verschiedener Substanzen wie Benzoesäure oder Serinol induziert werden. ${ }^{39} \mathrm{Ob}$ in Zukunft eine effizientere mikrobiologische Darstellung von Taxol z. B. durch die Zugabe bestimmter Aktivatoren möglich sein wird bleibt abzuwarten.

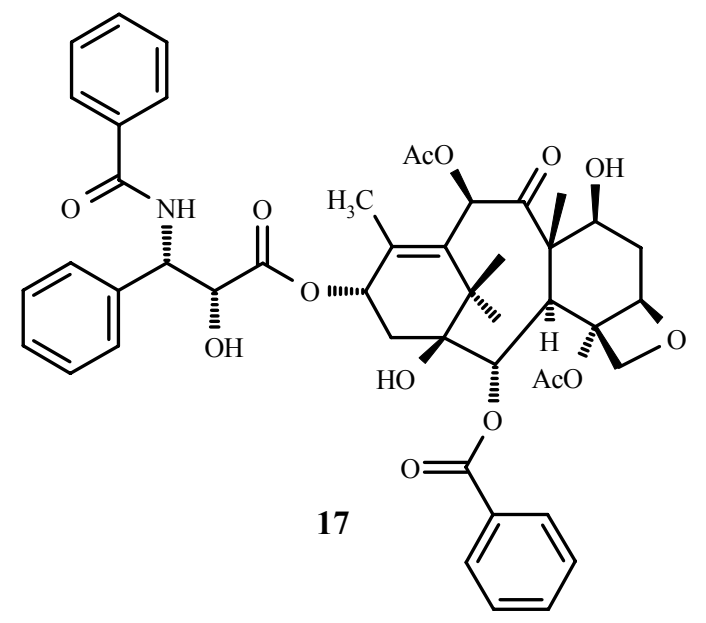

Diese Beispiele zeigen, dass endophytische Mikroorganismen ein großes Potential für die Bildung biologisch aktiver Naturstoffe besitzen. Trotzdem bleibt in den meisten Fällen unklar ob die von Endophyten unter Laborbedingungen produzierten Substanzen auch in der Natur gebildet werden. Es ist aber durchaus vorstellbar, dass Endophyten ihre Wirtspflanze durch die Bildung bioaktiver Metabolite vor Pathogenen oder Fraßfeinden schützen und sich so ihre biologische Nische erhalten. Leider ist bisher wenig über die molekulare Wechselwirkung zwischen mikrobiellen Endophyten und Pflanzen bekannt. Ein besseres Verständnis könnte die gezielte Suche nach neuen Wirkstoffen ermöglichen, da die zu untersuchenden Organismen nach rationalen Kriterien ausgewählt werden könnten. 


\section{Projektbeschreibung}

Die vorliegende Arbeit wurde im Rahmen des BMBF-Projektes „Endophytische Algen und Pflanzen verschiedener Meeresbiotope“ (FKZ 03F0360A) durchgeführt. Dieses Projekt basiert auf einer Kooperation dreier Hochschularbeitsgruppen unter der Federführung der BASF AG. Ziel des Projektes ist es, neue Naturstoffe, die sich für den Einsatz im Pflanzenschutz eignen oder Ausgangspunkt für Leitstrukturvariationen sein können, zu identifizieren.

Das die Arbeitsgruppe ZEECK betreffende Teilprojekt „Strukturaufklärung von Sekundärmetaboliten aus endophytischen Pilzen mariner Biotope und Metabolitenvariation unter veränderten Kultivierungsbedingungen (OSMAC-Ansatz)“ hatte die Erschließung neuer Naturstoffe aus einer originellen Quelle zum Ziel.

Die Isolierung der endophytische Pilze aus Algen und Pflanzen verschiedener Meeresbiotope erfolgte im Arbeitskreis AUST (TU Braunschweig, Frau Dr. Schulz). Kultiviert wurden die isolierten Pilze sowohl im Arbeitskreis AUST als auch im Arbeitskreis ZEECK. Die BASF AG übernahm die Prüfung der bereitgestellten Extrakte und Reinsubstanzen auf fungizide, insektizide und herbizide Wirkung. Im Test auffällige Pilzstämme wurden in Braunschweig bzw. Göttingen im größeren Maßstab kultiviert. Aus den Rohextrakten wurden dann im Arbeitskreis KROHN (Universität Paderborn) und im Arbeitskreis ZEECK die aktiven Komponenten isoliert und deren Wirkung kontrolliert.

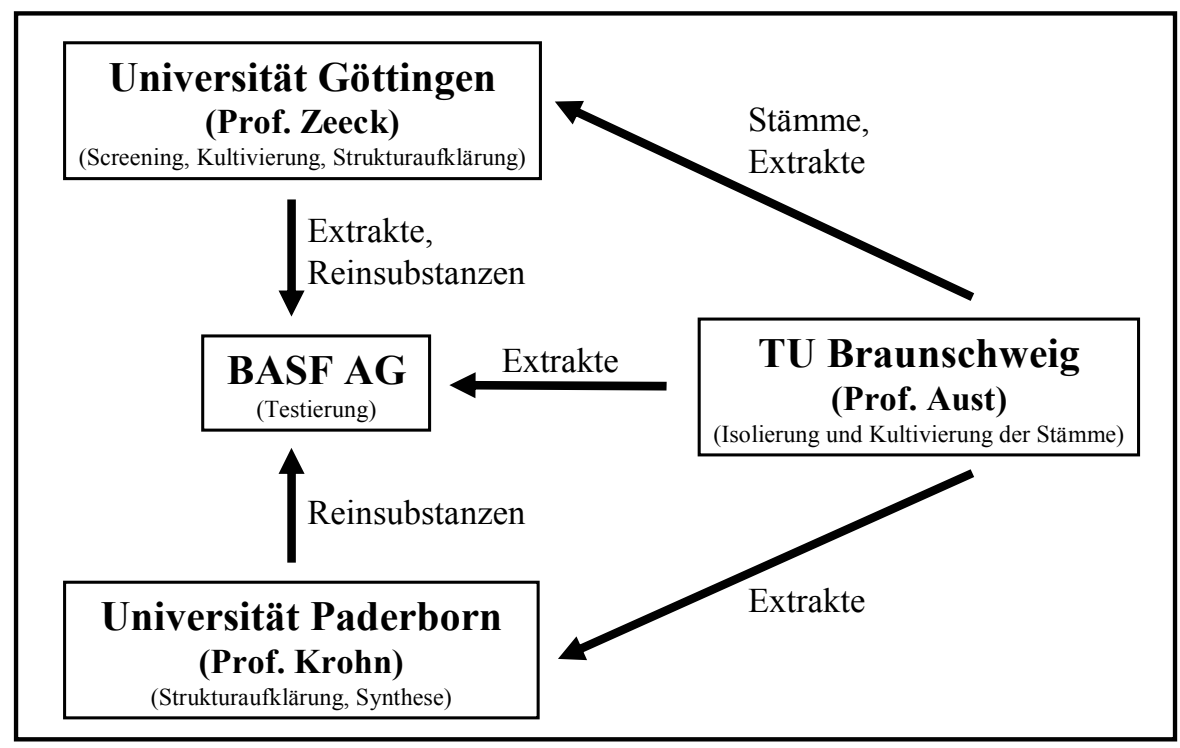

Abbildung 1: Kooperationen im BMBF-Projekt. 


\section{Aufgabenstellung}

Im Mittelpunkt dieser Arbeit steht die Untersuchung von endophytischen Pilzen aus marinen Biotopen hinsichtlich ihrer Sekundärstoffbildung.

Zunächst waren die Pilzstämme zu bearbeiten, die im Rahmen des Projektes durch ihre Aktivität im Screening auffielen. Mit Hilfe chromatographischer Trennverfahren sollten die aktiven Komponenten isoliert und durch moderne spektroskopische Methoden in ihrer Struktur aufgeklärt werden.

Um Engpässe in der Anfangsphase zu überbrücken und die Suche nach biologisch aktiven Mikroorganismen zu beschleunigen, sollten ausgewählte Pilzstämme auch im Chemischen Screening untersucht und die dabei gewonnenen Extrakte bei der BASF AG getestet werden. Stämme, die sich durch ein auffälliges Metabolitenmuster oder biologische Aktivität auszeichneten, sollten in größerem Maßstab kultiviert werden, um so ausreichende Rohproduktmengen für die Isolierung zu erhalten.

Um die Substanzbasis zu verbreitern, sollten aus zwei selbst gesammelten Algenproben endophytische Pilze isoliert und somit die Isolierungsmethodik erlernt und angewendet werden. Auf diesem Weg erhaltene Pilzstämme wurden in das Test und Isolierungsverfahren des Projektes eingebunden. Hier stand jedoch aus Zeitgründen das chemische Screening am Anfang und die Bedienung der Testsysteme war nachgeschaltet.

Bei Stämmen mit interessantem Metabolitenmuster sollte durch Variation der Kultivierungsbedingungen (OSMAC-Methode ${ }^{40}$ ) die biosynthetische Leistungsfähigkeit der Produzenten überprüft werden. Außerdem galt es, bei neuen Sekundärmetaboliten auch die Biosynthese durch Fütterung isotopenmarkierter Verbindung zu untersuchen.

Alle isolierten Reinsubstanzen sollten je nach verfügbarer Menge von der BASF AG auf die mögliche Anwendung im Pflanzenschutz hin vertieft geprüft werden. Parallel dazu sollten die antimikrobielle Wirkung der Substanzen ermittelt werden.

Am Ende sollte der eingeschlagene Weg zur Auffindung neuer Naturstoffe bzw. neuer Wirkstoffe bewertet und in den Gesamtzusammenhang der Wirkstoffsuche eingeordnet werden. 


\section{Herkunft und Screening der endophytischen Pilzen}

\section{Screening der endophytischen Pilze}

\subsection{Screeningmethoden}

Bei der Suche nach neuen Naturstoffen in Extrakten von Mikroorganismen können zwei verschiedene Screeningmethoden zum Einsatz kommen. Zum einen kann anhand einer biologischen Aktivität der Extrakte auf interessante Inhaltsstoffe geschlossen werden, zum anderen lassen sich die gebildeten Sekundärmetabolite mit physikochemischen Methoden nachweisen.

Das auf WAKSMAN zurückgehende „Biologische Screening ${ }^{641}$ zielt darauf ab, Substanzen mit bestimmten biologischen Eigenschaften $\mathrm{zu}$ finden. Dazu werden die Extrakte mit Testsystemen hinsichtlich ihrer biologischen Aktivität geprüft und die Metabolite wirkungsorientiert isoliert. Anfangs arbeitete man mit dem Plattendiffusionstest. Mit zunehmenden Verständnis von Krankheiten auf genetischer und molekularer Ebene entwickelte sich ein Target-orientiertes-Screening ${ }^{42}$, bei dem durch den Einsatz von Enzymund Rezeptorassays gezielter auf einzelne Krankheiten und pharmazeutische Anwendung getestet werden kann. In der Industrie wird diese Vorgehensweise heute im Rahmen des High-Throughput-Screenings (HTS) angewandt. ${ }^{43}$ Dieses wird mit Testrobotern, die durch den Einsatz von Mikrotiterplatten große Substanzbibliotheken oder zahlreiche Extraktproben in kürzester Zeit bewältigen können, durchgeführt. Nachteilig bei diesem Ansatz ist die Abhängigkeit von bestimmten Testsystemen, deren Relevanz bei bestimmten Krankheiten keineswegs immer geklärt ist. Interessante Sekundärmetabolite, die in den Tests nicht ansprechen, z. B. auf Grund zu geringer Wirkstoffkonzentration, werden auf diesem Weg nicht erkannt. Zudem können durch synergistische Effekte falsch positive Ergebnisse erzielt werden, was die Auswertung zusätzlich erschwert.

Im Gegensatz zur wirkungsorientierten Isolierung aktiver Substanzen im Biologischen Screening werden im „Chemischen Screening“ alle Substanzen angezeigt, die aufgrund chemischer bzw. physikochemischer Methoden nachgewiesen werden können. Dieser Ansatz wurde 1970 von UMEZAWA ${ }^{44}$ eingeführt und in den Arbeitsgruppen von ZÄHNER ${ }^{45}$ und ZEECK $^{46}$ weiterentwickelt. Bei dieser Methode werden Sekundärmetabolite durch ihr chemisches Verhalten gegenüber Sprühreagenzien oder durch UV-Aktivität sichtbar gemacht. 
Der entscheidende Vorteil des Chemischen Screenings ist dabei, dass im weiteren Verlauf die Reinsubstanzen in allen verfügbaren Testsystemen auch noch nach mehreren Jahren geprüft werden können. Auf diese Weise können auch gering konzentrierte Metabolite untersucht sowie falsch positive Testergebnisse vermieden werden.

Nachteilig am Chemischen Screening sind die nur halbautomatisierte Durchführbarkeit und der hohe Anteil nicht digitalisierbarer Daten. Außerdem werden wie beim Biologischen Screening immer wieder bereits bekannte Metabolite isoliert. Um die Wiederfindungsrate zu verringern wurde das Chemische Screening zum Physikochemischen Screening, in dem die Detektion der Metabolite mit Hilfe gekoppelter HPLC-Systeme erfolgt, weiterentwickelt. Durch Einsatz von Datenbanken lassen sich bereits bekannte Metabolite anhand ihrer Retentionszeiten und physikalischen Daten frühzeitig erkennen. Methodisch kommen hier die gekoppelten Methoden HPLC-DAD ${ }^{47}$, HPLC-MS ${ }^{48,49}$ und HPLC-NMR ${ }^{50}$ zum Einsatz.

Um die Vorteile sowohl des Biologischen als auch des Chemischen Screenings auszunutzen, wurden in den letzten Jahren kombinierte Systeme entwickelt. Bei diesen erfolgt parallel zu einer HPLC-Trennung mit physikochemischen Detektoren die biologische Testierung. Tritt eine biologische Aktivität auf, so lassen sich dieser z. B. UV- und MS-Daten zuordnen, was die Isolierung der für die Wirkung verantwortlichen Substanz erleichtert. Auch können die gewonnenen Daten mit Datenbanken abgeglichen werden, um bekannte Substanzen bereits frühzeitig zu identifizieren. In der Anwendung ist z.B. ein mit UV- und Massendetektion gekoppeltes HPLC-System, bei dem es gleichzeitig gelingt auf Hemmung der Acetylcholinesterase zu prüfen. ${ }^{51}$

\subsection{Durchführung des chemisch-biologischen Screenings}

Um die Potenz der Sekundärstoffbildung zu prüfen, wurden die Pilzstämme im Chemischen Screening in fünf verschiedenen Medien (1158, E2, G20, 1549 und SGG) in Schüttelkolben kultiviert und die Kulturbrühen standardisiert aufgearbeitet (siehe Abbildung 2). Die Analyse des Metabolitenmusters erfolgte über visuelle Detektion bei farbigen Substanzen, UV-Absorption/Fluoreszenz bei 254 und 366 nm, Farbreaktionen mit unterschiedlichen Sprühreagenzien und Laufverhalten auf Dünnschichtchromatogrammen. Mit Anisaldehyd, Ehrlich's Reagenz und Orcin als Sprühreagenzien wird gewährleistet, dass möglichst viele unterschiedliche funktionelle Gruppen sichtbar gemacht werden können. So lassen sich z. B. mit Anisaldehyd Steroide und höhere Alkohole, mit Ehrlich's Reagenz Amine und mit Orcin Zucker nachweisen. ${ }^{52}$ 


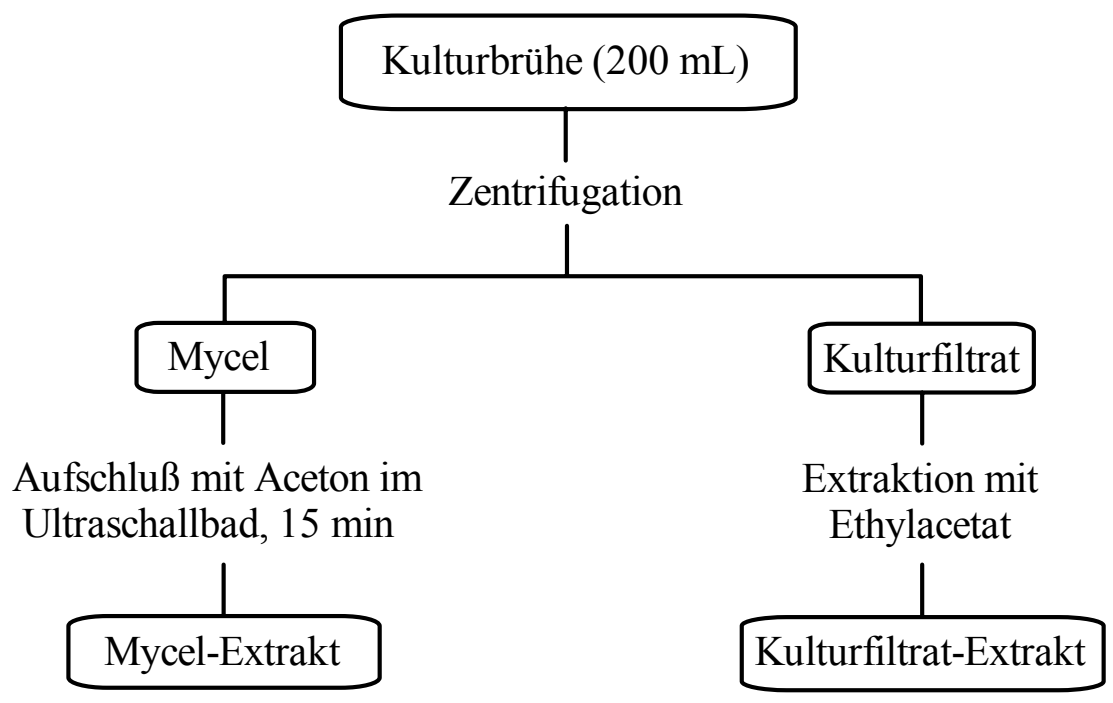

Abbildung 2: Aufarbeitungsschema der endophytischen Pilze.

Pilze die in diesem primären Screening durch ein interessantes Metabolitenmuster auffielen, wurden zusätzlich unter Zusatz von Natriumchlorid (33 g/L) kultiviert, um zu testen, in wie weit sie an das marine Habitat angepasst waren. Außerdem wurden bei auffälligen Pilzstämmen Ruhefermentationen in P-Kolben mit fünf verschiedenen Medien (1158, E2, G20, 1549 und SGG) durchgeführt.

Neben den chemischen Nachweisverfahren wurden die Extrakte bei der BASF AG auf ihre biologische Aktivität hin untersucht. Geprüft wurde dabei hauptsächlich auf fungizide Aktivität im Mikrotest oder im Ganzpflanzentest (Gewächshaus) gegen die Pathogene Phytophthora infestans, Botrytis cinerea, Pyricularia oryzae und Septoria tritici. Extrakte mit auffälliger biologischer Aktivität wurden anschließend im HPLC-Mikrotest bearbeitet. Dazu wurden die Extrakte zunächst in einem standardisierten HPLC-Lauf direkt auf eine Mikrotiterplatte in 80 Fraktionen getrennt und die einzelnen Fraktionen des Extraktes im Mikrotest geprüft. Als Detektionssystem ist an der HPLC neben einem UV- auch ein Lichtstreudetektor angeschlossen, so dass auch nicht UV-aktive Substanzen erkannt werden können. Treten im Mikrotest eine oder mehrere aktive Fraktionen auf, so können diesen anhand der chromatographischen Parameter und der UV-Daten Substanzen zugeordnet werden. Ein Beispiel für eine Fraktionierung und Substanzisolierung anhand der HPLCMikrotestergebnisse ist in Kapitel A.IV.3. beschrieben. 
Die Auswahlkriterien anhand derer entschieden wurde, ob ein Pilzstamm, der im Chemischen oder Biologischen Screening auffiel, im größeren Maßstab kultiviert werden sollte, sind in Abbildung 3 dargestellt. Im Idealfall besteht eine Korrelation zwischen den Ergebnissen aus dem Chemischen und dem Biologischen Screening, aber auch bei positiven Ergebnissen aus einem der Screeningsysteme kann eine weitere Bearbeitung in Betracht gezogen werden.

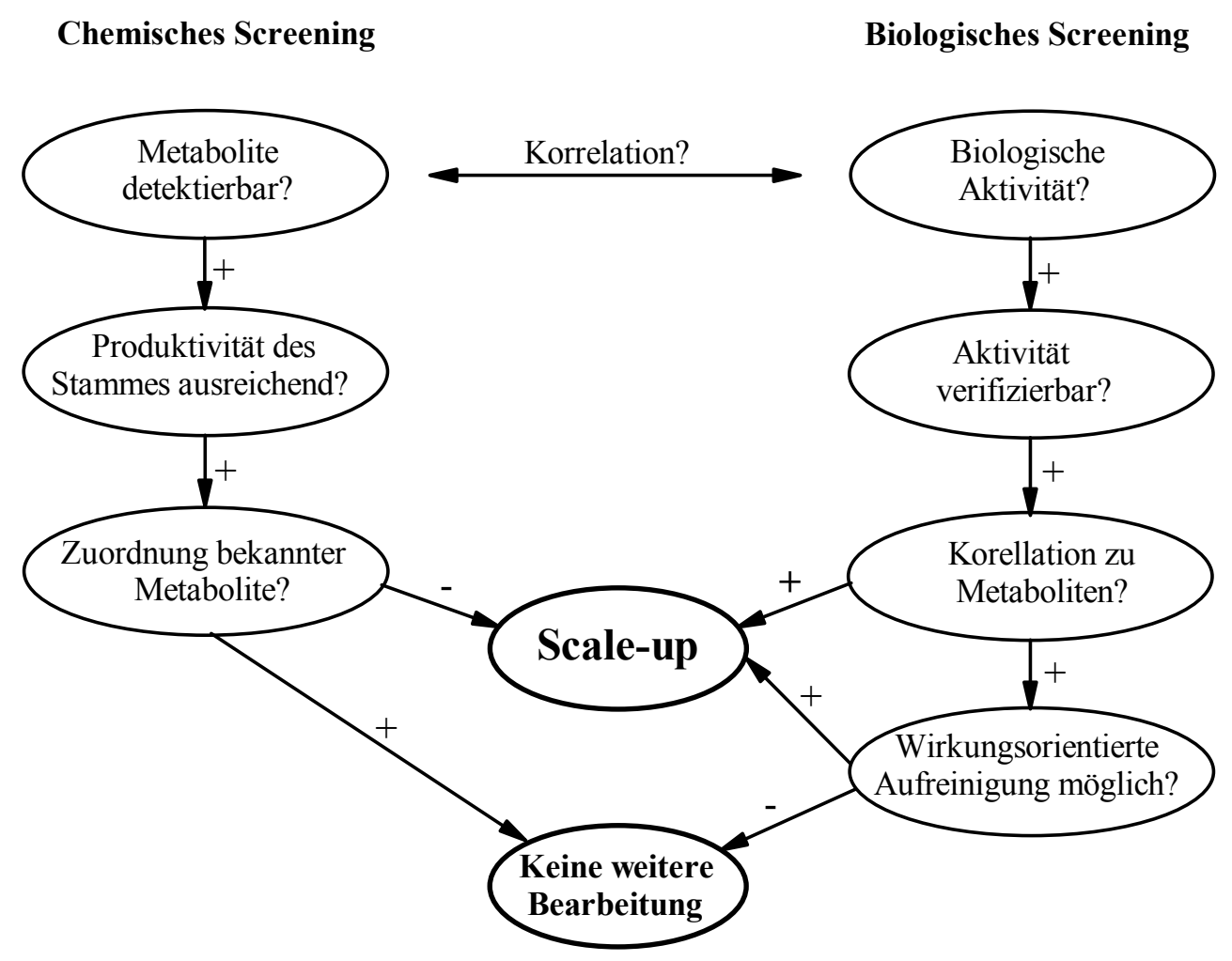

Abbildung 3: Auswahlkriterien für die weitere Bearbeitung von im Screening auffälligen Pilzen.

Um bekannte Substanzen rasch zu identifizieren, hat sich der Einsatz von Datenbanken bewährt. Sobald NMR-Spektren und/ oder Massenspektren vorlagen wurden Suchen in den Datenbanken „Dictionary of Natural Products“ (DNP, Chapman\&Hall/ CRC) ${ }^{53}$ und „Antibase“ (Chemical Concepts) ${ }^{54}$ durchgeführt. Beispiele für diese Vorgehensweise sind bei der Charakterisierung der isolierten bekannten Metabolite beschrieben. Aber auch bei neuen Substanzen können Datenbanken durch die Suche nach Aglyka bzw. Strukturfragmenten erste Hinweise auf das Grundgerüst liefern und so die Strukturaufklärung vereinfachen. 


\section{Herkunft der bearbeiteten endophytischen Pilze und Stammauswahl}

Im Chemischen Screening wurden im Rahmen dieser Arbeit insgesamt 56 Pilzstämme untersucht, deren Herkunft sich in drei Teilbereiche gliedert:

in Braunschweig isolierte endophytische Pilze:

in Göttingen isolierte endophytische Pilze:

ein Pilz aus der Göttinger Stammsammlung:
11 Stämme

44 Stämme

A6651

\subsection{In Braunschweig isolierte endophytische Pilzstämme}

Die aus Braunschweig stammenden endophytischen Pilze wurden im Arbeitskreis AUST (TU Braunschweig) aus Algen und Pflanzen mariner Biotope isoliert. Dabei sortierte man ubiquitär verbreitetete Pilzgattungen wie Alternaria, Fusarium und Aspergillus in der Regel im Rahmen einer Vorauswahl aus, da hier die Gefahr, auf bekannte Metabolite zu treffen, besonders hoch ist. In Ausnahmefällen, wenn z. B. auffällige morphologische Merkmale zu Tage traten, kamen auch als Ubiquitisten bekannte Pilze in die weitere Bearbeitung. Gemäß den Projektvorgaben sollten die isolierten Pilzstämme in Braunschweig kultiviert und Extrakte an die BASF AG zum Test abgegeben werden. Aus auffälligen Stämmen sollten die Arbeitsgruppen ZEECK (Universität Göttingen) und KROHN (Universität Paderborn) dann die für die Wirkung verantwortlichen Metabolite isolieren.

Der Schwerpunkt der Arbeiten bei uns sollte dabei in der Strukturaufklärung sowie in der Verbreiterung des Metabolitenprofils interessanter Pilzstämme durch Anwendung des OSMAC-Ansatzes liegen. Für die Isolierung neuer Substanzen sollte hauptsächlich auf in Braunschweig erstellte Rohextrakte zurückgegriffen werden.

Da zu Beginn des Projektes noch keine positiven Testergebnisse vorlagen und wir aus Braunschweig kaum Rohextrakte erhielten, wurden, um die Suche nach biologisch aktiven Mikroorganismen zu beschleunigen, elf der in Braunschweig isolierten endophytischen Pilze im Chemischen Screening untersucht. Sechs dieser Stämme zeigten ein auffälliges Metabolitenmuster und kamen in die weitere Bearbeitung (siehe Kapitel A.III.). Eine Übersicht über die aus Braunschweig erhaltenen Stämme und die mit diesen erzielten Ergebnisse gibt Tabelle 1. 


\begin{tabular}{|c|c|c|c|}
\hline $\begin{array}{l}\text { Stamm- } \\
\text { Nr. }\end{array}$ & Taxon & Herkunft & isolierte Substanzen \\
\hline 6288 & Microsphaeropsis sp. & $\begin{array}{c}\text { Ceramium sp., } \\
\text { Ostsee bei Travemünde }\end{array}$ & $\begin{array}{c}2 \text { Spiciferone }(\mathbf{1 7} \text { und } \mathbf{1 8}) \\
4 \text { a-Pyrone }(\mathbf{2 2}-\mathbf{2 5}) \\
4 \text { Betaenone }(39-\mathbf{4 2}) \\
\text { 3 Solanapyrone }(\mathbf{4 8}, \mathbf{4 9}, \mathbf{5 1})\end{array}$ \\
\hline 6303 & Ascotricha chartarum & $\begin{array}{c}\text { Fucus sp., } \\
\text { Nordsee bei Cuxhaven }\end{array}$ & Ergosterol (74) \\
\hline 6325 & nicht sporulierend & $\begin{array}{c}\text { Fucales serratus } \\
\text { Mittelmeer, Mallorca }\end{array}$ & $\begin{array}{l}2 \text { Furane ( } 75 \text { und } 76) \\
\text { Phenylessigsäure }(77)\end{array}$ \\
\hline 6587 & $\begin{array}{l}\text { Alternaria sp. } \\
\text { (großsporig) }\end{array}$ & $\begin{array}{l}\text { Enteromorpha linza, } \\
\text { Ostsee bei Ahrenshop }\end{array}$ & identisch mit Stamm 6588 \\
\hline 6588 & Alternaria sp. & $\begin{array}{l}\text { Enteromorpha linza, } \\
\text { Ostsee bei Ahrenshop }\end{array}$ & $\begin{array}{c}\text { Alternariol }(\mathbf{5 5}) \\
\text { Alternariol-9-methylether }(\mathbf{5 6}) \\
3 \text { Perylenchinone }(\mathbf{5 7}-\mathbf{5 9}) \\
\text { Sphaerolon }(\mathbf{7 0}) \\
3 \text { Naphtalene }(\mathbf{6 4}, \mathbf{6 5}, \mathbf{6 9}) \\
\end{array}$ \\
\hline 6615 & Phomopsis sp. & $\begin{array}{c}\text { Sanddorn, } \\
\text { Ostsee bei Ahrenshop }\end{array}$ & $\begin{array}{c}\text { 2-Phenylethanol (78) } \\
\text { Tryptophol (79) }\end{array}$ \\
\hline 6634 & nicht sporulierend & $\begin{array}{c}\text { Salsola kali } \\
\text { Ostsee bei Ahrenshoop }\end{array}$ & \\
\hline 6660 & Arthrinium sp. & $\begin{array}{c}\text { Artemisia vulgaris } \\
\text { Ostsee bei Ahrenshoop }\end{array}$ & \\
\hline 6747 & Ascochyta sp. & $\begin{array}{l}\text { Achillea mallifolia, } \\
\text { Ostsee bei Wustrow }\end{array}$ & \\
\hline 6768 & Diaporthe sp. & $\begin{array}{c}\text { Salix vinimalis, } \\
\text { Ostsee bei Wustrow }\end{array}$ & \\
\hline 6907 & Fusarium sp. & $\begin{array}{l}\text { Fucus vesiculosus, } \\
\text { Nordsee bei Cuxhaven }\end{array}$ & \\
\hline
\end{tabular}

Tabelle 1: Zusammenfassung der Ergebnisse für die in Braunschweig isolierten Pilzstämme.

\subsection{In Göttingen isolierte endophytische Pilzstämme}

Eine Rotalge aus einer Bucht in der Nähe von Kissamos (Kreta, Griechenland) sowie eine weitere Rotalge von der Insel Gozo (Malta) waren das Material für die Isolierung von 44 endophytischen Pilzen in Göttinger Regie. Bei den beiden Algen handelt es sich sehr wahrscheinlich um dieselbe Spezies: Gracillaria tikvahiae (Dr. ScHULz, TU Braunschweig). Um aus Algen endophytischen Pilze zu erhalten, wurde zunächst die Oberfläche nach der von B. ScHulz et al. beschriebenen Methode ${ }^{55}$ sterilisiert. Dazu wurden die Algen für 30 Sekunden in eine 70\%ige Ethanollösung getaucht und anschließend mit sterilem Wasser gewaschen. Um den Erfolg der Oberflächensterilisation zu kontrollieren, drückte man die Algen kurz auf Agarplatten, die dann inkubiert wurden. 
Die sterilisierten Algen wurden in kleine Stückchen zerschnitten und auf Agarplatten aufgebracht. Als Isolierungsmedien dienten verschiedene Medien sowohl ohne als auch mit Zusatz von Natriumchlorid (33 g/L). Um das Anwachsen von Bakterien zu verhindern, wurden den Medien jeweils $250 \mathrm{mg} / \mathrm{L}$ Penicillin G und $250 \mathrm{mg} / \mathrm{L}$ Streptomycin zugesetzt. Nach zwei bis drei Tagen waren erste Pilzkolonien zu erkennen, die mehrmals vereinzelt wurden. Nach dieser Methode konnten aus den von Kreta und Gozo stammenden Algen 11 bzw. 33 endophytische Pilze isoliert werden. Die Bestimmung der einzelnen Pilze erfolgte durch Dr. DRAEGER (Arbeitskeis AUST, TU Braunschweig) nach morphologischen Gesichtspunkten.

Die endophytischen Pilze wurden im Chemischen Screening untersucht und die Extrakte an die BASF AG zur Prüfung der biologischen Aktivität übergeben. Dabei fielen einige Extrakte im Mikrotest durch fungizide Aktivität gegen einen oder mehrere der vier Testorganismen Phytophthora infestans, Botrytis cinerea, Pyricularia oryzae und Septoria tritici auf. Eine Übersicht über die aus den beiden Algen isolierten endophytischen Pilze ist in den Tabellen 2 und 3 dargestellt.

\begin{tabular}{|c|c|c|c|}
\hline Stamm-Nr. & Taxon & $\begin{array}{c}\text { Aktivität im Mikrotest } \\
\text { (fungizid) der BASF AG }\end{array}$ & $\begin{array}{c}\text { Chemisches } \\
\text { Screening }\end{array}$ \\
\hline Gö 100/1 & Aspergillus niger & & - \\
\hline Gö 100/2 & Chaetomium sp. & PHYTIN & +++ \\
\hline Gö 100/3 & Phoma sp. & PHYTIN & + \\
\hline Gö 100/4 & Aspergillus flavus & PHYTIN & +++ \\
\hline Gö 100/5 & Aspergillus niger & & - \\
\hline Gö 100/6 & Penicillium sp. & PYRIOR & + \\
\hline Gö 100/7 & Nodulisporium sp. & PYRIOR & ++ \\
\hline Gö 100/8 & Dendryphiella sp. & PYRIOR & +++ \\
\hline Gö 100/9 & Chaetomium sp. & PHYTIN, SEPTTR & + \\
\hline Gö 100/10 & Chaetomium sp. & PYRIOR & +++ \\
\hline Gö $100 / 11$ & Chaetomium sp. & PHYTIN & + \\
\hline
\end{tabular}

Testorganismen: P. infestans (PHYTIN), B. cinerea (BORTR), P. oryzae (PYRIOR) und S. tritici (SEPTTR)

Tabelle 2: Zusammenfassung der Bearbeitung Göttinger Pilzstämme aus der bei Kreta gesammelten Alge. 


\begin{tabular}{|c|c|c|c|}
\hline Stamm-Nr. & Taxon & $\begin{array}{l}\text { Aktivität im Mikrotest } \\
\text { (fungizid) der BASF AG }\end{array}$ & $\begin{array}{l}\text { Chemisches } \\
\text { Screening }\end{array}$ \\
\hline Gö 101/1 & Aspergillus niger & & + \\
\hline Gö 101/2 & Aspergillus flavus & PHYTIN & ++ \\
\hline Gö 101/3 & $\begin{array}{l}\text { leere Ascocarpe, } \\
\text { nicht bestimmbar }\end{array}$ & & - \\
\hline Gö 101/4 & Aspergillus alliaceus & & - \\
\hline Gö 101/5 & Aspergillus niger & & - \\
\hline Gö 101/6 & Penicillium sp. & & + \\
\hline Gö 101/7 & Aspergillus flavipes & PHYTIN, PYRIOR & ++ \\
\hline Gö 101/8 & Dendryphiella sp. & & - \\
\hline Gö 101/9 & Aspergillus niger & & ++ \\
\hline Gö 101/10 & Aspergillus flavipes & PHYTIN & ++ \\
\hline Gö 101/11 & Penicillium sp. & PYRIOR & ++ \\
\hline Gö 101/12 & Dendryphiella sp. & & - \\
\hline Gö 101/13 & Penicillium sp. & PHYTIN, PYRIOR, SEPTTR & ++ \\
\hline Gö 101/14 & Aspergillus flavus & PYRIOR & +++ \\
\hline Gö 101/15 & Aspergillus niger & & ++ \\
\hline Gö 101/16 & Aspergillus melleus & & - \\
\hline Gö 101/17 & nicht sporulierend & & - \\
\hline Gö 101/18 & Fusarium sp. & & + \\
\hline Gö 101/19 & Trichoderma sp. & PHYTIN, PYRIOR & ++ \\
\hline Gö 101/20 & Aspergillus ochraceus & & - \\
\hline Gö 101/21 & Chaetomium sp. & & - \\
\hline Gö 101/22 & Aspergillus niger & & + \\
\hline Gö 101/23 & $\begin{array}{l}\text { Eurotium sp./Aspergillus } \\
\text { glaucus-Gruppe }\end{array}$ & & + \\
\hline Gö 101/24 & Dendryphiella $\mathrm{sp}$. & & - \\
\hline Gö 101/25 & Dendryphiella sp. & & - \\
\hline Gö 101/26 & Humicola grisea & PYRIOR, SEPTTR & ++ \\
\hline Gö 101/27 & Aspergillus ustus & PHYTIN, PYRIOR & + \\
\hline Gö 101/28 & Sporormiella sp. & & - \\
\hline Gö 101/29 & Drechslera $\mathrm{sp}$ & PHYTIN, PYRIOR, SEPTTR & +++ \\
\hline Gö 101/30 & Verticillium cinnabarinum & & - \\
\hline Gö 101/31 & Aspergillus versicolor & & + \\
\hline Gö 101/32 & Dendryphiella sp. & & - \\
\hline Gö 101/33 & nicht sporulierend & & - \\
\hline
\end{tabular}

Testorganismen: P. infestans (PHYTIN), B. cinerea (BORTR), P. oryzae (PYRIOR) und S. tritici (SEPTTR)

Tabelle 3: Zusammenfassung der Bearbeitung Göttinger Pilzstämme aus der bei Gozo gesammelten Alge.

Durch die eigenständige Isolierung endophytischer Pilze gab es mehr Pilzstämme deren Inhaltsstoffe im Chemischen Screening und/ oder durch eine biologische Aktivität auffielen. Dies ermöglichte es Ranglisten zu erstellen und gezielt besonders interessante Stämme zu bearbeiteten. Die zum Einsatz kommenden Auswahlkriterien wurden bereits im Kapitel A.I.1. dargelegt, besonders wichtig war dabei die Korrelation zwischen biologischer Aktivität 
und Chemischen Screening. Außerdem befanden sich unter den isolierten Stämmen auch ubiquitär verbreitetete Pilze wie Aspergillus sp. und Penicillium sp., die nur bearbeitet wurden, wenn die biologische Aktivität durch einen HPLC-Mikrotest bestätigt werden konnte. Durch dieses Vorgehen sollte eine gezielte Isolierung der aktiven Komponenten ermöglicht werde.

Das Metabolitenmuster der in Göttingen isolierten endophytischen Pilze wird in Kapitel A.IV. beschrieben. Eine Übersicht über die bearbeiteten Stämme und die mit diesen erzielten Ergebnisse gibt Tabelle 4.

\begin{tabular}{|c|c|c|c|}
\hline $\begin{array}{c}\text { Stamm- } \\
\text { Nr. }\end{array}$ & Taxon & Herkunft & isolierte Substanzen \\
\hline Gö 100/2 & Chaetomium sp. & Alge von Kreta & Chaetocyclinone A - C (82-84) \\
\hline Gö 100/4 & Aspergillus flavus & Alge von Kreta & $\begin{array}{c}\text { Kojic acid }(\mathbf{9 1}) \\
2 \text { Miyakamide }(\mathbf{9 2} \text { und } 93) \\
\text { Arthrographol }(\mathbf{9 6}) \\
\text { Arthrolacton }(\mathbf{9 8})\end{array}$ \\
\hline Gö 100/9 & Chaetomium sp. & Alge von Kreta & 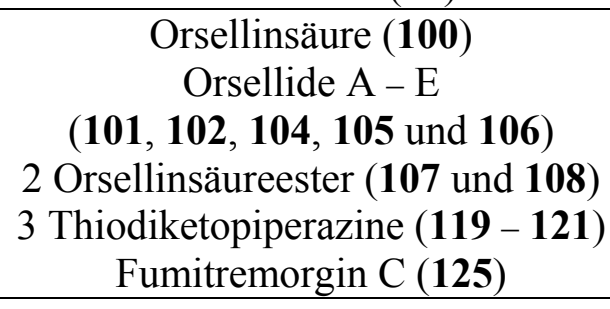 \\
\hline Gö 101/19 & Trichoderma sp. & Alge von Gozo & Sorbicillin $(\mathbf{1 2 6})$ \\
\hline Gö 101/26 & Humicola grisea, & Alge von Gozo & $\begin{array}{l}\text { 6-Methoxysterigmatocystin (127) } \\
\text { Fuscoatrosid (128) } \\
\end{array}$ \\
\hline
\end{tabular}

Tabelle 4: Zusammenfassung der Ergebnisse für die in Göttingen isolierten Pilzstämme.

\subsection{Der Stamm A6651}

Der aus der Göttinger Stammsammlung stammende Pilz A6651 wurde zu Beginn dieser Doktorarbeit bearbeitet, da noch keine im Rahmen des BMBF-Projektes isolierten Endophyten für Untersuchungen zur Verfügung standen. Im Rahmen einer Kooperation mit der Firma Hoechst (heute: Sanofi-Aventis) konnte S. PHILIPS Dihydrobipolaroxin (129) aus Kulturen des Stammes isolieren. In der vorliegenden Doktorarbeit sollte untersucht werden, ob der Stamm A6651 durch Variation der Fermentationsbedingungen zur Produktion weiterer Sekundärmetabolite angeregt werden kann. Das Metabolitenmuster dieses Stammes wird in Kapitel A.V.1. beschrieben. 


\section{Sekundärmetabolite in Braunschweig isolierter endophytischer Pilze}

\section{Microsphaeropsis sp. Stamm 6288}

Der Pilz Microsphaeropsis sp. Stamm 6288 wurde aus einer bei Travemüde in der Ostsee gesammelten Rotalge Ceramium sp. isoliert. Im chemischen Screening fiel dieser Stamm bei der Kultivierung in Schüttelkultur im Medium 1549 durch die Bildung einer gelben bei UVLicht (366 nm) stark fluoreszierenden Verbindung auf. Aus einer 2 L-Fermentation konnten neben der fluoreszierenden Substanz (18) noch 17 isoliert werden. Um das Biosynthesepotential dieses Stammes weiter $\mathrm{zu}$ erschließen, wurden Kultivierung in Ruhekultur (P-Kolben) durchgeführt, bei denen besonders die Bildung von drei bei $254 \mathrm{~nm}$ UVlöschenden Zonen auffiel, die mit Anisaldehyd blau anfärbten. Interessanterweise konnte die Ausbeute der zugehörigen Metabolite durch den Zusatz von Natriumchlorid (33 g/L) deutlich gesteigert werden.

In Abhängigkeit von den Kultivierungsvariationen ließen sich insgesamt dreizehn verschiedene Sekundärmetabolite isolieren, von denen nur zwei in der Literatur beschrieben waren. Die isolierten Sekundärmetabolite gehören vier verschiedenen Substanzklassen an: den Spiciferonen (17 und 18), den $\alpha$-Pyronen (22 - 25), den Betaenonen (39-42) und den Solanapyronen (48, 49 und 50). Eine Übersicht über die Ausbeuten der isolierten Substanzen aus den verschiedenen Kultivierungsansätzen gibt Tabelle 5.

\begin{tabular}{|c|c|c|c|c|c|c|}
\hline & $\begin{array}{c}\text { SK } \\
(1549)\end{array}$ & $\begin{array}{c}\text { PK } \\
(\mathrm{G} 20)\end{array}$ & $\begin{array}{c}\text { PK } \\
(\mathrm{G} 20+\mathrm{NaCl})\end{array}$ & $\begin{array}{c}\text { PK } \\
(\mathrm{E} 2)\end{array}$ & $\begin{array}{c}\text { PK } \\
(\mathrm{SGG})\end{array}$ & $\begin{array}{c}\text { PK } \\
(\mathrm{SGG}+\mathrm{NaCl})\end{array}$ \\
\hline $\mathbf{1 7}$ & 2.0 & - & - & - & - & - \\
\hline $\mathbf{1 8}$ & 27.0 & - & - & - & - & - \\
\hline $\mathbf{2 2}$ & - & 20.0 & 25.0 & 5.0 & 10.0 & - \\
\hline $\mathbf{2 3}$ & - & 25.0 & - & - & - & - \\
\hline $\mathbf{2 4}$ & - & - & 3.5 & - & - & - \\
\hline $\mathbf{2 5}$ & - & - & - & 1.0 & - & - \\
\hline $\mathbf{3 9}$ & - & - & 4.0 & - & - & 5.0 \\
\hline $\mathbf{4 0}$ & - & - & 1.5 & - & - & 2.0 \\
\hline $\mathbf{4 1}$ & - & - & 1.0 & - & - & 1.5 \\
\hline $\mathbf{4 2}$ & - & - & 1.0 & - & - & 1.5 \\
\hline $\mathbf{4 8}$ & - & - & 5.0 & - & - & - \\
\hline $\mathbf{4 9}$ & - & - & 1.5 & - & - & - \\
\hline $\mathbf{5 1}$ & - & - & 3.5 & - & - & - \\
\hline
\end{tabular}

Tabelle 5: Übersicht über die Ausbeuten $(\mathrm{mg} / \mathrm{L})$ der isolierten Substanzen aus verschiedenen Kultivierungen des Stammes 6288 (SK = Schüttelkolben, PK = P-Kolben). 


\subsection{Spiciferone}

Aus einer Fermentation in Schüttelkultur mit dem Medium 1549 ließen sich die Verbindungen 17 und 18 gewinnen.

\subsubsection{Spiciferon A (17)}

Die farblose kristalline Verbindung zeigt im EI-Massenspektrum den Peak höchster Masse bei $\mathrm{m} / \mathrm{z}=232$. Dem ${ }^{13} \mathrm{C}-\mathrm{NMR}-$ Spektrum sind Signale für vier Methyl-, eine Methylen- und zwei Methingruppen sowie sieben quartäre Kohlenstoffatome $\mathrm{zu}$ entnehmen. Mit Hilfe dieser Daten lässt sich für 17 die Summenformel $\mathrm{C}_{14} \mathrm{H}_{16} \mathrm{O}_{3}$ aufstellen, die durch ein hochaufgelöstes Massenspektrum bestätigt wurde. Die Protonen der Methylgruppen findet man bei $\delta_{\mathrm{H}}=0.68$, 1.44, 2.02 und 2.38, wobei die drei letzteren als Singuletts erscheinen. Die Methylgruppe bei $\delta_{\mathrm{H}}=0.68$ ist zum Triplett aufgespaltet, was die Nachbarschaft zu einer Methylengruppe bei $\delta_{\mathrm{H}}=1.97$ und 2.13 anzeigt. Die Kopplungskonstante von ${ }^{3} J=10.0 \mathrm{~Hz}$ der beiden Methingruppen bei $\delta_{\mathrm{H}}=6.17$ und 7.90 lässt auf eine cis-konfigurierte Doppelbindung schließen und die Differenz in der chemischen Verschiebung deutet auf die Nachbarschaft zu einer Carbonylgruppe hin. Eine mit diesen Daten durchgeführte Literatursuche konnte 17 eindeutig als Spiciferon $A^{56}$ identifizieren. Die absolute Stereochemie von Spiciferon A wurde für ein an C-7 reduziertes Derivat durch Röntgenstrukturanalyse und die Anwendung der Mosher-Methode bestimmt. ${ }^{57}$<smiles>CCC1(C)C(=O)C=Cc2c1oc(C)c(C)c2=O</smiles>

\subsubsection{Prospiciferon A (18)}

Die im Vergleich zu 17 polarere Komponente besitzt eine gelbe Eigenfarbe und zeigt die erwähnte starke Fluoreszenz bei $366 \mathrm{~nm}$. Im ${ }^{1} \mathrm{H}-\mathrm{NMR}-$ Spektrum finden sich die Signale von vier Methylgruppen bei $\delta_{\mathrm{H}}=0.66,1.18,1.22$ und 2.10, welche als Multipletts aufgespalten sind, was auf das Vorliegen mehrerer Konformere zurückzuführen sein könnte. Tatsächlich 
vereinfacht sich das NMR-Spektrum durch Messung bei höherer Temperatur $\left(50^{\circ} \mathrm{C}\right)$. Das Signal bei $\delta_{\mathrm{H}}=0.66$ wird zum Triplett, das bei $\delta_{\mathrm{H}}=1.18$ zum Singulett. Bei den beiden übrigen Methylgruppen tritt jedoch keine Vereinfachung ein. Ein ${ }^{1} \mathrm{H},{ }^{1} \mathrm{H}-\mathrm{COSY}$-Experiment ergibt, dass die Methylgruppe bei $\delta_{\mathrm{H}}=0.66$ wie erwartet mit einer Methylengruppe bei $\delta_{\mathrm{H}}=1.81$ und 1.90 verknüpft ist. Die beiden Methylgruppen bei $\delta_{\mathrm{H}}=1.18$ und 2.10 zeigen keinerlei Korrelationen und die Methylgruppe mit der Verschiebung von $\delta_{\mathrm{H}}=1.22$ weist eine Kopplung mit einer Methingruppe bei $\delta_{\mathrm{H}}=4.25$ auf. Dieses Signal wurde zunächst für eine Verunreinigung gehalten, da das zugehörige Integral nur einem halben Proton entspricht. Zusammen mit der Aufspaltung der Methylgruppen bei $\delta_{\mathrm{H}}=1.18$ und 2.10 könnte dies ein Hinweis auf eine Keto-Enol-Tautomerie sein, die zur Abschwächung der Intensität des Signals bei $\delta_{\mathrm{H}}=4.25$ führt. Diese Vermutung wurde durch Zusatz von $\mathrm{FeCl}_{3}$-Lösung, wobei eine Farbänderung von gelb nach grün eintrat, bestätigt. Des Weiteren treten im ${ }^{1} \mathrm{H}-\mathrm{NMR}$ Spektrum noch die Signale einer Doppelbindung bei $\delta_{\mathrm{H}}=5.53$ und 7.96 auf. Diese ist auf Grund der Kopplungskonstante von ${ }^{3} J=10.0 \mathrm{~Hz}$ cis-konfiguriert und die Verschiebungen der beiden Protonen lassen auf eine benachbarte Carbonylgruppe schließen. Ein ESIMassenspektrum liefert ein [M-H]--Ion bei $\mathrm{m} / \mathrm{z}=249$, dessen Hochauflösung die Summenformel $\mathrm{C}_{14} \mathrm{H}_{18} \mathrm{O}_{4}$ ergab. Im ${ }^{13} \mathrm{C}$-NMR-Spektrum treten wie erwartet Signale für vier Methyl-, eine Methylen- und vier Methingruppen sowie für fünf quartäre Kohlenstoffatome auf. Dies führte zusammen mit der Summenformel zu der Vermutung, dass sich 18 von 17 durch die Addition von Wasser unterscheidet. Im Einklang mit den Informationen aus den NMR-Spektren ergibt sich die Struktur von 18, die durch ein HMBC-Experiment bestätigt wurde. Die Stereochemie an C-9 wurde in Analogie zu Spiciferon A (17) angegeben. Da es sich bei 18 offensichtlich um einen Vorläufer von 17 handelt, wird der Name Prospiciferon A vorgeschlagen.

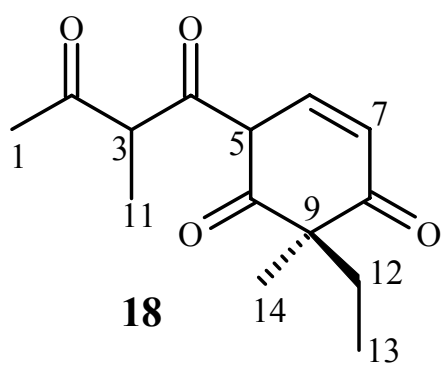


In den ${ }^{1} \mathrm{H}-\mathrm{NMR}-$ Spektren zeigt sich das Vorliegen einer Keto-Enol-Tautemerie an der verringerten Intensität des Signals für 3-H. Dabei ist die Gleichgewichtslage vom Lösungsmittel abhängig. In Methanol besitzt das Integral von 3-H eine Intensität von 0.5 wohingegen in Aceton eine Intensität von 0.8 Protonen beobachtet wird. Ein Signal für 5-H kann in keinem der beiden Lösungsmittel beobachtet werden. Diese Ergebnisse sprechen dafür, dass 18 in Form der Tautomeren 18a und 18b vorliegt. Weitere Tautomere bei denen es zusätzlich zur Enolisierung zwischen C-2 und C-3 bzw. zwischen C-3 und C-4 kommt, sind auf Grund der geringeren Azidität von 3-H im Vergleich mit der von 5-H weniger relevant.<smiles>CCC1(CC)C(=O)C=CC2=C1O[CH]OC2C([Tl])C(C)=O</smiles>

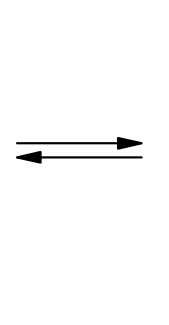<smiles>C=CC(=O)C(I)C(=O)C1C=CC(=O)[C@](C)(CC)C1=O</smiles><smiles></smiles>

Abbildung 4: Beobachtete Tautomere von Prospiciferon A (18). 


\subsubsection{Biosynthese von Prospiciferon A (18) und Spiciferon A (17)}

Spiciferon A (17) konnte 1989 aus dem pflanzenpathogenen Pilz Cochliobolus spicifer isoliert werden. ${ }^{56}$ Wenig später gelang die Isolierung der strukturell verwandten Spiciferone B und C, die sich von 17 durch Hydroxylierung der Methylgruppen an C-2 und C-3 ableiten. ${ }^{58}$ Die Biosynthese von Spiciferon A wurde durch Fütterung von $\left[1-{ }^{13} \mathrm{C}\right]-,\left[2-{ }^{13} \mathrm{C}\right]-$, , $\left.1,2-{ }^{13} \mathrm{C}_{2}\right]-$ und $\left[1-{ }^{13} \mathrm{C}^{2} \mathrm{H}_{3}\right]$ Acetat sowie von $\left[\mathrm{S}-{ }^{13} \mathrm{CH}_{3}\right]-\mathrm{L}-$ Methionin untersucht. ${ }^{59}$ Diese Untersuchungen ergaben, dass 17 aus einem Hexaketid und zwei aus Methionin stammenden $\mathrm{C}_{1}$-Einheiten aufgebaut wird. Mit diesem Wissen wurde ein Biosyntheseweg vorgeschlagen, bei dem es, nach einer ersten Cyclisierung zu einem 10-gliedrigen Ringsystem, zu einer Retro-AldolUmlagerung kommt. Dadurch entsteht ein Intermediat, welches mit Prospiciferon A (18) identisch ist. 18 kann anschließend unter Freisetzung von Wasser zu Spiciferon A umgesetzt werden (Abbildung 5).

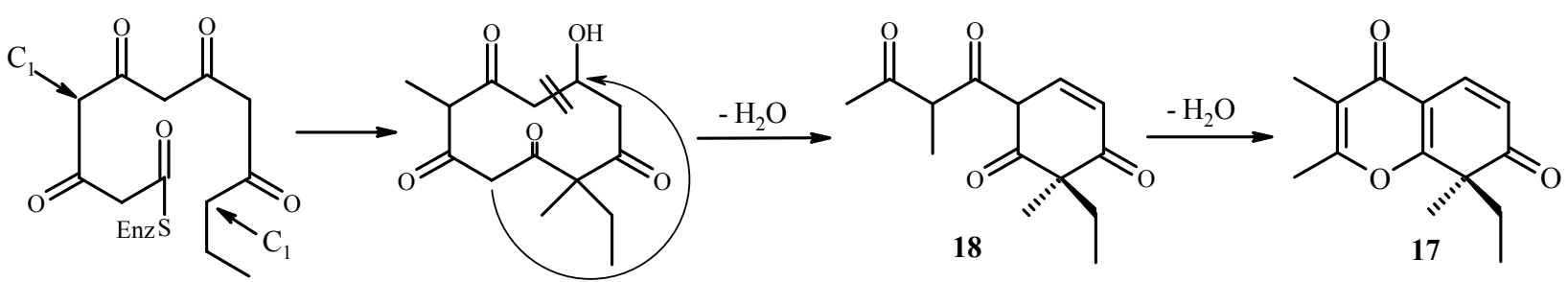

Abbildung 5: Biosynthese von Spiciferon A (17) und Prospiciferon A (18).

Durch die Isolierung von Prospiciferon A aus Microsphaeropsis sp. Stamm 6288 kann der formulierte Biosyntheseweg bestätigt werden. Die Tatsache, dass 18 die Hauptkomponente war, kann durch eine beim Stamm 6288 vorliegende Hemmung des letzten Cyclisierungsschrittes in der Biosynthese von 17 erklärt werden. In wässriger Lösung ( $\mathrm{pH}-$ Wert $=$ 5, 7 und 9) wurde keine Umwandlung von 18 in 17 beobachtet, d.h. eine pHabhängige spontane Cyclisierung scheidet aus. 


\section{2. $\alpha$-Pyrone}

Aus Ruhekulturen (P-Kolben) von Microsphaeropsis sp. Stamm 6288 konnten vier $\alpha$-Pyrone $(\mathbf{2 2}-\mathbf{2 5})$ isoliert werden, von denen drei $(\mathbf{2 3}-\mathbf{2 5})$ neu waren.

\subsubsection{Taiwapyron (22)}

Das EI-Massenspektrum des farblosen Feststoffes zeigt bei $\mathrm{m} / \mathrm{z}=198$ den Peak höchster Masse, dessen Hochauflösung zur Summenformel $\mathrm{C}_{10} \mathrm{H}_{14} \mathrm{O}_{4}$ führt. Im ${ }^{13} \mathrm{C}-\mathrm{NMR}-$ Spektrum treten Signale für eine Methyl-, drei Methylen- und drei Methingruppen sowie für drei quartäre Kohlenstoffatome auf. Bei einer Datenbanksuche in Antibase ${ }^{54}$ findet man 21 Einträge dieser Summenformel. Anhand der Konnektivitäten der C-Atome lassen sich 17 der gefundenen Strukturen ausschließen. Unter den anderen Substanzen finden sich drei Ramulosin-Derivate $(\mathbf{1 9}-\mathbf{2 1})^{60}$ und Taiwapyron $(\mathbf{2 2})^{61}$.<smiles>[R]C1CC(O)=C2C(=O)O[C@H](C)C([R])[C@@H]2[C@H]1[R]</smiles>

\begin{tabular}{|c|c|c|c|}
\hline & $\mathrm{R}^{1}$ & $\mathrm{R}^{2}$ & $\mathrm{R}^{3}$ \\
\hline $\mathbf{1 9}$ & $\mathrm{OH}$ & $\mathrm{H}$ & $\mathrm{H}$ \\
\hline $\mathbf{2 0}$ & $\mathrm{H}$ & $\mathrm{OH}$ & $\mathrm{H}$ \\
\hline $\mathbf{2 1}$ & $\mathrm{H}$ & $\mathrm{H}$ & $\mathrm{OH}$ \\
\hline
\end{tabular}<smiles>CCC[C@H](O)c1ccc(=O)oc1CO</smiles>

Im Protonenspektrum erscheint die Methylgruppe bei $\delta_{\mathrm{H}}=0.89$ als Triplett, sie muss daher $\mathrm{zu}$ einer $\mathrm{CH}_{2}$-Gruppe benachbart sein. Außerdem finden sich im Tieffeldbereich bei $\delta_{\mathrm{H}}=6.24$ und $\delta_{\mathrm{H}}=7.52$ die Signale einer Doppelbindung, die auf Grund der Kopplungskonstante von ${ }^{3} J=10.0 \mathrm{~Hz}$ cis-konfiguriert ist. Dadurch können 19-21 als Lösungen ausgeschlossen werden. Durch Vergleich mit den Literaturdaten ließ sich die Verbindung eindeutig als Taiwapyron $^{61}$ (22) identifizieren. Der gemessene Drehwert stimmt mit dem Literaturwert überein und bestätigt somit das Vorliegen der $S$-Konfiguration an C-1'. 


\subsubsection{Dehydrotaiwapyron (23)*}

Dehydrotaiwapyron wurde als farbloser Feststoff isoliert, der mit Anisaldehyd in der Wärme grünblau anfärbt. Aus dem EI-Massenspektrum erhält man eine im Vergleich zu 22 um zwei Einheiten geringere Masse. Die sich daraus ergebende Summenformel $\mathrm{C}_{10} \mathrm{H}_{12} \mathrm{O}_{4}$ ließ sich durch eine Hochauflösung bestätigen. In Analogie zu 22 lassen sich dem ${ }^{13} \mathrm{C}-\mathrm{NMR}-$ Spektrum Signale einer Methyl- und dreier Methylengruppen sowie für drei quartäre Kohlenstoffatome entnehmen. Bei den Methingruppen fehlt im Vergleich zu 22 das Signal von C-1' $\left(\delta_{\mathrm{C}}=66.9\right)$, stattdessen findet man eine zusätzliche Ketogruppe bei $\delta_{\mathrm{C}}=196.5$. Dies spricht dafür, dass es sich bei 23 um ein an C-1' oxidiertes Derivat von 22 handelt. Aus einem ${ }^{1} \mathrm{H}-{ }^{1} \mathrm{H}-\mathrm{COSY}$ Experiment kann man drei Spinsysteme ableiten, die sich durch ein HMBC-Experiment

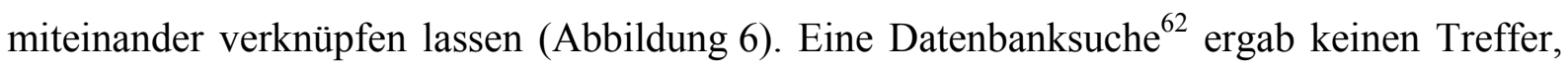
so dass es sich bei Dehydrotaiwapyron (23) um eine neue Verbindung handelt.
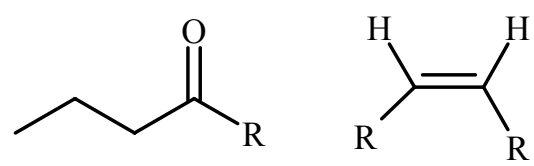

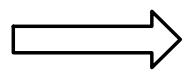

Abbildung 6: Aus dem COSY abgeleitete Strukturfragmente von 23 und deren Verknüpfung über ausgewählte HMBC-Korrelationen.

\subsubsection{5,6-Dihydrotaiwapyron (24)}

Das ${ }^{13} \mathrm{C}-\mathrm{NMR}-$ Spektrum des farblosen Naturstoffes hat große Ähnlichkeit zu dem Spektrum von 22, wobei anstelle der Signale der quartären Kohlenstoffatome bei $\delta_{C}=120.1$ und $\delta_{\mathrm{C}}=158.3$ zwei zusätzliche Methinsignale bei $\delta_{\mathrm{C}}=54.0$ und $\delta_{\mathrm{C}}=78.9$ zu erkennen sind. In Verbindung mit der durch eine ESI-Hochauflösung bestimmten Summenformel $\left(\mathrm{C}_{10} \mathrm{H}_{16} \mathrm{O}_{4}\right)$ erscheint es plausibel, dass hier ein 5,6-Dihydro-Derivat von 22 vorliegt. Diese Vermutung kann durch ein ${ }^{1} \mathrm{H}-{ }^{1} \mathrm{H}$-COSY-Experiment und ein $\mathrm{HMBC}$-Experiment bestätigt werden und führt zu der Struktur von 5,6-Dihydrotaiwapyron, entsprechend Formel 24.

\footnotetext{
* fehlerhafte Struktur, Korrektur siehe Seite 30.
} 
<smiles>CCC[C@H](O)[C@H]1C=CC(=O)O[C@@H]1CO</smiles>

Für die Stereochemie an C-1' wurde angenommen, dass hier wie bei 22 die $S$-Konfiguration vorliegt. Über die relative Stereochemie an C-5 und C-6 können die Kopplungskonstanten Auskunft geben. Die Kopplungskonstante ist abhängig von der Größe des Torsionswinkels zwischen zwei Protonen, dieser Zusammenhang ist in der Karplus-Kurve dargestellt. Im Umkehrschluß lässt sich mit Hilfe dieser Kurve aus der beobachteten Kopplungskonstante der Winkel zwischen zwei Protonen ableiten. Die Kopplungskonstante von ${ }^{3} J=6.0 \mathrm{~Hz}$ zwischen den beiden Protonen an C-5 und C-6 entspricht einem Diederwinkel von 30 bzw. 150 ${ }^{\circ}$. Die Auswertung im Fall von $\mathbf{2 4}$ wird durch das Vorliegen eines Gleichgewichts zwischen zwei Konformeren erschwert, bei denen C-6 entweder oberhalb oder unterhalb der durch den Rest des Pyranringes aufgespannten Ebene liegt. Da sich die beiden Konformeren sehr schnell in einander umwandeln, kann man sie im NMR-Experiment nicht beobachten und die Kopplungskonstante ergibt sich aus dem Mittelwert. Ein mittlerer Diederwinkel von $30^{\circ} \mathrm{kann}$ nur erreicht werden, wenn beide Protonen auf einer Seite, entweder ober- oder unterhalb des Pyranringes liegen. Ein zusätzlich durchgeführtes eindimensionales NOE-Experiment bestätigt diese Annahme. Die relative Stereochemie zwischen C-5 und C-1' konnte auf Grund der freien Drehbarkeit um diese Bindung nicht bestimmt werden.
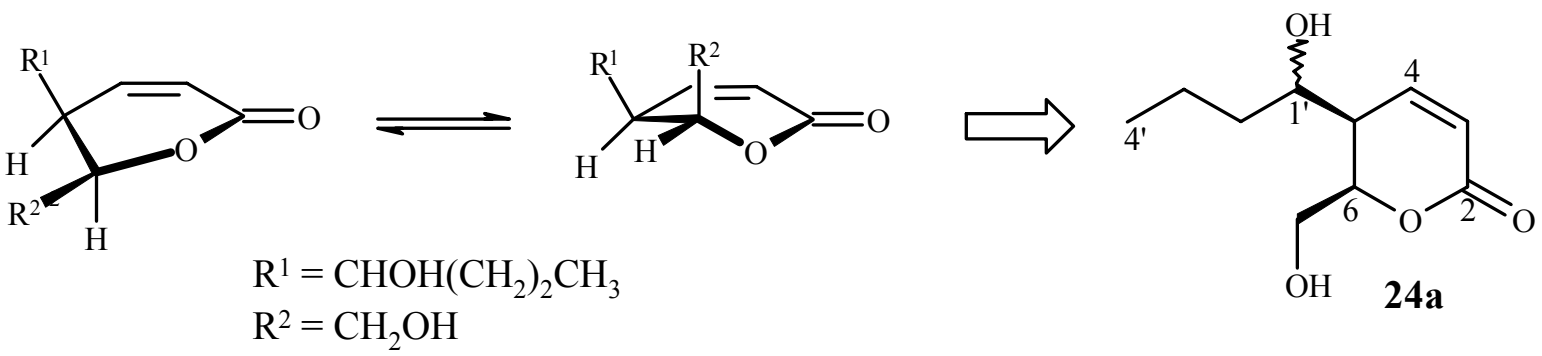


\subsubsection{7-(1-Methyl-ethyliden)-taiwapyron (25)*}

Der farblose Naturstoff wurde aus einer Ruhefermentation mit E2 als Nährmedium isoliert. Das ${ }^{1}$ H-NMR-Spektrum besitzt große Ähnlichkeit zu dem von 23. Lediglich das Signal der Hydroxymethylengruppe fehlt, dafür treten zusätzlich Singuletts für zwei Methylgruppen bei $\delta_{\mathrm{H}}=2.06$ und $\delta_{\mathrm{H}}=2.30$ auf. Es scheint sich somit um ein weiteres $\alpha$-Pyron zu handeln, das an der Hydroxymethylengruppe an C-6 verändert ist. Die Summenformel ergibt sich aus dem hochaufgelösten EI-Massenspektrum $\left(\mathrm{C}_{13} \mathrm{H}_{16} \mathrm{O}_{4}\right)$. Im ${ }^{13} \mathrm{C}-\mathrm{NMR}$-Spektrum finden sich die Signale der beiden neuen Methylgruppen bei $\delta_{\mathrm{C}}=17.2$ und $\delta_{\mathrm{C}}=19.9$ sowie für zwei zusätzlichen Kohlenstoffatome bei $\delta_{\mathrm{C}}=135.0$ und $\delta_{\mathrm{C}}=145.3$. Diese Ergebnisse sprechen dafür, dass 25 an Stelle der Hydroxymethylen- der anderen $\alpha$-Pyrone eine HydroxyisobutenylGruppe trägt. Durch ein HMBC-Experiment konnte die Verknüpfung des PyronGrundgerüstes mit dem Hydroxyisobutenylrest eindeutig bewiesen werden. Ein derartiges $\alpha$-Pyron ist in der Literatur bisher nicht beschrieben worden.

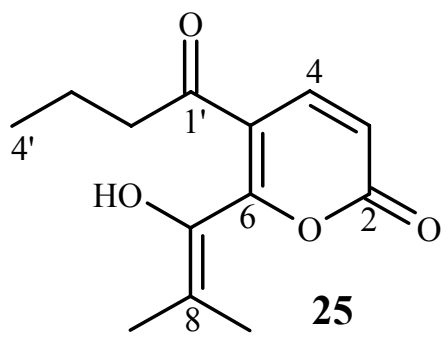

Vergleicht man die ${ }^{13} \mathrm{C}$-NMR-Daten der vier isolierten $\alpha$-Pyrone, so fallen deutliche Unterschiede in den Verschiebungen auf. Die Signale für C-2, C-7 und C-1' weisen bei 5,6Dihydrotaiwapyron (24) im Vergleich mit Taiwapyron (22) eine Tieffeldverschiebung auf (Tabelle 6). Diese kann durch die fehlende Doppelbindung zwischen C-5 und C-6 erklärt werden. Bei der Gegenüberstellung der Daten von 22 und $\mathbf{2 3}$ fällt die deutliche Tieffeldverschiebung von C-6 um 42.2 ppm und die von C-7 um 17.4 ppm auf. Diese starke Verschiebung kann möglicherweise durch die in Abbildung 7 dargestellten mesomeren Grenzstrukturen, die zu einer Verstärkung der positiven Polarisierung von C-6 führen, erklärt werden. Für das Vorliegen von mesomeren Grenzstrukturen spricht, dass die Signale der Kohlenstoffatome C-6 und C-1' von 25 gegenüber denen von 23 eine deutliche Hochfeldverschiebung aufweisen, da die neue Doppelbindung an C-7 zusätzliche Elektronendichte bereitstellt. Ein weiteres Indiz für die dargestellte Polarisierung von

\footnotetext{
${ }^{*}$ fehlerhafte Struktur, Korrektur siehe Seite 30.
} 
Dehydrotaiwapyron (23) ist, dass vergleichbare $\alpha$-Pyrone bei Temperaturen $>500^{\circ} \mathrm{C}$ unter Ringöffnung in ein Keten umlagern, welches zu dem in Abbildung 7 dargestellten analog ist. ${ }^{63}$ Ein Vergleich der ermittelten Daten mit Literaturdaten ist leider nicht möglich, da für vergleichbare Pyrone keine NMR-Spektren vorliegen. ${ }^{64}$

\begin{tabular}{|c|c|c|c|c|}
\hline & $\mathbf{2 2}^{\mathbf{a}}$ & $\mathbf{2 3}^{\mathbf{b}}$ & $\mathbf{2 4}^{\mathbf{b}}$ & $\mathbf{2 5}^{\mathbf{b}}$ \\
\hline C-2 & 164.2 & 171.6 & 170.9 & 171.6 \\
\hline C-3 & 116.3 & 119.9 & 123.0 & 120.2 \\
\hline C-4 & 145.8 & 132.2 & 147.7 & 132.5 \\
\hline C-5 & 121.8 & 112.8 & 54.0 & 115.2 \\
\hline C-6 & 159.8 & 202.0 & 78.9 & 185.5 \\
\hline C-7 & 58.9 & 76.3 & 73.9 & 145.3 \\
\hline C-8 & & & & 135.0 \\
\hline C-9 & & & & 17.2 \\
\hline C-10 & & & & 19.9 \\
\hline C-1 & 67.8 & 196.5 & 85.0 & 183.1 \\
\hline C-2 & 40.5 & 31.6 & 36.5 & 30.5 \\
\hline C-3 & 19.9 & 21.0 & 19.3 & 21.3 \\
\hline C-4 & 14.2 & 14.0 & 14.1 & 14.0 \\
\hline
\end{tabular}

Tabelle 6: Vergleich der ${ }^{13} \mathrm{C}-\mathrm{NMR}-\mathrm{Daten}$ der vier isolierten $\alpha$-Pyrone (22 - 24) $\left(\mathrm{CD}_{3} \mathrm{OD},{ }^{\mathrm{a}} 150.8 \mathrm{MHz},{ }^{\mathrm{b}} 125.7 \mathrm{MHz}\right)$.<smiles>CCCC(=O)C(=CC=[N+](C)C)C(=O)CO</smiles>

Abbildung 7: Mesomere Grenzstrukturen von Dehydrotaiwapyron (23).

Nach Beendigung der Dissertation stellte sich durch eine Röntgenstrukturanalyse heraus, dass die Strukturen von 23 und 25 fehlerhaft sind. Bei den beiden Verbindungen handelt es sich vielmehr um die bisher nicht in der Literatur beschriebenen Furanone 23a und 25a.

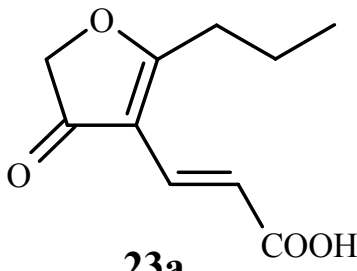

$23 \mathbf{a}$<smiles>CCCC1=C(/C=C/C(=O)O)C(=O)C(=C(C)C)O1</smiles>

25a 


\subsection{5. $\alpha$-Pyrone vom Typ des Taiwapyrons}

Taiwapyron (22) wurde erstmals 1976 aus dem pflanzenpathogenen Pilz Cercospora taiwanensis isoliert. ${ }^{61}$ Von Pilzen werden eine Vielzahl relativ einfacher $\alpha$-Pyrone wie das Taiwapyron gebildet, die trotz ihrer strukturellen Einfachheit diverse biologische Aktivitäten besitzen. Dazu gehören die von Penicillium waksmanii gebildeten und dem Taiwapyron strukturell verwandten Pyrenocine A-E. ${ }^{65}$ Der Produzentenstamm wurde ebenso wie Microsphaeropsis sp. Stamm 6288 aus einer Alge isoliert und zwar aus der Braunalge Sargassum ringgoldianum. Pyrenocin A (26) und B (27) besitzen breite antibiotische Aktivität gegen Pflanzen, Pilze und Bakterien. ${ }^{66}$

1998 konnten aus einem nicht bestimmten Pilz vier weitere, dem Taiwapyron strukturell nahe stehende $\alpha$-Pyrone ${ }^{67}(\mathbf{2 8}-\mathbf{3 1})$, isoliert werden, die sich nur in ihrer Seitenkette unterscheiden. Bei den Substanzen 28 - 30 handelt es sich um Liganden des Androgenrezeptors, wohingegen 31 keine Affinität zum Rezeptor zeigte. Die Wirkung dieser Verbindungen wurde durch eine strukturelle Ähnlichkeit zum Testosteron erklärt.

Natürlich gibt es noch viele weitere Metabolite, die $\alpha$-Pyrone enthalten. Diese sind zum Teil wesentlich komplexer, wie die Solanapyrone (siehe Kapitel A.III.1.4.), die Aurovertine ${ }^{68}$ oder das Myxopyronin $\mathrm{A}^{69}$ (32) oder weisen wie die antibakteriell wirksamen Gibepyrone A-D ${ }^{70}$ (33 - 36) im Vergleich zu den bisher besprochenen $\alpha$-Pyronen ein verändertes Grundgerüst auf.<smiles>[R]c1cc(=O)oc(C)c1OC</smiles><smiles>[R]OC(=O)/C=C\C(=O)CC(C)O</smiles><smiles>[R]c1ccc(=O)oc1C</smiles><smiles>[R][R]=CC=CCCC</smiles><smiles>CCC/C(C)=C/C=C(\C)C(=O)c1c(O)cc(C(C)/C=C/C=C/NC(C)=O)oc1=O</smiles><smiles>[R]C=C(C)c1ccc(C)c(=O)o1</smiles>

$33 \mathrm{R}=\mathrm{CH}_{3}$ $34 \mathrm{R}=\mathrm{CH}_{2} \mathrm{OH}$ $35 \mathrm{R}=\mathrm{CHO}$ $36 \mathrm{R}=\mathrm{CO}_{2} \mathrm{H}$

Abbildung 8: Ausgewählte Metabolite aus der Substanzklasse der $\alpha$-Pyrone. 


\subsection{6. Überlegungen zur Biosynthese der $\alpha$-Pyrone}

Die Biosynthese von $\alpha$-Pyronen, die dasselbe Kohlenstoffgerüst wie Taiwapyron (22) besitzen, wurde anhand der Pyrenocine A(26) und B(27) untersucht. Aus dem Produzentenstamm Penicillium citreo-viride konnte neben Pyrenocin A (26) und B (27) noch das Citreothiolacton (37), ein schwefelhaltiges $\alpha$-Pyrone, isoliert werden. ${ }^{71}$ Zur Untersuchung der Biosynthese wurde $\left[1,2-{ }^{13} \mathrm{C}_{2}\right]$ Acetat an den Produzentenstamm verfüttert. Aus dem beobachteten Einbau wurde dann der in Abbildung 9 dargestellte Biosyntheseweg abgeleitet. Dieser geht davon aus, dass 26, 27 und 37 aus zwei Polyketidketten, einem Di- und einem Triketid, entstehen.
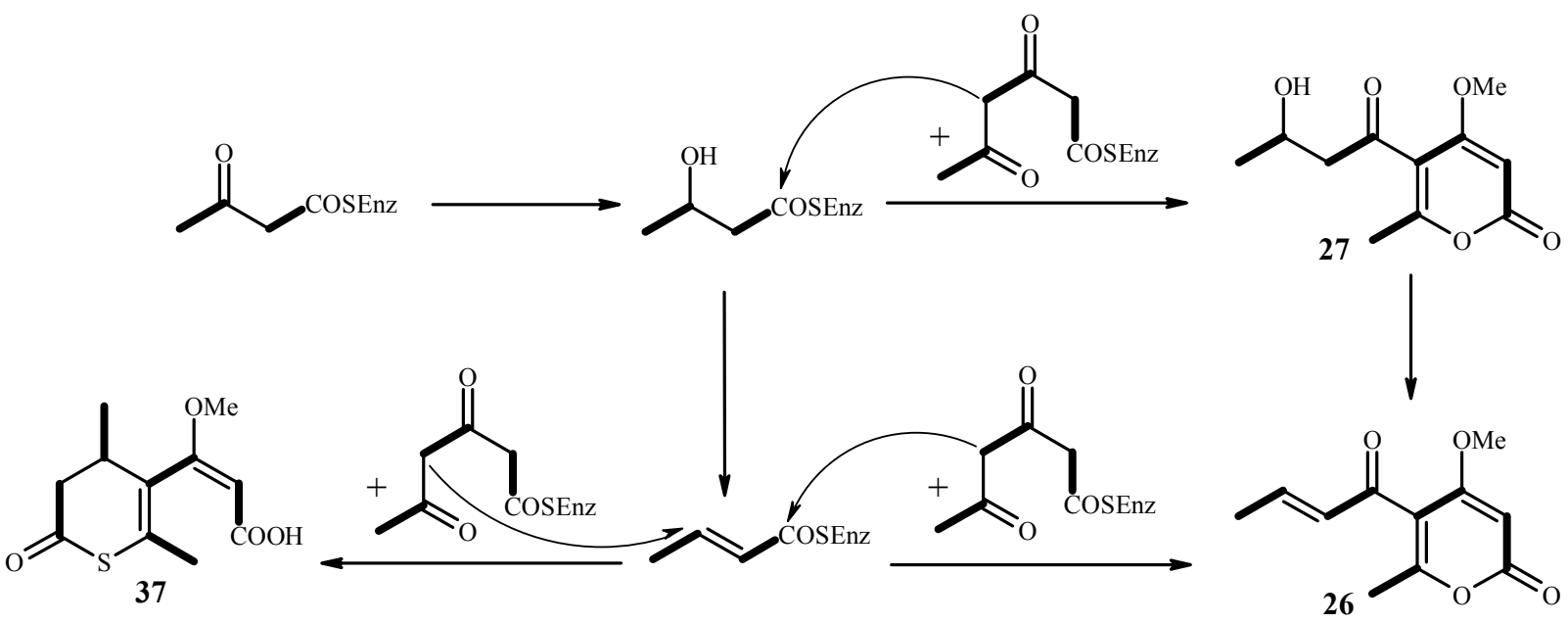

Abbildung 9: Biosynthese von 26, 27 und 37 aus zwei Polyketidketten.

Leider wurde damals ein Fehler bei der Strukturaufklärung von 37 gemacht und fälschlicherweise die Positionen einer Methylgruppe und einer Ketofunktion vertauscht. ${ }^{72}$ Bei der isolierten Verbindung handelt es sich nicht um ein $\alpha$-Pyron sondern um Citreothiopyran B (38). Aber auch unter Berücksichtigung der korrekten Struktur von 38 kann das gefundene Einbaumuster durch die Bildung aus zwei Polyketidketten erklärt werden.

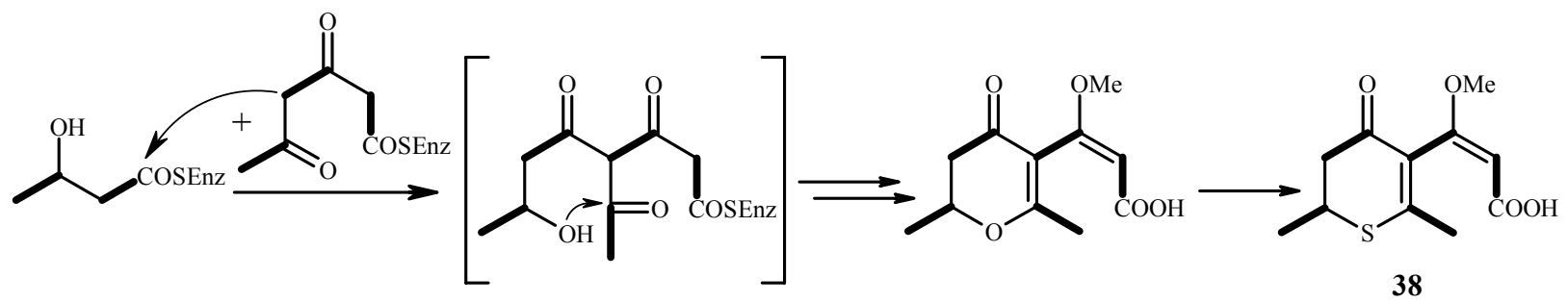

Abbildung 10: Biosynthese von 38 aus zwei Polyketidketten. 
Allerdings ist das gefundene Einbaumuster kein Beweis für die vorgeschlagene Biosynthese aus einem Di- und einem Triketid, da es auch durch die Bildung aus einem Pentaketid erklärt werden kann. In diesem Fall entsteht zunächst ein Aromat, der oxidativ geöffnet wird. Die dabei gebildete Carbonsäuregruppe bildet dann durch Angriff einer Carbonylgruppe den Pyranring aus. Auf einem analogen Weg kann die Bildung von 38 erfolgen. Hier müsste zunächst das Pentaketid, unter Verbleib des Schwefels im Molekül, vom Enzym abgespalten werde. Nach einem ersten Ringschluß und der Bildung des Thiolactons wird der Sechsring in Analogie zur Biosynthese von $\mathbf{2 6}$ und $\mathbf{2 7}$ oxidativ gespalten und es kommt zur Bildung von Citreothiopyran B (38) (Abbildung 11).

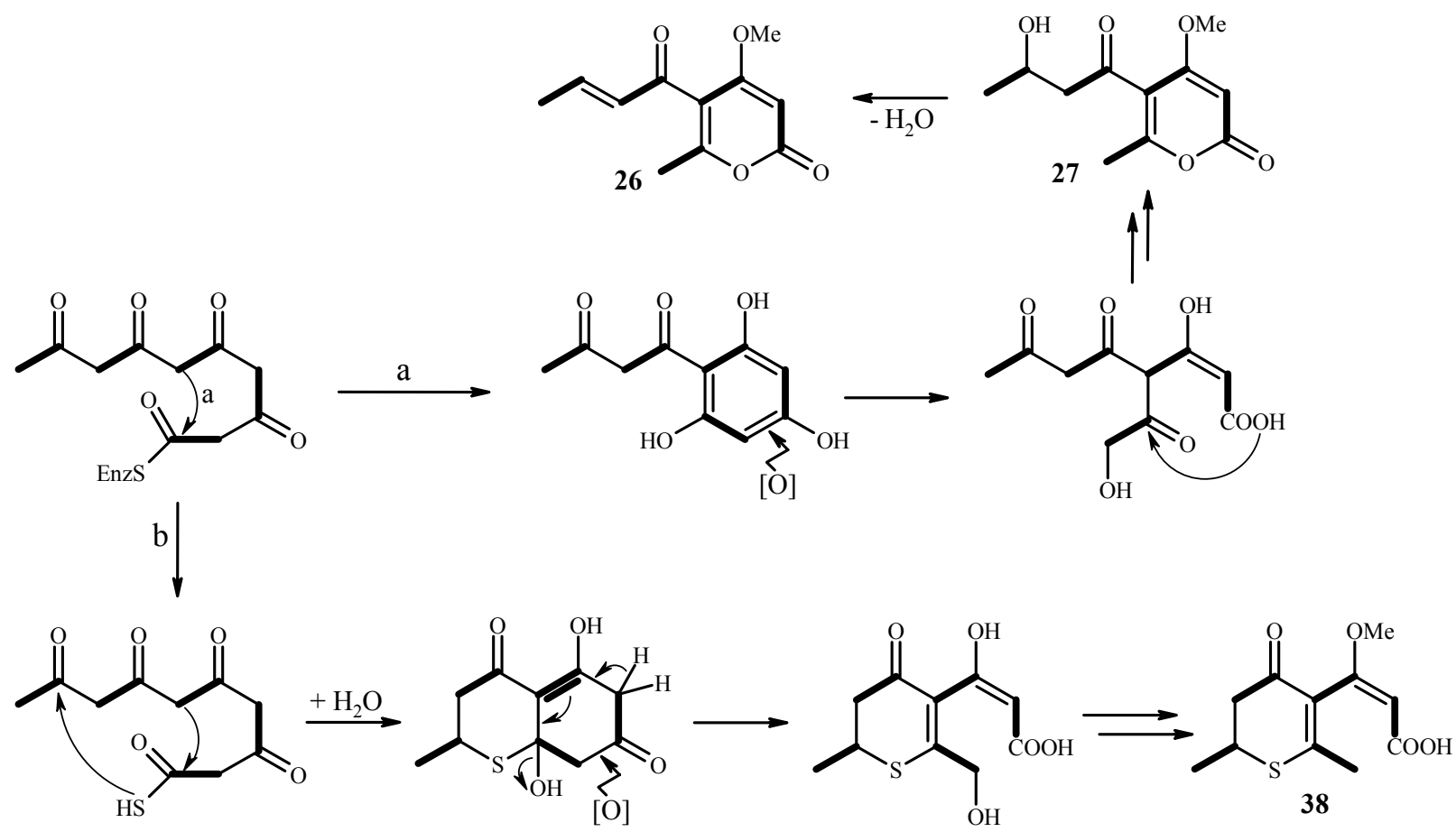

Abbildung 11: Biosynthese von 26, 27 und 38 aus einem Pentaketid.

Die Bildung von 38 aus einem Pentaketid hat im Gegensatz zur Bildung aus zwei Polyketidketten den Vorteil, dass sie den Einbau von Schwefel erklären kann.

Da die von Microsphaeropsis sp. Stamm 6288 gebildeten $\alpha$-Pyrone dasselbe Kohlenstoffgerüst wie die Pyrenocin A (26) und B (27) besitzen, sind die beiden vorgestellten Biosynthesewege auch für die Beiden Hauptmetaboliten des Stammes 6288 Taiwapyron (22) und Dehydrotaiwapyron (23) Verbindungen denkbar (siehe Abbildung 12 und 13). 


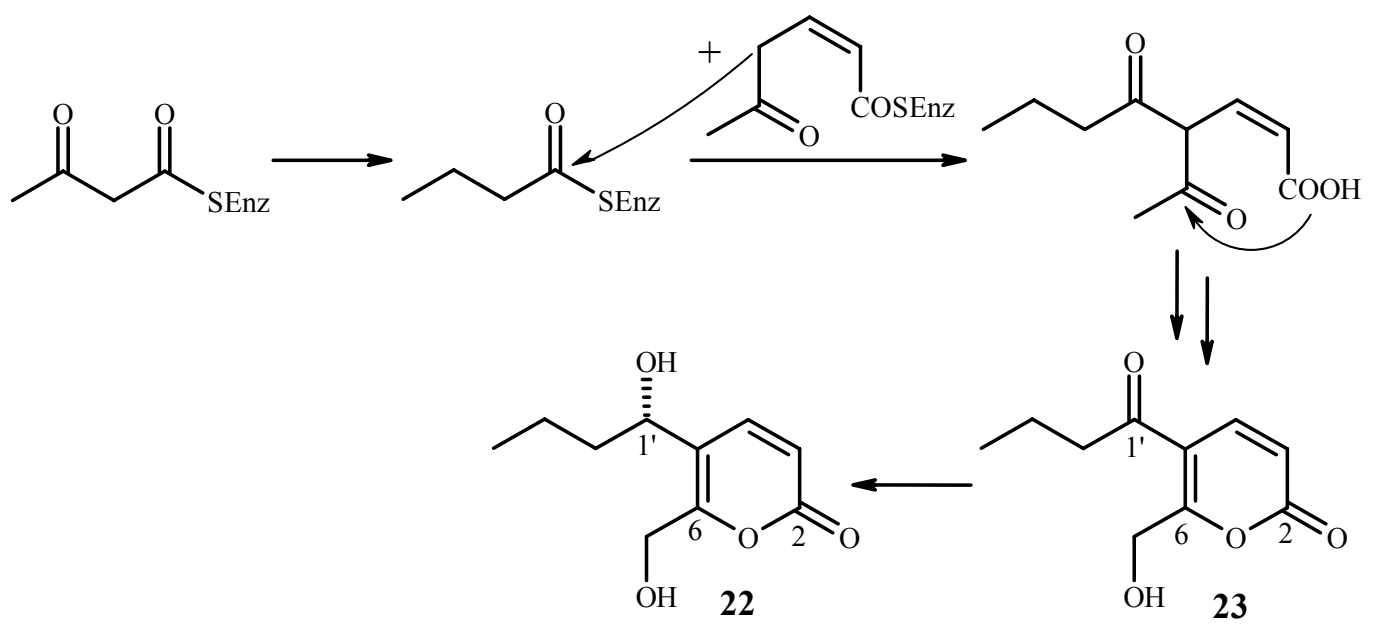

Abbildung 12: Biosynthese von 22 und 23 aus zwei Polyketidketten.

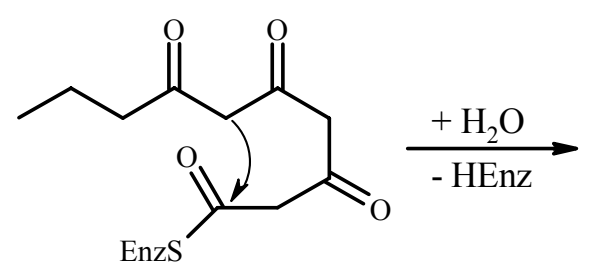<smiles>CC(=O)CC(=O)c1c(O)cc(O)cc1O</smiles><smiles>CC(=O)CC(=O)C(C(=O)CO)=C(O)CC(=O)O</smiles><smiles>CCC[C@H](O)c1ccc(=O)oc1CO</smiles><smiles>CCCC(=O)c1ccc(=O)oc1CO</smiles>

Abbildung 13: Biosynthese von 22 und 23 aus einem Pentaketid.

Anhand der Fütterung von ${ }^{13} \mathrm{C}$ markiertem Acetat ist eine Unterscheidung zwischen den beiden Biosynthesenwegen nicht möglich, da sie zum identischen Einbaumuster führen. Die Fütterung von deuteriertem Acetat hingegen sollte es ermöglichen zwischen den beiden denkbaren Biosynthesewegen zu unterscheiden. Bei dem Aufbau des $\alpha$-Pyron-Grundgerüstes aus zwei Polyketidketten sollte die Hydroxymethylengruppe an C-6 von 22 bzw. 23 drei Deuteriumatome tragen. Verläuft die Biosynthese hingegen über eine Sechsringzwischenstufe, dürfte sich an der Hydroxymethylengruppe unter Berücksichtigung der KetoEnol-Tautomerie kein Deuteriumatom mehr befinden. 


\subsubsection{Zur Biosynthese der von Stamm 6288 gebildeten $\alpha$-Pyrone}

Die $\alpha$-Pyrone des Stammes 6288 werden ausschließlich bei Ruhefermentationen in P-Kolben gebildet. Da die Fermentationsdauer solcher Ruhekulturen vier Wochen beträgt, sind diese Bedingungen für die Fütterung markierter Vorläufer wenig geeignet. Fütterungsexperimente über einen langen Zeitraum führen zu einem Scrembling der Markierung. Daher wurde nach geeigneteren Fermentationsbedingungen gesucht, um kurze Fütterungszeiten zu ermöglichen. So wurden Kultivierungen in Schüttelkolben mit unterschiedlichen Fermentationsdauern und Schüttelgeschwindigkeiten durchgeführt, jedoch konnte bei keinem der Versuch die Produktion eines der vier vom Stamm gebildeten $\alpha$-Pyrone beobachtet werden. Als nächstes wurde untersucht, ab welchem Zeitpunkt die Bildung der $\alpha$-Pyrone in Ruhekultur beginnt. Bei den Versuchen mit Schüttelkulturen des Stammes 6288 hatte sich gezeigte, dass er in Schüttelkultur sehr viel schneller anwächst. So wurde der Stamm zunächst in Schüttelkolben (24 h, $180 \mathrm{rpm}$ ) angezogen und dann als Ruhekultur weitergeführt. Unter diesen Fermentationsbedingungen konnte die Bildung von Dehydrotaiwapyron (23) beobachtet werden. Die Produktion von 23 setzte nach zwölf Tagen ein und nach 14 Tagen konnten $2.0 \mathrm{mg} 23$ aus einem Liter Kulturbrühe isoliert werden. Da dieses Ergebnis reproduzierbar war, wurde zunächst $\left[1-{ }^{13} \mathrm{C}\right]$ Acetat gefüttert. Für fünf Kohlenstoffatome von $\mathbf{2 3}$ trat eine Erhöhung der Signalintensitäten im ${ }^{13} \mathrm{C}$-NMR-Spektrum auf. Das Einbaumuster und die beobachteten Einbauraten sind in Abbildung 14 dargestellt.

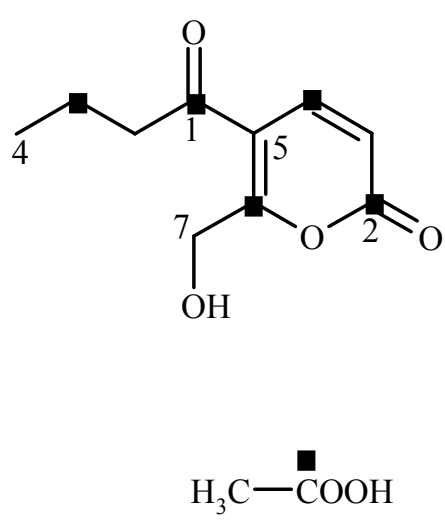

\begin{tabular}{|c|c|c|}
\hline & $\begin{array}{c}\text { Verschiebung } \\
{[\mathrm{ppm}]}\end{array}$ & $\begin{array}{c}\text { Spez. Einbau } \\
{[\%]}\end{array}$ \\
\hline C-2 & 171.6 & $\mathbf{2 . 7}$ \\
\hline C-3 & 119.9 & 0.0 \\
\hline C-4 & 132.2 & $\mathbf{3 . 7}$ \\
\hline C-5 & 112.8 & 0.0 \\
\hline C-6 & 202.0 & $\mathbf{1 . 8}$ \\
\hline C-7 & 76.3 & 0.0 \\
\hline C-1' & 196.5 & $\mathbf{8 . 9}$ \\
\hline C-2' & 31.6 & 0.2 \\
\hline C-3' & 21.0 & $\mathbf{2 . 6}$ \\
\hline C-4' & 14.0 & 0.5 \\
\hline
\end{tabular}

Abbildung 14: Einbaumuster und Einbauraten der $\left[1-{ }^{13} \mathrm{C}\right]$ Acetat-Fütterung. ${ }^{*}$

\footnotetext{
* fehlerhafte Struktur, Korrektur siehe Seite 30.
} 
Das gefundene Einbaumuster bestätigt, dass es sich bei $\mathbf{2 3}$ wie erwartet um ein Pentaketid handelt, wobei die höchste Einbaurate mit $8.9 \%$ bei C-1' auftrat, während die Anreicherung der Kohlenstoffatome C-2, C-4, C-6 und C-3' zwischen 1.8 und 3.7 \% liegt. Auf Grund dieser recht niedrigen Einbauraten wurde auf die Fütterung von deuteriertem Acetat verzichtet, da sich eine Deuteriummarkierung bei einem Einbau in vergleichbarer Höhe schlecht nachweisen lässt.

Um hier zum Erfolg zu kommen, müsste die Produktion der vom Stamm 6288 gebildeten $\alpha$ Pyrone zunächst weiter optimiert werden, wozu im Rahmen dieser Arbeit die Zeit fehlte.

\subsection{Betaenone}

In Ruhefermentationen in P-Kolben (Medien: $\mathrm{G} 20+\mathrm{NaCl}$ und $\mathrm{SGG}+\mathrm{NaCl}$ ) produzierte Microsphaeropsis sp. Stamm 6288 vier neue Betaenone (39-42).

\subsubsection{Betaenon G (39)}

Die als farbloser Feststoff vorliegende Substanz färbt mit Anisaldehyd in der Wärme intensiv blau an. 39 liefert im ESI-Massenspektrum das $[\mathrm{M}+\mathrm{Na}]^{+}$-Ion bei $\mathrm{m} / \mathrm{z}=375$, dessen Hochauflösung zur Summenformel $\mathrm{C}_{21} \mathrm{H}_{36} \mathrm{O}_{4}$ führt. Dieses Ergebnis wird durch das ${ }^{13} \mathrm{C}$ NMR-Spektrum, in dem die Signale von fünf Methyl-, fünf Methylen-, sieben aliphatischen und einer olefinischen Methingruppe sowie für drei quartäre Kohlenstoffatome identifiziert werden können, bestätigt. Im ${ }^{1}$ H-NMR-Spektrum werden die Signale der fünf Methylgruppen im Bereich von $\delta_{\mathrm{H}}=0.62-1.19$ gefunden. Ein Signal tritt dabei als Triplett, drei als Dublett und ein weiteres als Singulett auf. Die Methylgruppen sind somit mit einer Methylen- und drei Methingruppen sowie einem quartären Kohlenstoffatom verknüpft. Die Verschiebung zweier Methylen- und einer Methingruppe zwischen $\delta_{\mathrm{H}}=3.00$ und $\delta_{\mathrm{H}}=4.12$ weisen auf die Anknüpfung an Heteroatomen hin, wobei die Methylengruppe mit Signalen bei $\delta_{\mathrm{H}}=2.75$ und 2.88 koppelt, die zu einer weiteren Methylengruppe gehören. Bei den Heteroatomen muss es sich auf Grund der Summenformel und der Konnektivitäten aus dem ${ }^{13} \mathrm{C}-\mathrm{NMR}-$ Spektrum um Hydroxygruppen handeln. Weiterhin erkennt man im ${ }^{1} \mathrm{H}-\mathrm{NMR}-$ Spektrum das Signal der olefinischen Methingruppe bei $\delta_{\mathrm{H}}=6.05$. Durch die Kopplungskonstanten des ${ }^{1} \mathrm{H}-\mathrm{NMR}$ Spektrums und durch ein ${ }^{1} \mathrm{H},{ }^{1} \mathrm{H}$-COSY-Experiment konnten die in Abbildung 16 gezeigten Fragmente aufgestellt werde. Durch ein HMBC-Experiment gelingt es die Fragmente eindeutig miteinander zu verknüpfen. 


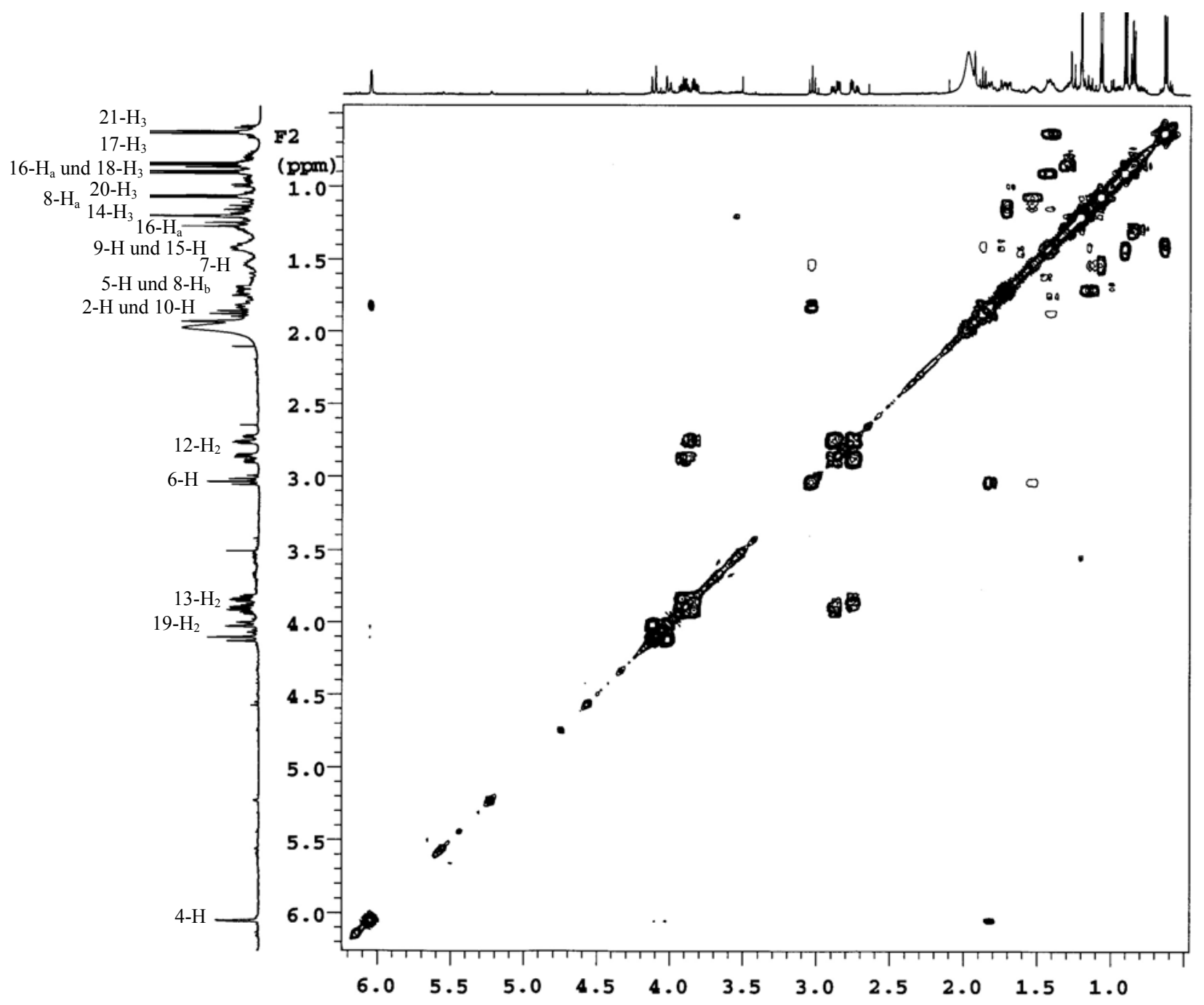

Abbildung 15: ${ }^{1} \mathrm{H},{ }^{1} \mathrm{H}-\mathrm{COSY}$-Experiment $\left(600 \mathrm{MHz}, \mathrm{CDCl}_{3}\right)$ von Betaenon $\mathrm{G}(\mathbf{3 9})$.<smiles>[R]C([R])C(C)CC</smiles><smiles>[R]C([R])=CC1C([R])C(C)CC(C)C1O</smiles><smiles>[R]CCO</smiles><smiles>[R]CO</smiles><smiles>c1ccccc1</smiles>

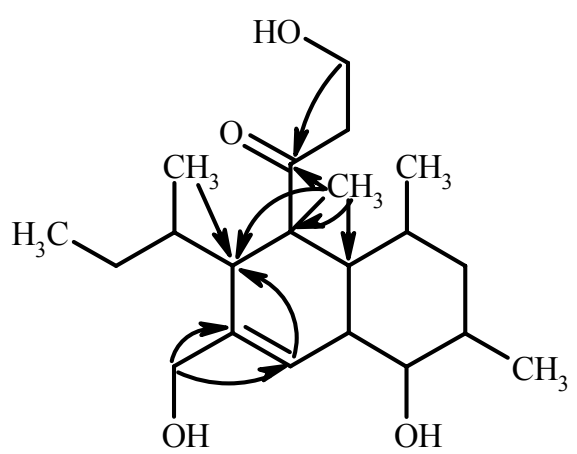

39

Abbildung 16: Aus dem COSY abgeleitete Strukturfragmente von Betaenon G (39) und deren Verknüpfung über ausgewählte HMBC-Korrelationen. 
Eine Datenbanksuche ${ }^{62}$ zeigte, dass 39 bisher nicht in der Literatur beschrieben wurde. Allerdings gibt es Verbindungen gleichen Grundgerüstes, die zur Substanzklasse der Betaenone gehören. Daher wird für 39 der Name Betaenon G vorgeschlagen. Die relative Stereochemie von 39 lässt sich nur teilweise aus den Kopplungskonstanten herleiten. Die Kopplungskonstante zwischen $5-\mathrm{H}$ und $10-\mathrm{H}$ beträgt ${ }^{3} J=9.5 \mathrm{~Hz}$, folglich müssen die beiden Protonen in trans-Stellung zueinander stehen. Das Proton an C-6 weist sowohl zu 5-H, als auch zu 7-H eine Kopplungskonstante von ${ }^{3} J=9.5 \mathrm{~Hz}$ auf, so dass diese Protonen ebenfalls trans zueinander stehen. Gleiches gilt für die Protonen $9-\mathrm{H}$ und $10-\mathrm{H}\left({ }^{3} J=10.0 \mathrm{~Hz}\right)$. Um diese Ergebnisse abzusichern und die relative Konfiguration an C-1 zu klären, wurden 1DNOE-Korrelationen gemessen. Diese bestätigen die aus den Kopplungskonstanten abgeleiteten Ergebnisse und zeigen zusätzlich eine Korellationen zwischen der Methylgruppe an C-1, dem Protonen an C-2 und C-5. Die sich daraus ergebende relative Stereochemie ist in Abbildung 17 dargestellt.

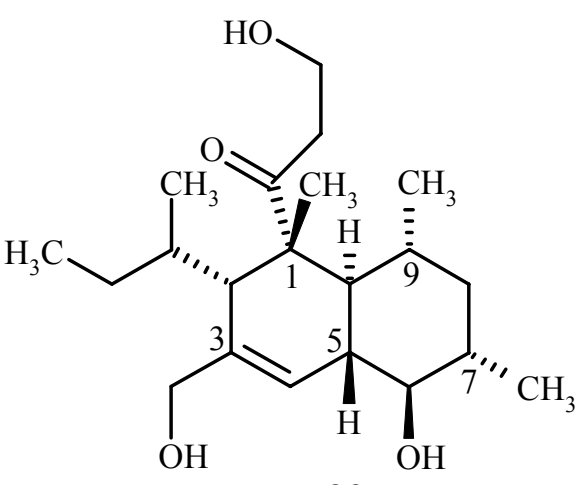

39

Abbildung 17: Relative Stereochemie von Betaenon G (39).

\subsubsection{Betaenon H (40)}

Die im Vergleich zu 39 unpolarere Komponente färbt mit Anisaldehyd in der Wärme ebenso wie Betaenon G intensiv blau an. Im ESI-Massenspektrum findet sich der Quasimolekülpeak bei $\mathrm{m} / \mathrm{z}=359[\mathrm{M}+\mathrm{Na}]^{+}$. Die im Vergleich zu 39 um 16 Einheiten kleinere Masse deutet auf das Fehlen einer Hydroxygruppe hin. Das ${ }^{13} \mathrm{C}-\mathrm{NMR}-$ Spektrum hat große Ähnlichkeit zu dem von 39, lediglich das Signal der Methingruppe bei $\delta_{\mathrm{C}}=79.0$ fehlt. Stattdessen findet sich das Signal einer zusätzlichen Methylengruppe bei $\delta_{C}=41.5$. Auch das ${ }^{1} H-N M R-S p e k t r u m$ von 40 ist mit dem von 39 vergleichbar bis auf die neue Methylengruppe bei $\delta_{\mathrm{H}}=0.95-1.00$ und 1.85. Somit ist Betaenon H (40) im Vergleich zu 39 an C-6 desoxygeniert. Die relative 
Stereochemie von 40 konnte wie für 39 beschrieben aus den Kopplungskonstanten im ${ }^{1} \mathrm{H}$ NMR-Spektrum sowie aus NOE-Korrelationen abgeleitet werden.

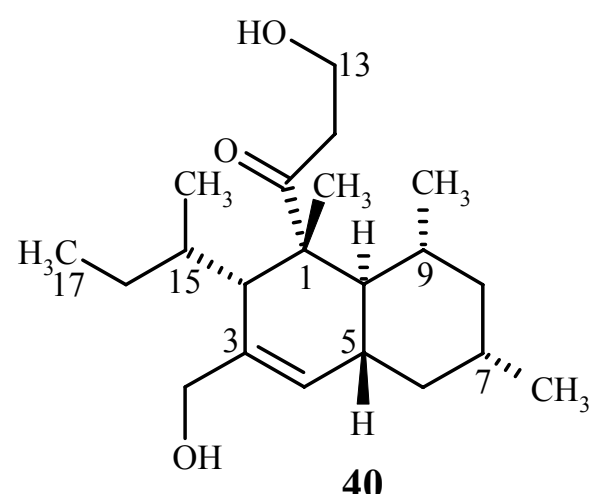

\subsubsection{Betaenon I (41)}

Die dritte Verbindung ist mit einem $\mathrm{R}_{\mathrm{f}}-$ Wert von $0.21\left(\mathrm{CHCl}_{3} / \mathrm{MeOH} 9: 1\right)$ polarer als 39, färbt aber ebenso wie sie mit Anisaldehyd in der Wärme intensiv blau an. Ein hochaufgelöstes ESIMassenspektrum führte zur Summenformel $\mathrm{C}_{21} \mathrm{H}_{36} \mathrm{O}_{4}$, es liegt somit ein Isomer von 39 vor. Tatsächlich besitzen die NMR-Daten von $\mathbf{4 1}$ große Ähnlichkeit mit den für 39 ermittelteten. Im ${ }^{1}$ H-NMR-Spektrum erkennt man jedoch, dass die Methylgruppe an C-7 keinen Kopplungspartner mehr hat und als Singulett auftritt. Das quartäre Kohlenstoffatom C-7 besitzt im ${ }^{13} \mathrm{C}$-NMR-Spektrum eine Verschiebung von $\delta_{\mathrm{C}}=70.5$, was auf das Vorhandensein einer Hydroxygruppe schließen lässt. Dem Kohlenstoffatom C-6 kann in $\mathbf{4 1}$ eine Methylengruppe bei $\delta_{\mathrm{C}}=45.8$ zugeordnet werden. Somit befindet sich die Hydroxygruppe an C-7 und nicht wie bei 39 an C-6. Die relative Stereochemie von Betaenon I (41) wurde wie bei den Betaenonen G (39) und H (40) aus den Kopplungskonstanten des ${ }^{1} \mathrm{H}-\mathrm{NMR}-$ Spektrums und NOE-Korrelationen abgeleitet.

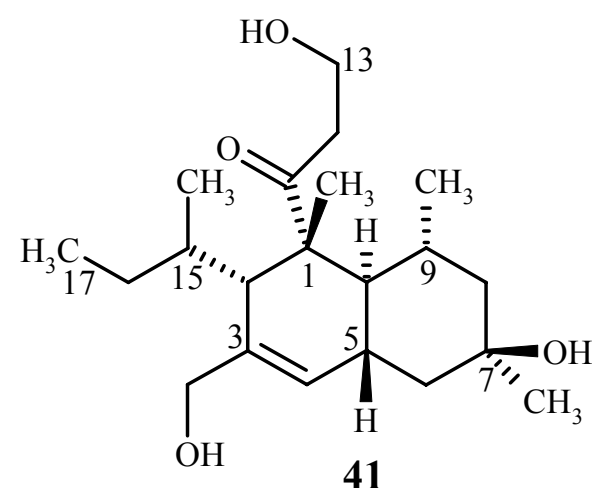




\subsubsection{Betaenon $J(42)$}

Die vierte Verbindung besitzt denselben $\mathrm{R}_{\mathrm{f}}$-Wert wie Betaenon I (41) und färbt ebenfalls mit Anisaldehyd in der Wärme intensiv blau an. Die Summenformel $\mathrm{C}_{21} \mathrm{H}_{36} \mathrm{O}_{4}$ wurde durch ein hochaufgelöstes ESI-Massenspektrum bestimmt und ist somit mit der von Betaenon G (39) und I (41) identisch. Die NMR-Spektren von 42 lassen sofort erkennen, dass es sich um ein weiteres Betaenon-Derivat handelt. Das ${ }^{13} \mathrm{C}-\mathrm{NMR}-$ Spektrum besitzt die größte Ähnlichkeit zu dem von 40, wobei jedoch das Signal der Methylgruppe C-17 fehlt. Dafür ist bei $\delta_{C}=61.5$ das Signal einer zusätzlichen Methylengruppe zu erkennen, der im ${ }^{1} \mathrm{H}-\mathrm{NMR}$-Spektrum Signale bei $\delta_{\mathrm{H}}=3.59$ und 3.68 zugeordnet werden können. Dies legt den Schluss nahe, dass 42 im Vergleich zu 40 an C-17 hydroxyliert ist. Die Signalverschiebung der benachbarten Methylengruppe um 10 ppm (C-16) ins Tieffeld bekräftigt zusätzlich die Position der Hydroxygruppe. Ebenso wie bei den anderen von Microsphaeropsis sp. Stamm 6288 gebildeten Betaenonen konnte die relative Stereochemie von Betaenon J (42) aus den Kopplungskonstanten des ${ }^{1} \mathrm{H}-\mathrm{NMR}$-Spektrum und NOE-Korrelationen hergeleitet werden.

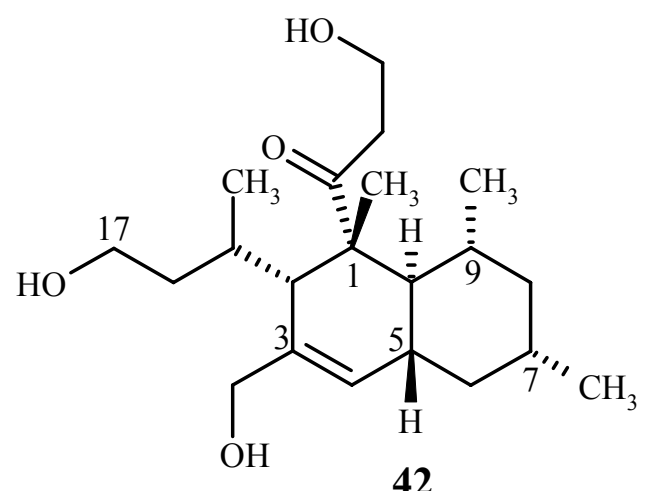




\subsubsection{Die Gruppe der Betaenone und Stemphyloxine}

1983 wurden die Betaenone A (43), B und C (44) aus dem Pilz Phoma betae isoliert und mit Hilfe einer Röntgenstrukturanalyse in ihrer Struktur aufgeklärt. ${ }^{73} \mathrm{Im}$ selben Jahr wurde von einer israelischen Forschungsgruppe aus dem Pilz Stemphylium botryosum eine Verbindung isoliert, die sich nur durch eine Hydroxymethylengruppe an C-15 von 44 unterscheidet und den Namen Stemphyloxin I (45) erhielt. ${ }^{74}$ Im folgenden Jahr konnte Stemphyloxin II (46) isoliert werden, ein Strukturanaloges zu 43. ${ }^{75}$ Bis heute wurden insgesamt 11 Vertreter dieser Substanzklasse als Phytotoxine beschrieben. ${ }^{76}$ Eine vergleichende Studie zur biologischen Aktivität der Vertreter dieser Verbindungsklasse zeigte, dass die strukturell verwandten Verbindungen 44 und 45 das Wachstum von Tomatenzellen am stärksten hemmen. ${ }^{77}$ Dabei war die hydroxylierte Verbindung $\mathbf{4 5}$ etwas stärker wirksam als $\mathbf{4 4}$. Die Hydroxymethylengruppe an C-15 scheint somit zu einer Verstärkung der Pytotoxizität zu führen.

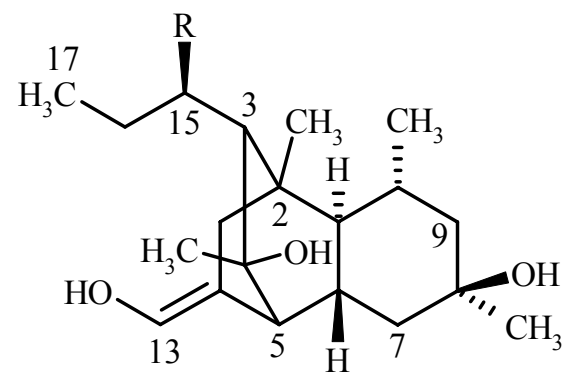

$43 \mathrm{R}=\mathrm{CH}_{3}$ $46 \mathrm{R}=\mathrm{CH}_{2} \mathrm{OH}$

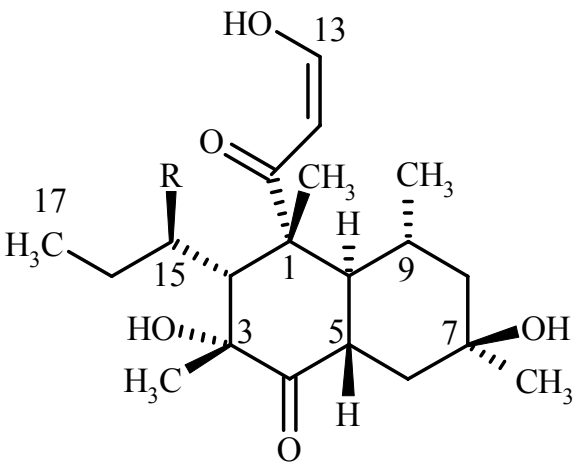

$44 \mathrm{R}=\mathrm{CH}_{3}$ $45 \mathrm{R}=\mathrm{CH}_{2} \mathrm{OH}$

Abbildung 18: Vergleich der Strukturen von Betaenone A (43) und C (44) mit denen von Stemphyloxin I (45) und Stemphyloxin II (46). 
Durch Biosyntheseuntersuchungen mit isotopenmarkierten Vorläufern konnte gezeigt werden, dass die Betaenone aus acht Acetateinheiten und fünfmal Methionin aufgebaut werden. ${ }^{78}$ Auch die Herkunft der Sauerstoffmoleküle ließ sich durch die Fütterung von $\left[1-{ }^{13} \mathrm{C},{ }^{18} \mathrm{O}_{2}\right]-$ Acetat und Fermentationen unter Zusatz des P-450-Inhibitors Ancymidol aufklären. ${ }^{79}$ Durch den Einsatz von Ancymidol kam es zur Akkumulation eines Biosynthesevorläufers der Betaenone. Dieses Probetaenon I (47) weist auf die Beteiligung einer Diels-Alder-Reaktion an der Biosynthese der Betaenone hin und scheint außerdem auch beim Stamm 6288 ein Vorläufer der isolierten Betaenone zu sein (Abbildung 19).

1 Acetyl-CoA 7 Malonyl-CoA 5 Methionin

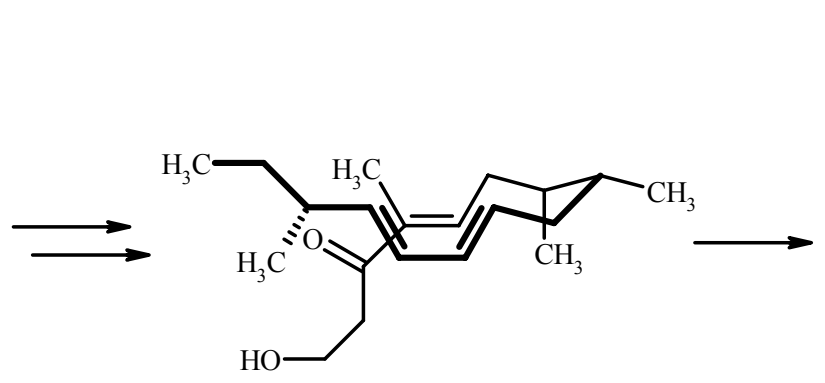<smiles>CC[C@H](C)[C@H]1C(CO)=C[C@H]2[C@H](O)[C@H](C)C[C@@H](C)[C@]2(C)[C@@]1(C)C(=O)CCO</smiles>

39<smiles>CC[C@H](C)[C@H]1C(CO)=C[C@@H]2C[C@@](C)(O)C[C@@H](C)[C@]2(C(=O)CCO)[C@H]1C</smiles>

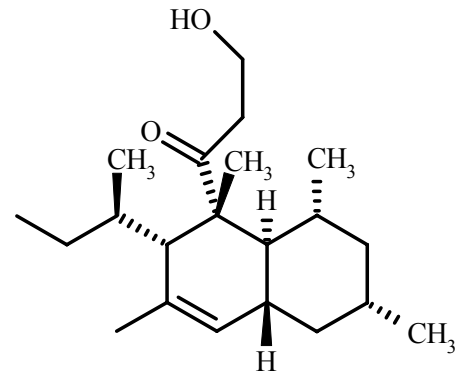

47<smiles>CC[C@H](C)[C@H]1C(CO)=C[C@@H]2C[C@@H](C)C[C@H](C)[C@H]2[C@]1(C)C(=O)CCO</smiles>

40

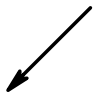<smiles>C[C@@H](CCO)[C@H]1C(CO)=C[C@@H]2C[C@H](C)C[C@H](C)[C@H]2[C@@]1(C)C(=O)CCO</smiles>

Abbildung 19: Gemeinsame Biosynthese der aus dem Stamm 6288 isolierten Betaenone. 


\subsection{Solanapyrone}

Aus Ruhefermentationen in P-Kolben (Medium: G20+NaCl) konnten aus dem Stamm 6288 drei neue Solanapyrone $(\mathbf{4 8}, \mathbf{4 9}$ und $\mathbf{5 1})$ isoliert werden.

\subsubsection{Solanapyron H (48)}

Solanapyron $\mathrm{H}$ wurde als farbloses Öl isoliert, das mit Anisaldehyd in der Wärme grün anfärbt. Ein EI-Massenspektrum liefert das Molekülion bei $\mathrm{m} / \mathrm{z}=262$, dessen Hochauflösung zur Summenformel $\mathrm{C}_{16} \mathrm{H}_{22} \mathrm{O}_{3}$ führt. Im ${ }^{13} \mathrm{C}$-NMR zeigen sich dementsprechend die Signale von 16 Kohlenstoffatomen, die einer Methyl-, fünf Methylen-, fünf aliphatischen und drei olefinischen Methingruppen sowie zwei quartären Kohlenstoffatomen entsprechen. Das Signal der Methylgruppe tritt im ${ }^{1}$ H-NMR-Spektrum bei $\delta_{\mathrm{H}}=0.97$ als Dublett auf und ist somit mit einer Methingruppe verknüpft. Im Bereich von $\delta_{\mathrm{H}}=1.36-2.58$ erkennt man insgesamt 12 aliphatische Protonen die zu je vier Methylen- und Methingruppen gehören. Des Weiteren treten bei $\delta_{\mathrm{H}}=4.10$ eine Methin- und bei $\delta_{\mathrm{H}}=4.49$ eine Methylengruppe auf, deren Verschiebungen für eine Verknüpfung mit Sauerstoff sprechen. Von den Signalen der drei olefinischen Methingruppen bei $\delta_{\mathrm{H}}=5.42,5.47$ und 5.61 zeigend die beiden letzteren eine Kopplungskonstante von ${ }^{3} J=10.0 \mathrm{~Hz}$, was auf eine cis konfigurierten Doppelbindung hindeutet. Im ${ }^{13} \mathrm{C}$-NMR-Spektrum findet man, neben den Signalen dieser Doppelbindung bei $\delta_{\mathrm{C}}=131.1$ und 131.2 noch zwei weitere Signale bei $\delta_{\mathrm{C}}=106.7$ und 179.1. Diese können zusammen mit einem Signal bei $\delta_{\mathrm{C}}=192.1$ einem $\alpha, \beta$ ungesättigtem Keton zugeordnet werden. Aus einem ${ }^{1} \mathrm{H}-{ }^{1} \mathrm{H}-\mathrm{COSY}$-Experiment kann man drei Spinsysteme ableiten die sich durch ein HMBC-Experiment eindeutig miteinander verknüpft lassen (Abbildung 20).<smiles>[R]OC1CCC2C(C=CC(C)C2[R])C1</smiles>
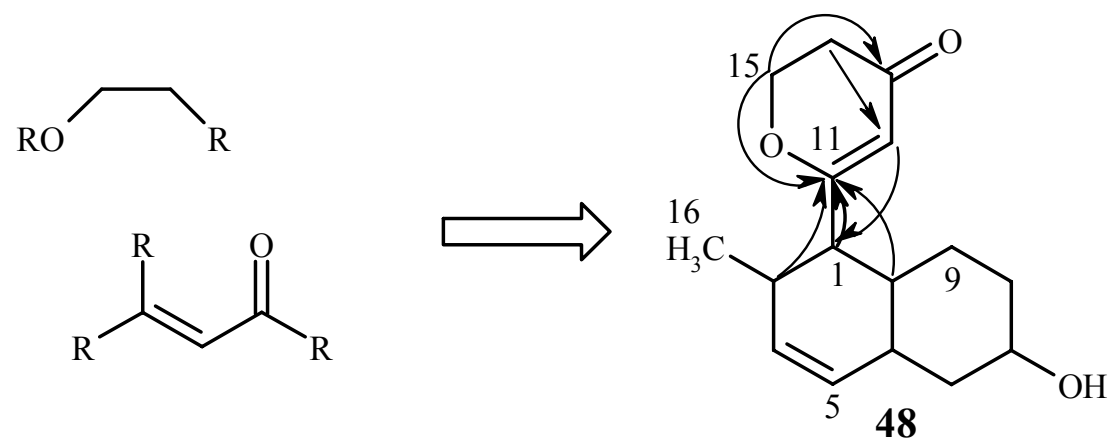

Abbildung 20: Aus dem COSY abgeleitete Strukturfragmente von 48 und deren Verknüpfung über ausgewählte HMBC-Korrelationen. 
Das Vorliegen eines Dihydro- $\gamma$-pyrons wird zusätzlich durch die gute Übereinstimmung der ${ }^{13} \mathrm{C}$-NMR-Daten mit denen von 5,6-Dihydro-6-methyl-pyran-4-on ${ }^{80}$ bestätigt. Die relative

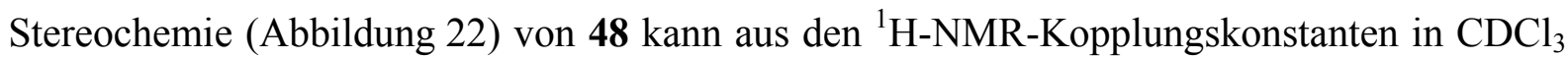
und Pyridin- $\mathrm{d}_{5}$ abgeleitet werden und wurde zusätzlich durch 1D-NOE-Korrellationen bestätigt.

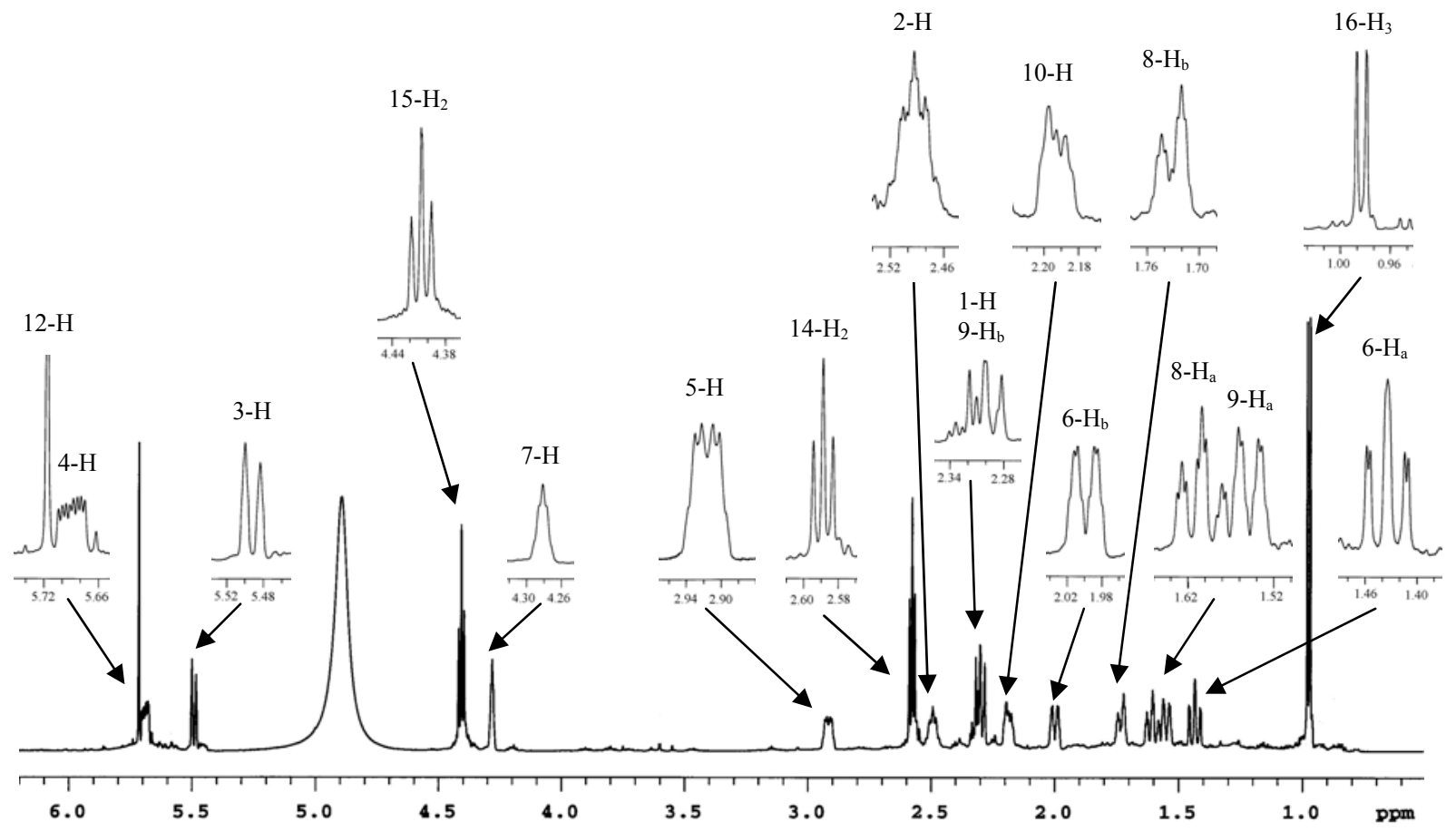

Abbildung 21: ${ }^{1} \mathrm{H}-\mathrm{NMR}-$ Spektrum (600 MHz, Pyridin-d ${ }_{5}$ ) von Solanapyron H (48).
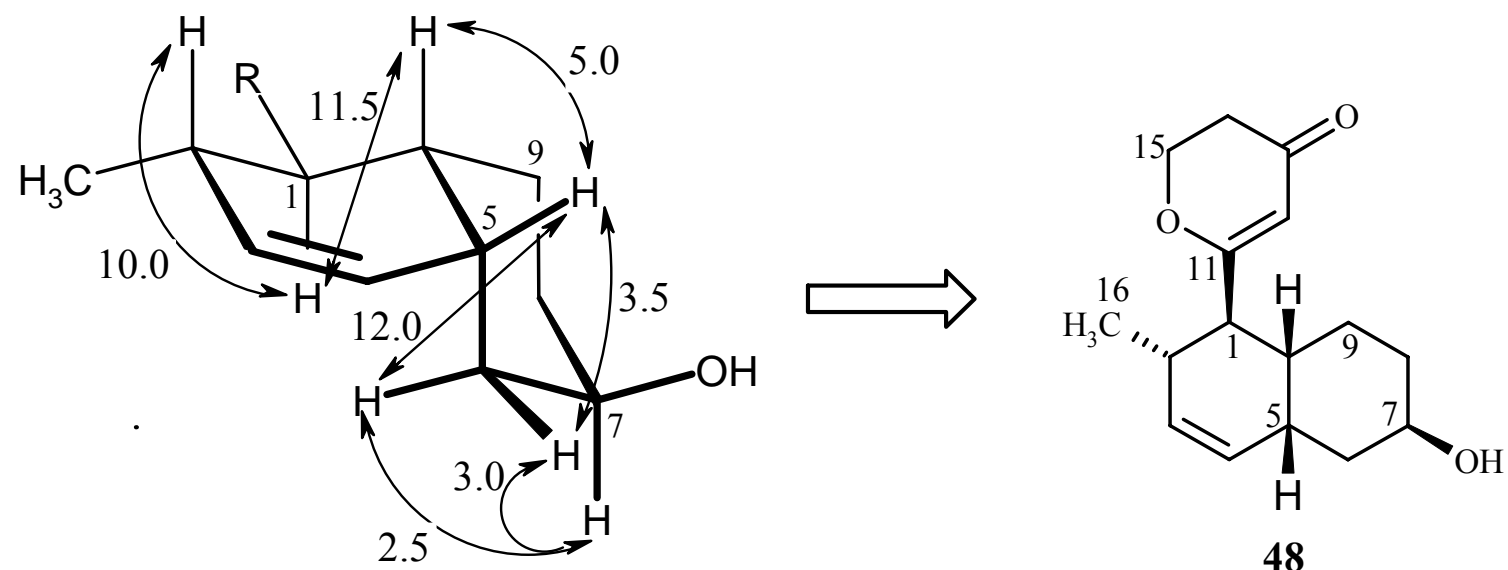

48

Abbildung 22: Ausgewählte Kopplungskonstanten in $\mathrm{Hz}$ und die sich daraus ergebende relative Stereochemie von Solanapyron H (48). 


\subsubsection{Solanapyron I (49)}

Ein EI-Massenspektrum der als farbloses Öl isolierten Substanz ergab die Molmasse 347, deren ungerader Wert auf die Anwesenheit von Stickstoff hinweist. Dem ${ }^{13} \mathrm{C}-\mathrm{NMR}-$ Spektrum sind Signale für eine Methyl-, fünf Methylen-, fünf aliphatische und drei olefinische Methingruppen sowie vier quartäre Kohlenstoffatome zu entnehmen. Ein weiteres Signal bei $\delta_{\mathrm{C}}=191.3$ kann einer Aldehydfunktion zugeordnet werden. Mit Hilfe dieser Daten lässt sich für 49 die Summenformel $\mathrm{C}_{19} \mathrm{H}_{25} \mathrm{NO}_{5}$ aufstellen, die durch HREI-MS bestätigt wurde. Das ${ }^{13}$ C-NMR-Spektrum ist dem von Solanapyron H (48) sehr ähnlich, im Bereich des bicyclischen Ringsystems sind die Spektren praktisch identisch. Aufgrund der Summenformel und der Konnektivitäten aus dem HSQC-Spektrum müssen im Protonenspektrum drei austauschbare Protonen vorhanden sein, von denen aber nur ein Signal bei $\delta_{\mathrm{H}}=10.82$ detektiert werden kann. Weiterhin finden sich im ${ }^{1}$ H-NMR-Spektrum, neben den Signalen des Ringsystems, zwei mit einander verknüpfte Methylengruppen bei $\delta_{\mathrm{H}}=3.55$ und 3.90, das Signal einer Methingruppe bei $\delta_{\mathrm{H}}=6.02$ und das Proton der Aldehydfunktion bei $\delta_{\mathrm{H}}=9.98$. Eine mit diesen Informationen durchgeführte Datenbankrecherche ${ }^{53}$ führte $\mathrm{zu}$ keinem Ergebnis. Lässt man jedoch die Hydroxylgruppe am bicyclischen Ringsystem weg, so erhält man eine mögliche Lösung: Solanapyron $C^{81}(\mathbf{5 0})$. Ein Vergleich der spektroskopischen Daten offenbart, dass 49 dasselbe Pyronsystem wie $\mathbf{5 0}$ besitzt und es sich somit um das an C-7 hydroxylierte Derivat von Solanapyron C handeln muss. Die Stereochemie ist in Anlehnung an 48 und 50 dargestellt.

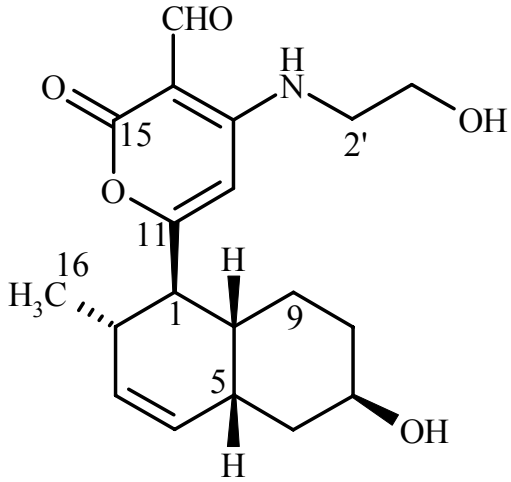

49

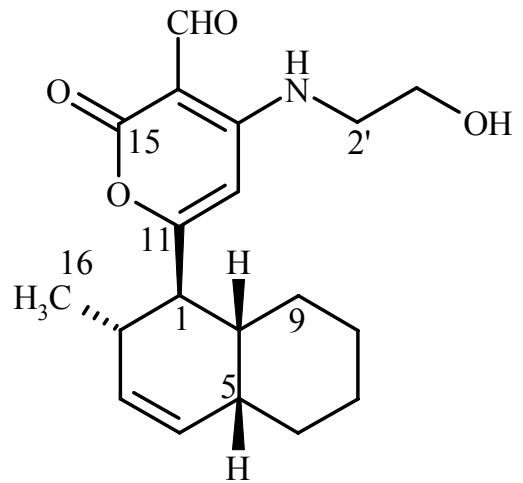

50 


\subsubsection{Solanapyron J (51)*}

Die Substanz wurde als gelber Feststoff aus Ruhefermentationen unter Zusatz von $\mathrm{NaCl}$ isoliert. Ein ESI-Massenspektrum ergab nach Hochauflösung die Summenformel $\mathrm{C}_{28} \mathrm{H}_{32} \mathrm{O}_{8}$. Im ${ }^{13}$ C-NMR-Spektrum erkennt man die erwarteten 28 Signale, die drei Methyl-, fünf Methylen-, fünf aliphatischen und sechs olefinischen Methingruppen sowie neun quartären Kohlenstoffatomen zugeordnet werden können. Im ${ }^{1} \mathrm{H}-\mathrm{NMR}-$ Spektrum fallen bei $\delta_{\mathrm{H}}=5.46$ und 5.61 zwei Signale auf, die typisch für die Doppelbindung des bicyclischen Systems der Solanapyrone H (48) und I (49) sind. Die Vermutung, dass 51 dasselbe Ringsystem wie die beiden anderen Verbindungen besitzt, wird durch die NMR-Spektren bestätigt. Im ${ }^{1} \mathrm{H}-\mathrm{NMR}$ Spektrum erkennt man eine weitere Doppelbindung mit Resonanzen von $\delta_{H}=7.03$ und 7.36, die eigentlich typisch für die ebenfalls aus dem Stamm 6288 isolierten $\alpha$-Pyrone ist. Tatsächlich findet man in den NMR-Spektren alle zum Dehydrotaiwapyron (23) gehörenden Signale, lediglich das Signal der Hydroxymethylengruppe an C-7 fehlt. Im ${ }^{1}$ H-NMRSpektrum verbleiben somit nur noch die Signale einer Methoxygruppe bei $\delta_{\mathrm{H}}=3.98$ und zwei Singuletts bei 6.11 und 6.81, die keinem der beiden Spinsysteme zugeordnet werden konnten. Im ${ }^{13} \mathrm{C}$-NMR-Spektrum wurden insgesamt acht Signale noch nicht zugeordnet. Durch ein HMBC-Experiment können diese acht Kohlenstoffatome zu einem Pyronring und einer an diesen gebundene Doppelbindung verknüpft werden. Die Struktur des Pyronrings kann zusätzlich durch einen Vergleich der ${ }^{13} \mathrm{C}$-NMR-Daten mit Solanapyron $\mathrm{A}^{81}$ abgesichert werden. Des Weiteren finden sich im HMBC Korrelationen durch die die drei Strukturfragmente zusammengefügt werden können. Die Stereochemie ist in Anlehnung an Solanapyron I (49) dargestellt, die Konformation der Doppelbindung konnte leider nicht bestimmt werden. Aus sterischen Gründen sollte die Z-Konfiguration bevorzugt sein.

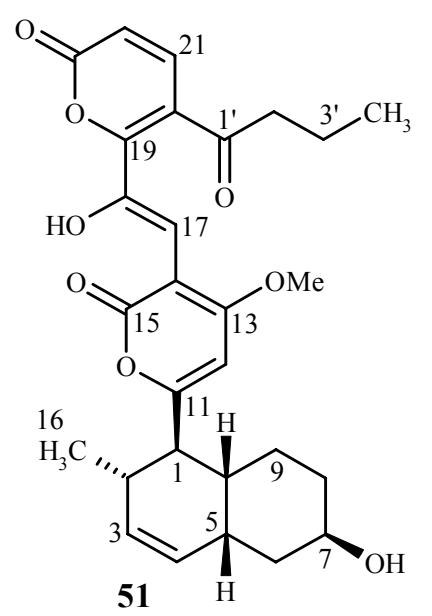

\footnotetext{
* Struktur im Pyronteil fehlerhaft, Korrektur siehe Seite 30.
} 


\subsubsection{Die Gruppe der Solanapyrone}

Bei den Solanapyronen handelt es sich um phytotoxische Substanzen aus dem Pilz Alternaria solani. ${ }^{81}$ Dieser verursacht bei Kartoffeln die so genannte Dürrfleckenkrankheit, bei der sich auf dem Kartoffellaub zunächst punktartige, sich schnell vergrößernde, dunkelbraune Blattflecken bilden. Beschrieben sind bisher die Solanapyrone A-G. Durch Biosyntheseuntersuchungen konnte gezeigt werden, dass der Hauptmetabolit, Solanapyron A (52), aus acht Acetateinheiten und zweimal Methionin aufgebaut wird. Schon früh wurde vermutet, dass die Solanapyrone ebenso wie die Betaenone über eine intramolekulare DielsAlder-Reaktion cyclisieren. Diese Vermutung wurde durch die Isolierung zweier Minderkomponenten, Solanapyron D (53) und E, erhärtet, bei denen es sich um Diastereomere der Solanapyrone A und C handelt. Die Bildung dieser Minderkomponenten, die im Verhältnis 1:6 vorliegen, kann durch zwei unterschiedliche Übergangszustände während der Diels-Alder-Reaktion erklärt werden (Abbildung 23).

1 Acetyl-CoA

7 Malonyl-CoA

2 Methionin
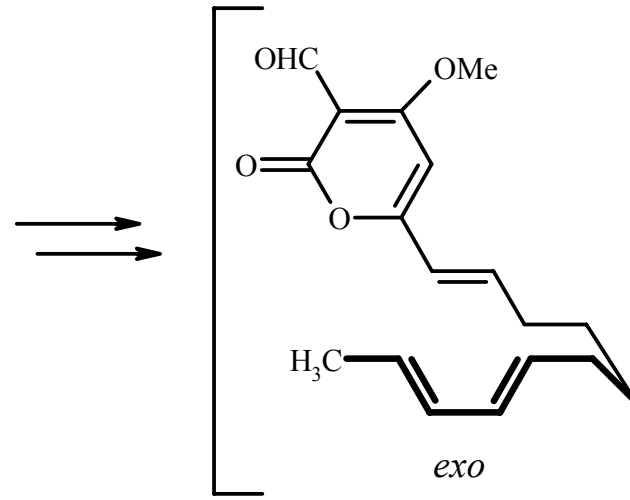

exo
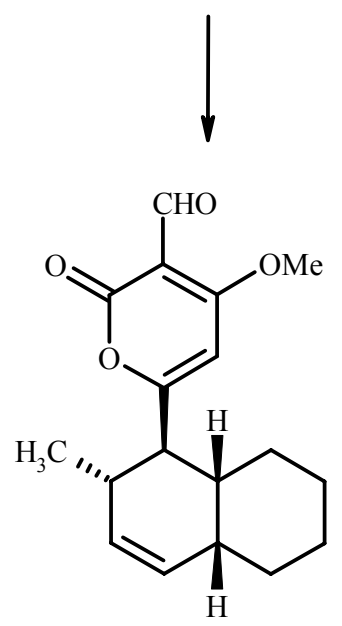

52

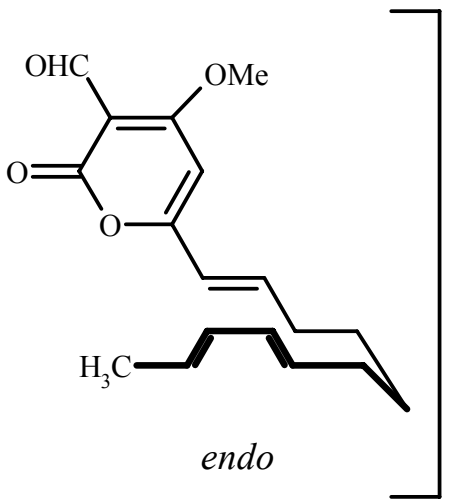

$$
\downarrow
$$<smiles>COc1cc([C@H]2[C@@H](C)C=C[C@H]3CCCC[C@H]32)oc(=O)c1C=O</smiles>

53

Abbildung 23: Bildung der diastereomeren Solanapyrone A (52) und D (53) durch unterschiedliche Übergangszustände bei der Cyclisierung. 
In wässriger Lösung reagiert das im Übergangszustand dargestellte Prosolanapyron ebenfalls zu den beiden Produkten allerdings liegt hier das Verhältnis mit 3:97 auf der Seite der Minderkomponente 53. ${ }^{82}$ Der endo-Übergangszustand scheint also der thermodynamisch begünstigte zu sein. Wird das Prosolanapyron hingegen $\mathrm{zu}$ einem zellfreien Extrakt des Stammes Alternaria solani gegeben, so überwiegt das exo-Produkt. Diese Ergebnisse zeigen eindeutig, dass an der Bildung der Solanapyrone ein exo-selektives Enzym beteiligt sein muss.

Bereits 1996 stellte LASCHAT die Frage, ob die Natur eine Diels-Alder-Reaktion kennt ${ }^{83}$ und noch immer wird die Existenz einer Diels-Alderase, deren alleinige Funktion die Katalyse einer Diels-Alder-Reaktion ist, diskutiert. ${ }^{84}$ Alternativ besteht die Möglichkeit, dass das Enzym, welches in der Biosynthese den vorhergehenden Reaktionsschritt katalysiert, auch die sich anschließende Diels-Alder-Reaktion ermöglicht. Dies könnte durch eine dem Übergangszustand ähnelnde räumliche Organisation des Edukts geschehen. Das Problematische an einer Diels-Alderase ist, dass der Übergangszustand der Struktur des Produktes relativ ähnlich sein müsste und somit das Produkt an das Enzym binden könnte, was ein Fortschreiten der Reaktion erschweren würde.

Im Falle der Solanapyrone konnte durch die Isolierung des für die Diels-Alder-Reaktion verantwortlichen Enzyms gezeigt werden, dass dieses Enzym keine reine Diels-Alderase ist. Es katalysiert neben der Diels-Alder-Reaktion noch die zur Bildung der Aldehydgruppe an C-17 des Prosolanapyrons notwendige Oxidation. Somit überführt dieses an der Biosynthese der Solanapyrone beteiligte Enzym das Edukt nur in einen für die Diels-Alder-Reaktion günstigen Übergangszustand.

\section{5. Überlegungen zur Bildung von Solanapyron $\mathbf{J}$ (51) und 7-(1-Methyl-ethyliden)- taiwapyron (25)*}

Das aus Kulturen von Stamm 6288 isolierte Solanapyron J (51) besteht aus zwei Strukturelementen. Der untere Teil der Struktur von $\mathbf{5 1}$ ist weitgehend mit Solanapyron A (54) identisch, der obere mit Dehydrotaiwapyron (23). Man kann vermuten, dass 51 durch die Verknüpfung von 54 mit 23 entsteht. In Analogie dazu könnte 25 durch die Verknüpfung von 23 mit einem $\mathrm{C}_{3}$-Körper wie Aceton entstehen (Abbildung 24).

\footnotetext{
* Die Strukturen von 25 und $\mathbf{5 1}$ sind fehlerhaft, Korrektur siehe Seite 30.
} 


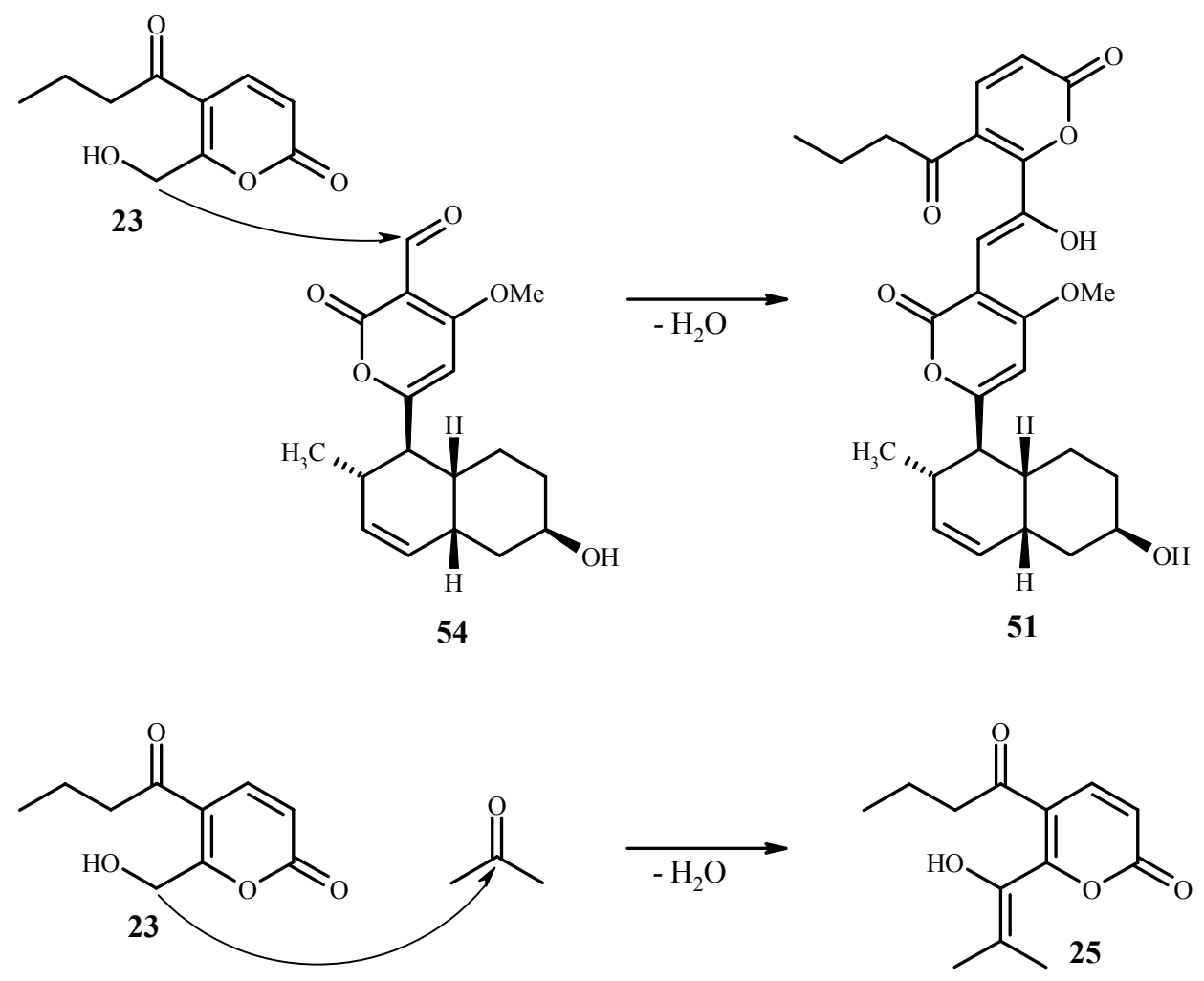

Abbildung 24: Hypothetische Bildung von Solanapyron J (51) und 7-(1-Methyl-ethyliden)-taiwapyron (25).

Um zu überprüfen, ob letztere Reaktion spontan abläuft, wurde eine Lösung von Dehydrotaiwapyron (23) in Aceton für mehrere Stunden bei verschiedenen pH-Werten ( $\mathrm{pH}=4.0,7.0$ und 9.0) gerührt. Dabei kam es unter basischen Bedingungen zur Zersetzung von 23 in mehrere undefinierte Produkte, von denen keines mit 25 identisch war. Auch unter den anderen Bedingungen konnte keine Bildung von 25 beobachtet werden. In einem weiteren Versuch wurde versucht $\mathbf{2 3}$ mit Benzaldehyd umzusetzen, auch hier trat keine Reaktion ein.

Es ist somit plausibel, wenn man annimmt, dass die Kondensation von Dehydrotaiwapyron (23) mit einer aktiven Carbonylverbindung enzymatisch katalysiert wird. Für die Bildung von 25 gibt es zwei verschiedene Möglichkeiten, zum einen kann 25 in Analogie zur Bildung von 51 durch Kondensation von 23 mit einem $\mathrm{C}_{3}$-Körper entstehen. Zum andern ist auch die Bildung aus einer Isoprenoid-Einheit und viermal Acetat denkbar. Welche dieser beiden Möglichkeiten zutrifft, könnte durch die Fütterung von markiertem Acetat entschieden werden. Da 25 aus Ruhefermentationen in P-Kolben (Medium: E2) lediglich als Minderkomponente in Ausbeuten von $1.0 \mathrm{mg} / \mathrm{L}$ anfällt, wurde von einer Fütterung mit markiertem Acetat abgesehen. 


\subsection{Beurteilung der Biosyntheseleistung des Stammes}

Aus verschiedenen Kultivierungsansätzen des Stammes 6288 konnten insgesamt 13 Sekundärmetabolite isoliert werden, von denen elf neu waren. Bei allen Metaboliten handelt es sich um Polyketide, die allerdings unterschiedliche Grundstrukturen besitzen.

Die beiden in Schüttelkultur gebildeten Spiciferonen sind Hexaketide aus sechs Acetat- und zwei zusätzlichen $\mathrm{C}_{1}$-Einheiten. Dabei konnte mit Prospiceferon A (18) der bisher nur postulierte Biosynthesevorläufer von Spiciferon A (17) isoliert werden. Durch die Fermentationsbedingungen scheint die Biosynthese von $\mathbf{1 7}$ gehemmt zu werden, so dass 18 zur Haupkomponente wird.

Für die Erschießung des Metabolitenspektrums des Stammes 6288 erwiesen sich Ruhekulturen als besonders wertvoll. Durch Verwendung verschiedener Nährmedien konnte der Stamm zur Bildung von elf weiteren Sekundärmetaboliten, die zu drei Substanzklassen gehören, angeregt werden.

Die vier isolierten $\alpha$-Pyronen $(\mathbf{2 2}$ - 24) werden aus fünf Acetat-Einheiten aufgebaut. Das $\alpha$-Pyron 25 kann entweder durch Kondensation von 23 mit einer $\mathrm{C}_{3}$-Einheitt, deren Herkunft noch nicht geklärt ist, oder aus einer Isoprenoid-Einheit und viermal Acetat gebildet werden. Bei den vier Betaenone (39-42) handelt es sich um Oktaketide aus acht Acetat- und fünf $\mathrm{C}_{1^{-}}$ Einheiten. Die Betaenone werden höchstwahrscheinlich über eine Diels-Alder-Reaktion cyclisiert und dann unterschiedlich oxidiert. Dabei gehen die Betaenone G (39), I (41) und $\mathrm{J}$ (42) durch regiopezifische Oxidationen aus Betaenon H (40) hervor.

Solanapyron H (48) und I (49) werden aus acht Acetat-Einheiten aufgebaut, wobei 49 zusätzlich durch eine $\mathrm{C}_{1}$-Einheit und eine vermutlich aus Glycin stammende Seitenkette ergänzt wird. Für die Gruppe der Solanapyrone wurde durch verschiedene Experimente bewiesen, dass sie durch eine intramolekulare Diels-Alder-Reaktion cyclisieren. ${ }^{82}$ Solanapyron J (51) wird höchstwahrscheinlich durch Kondensation von Solanapyron A (54) mit Dehydrotaiwapyron (23) gebildet und ist ein hybrider Naturstoff, mit Strukturelementen zweier Substanzklassen.

Zusammenfassend kann gesagt werden, dass sich der Stamm 6288 bei der Variation der Fermentationsbedingungen als sehr variabel in seinem Metabolitenmuster erwiesen hat. Die vier für den Aufbau der verschiedenen Metabolite benötigten Biosynthesewege werden bei unterschiedlichen Bedingungen aktiviert. 


\section{Alternaria sp. Stamm 6587 und 6588}

Die Pilze Alternaria sp. Stamm 6587 und 6588 wurden aus der in der Ostsee bei Travemüde gesammelten Grünalge Enteromorpha linza isoliert. Die beiden Pilzstämme unterscheiden sich in ihrer Morphologie, zeigten aber bei Kultivierung in Schüttel- oder Ruhekultur im chemischen Screening ein identisches Metabolitenmuster.

Alternaria sp. Stamm 6588 fiel bei der Kultivierung in Schüttelkolben in den Medien 1158, 1549 und M2 durch die Bildung von drei unter UV-Licht (366 nm) orange fluoreszierender Metabolite (57 - 59) auf und produzierte in Ruhekultur zwei weitere Substanzen (55 und 56).

Durch Zugabe von Enzyminhibitoren konnte der Stamm 6588 zur Bildung von vier zusätzlichen Metaboliten (64, 65, 69 und 70) gebracht werden. Alle neun Verbindungen erwiesen sich als literaturbekannt.

Bei der biologischen Testierung durch die BASF AG fiel ein Extrakt durch fungizide Eigenschaften gegen Pyricularia oryzae auf. Ob eine der isolierten Substanzen für diese Wirkung verantwortlich ist, konnte aufgrund ausstehender Testergebnisse noch nicht geklärt werden.

\subsection{Alternariol (55)}

Die als farbloser Feststoff isolierte Substanz liefert im ESI-Massenspektrum den Pseudomolekülpeak bei m/z=257 [M-H] $]^{-}$und zeigt im ${ }^{13} \mathrm{C}-\mathrm{NMR}-$ Spektrum Signale für 14 Kohlenstoffatome. Im ${ }^{1}$ H-NMR-Spektrum sind die Signale von einer Methylgruppe $\left(\delta_{\mathrm{H}}=2.55\right)$, vier Methingruppen $\left(\delta_{\mathrm{H}}=6.88-7.42\right)$ und drei austauschbaren Protonen $\left(\delta_{\mathrm{H}}=8.72,12.50\right.$ und 12.59) $\mathrm{zu}$ erkennen. Aus diesen Ergebnissen lässt sich die Summenformel $\mathrm{C}_{14} \mathrm{H}_{10} \mathrm{O}_{5}$ ableitet, die durch ein hochaufgelöstes Massenspektrum bestätigt werden konnte. Die vier Methingruppen gehören auf Grund einer Kopplungskonstante von jeweils ${ }^{4} J=2.0 \mathrm{~Hz}$ zu zwei tetrasubstituierten aromatischen Systemen, die die beiden Methinprotonen jeweils in meta-Position tragen. Durch eine mit diesen Informationen durchgeführte Datenbankrecherche ${ }^{54}$ und den Vergleich mit Literaturdaten konnte $\mathbf{5 5}$ als Alternariol $^{85}$ identifiziert werden. 


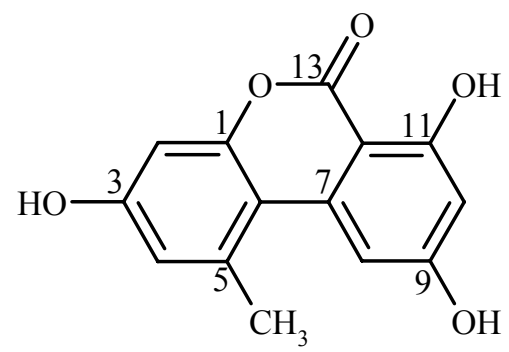

55

\subsection{Alternariol-9-methylether (56)}

Die ${ }^{1} \mathrm{H}$ - und ${ }^{13} \mathrm{C}-\mathrm{NMR}-$ Spektren von 55 und der als weißen Feststoff erhaltenen Verbindung stimmen fast vollständig überein. Es fehlt lediglich einer Hydroxygruppe $\left(\delta_{\mathrm{H}}=8.72\right)$ an deren Stelle eine Methoxygruppe $\left(\delta_{\mathrm{H}}=3.82, \delta_{\mathrm{C}}=55.8\right)$ tritt. Durch ein ESI-Massenspektrum kann die vermutete Summenformel $\mathrm{C}_{15} \mathrm{H}_{12} \mathrm{O}_{5}$ bestätigt werden. Die Position der Methylgruppe ergibt sich aus einem HMBC-Experiment, welches eine Kopplung zwischen der Methoxygruppe und C-9 zeigt. Bei 56 handelt es sich somit um Alternariol-9-methylether ${ }^{86}$.

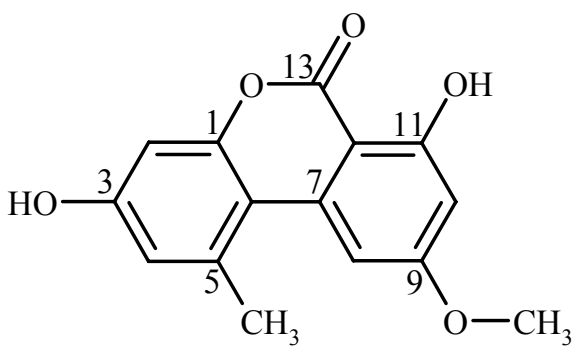

56

Die beiden Verbindungen 55 und 56 sind typische Alternaria-Mykotoxine, deren akute Toxizität in Mäusen mit LD L -Werten von 400 mg/kg (55) und 200 mg/kg (56) beträgt. $^{85,86}$ 55 und 56 lassen sich auch in von Alternaria sp. befallenen Nutzpflanzen, wie Tomaten oder verschiedenen Getreidesorten nachweisen und sind möglicherweise für Vergiftungen von Nutztieren verantwortlich. ${ }^{87}$ Beide Naturstoffe gehören zu den Heptaketiden, wobei zunächst 55 durch Kondensation von Acetyl-CoA und sechs Einheiten Malonyl-CoA gebildet und anschließend durch Methylierung in 56 überführt wird (Abbildung 25). ${ }^{88}$ 

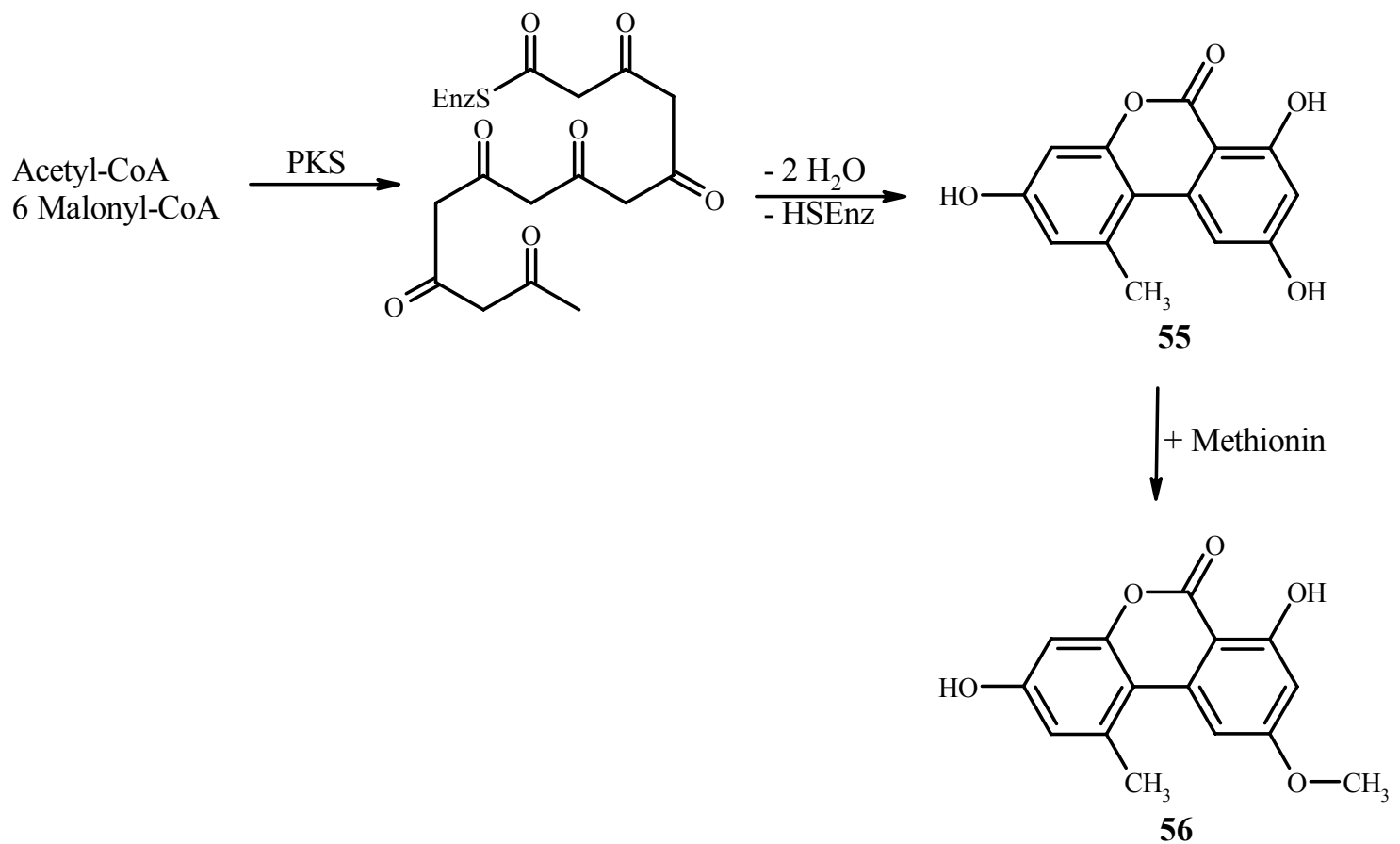

Abbildung 25: Biosynthese von Alternariol (55) und Alternariol-9-methylether (56).

\subsection{Perylenchinone}

Stamm 6588 produzierte in Schüttelkultur und bei sämtlichen Ruhefermentationen in P-Kolben drei Perylenchinone (57 - 59).

\subsubsection{Alterperylenol (57)}

Der gelbe, unter UV-Licht $(366 \mathrm{~nm})$ stark fluoreszierende Festsoff zeigt im ESIMassenspektrum bei $\mathrm{m} / \mathrm{z}=349$ das $[\mathrm{M}-\mathrm{H}]$--Ion. Im ${ }^{13} \mathrm{C}-\mathrm{NMR}-\mathrm{Spektrum}$ erkennt man die Signale für eine Methylen-, zwei aliphatische und sechs olefinische Methingruppen sowie für elf quartäre Kohlenstoffatome. Aus der durch ein hochaufgelöstes ESI-Massenspektrum bestimmten Summenformel $\mathrm{C}_{20} \mathrm{H}_{14} \mathrm{O}_{6}$ ergibt sich, dass das Molekül vier austauschbare Protonen enthalten muss, die man im ${ }^{1} \mathrm{H}-\mathrm{NMR}-S p e k t r u m$ bei $\delta_{\mathrm{H}}=4.91,5.05,12.50$ und 12.58 findet. Die Verschiebung der beiden Hydroxygruppen bei $\delta_{\mathrm{H}}=12.50$ und 12.58 weist auf ihre peri-Stellung zu Carbonylgruppen hin. Die sechs olefinischen Methingruppen treten sämtlich als Dublett im Bereich von $\delta_{\mathrm{H}}=6.34-8.07$ auf. Eine mit der Summenformel und den oben genannten Strukturelementen durchgeführte Datenbanksuche ${ }^{54}$ führt zu Alterperylenol ${ }^{89}$ (57). 


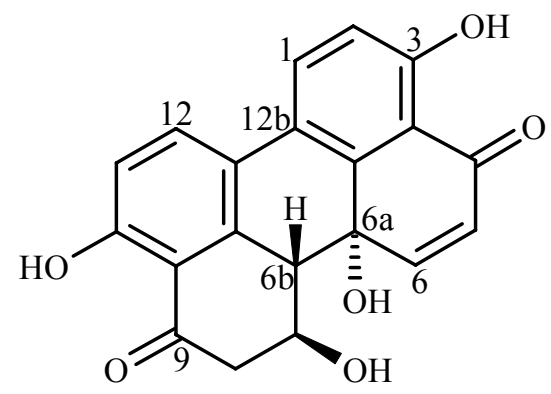

57

\subsubsection{5,6-Dihydroalterperylenol (58)}

Wie 57 zeigt auch 58 unter UV-Licht $(366 \mathrm{~nm})$ starke Fluoreszenz. Die Summenformel konnte durch ein hochaufgelöstes ESI-Massenspektrum mit $\mathrm{C}_{20} \mathrm{H}_{16} \mathrm{O}_{6}$ bestimmt werden. Die 20 Kohlenstoffe lassen sich anhand des ${ }^{13} \mathrm{C}-\mathrm{NMR}-$ Spektrums als drei Methylen-, zwei aliphatische und vier olefinische Methingruppen sowie elf quartäre Kohlenstoffatome identifizieren. Dies führte zusammen mit der Summenformel zu der Vermutung, dass es sich um ein hydriertes Derivat von 57 handelt. Tatsächlich fehlen im ${ }^{1}$ H-NMR-Spektrum die Signale der Doppelbindung zwischen C-5 und C-6. Stattdessen findet man bei $\delta_{\mathrm{H}}=2.41$ und 2.68 sowie bei $\delta_{\mathrm{H}}=3.03$ und 3.15 die Signale der beiden zusätzlichen Methylengruppen. Es liegt somit 5,6-Dihydroalterperylenol (58) vor, was sich beim Vergleich mit Literaturdaten ${ }^{89}$ bestätigte. Auch der gemessene Drehwert stimmt mit dem Literaturwert überein und bestätigt somit, dass C-6a R- und C-6b sowie C-7 S-konfiguriert sind.<smiles></smiles>

58

\subsubsection{Stemphyperylenol (59)}

Die unter UV-Licht (366 nm) ebenfalls stark fluoreszierende Substanz zeigt im ${ }^{13} \mathrm{C}-\mathrm{NMR}$ Spektrum zehn Signale, die einer Methylen-, zwei aliphatischen und zwei olefinischen Methingruppen sowie fünf quartären Kohlenstoffatomen entsprechen. Im ${ }^{1} \mathrm{H}-\mathrm{NMR}-\mathrm{Spektrum}$ 
treten die Signale der aliphatischen Methingruppen bei $\delta_{\mathrm{H}}=3.76$ und 4.76 auf, das Signal der Methylengruppe ist diastereotop aufgespalten. Die beiden olefinischen Methingruppen bei $\delta_{\mathrm{H}}=6.82$ und 8.15 sind mit einer Kopplungskonstante von ${ }^{3} J=8.5 \mathrm{~Hz}$ zum Dublett aufgespalten. Ein ESI-Massenspektrum liefert den Peak höchster Masse ([M-H] $]^{-}$bei $\mathrm{m} / \mathrm{z}=351$, aus dem durch Hochauflösung die Summenformel $\mathrm{C}_{20} \mathrm{H}_{16} \mathrm{O}_{6}$ abgeleitet werden kann. Da im ${ }^{13} \mathrm{C}$-NMR-Spektrum lediglich zehn C-Atome beobachtet werden konnte, muss es sich bei 59 um ein symmetrisches Molekül handeln. Durch eine Datenbanksuche ${ }^{54}$ konnte 59 als Stemphyperylenol ${ }^{90}$ identifiziert werden.

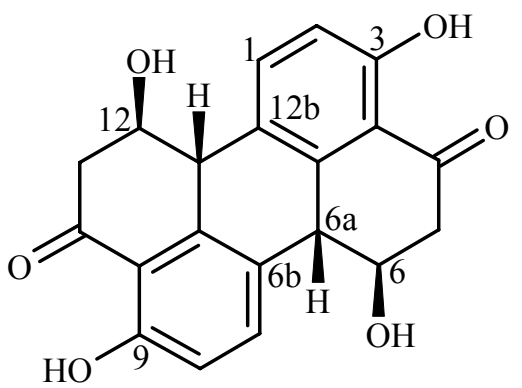

59

\subsubsection{Zur Biosynthese der isolierten Perylenchinone}

Die drei aus dem Stamm 6588 isolierten Perylenchinone (57 - 59) gehören ebenso wie 55 und $56 \mathrm{zu}$ den häufig isolierten Alternaria-Mycotoxinen. In der Literatur werden sie als phytotoxisch und antifungisch wirksam beschrieben. 58 ist außerdem mutagen (erbgutverändernd), ${ }^{91}$ wobei davon ausgegangen werden kann, dass dies auch für die beiden anderen Perylenchinone (57 und 59) gilt.

Durch Fütterungsexperimente mit $\left[2-{ }^{13} \mathrm{C}\right]$ Acetat konnte gezeigt werden, dass Alterperylenol (57) ebenso wie andere Perylenchinone aus zwei identischen Pentaketideinheiten aufgebaut wird. ${ }^{92,93}$ Dieses Pentaketid wird dann in Analogie zur Melanin-Biosynthese (siehe Kapitel A.III.2.4) über mehrere Zwischenstufen in 1,8-Dihydroxynaphthalen (DHN) umgewandelt. Durch oxidative Kupplung von zwei Einheiten DHN entsteht dann 4,9Dihydroxyperylen-3,10-chinon (60), dessen weitere Oxidation an unterschiedlichen Positionen zu 57 und 58 oder 59 führt. Unterstützt wird der vorgeschlagene Biosyntheseweg durch die Tatsache, dass neben $\mathbf{6 0}$ auch die Zwischenprodukte $\mathbf{6 1}$ und $\mathbf{6 2}$ isoliert werden konnten. ${ }^{89,94}$ 

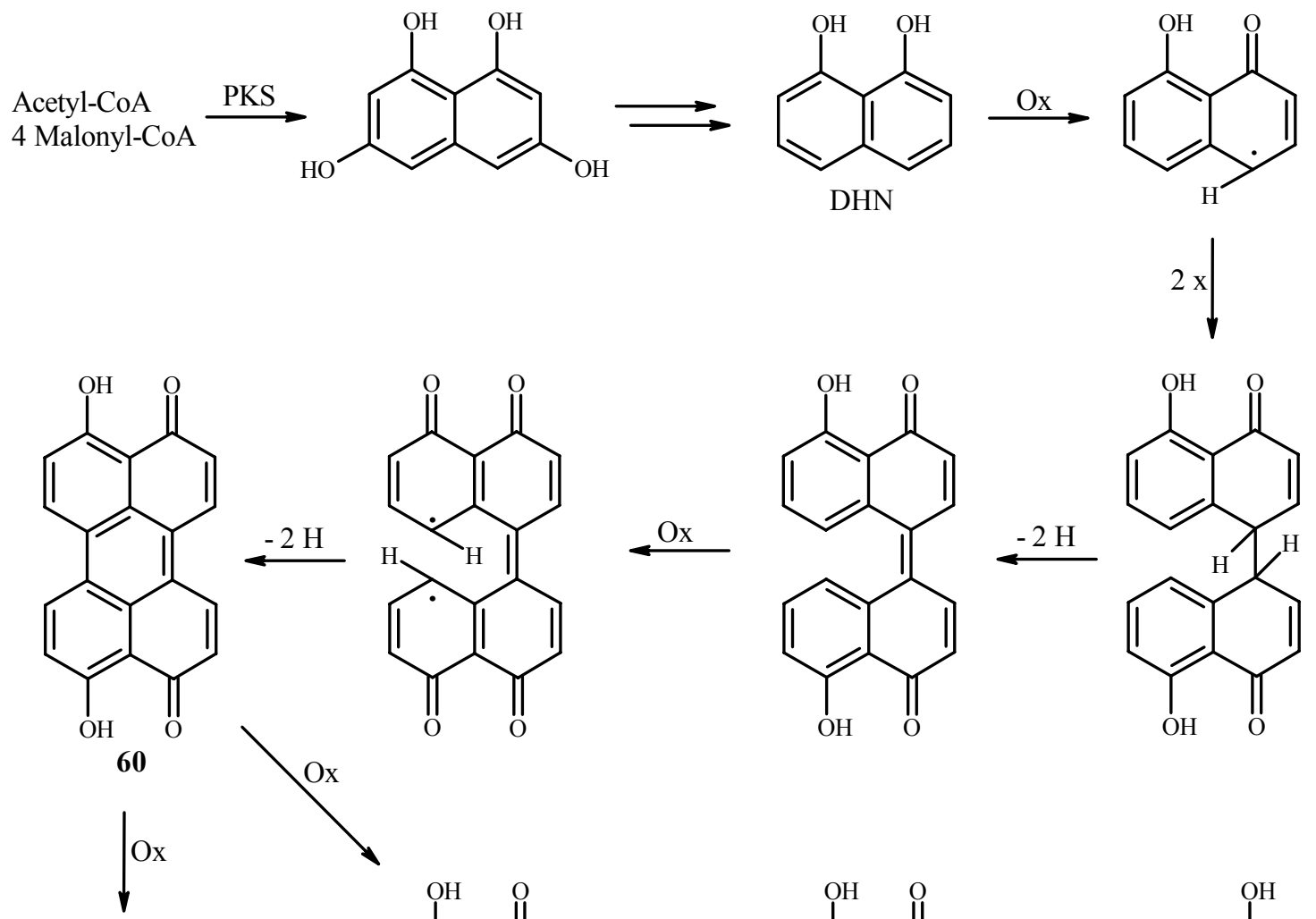<smiles></smiles><smiles></smiles><smiles>CC1C=CC(=O)c2c(O)cccc2C1C</smiles>

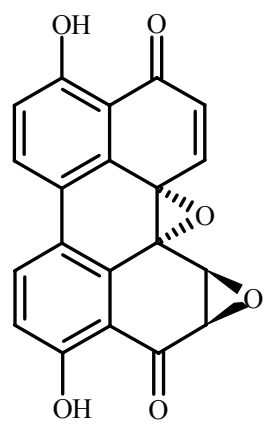

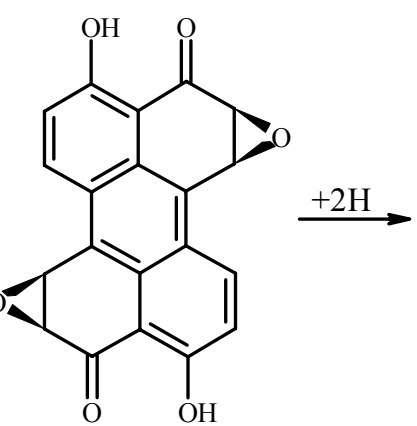<smiles>O=C1c2c(O)ccc3c2[C@@H](c2ccc(O)c4c2[C@H]3[C@H]2OC2C4=O)C2OC12</smiles>

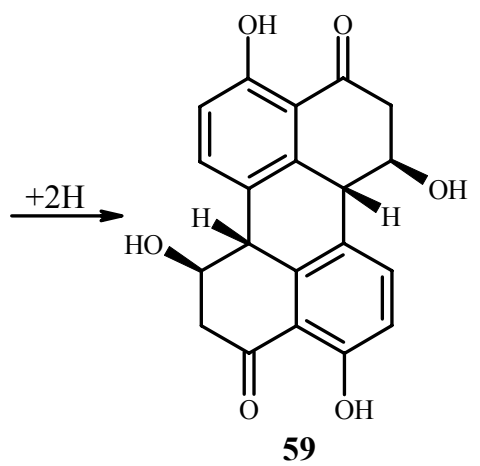<smiles>[3H][13CH3]</smiles>

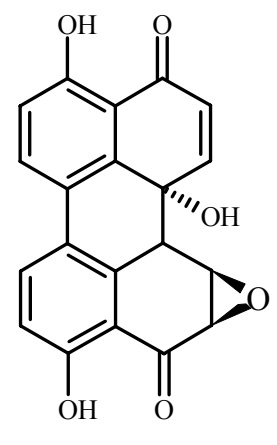

62<smiles>O=C1C[C@H](O)C2c3c(ccc(O)c31)-c1ccc(O)c3c1[C@]2(O)C=CC3=O</smiles>

57<smiles>CC(C)[C@H]1c2ccc(O)c3c2C2c4c(c(O)ccc41)C(=O)CC[C@]2(O)CCC3=O</smiles>

Abbildung 26: Gemeinsame Biosynthese der drei von Alternaria sp. Stamm 6588 gebildeten Perylenchinone $(\mathbf{5 7}-\mathbf{5 9})$. 


\subsection{Variation des Metabolitenmusters durch Enzyminhibitoren}

Wie im vorherigen Kapitel dargestellt werden die vom Stamm 6588 gebildeten Perylenchinone durch Dimerisierung von zwei Einheiten 1,8-Dihydroxynaphtalen (DHN) aufgebaut. Dieses dient vielen Pilzen als Vorläufer zum Aufbau von DHN-Melanin. Melanin ist ein wichtiger Faktor für die Pathogenität verschiedener pflanzen- und humanpathogener Pilze wie Magnaporthe grisea, Colletotrichum lagenarium, Exhophiala dermatidis, Aspergillus fumigatus und Alternaria alternata, da ihre Zellwand durch Einlagerung von Melanin stabilisiert werden kann. ${ }^{95}$ Aktuell wird sogar diskutiert, ob es sich bei Melanin um einen Virulenzfaktor von Aspergillus fumigatus, ${ }^{96,97} \mathrm{dem}$ Verursacher der lebensbedrohlichen invasiven Aspergillose handelt. ${ }^{98}$ Das Verständnis der DHN-Biosynthese ist daher die Grundlage um dieses wichtige Target für die Bekämpfung der oben genannten Pathogene nutzen zu können. Das Pentaketid DHN entsteht durch die Kondensation von Acetyl-CoA mit vier Einheiten Malonyl-CoA zum 1,3,6,8-Tetrahydroxynaphthalen (63). Dieses wird via Scytalone (64), 1,3,8-Trihydroxynaphthalen (65) und Vermelone (66) in 1,8-Dihydroxynaphtalen umgewandelt (Abbildung 27).<smiles>O=C1OCc2c(Cl)c(Cl)c(Cl)c(Cl)c21</smiles>

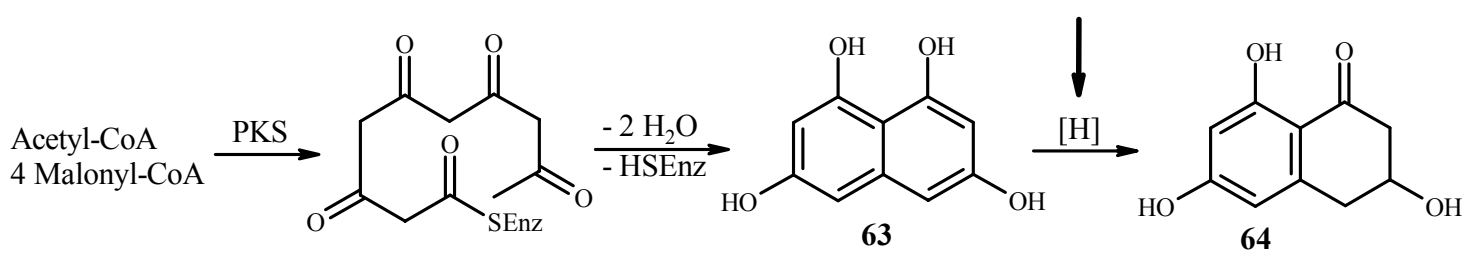<smiles>Cc1cccc2sc3nncn3c12</smiles>

Abbildung 27: Biosynthese von Dihydroxynaphtalen (DHN). 
Das Fungizid Tricyclazol (68) inhibiert die 1,3,8-Trihydroxynaphtalenreduktase, ${ }^{99}$ welche die Reduktion von 65 zu 66 katalysiert. Ein weiterer Inhibitor der DHN-Biosynthese ist Fthalid (67), welches den Reduktionschritt von 63 zu 64 blockiert. ${ }^{100}$ Die Zugabe der beiden Enzyminhibitoren führte dazu, dass der Stamm 6588 keine Perylenchinone mehr produzierte. Stattdessen konnte aus einer Kultivierung unter Zusatz von Tricyclazol Sphaerolon (70) neben den erwarteten Metabolien 1,3,8-Trihydroxynaphthalen (65) und 2-Hydroxyjuglon (69) isoliert werden. Die Zugabe von Fthalid führt zur Akkumulation von Scytalon (64).

\subsubsection{1,3,8-Trihydroxynaphthalen (65)}

Im ${ }^{1}$ H-NMR-Spektrum dieser Substanz erkennt man im Bereich von $\delta_{\mathrm{H}}=6.45-7.12$ die Signale von fünf Methingruppen sowie für drei austauschbare Protonen $\left(\delta_{\mathrm{H}}=8.73\right.$ und 10.27). Anhand der Kopplungsmuster können drei Protonen einem 1,2,3-trisubstituierten und die beiden anderen Protonen einem 1,2,3,5-tetrasubstituierten Aromaten zugeordnet werden. Dies spricht dafür, dass es sich bei $\mathbf{6 5}$ um das bei Zufütterung von Tricyclazol erwarteten 1,3,8-Trihydroxynaphthalen handelt. Durch ein ${ }^{13} \mathrm{C}$-NMR-Spektrum, welches Signale für zehn Kohlenstoffatome enthält und durch Vergleich mit Literaturdaten ${ }^{101}$ konnte diese Vermutung bestätigt werden.<smiles>Oc1cc(O)c2c(O)cccc2c1</smiles>

\subsubsection{2-Hydroxyjuglon (69)}

Die Summenformel der leicht gelblichen Substanz kann durch eine EI-Hochauflösung zu $\mathrm{C}_{10} \mathrm{H}_{6} \mathrm{O}_{4}$ bestimmt werden. Das in $\mathrm{CD}_{3} \mathrm{OD}$ aufgenommene ${ }^{1} \mathrm{H}-\mathrm{NMR}$-Spektrum zeigt Signale von vier Protonen im Bereich von $\delta_{\mathrm{H}}=5.65-7.47$. Dabei tritt das Signal bei $\delta_{\mathrm{H}}=5.65$ als Singulett auf und die andern drei Protonen können einem 1,2,3-trisubstituierten Aromaten zugeordnet werden. Durch Vergleich mit Literaturdaten ${ }^{102}$ konnte 69 eindeutig als 2-Hydroxyjuglon identifiziert werden. 
<smiles>O=C1C=C(O)C(=O)c2c(O)cccc21</smiles>

\subsubsection{Sphaerolon (70)}

Die als roter Feststoff isolierte Substanz zeigt im EI-Massenspektrum den Peak höchster Intensität bei $\mathrm{m} / \mathrm{z}=348$, der durch Hochauflösung zur Summenformel $\mathrm{C}_{20} \mathrm{H}_{12} \mathrm{O}_{6}$ führt. Dem ${ }^{13} \mathrm{C}-\mathrm{NMR}-S p e k t r u m$ sind Signale für eine Methylen-, sieben olefinische Methingruppen und zwölf quartäre Kohlenstoffatome zu entnehmen. Wobei zwei quartäre C-Atome mit Verschiebungen bei $\delta_{\mathrm{C}}=192.9$ und 202.3 für das Vorhandensein zweier Carbonylgruppen sprechen. Die Methylengruppe tritt im ${ }^{1} \mathrm{H}-\mathrm{NMR}-$ Spektrum bei $\delta_{\mathrm{H}}=3.54$ auf und die Methingruppen sind im Bereich von $\delta_{\mathrm{H}}=5.96-8.10 \mathrm{zu}$ erkennen. Das Signal bei $\delta_{\mathrm{H}}=5.96$ erscheint als Singulett, die verbleibenden sechs Methingruppen müssen aufgrund ihres Kopplungsmusters zu zwei 1,2,3-trisubstituierten Aromaten gehören. Außerdem sind dem ${ }^{1}$ H-NMR-Spektrum noch die Signal für zwei austauschbare Protonen bei $\delta_{\mathrm{H}}=12.36$ und $12.69 \mathrm{zu}$ entnehmen, die anhand ihrer Verschiebungen zwei chelatisierten Hydroxygruppen zugeordnet werden. Durch eine Datenbanksuche ${ }^{54}$ konnte 70 als Sphaerolon ${ }^{103}$ identifiziert werden.<smiles>O=C1C=C2OC3(O)CC(=O)c4c(O)cccc4C3=C2c2cccc(O)c21</smiles> 
Sphaerolon wurde erstmals von H. B. BodE aus dem Stamm Sphaeropsidales sp. F-24'707 isoliert. ${ }^{103}$ Dieser Pilzstamm produziert mehrere Spirobisnaphtalene, die ebenso wie die von Alternaria sp. Stamm 6588 gebildeten Perylenchinone aus zwei Einheiten 1,8-Dihydroxynaphtalen (DHN) aufgebaut werden. Durch Inhibition der DHN-Biosynthese mit Tricyclazol konnte Sphaeropsidales sp. F-24'707 zur Bildung von zwei neuen Metaboliten, Sphaerolon (70) und Dihydrosphaerolon (71), angeregt werden. Für die beiden Metabolite wurde eine Biosynthese ausgehend von 1,3,8-Trihydroxynaphtalen (65), welches durch Inhibition der 1,3,8-Trihydroxynaphtalenreduktase mit Tricyclazol akkumuliert wird, vorgeschlagen. Unter aeroben Bedingungen kann 65 leicht zum 2-Hydroxyjuglon (69) oder aber zum Radikal 72 oxidiert werden. Dieses Radikal kann zum Zwischenprodukt 73 dimerisieren, welches wiederum zu 70 oxidiert wird. Durch Reduktion der Carbonylfunktion an C-1' wird dann schließlich Dihydrosphaerolon (71) gebildet.<smiles>Oc1cc(O)c2c(O)cc(O)cc2c1</smiles>

63<smiles>O=C1CC(O)Cc2cc(O)cc(O)c21</smiles>

64<smiles>Oc1cc(O)c2c(O)cccc2c1</smiles>

65<smiles>O=C1C=C(O)C(C2C(O)=CC(=O)c3c(O)cccc32)c2cccc(O)c21</smiles><smiles>Oc1cc(O)c2c(O)cccc2c1</smiles><smiles>C[14CH3]</smiles><smiles>O=C1C=C(O)C(=O)c2ccccc21</smiles>

73<smiles>C[13CH]</smiles><smiles>O=C1C=C(O)C(=C2C(O)=CC(=O)c3c(O)cccc32)c2cccc(O)c21</smiles><smiles>C=C</smiles><smiles>C=CC=C1C(=O)CC(=O)c2c(O)cccc2/C1=C1\C(O)=CC(=O)c2c(O)cccc21</smiles><smiles>O=C1C=C2OC3(O)CC(=O)c4c(O)cccc4C3=C2c2cccc(O)c21</smiles><smiles>O=C1C=C2OC3(O)CC(O)c4c(O)cccc4C3=C2c2cccc(O)c21</smiles>

Abbildung 28: Gemeinsame Biosynthese von Sphaerolon (70) und Dihydrosphaerolon (71). ${ }^{103}$ 


\subsubsection{Scytalon (64)}

Dem ${ }^{13} \mathrm{C}-\mathrm{NMR}-$ Spektrum sind die Signale für zwei Methylen-, eine aliphatische und zwei olefinische Methingruppen sowie für fünf quartäre Kohlenstoffatome zu entnehmen. Im Bereich von $\delta_{\mathrm{C}}=101.7-166.8$ befinden sich sechs Signale, die auf die Anwesenheit eines mit Sauerstoff substituierten Benzolringes hindeuten. Zwei Kohlenstoffatome mit chemischen Verschiebungen von $\delta_{C}=166.5$ und 166.8 bestätigen diese Vermutung. Ein weiteres Signal bei $\delta_{\mathrm{C}}=202.4$ kann einer Carbonylgruppe zugeordnet werden. Das ${ }^{1} \mathrm{H}-\mathrm{NMR}-\mathrm{Spektrum}$ zeigt die beiden olefinischen Methingruppen bei $\delta_{\mathrm{H}}=6.09$ und 6.21, die sich aufgrund einer Kopplungskonstante von ${ }^{4} J=2.5 \mathrm{~Hz}$ in meta Position $\mathrm{zu}$ einander, an einem tetrasubstituierten Aromaten befinden sollten. Die aliphatische Methingruppe $\left(\delta_{\mathrm{H}}=4.24\right)$ kann durch ein ${ }^{1} \mathrm{H},{ }^{1} \mathrm{H}-\mathrm{COSY}$-Experiment mit den beiden Methylengruppen verknüpft werden. Zusammen mit dem durch ein EI-Massenspektrum bestimmten Molekulargewicht von $194 \mathrm{~g} / \mathrm{mol}$ wurde 64 als Scytalon ${ }^{104}$ identifiziert.

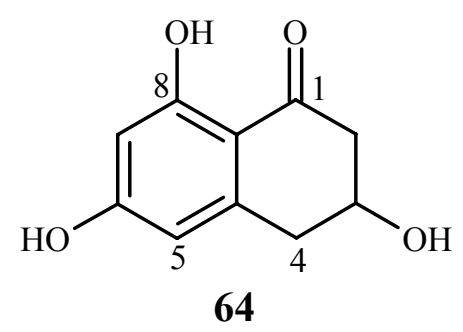

Bei Kultivierung unter Zusatz von Fthalid (67) war eigentlich mit der Akkumulation von 1,3,6,8-Tetrahydroxynaphthalen (63) oder dessen Oxidationsprodukt Flaviolin gerechnet worden, da $\mathbf{6 7}$ die 1,3,6,8-Tetrahydroxynaphthalenreduktase inhibieren sollte. Dass stattdessen Scytalon (64) isoliert wird, spricht eher für die Inhibierung der Scytalondehydratase. Eine mögliche Erklärung ist, dass Stamm 6588 über eine alternative Reduktase verfügt, die das gebildete $\mathbf{6 3}$ als Substrat akzeptiert und es somit zur Bildung von Scytalon (64) kommt. Allerdings müsste auch hier eine Inhibierung der Scytalondehydratase vorliegen, da ansonsten kein Scytalon akkumuliert werden dürfte. 


\section{Weitere in Braunschweig isolierte endophytische Pilze}

\subsection{Ascotricha chartarum Stamm 6303}

Der Pilz Ascotricha chartarum Stamm 6303 wurde aus einer Braunalge (Fucus serratus), die bei Cuxhaven in der Nordsee gefunden wurde, isoliert. Der Stamm fiel durch ein besseres Wachstum bei der Kultivierung unter Zusatz von Natriumchlorid (33 g/L) auf. Im Chemischen Screening erwies er sich jedoch als relativ uninteressant, da keine Metabolite in nennenswerter Konzentration nachzuweisen waren. Aus einer in Braunschweig durchgeführten Kultivierung auf Festmedium (5 L, Zusatz von Natriumchlorid) konnte als einziger Metabolit Ergosterol (74) in einer Ausbeute von $520 \mathrm{mg}$ isoliert und durch Vergleich mit Literaturspektren ${ }^{105}$ identifiziert werden. Ergosterol kommt ubiquitär in Pilzen als Bestandteil der Zytoplasmamembran vor und ist daher kein Sekundärmetabolit im eigentlichen Sinne. Da keiner der aus Kulturen des Stammes 6303 hergestellten Extrakte durch eine Aktivität in den Testsystemen der BASF AG auffiel, wurde die Bearbeitung dieses Stammes eingestellt.

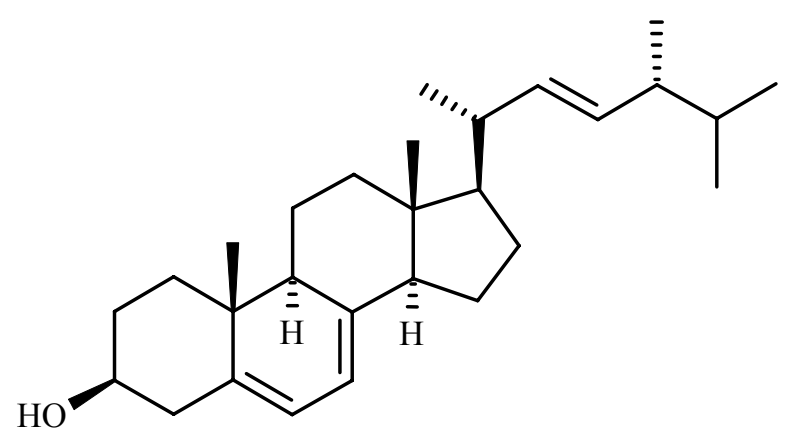

74

\subsection{Stamm 6325}

Der Pilz mit der Stammnummer 6325 wurde aus einer bei Mallorca gesammelten Braunalge der Gattung Fucus isoliert. Er sporuliert nicht und konnte daher nicht bestimmt werden. Der Stamm 6325 wächst vergleichsweise langsam, so dass die Kultivierungsdauer auf sieben Tage erhöht wurde. Im Chemisch Screening fiel der Stamm bei Kultivierung in Schüttelkolben (Medium: G20) durch die Bildung einer UV-löschenden und einer mit Anisaldehyd braun anfärbbaren Substanz auf. Die beiden Substanz konnte als 2-Formyl-5-hydroxymethylfuran (75) und 2,5-Bishydroxymethylfuran (76) identifiziert werden, die allerdings keine 
Sekundärmetaboliten darstellen, sondern beim Erhitzen von Glucose entstehen. ${ }^{106}$ Weiterhin konnte Phenylessigsäure ${ }^{107}(\mathbf{7 7})$ isoliert werden.

Da der Stamm 6325 nicht durch eine Aktivität in den Testsystemen der BASF AG auffiel, wurde er nicht weiter bearbeitet.<smiles>O=Cc1ccc(CO)o1</smiles>

75<smiles>OCc1ccc(CO)o1</smiles>

76<smiles>O=C(O)Cc1ccccc1</smiles>

77

\subsection{Phomopsis sp. Stamm 6615}

Der Pilz Phomopsis sp. Stamm 6615 wurde aus einem bei Ahrenshop wachsenden Sanddorn isoliert und fiel bei Kultivierung in Ruhekultur (Medium: $1549+\mathrm{NaCl}$ ) durch die Bildung von zwei mit Anisaldehyd rötlich anfärbbaren Metaboliten auf. Die beiden Substanzen konnten durch ein ${ }^{1} \mathrm{H}-\mathrm{NMR}$-Spektrum und den Vergleich mit Literaturdaten als 2-Phenylethanol ${ }^{108}$ (78) und Tryptophol ${ }^{109}$ (79) identifiziert werden. In den Testsystemen der BASF AG konnte für die Extrakte dieses Stammes keine Aktivität beobachtet werden.<smiles>OCCc1ccccc1</smiles>

78<smiles>OCCc1c[nH]c2ccccc12</smiles>

79 


\section{Sekundärmetabolite in Göttingen isolierter endophytischer Pilze}

\section{Chaetomium sp. Stamm Gö 100/2}

Der Pilz Chaetomium sp. Stamm Gö 100/2 fiel im chemischen Screening bei Kultivierung in Schüttelkultur (Medium: 1158) durch die Bildung einer gelben, UV-Licht der Wellenlänge $254 \mathrm{~nm}$ löschenden Verbindung auf. Ein Extrakt dieses Stammes wies zudem bei der biologischen Testierung durch die BASF AG eine Aktivität gegen Phytophthora infestans auf. S. LÖSGEN konnte in ihrer Diplomarbeit insgesamt sechs Sekundärmetabolite aus diesem Stamm isolieren. Dazu gehören neben den drei literaturbekannten Verbindungen Tryptophol (79), Toluquinol (80) und Aurasperon B (81) drei bisher nicht in der Literatur beschriebene Substanzen, die Chaetocyclinone A-C $\quad(\mathbf{8 2}-\mathbf{8 4}) .{ }^{110}$ Chaetocyclinon A (82) hemmt Phytophthora infestans bis zu eine Konzentration von $31 \mathrm{ppm}$ und scheint somit für die fungizide Aktivität dieses Stammes verantwortlich zu sein.

Des Weiteren konnte S. LÖSGEN durch Fütterungsexperimente mit $\left[1-{ }^{13} \mathrm{C}\right]-$ und $\left[1,2-{ }^{13} \mathrm{C}_{2}\right]$ Acetat die Bildung von Chaetocyclinon A (82) auf dem Polyketidweg beweisen und aufbauend auf diesen Ergebnissen einen Biosyntheseweg für Chaetocyclinon C (84) vorschlagen. Im Folgenden möchte ich ihre Arbeiten mit Chaetomium sp. Stamm Gö 100/2 vorstellen.

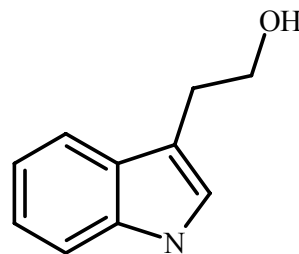

79<smiles>Cc1cc(O)ccc1O</smiles>

80<smiles>COc1cc(OC)c2c(O)c3c(c(-c4c(OC)cc5cc6c(c(O)c5c4OC)C(=O)CC(C)(O)O6)c2c1)OC(C)(O)CC3=O</smiles>

81

Abbildung 29: Vom Stamm Gö 100/2 gebildete literaturbekannte Verbindungen Tryptophol (79), Toluquinol (80) und Aurasperon B (81). 


\subsection{Die Chaetocyclinone A, B und C (82 - 84)}

Die Strukturformeln der von Chaetomium sp. Stamm Gö 100/2 gebildeten Chaetocyclinone A - C (82 - 84) sind in Abbildung 30 dargestellt. Chaetocyclinone A (82) unterscheidet sich von Chaetocyclinon B (83) lediglich durch die Methoxygruppe an C-1, besitzt ansonsten aber dasselbe Pyrano-Chromen Grundgerüst. Ebenfalls ein Pyrano-Chromen Grundgerüst besitzt die strukturell verwandte Verbindung Anhydrofulvinsäure (85). Diese unterscheidet sich aber von $\mathbf{8 2}$ und $\mathbf{8 3}$ in der Substitution an C-6 und C-8, außerdem ist keine der Hydroxygruppen methyliert. Anhydrofulvinsäure (85) wird von Penicillium afacidum produziert. ${ }^{111} \mathbf{8 5}$ besitzt antifungische Eigenschaften, die auf eine Hemmung des Elektronentransports in den Mitochondrien zurückgeführt werden. Im Unterschied zu den Chaetocylinonen A und B ist Chaetocyclinon C (84) aus der doppelte Anzahl an Kohlenstoffatomen aufgebaut, weist aber deutliche strukturelle Ähnlichkeiten zu den beiden anderen Verbindungen auf. Insbesondere enthält 84 zweimal den Chromen-Teil der Chaetocyclinone A und B und kann daher als ein „dimeres“ Chaetocyclinon betrachtet werden. Dasselbe Grundgerüst wie Chaetocyclinon C besitzt das 1991 aus Penicillium vinaceum isolierte Vinaxanthon (86), welches ein Inhibitor der Phospholipase C ist. ${ }^{112}$<smiles></smiles><smiles>COC(=O)c1cc(OC)c(O)c2oc3c(c(=O)c12)COC(C)=C3</smiles><smiles></smiles><smiles>COC(=O)c1cc(OC)c(O)c2occ(-c3c(C(C)=O)cc4c(=O)c5c(C(=O)OC)cc(OC)c(O)c5oc4c3C(C)=O)c(=O)c12</smiles><smiles>CC(=O)c1cc2c(=O)c3c(C(=O)O)cc(O)c(O)c3oc2c(C(C)=O)c1-c1coc2c(O)c(O)cc(C(=O)O)c2c1=O</smiles>

Abbildung 30: Die vom Stamm Gö 100/2 gebildeten Chaetocyclinone A - C (82 - 84) sowie strukturell verwandte Verbindungen Anhydrofulvinsäure (85) und Vinaxanthon (86). 


\subsection{Untersuchungen zur Biosynthese von Chaetocyclinon $\mathbf{A}$}

Durch Fütterung von $\left[1-{ }^{13} \mathrm{C}\right]-$ und $\left[1,2-{ }^{13} \mathrm{C}_{2}\right]$ Acetat konnte gezeigt werden, dass Chaetocyclinon A (82) aus 7 Acetateinheiten aufgebaut wird. Das gefundene Einbaumuster legt einen Mechanismus nahe, bei dem zunächst aus einem Acetyl-CoA und sechs Einheiten Malonyl-CoA ein lineares Heptaketid aufgebaut und zur Zwischenstufe (87) cyclisiert wird. Im Anschluss an eine oxidative Ringöffnung und Drehung der aromatischen Ringsystems wird unter Abspaltung von Wasser das Pyrano-Chromen Grundgerüst (88) ausgebildet, aus dem durch dreifache Methylierung mit Methionin Chaetocyclinon A (82) entsteht.

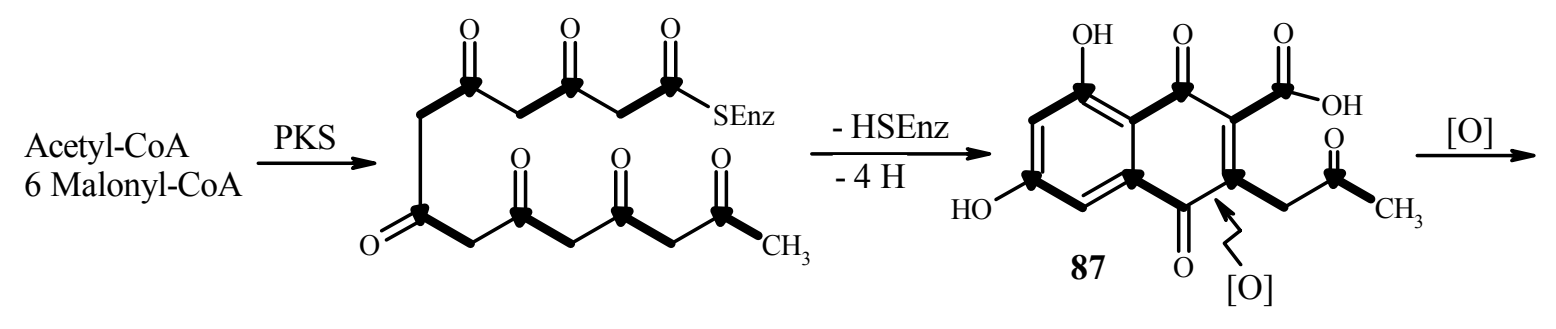

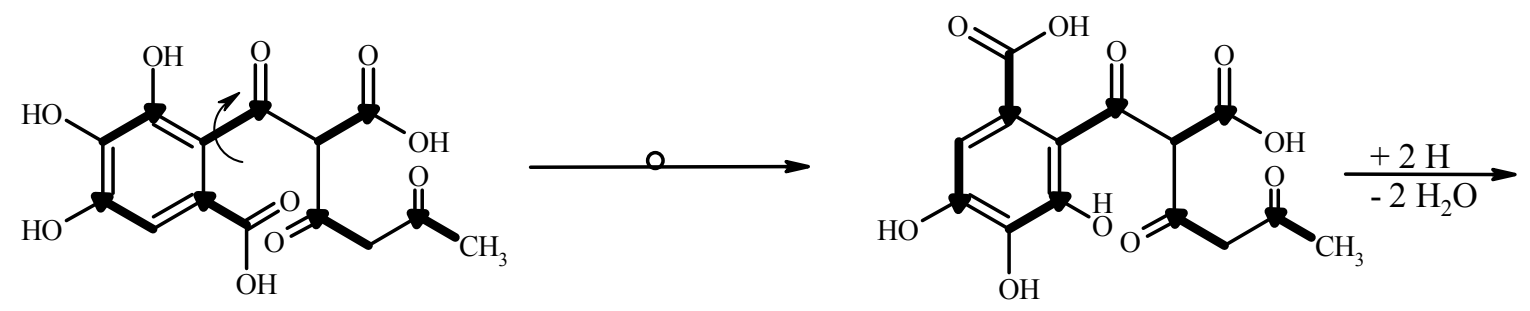

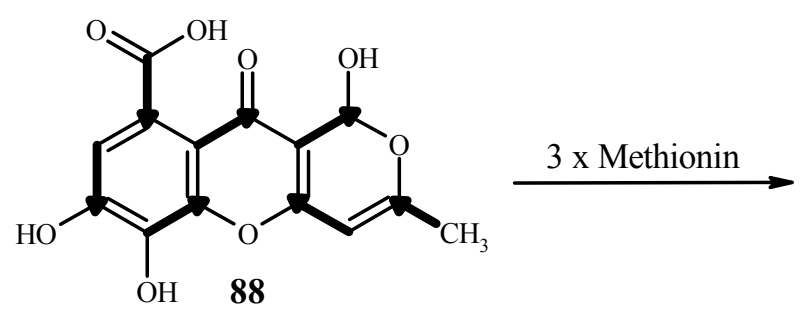

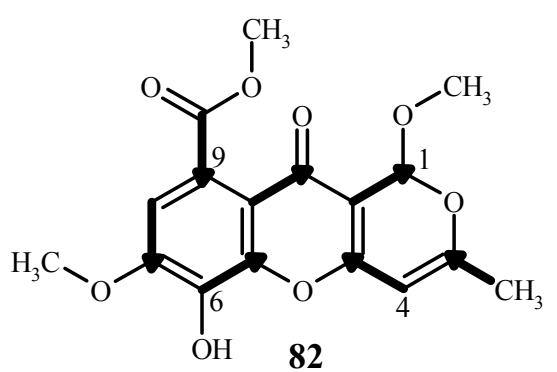

Abbildung 31: Biosynthese von Chaetocyclinon A (82). 


\section{3. Überlegungen zur Biosynthese von Chaetocyclinon B (83) und C (84)}

Die beiden Chaetocyclinone B (83) und C (84) werden von Chaetomium sp. Stamm Gö 100/2 lediglich in geringen Ausbeuten gebildet, daher konnte aus den Acetatfütterungen keine für die Bestimmung von Einbauraten ausreichenden Mengen an 83 und 84 isoliert werden. Jedoch kann man anhand der mit Chaetocyclinon A (82) erzielten Ergebnisse Überlegungen zur Biosynthese der Chaetocyclinone B und $\mathrm{C}$ anstellen.

Chaetocyclinon B (83) unterscheidet sich von 82 lediglich durch die Methoxygruppe an C-1 und sollte daher auf demselben Weg gebildet werden. Der Punkt, an dem die Biosynthese von 83 von der von 82 abzweigt, kann nicht genau bestimmt werden. Am wahrscheinlichsten erscheint allerdings die Reduktion der Hydroxygruppe an C-1 auf dem Stand der Vorstufe 88. Anschließende Methylierung mit Methionin führt dann zum Chaetocyclinon B (83).

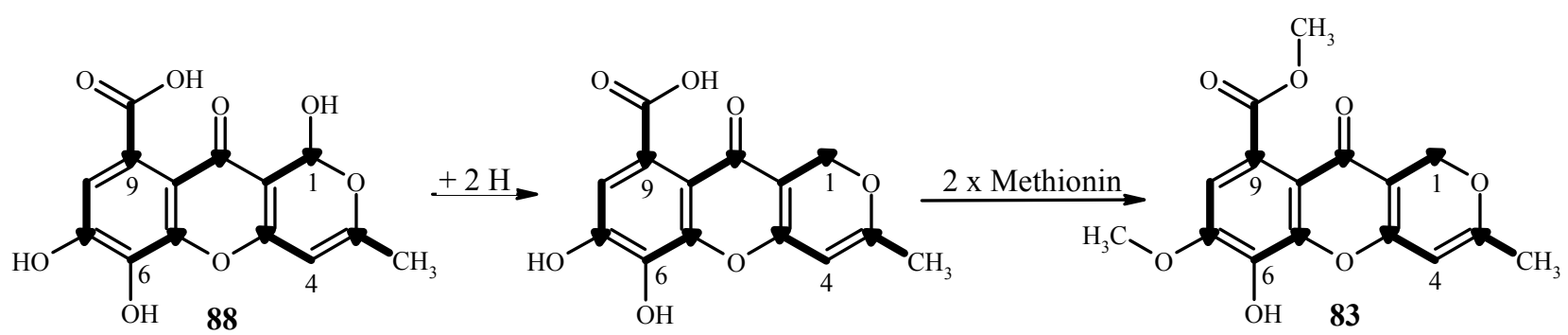

Abbildung 32: Hypothese für die Biosynthese von Chaetocyclinon B (83)

Chaetocyclinon C (84) besitzt deutliche strukturelle Ähnlichkeiten zu Chaetocyclinon A (82). Anhand dieser Ähnlichkeiten kann man sich für 84 eine Biosynthese ausgehend von zwei Heptaketiden vorstellen. Im Unterschied zur Biosynthese von 82 müssten diese allerdings anders gefaltet werden, so dass eine längere Seitenkette entsteht. In Analogie zur Biosynthese von 82 gelangt man nach oxidativer Ringöffnung und einem Reduktionsschritt zur monomeren Zwischenstufe (89), die im Gleichgewicht mit der cyclischen Halbacetalform (90) steht. Durch Dimerisierung zweier monomerer Einheiten entsteht unter Ausbildung eines aromatischen Systems Vinaxanthon (86). Im letzten Schritt erfolgt dann die Methylierung von 86 zum Chaetocyclinon C (84) (siehe Abbildung 33). 
<smiles>CC(=O)CC(=O)CC(=O)CC(=O)CC(=O)CC(=O)CC(=O)CC(=O)CC(=O)CC(C)C</smiles>

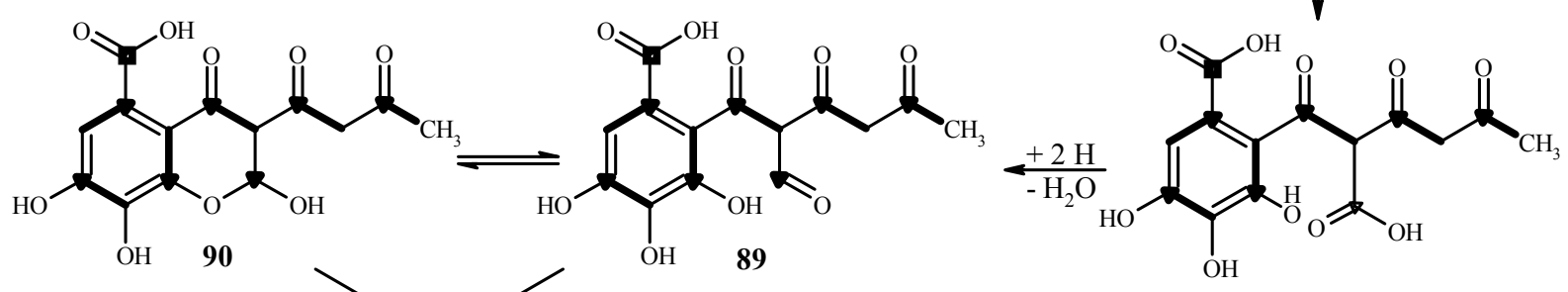

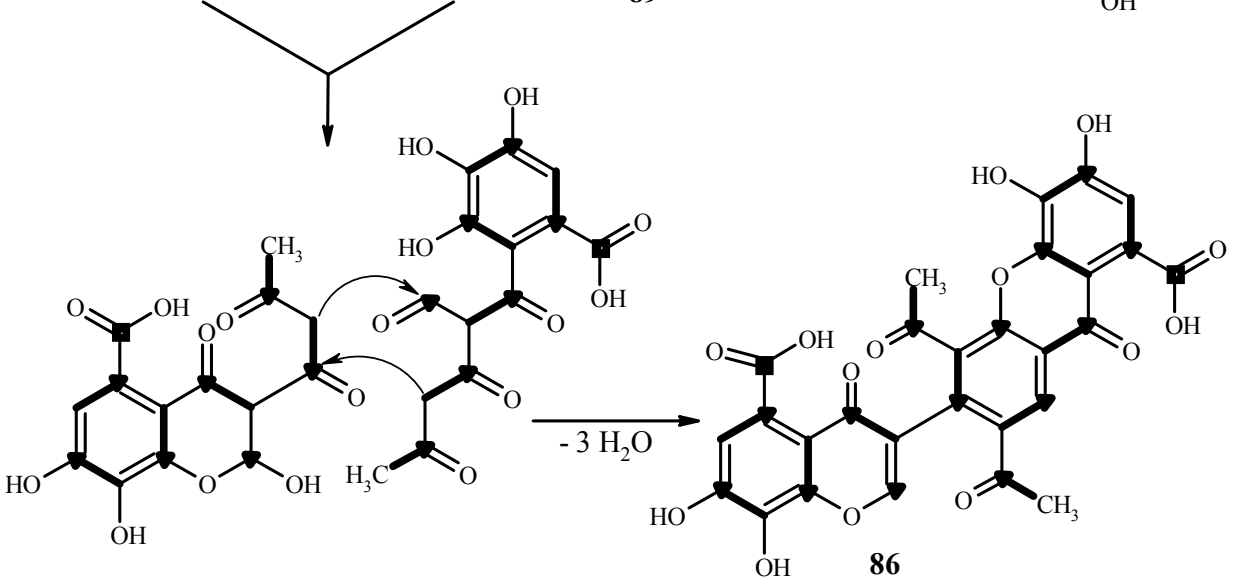

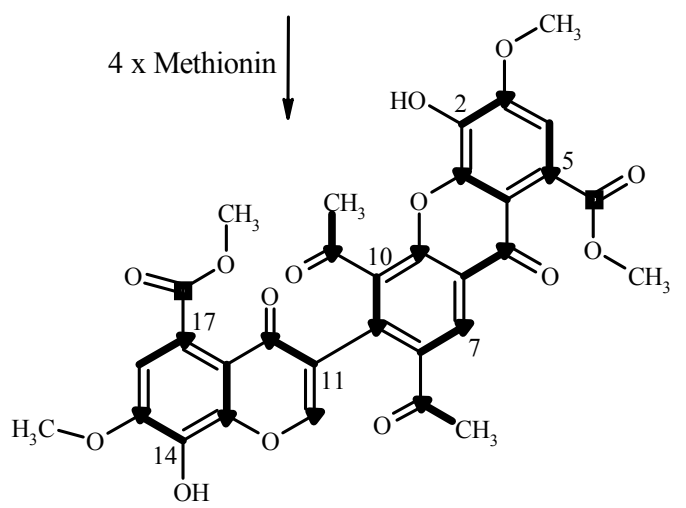

84

Abbildung 33: Hypothese für die Biosynthese von Chaetocyclinon C (84).

Um den hypothetischen Biosynthesemechanismus von Chaetocyclinon C (84) durch Fütterungsexperimente zu überprüfen, muss zunächst die Produktion an $\mathbf{8 4}$ soweit gesteigert werden, dass ausreichende Ausbeuten erzielt werden um die isotopenmarkierte Vorläufer auch effizient einsetzen zu können. Versuche dazu werden momentan von S. Lösgen im Rahmen ihrer Doktorarbeit durchgeführt. 


\section{Aspergillus flavus Stamm Gö 100/4}

Im chemischen Screening fiel der Pilz Aspergillus flavus Stamm Gö 100/4 bei Kultivierung in Schüttelkultur durch die Bildung einer stark UV-löschenden Zone (91) auf. Aus einer Schüttelkultur im Medium SGG konnten insgesamt fünf Metabolite (91, 92, 93, 96 und 98) isoliert werden, von denen lediglich 98 neu war. Die Biogenese von Arthrographol (96) und Arthrolacton (98) auf dem Polyketidweg konnte durch Fütterung von $\left[1-{ }^{13} \mathrm{C}\right]$ - und $\left[1,2-{ }^{13} \mathrm{C}_{2}\right]$-Acetat bewiesen werden.

In den Testsystemen der BASF AG fielen mehrere Extrakte des Stammes Gö 100/4 durch eine Aktivität gegen Phytophthora infestans auf. Durch einen HPLC-Mikrotest konnte diese Aktivität bestätigt und einer Substanz mit einer Retentionszeit von ca. neun Minuten zugeordnet werden. Bei der aktiven Substanz könnte es sich um Arthrographol (96) handeln, das in der Literatur mit fungiziden Eigenschaften ${ }^{113}$ beschrieben wird. Die Testergebnisse der Reinsubstanz stehen allerdings noch aus.

\subsection{Kojic acid (91)}

Die farblose, UV-Licht der Wellenlänge $254 \mathrm{~nm}$ löschende Verbindung wurde aus Fermentationen in Schüttelkultur in Ausbeuten von $200 \mathrm{mg} / \mathrm{L}$ isoliert. Die Summenformel lautet $\mathrm{C}_{6} \mathrm{H}_{6} \mathrm{O}_{4}$ (HREI-Massenspektrum). Im ${ }^{1} \mathrm{H}-\mathrm{NMR}$-Spektrum sind die Signale von einer Methylen- $\left(\delta_{\mathrm{H}}=4.62\right)$ und zwei Methingruppen $\left(\delta_{\mathrm{H}}=6.92\right.$ und 8.09) zu erkennen und das ${ }^{13} \mathrm{C}-\mathrm{NMR}-$ Spektrum zeigt neben den Signalen der Methylengruppe $\left(\delta_{\mathrm{C}}=60.5\right)$ und der beiden Methingruppen $\left(\delta_{C}=110.5\right.$ und 139.4) drei quartäre Kohlenstoffatome bei $\delta_{C}=147.6,169.0$ und 175.4. Eine Datenbanksuche ${ }^{54}$ und der Vergleich mit Literaturdaten ${ }^{114}$ ergaben, dass es sich bei 91 um Kojic acid handelt.<smiles>O=c1cc(CO)occ1O</smiles>

91

91 wird häufig von Aspergillus sp. gebildet und besitzt eine schwache antibakterielle Wirkung. Bereits 1953 konnte durch Markierungexperimente gezeigt werden, dass Kojic acid aus Glucose entsteht. ${ }^{115}$ 


\subsection{Miyakamide}

Aus Schüttelkulturen (Medium: SGG) des Stammes Gö 100/4 konnten die beiden literaturbekannten Miyakamide $\mathrm{A}_{2}$ und $\mathrm{B}_{1}$ in Ausbeuten von jeweils $0.5 \mathrm{mg} / \mathrm{L}$ isoliert werden.

\subsubsection{Miyakamid $A_{2}(92)$}

Dem leicht gelblichen Feststoff kann durch ein hochaufgelöstes ESI-Massenspektrum die Summenformel $\mathrm{C}_{31} \mathrm{H}_{32} \mathrm{~N}_{4} \mathrm{O}_{3}$ zugeordnet werden. Dem ${ }^{13} \mathrm{C}-\mathrm{NMR}-$ Spektrum sind Signale für zwei Methyl-, zwei Methylen-, zwei aliphatische und siebzehn olefinische Methingruppen sowie acht quartäre Kohlenstoffatome zu entnehmen. Im ${ }^{1} \mathrm{H}-\mathrm{NMR}-$ Spektrum finden sich die Signale der olefinischen Methingruppen im Bereich von $\delta_{\mathrm{H}}=6.51-7.75$. Durch ein ${ }^{1} \mathrm{H},{ }^{1} \mathrm{H}$-COSY-Experiment können sie zwei monosubstituierten und einem 1,2-disubstituierten Benzolring zugeordnet werden. Zwei der drei verbleibenden olefinischen Methinprotonen $\left(\delta_{\mathrm{H}}=6.51\right.$ und 7.48) deuten durch ihre Kopplungskonstante von ${ }^{3} J=15.0 \mathrm{~Hz}$ auf eine transkonfigurierten Doppelbindung hin. Des Weiteren erkennt man bei $\delta_{\mathrm{H}}=8.69$ und 10.02 die Signale von zwei austauschbaren Protonen, wobei das Tieffeldverschobene mit einem der Doppelbindungsprotonen $\left(\delta_{\mathrm{H}}=7.48\right)$ koppelt. Die beiden aliphatischen Methingruppen müssen auf Grund ihrer Verschiebung ins Tieffeld $\left(\delta_{\mathrm{H}}=4.81\right.$ und 5.04) an Sauer- oder Stickstoff gebunden sein. Weiterhin ergibt sich aus dem ${ }^{1} \mathrm{H},{ }^{1} \mathrm{H}-\mathrm{COSY}$-Experiment, dass sie jeweils mit einer Methylengruppe verknüpft sind. Durch eine Datenbanksuche ${ }^{53}$ konnte die Verbindung als Miyakamid $\mathrm{A}_{2}{ }^{116}$ (92), das aus E-Didehydrotryptamin (dhTra), N-Methyl-LPhenylalanin (MePhe) und N-Acetyl-L-Phenylalanin (AcPhe) aufgebaut ist, identifiziert werden.

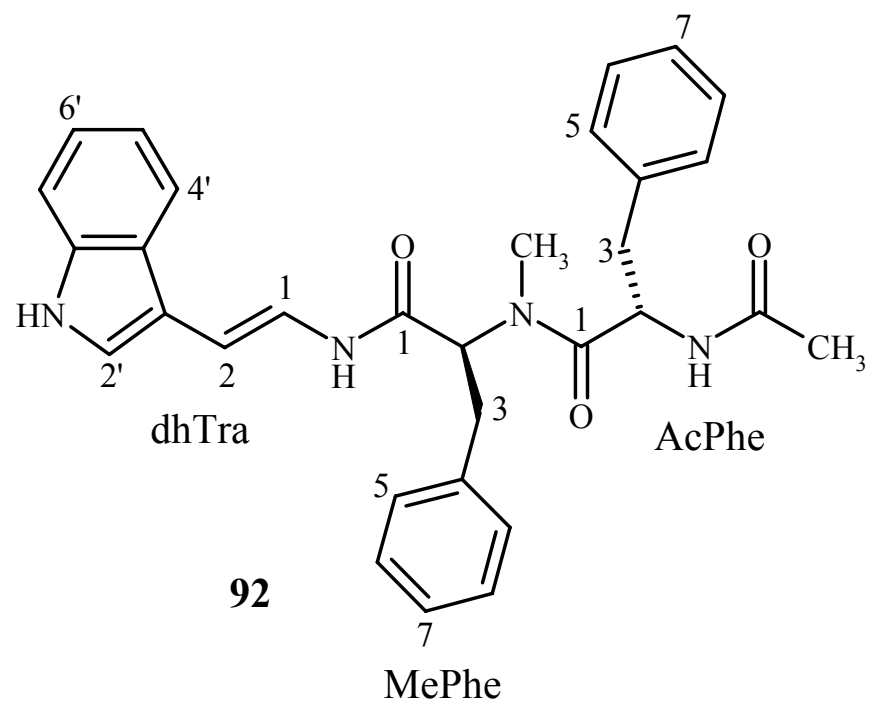




\subsubsection{Miyakamid $B_{1}(93)$}

Das IR-Spektrum von 93 zeigt ebenso wie das von 92 im Bereich von $1630-1650 \mathrm{~cm}^{-1}$ die typischen Schwingungsbanden von Säureamiden. Ein ESI-Massenspektrum liefert bei $\mathrm{m} / \mathrm{z}=547$ das $[\mathrm{M}+\mathrm{Na}]^{+}$-Ion, das durch Hochauflösung zur Summenformel $\mathrm{C}_{31} \mathrm{H}_{32} \mathrm{~N}_{4} \mathrm{O}_{4}$ führt. Das ${ }^{1}$ H-NMR-Spektrum weist große Ähnlichkeit zu dem von Miyakamid $\mathrm{A}_{2}$ (92) auf. Im Unterschied zu 92 fallen besonders zwei ins Hochfeld verschobene Dubletts bei $\left(\delta_{\mathrm{H}}=6.54\right.$ und 6.79) auf, die zu je zwei Methingruppen gehören und auf das Vorliegen eines parasubstituierten Aromaten hinweisen. Ebenfalls ins Hochfeld verschoben sind die Signale einer Doppelbindung bei $\delta_{\mathrm{H}}=6.02$ und 6.88. An der Kopplungskonstante von ${ }^{3} J=9.0 \mathrm{~Hz}$ erkennt man, dass die Doppelbindung im Gegensatz zu der von 92, cis-konfiguriert seien muss. Ein ${ }^{1} \mathrm{H},{ }^{1} \mathrm{H}$-COSY-Experiment ergibt, dass neben dem para-substituierten noch ein monosubstituierter und einem 1,2-disubstituierten Benzolring vorliegen. Weiterhin kann aus dem ${ }^{1} \mathrm{H},{ }^{1} \mathrm{H}$-COSY-Experiment abgeleitet werden, dass die beiden aliphatischen Methingruppen $\left(\delta_{\mathrm{H}}=4.97\right.$ und 5.16) ebenso wie bei 92 mit je einer Methylengruppe verknüpft sind. Mit Hilfe der so bestimmten Strukturelemente kann 93 als Miyakamid $\mathrm{B}_{1}{ }^{116}$ identifiziert werden. 93 besteht aus Z-Didehydrotryptamin (dhTra), N-Methyl-L-Phenylalanin (MePhe) und N-Acetyl-L-Tyrosin (AcTyr).

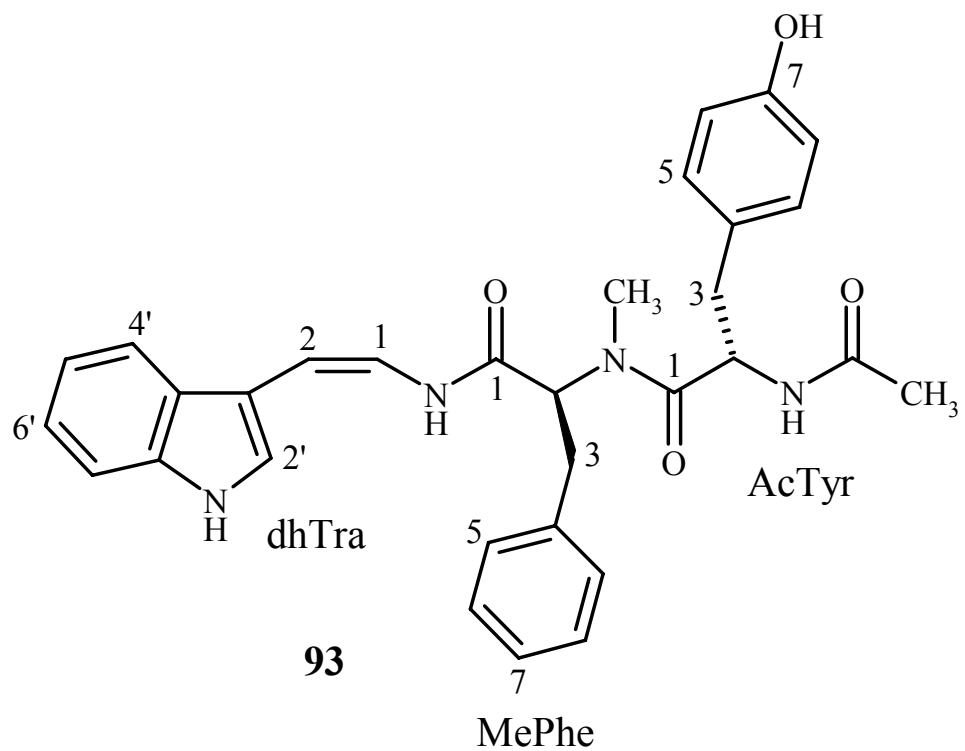




\subsubsection{Miyakamide in der Literatur}

SHIOMI et al. berichteten 2002 über die Isolierung der vier Miyakamide $A_{1}, A_{2}, B_{1}$ und $B_{2}$ aus einem in Schüttelkultur kultivierten Aspergillus flavus Stamm. ${ }^{116}$ Die Miyakamide der AReihe sind aus Didehydrotryptamin und zweimal Phenylalanin aufgebaut. Während bei den Miyakamiden der B-Reihe ein Phenylalanin durch Tyrosin ersetzt wurden. Weiterhin unterscheiden sich die vier Metabolite in der Konformation der Doppelbindung des Didehydrotryptamins, so liegt bei den Miyakamiden $\mathrm{A}_{1}$ und $\mathrm{B}_{1}$ das Didehydrotryptamin in der cis- und bei den Miyakamiden $\mathrm{A}_{2}$ und $\mathrm{B}_{2}$ in der trans-Konformation vor. Bei Untersuchungen zur biologischen Aktivität der Miyakamide konnte gezeigt werden, dass sie das Wachstum von Artemia salina inhibieren. Dabei besaßen die Miyakamide $\mathrm{A}_{1}$ und $\mathrm{A}_{2}$ mit einem MICWert von $5 \mu \mathrm{g} / \mathrm{ml}$ eine bessere Wirksamkeit als die Miyakamide $\mathrm{B}_{1}$ und $\mathrm{B}_{2}$ (MIC: $20 \mu \mathrm{g} / \mathrm{ml}$ ). In der Publikation wird außerdem berichtet, dass sich die Miyakamide $\mathrm{A}_{1}(\mathbf{9 2})$ und $\mathrm{B}_{1}(\mathbf{9 4})$ in die beiden Miyakamide $\mathrm{A}_{2}$ (95) und $\mathrm{B}_{2}$ (93) umwandeln, wenn sie eine Woche in Methanol gelöst bei Raumtemperatur und unter Beleuchtung stehen gelassen werden. Dies spricht dafür, dass die vom Pilz gebildeten Metabolite die Miyakamide $A_{1}$ und $B_{1}$ sind und die beiden anderen Miyakamide während der Aufarbeitung gebildet werden. Somit sollte auch Aspergillus flavus Stamm Gö 100/4 ursprünglich die beiden Miyakamide $\mathrm{A}_{1}$ und $\mathrm{B}_{1}$ gebildet haben. Das sich lediglich bei einem der beiden isolierten Miyakamide (93) das trans-Didehydrotryptamin in das cis-Didehydrotryptamin umgewandelt hat, kann durch Unterschiede in der Aufarbeitung erklärt werden.
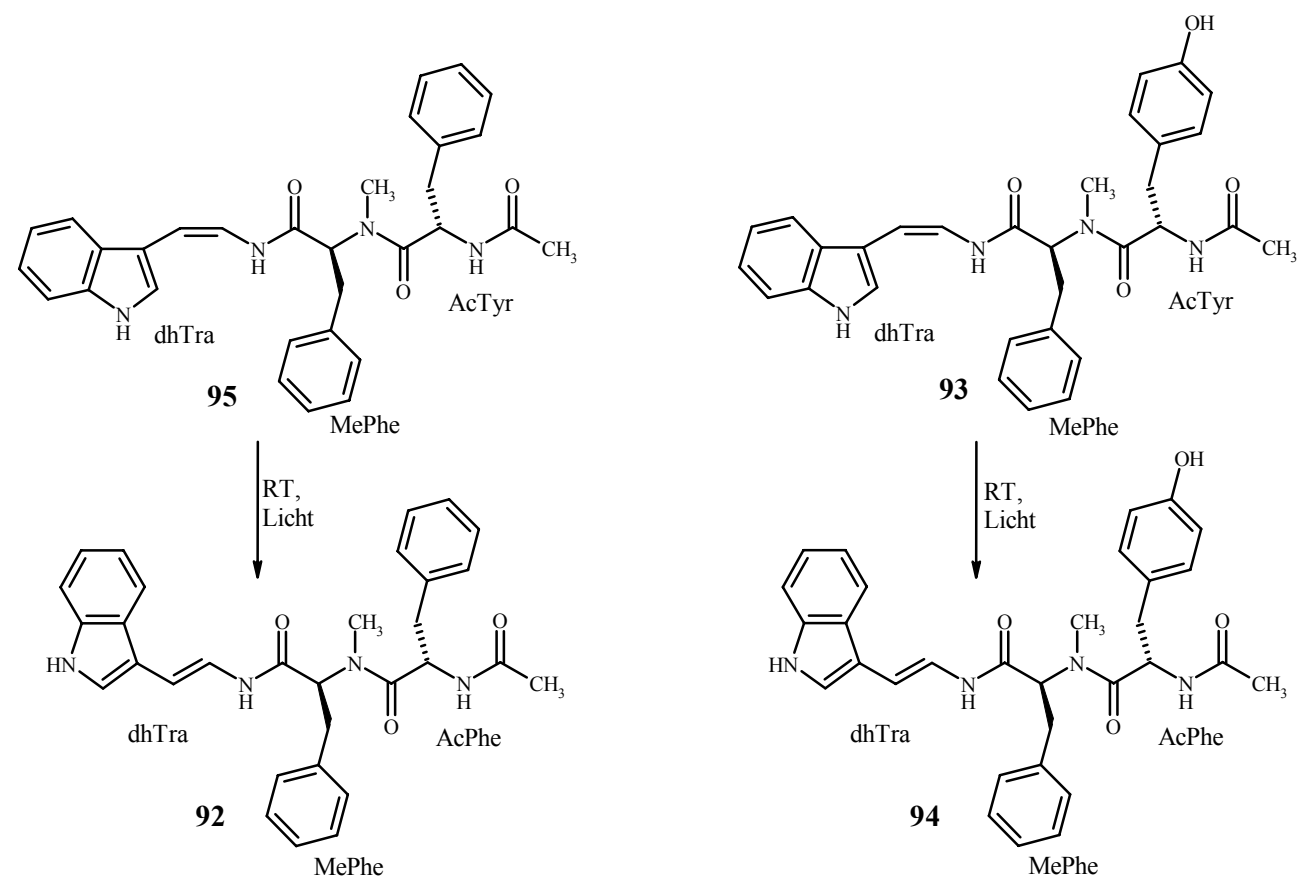

Abbildung 34: Bildung der Miyakamide $A_{2}$ und $B_{2}$ durch Isomerisierung. 


\subsection{Arthrographol (96)}

Die in Ausbeuten von $2.5 \mathrm{mg} / \mathrm{L}$ isolierte Substanz zeigt im EI-Massenspektrum den Peak höchster Masse bei $\mathrm{m} / \mathrm{z}=218$. Dem ${ }^{13} \mathrm{C}$-NMR-Spektrum sind Signale für eine Methyl-, eine Methylen-, eine aliphatische und sechs olefinische Methingruppen sowie vier quartäre Kohlenstoffatome zu entnehmen. Mit Hilfe dieser Daten lässt sich für 96 die Summenformel $\mathrm{C}_{13} \mathrm{H}_{14} \mathrm{O}_{3}$ aufstellen, die durch ein hochaufgelöstes Massenspektrum bestätigt wurde. Im ${ }^{1}$ H-NMR-Spektrum treten Signale für insgesamt zwölf Protonen auf. Im Molekül müssen folglich 2 Hydroxygruppen enthalten sein, deren Protonen nicht detektiert werden können. Durch ein ${ }^{1} \mathrm{H},{ }^{1} \mathrm{H}-\mathrm{COSY}$-Experiment können zehn Protonen zu einer Alkylkette mit zwei trans-konfigurierten Doppelbindungen verknüpft werden. Die zwei verbleibenden Protonen bei $\delta_{\mathrm{H}}=6.28$ und 6.30 gehören aufgrund einer Kopplungskonstante von ${ }^{4} J=2.0 \mathrm{~Hz}$ zu einem tetrasubstituierten Aromaten, bei dem sich zwei Protonen in meta Position zueinander befinden. Die Verknüpfung dieser beiden Spinsysteme führt zu zwei denkbaren Benzofuranen (96 und 97). Ein Vergleich insbesondere der ${ }^{13} \mathrm{C}-\mathrm{NMR}-D a t e n$ mit Literaturdaten ${ }^{113}$ zeigt, dass es sich bei der isolierten Verbindung um Arthrographol (96) handelt. Die Stereochemie an C-2 wurde durch Vergleich der CD-Spektren mit 4,6-Dimethoxy-2-vinyldihydrobenzofuran als $\mathrm{R}$ bestimmt.

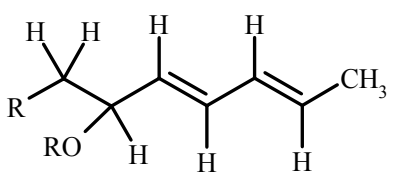<smiles>[R]c1ccc([R])c([R])c1[R]</smiles>
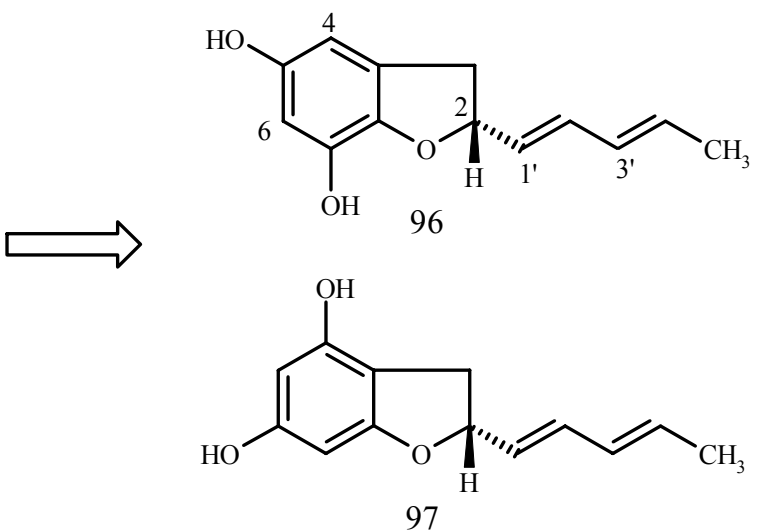

Abbildung 35: Strukturfragmente von 96 und deren mögliche Verknüpfungen.

96 wurde 1990 von zwei Arbeitsgruppen unter den Namen Arthrographol ${ }^{117}$ bzw. Asperfuran ${ }^{113}$ publiziert. Es besitzt antifungische Aktivität gegen eine Vielzahl verschiedener Pilzstämme und bewirkt bei Mucor miehei ab Konzentrationen von 20 ng/Testplättchen eine Veränderungen der Morphologie. Die phenolischen Hydroxygruppen scheinen für die antifungischen Aktivität von entscheidender Bedeutung zu sein, da die Methylierung oder Acetylierung zu einem Verlust dieser Aktivität führt. ${ }^{113}$ 


\subsection{Arthrolacton (98)}

Die Summenformel von 98 konnte durch ein hochaufgelöstes ESI-Massenspektrum als $\mathrm{C}_{14} \mathrm{H}_{14} \mathrm{O}_{4}$ bestimmt werden. Im ${ }^{1} \mathrm{H}-\mathrm{NMR}$-Spektrum erkennt man die Signale für eine Methyl-, eine Methylen-, eine aliphatische und sechs olefinische Methingruppen. Diese können durch ein ${ }^{1} \mathrm{H},{ }^{1} \mathrm{H}$-COSY-Experiment zu der für 96 beschriebenen Alkylkette mit zwei transkonfigurierten Doppelbindungen und zwei Methingruppen, die ebenso wie bei 96 zu einem tetrasubstituierten Aromaten gehören sollten, zusammengefügt werden. Das ${ }^{13} \mathrm{C}-\mathrm{NMR}$ Spektrum besitzt große Ähnlichkeit zu dem von 96, auffällig ist ein zusätzliches quartäres CAtom bei $\delta_{\mathrm{C}}=169.8$. Außerdem sind die Signale der phenolischen Hydroxygruppen an C-5 und C-7 um 12 bzw. 24 ppm ins Tieffeld und Signale für C-3a und C-7a um 14 bzw. 38 ppm ins Hochfeld verschoben. Dies deutet zusammen mit der Summenformel darauf hin, dass sich an C-7a eine Carboxylgruppe befindet. Durch Acetylierung der beiden phenolischen Hydroxygruppen konnte gezeigt werden, das diese Carbonylgruppe mit der Hydroxygruppe an C-2 einen Lactonring bildet.

Auf Grund der Ähnlichkeit der Struktur von 98 und Arthrographol (96) wird für 98 der Name Arthrolacton vorgeschlagen.<smiles>CC=CC=CC1Cc2cc(O)cc(O)c2C(=O)O1</smiles>

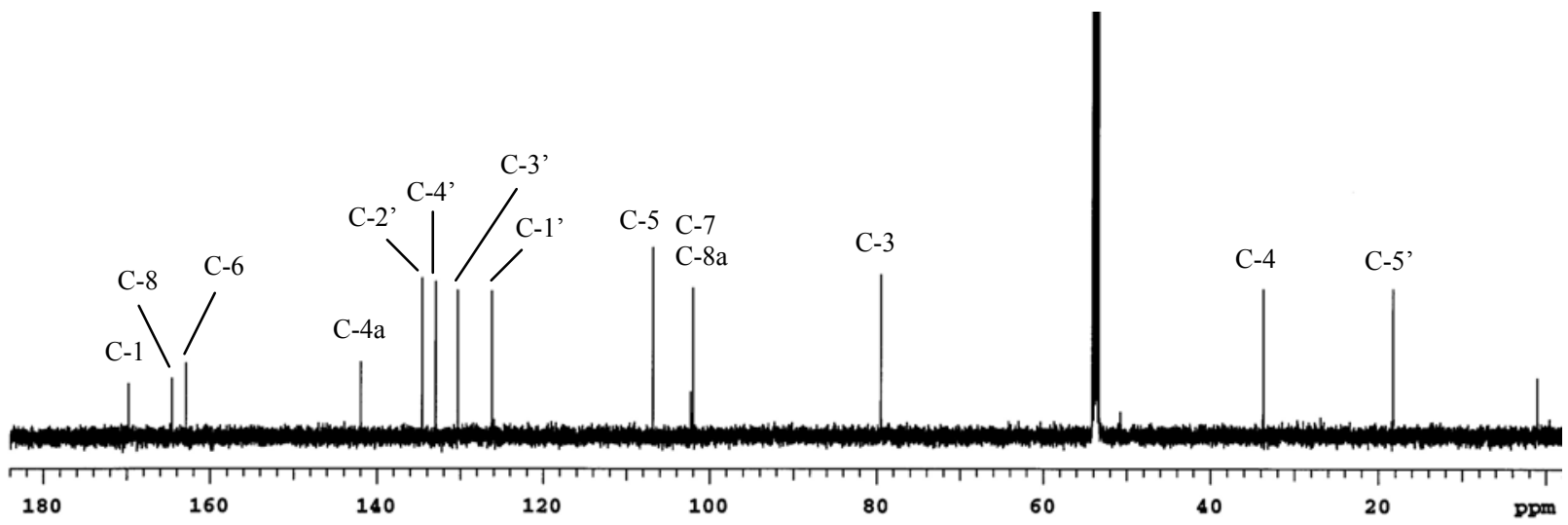

Abbildung 36: ${ }^{13} \mathrm{C}-\mathrm{NMR}-$ Spektrum $\left(150.8 \mathrm{MHz}, \mathrm{CD}_{2} \mathrm{Cl}_{2}\right)$ von Arthrolacton (98). 


\subsection{Untersuchungen zur Biosynthese von Arthrographol (96)}

Bei Arthrographol (96) handelt es sich um ein Benzofuran mit einer Alkylseitenkette. Für den Aufbau eines Benzofurans werden in der Literatur zwei mögliche Biosynthesewege beschrieben. Zum einen wird für verschiedene Pilze aber auch für Pflanzen die Biosynthese des Benzofuransystems auf dem Shikimatweg über Phenylalanin und Zimtsäure beschrieben. ${ }^{118}$ Zum anderen gibt es Beispiele für die Bildung von Benzofuranen auf dem Polyketidweg. ${ }^{119} \mathrm{Um}$ zu klären welcher der beiden möglichen Wege im Falle von 96 beschritten wird, sollten Fütterungsexperimente mit isotopenmarkierten Vorläufermolekülen durchgeführt werden.

Da Aspergillus flavus Stamm Gö 100/4 neben Arthrographol (96) auch Arthrolacton (98) bildet, welches sich von einem Biosynthesvorläufer von 96 ableiten könnte, wurde vermutet, dass es sich bei 96 um ein Heptaketid handelt, aus welchem im Verlauf der Biosynthese $\mathrm{CO}_{2}$ abgespalten wird. Daher sollte zunächst $\left[1-{ }^{13} \mathrm{C}\right]$ Acetat an Kulturen des Stammes Gö 100/2 verfüttert werden. Auch bei einer Bildung auf dem Shikimatweg ist eine Markierung von 96 durch $\left[1-{ }^{13} \mathrm{C}\right]$ Acetat zu erwarten, da in diesem Fall die Seitenkette über Malonyl-CoA aufgebaut werden sollte.

\section{Fermentationsverlauf}

Für Biosyntheseuntersuchungen ist es wichtig, den zeitabhängigen Produktionsverlauf der Sekundärmetabolite zu kennen. Erkenntnisse über den pH-Verlauf und die Produktbildung liefern notwendige Hinweise, um die Länge der Kultivierungszeit festzulegen und markierte Vorläufer zum richtigen Zeitpunkt in die spezifischen Stoffwechselwege eines Sekundärmetaboliten einschleusen zu können, damit eine genügend hohe Anreicherung im Zielmolekül erreicht wird. Daher erfolgt die Fütterung der markierten Vorläufer meist zu Beginn der Sekundärstoffproduktion und endet mit dem Erreichen der maximalen Konzentration eines Metaboliten.

Da Arthrographol (96) aus Schüttelkulturen von Aspergillus flavus Stamm Gö 100/4 in einer für Fütterungsexperimente ausreichenden Ausbeuten von $2.5 \mathrm{mg} / \mathrm{L}$ isoliert werden konnte, wurde auf eine Optimierung der Fermentationsbedingungen verzichtet. Um den optimalen Fütterungszeitpunkt zu bestimmt wurden von der sechsten bis zur 158. Stunde der Fermentation Proben von einer Schüttelkultur des Stammes genommen und mit Hilfe der HPLC analysiert. Die sich daraus ergebende Fermentationskurve ist in Abbildung 37 dargestellt 


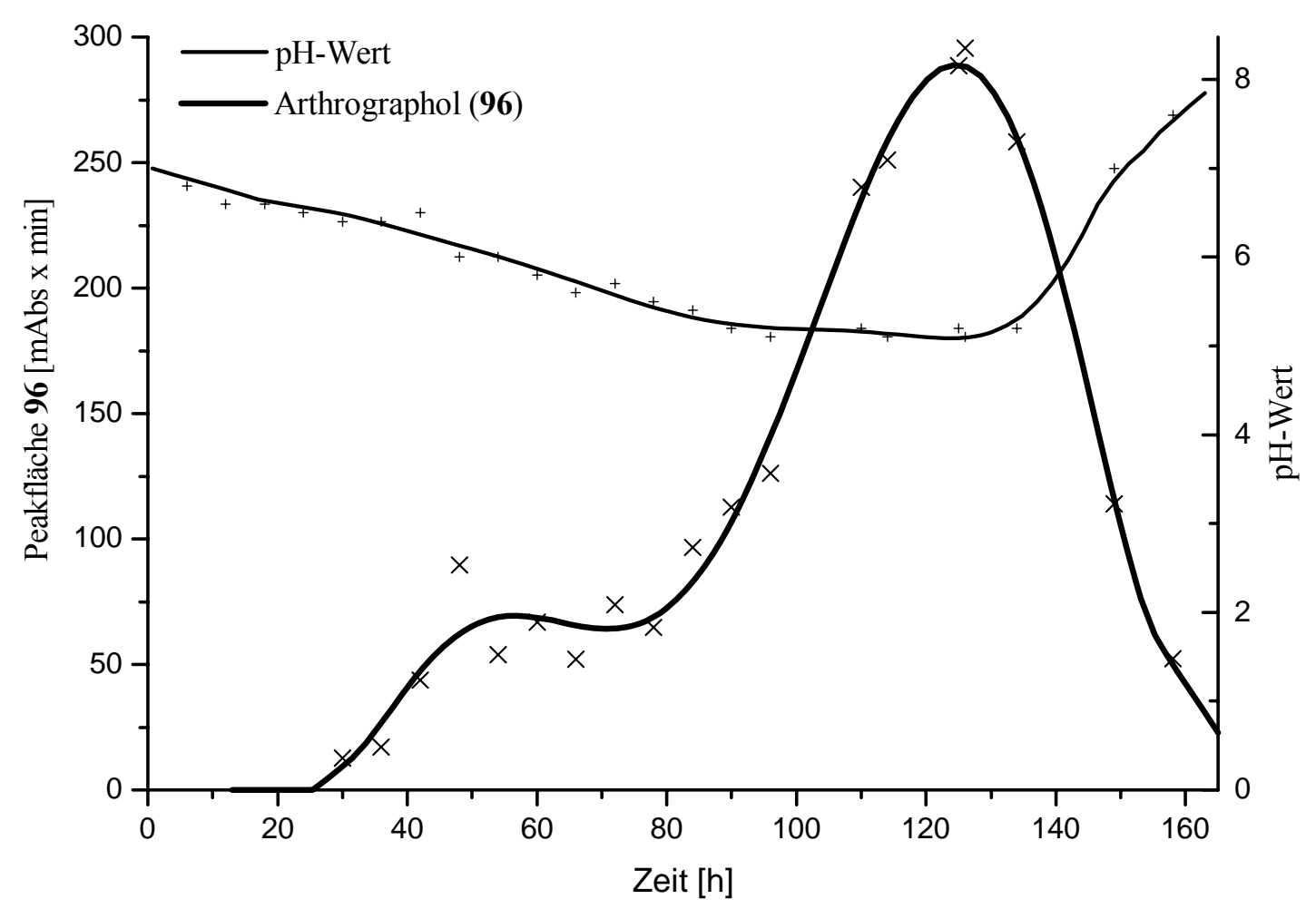

Abbildung 37: Verlauf der Bildung von Arthrographol (96).

Aus Abbildung 37 ist ersichtlich, dass die Produktion von Arthrographol ungefähr ab der 40 . Stunde einsetzt und nach ca. 130 Stunden ein Maximum erreicht. Nach Erreichen des Maximums kommt es zu einem steilen Abfall des Gehalts an Arthrographol in den Kulturen. Ungefähr zur selben Zeit steigt der $\mathrm{pH}-$ Wert, der von $\mathrm{pH}=6.8 \mathrm{zu}$ Beginn der Fermentation auf einen Wert von 5.1 bis 5.2 fällt, steil bis auf einen Wert von $\mathrm{pH}=7.6$ an.

Da zwischen der 85. und der 120. Stunde ein exponentieller Anstieg der Bildung von 96 zu verzeichnen ist, wurden die markierten Vorläufer von der 90. bis zur 108. Stunde im PulseFeeding-Verfahren zugefüttert. Nach weiteren 12 Stunden wurden die Kulturen geerntet.

\section{Fütterung von $\left[1-{ }^{13} \mathrm{C}\right]$ Acetat}

Durch die Fütterung von $\left[1-{ }^{13} \mathrm{C}\right]$ Acetat konnte die Ausbeute an Arthrographol (96) verdoppelt werden. Die beobachteten Einbauraten sind mit 30 - 67 \% ungewöhnlich hoch und belegen, dass das gefütterte Acetat direkt in 96 eingebaut wird. Aspergillus flavus Stamm Gö 100/4 scheint für die Biosynthese von 96 auf das zugefütterte Acetat zurückzugreifen und fast vollständig auf die Requirierung von Acetat aus andern Quellen zu verzichten. Das Einbaumuster der $\left[1-{ }^{13} \mathrm{C}\right]$ Acetat-Fütterung ist in Abbildung 39 dargestellt. 


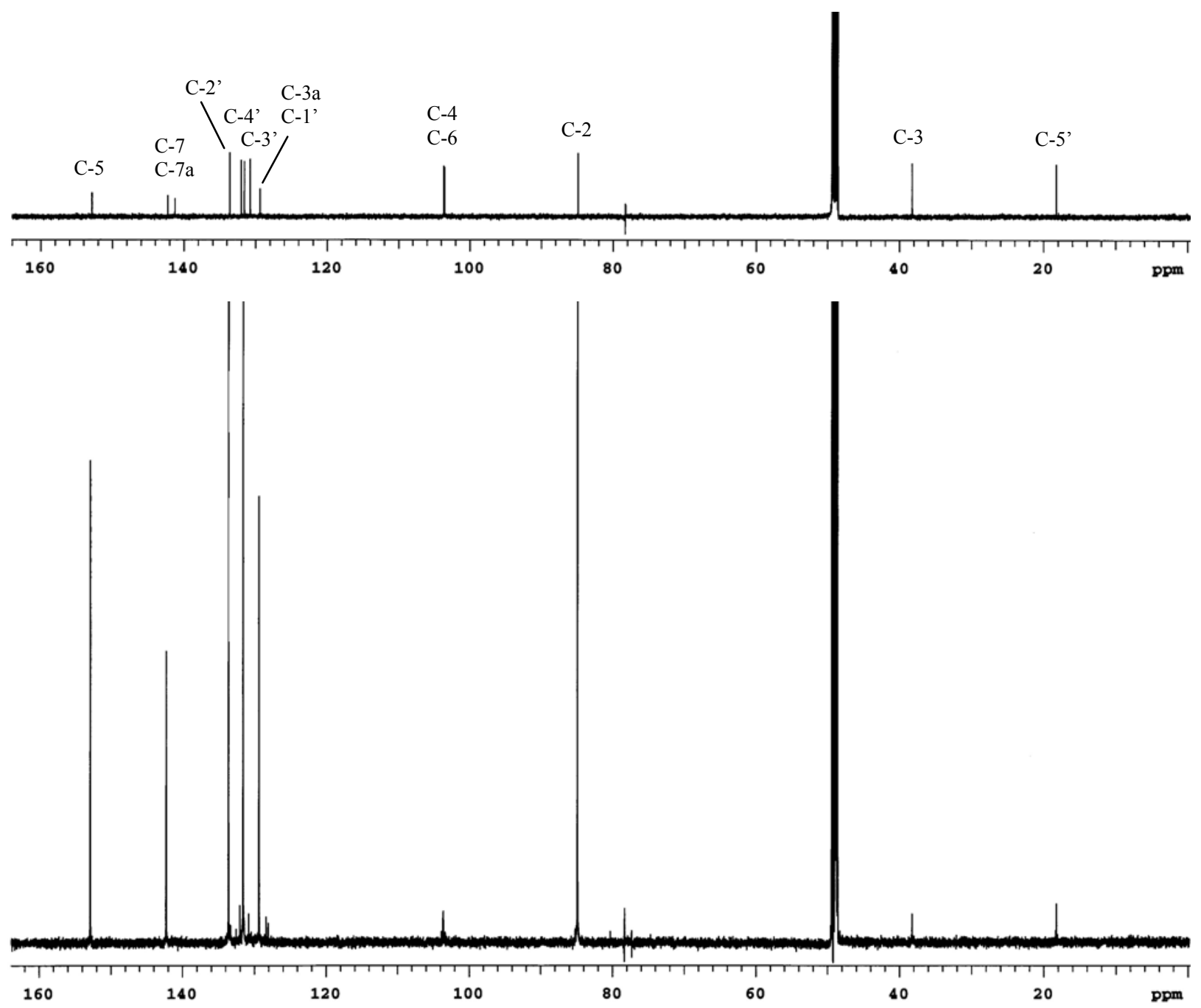

Abbildung 38: ${ }^{13} \mathrm{C}-\mathrm{NMR}-$ Spektrum $\left(150.8 \mathrm{MHz}, \mathrm{CD}_{3} \mathrm{OD}\right)$ von Arthrographol (96) aus der $\left[1-{ }^{13} \mathrm{C}\right]$ Acetat-Fütterung im Vergleich zum Referenzspektrum.

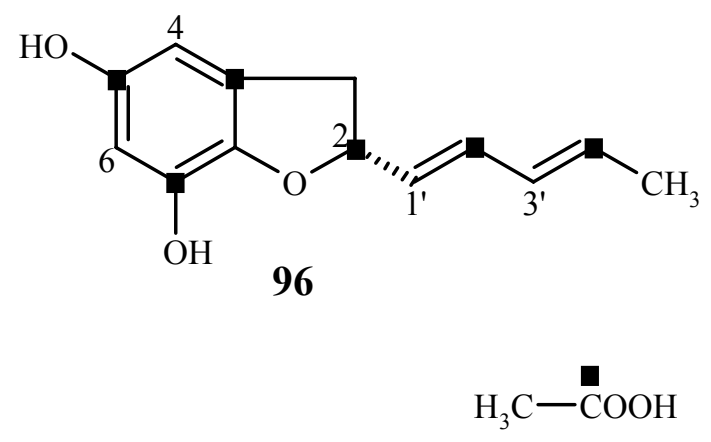

\begin{tabular}{|c|c|c|}
\hline C-Atom & $\begin{array}{c}\text { Verschiebung } \\
{[\mathrm{ppm}]}\end{array}$ & $\begin{array}{c}\text { Spez. Einbau } \\
{[\%]}\end{array}$ \\
\hline C-2 & 84.6 & $\mathbf{3 3 . 2}$ \\
\hline C-3 & 37.3 & -0.3 \\
\hline C-3a & 128.1 & $\mathbf{3 5 . 4}$ \\
\hline C-4 & 103.6 & -0.2 \\
\hline C-5 & 150.4 & $\mathbf{5 2 . 6}$ \\
\hline C-6 & 102.5 & -0.2 \\
\hline C-7 & 140.1 & $\mathbf{2 2 . 6}$ \\
\hline C-7a & 140.2 & 0.0 \\
\hline C-1 ${ }^{\prime}$ & 128.5 & -0.4 \\
\hline C-2 & 133.4 & $\mathbf{4 5 . 1}$ \\
\hline C-3 & 130.3 & -0.1 \\
\hline C-4' & 131.8 & $\mathbf{6 7 . 3}$ \\
\hline C-5 & 18.2 & 0.0 \\
\hline
\end{tabular}

Abbildung 39: Einbaumuster und Einbauraten der $\left[1-{ }^{13} \mathrm{C}\right]$ Acetat-Fütterung. 
Fütterung von $\left[1,2-{ }^{13} \mathrm{C}_{2}\right]$-Acetat

Um zu entscheiden in welcher Richtung die Acetateinheiten eingebaut werden, wurde eine zweite Fütterung mit doppeltmarkiertem Acetat durchgeführt. Diese konnte den Einbau von sechs intakten Acetateinheiten bestätigen (siehe Abbildung 41). An C-7a findet sich eine einfache ${ }^{13} \mathrm{C}$-Markierung, die vom C-2 des Acetats stammen sollte. Durch Acetylierung des aus der $\left[1,2-{ }^{13} \mathrm{C}_{2}\right]$-Acetat-Fütterung erhaltenen Arthrographols und Vergleich mit unmarkiertem, ebenfalls acetyliertem Arthrographol, konnte für C-9 ein spezifischer Einbau von $7.3 \%$ ermittelt werden.

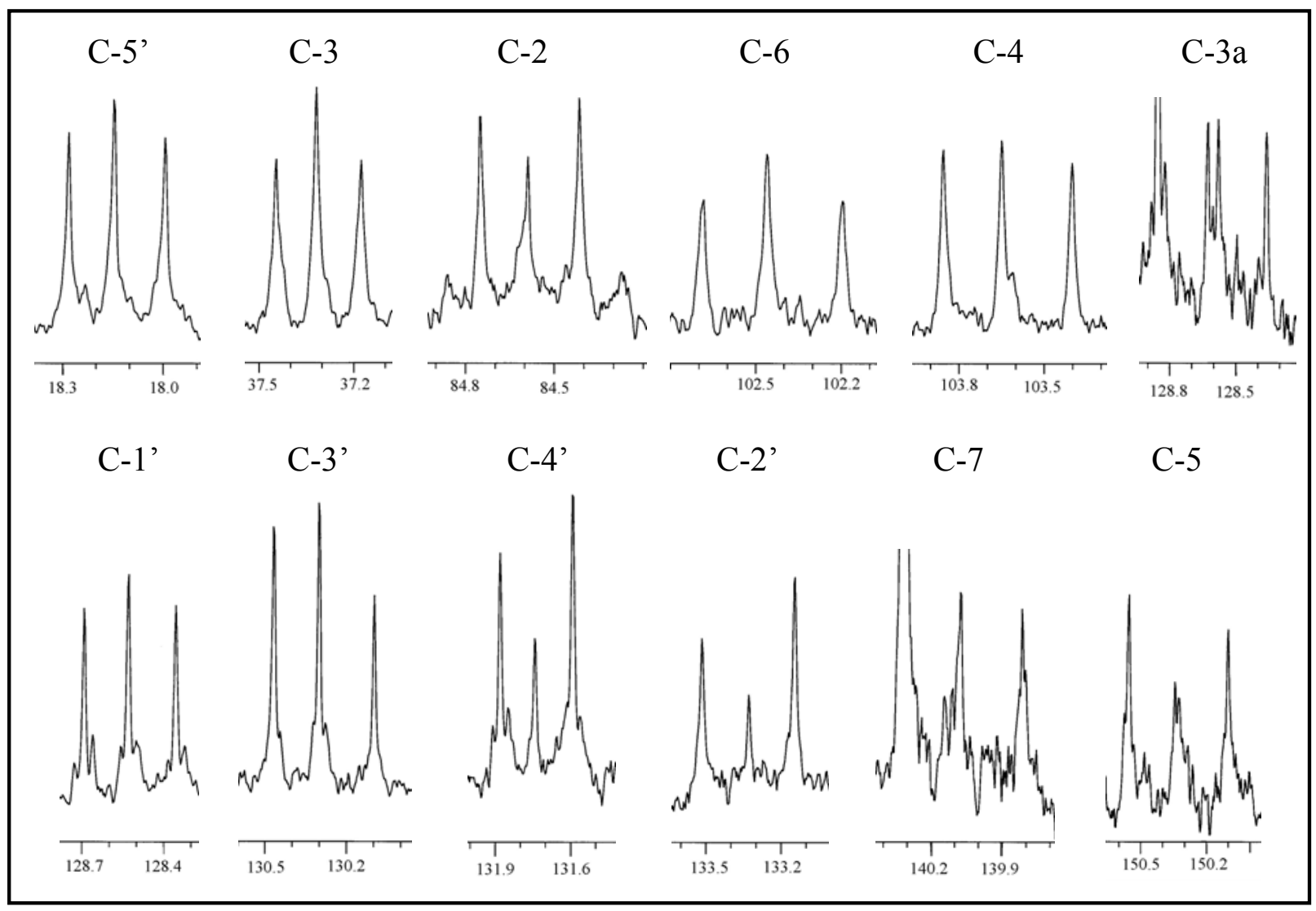

Abbildung 40: ${ }^{13} \mathrm{C}$-NMR-Signale von 96 aus der $\left[1,2-{ }^{13} \mathrm{C}_{2}\right]$-Acetat-Fütterung. 

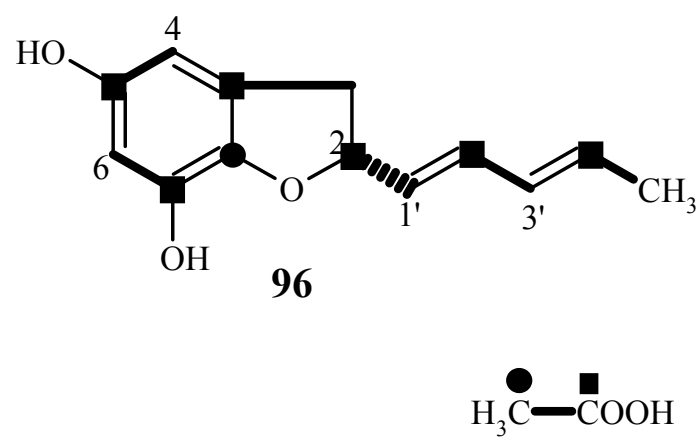

\begin{tabular}{|c|c|c|}
\hline C-Atom & $\begin{array}{c}\text { Verschiebung } \\
{[\mathrm{ppm}]}\end{array}$ & $\begin{array}{c}\text { Kopplung } \\
{[\mathrm{Hz}]}\end{array}$ \\
\hline $\mathrm{C}-2$ & 84.6 & 51 \\
\hline $\mathrm{C}-3$ & 37.3 & 41 \\
\hline $\mathrm{C}-3 \mathrm{a}$ & 128.1 & 41 \\
\hline $\mathrm{C}-4$ & 103.6 & 69 \\
\hline $\mathrm{C}-5$ & 150.4 & 69 \\
\hline $\mathrm{C}-6$ & 102.5 & 74 \\
\hline $\mathrm{C}-7$ & 140.1 & 74 \\
\hline $\mathrm{C}-7 \mathrm{a}$ & 140.2 & - \\
\hline $\mathrm{C}-{ }^{\prime}$ & 128.5 & 51 \\
\hline $\mathrm{C}-2^{\prime}$ & 133.4 & 56 \\
\hline $\mathrm{C}-3{ }^{\prime}$ & 130.3 & 56 \\
\hline $\mathrm{C}-4{ }^{\prime}$ & 131.8 & 44 \\
\hline C-5 & 18.2 & 44 \\
\hline
\end{tabular}

Abbildung 41: Einbaumuster und Kopplungskonstanten der $\left[1,2-{ }^{13} \mathrm{C}_{2}\right]$-Acetat-Fütterung.

Aus den Ergebnissen der beiden Fütterungen mit $\left[1-{ }^{13} \mathrm{C}\right]-$ und $\left[1,2-{ }^{13} \mathrm{C}_{2}\right]$ Acetat lässt sich ein Biosyntheseweg ableiten, bei dem durch eine pilzliche Polyketidsynthase zunächst aus einem Acetyl-CoA und 6 Einheiten Malonyl-CoA ein Heptaketid aufgebaut wird. Dieses wird im Anschluss zu einer Dihydroxybenzoesäure (99) cyclisiert, welche entweder durch Ausbildung eines Lactonringes zu 98 oder aber nach Decarboxylierung zum Benzofuran 96 reagieren kann. Da Aspergillus flavus Stamm Gö 100/4 Arthrographol (96) und nicht Arthrolacton (98) als Haupmetaboliten bildet, kann vermutet werden, dass die Decarboxylierung enzymatisch abläuft, da sonst die intramolekulare Lactonisierung zu 98 bevorzugt wäre. Ob diese Reaktion durch die Polyketidsynthase oder ein anderes Enzym katalysiert wird, kann allerdings anhand der durchgeführten Experimente nicht beantwortet werde.

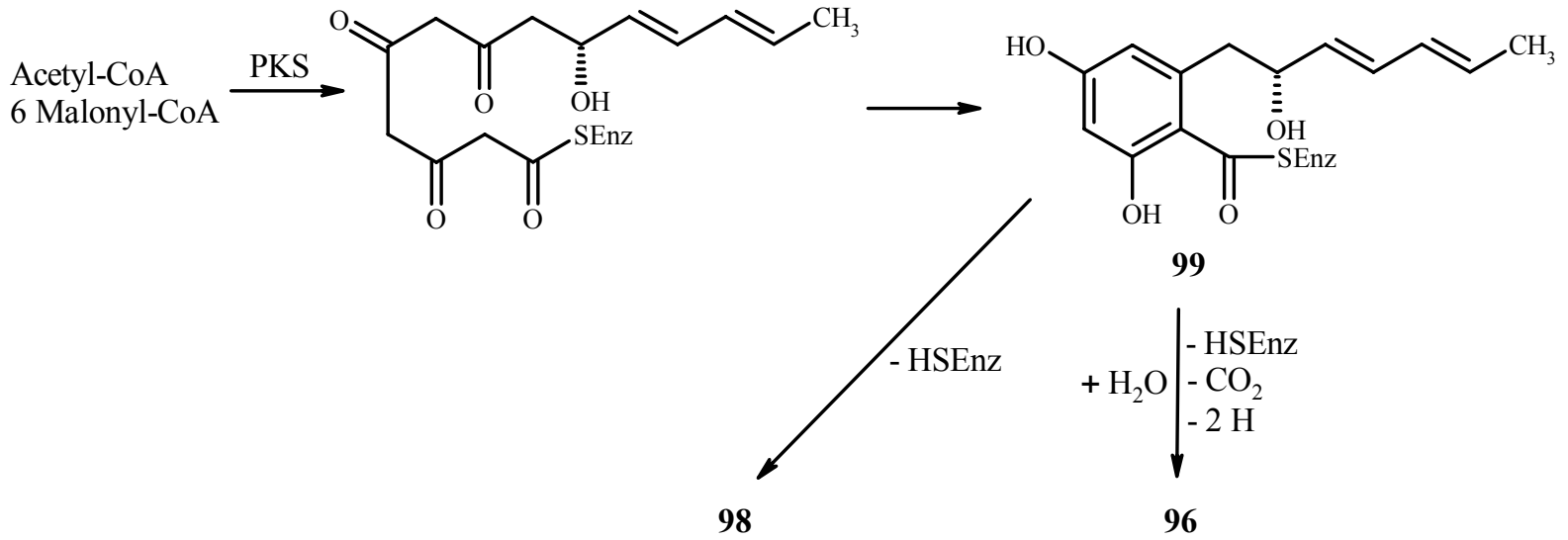

Abbildung 42: Biosynthese von Arthrographol (96) und Arthrolacton (98). 


\section{Chaetomium sp. Stamm Gö 100/9}

Der Pilz Chaetomium sp. Stamm Gö 100/9 fiel im Chemischen Screening bei Kultivierung in Schüttelkultur durch die Bildung mehrerer mit Anisaldehyd rot anfärbbaren, UV-löschenden Zonen auf. Aus einer Schüttelkultur im Medium SGG konnten neben den literaturbekannten Metaboliten Orsellinsäure (100) und Chetomin (119) sieben weitere, bisher nicht in der Literatur beschriebene Metabolite isoliert und charakterisiert werden. Unter diesen finden sich fünf Metabolite (101, 102, 104, 105 und 106) die aus Orsellinsäure sowie einem Desoxyzucker bestehen. Bei zwei weiteren Substanzen (107 und 108) handelt es sich vermutlich um Abbauprodukte von 104. Die Bildung von Desoxyzuckern wird bisher für Pilze nicht beschrieben, daher wurden Fütterungsexperimente mit $\left[1-{ }^{13} \mathrm{C}\right]$ Acetat und $\left[1-{ }^{13} \mathrm{C}\right]$ Glucose durchgeführt. Diese ergaben, dass sich die Desoxyzucker der isolierten Substanzen wie vermutet aus D-Glucose ableiten.

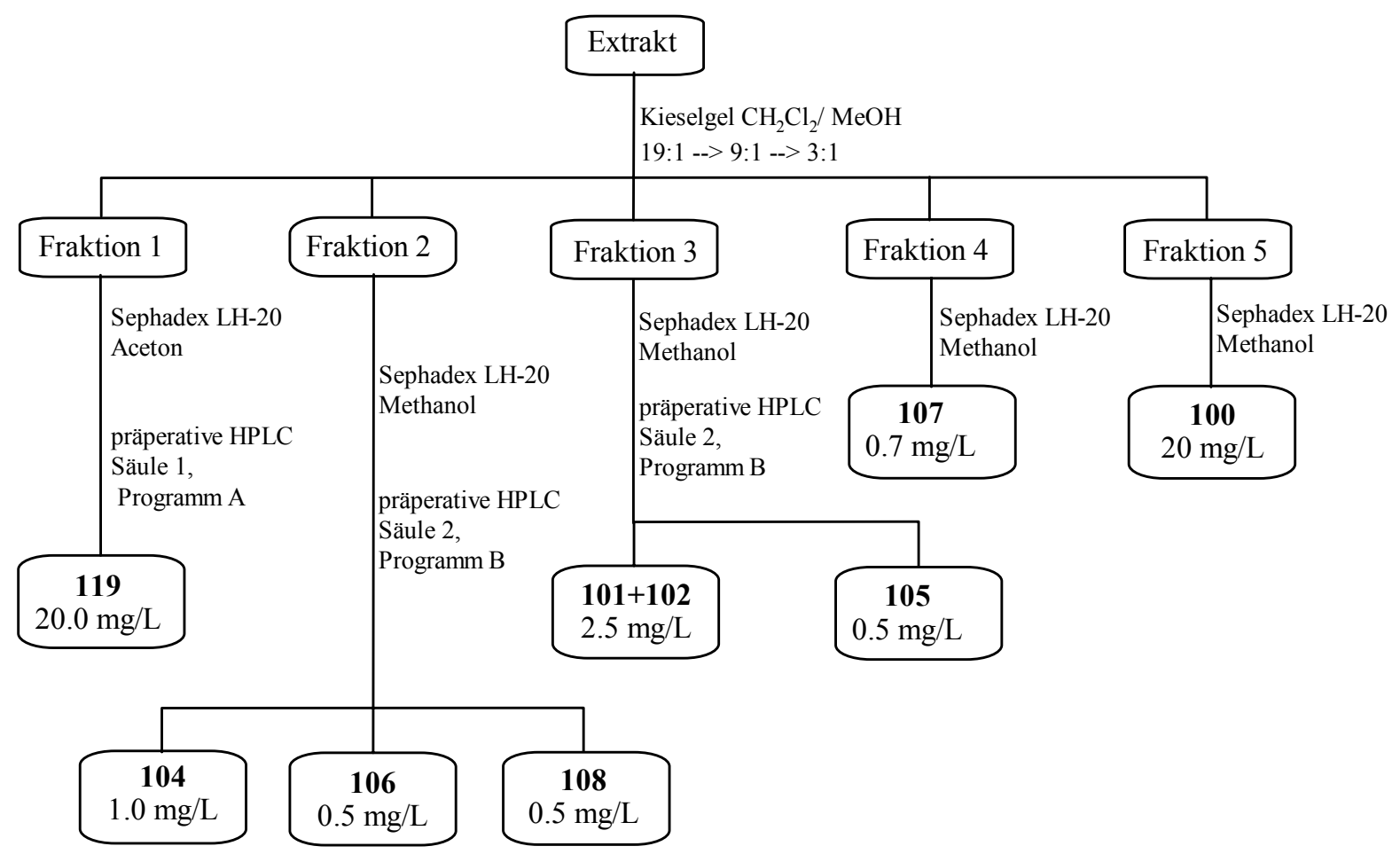

Abbildung 43: Aufreinigung des Extraktes aus der Schüttelkultur von Chaetomium sp. Stamm Gö 100/9 (Medium: SGG).

Bei einer Fermentation unter Zusatz von Acetat konnte die Ausbeute an 119 auf 135 mg/L gesteigert werden, daneben wurden drei weitere Metabolite (120, 121 und 125) in Ausbeuten von 10.5, 7.8 und $3.5 \mathrm{mg} / \mathrm{L}$ isoliert. $\mathbf{1 2 0}$ und $\mathbf{1 2 1}$ gehören zur Gruppe der Thiodiketopiperazine, wobei 121 bisher nicht in der Literatur beschrieben wurde. 
Somit konnten aus Chaetomium sp. Stamm Gö 100/9 insgesamt 12 Sekundärmetabolite, darunter acht neue, isoliert und in ihrer Struktur aufgeklärt werden.

In den Testsystemen der BASF AG fielen mehrere Extrakte des Stammes Gö 100/9 durch eine Aktivität gegen Phytophthora infestans und Septoria tritici auf. Durch einen HPLCMikrotest konnte diese Aktivität bestätigt und den Fraktionen 37-39 zugeordnet werden (Abbildung 44). Durch Vergleich der Retentionszeit der aktiven Komponente mit denen der isolierten Reinsubstanzen ließ sich die beobachtete Aktivität Chetomin (119) zuordnen, was durch anschließende Testierung bestätigt wurde. 119 hemmt $P$. infestans bis zu einer Konzentration von $0.5 \mathrm{ppm}$ und S.tritici bis zu einer Konzentration von $8.0 \mathrm{ppm}$.

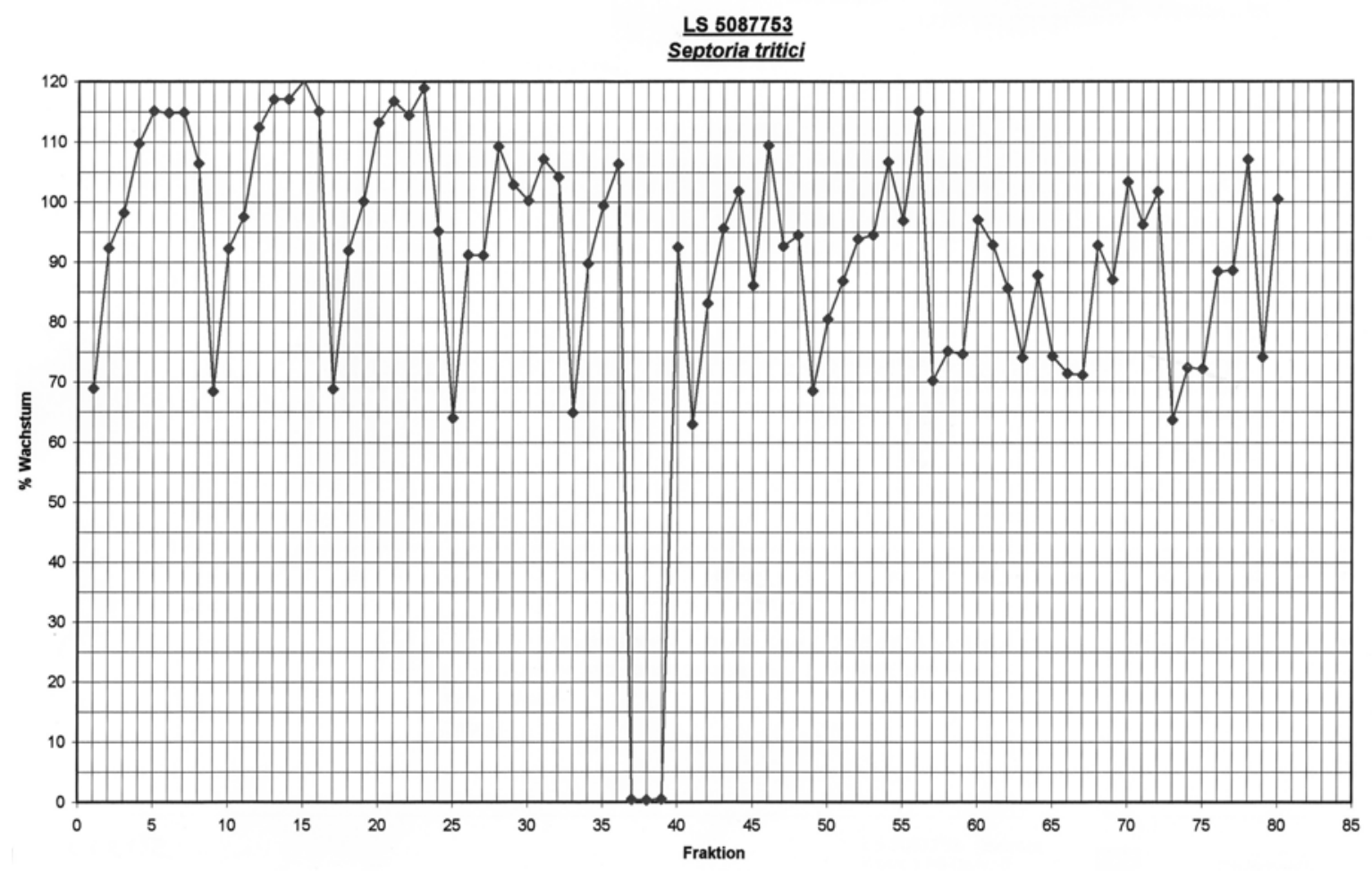

Abbildung 44: Hemmwirkung der einzelnen Fraktionen gegen Septoria tritici aus der HPLCTrennung des Stammes Gö 100/9.

\subsection{Orsellinsäure und mit Orsellinsäure acylierte Verbindungen}

Der Pilzstamm Gö 100/9 produzierte in Schüttelkultur (Medium: SGG) als Hauptmetaboliten Orsellinsäure (100). Daneben konnten sieben neue mit Orsellinsäure acylierte Sekundärmetabolite isoliert werden, wobei fünf dieser Metabolite einen Desoxyzucker enthalten. 


\subsubsection{Orsellinsäure (100)}

Das UV-Spektrum des Hauptmetaboliten zeigt im neutralen Bereich ein Maximum bei $270 \mathrm{~nm}$, das durch Zugabe von Natronlauge bathochrom auf $314 \mathrm{~nm}$ verschoben wird und somit auf das Vorhandensein phenolischer Hydroxygruppen hinweist. Im EI-Massenspektrum findet sich der Peak höchster Masse bei $\mathrm{m} / \mathrm{z}=168$, dessen Hochauflösung zur Summenformel $\mathrm{C}_{8} \mathrm{H}_{8} \mathrm{O}_{4}$ führt. Die acht Kohlenstoffatome können durch ein ${ }^{13} \mathrm{C}-\mathrm{NMR}$-Spektrum einer Methyl- und zwei Methingruppen sowie vier quartären Kohlenstoffatomen zugeordnet werden. Im ${ }^{1}$ H-NMR-Spektrum treten die Signale der Methylgruppe bei $\delta_{\mathrm{H}}=2.45$ und die der Methingruppen bei $\delta_{\mathrm{H}}=6.14$ und 6.17 auf. Die beiden Methingruppen sollten sich auf Grund einer Kopplungskonstante von ${ }^{4} J=2.0 \mathrm{~Hz}$ in meta Position zueinander an einem tetrasubstituierten Aromaten befinden. Eine mit diesen Informationen durchgeführte Datenbanksuche $^{53}$ führt zu Orsellinsäure ${ }^{120}(\mathbf{1 0 0})$.

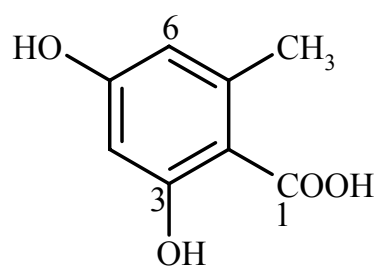

100

\subsubsection{Orsellide A (101) und B (102)}

Das UV-Spektrum der als farbloses Öl vorliegenden Verbindung zeigt große Ähnlichkeit zu dem von 100, was für das Vorhandensein desselben Chromophors spricht. Im ${ }^{1} \mathrm{H}-\mathrm{NMR}-$ Spektrum zeigen sich die Signalsätze von zwei Verbindungen, die im Verhältnis $3: 2$ vorliegen. Die beiden Verbindungen konnten zwar mit der präparativer HPLC getrennt werden, wandelten sich jedoch innerhalb kürzester Zeit wieder in einander um, was auf ein Gleichgewichts zwischen zwei strukturisomeren Verbindungen hinweist. Da eine Trennung nicht möglich war, wurden die Strukturen der Isomeren aus dem Gemisch heraus aufgeklärt. Die Summenformel $\left(\mathrm{C}_{15} \mathrm{H}_{18} \mathrm{O}_{8}\right)$ konnte durch ein hochaufgelöstes ESI-Massenspektrum bestimmt werden. Im ${ }^{1} \mathrm{H}-\mathrm{NMR}-\mathrm{Spektrum}$ können dem Hauptisomer $\mathbf{1 0 1}$ die Signale von drei Methyl- und sechs Methingruppen zugeordnet werden. Zwei ins Tieffeld verschobene Methingruppen bei $\delta_{\mathrm{H}}=6.22$ und 6.24 gehören auf Grund einer Kopplungskonstante von ${ }^{4} J=2.0 \mathrm{~Hz}$ zu einem 1,2,3,4-tetrasubstituiertem Aromaten und weisen zusammen mit der 
Methylgruppe bei $\delta_{\mathrm{H}}=2.51$ daraufhin, dass ein Orsellinsäurederivat vorliegt. Diese Vermutung wird durch das ${ }^{13} \mathrm{C}-\mathrm{NMR}-$ Spektrum, in dem sich acht Signale finden, deren Verschiebung zu denen von $\mathbf{1 0 0}$ passen, bestätigt. Die verbleibenden sieben Signale können einer Methyl-, einer Methoxy- und vier Methingruppen sowie einem Carbonylkohlenstoff bei $\delta_{\mathrm{C}}=200.0$ zugeordnet werden. Die Tieffeldverschiebungen der vier Methingruppen deuten auf die Verknüpfung mit Sauerstoff hin, wobei eine Methingruppe bei $\delta_{\mathrm{C}}=100.0$ an zwei Sauerstoffatome gebunden sein sollte. Aus einem ${ }^{1} \mathrm{H},{ }^{1} \mathrm{H}-\mathrm{COSY}$-Experiment konnten die in Abbildung 45 dargestellten Fragmente erhalten und durch ein HMBC-Experiment zu 101 verknüpft werden.<smiles>COC(=O)c1c(C)cc(Br)cc1OCCO</smiles><smiles>[R]C(=O)O</smiles><smiles>[R]C([R])C([R])O[K]</smiles><smiles>CO[C@H]1O[C@@H](C)[C@@H](O)C(=O)[C@H]1OC(=O)c1c(C)cc(O)cc1O</smiles>

Abbildung 45: Strukturfragmente von Orsellid A (101) und deren Verknüpfung über ausgewählte HMBC-Korrelationen.

Im ${ }^{13}$ C-NMR-Spektrum der Minderkomponente Orsellid B finden sich in Analogie zu 101 acht Signale, deren Verschiebung zu denen von Orsellinsäure (100) passen. Die verbleibenden sieben Signale können einer Methyl-, einer Methoxy- und vier Methingruppen sowie einem Carbonylkohlenstoff bei $\delta_{\mathrm{C}}=199.6$ zugeordnet werden. Durch ein ${ }^{1} \mathrm{H},{ }^{1} \mathrm{H}-\mathrm{COSY}$ - und ein HMBC-Experiment gelingt die Verknüpfung dieser sieben Kohlenstoffatome zu dem aus 101 bekannten Zuckerbestandteil. Der Unterschied liegt in der Verknüpfung mit Orsellinsäure (100). Bei Orsellid A (101) war es die Position 2', bei Orsellid B ist es 4', was zur Struktur 102 führt.

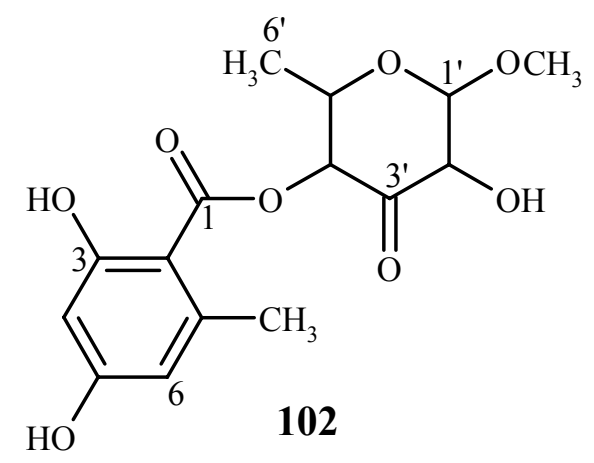


Die relative Stereochemie des Ketozuckers von 101 und 102 kann aus den in den ${ }^{1}$ H-NMRSpektren beobachteten Kopplungskonstanten und einem NOESY-Experiment abgeleitet werden. Auf Grund einer Kopplungskonstante von ${ }^{3} J=10.0 \mathrm{~Hz}$ müssen die Protonen 4'-H und 5'-H trans zu einander stehen. Die Kopplungskonstante von ${ }^{3} J=4.5 \mathrm{~Hz}$ zwischen 1'-H und 2'-H kann auf eine axial-äquatoriale Kopplung zurückgeführt werden. In einem NOESYExperiment können Korrelationen zwischen 2'-H und 4'-H sowie zwischen 5'-H und der Methoxygruppe an C-1' beobachtet werden. Daraus ergibt sich die in Abbildung 47 dargestellte relative Stereochemie der beiden Verbindungen 101 und 102.

Die axiale Position der Methoxygruppe an C-1' konnte zusätzlich durch ein gekoppeltes ${ }^{13} \mathrm{C}$ NMR-Spektrum, das eine C-H-Kopplungskonstante von ${ }^{1} J_{C, H}=175 \mathrm{~Hz}$ ergab, bestätigt werden. Bei einer äquatorialen Position der Methoxygruppe wäre ein Wert zwischen 160 und $170 \mathrm{~Hz}$ zu erwarten gewesen. ${ }^{121}$

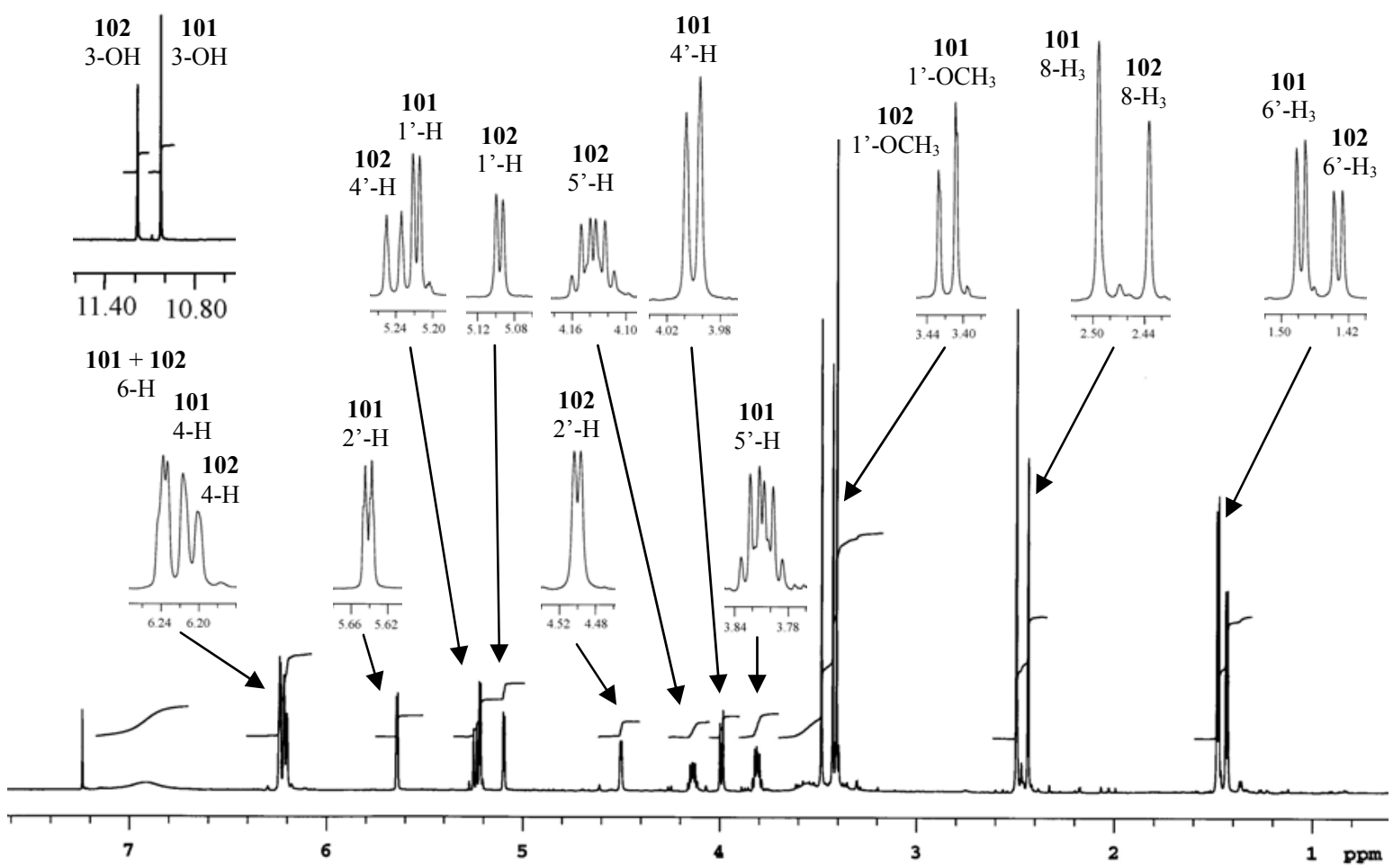

Abbildung 46: ${ }^{1} \mathrm{H}-\mathrm{NMR}-$ Spektrum $\left(600 \mathrm{MHz}, \mathrm{CDCl}_{3}\right)$ von Orsellide A (101) und B (102). 


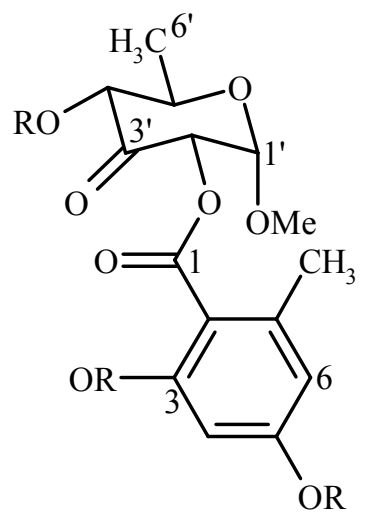

101a $\mathrm{R}=\mathrm{H}$

101b $\mathrm{R}=\mathrm{CH}_{3} \mathrm{CO}$

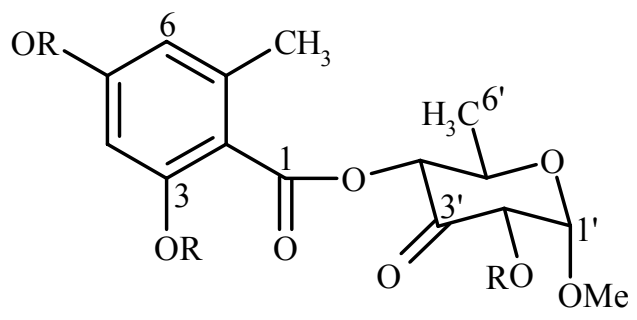

102a $\mathrm{R}=\mathrm{H}$

102b $\mathrm{R}=\mathrm{CH}_{3} \mathrm{CO}$

Abbildung 47: Relative Stereochemie der Orsellide A (101a) und B (102a).

Um die Orsellide A (101) und B (102) doch noch als Reinsubstanzen zu erhalten, wurde versucht sie mit Hilfe der präparativen HPLC zu trennen. Bei diesen Versuchen zeigte sich, dass eine Trennung zwar möglich ist, aber bereits nach dem Entfernen des HPLCLösungsmittels lagen 101 und 102 wieder im Gleichgewicht vor. Daher wurde das Gemisch der beiden Verbindungen acetyliert und anschließend per präparativer HPLC getrennt. Mit dieser Methode ist eine Trennung der acetylierten Orsellide A und B möglich. Erhalten wurden die Triacetate 101b und 102b. Anhand des Drehwertes $[\alpha]_{D}^{20}=+79^{\circ}(\mathrm{c}=0.1$, Methanol) von 101b kann durch Vergleich mit dem für Diethyl-(2,4-Di-O-acetyl-6-desoxy-3keto- $\alpha$-D-ribo-hexopyranosyl)-phosphat ${ }^{122}$ bestimmten Drehwertes $[\alpha]_{D}^{20}=+84.5^{\circ}(\mathrm{c}=1$, Chloroform) auf die absolute Stereochemie von 101 und 102 geschlossen werden. Danach sind die beiden Stereozentren an C-1' und C-2' S-konfiguriert während C-4' und C-5' die $R$-Konfiguration besitzen und der in 101 und 102 enthaltene Zuckerbestandteil kann als Methyl-6-desoxy-3-keto- $\alpha$-D-ribo-hexopyranose identifiziert werden.

Um das zwischen den Orselliden A (101) und B (102) vorliegende Gleichgewicht näher zu untersuchen, wurde ein Gemisch der beiden Substanzen per HPLC getrennt und das dabei erhaltenen 101 dann im Abstand von jeweils einer Stunde mittels HPLC untersucht. Auf diese Weise konnte die Einstellung des Gleichgewichts zwischen 101 und 102 beobachtet werden. Es zeigte sich, dass bei Raumtemperatur nach ca. 35 Stunden das Gleichgewicht zwischen den beiden Isomeren erreicht wird (Abbildung 48). 


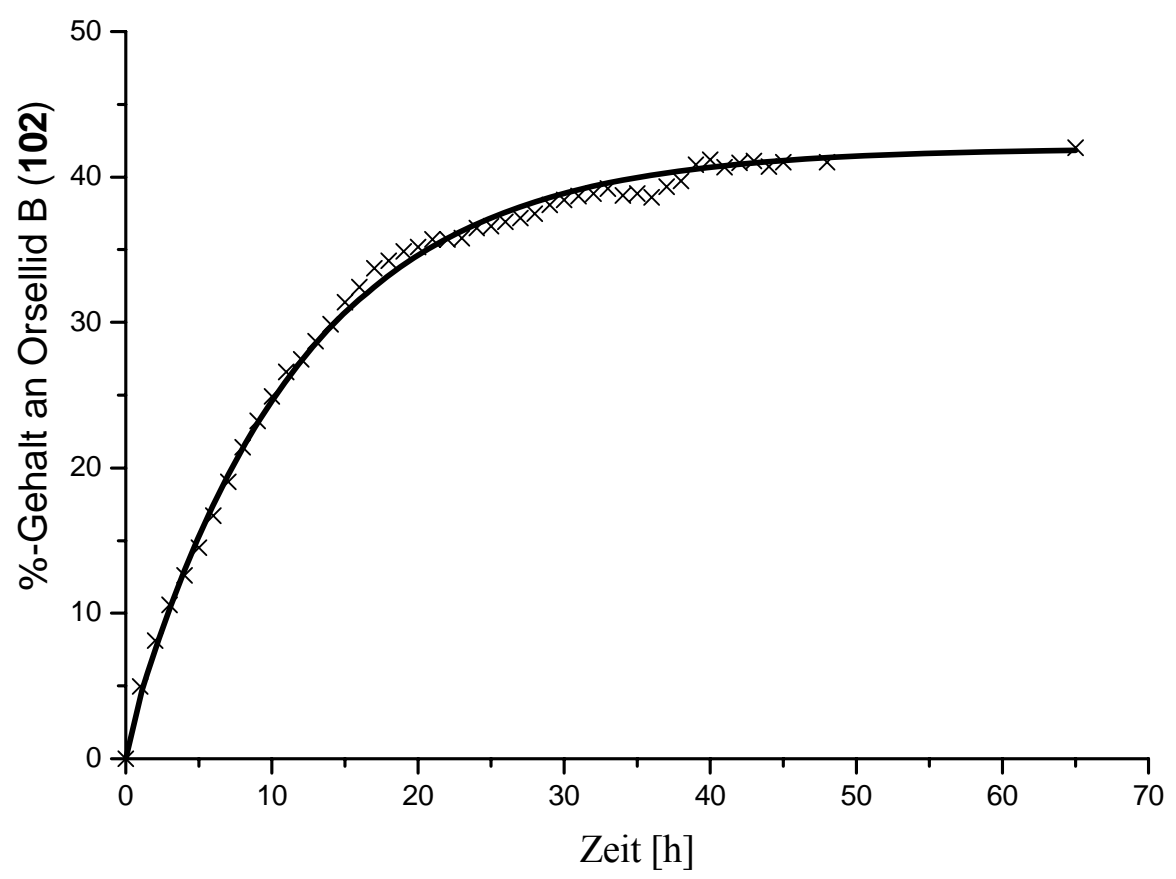

Abbildung 48: Einstellung des Gleichgewichts zwischen den Orselliden A (101) und B (102).

Bei der Umwandlung von 101 in 102 kommt es zu einer 1,3-Acylverschiebung, die mit Hilfe eines sechsgliedrigen Übergangszustandes erklärt werden kann. Um diesen zu erreichen, muss 101 zunächst von der Sessel- in die Wannenkonformation umklappen. In der Wannenkonformation kommen sich der Acylrest an C-2' und die Hydroxygruppe an C-4' relativ nah, so dass durch Angriff der Hydroxygruppe am Carbonylkohlenstoffatom des Acylrestes der sechsgliedrige Orthoester-Übergangszustand (103) ausgebildet wird. 103 vermittelt zwischen 101 und 102, wobei 101 etwas stabiler ist als 102.<smiles>[R]C(=O)O[C@H](OC)[C@@H](OC)C(=O)O</smiles><smiles>[R]C(=O)OC1C(C)C(=O)C(O)C1OC</smiles><smiles>[R]#Cc1c(C)cc(O)cc1O</smiles><smiles>[R]C(=O)O[C@H]1C(=O)[C@H](O)[C@@H](OC)O[C@H]1C</smiles>

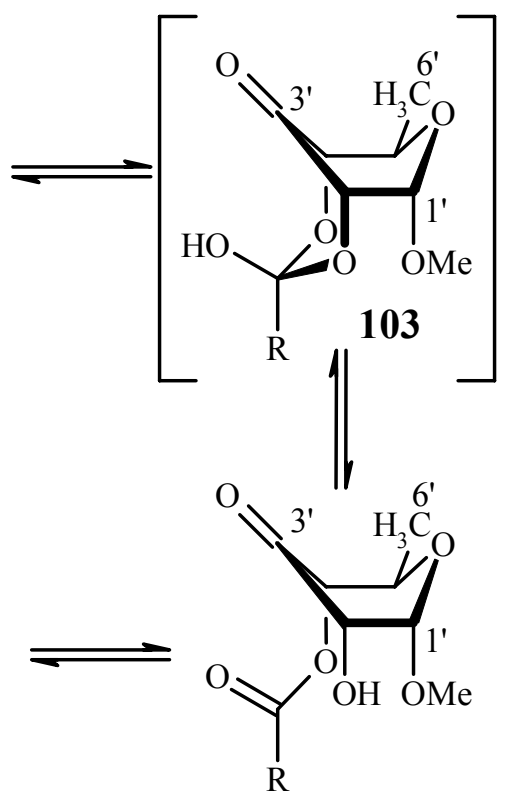

Abbildung 49: Mechanismus für die Umlagerung von 101 in 102. 


\subsubsection{Orsellid C (104)}

Anhand des UV-Spektrums dieser Verbindung, welches große Ähnlichkeit zu denen von 101 und 102 besitzt, kann vermutet werden, dass Orsellid C ebenfalls Orsellinsäure als Chromophor enthält. Diese Vermutung wird durch das ${ }^{13} \mathrm{C}-\mathrm{NMR}-\mathrm{Spektrum}$, in dem acht Signale auftreten deren Verschiebungen zu denen von Orsellinsäure passen, bestätigt. Unter den restlichen sieben Signalen findet sich das Signal eines Carbonylkohlenstoffs $\left(\delta_{C}=198.5\right)$ und das einer Methingruppe bei $\delta_{\mathrm{C}}=100.3$. Im Vergleich zu 101 und 102 fehlt das Signal einer Methingruppe, dafür tritt eine zusätzliche Methylengruppe bei $\delta_{\mathrm{C}}=48.5$ auf. Die Vermutung, dass es sich um ein Desoxyderivat von 101 oder 102 handelt, wird durch ein ESIMassenspektrum bestätigt. Dieses führt durch Hochauflösung des $[\mathrm{M}+\mathrm{Na}]^{+}$-Ion bei $\mathrm{m} / \mathrm{z}=333$ zur Summenformel $\mathrm{C}_{15} \mathrm{H}_{18} \mathrm{O}_{7}$. Im ${ }^{1} \mathrm{H}$-NMR-Spektrum finden sich die Signale der Methylengruppe bei $\delta_{\mathrm{H}}=2.50$ und 2.59. Durch ein ${ }^{1} \mathrm{H},{ }^{1} \mathrm{H}-\mathrm{COSY}$-Experiment kann diese Methylengruppe mit einer Methingruppe bei $\delta_{\mathrm{H}}=4.24$, die mit einer Methylgruppe bei $\delta_{\mathrm{H}}=1.37$ verbunden ist, verknüpft werden. Eine weitere Methingruppe bei $\delta_{\mathrm{H}}=5.56$ weist eine Kopplung mit dem Signal der anomeren Methingruppe $\left(\delta_{\mathrm{H}}=5.19\right)$ auf.

Somit handelt es sich bei Orsellid C um 4'-Desoxy-101 entsprechend Formel 104, was durch ein HMBC-Experiment bestätigt werden konnte. Die relative Stereochemie kann aus den Kopplungskonstanten des ${ }^{1}$ H-NMR-Spektrums abgeleitet werden. Durch den Vergleich des Drehwertes von $[\alpha]_{D}^{20}=+45^{\circ}(\mathrm{c}=0.1$, Methanol) mit dem von Methyl-2-O-Benzoyl-4,6didesoxy-3-keto- $\alpha$-D-erythro-hexopyranose ${ }^{123}\left([\alpha]_{D}^{20}=+66^{\circ}(\mathrm{c}=1.8\right.$, Chloroform $\left.)\right)$ kann die absolute Konfiguration an C-1' und C-2' mit $S$ und an C-5' mit $R$ bestimmt werden. Damit kann der Zuckerbestandteil von $\mathbf{1 0 4}$ als Methyl-4,6-didesoxy-3-keto- $\alpha$-D-erythrohexopyranose identifiziert werden.

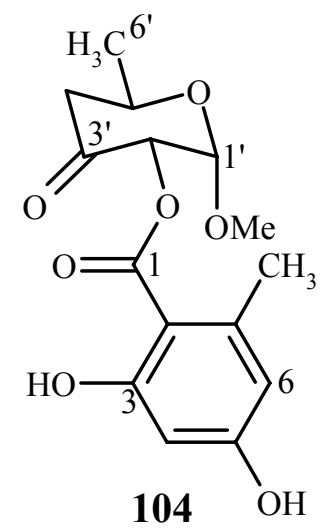




\subsubsection{Orsellid D (105)}

Das UV-Spektrum von Orsellid D weist durch Ähnlichkeit zu den vorher beschriebenen Orselliden A - C (101-104) darauf hin, dass der farblose Feststoff ebenfalls Orsellinsäure als Chromophor enthält. Dies wird durch das ${ }^{13}$ C-NMR-Spektrum, in dem acht Signale auftreten deren Verschiebungen zu denen von Orsellinsäure passen, bestätigt. Die restlichen sechs Signale können einer Methyl- und drei Methingruppen sowie zwei quartären Kohlenstoffatomen zugeordnet werden. Zwei der Methingruppen mit Verschiebungen von $\delta_{\mathrm{C}}=72.5$ und 80.8 sollten mit Sauerstoff verknüpft sein, wohingegen die andere Methingruppe $\left(\delta_{C}=128.7\right)$ zusammen mit den quartären Kohlenstoffatomen bei $\delta_{C}=157.8$ und $188.1 \mathrm{zu}$ einem $\alpha, \beta$-ungesättigtem Keton gehört. Durch ein ${ }^{1} \mathrm{H},{ }_{1}^{1} \mathrm{H}$-COSY-Experiment können die beiden aliphatischen Methingruppen $\left(\delta_{\mathrm{H}}=4.19\right.$ und 4.36) miteinander verknüpft werden. Das Signal bei $\delta_{\mathrm{H}}=4.36$ weist zusätzlich eine Kopplung mit einer Methylgruppe bei $\delta_{\mathrm{H}}=1.60$ auf. Anhand dieser Strukturelemente liegt die Vermutung nahe, dass Orsellid D durch Abspaltung von Methanol aus 101 hervorgeht. Diese Vermutung kann durch eine Hochauflösung des im ESI-Massenspektrum bei $\mathrm{m} / \mathrm{z}=317$ detektierten $[\mathrm{M}+\mathrm{Na}]^{+}$-Ion, die zur Summenformel $\mathrm{C}_{14} \mathrm{H}_{14} \mathrm{O}_{7}$ führt, bestätigt werden. Die relative Stereochemie lässt sich aus den Kopplungskonstanten des ${ }^{1} \mathrm{H}-\mathrm{NMR}-\mathrm{Spektrums}$ ableiten, während die absolute Konfiguration durch Vergleich des Drehwertes mit Werten ähnlicher Verbindungen ${ }^{122}$ ermittelt wurde. Daraus ergibt sich für C-4' und C-5' die R-Konfiguration und der in $\mathbf{1 0 5}$ enthaltene Zucker kann als 1,2-Anhydro-6-desoxy-3-keto- $\alpha$-D-ribo-hexopyraose identifiziert werden.

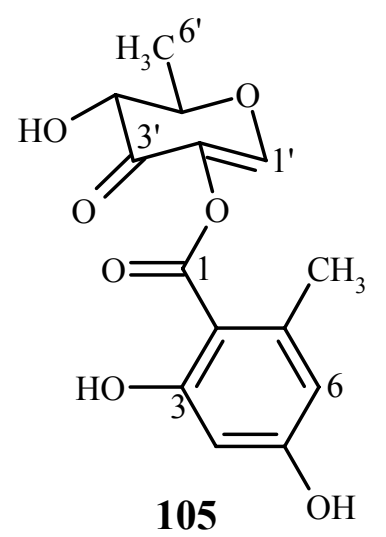




\subsubsection{Orsellid E (106)}

Durch ein hochaufgelöstes ESI-Massenspektrum kann für das als farbloses Öl vorliegende Orsellid E die Summenformel $\mathrm{C}_{14} \mathrm{H}_{14} \mathrm{O}_{6}$ bestimmt werden. Diese deutet darauf hin, dass es sich von 105 durch das Fehlen einer Hydroxygruppe unterscheidet. Das ${ }^{13} \mathrm{C}-\mathrm{NMR}-\mathrm{Spektrum}$ besitzt große Ähnlichkeit zu dem von 105, wobei das Signal einer Methingruppe fehlt und stattdessen bei $\delta_{\mathrm{C}}=42.5$ das Signal einer Methylengruppe beobachtet werden kann. Im ${ }^{1} \mathrm{H}$ NMR-Spektrum können der Methylengruppe Signale bei $\delta_{\mathrm{H}}=2.63$ und 2.74 zugeordnet werden. Durch ein ${ }^{1} \mathrm{H},{ }^{1} \mathrm{H}-\mathrm{COSY}$-Experiment kann die Methylengruppe mit einer Methingruppe bei $\delta_{\mathrm{H}}=4.75$, die wiederum an ein Methylgruppe bei $\delta_{\mathrm{H}}=1.52$ gebunden ist, verknüpft werden. Diese Beobachtungen bestätigen, dass Orsellid E entsprechend Formel 106 ein 4'-Desoxy-105 ist.

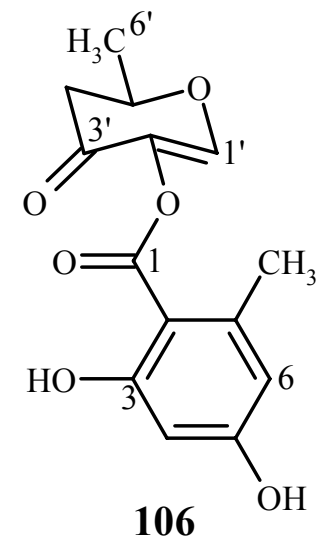

\subsection{6. (4-Hydroxy-pent-2-on-1-yl)-orsellinat (107)}

Das UV-Spektrum der Verbindung ähnelt denen der Orsellide A-E (101-106). Die Summenformel kann durch ein hochaufgelöstes ESI-Massenspektrum mit $\mathrm{C}_{13} \mathrm{H}_{16} \mathrm{O}_{6}$ bestimmt werden. Acht der 13 im ${ }^{13} \mathrm{C}-\mathrm{NMR}-$ Spektrum auftretenden Signale können anhand ihrer Verschiebungen Orsellinsäure zugeordnet werden. Die verbleibenden Signale gehören zu einer Methyl-, zwei Methylen- und einer Methingruppe sowie zu einem Carbonylkohlenstoff $\left(\delta_{\mathrm{C}}=204.3\right)$. Im ${ }^{1} \mathrm{H}-\mathrm{NMR}-\mathrm{Spektrum}$ weisen die Tieffeldverschiebungen der Methingruppe $\left(\delta_{\mathrm{H}}=4.23\right)$ und einer Methylengruppe bei $\delta_{\mathrm{H}}=5.00$ und 5.04 auf die Verknüpfung mit Sauerstoff hin. Die Signale der Methylgruppe und der verbleibenden Methylengruppe treten bei $\delta_{\mathrm{H}}=1.21$ bzw. bei $\delta_{\mathrm{H}}=2.57$ und 2.66 auf. Durch ein ${ }^{1} \mathrm{H},{ }^{1} \mathrm{H}-\mathrm{COSY}$ - und ein HMBCExperiment können die erhaltenen Fragmente zu der abgebildeten Struktur verknüpft und als $\mathbf{1 0 7}$ identifiziert werden. 
<smiles>Cc1cc(O)cc(O)c1C(=O)OCC(=O)CC(C)O</smiles>

107

\subsection{7. (Pent-3-en-2-on-1-yl)-orsellinat (108)}

Die Substanz konnte als farbloser Feststoff isoliert werden und besitzt ein mit 107 praktisch identisches UV-Spektrum. Im ESI-Massenspektrum kann ein $[\mathrm{M}-\mathrm{H}]^{-}-\mathrm{Ion}$ bei $\mathrm{m} / \mathrm{z}=249$ detektiert werden. Die im Vergleich zu 107 um 18 Einheiten kleinere Masse deutet auf die Abspaltung von Wasser hin, was durch eine Hochauflösung, die zur Summenformel $\mathrm{C}_{13} \mathrm{H}_{14} \mathrm{O}_{5}$ führt, bestätigt wird. Im ${ }^{1} \mathrm{H}-\mathrm{NMR}-$ Spektrum erkennt man die für Orsellinsäure typischen Signalen der beiden aromatischen Methinprotonen bei $\delta_{\mathrm{H}}=6.22$ und 6.25 sowie der Methylgruppe bei $\delta_{H}=2.52$. Daneben finden sich die Signale einer Methylgruppe $\left(\delta_{\mathrm{H}}=1.95\right)$ und einer mit Sauerstoff verknüpften Methylengruppe $\left(\delta_{\mathrm{H}}=5.08\right)$ sowie die von zwei Methinprotonen $\left(\delta_{\mathrm{H}}=6.23\right.$ und 7.03), die auf Grund einer Kopplungskonstante von ${ }^{3} J=16.0 \mathrm{~Hz}$ zu einer trans-konfigurierten Doppelbindung gehören. Im ${ }^{13} \mathrm{C}-\mathrm{NMR}-\mathrm{Spektrum}$ findet sich neben den acht Signalen der Orsellinsäure und denen der Methyl-, der Methylenund der beiden Methingruppen das Signal eines Carbonylkohlenstoffatoms bei $\delta_{\mathrm{C}}=191.9$. Durch ein HMBC-Experiment kann die abgebildete Struktur abgeleitet werden. Es handelt sich um (Pent-3-en-2-on-1-yl)-orsellinat (108).<smiles>CC=CC(=O)C(=O)OC(=O)c1c(C)cc(O)cc1O</smiles>

108 


\subsection{Literaturbekannte Verbindungen mit Desoxyzuckern}

Eine Datenbanksuche ${ }^{62}$ nach den in den Orselliden $\mathrm{A}-\mathrm{E}$ enthaltenen Zuckerbestandteilen erbrachte mehrere Treffer für die in 101 und 102 enthaltene Methyl-6-desoxy-3-keto- $\alpha$-Dribo-hexopyranose. Diese ist ein Intermediat bei der Bildung von Didesoxyzuckern und wurde auch synthetisch bearbeitet. ${ }^{124}$ Es gibt allerdings nur zwei Sekundärmetabolite die eine 6-Desoxy-3-keto-ribo-hexopyranose enthalten, das von Bacillus aurantinus gebildete Aurantinin $\mathrm{B}^{125}$ (109) und das aus Zweigen der Pflanze Datisca glomerata isolierte Datiscosid $^{126}$ (110). Wobei 110 die $\alpha$-L-Form enthält und $109 \beta$-verknüpft ist. Der in 104 enthaltene Zucker tritt als Bestandteil von $\operatorname{Spectinomycin}^{4}(\mathbf{1 1 1})$ sowie bei fünf Erythromycin-Derivaten $^{127}$ (112-116) auf. Die Glykal-artigen Zuckerbestandteile der Orsellide E (105) und F (106) sind bisher nicht als Bestandteil von Naturstoffen beschrieben worden.

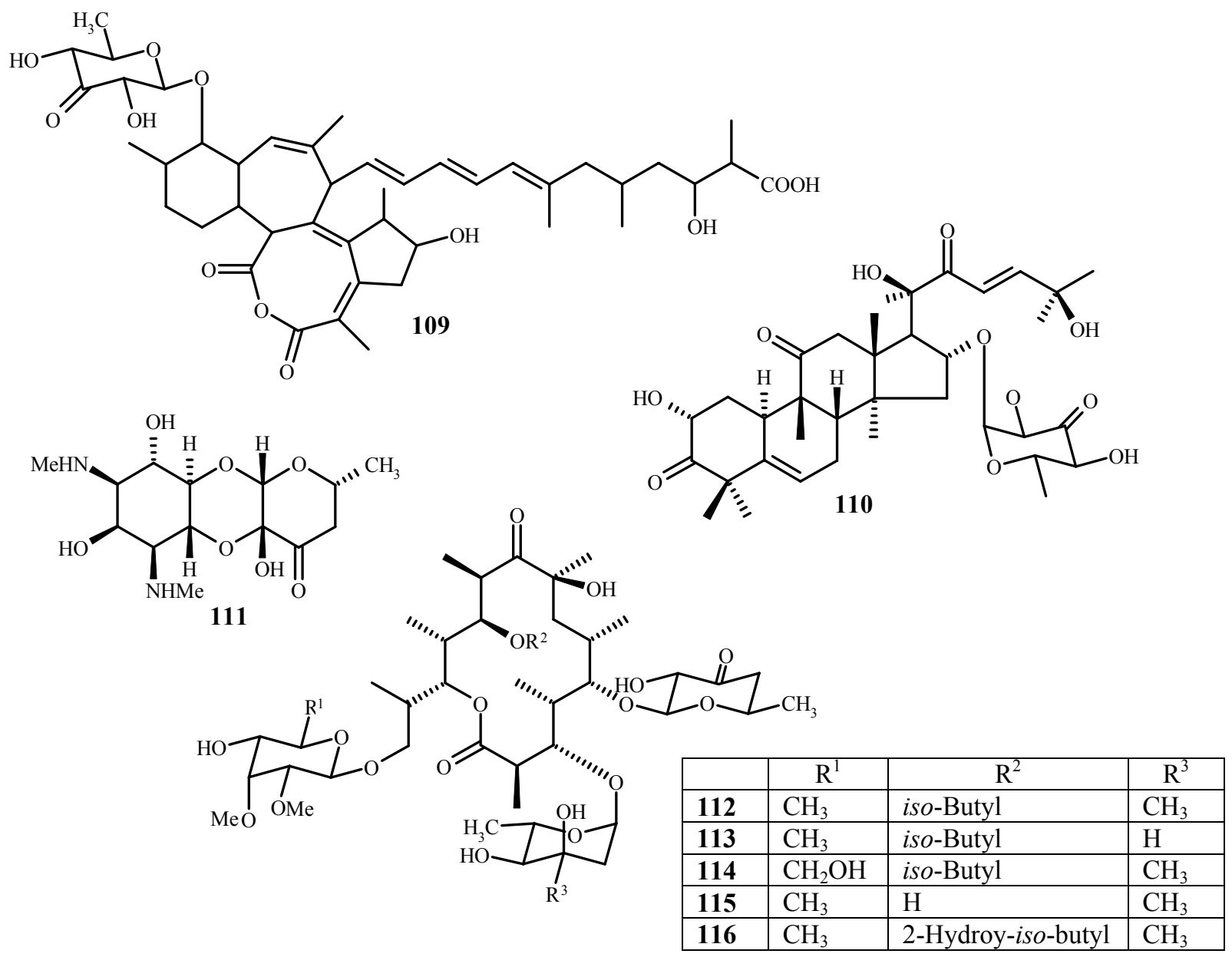

Abbildung 50: Literaturbekannte Verbindungen, die wie die Orsellide A - C (101, 102 und 104) 6-Desoxy-3-keto-ribo-hexopyranose oder 4,6-Didesoxy-3-keto-erythrohexopyranose als Zuckerbaustein enthalten. 


\subsection{Untersuchungen zur Biosynthese der Orsellide}

Die aus Schüttelkulturen des Pilzes Chaetomium sp. Stamm Gö 100/9 isolierten Orsellide A - E (101, 102 und 104 - 106)enthalten Desoxyzuckerbausteine. Während aus Bakterien die verschiedensten Desoxyzucker enthaltenden Sekundärmetabolite isoliert werden konnte, finden sich keine Publikationen über Desoxyzucker enthaltende Sekundärmetabolite aus Pilzen. Es gibt lediglich einen Bericht über ein Polysaccharid aus Kulturen des Pilzes Sphaeropsis sp., welches einen Desoxyzucker enthält. ${ }^{128}$

Daher stellt sich die Frage, ob der Zuckerbestandteil der Orsellide A - E tatsächlich aus Glucose gebildet wird. Alternativ wäre auch denkbar, dass es sich um Triketide, die zu einem Pyronring cyclisiert wurden, handelt. Um die Frage nach der Herkunft der in den Orselliden enthaltenen Desoxyzucker zu klären, sollten $\left[1-{ }^{13} \mathrm{C}\right]$ Acetat und $\left[1-{ }^{13} \mathrm{C}\right]$ Glucose an wachsende Kulturen des Stammes Gö 100/9 verfüttert werden.

\section{Fermentationsverlauf}

Um den optimalen Fütterungszeitpunkt zu bestimmen wurden ab Beginn der Fermentation bis zur 122. Stunde im Abstand von 6 Stunden Proben von einer Schüttelkultur des Stammes genommen und die Bildung der Orsellide A (101) und B (102) sowie von Orsellinsäure (100) mit Hilfe der HPLC analysiert. Dabei zeigt sich, dass die Bildung von 100 bereits 48 Stunden nach dem Animpfen beginnt und nach ca. 100 Stunden ein Maximum erreicht. Da 101 und 102 im Gleichgewicht miteinander stehen, konnte nur der Gesamtgehalt der beiden Verbindungen bestimmt werden. Die Produktion der beiden Orsellide beginnt ab der 66 . Stunde und steigt kontinuierlich bis zur 120. Stunde an. Der pH-Wert steigt ausgehend von einem Wert von $\mathrm{pH}=6.7$ während der Fermentation kontinuierlich bis auf einen Wert von 7.2 an. 


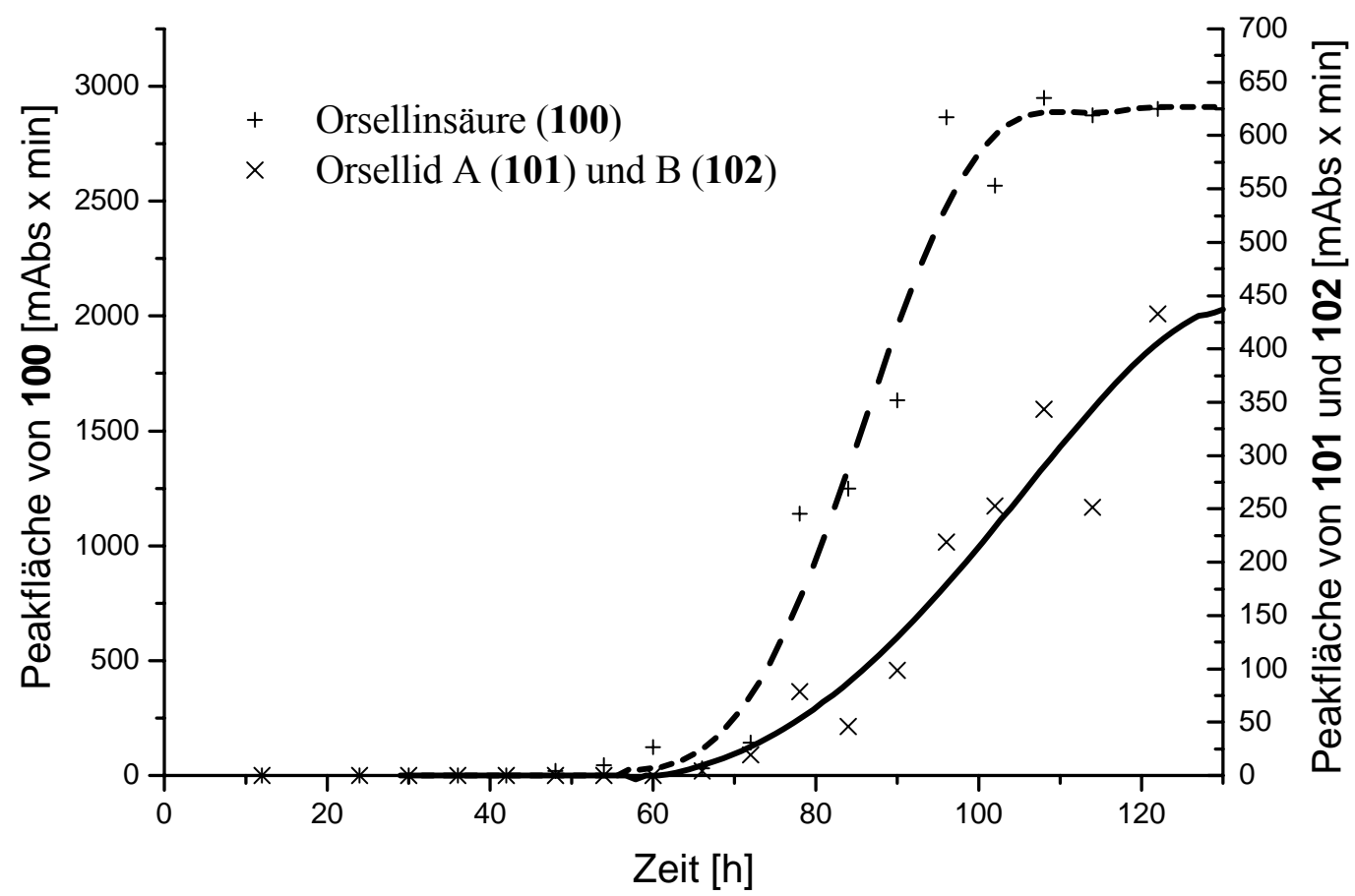

Abbildung 51: Verlauf der Produktion von Orsellinsäure (100) und der Orsellide A (101) und B (102).

Da der Gehalt der Orsellide A (101) und B (102) in den Kulturen von Chaetomium sp. Stamm Gö 100/9 75 bis 80 Stunden nach Fermentationsbeginn stark anzusteigen beginnt, wurden die markierten Vorläufer von der 78. bis zur 96. Stunde im Pulse-Feeding-Verfahren zugefüttert und die Kulturen nach weiteren zwölf Stunden geerntet.

\section{Fütterung von $\left[1-{ }^{13} \mathrm{C}\right]$ Acetat}

Aus der Fütterung von $\left[1-{ }^{13} \mathrm{C}\right]$ Acetat an Kulturen des Stammes Gö 100/9 konnte als Hauptmetabolit Orsellinsäure (100) isoliert werden, wobei die Ausbeute mit 40 mg/L doppelt so hoch wie bei Fermentationen ohne Zusatz von Acetat war. Wie erwartet weist 100 den Einbau von vier Acetat-Einheiten auf. Auch die Ausbeute der beiden im Gemisch vorliegenden Metaboliten 101 und 102 war gegenüber Fermentationen ohne Zusatz von Acetat von $2.5 \mathrm{mg} / \mathrm{L}$ auf $5.0 \mathrm{mg} / \mathrm{L}$ erhöht. Daneben konnten aus der Fütterung von $\left[1-{ }^{13} \mathrm{C}\right]$ Acetat auch die beiden Orsellide C (104) und D (105) in Ausbeuten von $2.5 \mathrm{mg} / \mathrm{L}$ und $1.5 \mathrm{mg} / \mathrm{L}$ isoliert werden. Beide Metaboliten treten in Fermentationen ohne Zusatz von Acetat lediglich als Minderkomponenten in wesentlich geringeren Ausbeuten auf.

Durch das Vorliegen der Orsellide A (101) und B (102) im Gleichgewicht wird die Auswertung von NMR-Spektren erschwert, da die Signale der beiden Verbindungen relativ dicht bei einander liegen und so Probleme bei der Zuordnung entstehen können. Weiterhin kann Orsellid D (105) nur durch präparative HPLC vom Gemisch der Orsellide A (101) und B 
(102) abgetrennt werden. Daher wurde das Gemisch der drei Verbindungen acetyliert und anschließend per präparativer HPLC aufgereinigt. Bei der Bestimmung der Einbauraten wurden dann auf die Acetylreste referenziert. Die für die Orsellide A - D (101, 102, 104 und 105) beobachteten Einbauraten sind in den Tabellen 7 und 8 angegeben.
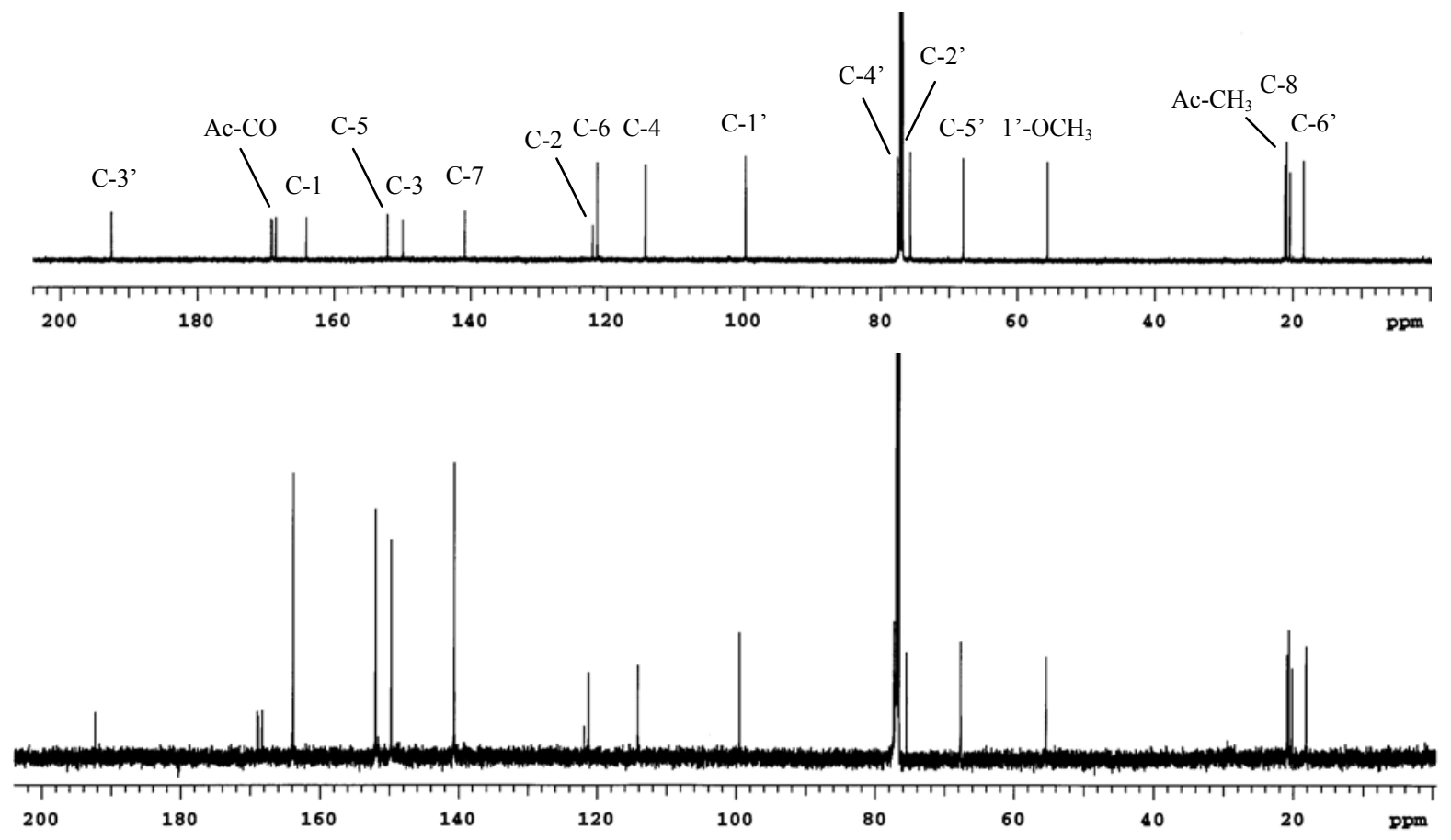

Abbildung 52: ${ }^{13} \mathrm{C}-\mathrm{NMR}-S p e k t r u m\left(150.8 \mathrm{MHz}, \mathrm{CDCl}_{3}\right)$ von $\left[1-{ }^{13} \mathrm{C}\right]$ Acetat angereichertem 3,5,4'-Tri-O-Acetyl-Orsellid A (101b) im Vergleich zum Referenzspektrum.

\begin{tabular}{|c|c|c|c|c|c|}
\hline \multicolumn{3}{|c|}{ Orsellid A (101) } & \multicolumn{3}{c|}{ Orsellid B (102) } \\
\hline C-Atom & $\begin{array}{c}\text { Verschiebung } \\
{[\mathrm{ppm}]}\end{array}$ & $\begin{array}{c}\text { Spez. } \\
\text { Einbau [\%] }\end{array}$ & C-Atom & $\begin{array}{c}\text { Verschiebung } \\
{[\mathrm{ppm}]}\end{array}$ & $\begin{array}{c}\text { Spez. } \\
\text { Einbau [\%] }\end{array}$ \\
\hline C-1 & 164.1 & $\mathbf{7 . 1}$ & C-1 & 164.2 & $\mathbf{5 . 1}$ \\
\hline C-2 & 122.1 & 0.1 & C-2 & 123.0 & 0.2 \\
\hline C-3 & 150.0 & $\mathbf{5 . 7}$ & C-3 & 149.1 & $\mathbf{4 . 8}$ \\
\hline C-4 & 114.4 & 0.1 & C-4 & 114.2 & 0.0 \\
\hline C-5 & 152.2 & $\mathbf{6 . 0}$ & C-5 & 151.9 & $\mathbf{4 . 5}$ \\
\hline C-6 & 121.4 & 0.0 & C-6 & 121.2 & 0.3 \\
\hline C-7 & 140.9 & $\mathbf{6 . 8}$ & C-7 & 139.7 & $\mathbf{5 . 1}$ \\
\hline C-8 & 21.1 & 0.2 & C-8 & 21.1 & 0.4 \\
\hline C-1' & 99.7 & 0.4 & C-1' & 99.7 & 0.3 \\
\hline C-2' & 75.7 & 0.2 & C-2' & 74.9 & 0.2 \\
\hline C-3' & 192.5 & 0.2 & C-3' & 192.5 & 0.2 \\
\hline C-4' & 77.6 & 0.6 & C-4' & 78.3 & 0.2 \\
\hline C-5' & 67.9 & 0.3 & C-5' & 67.8 & 0.3 \\
\hline C-6' & 18.4 & 0.4 & C-6' & 18.4 & 0.5 \\
\hline 1'-OMe $^{\prime}$ '-OM $^{\prime}$ & 55.6 & 0.2 & ''-OMe $^{\prime}$ & 55.6 & 0.3 \\
\hline
\end{tabular}

Tabelle 7: Einbauraten der $\left[1{ }^{13} \mathrm{C}\right]$ Acetat-Fütterung für die Orsellide A (101) und B (102). 


\begin{tabular}{|c|c|c|c|c|c|}
\hline \multicolumn{3}{|c|}{ Orsellid C (104) } & \multicolumn{3}{c|}{ Orsellid D (105) } \\
\hline C-Atom & $\begin{array}{c}\text { Verschiebung } \\
{[\mathrm{ppm}]}\end{array}$ & $\begin{array}{c}\text { Spez. } \\
\text { Einbau [\%] }\end{array}$ & C-Atom & $\begin{array}{c}\text { Verschiebung } \\
{[\mathrm{ppm}]}\end{array}$ & $\begin{array}{c}\text { Spez. } \\
\text { Einbau [\%] }\end{array}$ \\
\hline C-1 & 169.5 & $\mathbf{4 . 5}$ & C-1 & 163.5 & $\mathbf{3 . 1}$ \\
\hline C-2 & 103.8 & 0.1 & C-2 & 122.2 & 0.0 \\
\hline C-3 & 161.1 & $\mathbf{3 . 9}$ & C-3 & 149.6 & $\mathbf{2 . 8}$ \\
\hline C-4 & 101.2 & 0.0 & C-4 & 114.2 & 0.0 \\
\hline C-5 & 165.0 & $\mathbf{3 . 2}$ & C-5 & 152.3 & $\mathbf{2 . 9}$ \\
\hline C-6 & 11.7 & 0.1 & C-6 & 121.3 & 0.0 \\
\hline C-7 & 145.0 & $\mathbf{4 . 8}$ & C-7 & 140.5 & $\mathbf{3 . 0}$ \\
\hline C-8 & 24.1 & 0.2 & C-8 & 20.7 & 0.1 \\
\hline C-1' & 100.3 & 0.3 & C-1' & 156.1 & 0.3 \\
\hline C-2' & 76.0 & 0.2 & C-2' & 181.7 & 0.2 \\
\hline C-3' & 198.2 & 0.0 & C-3' & 130.3 & 0.0 \\
\hline C-4' & 48.5 & 0.2 & C-4' & 78.3 & 0.1 \\
\hline C-5' & 66.0 & 0.2 & C-5' & 72.6 & 0.3 \\
\hline C-6' & 21.2 & 0.2 & C-6' & 17.3 & 0.2 \\
\hline 1'-OMe $^{\prime}$ 'OM. & & & \\
\hline
\end{tabular}

Tabelle 8: Einbauraten der $\left[1-{ }^{13} \mathrm{C}\right]$ Acetat-Fütterung für die Orsellide C (104) und D (105).
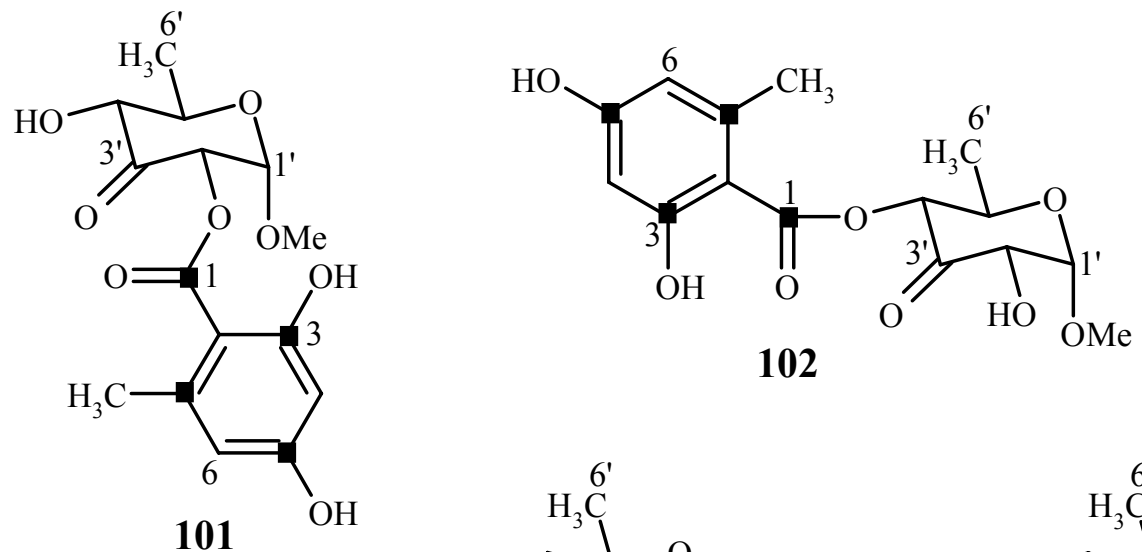

102
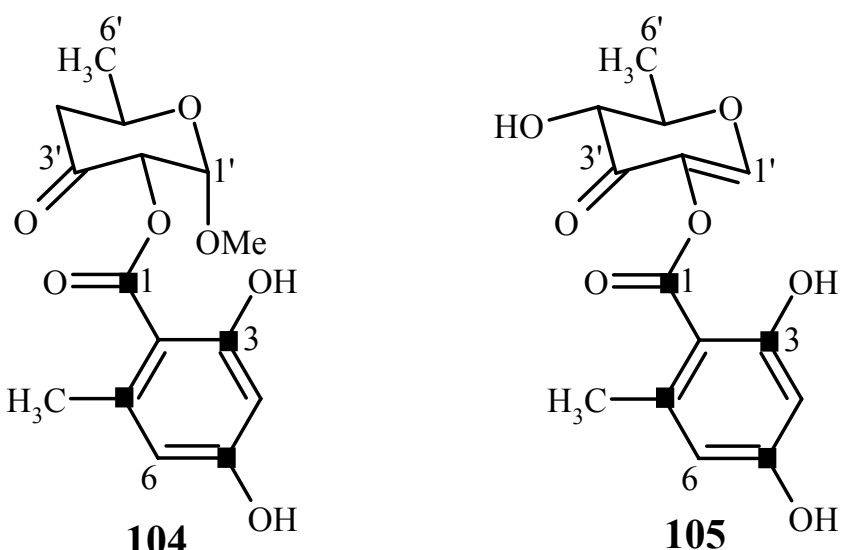

Abbildung 53: Einbaumuster der $\left[1-{ }^{13} \mathrm{C}\right]$ Acetat-Fütterung für die Orsellide A - D (101, 102, 104 und 105). 
Die Auswertung der ${ }^{13} \mathrm{C}-\mathrm{NMR}-$ Spektrum der vier Orsellide A - D (101, 102, 104 und 105) zeigt, dass lediglich vier Kohlenstoffatome der Orsellinsäure eine Erhöhung der Signalintensitäten aufweisen. Da bei keinem der isolierten Orsellide ein Einbau von $\left[1-{ }^{13} \mathrm{C}\right]$ Acetat in den Zuckerbestandteil stattgefunden hat, kann dieses kein Polyketid sein. Nun sollte durch die Fütterung markierter Glucose geklärt werden, ob diese ein Vorläufer für den Zuckerteil ist.

\section{Fütterung von $\left[1-{ }^{13} \mathrm{C}\right]$ Glucose}

Da durch die Fütterung von Acetat die Ausbeuten der Orsellide A - D, insbesondere die der Nebenkomponenten 104 und 105, gesteigert werden konnte, wurde zusätzlich zur markierten Glucose $(0.5 \mathrm{~g} / \mathrm{L})$ unmarkiertes Acetat in einer Konzentration von $1 \mathrm{~g} / \mathrm{L}$ gefüttert. Das nach einer ersten chromatographischen Aufreinigung erhaltene Gemisch der drei Orsellide A, B und $\mathrm{D}\left(\mathbf{1 0 1}, 102\right.$ und 105) wurde acetyliert und per präparativer HPLC getrennt. $\operatorname{Im}{ }^{13} \mathrm{C}$ NMR-Spektrums konnte für diese drei Verbindungen sowie für das ebenfalls aus der Fütterung isolierte Orsellid C (104) eine Markierung des Kohlenstoffatoms C-1' nachgewiesen werden. Der beobachtete spezifische Einbau liegt für 101 und 102 bei $4.4 \%$, bei 105 und 104 bei 5.1 bzw. $5.4 \%$.

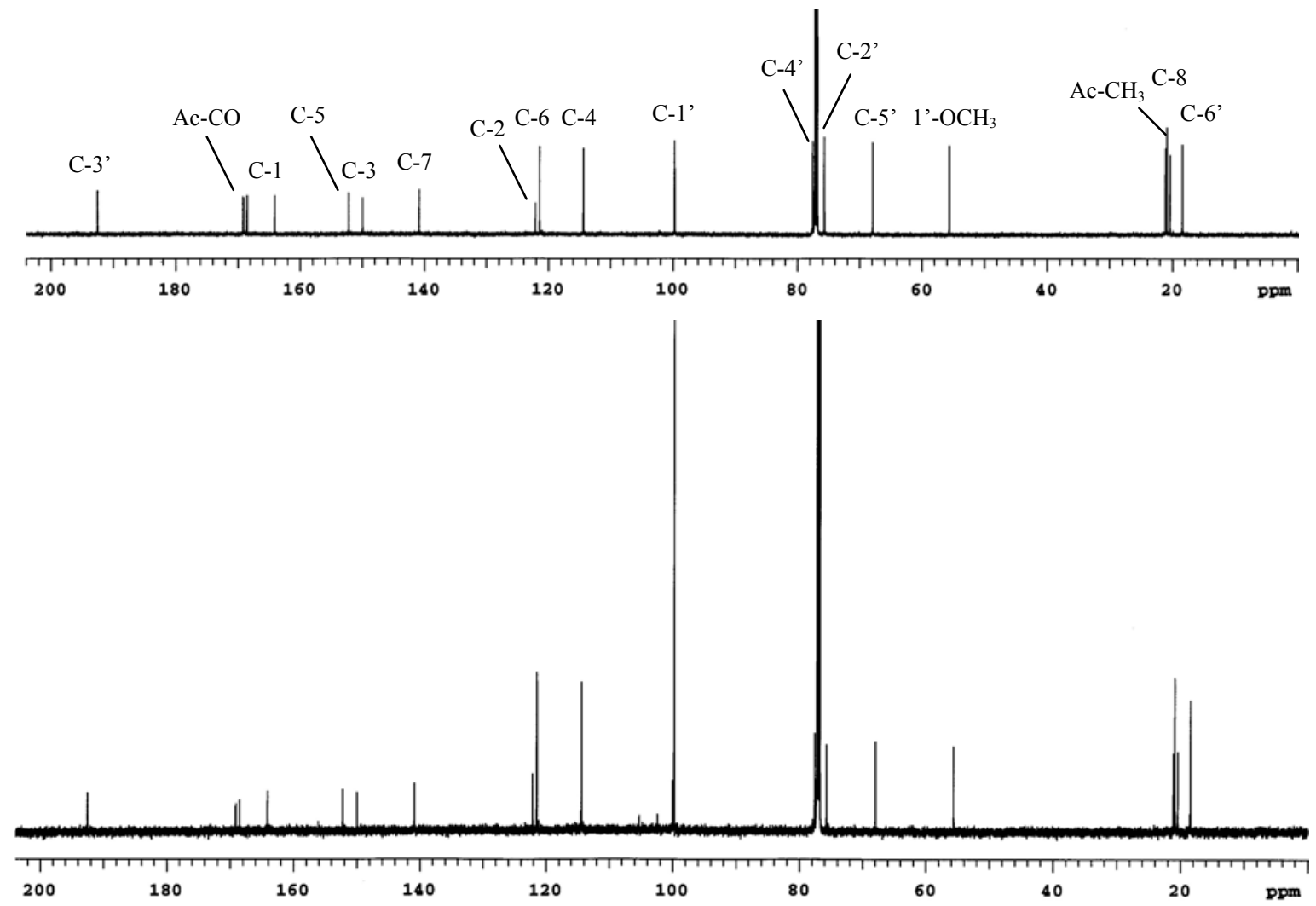

Abbildung 54: ${ }^{13} \mathrm{C}-\mathrm{NMR}-S p e k t r u m\left(150.8 \mathrm{MHz}, \mathrm{CDCl}_{3}\right)$ von $\left[1-{ }^{13} \mathrm{C}\right]$ Glucose angereichertem 3,5,4'-Tri-O-Acetyl-Orsellid A (101b) im Vergleich zum Referenzspektrum. 

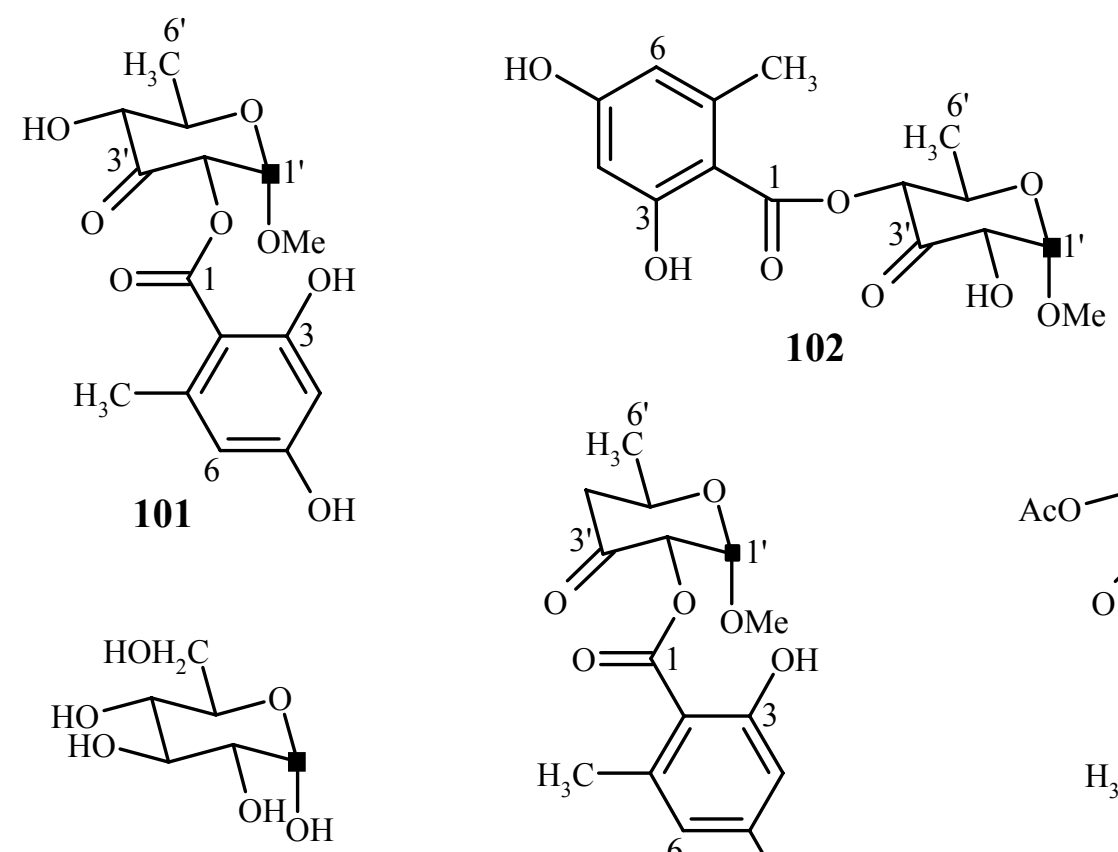

$\left[1-{ }^{13} \mathrm{C}\right]$-Glukose
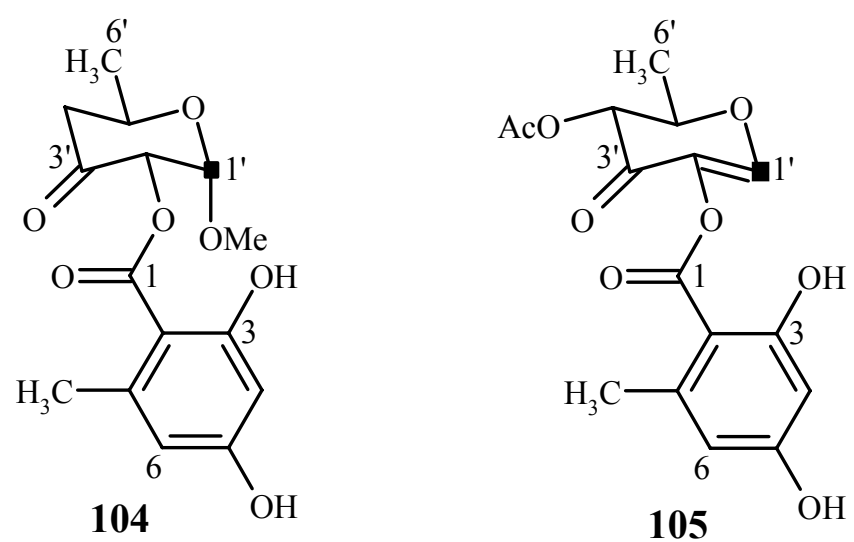

Abbildung 55: Das aus der $\left[1-{ }^{13} \mathrm{C}\right]$ Glucose-Fütterung für die Orsellide A - D (101, 102, 104 und 105) erhaltene Einbaumuster.

Durch den Einbau von $\left[1-{ }^{13} \mathrm{C}\right]$ Glucose in die vier Orsellide A - D (101, 102, 104 und 105) steht außer Frage, dass der Zuckerteil dieser Substanzen aus dem Kohlenhydrat-Stoffwechsel stammt. Bisher ist man davon ausgegangen, dass Pilze keine Desoxyzucker bilden können. Dass Chaetomium sp. Stamm Gö 100/9 dennoch über dies Fähigkeit verfügt, könnte auf einen horizontalen Gentransfer zurückzuführen sein. Die Sequenzierung der entsprechenden Gene und der anschließende Vergleich mit bakteriellen Enzymen sollte Aufschluss über die Herkunft dieser Gene liefern. 


\section{4. Überlegungen zur Biosynthese der Orsellide}

Die als Gemisch vorliegenden Orsellide A (101) und B (102) gehören zur Gruppe der 6-Desoxyzucker. Wohingegen Orsellid C (104) der Gruppe der relativ seltenen 4,6-Didesoxyzucker zuzuordnen ist. Bei den beiden Orselliden D (105) und E (106) handelt es sich um 2,3-Dihydro-4-pyrone, die wahrscheinlich durch Abspaltung von Methanol aus 101 bzw. 104 hervorgehen. Ausgehend von diesen Überlegungen ergibt sich der in Abbildung 56 dargestellte hypothetische Mechanismus für die Bildung der Orsellide A - E.
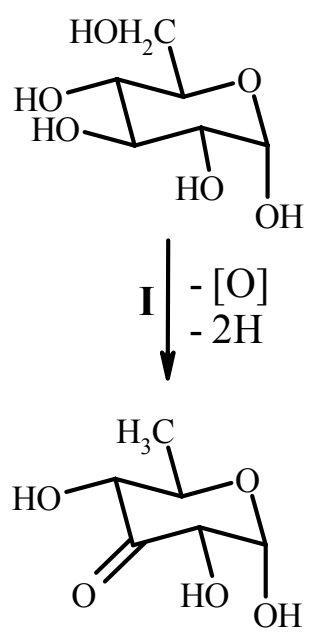

II $\downarrow-[\mathrm{O}]$

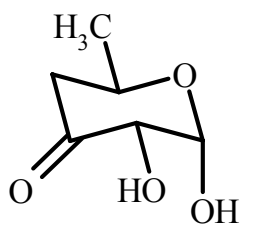

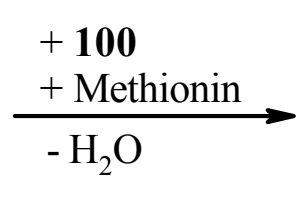

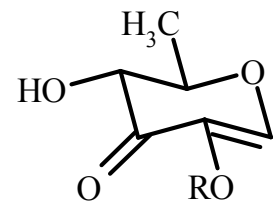

105
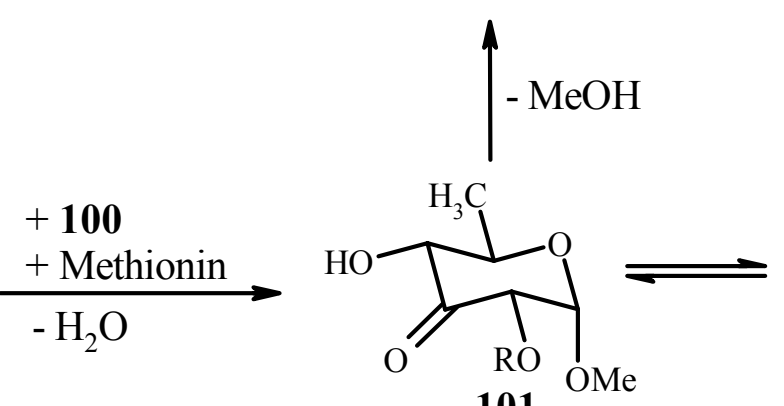

101

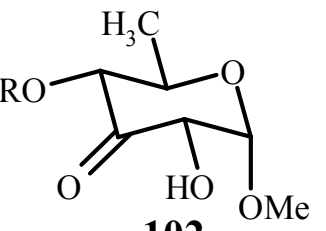

102

Abbildung 56: Hypothetischer Mechanismus für die Bildung der Orsellide A - E (101 - 102 und 104 - 106) $(\mathrm{R}=$ Orsellinsäure (100)).

Im ersten Reaktionsschritt des obigen Mechanismus wird aus Glucose ein 6-Desoxyzucker gebildet. Diese Reaktion wird von der NDP-Glucose-Oxidoreduktase (NDP = Nucleosiddiphosphat) katalysiert und ihr Mechanismus wurde in den vergangenen 40 Jahren intensiv untersucht. Durch Verwendung verschiedener Modellsysteme konnte sogar der stereochemische Verlauf dieser Reaktion geklärt werden. Im ersten Schritt wird das Proton an C-4 der NDP-gebundenen Glucose auf NAD ${ }^{+}$übertragen. Nach Abspaltung von Wasser wird dann eben dieses Proton auf das Kohlenstoffatom C-6 übertragen und die gebildete NDP-6- 
Desoxy-4-keto-D-xylo-hexopyranose steht mit dem in den Orselliden A und B enthaltenen 6-Desoxyzucker 117 im Gleichgewicht (Abbildung 57). ${ }^{129}$

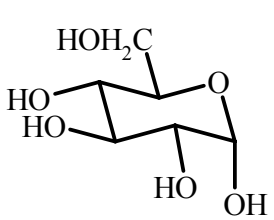<smiles>C[Nb+]</smiles>

$\mathrm{OH}$
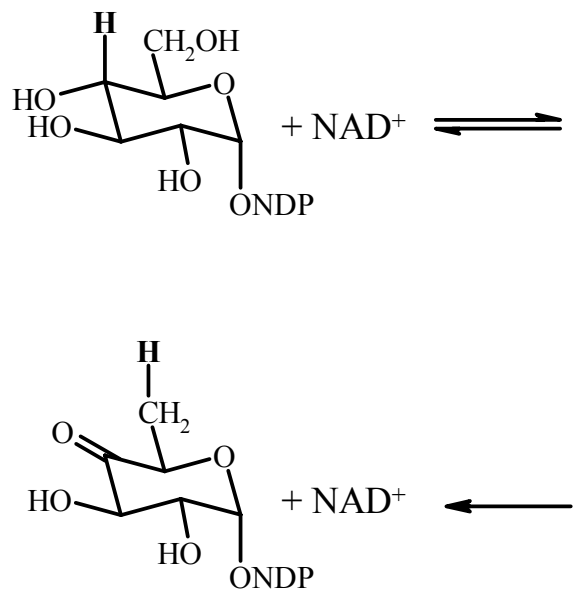

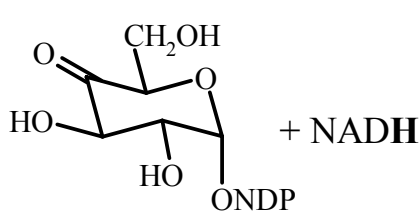

$$
\downarrow-\mathrm{H}_{2} \mathrm{O}
$$<smiles>C=C1OC([N+](=O)O)C(O)C(O)C1O</smiles>

Abbildung 57: Mechanismus für die Bildung von 6-Desoxyzuckern aus Glucose.

In Abbildung 56 kommt es während des mit II gekennzeichneten Reaktionsschrittes zur Abspaltung der Hydroxygruppe an C-4 und dadurch zur Bildung eines 4,6-Didesoxyzuckers. Dieser Didesoxyzucker wird als Intermediat bei der Biosynthese des D-Desosamin, welches ein Bestandteil von Erythromycin A ist, vermutet. In Analogie zu dem für D-Desosamin vorgeschlagenen Biosynthesemechanismus ${ }^{129}$ kann für den in Orsellid $\mathrm{C}$ enthaltenen 4,6-Didesoxyzucker ein Mechanismus ausgehen von NDP-6-Desoxy-3-keto-ribohexopyranose (117) aufgestellt werden. Nach Umlagerung von 117 in eine NDP-6-Desoxy-4keto-ribo-hexopyranose kommt es zur Abspaltung der Hydroxygruppe an C-4 und die dabei gebildet Doppelbindung wird reduziert. Das gebildete 118 kann dann mit Orsellinsäure zum Orsellid C (104) verknüpft werden.

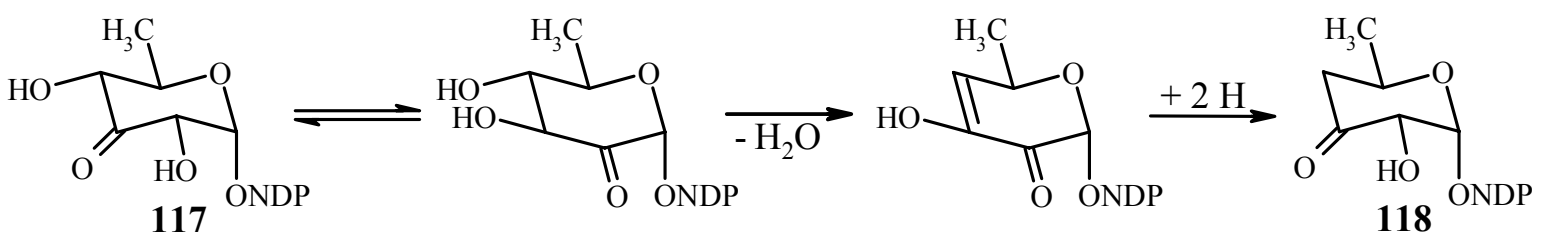

Abbildung 58: Mechanismus für die Bildung des 4,6-Didesoxyzuckern aus Orsellid C (104). 


\section{5. Überlegungen zur Bildung von (4-Hydroxy-pent-2-on-1-yl)-orsellinat (107) und (Pent-3-en-2-on-1-yl)-orsellinat (108)}

Anhand ihrer Struktur lässt sich für die beiden ebenfalls von Stamm Gö 100/9 gebildeten Metabolite 107 und 108 vermuten, dass sie sich von Orsellid E (106) ableiten. So könnte nach Öffnung des Pyranrings das Kohlenstoffatom C-1' zu einer Carbonsäurefunktion oxidiert werden. Durch eine Decarboxylierung entsteht dann (4-Hydroxy-pent-2-on-1-yl)-orsellinat (107) aus dem durch Freisetzung von Wasser (Pent-3-en-2-on-1-yl)-orsellinat (108) hervorgeht.

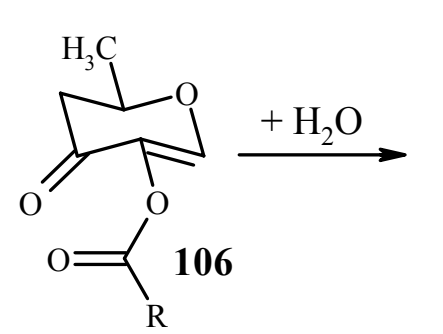<smiles>[R]C(=O)OC(C=O)C(=O)CC(C)O</smiles><smiles>[R]C(=O)OC(C(=O)O)(C(=O)O)C(=O)CC(C)O</smiles><smiles>[R]c1c(C)cc(O)cc1O</smiles><smiles>[R]C(=O)OCC(=O)/C=C/C</smiles><smiles>[R]C(=O)OCC(=O)CC(C)O</smiles>

Abbildung 59: Hypothetischer Mechanismus für die Bildung von (4-Hydroxy-pent-2-on-1yl)-orsellinat (107) und (Pent-3-en-2-on-1-yl)-orsellinat (108).

Unterstützt wird diese Hypothese durch die bei Fütterung von $\left[1-{ }^{13} \mathrm{C}\right]$ Glucose erhöhten Ausbeuten an 107 und 108. Da bei der Decarboxylierung das markierte Kohlenstoffatom verloren geht, tragen die aus der Fütterung isolierten Substanzen keine ${ }^{13} \mathrm{C}$-Markierung mehr. Alternativ könnten 1,4-Dihydroxy-2-pentanon bzw. 1-Hydroxy-3-Penten-2-on auch direkt mit Orsellinsäure verestert werden. Aufschluss über die Herkunft der beiden $\mathrm{C}_{5}$-Körper könnte die Fütterung von $\left[\mathrm{U}_{-}^{13} \mathrm{C}_{6}\right]$ Glucose liefern. Die Bildung von 107 und $\mathbf{1 0 8}$ nach dem in Abbildung 59 dargestellten Mechanismus kann allerdings erst durch eine Fütterung mit markiertem Orsellid E (106) bewiesen werden. 


\subsection{Thiodiketopiperazine}

Aus Schüttelkulturen (Medium: SGG) des Stammes Gö 100/9 konnte Chetomin (119) in einer Ausbeute von $20 \mathrm{mg} / \mathrm{L}$ isoliert werden. Durch Zufütterung von Acetat (1 g/L) von der 78. bis zur 96. Stunde (siehe Kapitel A.IV.3.3) konnte die Ausbeute an Chetomin auf $135 \mathrm{mg} / \mathrm{L}$ gesteigert werden. Daneben wurden zwei weitere Metabolite Chetoseminudin A (120) und das neue Chetomin B (121) in Ausbeuten von 10.5 bzw. $7.8 \mathrm{mg} / \mathrm{L}$ isoliert.

\subsubsection{Chetomin (119)}

Der farblose Feststoff mit dem Rf-Wert 0.82 ist in Chloroform und Aceton gut und in Methanol mäßig löslich. Im ${ }^{1}$ H-NMR-Spektrum erkennt man die Signale von drei Methylgruppen zwischen $\delta_{\mathrm{H}}=2.93$ und 3.15, die als Singulett auftreten. Die Verschiebungen dieser Signale sprechen für die Verknüpfung mit Sauer- oder Stickstoff. Des Weiteren erscheinen Signale für acht Protonen im Bereich von $\delta_{\mathrm{H}}=3.12$ bis 4.38 , die zu vier Methylengruppen gehören. Dem ${ }^{13}$ C-NMR-Spektrum sind Signale für drei Methyl-, vier Methylen- und zehn Methingruppen sowie für zehn quartäre Kohlenstoffatome zu entnehmen. Bei den quartären Kohlenstoffatomen fallen insbesondere fünf auf, deren Verschiebungen zwischen 70 und 80 ppm liegen. Diese deutet auf die Nachbarschaft zu elektronegativen Atomen wie Sauerstoff hin. Die Hochauflösung des im ESI-Massenspektrum gefundenen Molekülpeaks bei $733[\mathrm{M}+\mathrm{Na}]^{+}$führte zur Summenformel $\mathrm{C}_{31} \mathrm{H}_{30} \mathrm{~N}_{6} \mathrm{O}_{6} \mathrm{~S}_{4}$. Eine mit diesen Informationen durchgeführte Literatursuche ${ }^{53}$ liefert Chetomin als einzige passende Verbindungen und durch Vergleich mit den Literaturdaten ${ }^{130}$ konnte die Verbindung eindeutig als Chetomin (119) identifiziert werden.

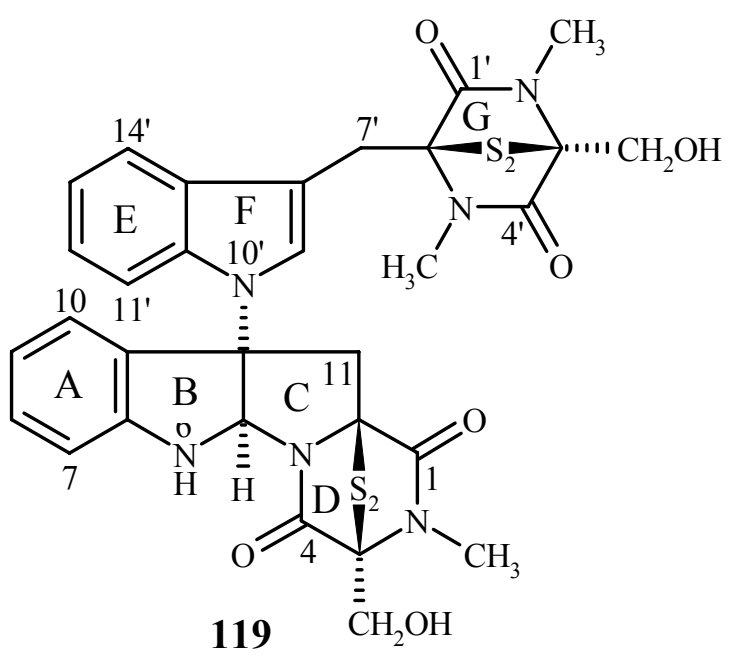




\subsubsection{Chetoseminudin A (120)}

Der farblose Feststoff ist ebenso wie 119 in Chloroform und Aceton gut und in Methanol mäßig löslich. Im ESI-Massenspektrum tritt bei $\mathrm{m} / \mathrm{z}=765$ ein $[\mathrm{M}+\mathrm{Na}]^{+}-$Ion auf, dessen Hochauflösung zur Summenformel $\mathrm{C}_{31} \mathrm{H}_{30} \mathrm{~N}_{6} \mathrm{O}_{6} \mathrm{~S}_{5}$ führt. Sowohl das ${ }^{1} \mathrm{H}-\mathrm{NMR}$ - als auch das ${ }^{13} \mathrm{C}-\mathrm{NMR}-S p e k t r u m$ von 120 besitzt große Ähnlichkeit mit denen von 119. Der auffälligste Unterschied ist die Tieffeldverschiebung des ${ }^{13} \mathrm{C}$-NMR-Signals von C-11 um 6.4 ppm. Des Weiteren sind die Signale C-3 und C-11a um 1.8 bzw. 2.0 ppm sowie die Carbonylkohlenstoffatome C-1 und C-3 um 2.8 bzw. 2.4 ppm ins Tieffeld verschoben. Die Verschiebungen aller anderen Kohlenstoffatome unterscheiden sich von denen von Chetomin (119) um weniger als $1.0 \mathrm{ppm}$. Dies führt zusammen mit der Summenformel zur Vermutung, dass es sich um eine mit 119 verwandte Verbindung handelt, die im Ring D statt einer Disulfid- eine Trisulfidbrücke enthält. Eine Datenbanksuche ${ }^{53}$ und der anschließenden Vergleich mit Literaturdaten ${ }^{131}$ führte zu Chetoseminudin A (120).

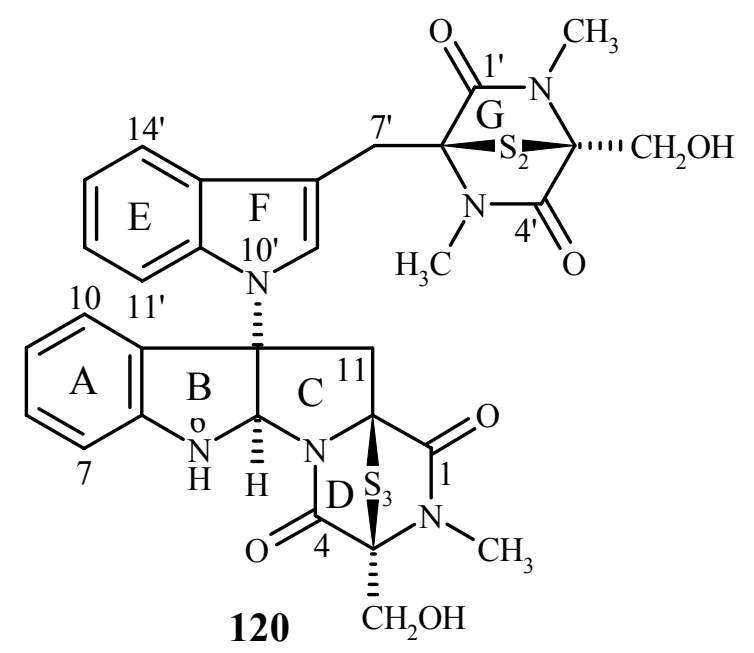

\subsubsection{Chetomin B (121)}

Die durch eine Hochauflösung bestimmte Summenformel $\mathrm{C}_{31} \mathrm{H}_{30} \mathrm{~N}_{6} \mathrm{O}_{6} \mathrm{~S}_{5}$ von 121 ist mit der von 120 identisch und führt zu der Vermutung, dass 121 ebenfalls ein Thiodiketopiperazin ist und sich von 120 durch Vertauschen der Di- und der Trisulfidbrücke ableitet. Im ${ }^{1} \mathrm{H}-\mathrm{NMR}$ Spektrum treten die Signalsätze von zwei Konformere im Verhältnis 6 zu 5 auf. Durch eine Messung bei höherer Temperatur $\left(120^{\circ} \mathrm{C}\right)$ kann die Einstellung des Gleichgewichts zwischen den beiden Konformern beschleunigt werden, so dass nur noch ein Konformer beobachtet wird, welches auch bei Raumtemperatur das Hauptkonformer ist. Im ${ }^{13} \mathrm{C}$-NMR-Spektrum 
können dem Hauptkonformer 31 Signale zugeordnet werden, wobei die Verschiebungen denen von Chetomin (119) stark ähneln. Die größten Unterschiede im Vergleich zu 119 sind dabei die Tieffeldverschiebung der Signale von C-1', C-3', C-7' und C-15' sowie die Hochfeldverschiebung der Signale von C-4' und C-6'. Das ${ }^{1}$ H-NMR-Spektrum besitzt ebenfalls große Ähnlichkeit zu dem von 119, wobei die signifikantesten Unterschiede ebenfalls im Bereich von Ring G auftreten. So ist die Hydroxymethlengruppe an C-3 um 2.0 und die Methylengruppe C-7 um 2.5 ppm ins Tieffeld verschoben. Somit sollte sich 121 von Chetomin durch eine Trisulfidbrücke im Ring G unterscheiden, wobei das Auftreten von zwei Signalsätzen durch zwei Konformere im Bereich der Trisulfidbrücke erklärt werden kann. Dasselbe Phänomen wurde bereits im Fall der Chaetocine $B$ und $\mathrm{C}^{132}$ sowie bei Emethallicin $\mathrm{D}^{133}$ beobachtet.

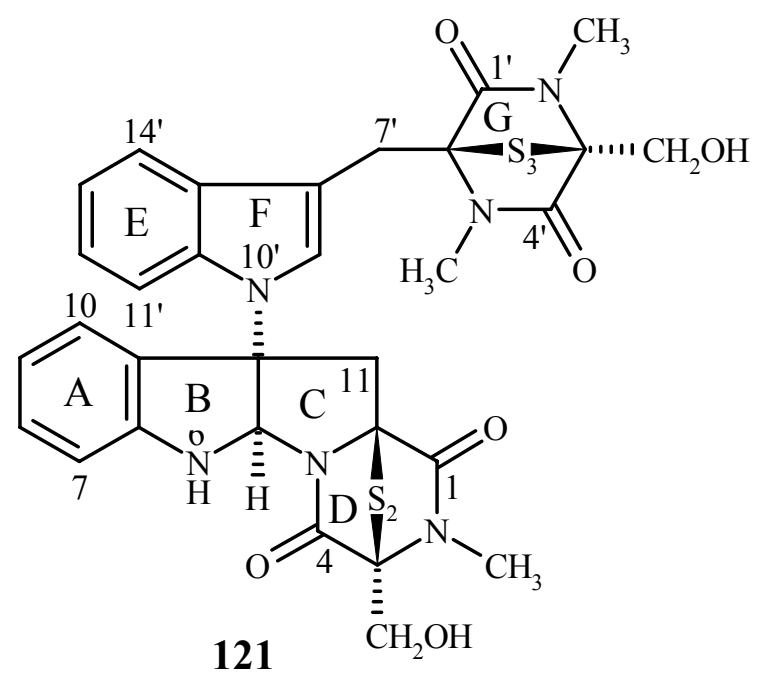

\subsubsection{Die Gruppe der Thiodiketopiperazine}

Bei einer Datenbankrecherche in Chapman\&Hall ${ }^{53}$ konnten über 100 Thiodiketopiperazine gefunden werden, unter denen sich neben Di- auch mehrere Tri- und Tetrasulfide befinden. Es gibt sowohl „dimere“ Verbindungen, die wie die von Chaetomium sp. Stamm Gö 100/9 gebildeten Thiodiketopiperazine zwei Diketopiperazinringe enthalten, als auch „monomere“ Verbindungen. Der älteste Vertreter dieser Substanzklasse ist Gliotoxin ${ }^{134}$ (122), welches Aktivität gegen Bakterien, Pilze und Viren sowie eine immunsuppressive Wirkung besitzt. ${ }^{135}$ Weitere Beispiele für „monomere“ Thiodiketopiperazine sind das fungizid wirksame Hyalodendrin $^{136}$ (123) und Sirodesmin ${ }^{137}$ (124), welches antibakterielle und antivirale Eigenschaften besitzt. 
Zu den „dimeren“ Thiodiketopiperazinen gehören z.B. die cytotoxischen Stereoisomere Chaetocin ${ }^{138}(\mathbf{1 2 5})$ und Verticillin $A^{139}$ (126). Bei diesen Verbindungen konnten, wie auch bei vielen andern Thiodiketopiperazinen, neben den Disulfid auch die Tri- und Tetrasuldfide isoliert werden. ${ }^{140}$ Die zur Gruppe der Thiodiketopiperazine gehörenden Substanzen besitzen fast alle antibiotische Aktivität, wobei Verbindungen bei denen die Schwefelbrücke geöffnet wurde weniger wirksam sind. Die Anzahl der Schwefelatome scheint hingegen keine Rolle für die Wirksamkeit der Verbindungen zu spielen. ${ }^{141}$ Für Gliotoxin (122) konnte gezeigt werden, dass der Wirkmechanismus auf einer kovalente Interaktion mit Proteinen durch die Bildung von gemischten Disulfiden beruht. ${ }^{142}$
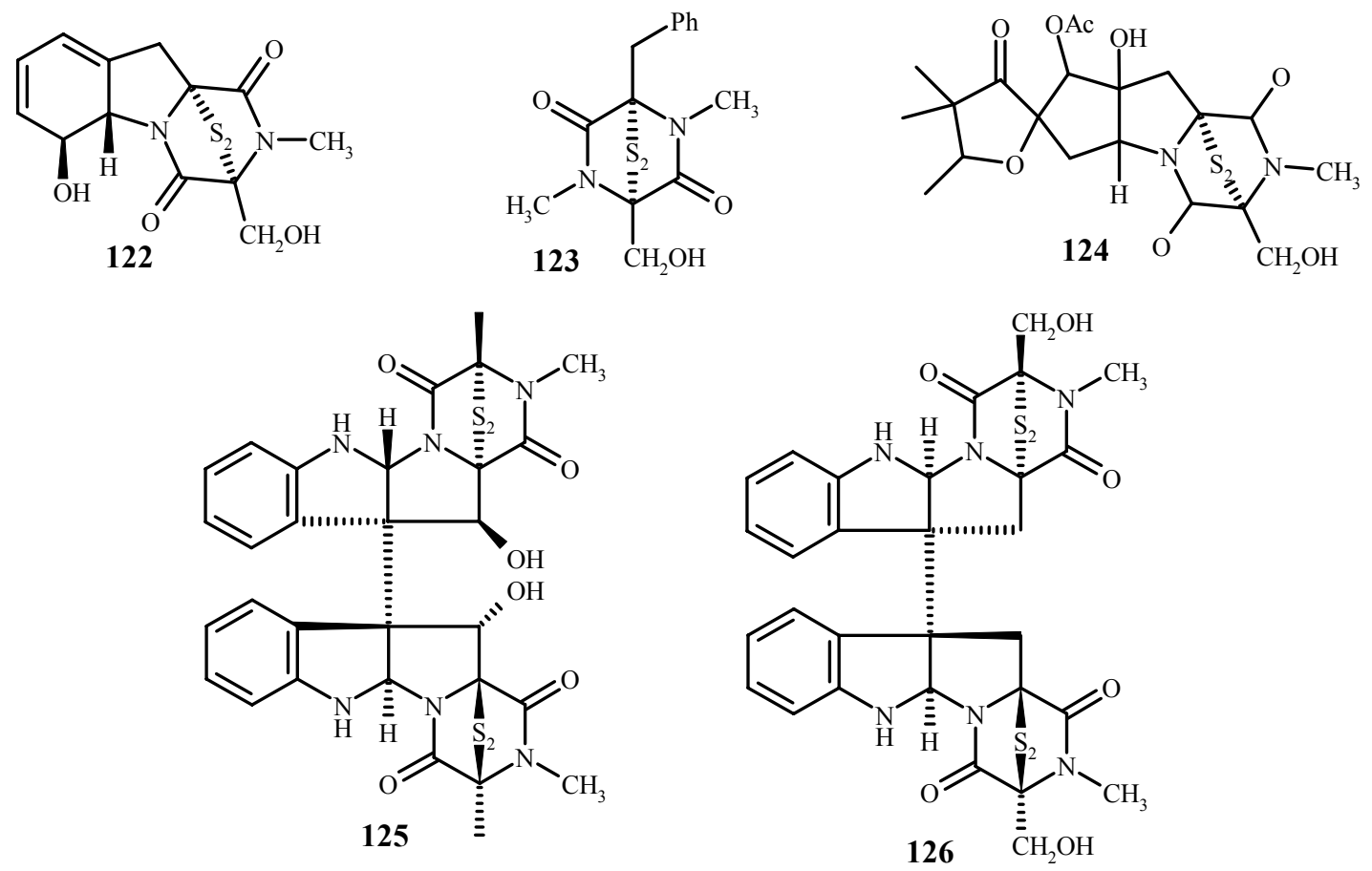

Abbildung 60: Ausgewählte Metabolite aus der Substanzklasse der Thiodiketopiperazinen.

Bereits im Jahre 1975 fanden die ersten Untersuchungen zur Biosynthese von Gliotoxin (122) statt. Bei diesen Experimenten konnte durch Fütterung radioaktiv markierter Vorläufer gezeigt werden, dass 122 aus Phenylalanin und Serin aufgebaut wird, die zunächst zu einem Schwefel freien Diketopiperazin cyclisiert werden. ${ }^{143}$ Auch für Hyalodendrin (123) konnte die Biosynthese ausgehend von Phenylalanin und Serin bewiesen werde. ${ }^{144}$ In Analogie dazu sollte Chetomin (119) aus Tryptophan und Serin aufgebaut werde.

Aufschluss über den weiteren Verlauf der Biosynthese der Thiodiketopiperazine, unter anderem darüber, wie der Schwefel in das Diketopiperazin eingeführt wird, könnten genetische Arbeiten liefern. So ist im letzten Jahr erstmals der Gencluster für ein Thiodiketopiperazine isoliert worden und zwar der für die Biosynthese von 
Sirodesmin (124). ${ }^{145}$ Neben den erwarteten Genen für den Aufbau des Diketopiperazins wurden auch Gene gefunden der Funktion noch nicht ganz klar ist und man darf gespannt sein welche Ergebnisse die weiteren Arbeiten an diesem Gencluster liefern.

\subsection{Fumitremorgin C (125)}

Der leicht gelbliche Feststoff konnte wie Chetoseminudin A (120) und Chetomin B (121) aus einer Fermentation des Stammes Gö 100/9 unter Zusatz von Acetat (1 g/L) in einer Ausbeute von $3.5 \mathrm{mg} / \mathrm{L}$ isoliert werden. Im ${ }^{1} \mathrm{H}-\mathrm{NMR}$-Spektrum von 125 treten die Signale von zwei Methylgruppen bei $\delta_{\mathrm{H}}=1.63$ und 1.97 sowie eine Methoxygruppe bei $\delta_{\mathrm{H}}=3.81$ auf. Im Tieffeldbereich sind zwei Signale bei $\delta_{\mathrm{H}}=6.80$ und $7.41 \mathrm{zu}$ erkennen, die $\mathrm{zu}$ einem mit Sauerstoff substituierten Aromaten gehören und auf Grund einer Kopplung von ${ }^{3} J=8.5 \mathrm{~Hz}$ ortho zu einander stehen sollten. Das Signal bei $\delta_{\mathrm{H}}=6.80$ weist zusätzlich eine Kopplung von ${ }^{4} J=2.0 \mathrm{~Hz}$ zu einem weiteren Proton bei $\delta_{\mathrm{H}}=6.84$ auf, was für einen 1,2,4-trisubstituierten Aromaten spricht. Aus dem Protonenspektrum können in Kombination mit einem ${ }^{1} \mathrm{H},{ }^{1} \mathrm{H}$ COSY-Experiment drei weitere Spinsysteme abgeleitet werden (Abbildung 61).<smiles>[R]OC</smiles><smiles>[R]c1ccc([R])c([R])c1</smiles><smiles>[R]CC([R])[R]</smiles><smiles>[R]C([R])C=C(C)C</smiles><smiles>[R]CCCC([R])[R]</smiles>

Abbildung 61: Aus einem ${ }^{1} \mathrm{H},{ }^{1} \mathrm{H}-\mathrm{COSY}$-Experiment abgeleitete Fragmente.

Dem ${ }^{13}$ C-NMR-Spektrum sind Signale für drei Methyl-, vier Methylen-, drei aliphatische und vier olefinische Methingruppen sowie für acht quartäre Kohlenstoffatome zu entnehmen. Zusammen mit dem im EI-Massenspektrum gefundenen Peak höchster Masse bei m/z=379 lässt sich daraus die Summenformel $\mathrm{C}_{22} \mathrm{H}_{25} \mathrm{~N}_{3} \mathrm{O}_{3}$ ableiten. Eine mit diesen Informationen durchgeführte Datenbanksuche ${ }^{53}$ führt zu Fumitremorgin $C^{146}(\mathbf{1 2 5})$.<smiles>COc1ccc2c3c([nH]c2c1)[C@@H](C=C(C)C)N1C(=O)[C@H]2CCCN2C(=O)[C@H]1C3</smiles> 


\section{Trichoderma sp. Stamm Gö 101/19}

Im chemischen Screening fiel der Pilz Trichoderma sp. Stamm Gö 100/19 bei Kultivierung in Schüttelkultur (Medium: 1158) durch die Bildung einer Zone mit gelber Eigenfarbe auf. Für die BASF AG waren die Extrakte aus dieser Kultivierung durch eine Aktivität gegen Phytophthora infestans und Pyricularia oryzae interessant.

\subsection{Sorbicillin (126)}

Die Substanz zeigt im EI-Massenspektrum den Peak höchster Masse bei $\mathrm{m} / \mathrm{z}=232$. Dem ${ }^{13}$ C-NMR-Spektrum sind Signale für drei Methyl- und fünf olefinische Methingruppen sowie sechs quartäre Kohlenstoffatome zu entnehmen. Mit Hilfe dieser Daten lässt sich die Summenformel $\mathrm{C}_{14} \mathrm{H}_{16} \mathrm{O}_{3}$ aufstellen, die durch ein hochaufgelöstes Massenspektrum bestätigt wurde. Im ${ }^{1} \mathrm{H}-\mathrm{NMR}-$ Spektrum treten die Signale der drei Methylgruppen bei $\delta_{\mathrm{H}}=1.89,2.13$ und 2.20 und die der fünf Methingruppen zwischen $\delta_{\mathrm{H}}=6.26$ und 7.44 auf. Außerdem sind die Signale von zwei Hydroxygruppen bei $\delta_{H}=7.42$ und 13.58 zuerkennen, wobei die Tieffeldverschiebung des einen Protons auf eine chelatisierte Hydroxygruppe hinweist. Anhand der im ${ }^{1}$ H-NMR-Spektrum auftretenden Kopplungskonstanten können sieben Protonen zu einer Alkylkette verknüpft werden. Die beiden in dieser Alkylkette enthaltenen Doppelbindungen sind aufgrund ihrer Kopplungskonstanten von ${ }^{3} J=14.5$ und $15.0 \mathrm{~Hz}$ transkonfiguriert. Eine Datenbanksuche ${ }^{54}$ und der anschließende Vergleich mit Literaturdaten ${ }^{147}$ führte zu Sorbicillin (126).<smiles>C/C=C/C=C/C(=O)c1cc(C)c(O)c(C)c1O</smiles> 


\section{Humicola grisea Stamm Gö 101/26}

Im chemischen Screening fiel der Pilz Humicola grisea Stamm Gö 101/26 bei Kultivierung in Schüttelkultur durch die Bildung mehrerer UV-Licht $(254 \mathrm{~nm})$ löschender Zonen auf. Aus einer Schüttelkultur (Medium: SGG) konnten zwei literaturbekannte Metabolite (127 und 128) isoliert werden. Bei der BASF AG fiel der Extrakte aus dieser Kultivierung durch eine Aktivität gegen Pyricularia oryzae und Septoria tritici auf.

\subsection{6-Methoxysterigmatocystin (127)}

Dem leicht gelben Feststoff kann durch ein hochaufgelöstes ESI-Massenspektrum die Summenformel $\mathrm{C}_{19} \mathrm{H}_{14} \mathrm{O}_{7}$ zugeordnet werden. Dem ${ }^{13} \mathrm{C}$-NMR-Spektrum sind Signale für zwei Methoxy- und sieben Methingruppen sowie für zehn quartäre Kohlenstoffatome zu entnehmen, wobei ein Signal bei $\delta_{\mathrm{C}}=181.9 \mathrm{zu}$ einer Carbonylgruppe gehört. Im ${ }^{1} \mathrm{H}-\mathrm{NMR}$ Spektrum treten die Signale der beiden Methoxygruppen bei $\delta_{\mathrm{H}}=3.91$ und 3.98 auf. Zwei Methinprotonen bei $\delta_{\mathrm{H}}=6.79$ und 7.18 stehen aufgrund ihrer Kopplungskonstante von ${ }^{3} J=9.0 \mathrm{~Hz}$ in ortho-Position an einem Aromaten. Weiterhin weist die Tieffeldverschiebung eines Protons bei $\delta_{\mathrm{H}}=13.45$ auf eine chelatisierte Hydroxygruppe hin. Durch eine mit diesen Informationen durchgeführte Datenbanksuche ${ }^{53}$ kann die Verbindung als 6-Methoxysterigmatocystin ${ }^{148}$ (127) identifiziert werden.

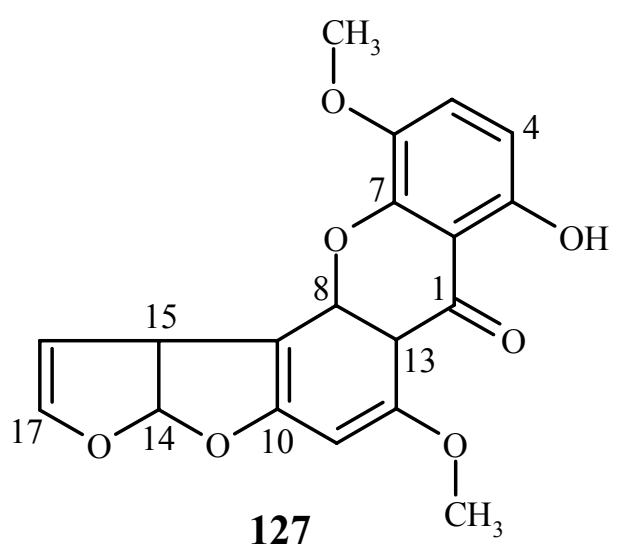




\subsection{Fuscoatrosid (128)}

Im ESI-Massenspektrum tritt bei $\mathrm{m} / \mathrm{z}=701$ ein $[\mathrm{M}+\mathrm{Na}]^{+}$-Ion auf, dessen Hochauflösung zur Summenformel $\mathrm{C}_{38} \mathrm{H}_{62} \mathrm{O}_{10}$ führt. Im ${ }^{1} \mathrm{H}-\mathrm{NMR}$-Spektrum tauchen im Bereich von $\delta_{\mathrm{H}}=0.73$ bis 2.00 insgesamt zehn Methylgruppen auf. Drei Methylgruppen sind zum Dublett aufgespalten, während die restlichen sieben als Singuletts vorliegen. Weiterhin treten zwischen $\delta_{H}=2.93$ und 4.98 die Signale von sieben Methin- und einer Methylengruppe auf, deren Verschiebungen auf die Verknüpfung mit Sauerstoff hinweisen. Sieben dieser Protonen konnten mittels eines ${ }^{1} \mathrm{H},{ }^{1} \mathrm{H}$-COSY-Experimentes einer Hexose zugeordnet werden. Dem ${ }^{13} \mathrm{C}$ NMR-Spektrum sind Signale für zehn Methyl-, acht Methylen- und elf Methingruppen sowie für neun quartäre Kohlenstoffatome zu entnehmen. Zwei Signale mit Verschiebungen von $\delta_{\mathrm{C}}=170.0$ und 175.1 können Carboxylgruppen zugeordnet werden. Zwei weiter quartäre Kohlenstoffatome bei $\delta_{\mathrm{C}}=133.4$ und 133.6 legen die Anwesenheit einer tetrasubstituierten Doppelbindung nahe. Die mit diesen Informationen durchgeführte Datenbankrecherche ${ }^{53}$ führte zu Fuscoatrosid (128). 128 wurde erstmals 2002 aus Humicola grisea isoliert und besitzt eine fungizide Wirkung gegen Aspergillus flavus. ${ }^{149}$

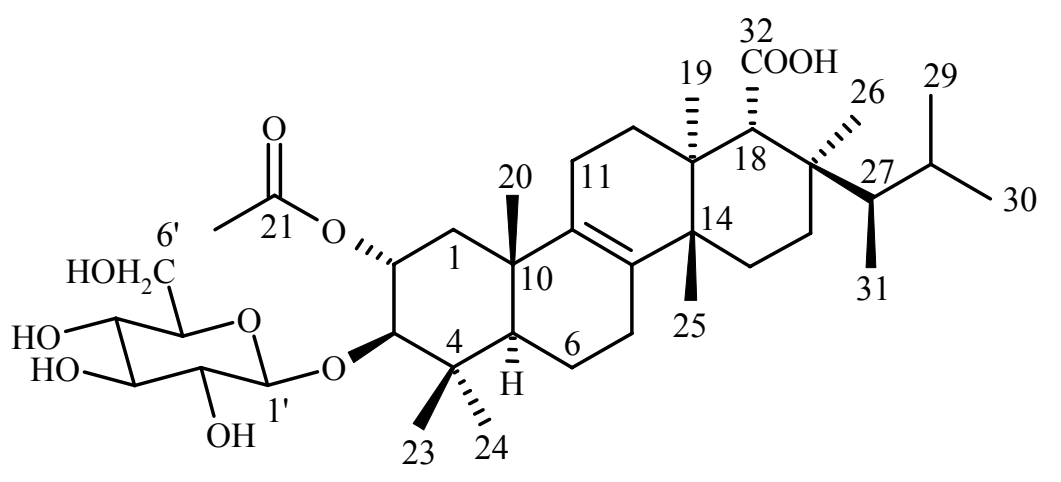




\section{Sekundärmetabolite weiterer untersuchter Pilze}

\section{Der Stamm A6651}

Zu Beginn dieser Doktorarbeit standen noch keine im Rahmen des BMBF-Projektes isolierten Pilze für Untersuchungen zur Verfügung, daher sollte zunächst der Stamm A6651 bearbeitet werden. Dieser wurde Anfang der 1990er Jahre von S. Philips im Rahmen einer Kooperation mit der Firma Hoechst (heute: Sanofi-Aventis) bearbeitet. Dabei konnte Dihydrobipolaroxin (129) aus Kulturen des Stammes isoliert werden. Im Rahmen der vorliegenden Doktorarbeit war zu prüfen, ob dieser Stamm durch Variation der Fermentationsbedingungen zur Produktion weiterer Sekundärmetabolite angeregt werden kann. Im chemischen Screening zeigte sich, dass der Stamm in Schüttelkultur (Medium: M2) eine Vielzahl weiterer Metabolite produziert, die UV-Licht $(254 \mathrm{~nm})$ löschen und mit Anisaldehyd blau anfärben. In anderen Medien (SGG, HA, SM, G20, E2 und 1158) zeigte der Stamm ein ähnliches Metabolitenmuster, die Produktivität in diesen Medien war jedoch deutlich geringer.

Aus einer Schüttelkultur (2 L, M2-Medium) konnten insgesamt acht Metabolite in Ausbeuten von 1.5 bis $12 \mathrm{mg}$ gewonnen werden, von denen nur die Verbindung 133 neu war. Durch weitere Variationen der Kultivierungsbedingungen im Sinne der OSMAC-Methode ${ }^{150}$ (One Strain/Many Compounds) konnte der Stamm nicht zur Produktion neuartiger Substanzen angeregt werden. Lediglich die Ausbeuten variierten je nach Fermentationsbedingungen.

\subsection{Eremophilane}

Aus Schüttelkulturen des Stammes A6651 konnten fünf Metabolite mit Eremophilan-Struktur in Ausbeuten von $3.0-6.0 \mathrm{mg} / \mathrm{L}$ isoliert werden.

\subsubsection{Dihydrobipolaroxin (129)}

Der farblose Feststoff färbt mit Anisaldehyd blau an und ist in Chloroform, Aceton und Methanol gut löslich. Im ${ }^{1} \mathrm{H}-\mathrm{NMR}-$ Spektrum treten die Signale für zwei Methylgruppen auf, bei $\delta_{\mathrm{H}}=1.17$ als Triplett $\left({ }^{3} J=7.0 \mathrm{~Hz}\right)$ und bei $\delta_{\mathrm{H}}=1.46$ als Singulett. Des Weiteren erscheinen Signale für 5 Protonen im Bereich von $\delta_{\mathrm{H}}=5.26$ bis 6.33, deren Verschiebungen charakteristisch für Doppelbindungen sind. Dem ${ }^{13} \mathrm{C}-\mathrm{NMR}-$ Spektrum sind Signale für zwei 
Methyl-, zwei Methylen-, zwei aliphatische und drei olefinische Methingruppen sowie fünf quartäre Kohlenstoffatome $\mathrm{zu}$ entnehmen. Dabei fällt die chemische Verschiebung einer Methylengruppen mit $\delta_{\mathrm{C}}=113.5$ besonders auf. Diese weist auf eine relativ selten vorkommende exocyclische Doppelbindung hin. Da Dihydrobipolaroxin, welches bereits von S. Philips aus diesem Stamm isoliert werden konnte, ebenfalls eine exocyclische Doppelbindung enthält, wurde vermutet, dass es sich bei der Verbindung um eben dieses handelt. Die Hochauflösung des im ESI-Massenspektrum gefundenen [M+Na] ${ }^{+}$-Ions bei 287 führt zur Summenformel $\mathrm{C}_{15} \mathrm{H}_{20} \mathrm{O}_{4}$. Durch Vergleich der NMR-Daten mit denen in der Literatur $^{151}$ konnte die Vermutung, dass es sich bei 129 um Dihydrobipolaroxin handelt, bestätigt werden.<smiles>C=C(CO)[C@]1(O)C[C@]2(C)C(=CC1=O)C(I)=C[C@@H](O)[C@@H]2C</smiles>

\subsubsection{Bipolaroxin (130)}

Der mit Anisaldehyd blau anfärbende farblose Feststoff ist in Chloroform und Aceton gut und in Methanol mäßig löslich. Im EI-Massenspektrum erscheint der Peak höchster Masse bei $\mathrm{m} / \mathrm{z}=262$, dessen Hochauflösung zur Summenformel $\mathrm{C}_{15} \mathrm{H}_{18} \mathrm{O}_{4}$ führt. Die NMR-Spektren besitzen große Ähnlichkeit mit denen von 129, allerdings fehlen die Signale für die Hydroxymethylengruppe bei $\delta_{\mathrm{H}}=4.13$ und 4.23 sowie bei $\delta_{\mathrm{C}}=64.1$. Dies und die um 2 Einheiten geringere Molekülmasse deuten darauf hin, dass anstelle des primären Alkohols von 129 an C-12 einen Aldehyd vorliegt. Tatsächlich finden sich in den NMR-Spektren die zusätzlichen Signale einer Aldehydfunktion $\left(\delta_{\mathrm{H}}=9.48\right.$ und $\left.\delta_{\mathrm{C}}=192.8\right)$. Bei der Substanz handelt es sich somit um Bipolaroxin ${ }^{151}$ (130).

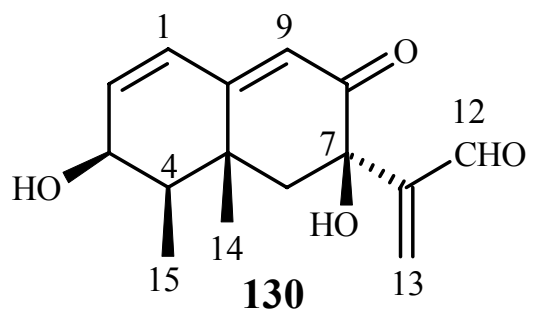




\subsubsection{Dendryphiellin E (131)}

Die Verbindung fiel als farbloses Öl an, das mit Anisaldehyd in der Wärme blau anfärbt. Eine Hochauflösung, des im ESI-Massenspektrum detektierten Ions bei $423[\mathrm{M}+\mathrm{Na}]^{+}$, ergab die Summenformel $\mathrm{C}_{24} \mathrm{H}_{32} \mathrm{O}_{5}$. Die NMR-Spektren dieser Verbindung zeigen einen doppelten Signalsatz, was zu der Vermutung führte, dass es sich um zwei sehr ähnliche Komponenten handelt. Diese konnten mit chromatographischen Methoden allerdings nicht von einander getrennt werden. Daher wurde versucht die Isomeren aus dem Gemisch heraus zu identifizieren. Im Folgenden wird zunächst die Strukturaufklärung für die Hauptkomponente beschrieben. Dieser konnten im ${ }^{13}$ C-NMR-Spektrum Signale für vier Methyl-, vier Methylen-, drei aliphatische und sieben olefinische Methingruppen sowie für sechs quartäre Kohlenstoffatome zugeordnet werden. Wie in den Spektren von 129 und 130 findet sich auch im ${ }^{13} \mathrm{C}$-NMR-Spektrum bei $\delta_{\mathrm{C}}=113.5$ ein Signal für eine exocyclische Doppelbindung. Ein Vergleich der ${ }^{13} \mathrm{C}-\mathrm{NMR}-$ Spektren der Verbindung mit denen von 129 ergibt große Ähnlichkeiten, allerdings treten bei 131 Signale für neun zusätzliche Kohlenstoffatome auf (zwei Methyl-, eine Methylen-, eine aliphatischen und vier olefinische Methingruppen sowie eine Estergruppe). Im ${ }^{1} \mathrm{H}-\mathrm{NMR}-$ Spektrum der Verbindung ist das Signal von 3-H um $1.25 \mathrm{ppm}$ ins Tieffeld verschoben. Dies spricht für eine Veresterung der Hydroxygruppe an C-3. Mit diesen Informationen und durch eine Datenbanksuche ${ }^{53}$ wurde die Verbindung eindeutig als Dendryphiellin $\mathrm{E}^{152}$ (131) identifiziert.

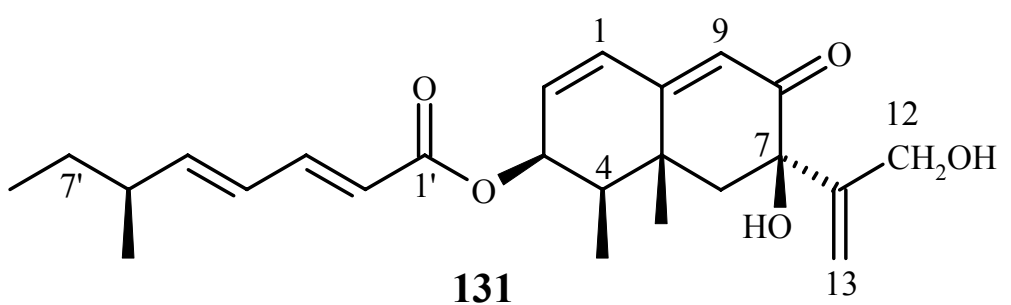

In der Literatur wird für $\mathbf{1 3 1}$ das Vorliegen eines Gleichgewichtes zwischen einer Keto(131a) und einer cyclischen Halbacetalform (131b), bei der die Hydroxygruppe an C-12 die Ketogruppe an C-8 angreift, beschrieben. Dies passt zu der Beobachtung, dass sich die Nebenkomponente von der Hauptkomponente durch das Fehlen des Signals der Carbonylgruppe an C-8 unterscheidet. An Stelle des Signals bei $\delta_{\mathrm{C}}=199.5$ tritt ein Signal bei 99.5 auf. Im ${ }^{1}$ H-NMR-Spektrum fällt besonders die Verschiebung der beiden Signale der Hydroxymethylengruppe ins Tieffeld auf. 
Aus dem in Chlorform gemessenen ${ }^{1} \mathrm{H}-\mathrm{NMR}-$ Spektrum ergibt sich ein Verhältnis von $8: 5$ zwischen Keto- (131a) und cyclischer Halbacetalform (131b). Die wichtigsten Unterschiede in den NMR-Daten der beiden Tautomeren sind in Abbildung 62 dargestellt.
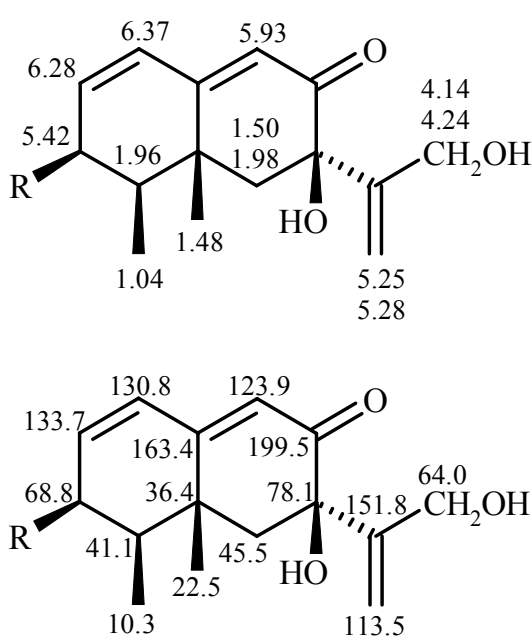

$131 \mathrm{a}$
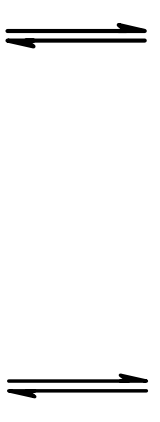

Abbildung 62: ${ }^{1} \mathrm{H}$ - und ${ }^{13} \mathrm{C}-\mathrm{NMR}-\mathrm{Daten}\left(\mathrm{CDCl}_{3}\right)$ der beiden Tautomeren 131a und 131b.

\subsubsection{KM-01 (132)}

Die als farbloses Öl vorliegende Substanz ist mit einem $\mathrm{R}_{\mathrm{f}}$-Wert von 0.77 (Chloroform/Methanol 9:1) unpolarer als 129 und 130. Sie färbt mit Anisaldehyd intensiv blau an und ihre NMR-Spektren weisen große Ähnlichkeit zu denen von 131 auf. Allerdings fehlen die Signale der Hydroxymethylengruppe bei $\delta_{\mathrm{H}}=4.14$ und 4.24 sowie bei $\delta_{\mathrm{C}}=64.0$. Dafür treten zusätzliche Signale bei $\delta_{\mathrm{H}}=9.50$ sowie bei $\delta_{\mathrm{C}}=192.7$ auf. Dieses legt die Vermutung nahe, dass diese Substanz anstelle des primären Alkohols von 131 einen Aldehyd trägt. Ein ESI-Massenspektrum, das durch Hochauflösung des bei $\mathrm{m} / \mathrm{z}=421$ detektierten $[\mathrm{M}+\mathrm{Na}]^{+}$-Ions zur Summenformel $\mathrm{C}_{24} \mathrm{H}_{30} \mathrm{O}_{5}$ führt, bestätigt diese Vermutung. Durch Vergleich mit Literaturdaten ${ }^{153}$ führte zu KM-01 (132).<smiles>C=C(C=O)[C@]1(O)C[C@]2(C)C(=CC1=O)C(I)=C[C@@H](O[14C](=O)/C=C/C=C/[C@H](C)CC)[C@@H]2C</smiles> 


\subsubsection{Metabolit A6651-E (133)}

Das farblose Ö1 mit dem $\mathrm{R}_{\mathrm{f}}$-Wert 0.19 (Chloroform/Methanol 9:1) färbt mit Anisaldehyd in der Wärme intensiv blau an. Sowohl die ${ }^{1} \mathrm{H}-\mathrm{NMR}$ - als auch die ${ }^{13} \mathrm{C}-\mathrm{NMR}-$ Spektren sind denen von 132 und 131 sehr ähnlich. Auch diese Verbindung besteht aus dem EremophilaneGrundgerüst an das wie bei 132 und 131 Dendryphiellinsäure A gebunden ist. Im Unterschied $\mathrm{zu}$ den zuvor beschriebenen Verbindungen fehlt allerdings im ${ }^{13} \mathrm{C}-\mathrm{NMR}-$ Spektrum das Signale der Ketogruppe an C-8 sowie das eines Aldehyds oder Alkohols an C-12, stattdessen treten neue Signale für quartäre Kohlenstoffatome bei $\delta_{\mathrm{C}}=127.7$ und $\delta_{\mathrm{C}}=168.9$ auf. Die Analyse der ${ }^{13} \mathrm{C}$-NMR-Daten und die Polarität der Verbindung sprechen dafür, dass sich an C-12 eine Carboxygruppe befindet, die unter Ausbildung eines Lactons mit der Ketogruppe an C-8 reagiert hat. Diese Annahme wird durch ein hochaufgelöstes ESI-Massenspektrum, welches zur Summenformel $\mathrm{C}_{24} \mathrm{H}_{30} \mathrm{O}_{6}$ führt, bestätigt. In der Literatur ist diese Verbindung bisher nicht bekannt. Die Stereochemie ist in Anlehnung an die anderen aus diesem Stamm isolierten Eremophilane (129 - 132) dargestellt.
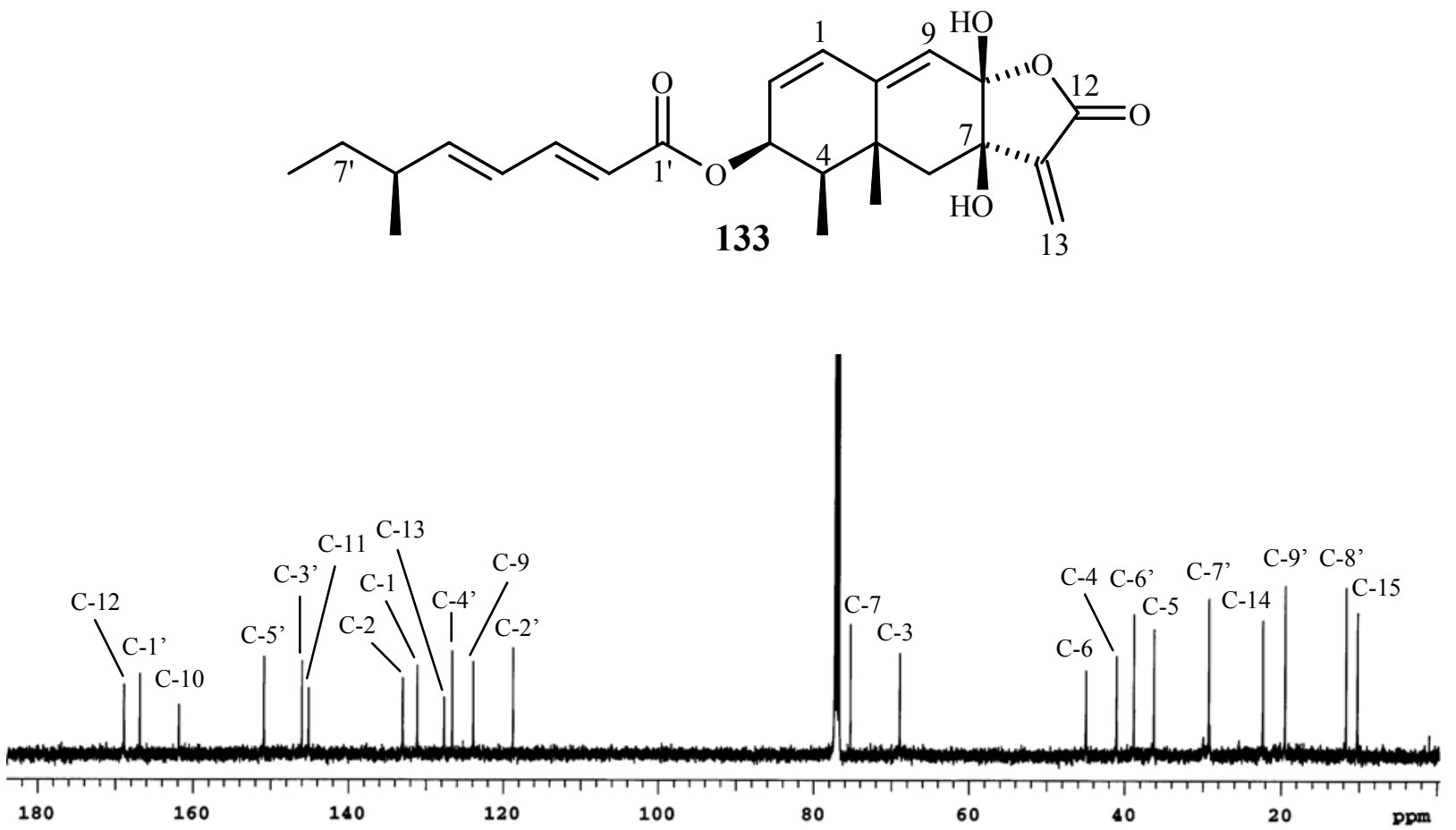

Abbildung 63: ${ }^{13} \mathrm{C}-\mathrm{NMR}-S p e k t r u m\left(125.7 \mathrm{MHz}, \mathrm{CDCl}_{3}\right)$ von Metabolit A6651-E (133). 


\subsubsection{Die Gruppe der Eremophilane}

Die Grundstruktur der Eremophilane besteht aus einem cis-Decalin-Grundgerüst mit zwei Kohlenstoffen an C-4 und C-5 sowie einer iso-Propylgruppe an C-7. Mittlerweile wurden mehr als 100 Eremophilane in der Literatur beschrieben, wobei die meisten aus Pflanzen und Pilzen stammen ${ }^{154}$. Viele Verbindungen dieser Substanzklasse, insbesondere die von pflanzenpathogenen Pilzen, wirken als Phytotoxine. In der Literatur wird für die aus dem Stamm A6651 isolierten Metabolite Bipolaroxin (130) und KM-01 (132) eine schädigende Wirkung auf verschiedene Gräser beschrieben. ${ }^{151,155}$ Bei den beiden Verbindungen Dihydrobipolaroxin (129) und Dendryphiellin E (131) konnte hingegen keine derartige Wirkung beobachtet werden, was darauf hinweist, dass die Aldehydfunktion in Position 12 essentiell für die Wirkung ist. Der Metabolit 133 ist neu, jedoch im rechten Teil Tulasnein ${ }^{156}$ (134) verwandt, das aus dem Pilz Podosordaria tulasnei stammt. 134 weist antibiotische Wirkung gegen verschiedene Pilze und Bakterien sowie eine schwache phytotoxische Wirkung auf. Für 133 konnte im Plattendiffusionstest jedoch keine antibiotische Wirkung beobachtet werden. Weitere mit 133 strukturell verwandte Verbindungen $(135,136)$ wurden semisynthetisch aus PR Toxin hergestellt und als Liganden des Progesteronrezeptors getestet. Dabei zeigte 136 mit $55 \mathrm{nM}$ die beste Affinität zum Rezeptor ${ }^{157}$.

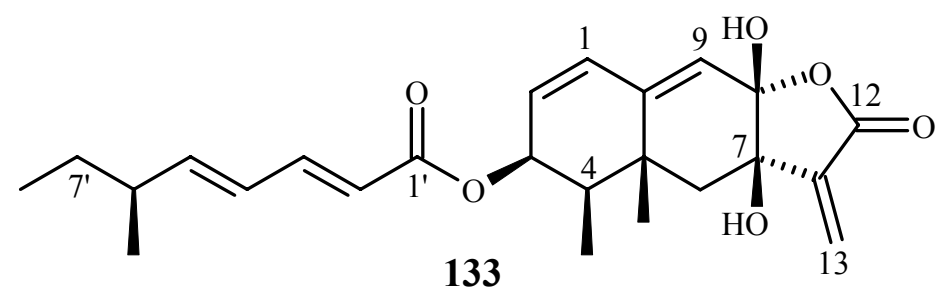<smiles>C=C1C(=O)O[C@H]2CC3CCCC(C)[C@@]3(C)C[C@]12O</smiles>

134<smiles>C=C1C(=O)OC2C=C3C(Cl)=C[C@H](OC(C)=O)[C@H](C)[C@]3(C)CC12</smiles>

135<smiles>CC(=O)O[C@H]1C=CC2=CC3OC(=O)C(C)=C3C[C@@]2(C)[C@@H]1C</smiles>

136

Abbildung 64: Metabolit A6651-E (133) und verwandte Verbindungen. 


\subsubsection{Zur Biosynthese der isolierten Eremophilane}

Bei den Eremophilanen handelt es sich um Sesquiterpene, die aus Farnesylpyrophosphat (FPP) aufgebaut werden. Da sich eine Methylgruppe an C-5 und nicht wie für Sesquiterpene erwartet an C-10 befindet, muss es bei der Ausbildung des Eremophilan-Grundgerüstes zu einer Methylgruppenwanderung kommen. Für diese wird ein Mechanismus angenommen, bei dem es zunächst unter Abspaltung des Phosphatrestes zur Ausbildung eines 10-gliedriegen Ringsystems (137) kommt, das dann nach Protonierung an C-6 erneut zwischen C-2 und C-7 cyclisiert wird. Auf einen Hydridshift von C-2 zu dem beim vorherigen Ringschluss gebildeten Carbokation an C-3 folgt die Wanderung der Methylgruppe von C-7 nach C-2. Nach Abspaltung des Protons an C-8 kommt es unter Ausbildung einer Doppelbindung zur Bildung von Aristolochen (138). Katalysiert wird diese Methylgruppenwanderung von der Aristolochen-Synthase, die unter anderem aus Aspergillus terreus und Penicillium roqueforti isoliert werden konnte und bei Inkubation mit trans, trans-Farnesylpyrophosphat Aristolochen (138) bildet. ${ }^{158}$ Durch gezielte Mutationen an der 5-Epi-Aristolochen-Synthase aus Tabak konnte außerdem gezeigt werden, dass es sich bei 137 tatsächlich um ein Intermediat bei der Ausbildung des Eremophilan-Grundgerüstes handelt, ${ }^{159}$ so dass angenommen werden darf, das der in Abbildung 65 vorgestellte Mechanismus der Realität entspricht.<smiles>CC1=CCCCCCCCCC(C)C1</smiles>
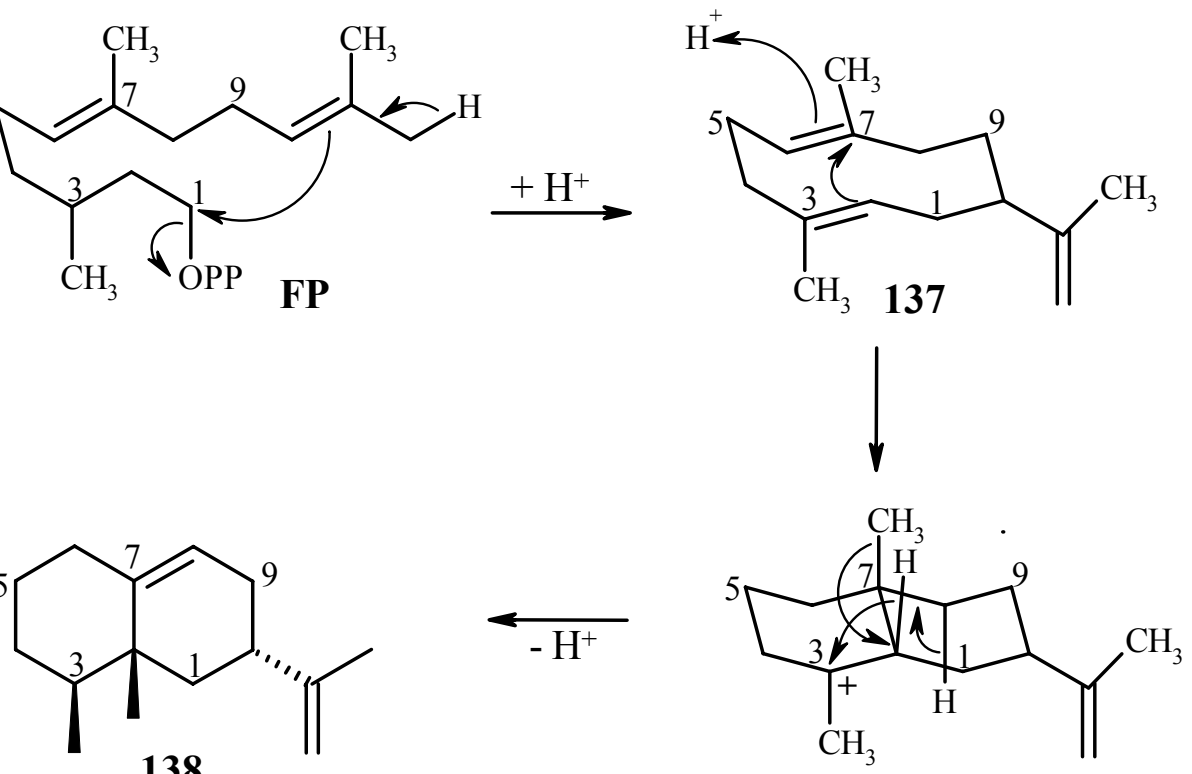

Abbildung 65: Biosynthese des Eremophilan-Grundgerüstes. 
Die aus dem Stamm A6651 isolierten Eremophilane sollten durch Folgereaktionen aus 138 hervorgehen. So erscheint es plausibel, dass zunächst Dihydrobipolaroxin (129) entsteht und aus diesem durch Oxidation an C-12 Bipolaroxin (130). Dendryphiellin E (131) sollte dann durch Veresterung von 129 mit Dendryphiellinsäure A (139) gebildet werden. Ob die Verbindung KM-01 (132) durch Oxidation von 131 oder durch Veresterung von 130 mit 139 entsteht, kann nicht entschieden werden. Ein Indiz dafür, dass 131 zu 132 oxidiert wird, liefert allerdings die Tatsache, dass nur $\mathbf{1 3 3}$ und keine analoge Verbindung ohne Seitenkette in den Extrakten gefunden wurde. Dies entspräche einer sukzessiven Oxidation von Dendryphiellin E (131) über 132 zu 133.<smiles>C=C(C)[C@@H]1CC=C2CCC[C@H](C)[C@]2(C)C1</smiles><smiles>C=[Te]</smiles><smiles>C=C(CO)[C@]1(O)[C@H](C)[C@@H](OC(=O)/C=C/C=C/[C@H](C)CC)C=CC2=CC(=O)[C@](O)(C(=C)CO)C[C@]21C</smiles><smiles>C=C(C=O)[C@]1(O)C[C@]2(C)C(=CC1=O)C=C[C@@H](O)[C@@H]2C</smiles>

130<smiles>C=C(C=O)[C@@]1(O)C[C@]2(C)C(=CC1=O)C=C[C@@H](OC(=O)/C=C/C=C/[C@H](C)CC)C(C)[C@@H]2OC(=O)/C=C/C=C/[C@H](C)CC</smiles>

133

Abbildung 66: Die späte Biosynthese der isolierten Eremophilane (129 - 133). 


\subsection{Dendryphiellinsäure A (139)}

Der mit Anisaldehyd in der Wärme blau anfärbbare farblose Feststoff (Ausbeute: $4.5 \mathrm{mg} / \mathrm{L}$ ) besitzt in Chloroform/ Methanol 9:1 einen $\mathrm{R}_{\mathrm{f}}$-Wert von 0.19. Ein EI-Massenspektrum lieferte den Peak höchster Masse mit m/z=154, dessen Hochauflösung zur Summenformel $\mathrm{C}_{9} \mathrm{H}_{14} \mathrm{O}_{2}$ führt. Das ${ }^{1} \mathrm{H}-\mathrm{NMR}$ zeigt Signale für zwei Methyl-, eine Methylen-, eine aliphatische und vier olefinische Methingruppen. Die chemischen Verschiebungen der Signale stimmen genau mit denen der Signale der Seitenkette aus den vorher beschriebenen Verbindungen (131 - 133) überein. Somit handelt es sich bei 139 um Dendryphiellinsäure $A^{152}$.<smiles>CCC(C)/C=C/C=C/C(=O)O</smiles>

139

\subsection{Cynodontin (140)}

Der rote Feststoff wurde aus einer Schüttelkultur im M2-Medium isoliert und ist in Chloroform gut, in Methanol und Aceton mäßig löslich. Das ${ }^{1} \mathrm{H}-\mathrm{NMR}-S p e k t r u m$ zeigt sieben Signale, die einer Methylgruppe bei $\delta_{\mathrm{H}}=2.40$ und drei aromatischen Protonen im Bereich von $\delta_{\mathrm{H}}=7.20$ bis 7.31 zugeordnet werden können. Des Weiteren finden sich vier Singuletts zwischen $\delta_{\mathrm{H}}=12.40$ und 12.83, die auf chelatisierte Hydroxygruppen hinweisen. Ein ESIMassenspektrum liefert das $[\mathrm{M}+\mathrm{H}]^{+}$-Ion bei $\mathrm{m} / \mathrm{z}=285$ der durch eine Hochauflösung zur Summenformel $\mathrm{C}_{15} \mathrm{H}_{10} \mathrm{O}_{6}$ führt. Eine mit diesen Daten durchgeführte Literatursuche konnte den roten Feststoff eindeutig als Cynodontin (140) identifizieren.<smiles>Cc1cc(O)c2c(c1O)C(=O)c1c(O)ccc(O)c1C2=O</smiles>

140 
140 wurde erstmals 1933 isoliert und in seiner Struktur aufgeklärt ${ }^{160}$ und wird von mehreren Flechten und Pilzen produziert. Bei biologischen Tests gegen verschiedene Pilze, unter anderem auch gegen Botrytis cinera (Grauschimmel bei Pflanzen), fiel Cynodontin durch eine mit den kommerziell erhältlichen Pestiziden Dicloran und Carbendazim vergleichbare Wirkung auf. ${ }^{161}$

Bei der Kultivierung des Stammes A6651 unter verschiedenen Bedingungen fiel auf, dass die Bildung von 140 durch Licht induziert wird. Zur Überprüfung wurde der Pilz im Dunkeln, bei Tageslicht und unter permanenter Beleuchtung auf Agarplatten angezogen. Bei der unter dem Einfluss von Tageslicht kultivierten Platte wies das Mycel nach wenigen Tagen eine Rotfärbung auf, wohingegen das Mycel der im Dunkeln und der unter permanenter Beleuchtung gelagerten Platten auch nach mehreren Wochen diese Verfärbung nicht zeigte. Diese Induktion der Anthrachinonbildung konnte bereits bei einem anderen Pilz, Curvularia pallescens, beobachtet werden. ${ }^{162}$ Die Frage, ob die Produktion von 140 einen Schutz vor ultravioletter Strahlung ermöglicht, muss leider offen bleiben.

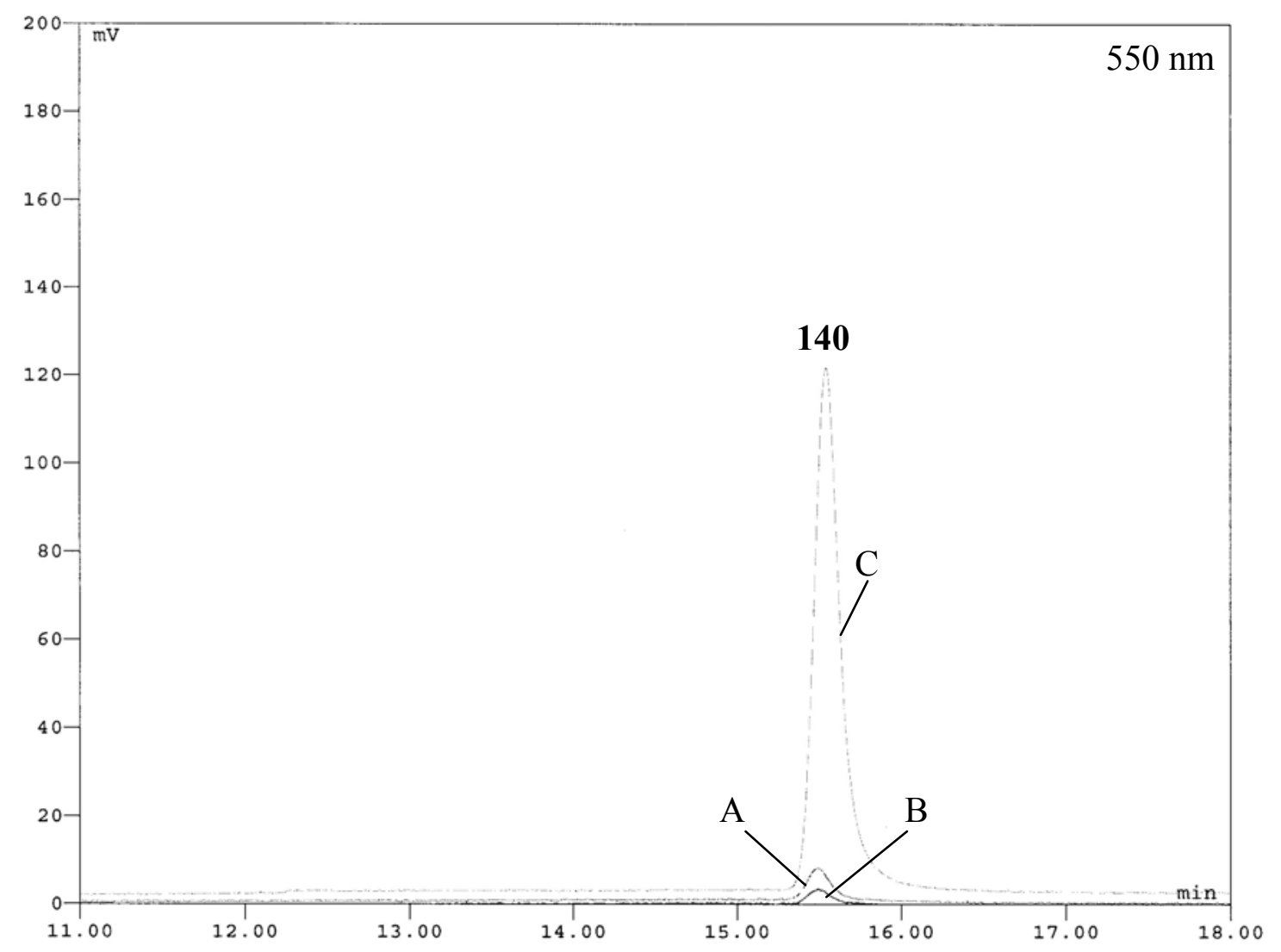

Abbildung 67: HPLC Chromatogramme von Extrakten des Stammes A6651 aus Kultivierungen im Dunkeln (A), bei permanenter Beleuchtung (B) und bei Tageslicht (C). 


\subsection{Cerebrosid C (141)}

Der farblose Feststoff fiel bei einer Kultivierung als Schüttelkultur (Medium: M2) in einer Ausbeute von $2.0 \mathrm{mg} / \mathrm{L}$ an. Er ist mit einem $\mathrm{R}_{\mathrm{f}}$-Wert von 0.06 (Chloroform/Methanol 9:1) recht polar. In den UV-Spektren sind lediglich End-Absorptionen zu erkennen, aus Banden im IR-Spektrum bei 3424 und 1651 bzw. $1542 \mathrm{~cm}^{-1}$ lässt sich auf die Anwesenheit von Hydroxyl- und Amidgruppen schließen. Die Summenformel konnte durch ein hochaufgelöstes ESI-Massenspektrum mit $\mathrm{C}_{43} \mathrm{H}_{79} \mathrm{NO}_{9}$ bestimmt werden. Im ${ }^{1} \mathrm{H}-\mathrm{NMR}-$ Spektrum erkennt man das Signal zweier Methylgruppen bei $\delta_{\mathrm{H}}=0.89$ sowie ein Signal für insgesamt 38 Protonen bei $\delta_{\mathrm{H}}=1.28$, was das Vorhandensein einer oder mehrerer langer Alkylketten impliziert. Weiterhin treten zwischen $\delta_{\mathrm{H}}=3.19$ und 4.43 Signale für 12 Protonen auf, deren Verschiebung auf die Verknüpfung mit Sauerstoff oder Stickstoff hinweist. Sieben dieser Protonen konnten mittels eines ${ }^{1} \mathrm{H},{ }^{1} \mathrm{H}$-COSY-Experimentes einer Hexose zugeordnet werden, die durch Vergleich mit Literaturdaten als $\beta$-D-Glucose identifiziert wurde. Zwischen $\delta_{\mathrm{H}}=5.14$ und 5.83 sind dem ${ }^{1} \mathrm{H}-\mathrm{NMR}$-Spektrum außerdem fünf Signale, die zu drei Doppelbindungen gehören, zu entnehmen. Zwei diese Doppelbindungen müssen auf Grund der Kopplungskonstante von ${ }^{3} J=15.0 \mathrm{~Hz}$ in der trans-Konfiguration vorliegen. Eine mit diesen Informationen durchgeführte Datenbankrecherche ${ }^{53}$ konnte 141 als Cerebrosid $C^{163}$ identifizieren, was im Einklang mit den ${ }^{13} \mathrm{C}-\mathrm{NMR}$-Daten steht.

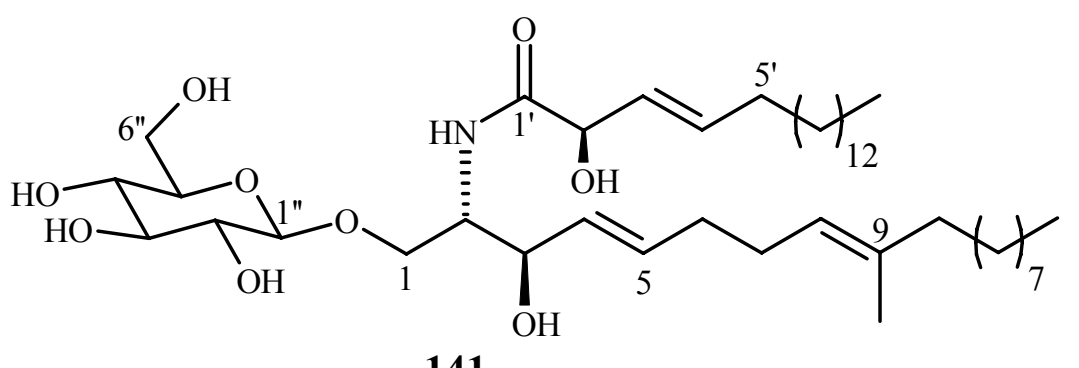

141

Cerebroside stellen eine Familie von Glycosphingolipiden dar, die in einer Vielzahl biologischer Systeme vorkommen. Unter anderem wurden Cerebroside aus marinen Schwämmen, verschiedenen Hohltieren, Ringelwürmern, Amphibien und Säugetieren, aber auch aus höheren Pflanzen sowie aus Pilzen und Bakterien isoliert. ${ }^{164}$ Sie besitzen eine Bedeutung für die strukturelle Stabilisierung von Zellmembranen und spielen bei einer Vielzahl biologischer Prozesse, wie intrazelluläre Kommunikation, Zellentwicklung und 
Zellagglutination eine Rolle. ${ }^{165}$ Relativ häufig werden Cerebroside aus Pilzen isoliert und können bei diesen die Ausbildung von Fruchtkörpern induzieren. ${ }^{166}$

Für diese Wirkung, die auch für das isolierte Cerebrosid C beschrieben wurde, ${ }^{167}$ scheinen die Methylgruppe an C-9 sowie die Doppelbindung zwischen C-4 und C-5 entscheidend zu sein. ${ }^{168}$ Des Weiteren verstärken Cerobrosid C und ähnliche Verbindungen die Wirkung von Aculeacin, einem Glucan-Synthetase Inhibitor. ${ }^{163}$ So zeigten verschiedene Cerebroside unter Zusatz von Aculeacin in Konzentrationen bei denen dieses nicht mehr wirkt, eine Wirkung gegen Candida albicans. Ohne Zugabe von Aculeacin besitzt keines der getesteten Cerebroside eine Candida-Aktivität auf.

\subsection{Variation der Fermentationsbedingungen}

Die aus dem Stamm isolierten Eremophilane (131 - 133) sind mit Dendryphiellinsäure A (139) verestert. Um Eremophilane mit anderen Carbonsäuren in der Seitenkette zu erhalten wurden sieben verschiedene Carbonsäuren (Ascorbinsäure, Essigsäure, Benzoesäure, Hexensäure, Nicotinsäure, Sorbinsäure und Zimtsäure) an den Stamm verfüttert. Dazu wurde der Stamm A6651 in Schüttelkolben fermentiert und nach einer Anwachsphase von 24 Stunden wurden die obigen Carbonsäuren in Konzentrationen von 0.1 und $1.0 \mathrm{~g} / \mathrm{L}$ zugesetzt. Die so erhaltenen Extrakte wurden dünnschichtchromatographisch und per HPLC-MS analysiert. Dabei wurde selektiv nach den, bei einem erfolgreichen Einbau, erwarteten Molmassen gesucht. Jedoch konnte in keinem der Ansätze die Bildung neuer Metabolite beobachtet werden. Das Enzym, welches die Veresterung mit Dendryphiellinsäure A katalysiert, scheint somit selektiv zu sein, da selbst die der Dendryphiellinsäure A recht ähnliche Sorbinsäure nicht akzeptiert wurde.

Zusätzlich wurden mit dem Stamm A6651 Ruhekultivierungen in P-Kolben unter Verwendung von sechs verschiedenen Nährmedien (M2, SGG, G20, 1158, 1549 und E2) durchgeführt. Auch hier gab es gegenüber der Kultivierung in Schüttelkolben keine Veränderungen im Metababolitenmuster, lediglich die Ausbeuten variierten in Abhängigkeit vom verwendeten Medium. Besonders auffällig war die gesteigerte Produktion von 140, was vermutlich auf die Tatsache zurückzuführen ist, dass die P-Kolben dem Tageslicht ausgesetzt waren (siehe Kapitel A.IV.1.3.). 


\section{Zur biologischen Aktivität der isolierten Naturstoffe}

In ersten Untersuchungen zur biologischen Aktivität wurden alle aus den Stämmen 6288, 6588, Gö 100/4, Gö 100/9, Gö 101/19 und Gö 101/26 isolierten Naturstoffe auf antimikrobielle Aktivität gegen Escherichia coli, Bacillus subtilis, Staphylococcus aureus und Candida albicans geprüft. Die Ergebnisse der aktiven Substanzen sind in Tabelle 9 dargestellt.

\begin{tabular}{|c|c|c|c|c|}
\hline Metabolit & E. coli & B. subtilis & S. aureus & C. albicans \\
\hline $\begin{array}{l}\text { 7-(1-Methyl-ethyliden)- } \\
\text { taiwapyron (25) }\end{array}$ & - & 12 & - & - \\
\hline Solanapyron H (48) & 7 & 21 & 9 & - \\
\hline Solanapyron I (49) & - & 14 & - & - \\
\hline 5,6-Dihydroalterperylenol (58) & - & 10 & - & - \\
\hline 1,3,8-Trihydroxynaphthalen (65) & - & 18 & - & - \\
\hline Kojic acid (91) & - & 15 & - & - \\
\hline Orsellinsäure (100) & - & 16 & - & - \\
\hline Orsellid A (101) und B (102) & 11 & 18 & 9 & - \\
\hline Orsellid C (104) & 14 & 30 & 12 & - \\
\hline Orsellid D (105) & 12 & 21 & 8 & - \\
\hline Orsellid E (106) & - & 13 & - & - \\
\hline 3,5,4'-Triacetyl-orsellid A (124) & 8 & 17 & 7 & - \\
\hline 3,5,2’-Triacetyl-orsellid B (125) & 10 & 25 & 10 & - \\
\hline 3,5,4'-Triacetyl-orsellid D (126) & 8 & 20 & 7 & - \\
\hline $\begin{array}{c}\text { (4-Hydroxy-pent-2-on-1-yl)- } \\
\text { orsellinat (107) }\end{array}$ & 8 & 22 & 9 & - \\
\hline $\begin{array}{c}\text { (Pent-3-en-2-on-1-yl)-orsellinat } \\
(\mathbf{1 0 8})\end{array}$ & 10 & 15 & 10 & - \\
\hline Chetomin (119) & 18 & 37 & 17 & - \\
\hline Chetoseminudin (120) & 17 & 35 & 16 & - \\
\hline Chetomin B (121) & 16 & 35 & 15 & - \\
\hline Fuscoatrosid (128) & 10 & 14 & 9 & \\
\hline Bipolaroxin (130) & - & 13 & - & - \\
\hline Dendryphiellin E (131) & - & 12 & - & - \\
\hline KM-01 (132) & - & 25 & 14 & - \\
\hline
\end{tabular}

Hemmhofdurchmesser in mm, getestete Substanzmenge: $15 \mu \mathrm{g}$, Testplättchendurchmesser $6 \mathrm{~mm}$

Tabelle 9: Ergebnisse im Plattendiffusionstest. 
Die aus dem Stamm Chaetomium sp. Gö 100/9 isolierten Orsellide A - E (101, 102, 104, 105 und 106) sind alle antimikrobiell wirksam, wobei Orsellid C (104) die besten Ergebnisse erzielt. Da auch (4-Hydroxy-pent-2-on-1-yl)-orsellinat (107) und (Pent-3-en-2-on-1-yl)orsellinat (108) wirksam sind, kann die Wirkung dieser Verbindungen möglicherweise durch eine bessere Verfügbarkeit der enthaltenen Orsellinsäure erklärt werden.

Die ebenfalls von dem Stamm Gö 100/9 gebildeten Thiodiketopiperazine (119 - 121) besitzen eine antimikrobielle Aktivität gegen E. coli, B. subtilis und S. aureus in ungefähr derselbe Größenordnung. Somit spielt die Anzahl der Schwefelatome für die Wirksamkeit eine untergeordnete Rolle.

Die Testergebnisse der aus dem Stamm A6651 isolierten Eremophilane (129-133) zeigen, dass für deren antimikrobielle Aktivität das Vorhandensein des Aldehyds an C-12 sowie der Seitenkette an C-3 entscheidend ist. Sind wie beim KM-01 (132) beide Merkmale vorhanden, wird eine maximale Hemmwirkung gegen B. subtilis und $S$. aureus beobachtet. Fehlen sie hingegen, wie beim Dihydrobipolaroxin (129), so ist keine Wirkung zu beobachten.

Alle isolierten Naturstoffe die in ausreichender Ausbeute (mindestens $1.0 \mathrm{mg}$ ) vorlagen, wurden bei der BASF AG auf eine mögliche Anwendung im Pflanzenschutz hin untersucht. Geprüft wurde dabei hauptsächlich auf fungizide Aktivität im Mikrotest gegen die Pathogene Phytophthora infestans, Botrytis cinerea, Pyricularia oryzae und Septoria tritici. Die Ergebnisse der aktiven Substanzen sind in Tabelle 10 dargestellt.

\begin{tabular}{|c|c|c|c|c|}
\hline Metabolit & P. infestans & B. cinera & P. oryzae & S. tritici \\
\hline Chaetomin (119) & $0.5 \mathrm{ppm}$ & - & - & $8 \mathrm{ppm}$ \\
\hline Bipolaroxin (130) & $125 \mathrm{ppm}$ & - & - & - \\
\hline KM-01 (131) & $8 \mathrm{ppm}$ & $125 \mathrm{ppm}$ & $31 \mathrm{ppm}$ & $8 \mathrm{ppm}$ \\
\hline Cerebrosid C (141) & $125 \mathrm{ppm}$ & - & - & - \\
\hline
\end{tabular}

Hemmkonzentration bis zu der eine Hemmung des Wachstums um mindestens $90 \%$ zu beobachten war

Tabelle 10: Ergebnisse aus dem Mikrotest (fungizid) der BASF AG.

Die Testergebnisse für weitere der Substanzen, insbesondere der Orsellide stehen noch aus. 


\section{Diskussion und Ausblick}

Im Rahmen des BMBF-Verbundprojektes sollten endophytische Pilze als Quelle neuer Naturstoffe erschlossen werden. Da diese bereits als Produzenten antifungischer und herbizider Substanzen bekannt sind, erhoffte man sich gerade in diesem Bereich interessante Ergebnisse. Zudem gehören sie zu den wenig untersuchten Spezies und man erwartete daher Metabolite mit neuen und ungewöhnlichen Strukturen, die durch auffällige biologische Wirkungen zu neuen Leitstrukturen führen können. Während der Durchführung des Projektes zeigten sich jedoch systematische Schwächen, die an den drei folgenden Beispielen erläutert werden sollen.

1) Zur Untersuchung der endophytischen Pilze sollte ein kombiniertes ChemischBiologisches Screening zur Anwendung kommen. Dabei sollte die Testierung von biologisch aktiven Extrakten im HPLC-Mikrotest zur Identifizierung der aktiven Metabolite führen. Das Problem bei der Durchführung dieses Screeningansatzes war allerdings, dass es drei bis sechs Monate dauerte, bis erste Testergebnisse vorlagen. So fielen die Extrakte des Stammes Gö 100/9 im HPLC-Mikrotest zu einem Zeitpunkt auf, als die aktive Komponente Chetomin (119) bereits aufgrund chemischer Detektionsmethoden isoliert worden war. Dieses Beispiel zeigt, dass insbesondere der HPLC-Mikrotest für die gezielte und schnelle Isolierung biologisch aktiver Metabolite ein optimaler Ansatz ist. Dazu sollten die Testergebnisse jedoch in einem vertretbaren Zeitrahmen vorliegen. Alternativ müsste man in einem vorgeschalteten Screening nach biologisch aktiven Extrakten von Pilzkulturen suchen, damit zu Beginn eines chemischen Teilprojektes bereits mehrere auffällige Pilzstämme zur Verfügung stehen.

2) Bei der Bearbeitung der endophytischen Pilze wurde auf Grund der langen Testzeit auf die chemische Detektion bei der Analyse des Metabolitenmusters zurückgegriffen. Dadurch ließen sich einige Metabolite zu Tage fördern, die im Rahmen des verwendeten biologisch orientierten Screeningansatzes nicht gefunden worden wären. So konnten aus Extrakten des Stammes 6288 durch Variation der Kultivierungsbedingungen insgesamt 13 Sekundärstoffe, von denen elf neu waren, isoliert werden. Auch die von Chaetomium sp. Gö 100/9 gebildeten antibakteriellen Orsellide A - E (101, 102, 104, 105 und 106) wären bei der Suche nach fungizider, herbizider oder insektizider Aktivität übersehen worden. 
Dieses Ergebnis verdeutlicht, dass die Auswahl von Stämmen allein nach biologischer Aktivität der Extrakte nicht sinnvoll ist, weil das Screening-Fenster zu eng ist. Interessante Pilze wie die Stämme 6288 oder Gö 100/9 bleiben in einem solchen Netz nicht hängen. Erfolgsversprechend erscheint vielmehr die Kombination der beiden Screeningansätze und die Prüfung isolierter Reinsubstanzen in allen verfügbaren Testsystemen bzw. ihre Verfügbarmachung in einem Naturstoffpool für die spätere Prüfung in neuen Testsystemen.

3) Ein wiederkehrendes Problem bei der Suche nach neuen Naturstoffen ist die Reisolierung bereits bekannter Substanzen. So wurden auch in diesem Projekt aus verschiedenen endophytischen Pilzen literaturbekannte Sekundärmetabolite isoliert. Diese konnten in der Regel zwar durch den Einsatz von Naturstoffdatenbanken wie „Dictionary of Natural Products“ (DNP, Chapman\&Hall/ CRC) ${ }^{53}$ und „Antibase“ (Chemical Concepts) ${ }^{54}$ relativ schnell identifiziert werden, mussten jedoch vorher mit einigem Aufwand aufgereinigt werden, um für eine Recherche belastbare Strukturdaten zu gewinnen. Die Identifizierung bekannter Substanzen aus Extrakten ist z.B. mit Hilfe einer HPLC-UV-MS-Kopplung möglich. Dazu müssten jedoch zunächst Datenbanken angelegt werden, die einen großen Teil der bekannten ca. 30000 mikrobiellen Sekundärmetabolite enthalten. Durch Vergleich und Kombination von Retentionszeiten, UV-Spektren, Molmassen und Fragmentierungsmustern könnten bekannte Substanzen schnell identifiziert werden. Dazu müssten die Rohextrakte jedoch einem vorgeschalteten Reinigungsschritt unterzogen werden, weil die je nach Stamm und Medium wechselnden Anteile lipophiler und hydrophiler Begleitsubstanzen die Trennungsergebnisse und Daten verfälschen können. Sofern man an eine Automatisierung der Screening- und Identifizierungsprogramme denkt, müssen alle Teilschritte sorgfältig validiert werden.

4) Der OSMAC-Ansatz, der darauf abzielt, stille Biosynthesegene für Sekundärstoffe durch veränderte Kultivierungsbedingungen $\mathrm{zu}$ aktivieren, führte nicht bei jedem Pilzstamm zum Erfolg. So war der Stamm A6651 nicht beeinflussbar, während der Stamm 6288 auf veränderte Bedingungen mit der Bildung mehrerer neuer Sekundärmetabolite reagierte. Aus den bisher bekannten Genomanalysen von Pilzen weiß man, dass Biosynthesegencluster für 20 - 30 verschiedene Sekundärstoffklassen auf deren Genom untergebracht sind. Ein besseres Verständnis der für die Regulation verantwortlichen 
molekularen Mechanismen könnte dabei helfen den OSMAC-Ansatz gezielter einzusetzen um neue Sekundärmetabolite zu erhalten.

Zusammenfassend ist festzustellen, dass trotz der beschriebenen Probleme in der verfügbaren Zeitspanne eine Vielzahl neuer Sekundärmetabolite isoliert wurde, was bestätigt, dass endophytische Pilze eine reiche Quelle für interessante Sekundärmetabolite darstellen. Allerdings fällt auf, dass sich die Metaboliten der endophytischen Pilze aus marinen Habitaten nicht wesentlich von denen terrestrischer Pilze unterscheiden. Die aus Chaetomium sp. Gö 100/9 isolierten Orsellide könnten ein Beispiel für spezifische endophytische Metabolite sein. 


\section{Zusammenfassung der Ergebnisse}

Im Rahmen dieser Arbeit wurden insgesamt 56 Pilzstämme in einem chemisch-biologischen Screening auf ihre Sekundärmetabolitenproduktion hin untersucht. Bei 55 dieser Pilzstämme handelt es sich um endophytische Pilze, die sowohl im Arbeitskreis AUST/ ScHULZ (TU Braunschweig) als auch in eigener Regie aus marinen Habitaten isoliert wurden. Ein weiterer Stamm wurde der Göttinger Stammsammlung entnommen. Die Kultivierung dieser Stämme führte zu 53 Naturstoffen, von denen 21 (40\%) neu waren. Das ist ein überraschend hoher Anteil. Die Ergebnisse aus der Bearbeitung der Pilzstämme wurden am Ende der Arbeit zusammenfassend diskutiert und bewertet.

\section{Microsphaeropsis sp. Stamm 6288:}

Der Stamm 6288 erwies sich als besonders variabel bezüglich seines Metabolitenmusters. Durch Kultivierung unter verschiedenen Bedingungen (OSMAC-Ansatz) konnten zwei literaturbekannte sowie elf neue Sekundärmetabolite isoliert und identifiziert werden, die zu vier verschiedenen Substanzklassen gehören.

1) Spiciferone: Aus Schüttelkulturen erhielt man das bekannte Spiciferon A (17) und Prospiciferon A (18).

2) $\alpha$-Pyrone: Neben Taiwapyron (22) wurden bei der Kultivierung in Ruhekultur die neuen a-Pyrone Dehydrotaiwapyron (23), 5,6-Dihydrotaiwapyron (24) und 7-(1-Methylethyliden)-taiwapyron (25) gebildet. Die Biosynthese der $\alpha$-Pyrone wurde diskutiert und erste Biosyntheseexperimente zeigten, dass es sich bei diesen um Pentaketide handelt.

3) Betaenone: Aus Ruhefermentationen in P-Kolben konnten die vier neuen Betaenone $\mathrm{G}-\mathrm{J}$ (39-42) isoliert werden, für deren gemeinsame Biosynthese ein hypothetischer Mechanismus aufgestellt wurde.

4) Solanapyrone: Drei bisher nicht in der Literatur beschriebenen Solanapyrone (48, 49 und 51) konnten aus Ruhekulturen erhalten werden. Solanapyron J (51) enthält Strukturelemente sowohl der $\alpha$-Pyrone als auch der Solanapyrone und wird vermutlich durch Kondensation von Solanapyron A (54) mit Dehydrotaiwapyron (23) gebildet. 
<smiles>CC[C@]1(C)C(=O)C=CC(C(=O)C(C)C(C)=O)C1=O</smiles><smiles>CCC(C)[C@H]1C(CO)=C[C@H]2C(O)[C@@H](C)C[C@@H](C)[C@]2(C)[C@H]1C(=O)CCO</smiles><smiles>CC(CCO)[C@H]1C(CO)=C[C@H]2C(O)[C@H](C)C[C@@H](C)[C@]2(C(=O)CCO)[C@@H]1C(=O)CCO</smiles><smiles>CCCC(=O)c1ccc(=O)oc1CO</smiles><smiles>CCC[C@H](O)C1C=CC(=O)OC1CO</smiles><smiles>CCCC(=O)c1ccc(=O)oc1C(O)=C(C)C</smiles><smiles>CCC(C)[C@H]1C(CO)=C[C@H]2C[C@@H](C)C[C@@H](C)[C@]2(C)[C@H]1C(=O)CCO</smiles><smiles>CCC(C)[C@H]1C(CO)=C[C@H]2C(O)[C@](C)(O)C[C@@H](C)[C@]2(C)[C@H]1C(=O)CCO</smiles><smiles>C[C@H]1C=C[C@H](C)[C@@H](CC[C@@H](C)O)C1c1cc(NCCO)c(C=O)c(=O)o1</smiles><smiles>[CH][C@@]12CC[C@@H](O)C[C@H]1C=C[C@@H](C)C2c1cc(OC)c(/C=C(\O)c2oc(=O)ccc2C(=O)CCC)c(=O)o1</smiles>

Alternaria sp. Stämme 6587 und 6588 :

Die beiden Pilzstämme 6587 und 6588 unterscheiden sich in ihrer Morphologie, zeigten aber bei Kultivierung als Schüttel- wie auch als Ruhekultur im chemischen Screening ein identisches Metabolitenmuster. Aus verschiedenen Kultivierungsansätzen konnten neun literaturbekannte Metabolite isoliert werden.

1) Aus Ruhekulturen in P-Kolben konnten Alternariol (55) und Alternariol-9-methylether (56) sowie drei Perylenchinone Alterperylenol (57), 5,6-Dihydroalterperylenol (58) und Stemphyperylenol (59) erhalten werden.

2) $\mathrm{Da}$ die Perylenchinone aus 1,8-Dihydroxynaphthalen hervorgehen, wurden zwei Inhibitoren, welche die 1,8-Dihydroxynaphthalenbiosynthese an unterschiedlichen Stellen unterbrechen, wachsenden Kulturen des Stammes zugesetzt. Die Zugabe von Tricyclazol und Fthalid führte dazu, dass keine Perylenchinone mehr produziert wurden. Stattdessen wurden im ersten Fall Sphaerolon (70), 1,3,8-Trihydroxynaphthalen (65) und 2-Hydroxyjuglon (69) gebildet, im zweiten Fall reicherte sich Scytalon (64) an. 


\section{Aspergillus flavus Stamm Gö 100/4}

Aus Schüttelkulturen des Stammes Gö 100/4 konnten fünf Sekundärmetabolite isoliert und identifiziert werden.

1) Beim Haupmetaboliten (200 mg/L) des Stammes handelt es sich um Kojic acid (91).

2) Zwei bekannte Peptide die insektizid wirksamen Miyakamide $A_{2}(92)$ und $B_{1}(94)$ wurden isoliert und identifiziert.

3) Neben Arthrographol (96) wurde das neue Arthrolacton (98) isoliert. Beide Metabolite stehen in einem engen Biosynthesezusammenhang. Durch Fütterung von $\left[1-{ }^{13} \mathrm{C}\right]-$ und $\left[1,2-{ }^{13} \mathrm{C}_{2}\right]$ Acetat konnte für 96 die Biosynthese auf dem Polyketidweg bewiesen werden. Ein Biosyntheseweg für 96 und 98 wurde postuliert.

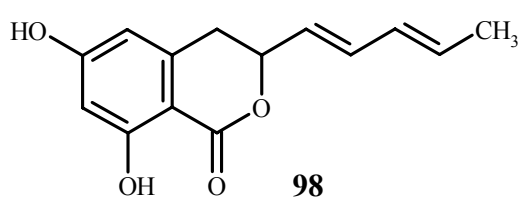

\section{Chaetomium sp. Stamm Gö 100/9:}

Der Stammes Gö 100/9 erwies sich als sehr ertragreich. Aus Schüttelkulturen konnten zwölf Sekundärmetabolite, von denen neun neu waren, isoliert werden.

1) Der Hauptmetabolit des Stammes war Orsellinsäure (100).

2) Die Orsellide A-E (101, 102, 104, 105 und 106) sind Ester aus Orsellinsäure und verschiedenen Desoxyzuckern. Es ist das erste Mal, dass Desoxyzucker als Bausteine von Pilzmetaboliten nachgewiesen wurden. Überraschenderweise zeigen die Orsellide eine signifikante antibakterielle Aktivität.

3) Die Struktur von zwei weiteren Orsellinsäureester (107 und 108) mit 1,4-Dihydroxy-2pentanon und 1-Hydroxy-3-Penten-2-on als Alkoholbaustein wurde aufgeklärt.

4) Fütterungsexperimente mit $\left[1-{ }^{13} \mathrm{C}\right]$ Acetat und $\left[1-{ }^{13} \mathrm{C}\right]$ Glucose zeigten, dass sich die Orsellide A-E (101, 102, 104, 105 und 106) aus Acetat und Glucose ableiten. Ein Biosyntheseweg für die Orsellide A-E sowie für 107 und 108 wurde aufgestellt und diskutiert. 
5) Die Orsellide A (101) und B (102) stehen über eine ungewöhnliche 1,3-Acylverschiebung miteinander im Gleichgewicht. Die Gleichgewichtseinstellung wurde untersucht und ein Mechanismus vorgeschlagen. Die Isomeren konnten nach Acetylierung getrennt und charakterisiert werden.

6) Die Thiodiketopiperazine Chetomin (119) und Chetoseminudin A (120) wurden identifiziert. Als neu erwies sich Chetomin B (121), das bezüglich der Schwefelbrücken isomer zu 120 ist. Alle drei Verbindungen besitzen interessante antibakterielle und antifungische Aktivitäten.

7) Aus einer Fermentation unter Zusatz von Acetat konnte Fumitremorgin C (125) isoliert werden.

8) Die antifungische Aktivität des Stammes Gö 100/9 konnte aufgrund eines HPLCMikrotestes auf Chetomin (119) zurückgeführt werden.<smiles>C=CCOC(OC(=O)c1c(C)cc(O)cc1O)C(OC)C(C)C(O)C(=O)O</smiles>

101<smiles></smiles><smiles>C/C=C/C(=O)COC(=O)c1c(C)cc(O)cc1O</smiles><smiles>COC1OC(C)CC(=O)C1OC(=O)c1c(C)cc(O)cc1O</smiles><smiles></smiles>

105

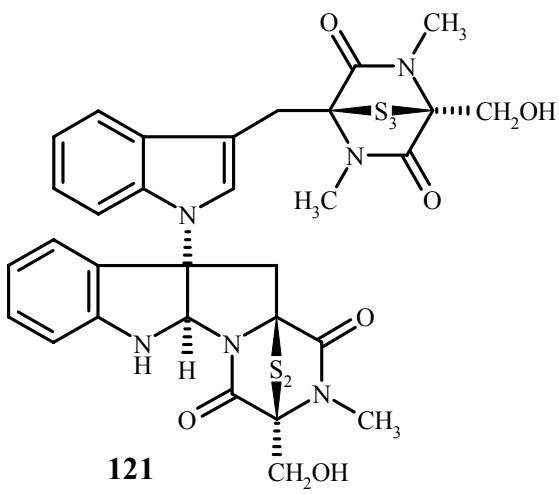

\section{Trichoderma sp. Stamm Gö 101/19}

Aus dem Kulturfiltratextrakt dieses Stammes konnte als einziger Metabolit Sorbicillin (126) isoliert und identifiziert werden. 


\section{Humicola grisea Stamm Gö 101/26}

Aus Extrakten des Stammes Gö 101/26 stammen die beiden bekannten Naturstoffe 6-Methoxysterigmatocystin (127) und Fuscoatrosid (128).

\section{$\underline{\text { Stamm A6651 }}$}

Aus Schüttelkulturen des Stammes A6651 konnten acht Sekudärmetabolite isoliert und identifiziert werden, von denen fünf zur Substanzklasse der Eremophilane gehören.

1) Neben dem erstmals beschriebenen Eremophilan 133 konnten die vier bekannten Vertreter Dihydrobipolaroxin (129), Bipolaroxin (130), Dendryphiellin E (131) und KM-01 (132) isoliert werden.

2) Die Biosynthese der isolierten Eremophilane wurde diskutiert und ein gemeinsamer Biosyntheseweg für die fünf Eremophilane vorgeschlagen.

3) Durch Fütterungsexperimente mit verschiedenen Carbonsäuren sollten Eremophilane mit neuer Seitenkette gewonnen werden. Diese Experimente waren nicht erfolgreich.

4) Biologische Tests zeigten, dass KM-01 (132) die besten antibakteriellen und antifungischen Eigenschaften unter den isolierten Eremophilane besitzt. Für die biologische Aktivität scheint das Vorhandensein des Aldehyds in Position 12 sowie der Seitenkette an C-3 eine entscheidende Rolle zu spielen.

5) Als weiterer Metabolit konnte Cerebrosid C (141) isoliert werden.

6) Für den literaturbekannten Metaboliten Cynodontin (140) wurde eine durch Licht induzierte Bildung beobachtet. Bei Kultivierung unter verschiedenen Beleuchtungsvariationen zeigte sich, dass 140 nur bei Tageslicht gebildet wird.

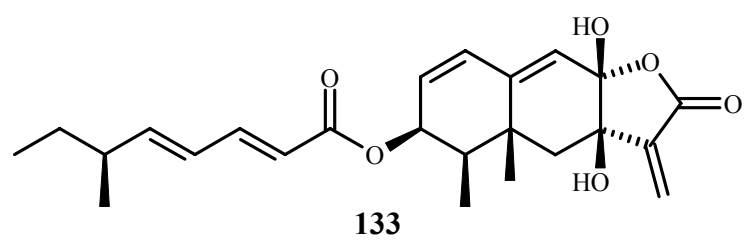




\section{B Experimenteller Teil}

\section{Allgemeines}

\section{Instrumentelle Analytik}

Massenspektren: EI-MS: Finnigan MAT 95, $70 \mathrm{eV}$, Hochauflösungen wurden mit Perfluorkerosin als Vergleichssubstanz gemessen, die relativen Intensitäten beziehen sich auf den Basispeak ( $\mathrm{I}=100 \%$ ) und sind in Klammern angegeben. DCI-MS: Finnigan MAT 95, $200 \mathrm{eV}$, Reaktandgas $\mathrm{NH}_{3}$. ESI-MS: Finnigan LC-Q. HR-ESI-MS: Bruker Apex-Q III, 7 Tesla.

Infrarotspektren (IR): Alle IR-Spektren wurden mit einem FT-IR-Spektrometer der Fa. Perkin-Elmer Modell 1600 als KBr-Presslinge gemessen, sh = Schulter.

Elektronenspektren (UV): Alle Elektronenspektren wurden mit einem Spektrometer der Fa. Varian Modell Cary 3E gemessen. Die Wellenlänge $\lambda$ ist in [nm] angegeben, der molare Extinktionskoeffizient $\varepsilon$ hat die Dimension $\left[1000 \mathrm{~cm}^{2} \mathrm{~mol}^{-1}\right]$, sh $=$ Schulter; Methanol/HCl bzw. Methanol/NaOH: Zu $2 \mathrm{ml}$ methanolischer Lösung wurde jeweils ein Tropfen $1 \mathrm{M} \mathrm{HCl}$ bzw. $1 \mathrm{M} \mathrm{NaOH}$ gegeben.

Drehwerte: Alle Drehwerte wurden mit einem Polarimeter der Fa. Perkin-Elmer Modell 343 bestimmt, die Drehwerte $[\alpha]_{\mathrm{D}}^{20}$ sind in $\left[10^{-1} \mathrm{deg} \mathrm{cm}^{2} \mathrm{~g}^{-1}\right]$ angegeben, die Konzentrationen $\mathrm{c}$ in $\left[10^{-2} \mathrm{~g} \mathrm{ml}^{-1}\right]$.

Circulardichroismus-Spektren (CD): Jasco J 500 mit Jasco IF 500 A/D-Wandler und BMC IF 800 Personalcomputer als Prozessor, die molaren Elliptizitäten $\theta$ sind in $\left[10^{-1} \mathrm{grad} \mathrm{cm}^{2} \mathrm{~mol}^{-1}\right]$ angegeben, $\mathrm{br}=$ breit. 
${ }^{1}$ H-NMR-Spektren: Varian Inova-600 (600 MHz), Varian Inova-500 (500 MHz), Varian Mercury-300 (300 MHz), Varian Unity-300 (300 MHz). Chemische Verschiebungen in $\delta$ Werten (ppm) relativ zum jeweiligen Lösungsmittel als internem Standard; Kopplungskonstanten $(J)$ in Hertz $(\mathrm{Hz})$.

Abkürzungen: $\mathrm{s}=$ Singulett, $\mathrm{d}=$ Dublett, $\mathrm{dd}=$ Dublett vom Dublett, $\mathrm{ddd}=$ Dublett vom Dublett vom Dublett, $\mathrm{ddt}=$ Dublett vom Dublett vom Triplett, $\mathrm{dt}=$ Dublett vom Triplett, $\mathrm{t}=$ Triplett, $\mathrm{q}=$ Quartett, $\mathrm{qd}=$ Quartett vom Dublett, quint $=$ Quintett, $\mathrm{m}=$ Multiplett, $\mathrm{br}=$ breit. Alle ${ }^{1}$ H-NMR-Spektren wurden näherungsweise als Spektren erster Ordnung interpretiert.

${ }^{13}$ C-NMR-Spektren: Varian Inova-600 (150.8 MHz), Varian Inova-500 (125.7 MHz), Varian Mercury-300 (75.5 MHz), Varian Unity-300 (75.5 MHz). Chemische Verschiebungen in $\delta$-Werten (ppm) relativ zum jeweiligen Lösungsmittel als internem Standard.

APT (Attached Proton Test): $\mathrm{CH}$ und $\mathrm{CH}_{3}$ stehen nach oben, $\mathrm{C}$ und $\mathrm{CH}_{2}$ stehen nach unten.

${ }^{13} \mathrm{C}-{ }^{1} \mathrm{H}$-Multiplizitäten sind aus HSQC-Experimenten in Verbindung mit APT-Experimenten ableitbar. Abkürzungen: $\mathrm{s}=$ Singulett, $\mathrm{d}=$ Dublett, $\mathrm{t}=$ Triplett, $\mathrm{q}=$ Quartett.

2D-NMR-Spektren: ${ }^{1} \mathbf{H},{ }^{1} \mathrm{H}-\mathrm{COSY}\left({ }^{1} \mathrm{H},{ }^{1} \mathrm{H}\right.$-Correlated Spectroscopy), HSQC (Heteronuclear Singular Quantum Coherence), HMBC (Heteronuclear Multiple Bond Connectivity), HMQC (Heteronuclear Multiple Quantum Coherence), NOESY (Nuclear Overhauser Effect Spectroscopy), TOCSY (Total Correlation Spectroscopy).

\section{Chromatographische Methoden}

Dünnschichtchromatographie (DC): Merck HPTLC-Fertigplatten Kieselgel 60 F $\mathrm{F}_{254}$ : 10 x $10 \mathrm{~cm}$, Schichtdicke $0.2 \mathrm{~mm}$; Merck DC-Alufolien Kieselgel $60 \mathrm{~F}_{254}: 20$ x $20 \mathrm{~cm}$, Schichtdicke $0.2 \mathrm{~mm}$; Merck DC-Alufolien RP-18 F254s: 20 x $20 \mathrm{~cm}$, Schichtdicke $0.2 \mathrm{~mm}$. Angegeben sind $\mathrm{R}_{\mathrm{f}}$-Werte (Laufhöhe relativ zur Laufmittelfront).

Lösungsmittel: Die Lösungsmittel für die Säulenchromatographie wurden zuvor destilliert. Für die HPLC wurden nur analysenreine Lösungsmittel und bidestilliertes Wasser verwendet. Lösungsmittel für die semipräparative HPLC wurden im Ultraschallbad (15 min) und anschließend durch Durchleiten von Helium (5 min) entgast. 
Säulen- und Gelchromatographie: ICN Kieselgel 60 (KG), $32-63 \mu \mathrm{m}$, Fluka Sephadex ${ }^{\circledR}$ LH-20.

Sprühreagenzien: Nach Merck, Anfärbereagenzien für die Dünnschichtchromatographie. ${ }^{169}$ Die DC-Platten wurden nach dem Ansprühen auf ca. $120^{\circ} \mathrm{C}$ erwärmt.

Anisaldehyd (Anis, Nr. 21): $1.0 \mathrm{~mL}$ Anisaldehyd wird in eine Lösung aus $85 \mathrm{~mL}$ Methanol, $10 \mathrm{~mL}$ Eisessig und $5 \mathrm{~mL}$ konz. Schwefelsäure gegeben.

Ehrlichs Reagenz (Ehrlich, Nr. 91): $1 \mathrm{~g}$ 4-Dimethylaminobenzaldehyd wird in einer Mischung von $25 \mathrm{~mL}$ Salzsäure (36\%) und $75 \mathrm{~mL}$ Methanol gelöst.

Orcin-Sprühreagenz (Orcin, Nr. 120-122): 1 g Eisen(III)-chlorid wird in 100 mL Schwefelsäure gelöst und zu gleichen Anteilen mit einer Orcinlösung (6 \% in Ethanol) gemischt.

\section{High Performance Liquid Chromatography (HPLC):}

LC-MS: Pumpe: Flux Instruments Rheos 4000; Autosampler: Jasco AS-851 (0 - $100 \mu \mathrm{L}$ variable Aufgabeschleife); UV-Detektor: Linear UVIS-205; Massendetektor: Finnigan LC-Q; Entgaser: Flux Instruments ERC-3415a; Steuersoftware HPLC: Flux Instruments Janeiro; Datensystem: Finnigan Xcalibur; Vorsäule: Grom Supersphere 100 RP-18 endcapped, $4 \mu \mathrm{m}$, 10 x 2 mm; Säule: Grom Supersphere 100 RP-18 endcapped, $4 \mu \mathrm{m}, 100$ x 2 mm.

Programm: von $20 \%$ Methanol auf $100 \%$ Methanol in $20 \mathrm{~min}, 5 \mathrm{~min} 100 \%$ Methanol, Flussrate: $300 \mu \mathrm{L} / \mathrm{min}$; UV-Detektion: $254 \mathrm{~nm}$, Massendetektion: positive und negative Ionen.

Präparative HPLC: Pumpe: Jasco PU-1587; UV-Detektor: Jasco UV 1575; manuelles Probenaufgabeventil: Rheodyne mit $2.0 \mathrm{~mL}$ Aufgabeschleife; Chromatographiesoftware: Jasco Borwin; Steuersoftware: Jasco Borwin HSS-1500;

Säulen: $\quad$ 1: Jasco Kromasil $100 \mathrm{C} 18,5 \mu \mathrm{m}, 20 \mathrm{~mm}$ x $250 \mathrm{~mm}$. 2: Jasco Nucleodur $100 \mathrm{C} 18,5 \mu \mathrm{m}, 20 \mathrm{~mm} \times 250 \mathrm{~mm}$.

Programm A: isokratisch $64 \% \mathrm{AcCN}$ mit $0.05 \%$ TFA und $36 \% \mathrm{H}_{2} \mathrm{O}$ mit $0.05 \%$ TFA; Flussrate: $12.0 \mathrm{~mL} / \mathrm{min}$; UV-Detektion: $280 \mathrm{~nm}$.

Programm B: isokratisch $40 \% \mathrm{AcCN}$ und $60 \% \mathrm{H}_{2} \mathrm{O}$; Flussrate: $15.0 \mathrm{~mL} / \mathrm{min}$; UVDetektion: $220 \mathrm{~nm}$.

Programm C: isokratisch $50 \% \mathrm{AcCN}$ und $50 \% \mathrm{H}_{2} \mathrm{O}$; Flussrate: $15.0 \mathrm{~mL} / \mathrm{min}$; UVDetektion: $220 \mathrm{~nm}$. 
Analytisce HPLC: Pumpe: Kontron 322 System; Autosampler: Kontron 360; Detektor Kontron Dioden Array Detektor 440; Mischkammer: Kontron HPLC 360, Datensystem: Kontron Kromasystem 2000 Version 1.60; analytische Aufgabenschleife;

Säulen: $\quad 3$ : Kromasil $100 \mathrm{C} 18,5 \mu \mathrm{m}, 4 \mathrm{~mm}$ x $250 \mathrm{~mm}$.

4: Nucleosil $100 \mathrm{C} 18,5 \mu \mathrm{m}, 3 \mathrm{~mm}$ x $250 \mathrm{~mm}$.

Programm D: Laufmittelsystem: Lösung A: $\mathrm{H}_{2} \mathrm{O}+0.1 \%$ TFA, Lösung B: Acetonitril +0.1 $\%$ TFA; Gradient von $15 \%$ B auf $100 \%$ B in 20 min, 5 min bei 100\% B; Flussrate: $0.5 \mathrm{~mL} / \mathrm{min}$.

Programm E: isokratisch $40 \%$ Acetonitril, $60 \% \mathrm{H}_{2} \mathrm{O}$; Flussrate: $0.5 \mathrm{~mL} / \mathrm{min}$.

Programm F: Laufmittelsystem: Lösung A: $\mathrm{H}_{2} \mathrm{O}+0.1 \%$ TFA, Lösung B: Acetonitril +0.1 $\%$ TFA; Gradient von $15 \%$ B auf $50 \%$ B in 25 min, von $50 \%$ B auf $100 \%$ B in $18 \mathrm{~min}, 5 \mathrm{~min}$ bei $100 \%$ B; Flussrate: $1.0 \mathrm{~mL} / \mathrm{min}$.

Programm G: Laufmittelsystem: Lösung A: $\mathrm{H}_{2} \mathrm{O}+0.1 \%$ TFA, Lösung B: Acetonitril +0.1 $\%$ TFA; Gradient von $30 \%$ B auf $100 \%$ B in 10 min, 8 min bei $100 \%$ B; Flussrate: $0.5 \mathrm{~mL} / \mathrm{min}$.

\section{Mikrobiologische Methoden}

Nährmedienbestandteile: Malzextrakt, Maltose, D-Glucose, Stärke, Caseinpepton, Fleischpepton und $\mathrm{CaCO}_{3}$ wurden von der Fa. Merck bezogen. Hefeextrakt stammt von der Fa. Oxoid, Cornsteep Powder von der Fa. Marcor, Soja fettarm von Henselwerk GmbH, Hafermehl (Holo Hafergold) von Neuform und BiTek Agar von der Fa. Difco.

Nährmedien: Für alle Nährmedien wurde demineralisiertes Wasser verwendet und der $\mathrm{pH}-$ Wert vor dem Autoklavieren mit $0.5 \mathrm{~N} \mathrm{NaOH}$ bzw. $0.5 \mathrm{~N} \mathrm{HCl}$ eingestellt. Die Nährmedien wurden durch Autoklavieren (feuchte Hitze, $121^{\circ} \mathrm{C}, 1$ bar Überdruck, $30 \mathrm{~min}$ ) sterilisiert. Festen Medien wurden $20 \mathrm{~g} / \mathrm{L}$ Agar zugesetzt.

M2: $\quad$ Malzextrakt $10 \mathrm{~g} / \mathrm{L}$, Hefeextrakt $4 \mathrm{~g} / \mathrm{L}$, Glucose $4 \mathrm{~g} / \mathrm{L}, \mathrm{pH}=7.0$;

Hafer (Ha): Hafermehl 20 g/L, Spur 112.5 ml/L, pH 7.2.

SGG: $\quad$ Glucose $10 \mathrm{~g} / \mathrm{L}$, Glycerin $10 \mathrm{~g} / \mathrm{L}$, Stärke $10 \mathrm{~g} / \mathrm{L}$, Cornsteep Powder $2.5 \mathrm{~g} / \mathrm{L}$, Caseinpepton $5 \mathrm{~g} / \mathrm{L}$, Hefeextrakt $2 \mathrm{~g} / \mathrm{L}, \mathrm{NaCl} 1 \mathrm{~g} / \mathrm{L}, \mathrm{CaCO}_{3} 3 \mathrm{~g} / \mathrm{L}, \mathrm{pH}=$ 7.0.

1158: 20 g Malzextrakt, $10 \mathrm{~g}$ Glucose, 2 g Hefeextrakt, $0.5 \mathrm{~g}$, Ammoniumhydrogenphosphat, $\mathrm{pH}=6.0$. 
E2: $\quad 20 \mathrm{~g}$ Glucose, $20 \mathrm{~g}$ Hafermehl, $20 \mathrm{~g}$ entfettetes Sojamehl.

G20: $\quad 20 \mathrm{~g}$ Glycerin, $10 \mathrm{~g}$ Malzextrakt, $4 \mathrm{~g}$ Hefeextrakt.

1549: $\quad 10 \mathrm{~g}$ Glucose, $10 \mathrm{~g}$ Maltose, $5 \mathrm{~g}$ Caseinpepton, $5 \mathrm{~g}$ Fleischpepton, $\mathrm{pH}=5.4$.

Spurenelementlösung Spur 11: $\mathrm{CaCl}_{2}$ × $2 \mathrm{H}_{2} \mathrm{O} 3 \mathrm{~g} / \mathrm{L}, \mathrm{Fe}(\mathrm{III})$-citrat $1 \mathrm{~g} / \mathrm{L}, \mathrm{MnSO}_{4} 0.2 \mathrm{~g} / \mathrm{L}$, $\mathrm{ZnCl}_{2} 0.1 \mathrm{~g} / \mathrm{L}, \mathrm{CuSO}_{4} \times 5 \mathrm{H}_{2} \mathrm{O} 25 \mathrm{mg} / \mathrm{L}, \mathrm{Na}_{2} \mathrm{~B}_{4} \mathrm{O}_{7}$ x $10 \mathrm{H}_{2} \mathrm{O} 20 \mathrm{mg} / \mathrm{L}, \mathrm{CoCl}_{2} 4 \mathrm{mg} / \mathrm{L}$, $\mathrm{Na}_{2} \mathrm{MoO}_{4}$ × $2 \mathrm{H}_{2} \mathrm{O} 10 \mathrm{mg} / \mathrm{L}$.

Isotopenmarkierte Verbindungen: $\left[1-{ }^{13} \mathrm{C}\right]$ Acetat wurde von der Firma Campro Scientific, $\left[1,2-{ }^{13} \mathrm{C}_{2}\right]$ Acetat und $\left[1-{ }^{13} \mathrm{C}\right]$ Glucose von der Firma Cambridge Isotope Lab. (CIL) bezogen. Alle Verbindungen waren zu 99\% mit markiertem Kohlenstoff angereichert.

Berechnung der ${ }^{13} \mathrm{C}$-Anreicherung (nicht miteinander koppelnde Kerne)

$\%$ Anreicherung $=1.1 \%$ x $\frac{\text { (Intensität Signal markierte Verbindung) }}{\text { (Intensität Signal Referenzverbindung) }}-1.1 \%$

Spezifischer Einbau $=\frac{(\% \text { Anreicherung }) \times 100}{(\% \text { Anreicherung der Vorstufe })}$

Zentrifugen: Sigma 2K1, Sigma 4K15, Sigma 210 M.

Schüttler: Braun Inkubationsschüttelschrank BS4, Braun Certomat RM, Braun Certomat BS-1.

Plattendiffusionstest: Auf die mit den verschiedenen Testkeimen angeimpfte Agarplatte wurden Filterplättchen (Durchmesser: $6 \mathrm{~mm}$, Dicke: $0.5 \mathrm{~mm}$ ) gelegt, die mit $15 \mu \mathrm{L}$ Substanzlösung (in Methanol oder Chloroform gelöst, $\mathrm{c}=1 \mathrm{mg} / \mathrm{mL}$ ) getränkt und vor dem Auflegen unter sterilen Bedingungen getrocknet worden waren. Als Testkeime dienten Bacillus subtilis, Escherichia coli, Staphylococcus aureus, und Candida albicans. Die Agarplatten wurden $24 \mathrm{~h}$ bei $25{ }^{\circ} \mathrm{C}$ (C. albicans) bzw. $37^{\circ} \mathrm{C}$ (B. subtilis, E. coli und S. aureus) inkubiert. 


\section{Zusammensetzung der Nährböden für die Plattendiffusionstests:}

B. subtilis und E. coli: $5 \mathrm{~g} / \mathrm{L}$ Glucose, $0.5 \mathrm{~g} / \mathrm{L}$ Trinatriumcitrat x $2 \mathrm{H}_{2} \mathrm{O}, 3 \mathrm{~g} / \mathrm{L} \mathrm{KH}_{2} \mathrm{PO}_{4}, 7 \mathrm{~g} / \mathrm{L}$ $\mathrm{K}_{2} \mathrm{HPO}_{4}, 0.1 \mathrm{~g} / \mathrm{L} \mathrm{MgSO}_{4} \times 7 \mathrm{H}_{2} \mathrm{O}, 1 \mathrm{~g} / \mathrm{L}\left(\mathrm{NH}_{4}\right)_{2} \mathrm{SO}_{4}$ und $15 \mathrm{~g} / \mathrm{L}$ Agar, keine pH-Einstellung.

S. aureus: $8 \mathrm{~g} / \mathrm{L}$ Bacto Nutrient Broth, $5 \mathrm{~g} / \mathrm{L} \mathrm{NaCl}$ und $15 \mathrm{~g} / \mathrm{L}$ Agar, keine pH-Einstellung. C. albicans: 4 g/L Hefeextrakt, $10 \mathrm{~g} / \mathrm{L}$ Malzextrakt, $4 \mathrm{~g} / \mathrm{L}$ Glucose und $15 \mathrm{~g} / \mathrm{L}$ Agar, $\mathrm{pH}=5.5$. Zum Ansetzen der Testplatten-Medien wurde demineralisiertes Wasser verwendet. Die Glucose wurde erst nach der 30 minütigen Sterilisation hinzugegeben.

\section{Allgemeine Arbeitsvorschriften (AAV)}

\section{AAV 1: Acetylierungen im 1-5 mg-Maßstab}

Die zu acetylierende Substanz wird in $1 \mathrm{ml}$ Pyridin gelöst und mit $2 \mathrm{ml}$ frisch destilliertem Acetanhydrid versetzt. Die Reaktionslösung wird 4-12 h bis zur quantitativen Umsetzung bei Raumtemperatur gerührt und anschließend mit $5 \mathrm{ml}$ Eiswasser hydrolisiert. Die wäßrige Phase wird dreimal mit Dichlormethan oder Ethylacetat extrahiert und die vereinigten organischen Phasen im Vakuum eingeengt. Reste Pyridin wurden azeotrop mit Toluol entfernt. 


\section{Sekundärmetabolite aus endophytischen Pilzstämme}

\section{Stammhaltung und Langzeitkonservierung}

Die aus Braunschweig (AK Aust) erhaltenen Endosymbionten wurden auf drei verschiedenen Medien $\left(1158, \mathrm{M}^{+}, \mathrm{E} 2\right)$ ausgestrichen und bei $28^{\circ} \mathrm{C} 5-8 \mathrm{~d}$ inkubiert. Das Medium, auf dem das beste Wachstum zu beobachten war, verwendete man zur Stammhaltung. Die Agarplatten wurden bei $4^{\circ} \mathrm{C}$ gelagert, die Langzeithaltung der Stämme erfolgte in flüssigem Stickstoff. Mit eigenen Isolaten aus Algen wurde analog verfahren.

\section{Durchführung des chemisch biologischen Screenings}

Die Kultivierung der zu untersuchenden Stämme erfolgte in fünf verschiedenen Medien (E2, 1158, 1549, G20 bzw. SGG) ohne und mit Zusatz von Natriumchlorid (33 g/L) in 250 oder 300 ml-Schüttelkolben (3 Schikanen, Wattestopfen), die jeweils mit $100 \mathrm{~mL}$ gefüllt waren.

Die Kulturen wurden mit einem $1 \mathrm{~cm}^{2}$ großem Stück einer gut bewachsenen Agarplatte beimpft und $96 \mathrm{~h}$ bei $28^{\circ} \mathrm{C}$ und $180 \mathrm{rpm}$ inkubiert. Es wurden je Nährmedium 2 Kolben angesetzt, die bei gleichem Aussehen und pH-Wert zusammen aufgearbeitet wurden.

Die vereinigte Kulturbrühe $(200 \mathrm{~mL})$ wurde zentrifugiert (4500 rpm, $15 \mathrm{~min})$, das Mycel $15 \mathrm{~min}$ im Ultraschallbad mit $100 \mathrm{~mL}$ Aceton extrahiert. Das Lösungsmittel wurde durch Zentrifugation (4500 rpm, $10 \mathrm{~min}$ ) abgetrennt und im Vakuum entfernt. Das Kulturfiltrat wurde mit Ethylacetat (2 x $200 \mathrm{~mL}$ ) extrahiert, anschließend wurde das Lösungsmittel im Vakuum entfernt. Die so erhaltenen Extrakte (Kulturfiltrat und Mycel) wurden jeweils in $2 \mathrm{ml}$ Methanol gelöst und $5 \mu \mathrm{l}$ dieser Lösung auf eine HPTLC-Kieselgelplatte aufgetragen. Die Dünnschichtchromatogramme wurden in Chlororform/ Methanol (9:1) entwickelt. Die Analyse des Metabolitenmusters erfolgte durch UV-Licht und mit Hilfe von Sprühreagenzien (Anisaldehyd, Ehrlich und Orcin).

Die Extrakte wurden anschließend der BASF AG zur weiteren Testierung übergeben. 


\section{Aufarbeitung von Fermentationsansätzen}

\subsection{Kultivierung in Schüttelkolben}

Fermentationen im größeren Maßstab wurden analog $\mathrm{zu}$ den unter B.II.2. beschriebenen Verfahren durchgeführt. In Schüttelkolben wurden zwischen 1 L und 4 L (10 - 40 Kolben) fermentiert.

Die Aufarbeitung erfolgte analog zu B.II.2., wobei das Mycel dreimal mit insgesamt ca. $500 \mathrm{~mL}$ Aceton je Liter Kulturlösung extrahiert und das Kulturfiltrat je Liter dreimal mit ca. $500 \mathrm{~mL}$ Ethylacetat extrahiert wurde.

\subsection{Kultivierung als Ruhekultur}

Stämme die nach dem chemischen Screening als interessant eingestuft wurden, wurden zusätzlich in fünf verschiedenen Medien (E2, 1158, 1549, G20 bzw. SGG) in P-Kolben (2 x $1 \mathrm{~L})$ bei Raumtemperatur kultiviert. Die Kulturen wurden mit 2 x 1/8 einer gut bewachsenen Agarplatte beimpft und direkt nach dem Animpfen und nach 7 Tagen kräftig geschüttelt, um ein gleichmäßiges Wachstum zu ermöglichen. Die Aufarbeitung erfolgte nach 28 Tagen nach dem unter B.II.3.1. beschriebenen Verfahren.

\section{Microsphaeropsis sp. Stamm 6288}

\subsection{Kultivierung und Aufarbeitung}

Der Stamm 6288 wurde wie unter B.II.3.1. beschrieben in Schüttelkolben (4 L, 40 x 100 mL) mit 1549 als Nährmedium kultiviert und aufgearbeitet. Aus dem Kulturfiltrat dieses Fermentationsansatzes konnten Spiciferon A (17) und Prospiciferon A (18) in Ausbeuten von $2.0 \mathrm{bzw} .27 .0 \mathrm{mg} / \mathrm{L}$ isoliert werden.

Weiterhin wurde der Stamm wie unter B.II.3.2. beschrieben in P-Kolben kultiviert. Als Nährmedium dienten die fünf Standardnährmedien (E2, 1158, 1549, G20 bzw. SGG) ohne und mit Zusatz von Natriumchlorid (33 g/L). Aus diesen Kultivierungsvariationen konnten elf verschiedene Metabolite erhalten werden. Eine Übersicht über die mit den unterschiedlichen Nährmedien erzielten Ergebnisse ist in Tabelle 5 (Kapitel A.III.1.) gegeben. 


\subsection{Variation der Kultivierungsbedingungen}

Um geeignete Bedingungen zur Untersuchung der Biosynthese der isolierten $\alpha$-Pyrone zu finden, wurde der Stamm 6288 in den Medien E2 und SGG ohne und mit Zusatz von Natriumchlorid (33 g/L) in 250 ml-Schüttelkolben (3 Schikanen, Wattestopfen) kultiviert. Die Schüttelkolben wurden mit 50 bzw. 100 mL Nährlösung befüllt und nach Beimpfen mit einem $1 \mathrm{~cm}^{2}$ großem Stück einer gut bewachsenen Agarplatte für $4-8$ Tage bei $28^{\circ} \mathrm{C}$ und $120 \mathrm{bzw}$. 180 rpm inkubiert. Die verschiedenen Ansätze wurden auf einen pH-Wert von 4-5 gebracht, mit derselben Menge Ethylacetat versetzt und $5 \mathrm{~min}$ mit dem Ultraturax behandelt. Anschließend wurde das Mycel abzentrifugiert und verworfen. Nach Abtrennen der organischen Phase wurde ein weiteres Mal mit Ethylacetat extrahiert und die organischen Phasen vereinigt. Die nach dem Entfernen des Lösungsmittels erhaltenen Extrakte wurden dünnschichtchromatographisch und per HPLC (Säule 4, Programm D) analysiert. Bei keinem der Versuche konnte die Produktion eines der vom Stamm gebildeten $\alpha$-Pyrone (22 - 25) beobachtet werden.

\subsection{Aufnahme einer Wachstumskurve}

Zur Aufnahme des zeitabhängigen Produktionsverlaufes der $\alpha$-Pyrone (22 - 25) in Ruhekultur wurde der Stamm 6288 für 24 h wie unter B.II.3.1. beschrieben in Schüttelkolben angezogen und anschließend als Ruhefermentation kultiviert. Ab dem 7. bis zum 14. Tag wurden täglich zwei Kolben entnommen und wie unter B.II.4.2. beschrieben aufgearbeitet. Die erhaltenen Extrakte wurden dünnschichtchromatographisch und per HPLC (Säule 4, Programm D) analysiert.

\subsection{Durchführung der Fütterungsexperimente}

Für die Fütterung von $\left[1-{ }^{13} \mathrm{C}\right]$ Acetat $(1.0 \mathrm{~g} / \mathrm{L}, 12.2 \mathrm{mmol})$ wurde der Stamm wie unter B.II.4.3. beschrieben kultiviert. Die markierten Vorläufer wurden in sterilem Wasser gelöst und die Lösung auf einen pH-Wert von 6.0 eingestellt. Vom 12. bis zum 14. Tag wurden die markierten Substanzen jeweils im Abstand von 12 Stunden im Pulse-Feeding-Verfahren zu den Kulturen des Stammes gegeben. Die Kulturen wurden nach weiteren zwölf Stunden geerntet und wie unter B.II.4.2. beschrieben aufgearbeitet. Die Isolierung von Dehydrotaiwapyron (23) erfolgte wie unter B.II.4.5. beschrieben. 


\subsection{Isolierung und Charakterisierung der Metabolite}

Spiciferon $A^{56,57}(17)$

17 konnte in einer Ausbeute von $2.0 \mathrm{mg} / \mathrm{L}$ aus dem Kulturfiltrat einer Fermentation in Schüttelkolben (Medium: 1549) erhalten werden. Die Aufreinigung erfolgte durch Chromatographie an Kieselgel $\left(\mathrm{CH}_{2} \mathrm{Cl}_{2} / \mathrm{MeOH} 19: 1 \rightarrow 9: 1 \rightarrow 3: 1\right)$, Sephadex ${ }^{\circledR}$ LH-20 (Aceton) und erneut an Kieselgel $\left(\mathrm{CH}_{2} \mathrm{Cl}_{2} / \mathrm{MeOH} 9: 1\right)$.

$\mathrm{C}_{14} \mathrm{H}_{16} \mathrm{O}_{3}(232.28)$

farbloser Feststoff

UV-löschend (254 nm)

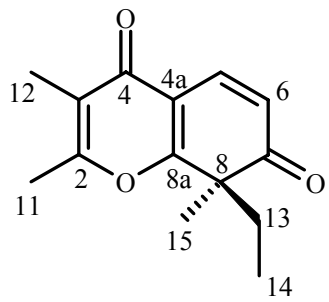

$\mathbf{R}_{\mathbf{f}}$-Wert: 0.76 (Chloroform/Methanol 9:1)

Smp.: $70^{\circ} \mathrm{C}$

EI-MS: m/z (\%) = $232(30)[\mathrm{M}]^{+}, 217$ (100), 204 (15), 189 (16), 175 (10).

HREI-MS: 232.1099 gefunden wie berechnet für $\mathrm{C}_{14} \mathrm{H}_{16} \mathrm{O}_{3}$.

IR(KBr): $\widetilde{v}=3426,2974,2920,2867,1670,1652,1630,1617,1458,1436,1382 \mathrm{~cm}^{-1}$.

UV: $(\mathrm{MeOH}): \quad\left[\lambda_{\max }(\lg \varepsilon)\right]=255(3.67), 315(3.16) \mathrm{nm}$;

$(\mathrm{MeOH} / \mathrm{HCl}): \quad\left[\lambda_{\max }(\lg \varepsilon)\right]=258(3.66), 313(3,13) \mathrm{nm} ;$

$(\mathrm{MeOH} / \mathrm{NaOH}):\left[\lambda_{\max }(\lg \varepsilon)\right]=245(3,70), 254(3,70), 307(3,28) \mathrm{nm}$.

CD (MeOH): $\lambda_{\max }([\Theta])=212$ (-4611), 233 (2939), 264 (7752), 296 (-5687), 333 (-1617).

${ }^{1}$ H-NMR (500 MHz, $\left.\mathbf{C D C l}_{3}\right): \delta=0.68\left(\mathrm{t}, J=7.5 \mathrm{~Hz}, 3 \mathrm{H}, 14-\mathrm{H}_{3}\right), 1.44$ (s, 3H, 15- $\left.\mathrm{H}_{3}\right), 1.97$ (dq, $\left.J=7.5,14.0 \mathrm{~Hz}, 1 \mathrm{H}, 13-\mathrm{H}_{\mathrm{a}}\right), 2.02\left(\mathrm{~s}, 3 \mathrm{H}, 12-\mathrm{H}_{3}\right), 2.13\left(\mathrm{dq}, J=7.5,14.0 \mathrm{~Hz}, 1 \mathrm{H}, 13-\mathrm{H}_{\mathrm{b}}\right)$, 2.38 (s, 3H, 11- $\left.\mathrm{H}_{3}\right), 6.17$ (d, $\left.J=10.0 \mathrm{~Hz}, 1 \mathrm{H}, 6-\mathrm{H}\right), 7.90$ (d, J=10.0 Hz, 1H, 5-H).

${ }^{13}$ C-NMR (125.7 MHz, $\left.\mathbf{C D C l}_{3}\right): \delta=9.3$ (q, C-14), 9.9 (q, C-12), 17.8 (q, C-11), 24.0 (q, C-15), 33.0 (t, C-13), 53.4 (s, C-8), 115.5 (s, C-4a), 120.9 (s, C-3), 123.7 (d, C-6), 137.8 (d, C-5), 161.1 (s, C-2), 170.7 (s, C-8a), 175.4 (s, C-4), 200.9 (s, C-7). 


\section{Prospiciferon A (18)}

18 konnte in einer Ausbeute von $27.0 \mathrm{mg} / \mathrm{L}$ aus dem Kulturfiltrat einer Fermentation in Schüttelkolben (Medium: 1549) erhalten werden. Die Aufreinigung erfolgte durch Chromatographie an Kieselgel $\left(\mathrm{CH}_{2} \mathrm{Cl}_{2} / \mathrm{MeOH} 19: 1 \rightarrow 9: 1 \rightarrow 3: 1\right)$, Sephadex ${ }^{\circledR}$ LH-20 $(\mathrm{MeOH})$ und erneut an Kieselgel $\left(\mathrm{CH}_{2} \mathrm{Cl}_{2} / \mathrm{MeOH} 9: 1\right)$.

$\mathrm{C}_{14} \mathrm{H}_{18} \mathrm{O}_{4}(250.29)$

gelber Feststoff

gelb fluoreszierend (366 nm)

$\mathbf{R}_{\mathbf{f}}$-Wert: 0.60 (Chloroform/Methanol 9:1)

Smp.: $85^{\circ} \mathrm{C}$

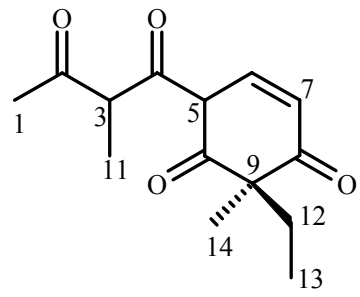

ESI-MS (positive Ionen): $\mathrm{m} / \mathrm{z}(\%)=273(100)[\mathrm{M}+\mathrm{Na}]^{+}$;

(negative Ionen): $\mathrm{m} / \mathrm{z}(\%)=249(100)[\mathrm{M}-\mathrm{H}]^{-}, 772(100)[3 \mathrm{M}+\mathrm{Na}-\mathrm{H}]^{-}$.

HRESI-MS: $251.1278[\mathrm{M}+\mathrm{H}]^{+}$.

IR(KBr): $\widetilde{v}=3427,2930,2547,1924,1651,1454,1351,997,834,701 \mathrm{~cm}^{-1}$.

UV: $(\mathrm{MeOH}): \quad\left[\lambda_{\max }(\lg \varepsilon)\right]=318(2.71), 401(2.70) \mathrm{nm}$;

$(\mathrm{MeOH} / \mathrm{HCl}): \quad\left[\lambda_{\max }(\lg \varepsilon)\right]=285(2.03) \mathrm{nm} ;$

$(\mathrm{MeOH} / \mathrm{NaOH}):\left[\lambda_{\max }(\lg \varepsilon)\right]=318(2.83), 400(2.81) \mathrm{nm}$.

CD (MeOH): $\lambda_{\max }([\Theta])=228$ (-433), 287 (1906), 332 (-1469).

${ }^{1}$ H-NMR (500 MHz, Aceton-d $\left.)_{6}\right): \delta=0.61\left(\mathrm{~m}, 3 \mathrm{H}, 13-\mathrm{H}_{3}\right), 1.18\left(\mathrm{~s}, 3 \mathrm{H}, 14-\mathrm{H}_{3}\right), 1.18(\mathrm{~d}$, $\left.J=7.0 \mathrm{~Hz}, 3 \mathrm{H}, 11-\mathrm{H}_{3}\right), 1.73\left(\mathrm{~m}, 1 \mathrm{H}, 12-\mathrm{H}_{\mathrm{a}}\right), 1.81\left(\mathrm{~m}, 1 \mathrm{H}, 12-\mathrm{H}_{\mathrm{b}}\right), 2.06\left(\mathrm{~s}, 3 \mathrm{H}, 1-\mathrm{H}_{3}\right), 4.18(\mathrm{q}$, $J=7.0 \mathrm{~Hz}, 1 \mathrm{H}, 3-\mathrm{H}), 5.40$ (d, $J=10.0 \mathrm{~Hz}, 1 \mathrm{H}, 7-\mathrm{H}), 7.82(\mathrm{~d}, J=10.0 \mathrm{~Hz}, 1 \mathrm{H}, 6-\mathrm{H})$.

${ }^{1}$ H-NMR (500 MHz, CD 3 OD): $\delta=0.66\left(\mathrm{~m}, 3 \mathrm{H}, 13-\mathrm{H}_{3}\right), 1.18\left(\mathrm{~m}, 3 \mathrm{H}, 14-\mathrm{H}_{3}\right), 1.22(\mathrm{~d}, J=7.0$ $\left.\mathrm{Hz}, 3 \mathrm{H}, 11-\mathrm{H}_{3}\right), 1.81\left(\mathrm{~m}, 1 \mathrm{H}, 12-\mathrm{H}_{\mathrm{a}}\right), 1.90\left(\mathrm{~m}, 1 \mathrm{H}, 12-\mathrm{H}_{\mathrm{b}}\right), 2.10\left(\mathrm{~s}, 3 \mathrm{H}, 1-\mathrm{H}_{3}\right), 4.25(\mathrm{q}, J=7.0$ $\mathrm{Hz}, 1 \mathrm{H}, 3-\mathrm{H}), 5.53$ (d, $J=10.0 \mathrm{~Hz}, 1 \mathrm{H}, 7-\mathrm{H}), 7.96$ (d, $J=10.0 \mathrm{~Hz}, 1 \mathrm{H}, 6-\mathrm{H})$.

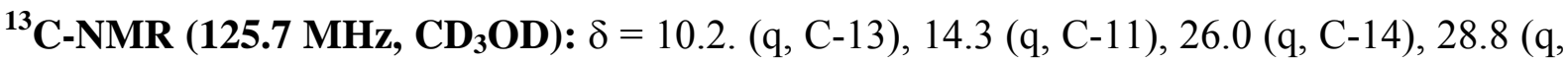
C-1), 33.3 (t, C-12), 58.0 (d, C-3), 61.9 (s, C-9), 109.3 (d, C-5), 111.5 (d, C-7), 150.6 (d, C-6), 194.7 (s, C-4), 202.4 (s, C-8), 205.4 (s, C-10), 208.0 (s, C-2). 


\section{Taiwapyron $^{61}(22)$}

22 konnte sowohl aus den Mycel- als auch aus den Kulturfiltratextrakten von Ruhefermentationen mit G20, G20 + NaCl, E2 und SGG als Nährmedien isoliert werden. Die Aufreinigung erfolgte durch Chromatographie an Kieselgel $\left(\mathrm{CH}_{2} \mathrm{Cl}_{2} / \mathrm{MeOH} 19: 1 \rightarrow 9: 1 \rightarrow 3: 1+\right.$ $0.1 \% \mathrm{AcOH})$, Sephadex ${ }^{\circledR} \mathrm{LH}-20(\mathrm{MeOH})$ und erneut an Kieselgel $\left(\mathrm{CH}_{2} \mathrm{Cl}_{2} / \mathrm{MeOH} 9: 1+0.1 \%\right.$ AcOH). Die Ausbeuten betrugen $20.0 \mathrm{mg} / \mathrm{L}$ (G20), $25.0 \mathrm{mg} / \mathrm{L}(\mathrm{G} 20+\mathrm{NaCl}), 5.0 \mathrm{mg} / \mathrm{L}$ (E2) und $10.0 \mathrm{mg} / \mathrm{L}$ (SGG).

$\mathrm{C}_{10} \mathrm{H}_{14} \mathrm{O}_{4}(198.22)$

farbloses Öl

UV-löschend (254 nm)

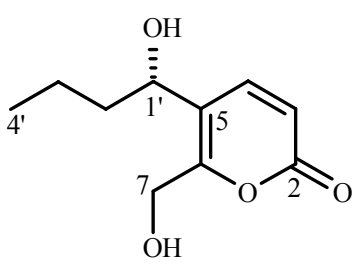

$\mathbf{R}_{\mathbf{f}}$-Wert: 0.36 (Chloroform/Methanol 9:1)

Anfärbeverhalten: braun (Anisaldehyd)

EI-MS: $\mathrm{m} / \mathrm{z}(\%)=198(8)[\mathrm{M}]^{+}, 155(55)\left[\mathrm{C}_{8} \mathrm{H}_{11} \mathrm{O}_{3}\right]^{+}, 137(100)\left[\mathrm{C}_{8} \mathrm{H}_{9} \mathrm{O}_{2}\right]^{+}, 109$ (12).

HREI-MS: 198.0892 gefunden wie berechnet für $\mathrm{C}_{10} \mathrm{H}_{14} \mathrm{O}_{4}$.

IR(KBr): $\widetilde{v}=3396,2960,2934,2872,2363,2342,1718,1638,1559,1459,1418,1381$, $1303,1227,1211,1181,1102,1067,1026,887,833 \mathrm{~cm}^{-1}$.

UV: $(\mathrm{MeOH}): \quad\left[\lambda_{\max }(\lg \varepsilon)\right]=211(\mathrm{sh}, 3.86), 296(3.80) \mathrm{nm}$;

$(\mathrm{MeOH} / \mathrm{HCl}): \quad\left[\lambda_{\max }(\lg \varepsilon)\right]=212(\mathrm{sh}, 3.74), 298(3.71) \mathrm{nm} ;$

$(\mathrm{MeOH} / \mathrm{NaOH}):\left[\lambda_{\max }(\lg \varepsilon)\right]=224(\mathrm{sh}, 3.43), 364(4.18) \mathrm{nm}$.

$[\alpha]_{D}^{20}=-13^{\circ}(\mathrm{c}=0.1$, Methanol).

CD (MeOH): $\lambda_{\max }([\Theta])=209$ (-13697), 241 (1782), 305 (-1410).

${ }^{1}$ H-NMR (500 MHz, $\left.\mathbf{C D C l}_{3}\right): \delta=0.89$ (t, $\left.J=7.0 \mathrm{~Hz}, 3 \mathrm{H}, 4{ }^{\prime}-\mathrm{H}_{3}\right), 1.19-1.26\left(\mathrm{~m}, 1 \mathrm{H}, 3\right.$ '- $\mathrm{H}_{\mathrm{a}}$ ), 1.32-1.40 (m, 1H, 3'- $\left.\mathrm{H}_{\mathrm{b}}\right), 1.46-1.52\left(\mathrm{~m}, 1 \mathrm{H}, 2^{\prime}-\mathrm{H}_{\mathrm{a}}\right), 1.69-1.76\left(\mathrm{~m}, 1 \mathrm{H}, 2^{\prime}-\mathrm{H}_{\mathrm{b}}\right), 3.3-4.1$ (s, br, 2H, 7-OH und 1'-OH), 4.34 (d, $\left.J=14.0 \mathrm{~Hz}, 1 \mathrm{H}, 7-\mathrm{H}_{\mathrm{a}}\right), 4.50$ (d, $\left.J=14.0 \mathrm{~Hz}, 1 \mathrm{H}, 7-\mathrm{H}_{\mathrm{b}}\right), 4.69$ (dd, $\left.J=7.0 \mathrm{~Hz}, 1 \mathrm{H}, 1^{\prime}-\mathrm{H}\right), 6.24$ (d, $\left.J=10.0 \mathrm{~Hz}, 1 \mathrm{H}, 3-\mathrm{H}\right), 7.52$ (d, $\left.J=10.0 \mathrm{~Hz}, 1 \mathrm{H}, 4-\mathrm{H}\right)$.

${ }^{13}$ C-NMR (125.7 MHz, $\left.\mathbf{C D C l}_{3}\right): \delta=13.7$ (q, C-4'), 18.7 (t, C-3'), 38.7 (t, C-2'), 58.2 (t, C-7), 66.9 (d, C-1'), 115.5 (d, C-3), 120.1 (s, C-5), 144.1 (d, C-4), 158.3 (s, C-6), 162.3 (s, C-2).

${ }^{13}$ C-NMR (150.8 MHz, CD 3 OD): $\delta=14.2$ (q, C-4'), 19.9 (t, C-3'), 40.5 (t, C-2'), 58.9 (t, C-7), 67.8 (d, C-1'), 116.3 (d, C-3), 121.8 (s, C-5), 145.8 (d, C-4), 159.8 (s, C-6), 164.2 (s, C-2). 


\section{Dehydrotaiwapyron (23) ${ }^{*}$}

23 konnte in einer Ausbeute von $25.0 \mathrm{mg} / \mathrm{L}$ aus einer Ruhefermentation mit G20 als Nährmedium isoliert werden. Die Aufreinigung erfolgte durch Chromatographie an Kieselgel $\left(\mathrm{CH}_{2} \mathrm{Cl}_{2} / \mathrm{MeOH} 19: 1 \rightarrow 9: 1 \rightarrow 3: 1+0.1 \% \mathrm{AcOH}\right)$, Sephadex ${ }^{\circledR} \mathrm{LH}-20(\mathrm{MeOH})$ und erneut an Kieselgel $\left(\mathrm{CH}_{2} \mathrm{Cl}_{2} / \mathrm{MeOH} 9: 1+0.1 \% \mathrm{AcOH}\right)$.

$\mathrm{C}_{10} \mathrm{H}_{12} \mathrm{O}_{4}(196.20)$

farbloser Feststoff

UV-löschend (254 nm)

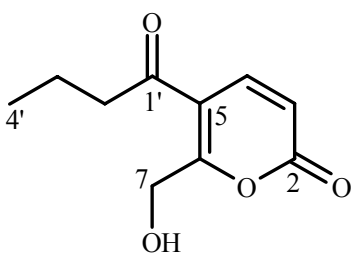

$\mathbf{R}_{\mathbf{f}}$-Wert: 0.33 (Chloroform/Methanol 9:1)

Anfärbeverhalten: grün-blau (Anisaldehyd)

Smp.: $134^{\circ} \mathrm{C}$

EI-MS: $\mathrm{m} / \mathrm{z}(\%)=196(40)[\mathrm{M}]^{+}, 178(24)\left[\mathrm{C}_{10} \mathrm{H}_{10} \mathrm{O}_{3}\right]^{+}, 163$ (100), 151 (12), 137 (14), 109 (24), 43 (20).

HREI-MS: 196.0736 gefunden wie berechnet für $\mathrm{C}_{10} \mathrm{H}_{12} \mathrm{O}_{4}$.

IR(KBr): $\widetilde{v}=3440,2966,2923,2868,2362,2327,1699,1634,1574,1417,1405,1384$, $1284,1190,1094,1037,1005,983,565,474 \mathrm{~cm}^{-1}$.

UV: $(\mathrm{MeOH}): \quad\left[\lambda_{\max }(\lg \varepsilon)\right]=268(3.83) \mathrm{nm}$;

$(\mathrm{MeOH} / \mathrm{HCl}): \quad\left[\lambda_{\max }(\lg \varepsilon)\right]=277(3.86) \mathrm{nm}$;

$(\mathrm{MeOH} / \mathrm{NaOH}):\left[\lambda_{\max }(\lg \varepsilon)\right]=264(3.80) \mathrm{nm}$.

${ }^{1}$ H-NMR (500 MHz, CD $\mathbf{C D}_{3}$ OD): $\delta=1.02$ (t, $J=7.5 \mathrm{~Hz}, 3 \mathrm{H}, 4^{\prime}-\mathrm{H}_{3}$ ), 1.76 (sextet, $J=7.5 \mathrm{~Hz}$, 2H, 3'- $\left.\mathrm{H}_{2}\right), 2.75$ (t, J=7.5 Hz, 2H, 2'- $\left.\mathrm{H}_{2}\right), 4.65$ (s, 2H, 7-H $)_{2}, 6.84$ (d, J=15.5 Hz, $\left.1 \mathrm{H}, 3-\mathrm{H}\right)$, $7.28(\mathrm{~d}, J=15.5 \mathrm{~Hz}, 1 \mathrm{H}, 4-\mathrm{H})$.

${ }^{13}$ C-NMR (125.7 MHz, CD 3 OD): $\delta=14.0$ (q, C-4'), 21.0 (t, C-3'), 31.6 (t, C-2'), 76.3 (t, C-7), 112.8 (s, C-5), 119.9 (d, C-3), 132.2 (d, C-4), 171.6 (s, C-2), 196.5 (s, C-1'), 202.0 (s, C-6).

${ }^{1}$ H-NMR (500 MHz, $\mathbf{C D C l}_{3}$ ): $\delta=1.04\left(\mathrm{t}, J=7.5 \mathrm{~Hz}, 3 \mathrm{H}, 4^{\prime}-\mathrm{H}_{3}\right.$ ), 1.77 (sextet, $J=7.5 \mathrm{~Hz}$, $2 \mathrm{H}, 3$ '- $\left.\mathrm{H}_{2}\right), 2.71$ (t, $\left.J=7.5 \mathrm{~Hz}, 2 \mathrm{H}, 2^{\prime}-\mathrm{H}_{2}\right), 4.60$ (s, 2H, 7-H $), 6.98$ (d, J=15.5 Hz, $\left.1 \mathrm{H}, 3-\mathrm{H}\right)$, $7.36(\mathrm{~d}, J=15.5 \mathrm{~Hz}, 1 \mathrm{H}, 4-\mathrm{H})$.

${ }^{13}$ C-NMR (150.8 MHz, $\mathbf{C D C l}_{3}$ ): $\delta=13.7$ (q, C-4'), 19.9 (t, C-3'), 30.8 (t, C-2'), 74.8 (t, C-7), 111.7 (s, C-5), 117.4 (d, C-3), 132.7 (d, C-4), 172.6 (s, C-2), 195.0 (s, C-1'), 199.3 (s, C-6).

\footnotetext{
${ }^{*}$ fehlerhafte Struktur, Korrektur siehe Seite 30.
} 


\section{5,6-Dihydrotaiwapyron (24)}

24 konnte in einer Ausbeute von $3.5 \mathrm{mg} / \mathrm{L}$ aus einer Fermentation in P-Kolben mit G20 + $\mathrm{NaCl}$ als Nährmedium isoliert werden. Die Aufreinigung erfolgte durch Chromatographie an Kieselgel $\left(\mathrm{CH}_{2} \mathrm{Cl}_{2} / \mathrm{MeOH}\right.$ 19:1 $\left.\rightarrow 9: 1 \rightarrow 3: 1+0.1 \% \mathrm{AcOH}\right)$, Sephadex ${ }^{\circledR} \mathrm{LH}-20(\mathrm{MeOH})$ und erneut an Kieselgel $\left(\mathrm{CH}_{2} \mathrm{Cl}_{2} / \mathrm{MeOH} 9: 1+0.1 \% \mathrm{AcOH}\right)$.

$\mathrm{C}_{10} \mathrm{H}_{16} \mathrm{O}_{4}(200.23)$

farbloses Ö1

UV-löschend (254 nm)

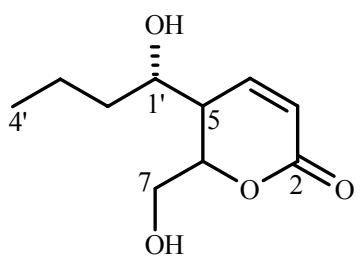

$\mathbf{R}_{\mathbf{f}}$-Wert: 0.21 (Chloroform/Methanol 9:1)

Anfärbeverhalten: braun (Anisaldehyd)

ESI-MS (positive Ionen): $\mathrm{m} / \mathrm{z}(\%)=223(50)[\mathrm{M}+\mathrm{Na}]^{+}, 423(80)[2 \mathrm{M}+\mathrm{Na}]^{+}, 623(100)$ $[3 \mathrm{M}+\mathrm{Na}]^{+}$

(negative Ionen): $\mathrm{m} / \mathrm{z}(\%)=199(20)[\mathrm{M}-\mathrm{H}]^{-}, 421(100)[2 \mathrm{M}+\mathrm{Na}-2 \mathrm{H}]^{-}, 643(80)$

$[3 \mathrm{M}+2 \mathrm{Na}-3 \mathrm{H}]^{-}, \quad 865 \quad(60) \quad[4 \mathrm{M}+3 \mathrm{Na}-4 \mathrm{H}]^{-}, \quad 1087$

$[5 \mathrm{M}+4 \mathrm{Na}-5 \mathrm{H}]^{-}$.

HRESI-MS: 223.09408 gefunden wie berechnet für $\left[\mathrm{C}_{10} \mathrm{H}_{16} \mathrm{O}_{4} \mathrm{Na}\right]^{+}$.

IR(KBr): $\widetilde{v}=3418,2960,2930,2870,1693,1641,1561,1436,1384,1235,1030 \mathrm{~cm}^{-1}$.

UV: $(\mathrm{MeOH}): \quad\left[\lambda_{\max }(\lg \varepsilon)\right]=291(3.29) \mathrm{nm}$;

$(\mathrm{MeOH} / \mathrm{HCl}): \quad\left[\lambda_{\max }(\lg \varepsilon)\right]=315(3.39) \mathrm{nm}$;

$(\mathrm{MeOH} / \mathrm{NaOH}):\left[\lambda_{\max }(\lg \varepsilon)\right]=291(3.67) \mathrm{nm}$.

CD (MeOH): $\lambda_{\max }([\Theta])=205$ (-11022), 239 (8015).

${ }^{1}$ H-NMR (600 MHz, CD CD) $_{3} \delta=0.90$ (t, $\left.J=7.0 \mathrm{~Hz}, 3 \mathrm{H}, 4^{\prime}-\mathrm{H}_{3}\right), 1.32-1.38$ (m, 1H, 3'- $\mathrm{H}_{\mathrm{a}}$ ), 1.43-1.50 (m, 1H, 3'- $\left.\mathrm{H}_{\mathrm{b}}\right), 1.55-1.62\left(\mathrm{~m}, 2 \mathrm{H}, 2^{\prime}-\mathrm{H}_{2}\right), 3.59$ (ddd, $J=5.0,7.5,8.5 \mathrm{~Hz}, 1 \mathrm{H}, 1$ '-H), $3.66(\mathrm{ddd}, J=5.0,6.0,9.5 \mathrm{~Hz}, 1 \mathrm{H}, 5-\mathrm{H}), 3.70\left(\mathrm{dd}, J=4.5,9.5 \mathrm{~Hz}, 1 \mathrm{H}, 7-\mathrm{H}_{\mathrm{a}}\right), 3.89$ (dd, $\left.J=6.5,9.5 \mathrm{~Hz}, 1 \mathrm{H}, 7-\mathrm{H}_{\mathrm{b}}\right), 4.13$ (ddd, $\left.J=4.5,6.0,6.5 \mathrm{~Hz}, 1 \mathrm{H}, 6-\mathrm{H}\right), 5.92(\mathrm{~d}, J=11.5 \mathrm{~Hz}, 1 \mathrm{H}$, 3-H), 5.99 (dd, $J=9.5,11.5 \mathrm{~Hz}, 1 \mathrm{H}, 4-\mathrm{H})$.

${ }^{13}$ C-NMR (125.7 MHz, CD 3 OD): $\delta=14.1$ (q, C-4'), 19.3 (t, C-3'), 36.5 (t, C-2'), 54.0 (d, C-5), 73.9 (t, C-7), 78.9 (d, C-6), 85.0 (d, C-1'), 123.0 (d, C-3), 147.7 (d, C-4), 170.9 (s, C-2). 


\section{7-(1-Methyl-ethyliden)-taiwapyron (25)}

25 konnte in einer Ausbeute von $1.0 \mathrm{mg} / \mathrm{L}$ aus dem Mycelextrakt einer Fermentation in P-Kolben mit E2 als Nährmedium isoliert werden. Die Aufreinigung erfolgte durch Chromatographie an Kieselgel $\left(\mathrm{CH}_{2} \mathrm{Cl}_{2} / \mathrm{MeOH} 19: 1 \rightarrow 9: 1 \rightarrow 3: 1+0.1 \%\right.$ AcOH$)$, Sephadex ${ }^{\circledR}$ LH-20 (MeOH) und erneut an Kieselgel $\left(\mathrm{CH}_{2} \mathrm{Cl}_{2} / \mathrm{MeOH} 9: 1+0.1 \% \mathrm{AcOH}\right)$.

$\mathrm{C}_{13} \mathrm{H}_{16} \mathrm{O}_{4}(236.27)$

farbloses Ö1

UV-löschend (254 nm)

$\mathbf{R}_{\mathbf{f}}$-Wert: 0.25 (Chloroform/Methanol 9:1)

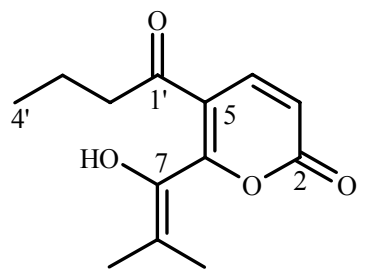

Anfärbeverhalten: orange (Anisaldehyd)

EI-MS: $\mathrm{m} / \mathrm{z}(\%)=236(48)[\mathrm{M}]^{+}, 218(40)\left[\mathrm{C}_{13} \mathrm{H}_{14} \mathrm{O}_{3}\right]^{+}, 189$ (100), 163 (16), 91 (10), 71 (34), $44(82)$.

HREI-MS: 236.1049 gefunden wie berechnet für $\mathrm{C}_{13} \mathrm{H}_{16} \mathrm{O}_{4}$.

IR(KBr): $\widetilde{v}=3405,2940,2834,1700,1637,1405,1384,1029 \mathrm{~cm}^{-1}$.

UV: $(\mathrm{MeOH}): \quad\left[\lambda_{\max }(\lg \varepsilon)\right]=267(3.87) \mathrm{nm}$;

$(\mathrm{MeOH} / \mathrm{HCl}): \quad\left[\lambda_{\max }(\lg \varepsilon)\right]=272(3.94) \mathrm{nm} ;$

$(\mathrm{MeOH} / \mathrm{NaOH}):\left[\lambda_{\max }(\lg \varepsilon)\right]=268(3.95), 304(\mathrm{sh}, 3.55) \mathrm{nm}$.

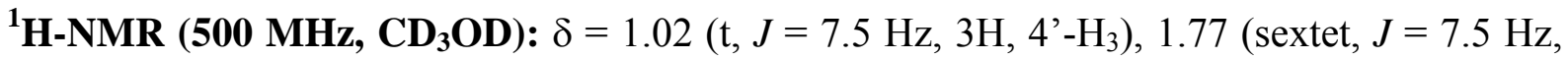
$2 \mathrm{H}, 3$ ' $\left.-\mathrm{H}_{2}\right), 2.06\left(\mathrm{~s}, 3 \mathrm{H}, 9-\mathrm{CH}_{3}\right.$ oder 10- $\left.\mathrm{CH}_{3}\right), 2.30$ (s, 3H, 9- $\mathrm{CH}_{3}$ oder 10- $\left.\mathrm{CH}_{3}\right), 2.77$ (t, $\left.J=7.5 \mathrm{~Hz}, 2 \mathrm{H}, 2^{\prime}-\mathrm{H}_{2}\right), 6.83$ (d, $\left.J=15.5 \mathrm{~Hz}, 1 \mathrm{H}, 3-\mathrm{H}\right), 7.30$ (d, $\left.J=15.5 \mathrm{~Hz}, 1 \mathrm{H}, 4-\mathrm{H}\right)$.

${ }^{13}$ C-NMR (125.7 MHz, CD ${ }_{3}$ OD): $\delta=14.0$ (q, C-4'), 17.2 (q, C-9 oder C-10), 19.9 (q, C-9 oder C-10), 21.3 (t, C-3'), 30.5 (t, C-2'), 115.2 (s, C-5), 120.2 (d, C-3), 132.5 (d, C-4), 135.0 (s, C-8), 145.3 (s, C-7), 171.6 (s, C-2), 183.1 (s, C-1'), 185.5(s, C-6).

\footnotetext{
* fehlerhafte Struktur, Korrektur siehe Seite 30.
} 


\section{Betaenon G (39)}

39 konnte aus den Kulturfiltraten von Ruhefermentation in P-Kolben in Ausbeute von $5.0 \mathrm{mg} / \mathrm{L}$ (Medium: $\mathrm{SGG}+\mathrm{NaCl}$ ) bzw. $4.0 \mathrm{mg} / \mathrm{L}$ (Medium: $\mathrm{G} 20+\mathrm{NaCl}$ ) erhalten werden. Die Aufreinigung erfolgte durch Chromatographie an Kieselgel $\left(\mathrm{CH}_{2} \mathrm{Cl}_{2} / \mathrm{MeOH}\right.$ $19: 1 \rightarrow 9: 1 \rightarrow 3: 1)$, Sephadex ${ }^{\circledR}$ LH-20 $(\mathrm{MeOH})$ und mit der MPLC an RP-Kieselgel $\left(\mathrm{MeOH} / \mathrm{H}_{2} \mathrm{O} 4: 1\right)$.

$\mathrm{C}_{21} \mathrm{H}_{36} \mathrm{O}_{4}(352.51)$

farbloser Feststoff

$\mathbf{R}_{\mathbf{f}}$-Wert: 0.30 (Chloroform/Methanol 9:1)

Anfärbeverhalten: blau (Anisaldehyd)

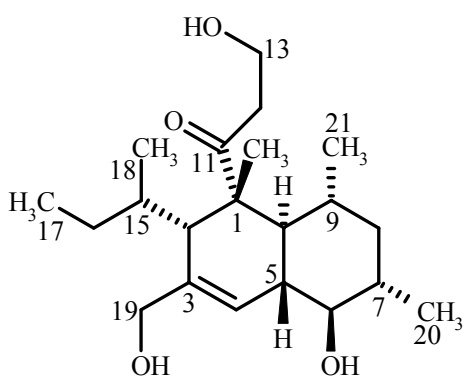

ESI-MS (positive Ionen): $\mathrm{m} / \mathrm{z}(\%)=375(10)[\mathrm{M}+\mathrm{Na}]^{+}, 727(100)[2 \mathrm{M}+\mathrm{Na}]^{+}$.

HRESI-MS: $353.26864[\mathrm{M}+\mathrm{H}]^{+}, 375.25058[\mathrm{M}+\mathrm{Na}]^{+}$.

IR(KBr): $\widetilde{v}=3424,2957,2926,2873,1696,1634,1458,1404,1383,1352,1119 \mathrm{~cm}^{-1}$.

UV: $(\mathrm{MeOH}): \quad\left[\lambda_{\max }(\lg \varepsilon)\right]=226(\mathrm{sh}, 3.46), 273(2.51) \mathrm{nm}$.

$[\alpha]_{D}^{20}=-18^{\circ}(\mathrm{c}=0.1$, Methanol).

CD (MeOH): $\lambda_{\max }([\Theta])=292(-1928)$.

${ }^{1}$ H-NMR (500 MHz, $\left.\mathbf{C D C l}_{3}\right): \delta=0.62\left(\mathrm{~d}, J=7.0 \mathrm{~Hz}, 3 \mathrm{H}, 21-\mathrm{H}_{3}\right), 0.80\left(\mathrm{~m}, 1 \mathrm{H}, 16-\mathrm{H}_{\mathrm{a}}\right), 0.84$ (dd, $\left.J=7.0 \mathrm{~Hz}, 3 \mathrm{H}, 17-\mathrm{H}_{3}\right), 0.89$ (d, $\left.J=7.0 \mathrm{~Hz}, 3 \mathrm{H}, 18-\mathrm{H}_{3}\right), 1.06$ (d, $\left.J=6.5 \mathrm{~Hz}, 3 \mathrm{H}, 20-\mathrm{H}_{3}\right)$, $1.13\left(\mathrm{~m}, 1 \mathrm{H}, 8-\mathrm{H}_{\mathrm{a}}\right), 1.19\left(\mathrm{~s}, 3 \mathrm{H}, 14-\mathrm{H}_{3}\right), 1,30\left(\mathrm{~m}, 1 \mathrm{H}, 16-\mathrm{H}_{\mathrm{b}}\right), 1.40(\mathrm{~m}, 2 \mathrm{H}, 9-\mathrm{H}$ und 15-H), 1.50 (m, $1 \mathrm{H}, 7-\mathrm{H}), 1.70$ (ddd, $\left.J=3.0,5.0,13.5 \mathrm{~Hz}, 1 \mathrm{H}, 8-\mathrm{H}_{\mathrm{b}}\right), 1.77$ (dd, $\left.J=9.5 \mathrm{~Hz}, 1 \mathrm{H}, 5-\mathrm{H}\right)$, 1.85 (s, 1H, 2-H), $1.86(\mathrm{dd}, J=9.5,10.0 \mathrm{~Hz}, 1 \mathrm{H}, 10-\mathrm{H}), 2.74(\mathrm{ddd}, J=3.5,6.0,18.5 \mathrm{~Hz}, 1 \mathrm{H}$, $\left.12-\mathrm{H}_{\mathrm{a}}\right), 2.87\left(\mathrm{ddd}, J=3.5,7.5,18.5 \mathrm{~Hz}, 1 \mathrm{H}, 12-\mathrm{H}_{\mathrm{b}}\right), 3.00(\mathrm{dd}, J=9.5 \mathrm{~Hz}, 1 \mathrm{H}, 6-\mathrm{H}), 3.84$ $\left(\mathrm{ddd}, J=3.5,6.0,11.5 \mathrm{~Hz}, 1 \mathrm{H}, 13-\mathrm{H}_{\mathrm{a}}\right), 3.90\left(\mathrm{ddd}, J=3.5,7.5,11.5 \mathrm{~Hz}, 1 \mathrm{H}, 13-\mathrm{H}_{\mathrm{b}}\right), 4.02(\mathrm{~d}$, $\left.J=13.5 \mathrm{~Hz}, 1 \mathrm{H}, 19-\mathrm{H}_{\mathrm{a}}\right), 4.12\left(\mathrm{~d}, J=13.5 \mathrm{~Hz}, 1 \mathrm{H}, 19-\mathrm{H}_{\mathrm{b}}\right), 6.05(\mathrm{~d}, J=3.0 \mathrm{~Hz}, 1 \mathrm{H}, 4-\mathrm{H})$.

${ }^{13}$ C-NMR (125.7 MHz, $\mathbf{C D C l}_{3}$ ): $\delta=13.0$ (q, C-17), 17.9 (q, C-14), 18.6 (q, C-20), 21.3 (q, C-18), 23.0 (q, C-21), 27.1 (t, C-16), 34.4 (d, C-15), 37.4 (d, C-9), 40.9 (d, C-7), 41.4 (d, C-10), 41.8 (t, C-12), 43.8 (t, C-8), 48.3 (d, C-5), 51.9 (d, C-2), 53.4 (s, C-1), 58.1 (t, C-13), 67.3 (t, C-19), 79.0 (d, C-6), 121.9 (d, C-4), 136.2 (s, C-3), 215.6 (s, C-11). 


\section{Betaenon H (40)}

40 konnte aus den Kulturfiltraten von Ruhefermentation in P-Kolben in Ausbeute von $2.0 \mathrm{mg} / \mathrm{L}$ (Medium: SGG $+\mathrm{NaCl}$ ) und $1.5 \mathrm{mg} / \mathrm{L}$ (Medium: $\mathrm{G} 20+\mathrm{NaCl}$ ) erhalten werden. Die Aufreinigung erfolgte durch Chromatographie an Kieselgel $\left(\mathrm{CH}_{2} \mathrm{Cl}_{2} / \mathrm{MeOH} 19: 1 \rightarrow 9: 1 \rightarrow 3: 1\right)$, Sephadex ${ }^{\circledR}$ LH-20 (MeOH) und mit der MPLC an RP-Kieselgel (MeOH/ $\left.\mathrm{H}_{2} \mathrm{O} 4: 1\right)$.

$\mathrm{C}_{21} \mathrm{H}_{36} \mathrm{O}_{3}(336.51)$

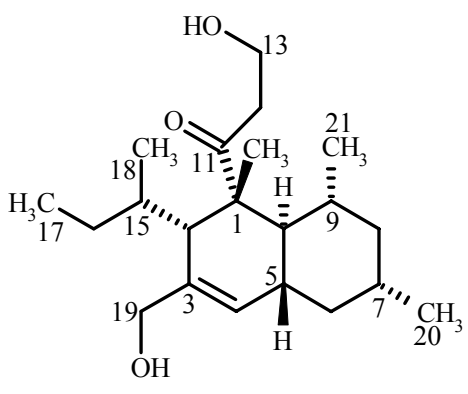

Anfärbeverhalten: blau (Anisaldehyd)

ESI-MS (positive Ionen): $\mathrm{m} / \mathrm{z}(\%)=359(30)[\mathrm{M}+\mathrm{Na}]^{+}, 695(100)[2 \mathrm{M}+\mathrm{Na}]^{+}$.

HRESI-MS: : 359.25624 gefunden wie berechnet für $\left[\mathrm{C}_{21} \mathrm{H}_{36} \mathrm{O}_{3} \mathrm{Na}\right]^{+}$.

IR(KBr): $\widetilde{v}=3441,2954,2926,2906,1699,1636,1458,1383,1061 \mathrm{~cm}^{-1}$.

UV: $(\mathrm{MeOH}): \quad\left[\lambda_{\max }(\lg \varepsilon)\right]=267(2.39) \mathrm{nm}$.

$[\alpha]_{D}^{20}=-18^{\circ}(\mathrm{c}=0.1$, Methanol).

CD (MeOH): $\lambda_{\max }([\Theta])=291(-6350)$.

${ }^{1}$ H-NMR (500 MHz, $\left.\mathbf{C D C l}_{3}\right): \delta=0.63\left(\mathrm{~d}, J=7.0 \mathrm{~Hz}, 3 \mathrm{H}, 21-\mathrm{H}_{3}\right), 0.78\left(\mathrm{~m}, 1 \mathrm{H}, 16-\mathrm{H}_{\mathrm{a}}\right), 0.86$ (dd, $\left.J=7.0 \mathrm{~Hz}, 3 \mathrm{H}, 17-\mathrm{H}_{3}\right), 0.90$ (d, $\left.J=7.0 \mathrm{~Hz}, 3 \mathrm{H}, 18-\mathrm{H}_{3}\right), 0.94$ (d, $J=7.0 \mathrm{~Hz}, 3 \mathrm{H}, 20-\mathrm{H}_{3}$ ), 0.95-1.00 (m, 1H, 6- $\left.\mathrm{H}_{\mathrm{a}}\right), 1.04\left(\mathrm{ddd}, J=12.0,13.0 \mathrm{~Hz}, 1 \mathrm{H}, 8-\mathrm{H}_{\mathrm{a}}\right), 1.20$ (s, 3H, 14- $\left.\mathrm{H}_{3}\right), 1,33(\mathrm{~m}$, 2H, 9-H und 16- $\left.\mathrm{H}_{\mathrm{b}}\right), 1.45(\mathrm{~m}, 2 \mathrm{H}, 15-\mathrm{H}), 1.65(\mathrm{~m}, 1 \mathrm{H}, 7-\mathrm{H}), 1.69$ (ddd, $J=2.0,4.5,13.0 \mathrm{~Hz}$, $\left.1 \mathrm{H}, 8-\mathrm{H}_{\mathrm{b}}\right), 1.80\left(\mathrm{~m}, 2 \mathrm{H}, 5-\mathrm{H}\right.$ und 10-H), $1.85\left(\mathrm{ddd}, J=2.0,4.0,12.0 \mathrm{~Hz}, 1 \mathrm{H}, 6-\mathrm{H}_{\mathrm{b}}\right), 1.94(\mathrm{~s}$, br, 1H, 2-H), 2.75 (ddd, $J=3.5,6.0,18.5 \mathrm{~Hz}, 1 \mathrm{H}, 12-\mathrm{H}_{\mathrm{a}}$ ), 2.88 (ddd, $J=3.5,7.5,18.5 \mathrm{~Hz}$, $\left.1 \mathrm{H}, 12-\mathrm{H}_{\mathrm{b}}\right), 3.84\left(\mathrm{ddd}, J=3.5,6.0,11.5 \mathrm{~Hz}, 1 \mathrm{H}, 13-\mathrm{H}_{\mathrm{a}}\right), 3.91$ (ddd, $J=3.5,7.5,11.5 \mathrm{~Hz}, 1 \mathrm{H}$, $\left.13-\mathrm{H}_{\mathrm{b}}\right), 4.00\left(\mathrm{~d}, J=13.0 \mathrm{~Hz}, 1 \mathrm{H}, 19-\mathrm{H}_{\mathrm{a}}\right), 4.06\left(\mathrm{~d}, J=13.0 \mathrm{~Hz}, 1 \mathrm{H}, 19-\mathrm{H}_{\mathrm{b}}\right), 5.54$ (d, $J=2.0$ $\mathrm{Hz}, 1 \mathrm{H}, 4-\mathrm{H})$.

${ }^{13}$ C-NMR (125.7 MHz, $\mathbf{C D C l}_{3}$ ): $\delta=13.0$ (q, C-17), 17.8 (q, C-14), 21.8 (q, C-18), 22.2 (q, C-20), 23.4 (q, C-21), 27.1 (t, C-16), 33.7 (d, C-7), 34.5 (d, C-15), 37.7 (d, C-9), 44.5 (d, C-10), 40.8 (t, C-12), 41.5 (t, C-6), 42.0 (d, C-5), 46.2 (t, C-8), 52.2 (d, C-2), 53.3 (s, C-1), 58.2 (t, C-13), 67.4 (t, C-19), 128.1 (d, C-4), 134.8 (s, C-3), 216.0 (s, C-11). 


\section{Betaenon I (41)}

41 konnte aus den Kulturfiltraten von Ruhefermentation in P-Kolben in Ausbeute von $1.5 \mathrm{mg} / \mathrm{L}$ (Medium: $\mathrm{SGG}+\mathrm{NaCl}$ ) bzw. $1.0 \mathrm{mg} / \mathrm{L}$ (Medium: $\mathrm{G} 20+\mathrm{NaCl}$ ) erhalten werden. Die Aufreinigung erfolgte durch Chromatographie an Kieselgel $\left(\mathrm{CH}_{2} \mathrm{Cl}_{2} / \mathrm{MeOH}\right.$ $19: 1 \rightarrow 9: 1 \rightarrow 3: 1)$, Sephadex ${ }^{\circledR}$ LH-20 $(\mathrm{MeOH})$ und mit der MPLC an RP-Kieselgel $\left(\mathrm{MeOH} / \mathrm{H}_{2} \mathrm{O} 4: 1\right)$.

$\mathrm{C}_{21} \mathrm{H}_{36} \mathrm{O}_{4}(352.51)$

farbloser Feststoff

$\mathbf{R}_{\mathbf{f}}$-Wert: 0.21 (Chloroform/Methanol 9:1)

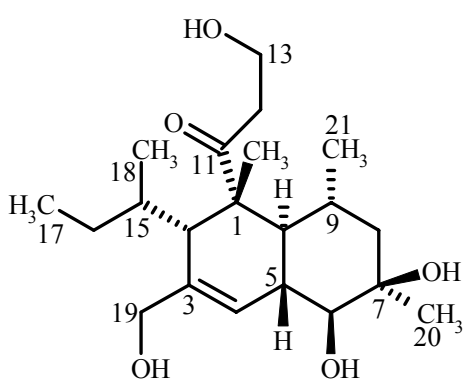

Anfärbeverhalten: blau (Anisaldehyd)

ESI-MS (positive Ionen): $\mathrm{m} / \mathrm{z}(\%)=375(30)[\mathrm{M}+\mathrm{Na}]^{+}, 727(100)[2 \mathrm{M}+\mathrm{Na}]^{+}$.

HRESI-MS: 375.25110 gefunden wie berechnet für $\left[\mathrm{C}_{21} \mathrm{H}_{36} \mathrm{O}_{4} \mathrm{Na}\right]^{+}$.

IR(KBr): $\widetilde{v}=3432,2960,2930,2873,1728,1630,1458,1384,1288,1125,1073 \mathrm{~cm}^{-1}$.

UV: $(\mathrm{MeOH}): \quad\left[\lambda_{\max }(\lg \varepsilon)\right]=217(\mathrm{sh}, 3.15) \mathrm{nm}$.

$[\alpha]_{D}^{20}=-3^{\circ}(\mathrm{c}=0.1$, Methanol).

CD (MeOH): $\lambda_{\max }([\Theta])=288(-1271)$.

${ }^{1}$ H-NMR (500 MHz, $\left.\mathbf{C D C l}_{3}\right): \delta=0.63\left(\mathrm{~d}, J=7.0 \mathrm{~Hz}, 3 \mathrm{H}, 21-\mathrm{H}_{3}\right), 0.78\left(\mathrm{~m}, 1 \mathrm{H}, 16-\mathrm{H}_{\mathrm{a}}\right), 0.86$ $\left(\mathrm{dd}, J=7.0 \mathrm{~Hz}, 3 \mathrm{H}, 17-\mathrm{H}_{3}\right), 0.91$ (d, $\left.J=7.0 \mathrm{~Hz}, 3 \mathrm{H}, 18-\mathrm{H}_{3}\right), 1.24\left(\mathrm{~s}, 3 \mathrm{H}, 14-\mathrm{H}_{3}\right), 1.29(\mathrm{~s}, 3 \mathrm{H}$, $\left.20-\mathrm{H}_{3}\right), 1,32\left(\mathrm{~m}, 1 \mathrm{H}, 16-\mathrm{H}_{\mathrm{b}}\right), 1.40\left(\mathrm{dd}, J=13.0 \mathrm{~Hz}, 1 \mathrm{H}, 6-\mathrm{H}_{\mathrm{a}}\right), 1.44(\mathrm{~m}, 1 \mathrm{H}, 15-\mathrm{H}), 1.47$ (dd, $\left.J=12.0,14.0 \mathrm{~Hz}, 1 \mathrm{H}, 8-\mathrm{H}_{\mathrm{a}}\right), 1.64\left(\mathrm{ddd}, J=2.5,14.0 \mathrm{~Hz}, 1 \mathrm{H}, 8-\mathrm{H}_{\mathrm{b}}\right), 1.69$ (m, 1H, 9-H), 1.79 (ddd, $\left.J=2.5,13.0 \mathrm{~Hz}, 1 \mathrm{H}, 6-\mathrm{H}_{\mathrm{b}}\right), 1.79$ (d, $\left.J=9.5 \mathrm{~Hz}, 1 \mathrm{H}, 10-\mathrm{H}\right), 1.98$ (s, br, 1H, 2-H), 2.29 (dddd, $J=2.5,3.0,9.5,13.0 \mathrm{~Hz}, 1 \mathrm{H}, 5-\mathrm{H}), 2.76\left(\mathrm{ddd}, J=3.5,6.0,18.5 \mathrm{~Hz}, 1 \mathrm{H}, 12-\mathrm{H}_{\mathrm{a}}\right.$ ), 2.89 $\left(\mathrm{ddd}, J=3.5,7.5,18.5 \mathrm{~Hz}, 1 \mathrm{H}, 12-\mathrm{H}_{\mathrm{b}}\right), 3.85\left(\mathrm{ddd}, J=3.5,6.0,11.5 \mathrm{~Hz}, 1 \mathrm{H}, 13-\mathrm{H}_{\mathrm{a}}\right), 3.92$ (ddd, $\left.J=3.5,7.5,11.5 \mathrm{~Hz}, 1 \mathrm{H}, 13-\mathrm{H}_{\mathrm{b}}\right), 4.01\left(\mathrm{~d}, J=13.0 \mathrm{~Hz}, 1 \mathrm{H}, 19-\mathrm{H}_{\mathrm{a}}\right), 4.07$ (d, $J=13.0 \mathrm{~Hz}$, $\left.1 \mathrm{H}, 19-\mathrm{H}_{\mathrm{b}}\right), 5.51(\mathrm{~d}, J=3.0 \mathrm{~Hz}, 1 \mathrm{H}, 4-\mathrm{H})$.

${ }^{13}$ C-NMR (125.7 MHz, CDCl 3 ): $\delta=13.0$ (q, C-17), 17.9 (q, C-14), 21.3 (q, C-18), 23.0 (q, C-21), 27.2 (t, C-16), 31.4 (q, C-20), 33.3 (d, C-9), 34.6 (d, C-15), 36.9 (d, C-5), 40.8 (t, C-12), 44.8 (d, C-10), 45.8 (t, C-6), 50,3 (t, C-8), 52.1 (d, C-2), 53.3 (s, C-1), 58.1 (t, C-13), 67.3 (t, C-19), 70.5 (s, C-7), 127.6 (d, C-4), 135.5 (s, C-3), 216.0 (s, C-11). 


\section{Betaenon J (42)}

42 konnte aus den Kulturfiltraten von Ruhefermentation in P-Kolben in Ausbeute von $1.0 \mathrm{mg} / \mathrm{L}$ (Medium: $\mathrm{SGG}+\mathrm{NaCl}$ ) und $1.5 \mathrm{mg} / \mathrm{L}$ (Medium: $\mathrm{G} 20+\mathrm{NaCl}$ ) erhalten werden. Die Aufreinigung erfolgte durch Chromatographie an Kieselgel $\left(\mathrm{CH}_{2} \mathrm{Cl}_{2} / \mathrm{MeOH} 19: 1 \rightarrow 9: 1 \rightarrow 3: 1\right)$, Sephadex ${ }^{\circledR}$ LH-20 (MeOH) und mit der MPLC an RP-Kieselgel (MeOH/ $\left.\mathrm{H}_{2} \mathrm{O} 4: 1\right)$.

$\mathrm{C}_{21} \mathrm{H}_{36} \mathrm{O}_{4}(352.51)$

farbloser Feststoff

$\mathbf{R}_{\mathbf{f}}$-Wert: 0.21 (Chloroform/Methanol 9:1)

Anfärbeverhalten: blau (Anisaldehyd)

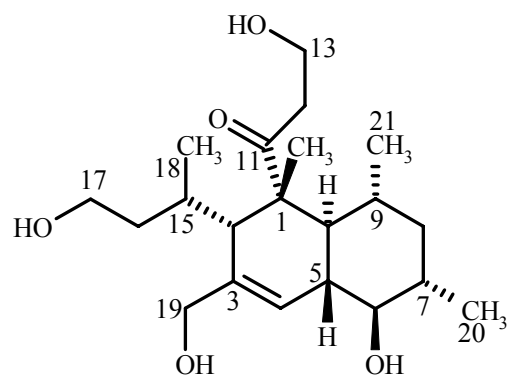

ESI-MS (positive Ionen): $\mathrm{m} / \mathrm{z}(\%)=375(20)[\mathrm{M}+\mathrm{Na}]^{+}, 727(100)[2 \mathrm{M}+\mathrm{Na}]^{+}$.

HRESI-MS: 375.25074 gefunden wie berechnet für $\left[\mathrm{C}_{21} \mathrm{H}_{36} \mathrm{O}_{4} \mathrm{Na}\right]^{+}$.

IR(KBr): $\widetilde{v}=3433,2924,1634,1384 \mathrm{~cm}^{-1}$.

$[\alpha]_{D}^{20}=-4^{\circ}(\mathrm{c}=0.1$, Methanol $)$.

CD (MeOH): $\lambda_{\max }([\Theta])=290(-6050)$.

${ }^{1}$ H-NMR (500 MHz, $\left.\mathbf{C D C l}_{3}\right): \delta=0.62\left(\mathrm{~d}, J=7.0 \mathrm{~Hz}, 3 \mathrm{H}, 21-\mathrm{H}_{3}\right), 0.94(\mathrm{~d}, J=7.0 \mathrm{~Hz}, 3 \mathrm{H}$, 20- $\left.\mathrm{H}_{3}\right), 0.98\left(\mathrm{~d}, J=7.0 \mathrm{~Hz}, 3 \mathrm{H}, 18-\mathrm{H}_{3}\right), 1.00\left(\mathrm{~m}, 1 \mathrm{H}, 6-\mathrm{H}_{\mathrm{a}}\right), 1.02(\mathrm{ddd}, J=12.0,13.0 \mathrm{~Hz}, 1 \mathrm{H}$, $\left.8-\mathrm{H}_{\mathrm{a}}\right), 1.10\left(\mathrm{~m}, 1 \mathrm{H}, 16-\mathrm{H}_{\mathrm{a}}\right), 1.22\left(\mathrm{~s}, 3 \mathrm{H}, 14-\mathrm{H}_{3}\right), 1,35(\mathrm{~m}, 1 \mathrm{H}, 9-\mathrm{H}), 1.64(\mathrm{~m}, 2 \mathrm{H}, 7-\mathrm{H}$ und 16- $\left.\mathrm{H}_{\mathrm{b}}\right), 1.67$ (m, 1H, 8- $\left.\mathrm{H}_{\mathrm{b}}\right), 1.74$ (m, 1H, 15-H), 1.83 (m, 1H, 5-H), 1.85 (m, 1H, 10-H), 1.88 (m, 1H, 6- $\mathrm{H}_{\mathrm{b}}$ ), 2.01 (s, br, 1H, 2-H), 2.77 (ddd, $J=3.5,6.0,18.5 \mathrm{~Hz}, 1 \mathrm{H}, 12-\mathrm{H}_{\mathrm{a}}$ ), 2.90 (ddd, $\left.J=3.5,7.5,18.5 \mathrm{~Hz}, 1 \mathrm{H}, 12-\mathrm{H}_{\mathrm{b}}\right), 3.59\left(\mathrm{ddd}, J=6.0,8.5,11.0 \mathrm{~Hz}, 1 \mathrm{H}, 17-\mathrm{H}_{\mathrm{a}}\right), 3.68$ (ddd, $\left.J=4.5,7.0,11.0 \mathrm{~Hz}, 1 \mathrm{H}, 17-\mathrm{H}_{\mathrm{a}}\right), 3.89\left(\mathrm{~m}, 2 \mathrm{H}, 13-\mathrm{H}_{2}\right), 4.08\left(\mathrm{~s}, 2 \mathrm{H}, 19-\mathrm{H}_{2}\right), 5.59(\mathrm{~d}, J=2.0$ $\mathrm{Hz}, 1 \mathrm{H}, 4-\mathrm{H})$.

${ }^{13}$ C-NMR (125.7 MHz, $\mathbf{C D C l}_{3}$ ): $\delta=18.0$ (q, C-14), 21.7 (q, C-18), 22.1 (q, C-20), 23.4 (q, C-21), 29.2 (d, C-15), 33.7 (d, C-7), 37.1 (t, C-16), 37.6 (d, C-9), 41.2 (t, C-12), 41.5 (t, C-6), 41.6 (d, C-5), 44.7 (d, C-10), 46.2 (t, C-8), 51.3 (d, C-2), 53.4 (s, C-1), 57.9 (t, C-13), 61.5 (t, C-17), 67.2 (t, C-19), 128.8 (d, C-4), 134.5 (s, C-3), 216.5 (s, C-11). 


\section{Solanapyron H (48)}

48 konnte in einer Ausbeute von $5.0 \mathrm{mg} / \mathrm{L}$ aus dem Kulturfiltrat einer Ruhefermentation in P-Kolben (Medium: G20 $+\mathrm{NaCl}$ ) erhalten werden. Die Aufreinigung erfolgte durch Chromatographie an Kieselgel $\left(\mathrm{CH}_{2} \mathrm{Cl}_{2} / \mathrm{MeOH} 19: 1 \rightarrow 9: 1 \rightarrow 3: 1\right)$, Sephadex ${ }^{\circledR}$ LH-20 $(\mathrm{MeOH})$ und erneut an Kieselgel $\left(\mathrm{CH}_{2} \mathrm{Cl}_{2} / \mathrm{MeOH} 9: 1\right)$.

$\mathrm{C}_{16} \mathrm{H}_{22} \mathrm{O}_{3}(262.35)$

farbloses Ö1

UV-löschend (254 nm)

$\mathbf{R}_{\mathbf{f}}$-Wert: 0.57 (Chloroform/Methanol 9:1)

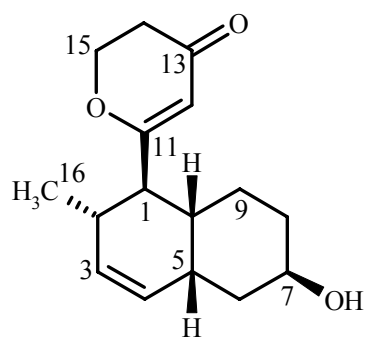

Anfärbeverhalten: grün (Anisaldehyd)

EI-MS: $\mathrm{m} / \mathrm{z}(\%)=262(90)[\mathrm{M}]^{+}, 244(80)\left[\mathrm{M}-\mathrm{H}_{2} \mathrm{O}\right]^{+}, 150$ (44), 145 (100), 105 (42), 91 (50), 43 (44), 41(78).

HREI-MS: 262.1569 gefunden wie berechnet für $\mathrm{C}_{10} \mathrm{H}_{12} \mathrm{O}_{4}$.

IR(KBr): $\widetilde{v}=3424,3011,2959,2927,2871,1667,1652,1595,1459,1404,1358,1253$, $1232,1189,1147,1072,1034,1009,985,941,894,822,728 \mathrm{~cm}^{-1}$.

UV: $(\mathrm{MeOH}): \quad\left[\lambda_{\max }(\lg \varepsilon)\right]=266(4.10) \mathrm{nm}$.

$[\alpha]_{D}^{20}=+43^{\circ}(\mathrm{c}=0.1$, Methanol).

${ }^{1}$ H-NMR (600 MHz, $\left.\mathbf{C D C l}_{3}\right): \delta=0.97\left(\mathrm{~d}, J=7.0 \mathrm{~Hz}, 3 \mathrm{H}, 16-\mathrm{H}_{3}\right), 1.36(\mathrm{ddd}, J=2.5,12.0$, $14.5 \mathrm{~Hz}, 1 \mathrm{H}, 6-\mathrm{H}_{\mathrm{a}}$ ), 1.41 (d, br, $J=14.0 \mathrm{~Hz}, 1 \mathrm{H}, 9-\mathrm{H}_{\mathrm{a}}$ ), 1.50 (dddd, $J=3.0,14.0 \mathrm{~Hz}, 1 \mathrm{H}$, $8-\mathrm{H}_{\mathrm{a}}$ ), 1.55 (ddd, $J=3.0,4.5,14.0 \mathrm{~Hz}, 1 \mathrm{H}, 8-\mathrm{H}_{\mathrm{b}}$ ), 1.79 (dddd, $J=2.5,3.0,3.5,14.5 \mathrm{~Hz}, 1 \mathrm{H}$, 6- $\mathrm{H}_{\mathrm{b}}$ ), 1.94 (dddd, $J=4.0,4.5,14.0 \mathrm{~Hz}, 1 \mathrm{H}, 9-\mathrm{H}_{\mathrm{b}}$ ), 2.11 (m, 2H, 1-H und 10-H), 2.44 (ddq, $J=1.0,7.0,8.0 \mathrm{~Hz}, 1 \mathrm{H}, 2-\mathrm{H}), 2.57$ (m, 1H, 5-H), 2.58 (t, $\left.J=7.0 \mathrm{~Hz}, 2 \mathrm{H}, 14-\mathrm{H}_{2}\right), 4.10$ (dddd, $J=2.5,3.0 \mathrm{~Hz}, 1 \mathrm{H}, 7-\mathrm{H}), 4.51\left(\mathrm{t}, J=7.0 \mathrm{~Hz}, 2 \mathrm{H}, 15-\mathrm{H}_{2}\right), 5.44$ (s, 1H, 12-H), 5.49 (dd, $J=1.0,10.0 \mathrm{~Hz}, 1 \mathrm{H}, 3-\mathrm{H}), 5.64$ (ddd, $J=2.5,5.0,10.0 \mathrm{~Hz}, 1 \mathrm{H}, 4-\mathrm{H})$.

${ }^{1}$ H-NMR (600 MHz, Pyridin-d $\left.\mathbf{d}_{5}\right): \delta=0.98\left(\mathrm{~d}, J=7.0 \mathrm{~Hz}, 3 \mathrm{H}, 16-\mathrm{H}_{3}\right), 1.43$ (ddd, $J=3.0$, $\left.14.0 \mathrm{~Hz}, 1 \mathrm{H}, 6-\mathrm{H}_{\mathrm{a}}\right), 1.55\left(\mathrm{ddd}, J=3.0,14.0 \mathrm{~Hz}, 1 \mathrm{H}, 9-\mathrm{H}_{\mathrm{a}}\right.$ ), 1.60 (dddd, $J=3.0,14.0 \mathrm{~Hz} 1 \mathrm{H}$, $\left.8-\mathrm{H}_{\mathrm{a}}\right), 1.73\left(\mathrm{ddd}, J=2.0,14.0 \mathrm{~Hz}, 1 \mathrm{H}, 8-\mathrm{H}_{\mathrm{b}}\right), 2.00\left(\mathrm{ddd}, J=2.5,14.0 \mathrm{~Hz}, 1 \mathrm{H}, 6-\mathrm{H}_{\mathrm{b}}\right), 2.19$ (dd, br, $J=5.0,11.5,1 \mathrm{H}, 10-\mathrm{H}), 2.30(\mathrm{dd}, J=10.0,11.5 \mathrm{~Hz}, 1 \mathrm{H}, 1-\mathrm{H}), 2.31$ (dddd, $J=4.0,4.5$, $\left.14.0 \mathrm{~Hz}, 1 \mathrm{H}, 9-\mathrm{H}_{\mathrm{b}}\right), 2.49$ (m, 1H, 2-H), 2.58 (dd, $\left.J=6.5 \mathrm{~Hz}, 2 \mathrm{H}, 14-\mathrm{H}_{2}\right), 2.92(\mathrm{dd}, J=5.0$, $12.0 \mathrm{~Hz}, 1 \mathrm{H}, 5-\mathrm{H}), 4.28$ (dd, $J=3.0 \mathrm{~Hz}, 1 \mathrm{H}, 7-\mathrm{H}), 4.41$ (dd, $\left.J=6.5 \mathrm{~Hz}, 2 \mathrm{H}, 15-\mathrm{H}_{2}\right), 5.49$ (dd, $J=1.0,10.0 \mathrm{~Hz}, 1 \mathrm{H}, 3-\mathrm{H}), 5.69$ (ddd, $J=2.5,5.0,10.0 \mathrm{~Hz}, 1 \mathrm{H}, 4-\mathrm{H}), 5.72$ (s, 1H, 12-H). 
${ }^{13}$ C-NMR (125.7 MHz, CDCl 3 ): $\delta=20.2$ (q, C-16), 21.5 (t, C-9), 27.6 (t, C-8), 30.3 (d, C-5), 34.6 (d, C-2), 35.8 (t, C-14), 35.8 (d, C-10), 35.9 (t, C-6), 46.5 (d, C-1), 65.7 (d, C-7), 68.0 (t, C-15), 106.7 (d, C-12), 131.1 (d, C-3), 131.2(d, C-4), 179.1 (s, C-11), 192.1 (s, C-13).

\section{Solanapyron I (49)}

49 konnte in einer Ausbeute von $1.5 \mathrm{mg} / \mathrm{L}$ aus dem Kulturfiltrat einer Ruhefermentation in P-Kolben (Medium: $\mathrm{G} 20+\mathrm{NaCl}$ ) erhalten werden. Die Aufreinigung erfolgte durch Chromatographie an Kieselgel $\left(\mathrm{CH}_{2} \mathrm{Cl}_{2} / \mathrm{MeOH} 19: 1 \rightarrow 9: 1 \rightarrow 3: 1\right)$, Sephadex ${ }^{\circledR}$ LH-20 $(\mathrm{MeOH})$ und erneut an Kieselgel $\left(\mathrm{CH}_{2} \mathrm{Cl}_{2} / \mathrm{MeOH} 9: 1\right)$.

$\mathrm{C}_{19} \mathrm{H}_{25} \mathrm{NO}_{5}$ (347.41)

farbloser Feststoff

UV-löschend (254 nm)

$\mathbf{R}_{\mathbf{f}}$-Wert: 0.25 (Chloroform/Methanol 9:1)

Anfärbeverhalten: gelb (Anisaldehyd)

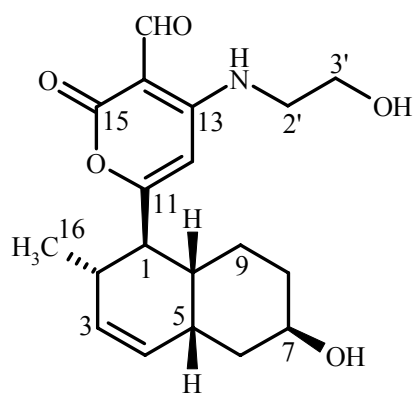

Smp.: $121^{\circ} \mathrm{C}$

EI-MS: $\mathrm{m} / \mathrm{z}(\%)=347$ (100) [M] $]^{+}, 319$ (40), 280 (18), 257 (22), 182 (50), 110 (54).

HREI-MS: 347.1733 gefunden wie berechnet für $\mathrm{C}_{19} \mathrm{H}_{25} \mathrm{O}_{5} \mathrm{~N}$.

IR(KBr): $\widetilde{v}=3425,3959,2927,2871,1698,1652,1576,1504,1452,1380,1343,1277$, $1233,1068,1009 \mathrm{~cm}^{-1}$.

UV: $(\mathrm{MeOH}): \quad\left[\lambda_{\max }(\lg \varepsilon)\right]=237(4.03), 247(\mathrm{sh}, 3.49), 281(3.56), 317(3.60) \mathrm{nm}$.

CD (MeOH): $\lambda_{\max }([\Theta])=238$ (9438), 314 (-3142).

${ }^{1}$ H-NMR (500 MHz, $\left.\mathbf{C D C l}_{3}\right): \delta=0.98\left(\mathrm{~m}, 3 \mathrm{H}, 16-\mathrm{H}_{3}\right), 1.29$ (d, br, $\left.J=15.0 \mathrm{~Hz}, 1 \mathrm{H}, 9-\mathrm{H}_{\mathrm{a}}\right)$, 1.37 (dd, br, $\left.J=12.0,14.0 \mathrm{~Hz}, 1 \mathrm{H}, 6-\mathrm{H}_{\mathrm{a}}\right), 1.49$ (d, br, $\left.J=14.0 \mathrm{~Hz}, 1 \mathrm{H}, 8-\mathrm{H}_{\mathrm{a}}\right), 1.55(\mathrm{~m}, 1 \mathrm{H}$, 8- $\left.\mathrm{H}_{\mathrm{b}}\right), 1.82\left(\mathrm{~d}, \mathrm{br}, J=14.5 \mathrm{~Hz}, 1 \mathrm{H}, 6-\mathrm{H}_{\mathrm{b}}\right), 1.94\left(\mathrm{~m}, 1 \mathrm{H}, 9-\mathrm{H}_{\mathrm{b}}\right), 2.30(\mathrm{~m}, 2 \mathrm{H}, 1-\mathrm{H}$ und 10-H), $2.62\left(\mathrm{~m}, 2 \mathrm{H}, 2-\mathrm{H}\right.$ und 5-H), 3.55 (t, $\left.J=7.0 \mathrm{~Hz}, 2 \mathrm{H}, 2^{\prime}-\mathrm{H}_{2}\right), 3.90$ (t, $\left.J=7.0 \mathrm{~Hz}, 2 \mathrm{H}, 3^{\prime}-\mathrm{H}_{2}\right)$, $4.10(\mathrm{~m}, 1 \mathrm{H}, 7-\mathrm{H}), 5.50(\mathrm{~d}, J=10.0 \mathrm{~Hz}, 1 \mathrm{H}, 3-\mathrm{H}), 5.64$ (ddd, $J=2.5,5.0,10.0 \mathrm{~Hz}, 1 \mathrm{H}, 4-\mathrm{H})$ 6.02 (s, 1H, 12-H), 9.98 (s, 1H, 17-H), 10.82 (s, br, 1'-NH).

${ }^{13}$ C-NMR (125.7 MHz, CDCl 3 ): $\delta=20.3$ (q, C-16), 21.5 (t, C-9), 27.6 (t, C-8), 30.3 (d, C-5), 35.2 (d, C-2), 35.4 (d, C-10), 36.0 (t, C-6), 44.9 (t, C-1'), 46.8 (d, C-1), 61.1 (t, C-2'), 65.6 (d, C-7), 94.9 (s, C-14), 95.9 (d, C-12), 130.9 (d, C-3), 131.1 (d, C-4), 160.6 (s, C-13), 164,0 (s, C-15), 172.0 (s, C-11), 191.3 (s, C-17). 


\section{Solanapyron J (51)}

51 konnte aus dem Kulturfiltrat einer Ruhefermentation in P-Kolben (Medium: G20 + NaCl) in einer Ausbeute von $3.5 \mathrm{mg} / \mathrm{L}$ erhalten werden. Die Aufreinigung erfolgte durch Chromatographie an Kieselgel $\left(\mathrm{CH}_{2} \mathrm{Cl}_{2} / \mathrm{MeOH} 19: 1 \rightarrow 9: 1 \rightarrow 3: 1\right)$, Sephadex ${ }^{\circledR}$ LH-20 $(\mathrm{MeOH})$ und erneut an Kieselgel $\left(\mathrm{CH}_{2} \mathrm{Cl}_{2} / \mathrm{MeOH} 9: 1\right)$.

$\mathrm{C}_{28} \mathrm{H}_{32} \mathrm{O}_{8}(496.56)$

gelber Feststoff

UV-löschend (254 nm)

gelb fluoreszierend $(366 \mathrm{~nm})$

Rf-Wert: 0.27 (Chloroform/Methanol 9:1)

Smp.: $102^{\circ} \mathrm{C}$

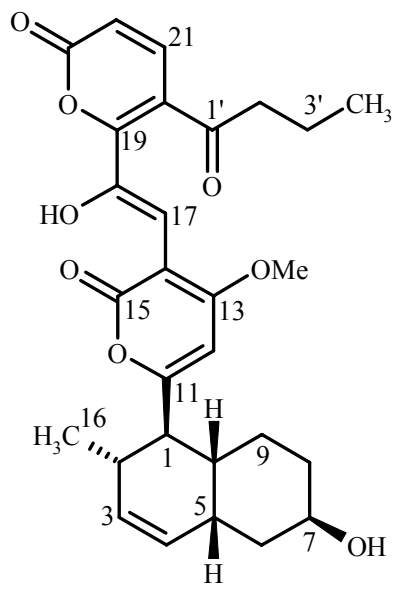

ESI-MS (positive Ionen): $\quad \mathrm{m} / \mathrm{z}(\%)=541(100)[\mathrm{M}-\mathrm{H}+2 \mathrm{Na}]^{+}, 1037(90)[2 \mathrm{M}-\mathrm{H}+2 \mathrm{Na}]^{+}$, $1059(100)[2 \mathrm{M}-2 \mathrm{H}+3 \mathrm{Na}]^{+}$;

(negative Ionen): $\quad \mathrm{m} / \mathrm{z}(\%)=495(100)[\mathrm{M}-\mathrm{H}]^{-}$.

HRESI-MS: 497.21699 gefunden wie berechnet für $\left[\mathrm{C}_{28} \mathrm{H}_{33} \mathrm{O}_{8}\right]^{+}$.

IR(KBr): $\widetilde{v}=3419,2944,2833,1634,1384,1027 \mathrm{~cm}^{-1}$.

UV: $(\mathrm{MeOH}): \quad\left[\lambda_{\max }(\lg \varepsilon)\right]=274(3.78), 387(\mathrm{sh}, 3.38), \mathrm{nm}$.

CD (MeOH): $\lambda_{\max }([\Theta])=209$ (14649).

${ }^{1}$ H-NMR (500 MHz, $\left.\mathbf{C D C l}_{3}\right): \delta=0.98\left(\mathrm{~d}, J=7.0 \mathrm{~Hz}, 3 \mathrm{H}, 16-\mathrm{H}_{3}\right), 1.01(\mathrm{t}, J=7.5 \mathrm{~Hz}, 3 \mathrm{H}$, $\left.4^{\prime}-\mathrm{H}_{3}\right), 1.13-1.27\left(\mathrm{~m}, 1 \mathrm{H}, 9-\mathrm{H}_{\mathrm{a}}\right), 1.28-1.40\left(\mathrm{~m}, 1 \mathrm{H}, 6-\mathrm{H}_{\mathrm{a}}\right), 1.43-1.59\left(\mathrm{~m}, 2 \mathrm{H}, 8-\mathrm{H}_{2}\right), 1.78$ (sextet, $\left.J=7.5 \mathrm{~Hz}, 2 \mathrm{H}, 3^{\prime}-\mathrm{H}_{2}\right), 1.78\left(\mathrm{~m}, 1 \mathrm{H}, 6-\mathrm{H}_{\mathrm{b}}\right), 1.91\left(\mathrm{~m}, 1 \mathrm{H}, 9-\mathrm{H}_{\mathrm{b}}\right), 1.96(\mathrm{dd}, \mathrm{br}, J=4.5$, $11.5 \mathrm{~Hz}, 1 \mathrm{H}, 10-\mathrm{H}), 2.39$ (dd, $J=10.0,12.0 \mathrm{~Hz}, 1 \mathrm{H}, 1-\mathrm{H}), 2.60(\mathrm{~m}, 2 \mathrm{H}, 2-\mathrm{H}$ und 5-H), 2.73 (t, $\left.J=7.5 \mathrm{~Hz}, 2 \mathrm{H}, 2^{\prime}-\mathrm{H}_{2}\right), 3.98\left(\mathrm{~s}, 13-\mathrm{CH}_{3}\right), 4.08(\mathrm{~m}, 1 \mathrm{H}, 7-\mathrm{H}), 5.46$ (d, $\left.J=10.0 \mathrm{~Hz}, 1 \mathrm{H}, 3-\mathrm{H}\right)$, $5.61(\mathrm{~m}, 1 \mathrm{H}, 4-\mathrm{H}), 6.11(\mathrm{~s}, 1 \mathrm{H}, 12-\mathrm{H}), 6.81(\mathrm{~s}, 1 \mathrm{H}, 17-\mathrm{H}), 7.03$ (d, J = $15.5 \mathrm{~Hz}, 1 \mathrm{H}, 3-\mathrm{H})$, $7.36(\mathrm{~d}, J=15.5 \mathrm{~Hz}, 1 \mathrm{H}, 4-\mathrm{H})$.

${ }^{13}$ C-NMR (125.7 MHz, $\mathbf{C D C l}_{3}$ ): $\delta=13.8$ (q, C-4'), 20.4 (q, C-16), 20.4 (t, C-3'), 21.6 (t, C-9), 27.9 (t, C-8), 30.0 (t, C-2'), 30.3 (d, C-5), 35.3 (d, C-2), 35.3 (d, C-10), 36.0 (t, C-6), 46.6 (d, C-1), 57.1 (q, 13-OMe), 65.6 (d, C-7), 97.8 (s, C-14), 95.5 (d, C-12), 104.2 (d, C-17), 112.8 (s, C-20), 119.9 (d, C-22), 130.8 (d, C-3), 132.8 (d, C-21), 131.2 (d, C-4), 145.0 (s, C-18), 169.1 (s, C-13), 161.0 (s, C-15), 171.8 (s, C-19), 172.0 (s, C-11), 183.6 (s, C-1'), 184.7 (s, C-23). 


\section{5. $\quad$ Alternaria sp. Stamm 6587 und 6588}

\subsection{Kultivierung und Isolierung der Metabolite}

Die Stämme 6587 und 6588 wurde wie unter B.II.3.1. beschrieben in Schüttelkolben und wie unter B.II.3.2. beschrieben in P-Kolben kultiviert. Als Nährmedium dienten die fünf Standardnährmedien (E2, 1158, 1549, G20 bzw. SGG) ohne und mit Zusatz von Natriumchlorid (33 g/L). Die beiden Pilzstämme unterscheiden sich in ihrer Morphologie, zeigten aber im chemischen Screening ein identisches Metabolitenmuster.

Alternaria sp. Stamm 6588 fielen bei Kultivierung in Schüttelkultur mit den Medien 1158, 1549 und M2 sowie bei alle Fermentationsansätze in Ruhekultur durch ein ähnliches Metabolitenmuster auf. Die höchsten Ausbeuten wurden bei einer Ruhefermentation mit 1549 als Nährmedium beobachtet. Aus diesem Fermentationsansatz konnten insgesamt fünf Metabolite (55 -59) isoliert werden. Die Isolierung der Metabolite ist in Abbildung 68 dargestellt.

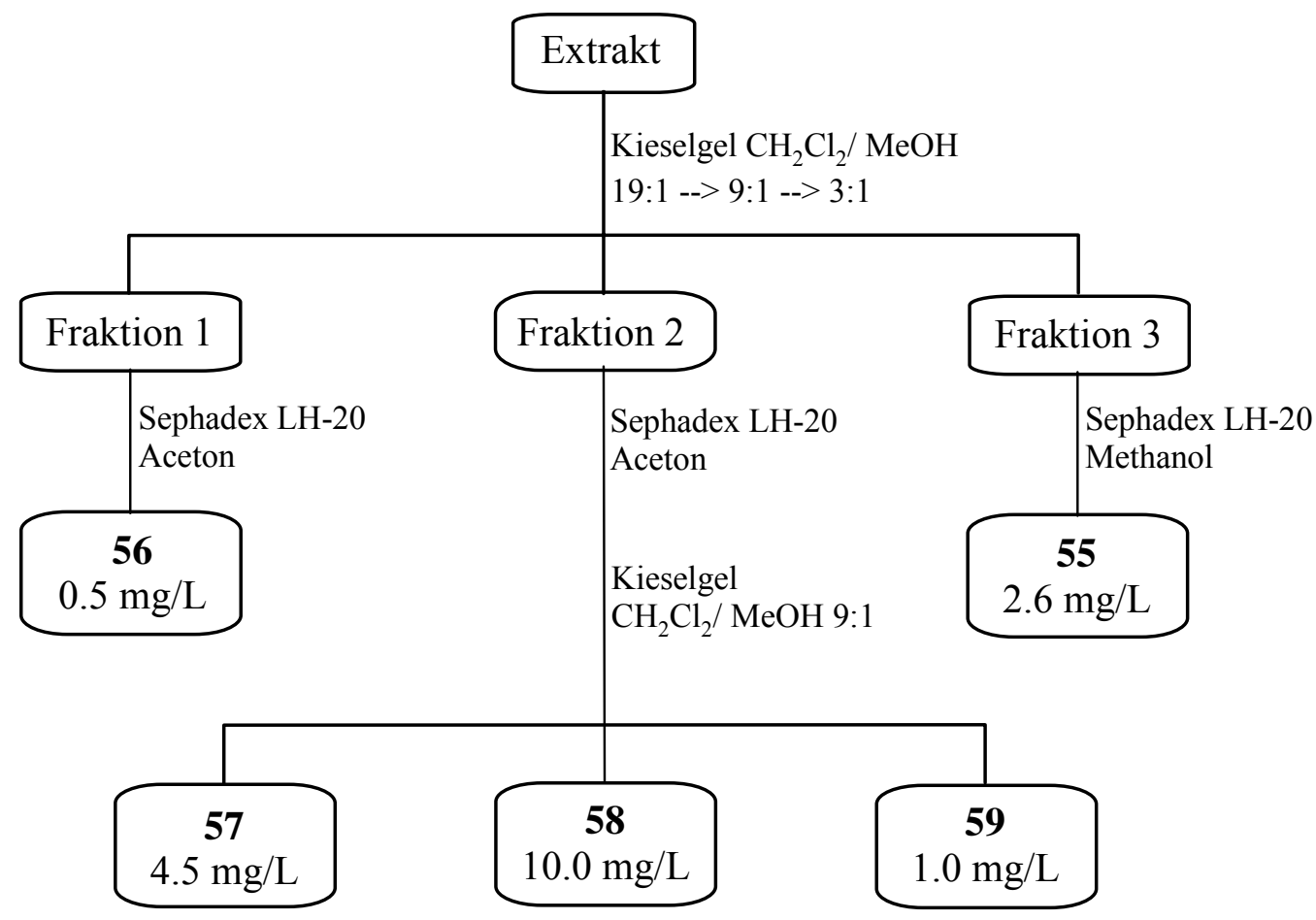

Abbildung 68: Aufreinigung des Extraktes aus der Fermentation von Alternaria sp. Stamm 6588 in Ruhekultur (Medium: 1549). 


\title{
5.2. Kultivierung unter Zusatz von Enzyminhibitoren
}

Um neue Bisnaphtalen zu erhalten, wurde der Stamm 6588 wie unter B.II.2. beschrieben in Schüttelkolben mit dem Nährmedium 1158 kultiviert. Die Enzyminhibitoren Tricyclazol (1.0, 5.0 und $10.0 \mathrm{mg} / \mathrm{L})$ bzw. Fthalid (10.0, 20.0 und $40.0 \mathrm{mg} / \mathrm{L})$ wurden dem Nährmedium nach dem Autoklavieren zugesetzt. Nach 96 h wurden die verschiedenen Ansätze auf einen pHWert von 4-5 gebracht, mit derselben Menge Ethylacetat versetzt und 5 min mit dem Ultraturax behandelt. Anschließend wurde das Mycel abzentrifugiert und verworfen. Nach Abtrennen der organischen Phase wurde ein weiteres Mal mit Ethylacetat extrahiert und die organischen Phasen vereinigt. Die nach dem Entfernen des Lösungsmittels erhaltenen Extrakte wurden dünnschichtchromatographisch und per HPLC analysiert.

Zur Isolierung der gebildeten Metabolite wurde der Stamm unter Zusatz von $10.0 \mathrm{mg} / \mathrm{L}$ Tricyclazol bzw. $20.0 \mathrm{mg} / \mathrm{L}$ Fthalid in Schüttelkolben (je $1 \mathrm{~L}, 10 \times 100 \mathrm{~mL}$ ) kultiviert und wie oben beschrieben aufgearbeitet. Die erste chromatographische Aufreinigung erfolgte an Kieselgel $\left(\mathrm{CH}_{2} \mathrm{Cl}_{2} / \mathrm{MeOH}\right.$ 19:1 $\left.\rightarrow 9: 1 \rightarrow 3: 1\right)$. Durch weiter Chromatographie an Sephadex ${ }^{\circledR}$ LH-20 (MeOH) und Kieselgel $\left(\mathrm{CH}_{2} \mathrm{Cl}_{2} / \mathrm{MeOH}\right.$ 9:1) ließen sich Sphaerolon (70), 1,3,8Naphtalentriol (65) und 2-Hydroxyjuglon (69) in Ausbeuten von 0.5, 8.0 und $5.0 \mathrm{mg} / \mathrm{L}$ erhalten. Aus der Fermentation unter Zusatz von Fthalid konnte nach Chromatographie an Kieselgel $\left(\mathrm{CH}_{2} \mathrm{Cl}_{2} / \mathrm{MeOH}\right.$ 19:1 $\left.\rightarrow 9: 1 \rightarrow 3: 1\right)$ Sephadex ${ }^{\circledR}$ LH-20 (MeOH) Scytalon $(64)$ in einer Ausbeute von $6.0 \mathrm{mg} / \mathrm{L}$ isoliert werden.

\subsection{Charakterisierung der Metabolite}

\author{
Alternariol $^{85}$ (55) \\ $\mathrm{C}_{14} \mathrm{H}_{10} \mathrm{O}_{5}(258.23)$ \\ farbloses Öl \\ UV-löschend (254 nm) \\ fluoreszierend (366 nm)
}

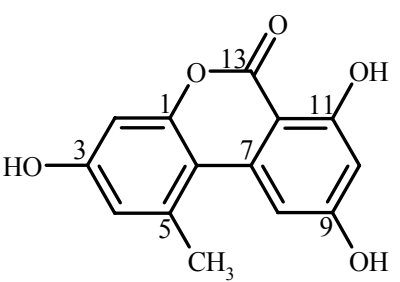

$\mathbf{R}_{\mathbf{f}}$-Wert: 0.53 (Chloroform/Methanol 9:1)

Anfärbeverhalten: schwach gelb (Anisaldehyd)

Smp.: $>200^{\circ} \mathrm{C}$

ESI-MS (negative Ionen): $\mathrm{m} / \mathrm{z}(\%)=257(100)[\mathrm{M}-\mathrm{H}]^{-}, 515(15)$ [2M-H]-

HRESI-MS: 257.04583 gefunden wie berechnet für $\left[\mathrm{C}_{14} \mathrm{H}_{9} \mathrm{O}_{5}\right]^{-}$. 
IR(KBr): $\widetilde{v}=3438,3169,2952,1610,1424,1351,1256,1165 \mathrm{~cm}^{-1}$.

UV: $(\mathrm{MeOH}): \quad\left[\lambda_{\max }(\lg \varepsilon)\right]=229(\mathrm{sh}, 3.68), 248(3.89) \mathrm{nm}$;

$(\mathrm{MeOH} / \mathrm{HCl}): \quad\left[\lambda_{\max }(\lg \varepsilon)\right]=227(\mathrm{sh}, 3.60), 249(3.87) \mathrm{nm} ;$

$(\mathrm{MeOH} / \mathrm{NaOH}):\left[\lambda_{\max }(\lg \varepsilon)\right]=235$ (sh, 4.08), 249 (3.93), $263(3.97) \mathrm{nm}$.

${ }^{1}$ H-NMR (600 MHz, Pyridin-d $): \delta=2.55$ (s, 3H, 5- $\left.\mathrm{CH}_{3}\right), 6.88(\mathrm{~d}, J=2.0 \mathrm{~Hz}, 1 \mathrm{H}, 10-\mathrm{H})$, $6.94(\mathrm{~d}, J=2.0 \mathrm{~Hz}, 1 \mathrm{H}, 2-\mathrm{H}), 6.98(\mathrm{~d}, J=2.0 \mathrm{~Hz}, 1 \mathrm{H}, 4-\mathrm{H}), 7.42(\mathrm{~d}, J=2.0 \mathrm{~Hz}, 1 \mathrm{H}, 8-\mathrm{H})$, 8.72 (s, 1H, 9-OH), 12.50 (s, 1H, 11-OH), 12.59 (s, br, 1H, 3-OH).

${ }^{13}$ C-NMR (150.8 MHz, Pyridin-d $)$ ): $\delta=25.3$ (q, 5- $\mathrm{CH}_{3}$ ), 98.7 (s, C-12), 102.1 (d, C-10), 102.7 (d, C-2), 105.4 (d, C-8), 110.2 (s, C-6), 118.6 (d, C-4), 138.9 (s, C-5 oder C-7), 139.4 (s, C-5 oder C-7), 153.9 (s, C-3), 160.2 (s, C-1), 165.8 (s, C-11), 166.0 (s, C-13), 167.2 (s, C-9).

Alternariol-9-methylether ${ }^{86}(56)$

$\mathrm{C}_{15} \mathrm{H}_{12} \mathrm{O}_{5}(272.26)$

farbloser Feststoff

UV-löschend (254 nm)

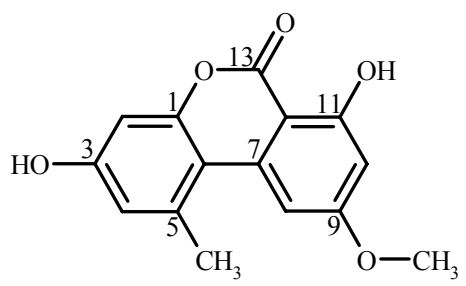

fluoreszierend (366 nm)

$\mathbf{R}_{\mathbf{f}}$-Wert: 0.76 (Chloroform/Methanol 9:1)

ESI-MS (positive Ionen): $\mathrm{m} / \mathrm{z}(\%)=295(15)[\mathrm{M}+\mathrm{Na}]^{+}, 567(100)[2 \mathrm{M}+\mathrm{Na}]^{+}$;

(negative Ionen): $\mathrm{m} / \mathrm{z}(\%)=271(100)[\mathrm{M}-\mathrm{H}]^{-}$.

HRESI-MS: 271.06084 gefunden wie berechnet für $\left[\mathrm{C}_{15} \mathrm{H}_{11} \mathrm{O}_{5}\right]^{-}$.

IR(KBr): $\widetilde{v}=3430,2925,1618,1384,1164 \mathrm{~cm}^{-1}$.

UV: $(\mathrm{MeOH}): \quad\left[\lambda_{\max }(\lg \varepsilon)\right]=223(\mathrm{sh}, 3.53), 257(3.91) \mathrm{nm}$;

$(\mathrm{MeOH} / \mathrm{HCl}): \quad\left[\lambda_{\max }(\lg \varepsilon)\right]=223(\mathrm{sh}, 3.54), 253(3.91) \mathrm{nm}$;

$(\mathrm{MeOH} / \mathrm{NaOH}):\left[\lambda_{\max }(\lg \varepsilon)\right]=226(\mathrm{sh}, 3.93), 241(3.59), 282(3.98) \mathrm{nm}$.

${ }^{1}$ H-NMR (600 MHz, Pyridin-d $): \delta=2.71\left(\mathrm{~s}, 3 \mathrm{H}, 5-\mathrm{CH}_{3}\right), 3.82\left(\mathrm{~s}, 3 \mathrm{H}, 9-\mathrm{OCH}_{3}\right), 6.76(\mathrm{~d}$, $J=2.0 \mathrm{~Hz}, 1 \mathrm{H}, 10-\mathrm{H}), 6.98(\mathrm{~d}, J=2.0 \mathrm{~Hz}, 1 \mathrm{H}, 2-\mathrm{H}), 7.00(\mathrm{~d}, J=2.0 \mathrm{~Hz}, 1 \mathrm{H}, 4-\mathrm{H}), 7.30$ (d, $J=2.0 \mathrm{~Hz}, 1 \mathrm{H}, 8-\mathrm{H}), 12.50$ (s, 1H, 11-OH), 12.58 (s, br, 1H, 3-OH).

${ }^{13}$ C-NMR (150.8 MHz, Pyridin-d $\left.\mathbf{d}_{5}\right): \delta=\delta=25.5$ (q, 5- $\mathrm{CH}_{3}$ ), 55.8 (q, 9- $\mathrm{CH}_{3}$ ), 99.8 (s, C-12), 99.6 (d, C-10), 102.8 (d, C-2), 104.3 (d, C-8), 110.0 (s, C-6), 118.8 (d, C-4), 138.9 (s, C-5 oder C-7), 139.0 (s, C-5 oder C-7), 153.9 (s, C-3), 160.3 (s, C-1), 165.7 (s, C-11), 166.0 (s, C-13), 166.9 (s, C-9). 


\section{Alterperylenol $^{89}$ (57)}

$\mathrm{C}_{20} \mathrm{H}_{14} \mathrm{O}_{6}(350.33)$

gelber Feststoff

UV-löschend (254 nm)

gelb fluoreszierend (366 nm)

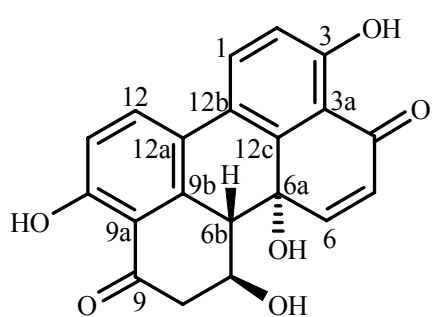

$\mathbf{R}_{\mathbf{f}}$-Wert: 0.48 (Chloroform/Methanol 9:1)

Anfärbeverhalten: braun (Anisaldehyd)

Smp.: $>200^{\circ} \mathrm{C}$

ESI-MS (positive Ionen): $\mathrm{m} / \mathrm{z}(\%)=351(100)[\mathrm{M}+\mathrm{H}]^{+}, 374(30)[\mathrm{M}+\mathrm{Na}]^{+}$;

(negative Ionen): $\mathrm{m} / \mathrm{z}(\%)=349(100)[\mathrm{M}-\mathrm{H}]^{-}, 721(10)[2 \mathrm{M}-2 \mathrm{H}+\mathrm{Na}]^{-}$.

HRESI-MS: 351.08687 gefunden wie berechnet für $\left[\mathrm{C}_{20} \mathrm{H}_{15} \mathrm{O}_{6}\right]^{+}$.

IR(KBr): $\widetilde{v}=3379,2946,2834,1653,1452,1383,1030 \mathrm{~cm}^{-1}$.

UV: $(\mathrm{MeOH}): \quad\left[\lambda_{\max }(\lg \varepsilon)\right]=213(\mathrm{sh}, 4.12), 244(4.04), 288$ (3.87), 295 (sh, 3.82), 363 (3.48), $541(3.15), 580(3.14) \mathrm{nm}$;

$(\mathrm{MeOH} / \mathrm{HCl}): \quad\left[\lambda_{\max }(\lg \varepsilon)\right]=218(\mathrm{sh}, 4.01), 258$ (4.04), 287 (sh, 3.84), 369 (3.57), 393 (sh, 3.51) nm;

$(\mathrm{MeOH} / \mathrm{NaOH}):\left[\lambda_{\max }(\lg \varepsilon)\right]=212(\mathrm{sh}, 4.12), 258$ (4.01), 282 (3.85), 296 (sh, 3.75), 362 (3.51), 419 (3.46), 442 (3.44), 611 (3.15) nm.

CD (MeOH): $\lambda_{\max }([\Theta])=214$ (-21465), 251 (23119), 345 (-3185), 410 (6437).

${ }^{1}$ H-NMR (600 MHz, Aceton-d $\left.)_{6}\right): \delta=3.03\left(\mathrm{dd}, J=12.0,16.0 \mathrm{~Hz}, 1 \mathrm{H}, 8-\mathrm{H}_{\mathrm{a}}\right), 3.10$ (dd, $\left.J=5.0,16.0 \mathrm{~Hz}, 1 \mathrm{H}, 8-\mathrm{H}_{\mathrm{b}}\right), 3.26$ (d, $\left.J=9.5 \mathrm{~Hz}, 1 \mathrm{H}, 6 \mathrm{~b}-\mathrm{H}\right), 4.78$ (ddd, $J=5.0,9.5,12.0 \mathrm{~Hz}$, 1H, 7-H), 4.91 (s, 2H, 6a-OH oder 7-OH), 5.05 (s, 2H, 6a-OH oder 7-OH), 6.34 (d, J = 10.5 $\mathrm{Hz}, 1 \mathrm{H}, 5-\mathrm{H}), 6.98$ (d, $J=8.5 \mathrm{~Hz}, 1 \mathrm{H}, 11-\mathrm{H}), 7.07$ (d, $J=8.5 \mathrm{~Hz}, 1 \mathrm{H}, 2-\mathrm{H}), 8.04$ (d, $J=8.5$ $\mathrm{Hz}, 1 \mathrm{H}, 1-\mathrm{H}), 8.07$ (d, $J=10.5 \mathrm{~Hz}, 1 \mathrm{H}, 6-\mathrm{H}), 8.08$ (d, $J=8.5 \mathrm{~Hz}, 1 \mathrm{H}, 12-\mathrm{H}), 12.50$ (s, 1H, 3-OH oder 10-OH), 12.58 (s, 1H, 3-OH oder 10-OH).

${ }^{13}$ C-NMR (150.8 MHz, Aceton-d ${ }_{6}$ ): $\delta=48.8$ (t, C-8), 52.8 (d, C-6b), 66.7 (d, C-7), 67.9 (s, C-6a), 114.3 (s, C-3a), 117.5 (d, C-11), 118.4 (s, C-9a), 119.1 (d, C-2), 126.1 (d, C-12b), 126.3 (d, C-5), 127.1 (s, C-12a), 133.1 (s, C-1), 133.4 (s, C-12), 138.9 (s, C-9b), 141.5 (s, C12c), 154.2 (d, C-6), 162.5 (s, C-3), 163.4 (s, C-10), 192.2 (s, C-4), 205.1 (s, C-9). 


\section{5,6-Dihydroalterperylenol ${ }^{89}(58)$}

$\mathrm{C}_{20} \mathrm{H}_{16} \mathrm{O}_{6}(352.34)$

gelber Feststoff

UV-löschend (254 nm)

gelb fluoreszierend (366 nm)

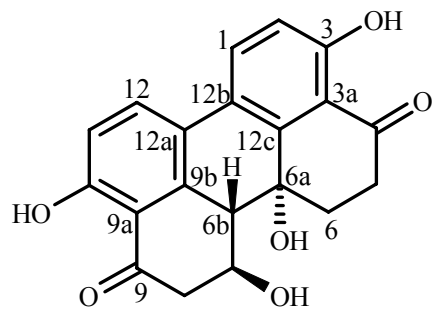

$\mathbf{R}_{\mathbf{f}}$-Wert: 0.55 (Chloroform/Methanol 9:1)

Anfärbeverhalten: braun (Anisaldehyd)

ESI-MS (negative Ionen): $\mathrm{m} / \mathrm{z}(\%)=351(100)[\mathrm{M}-\mathrm{H}]^{-}, 725(10)[2 \mathrm{M}-2 \mathrm{H}+\mathrm{Na}]^{-}$.

HRESI-MS: 351.08687 gefunden wie berechnet für $\left[\mathrm{C}_{20} \mathrm{H}_{15} \mathrm{O}_{6}\right]^{-}$.

IR(KBr): $\widetilde{v}=3418,2926,2857,1643,1459,1379,1232,1183,1058,1015 \mathrm{~cm}^{-1}$.

UV: $(\mathrm{MeOH}): \quad\left[\lambda_{\max }(\lg \varepsilon)\right]=214(4.34), 255(4.45), 286(4.12), 357(3.61) \mathrm{nm}$;

$(\mathrm{MeOH} / \mathrm{HCl}): \quad\left[\lambda_{\max }(\lg \varepsilon)\right]=214(4.33), 257(4.45), 286(4.12), 357(3.65) \mathrm{nm}$;

$(\mathrm{MeOH} / \mathrm{NaOH}):\left[\lambda_{\max }(\lg \varepsilon)\right]=260 \quad(4.09), \quad 283 \quad(4.09), \quad 396 \quad$ (3.78), 418 (3.79), $443(3.74) \mathrm{nm}$.

$[\alpha]_{D}^{20}=+224^{\circ}(\mathrm{c}=0.1$, Methanol $)$.

CD (MeOH): $\lambda_{\max }([\Theta])=216$ (-105185), 255 (81138), 282 (-6675), 312 (5302), 347 (-4727),

$$
385 \text { (14931). }
$$

${ }^{1}$ H-NMR (600 MHz, $\mathbf{C D C l}_{3}$ ): $\delta=2.41$ (ddd, $J=4.5,14.5,14.5 \mathrm{~Hz}, 1 \mathrm{H}, 6-\mathrm{H}_{\mathrm{a}}$ ), 2.68 (ddd, $\left.J=3.0,14.5,17.0 \mathrm{~Hz}, 1 \mathrm{H}, 6-\mathrm{H}_{\mathrm{b}}\right), 2.90\left(\mathrm{dd}, J=12.0,16.0 \mathrm{~Hz}, 1 \mathrm{H}, 8-\mathrm{H}_{\mathrm{a}}\right), 3.03$ (ddd, $J=3.0$, 14.5, $\left.14.5 \mathrm{~Hz}, 1 \mathrm{H}, 5-\mathrm{H}_{\mathrm{a}}\right), 3.06(\mathrm{~d}, J=9.0 \mathrm{~Hz}, 1 \mathrm{H}, 6 \mathrm{~b}-\mathrm{H}), 3.07$ (dd, $J=5.0,16.0 \mathrm{~Hz}, 1 \mathrm{H}$, $\left.8-\mathrm{H}_{\mathrm{b}}\right), 3.15\left(\mathrm{ddd}, J=4.5,14.5,17.0 \mathrm{~Hz}, 1 \mathrm{H}, 5-\mathrm{H}_{\mathrm{b}}\right), 4.73(\mathrm{ddd}, J=5.0,9.0,12.0 \mathrm{~Hz}, 1 \mathrm{H}, 7-\mathrm{H})$, $7.00(\mathrm{~d}, J=8.5 \mathrm{~Hz}, 1 \mathrm{H}, 1-\mathrm{H}), 7.06(\mathrm{~d}, J=8.5 \mathrm{~Hz}, 1 \mathrm{H}, 12-\mathrm{H}), 7.81(\mathrm{~d}, J=8.5 \mathrm{~Hz}, 1 \mathrm{H}, 2-\mathrm{H}$ oder 11-H), 7.82 (d, $J=8.5 \mathrm{~Hz}, 1 \mathrm{H}, 2-\mathrm{H}$ oder 11-H), 12.32 (s, 1H, 3-OH), 12.69 (s, 1H, $10-\mathrm{OH})$.

${ }^{13}$ C-NMR (150.8 MHz, CDCl 3 ): $\delta=34.0$ (t, C-5), 34.5 (t, C-6), 47.7 (t, C-8), 51.8 (d, C-6b), 66.0 (d, C-7), 69.2 (s, C-6a), 113.8 (s, C-3a), 116.9 (d, C-11), 117.4 (s, C-9a), 119.4 (d, C-2), 122.8 (d, C-12b), 124.2 (s, C-12a), 132.4 (s, C-12), 132.6 (s, C-1), 135.7 (s, C-12c), 139.1 (s, C-9b), 161.9 (s, C-10), 162.2 (s, C-3), 202.3 (s, C-9), 205.0 (s, C-4). 


\section{Stemphyperylenol ${ }^{90}(59)$}

$\mathrm{C}_{20} \mathrm{H}_{16} \mathrm{O}_{6}(352.34)$

gelber Feststoff

UV-löschend (254 nm)

gelb fluoreszierend (366 nm)

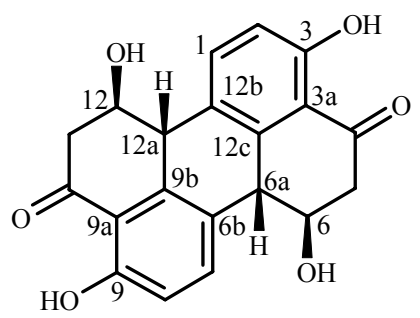

$\mathbf{R}_{\mathbf{f}}$-Wert: 0.43 (Chloroform/Methanol 9:1)

Anfärbeverhalten: braun (Anisaldehyd)

Smp.: $142^{\circ} \mathrm{C}$

ESI-MS (negative Ionen): $\mathrm{m} / \mathrm{z}(\%)=333(100)\left[\mathrm{M}-\mathrm{H}_{2} \mathrm{O}-\mathrm{H}\right]^{-}, 351(70)[\mathrm{M}-\mathrm{H}]^{-}$.

HRESI-MS: 351.08688 gefunden wie berechnet für $\left[\mathrm{C}_{20} \mathrm{H}_{15} \mathrm{O}_{6}\right]^{-}$.

IR(KBr): $\widetilde{v}=3428,2924,1642,1468,1357,1232,1167,1096,1068,1029 \mathrm{~cm}^{-1}$.

UV: $(\mathrm{MeOH}): \quad\left[\lambda_{\max }(\lg \varepsilon)\right]=215(\mathrm{sh}, 4.28), 258(4.15), 341(3.67) \mathrm{nm}$;

$(\mathrm{MeOH} / \mathrm{HCl}): \quad\left[\lambda_{\max }(\lg \varepsilon)\right]=218(\mathrm{sh}, 4.22), 258(4.12), 345(3.58) \mathrm{nm}$;

$(\mathrm{MeOH} / \mathrm{NaOH}):\left[\lambda_{\max }(\lg \varepsilon)\right]=210(\mathrm{sh}, 4.31), 266$ (4.20), 350 (3.74), 393 (3.80), 417

$$
\text { (3.81), } 441 \text { (3.75), } 567(3.25), 612(3.39) \mathrm{nm} \text {. }
$$

CD (MeOH): $\lambda_{\max }([\Theta])=218$ (58278), 232 (-37856), 259 (35923), 309 (16845).

${ }^{1}$ H-NMR (600 MHz, Aceton-d $\left.{ }_{6}\right): \delta=3.06\left(\mathrm{dd}, J=4.5,15.0 \mathrm{~Hz}, 2 \mathrm{H}, 5-\mathrm{H}_{\mathrm{a}}\right.$ und 11- $\left.\mathrm{H}_{\mathrm{a}}\right), 3.17$ $\left(\mathrm{dd}, J=12.5,15.0 \mathrm{~Hz}, 2 \mathrm{H}, 5-\mathrm{H}_{\mathrm{b}}\right.$ und 11- $\left.\mathrm{H}_{\mathrm{b}}\right), 3.76$ (d, $J=9.0 \mathrm{~Hz}, 2 \mathrm{H}, 6 \mathrm{a}-\mathrm{H}$ und 12a-H), 476 (ddd, $J=4.5,9.0,12.0 \mathrm{~Hz}, 2 \mathrm{H}, 6-\mathrm{H}$ und 12-H), 6.82 (d, $J=8.5 \mathrm{~Hz}, 2 \mathrm{H}, 1-\mathrm{H}$ und 8-H), 8.15 (d, $J=8.5 \mathrm{~Hz}, 2 \mathrm{H}, 2-\mathrm{H}$ und 7-H), 12.08 (s, 2H, 3-OH und 9-OH).

${ }^{13}$ C-NMR (150.8 MHz, Aceton-d $\left.{ }_{6}\right): \delta=45.9$ (d, C-6a und C-12a), 47.8 (t, C-5 und C-11), 68.2 (d, C-6 und C-12), 115.5 (d, C-2 und C-7), 115.9 (s, C-3a und C-9a), 130.9 (s, C-6b und C-12b), 135.7 (d, C-1 und C-8), 143.8 (s, C-9b und C-12c), 161.0 (s, C-3 und C-9), 204.1 (s, C-4 und C-10).

\section{1,3,8-Naphthalentriol ${ }^{101}$ (65)}

$\mathrm{C}_{10} \mathrm{H}_{8} \mathrm{O}_{3}(176.17)$

leicht gelbes Öl

UV-löschend (254 nm)<smiles>Oc1cc(O)c2c(O)cccc2c1</smiles>

gelb fluoreszierend (366 nm)

$\mathbf{R}_{\mathbf{f}}$-Wert: 0.30 (Chloroform/Methanol 9:1)

Anfärbeverhalten: blau (Anisaldehyd) 
Smp.: $>200^{\circ} \mathrm{C}$

${ }^{1}$ H-NMR (600 MHz, Aceton-d $\left.)_{6}\right): \delta=6.45(\mathrm{~d}, J=2.0 \mathrm{~Hz}, 1 \mathrm{H}, 2-\mathrm{H}), 6.55(\mathrm{dd}, J=1.0,8.0 \mathrm{~Hz}$, $1 \mathrm{H}, 7-\mathrm{H}), 6.66(\mathrm{~d}, J=2.0 \mathrm{~Hz}, 1 \mathrm{H}, 4-\mathrm{H}), 7.06$ (dd, $J=1.0,8.0 \mathrm{~Hz}, 1 \mathrm{H}, 5-\mathrm{H}), 7.12$ (dd, $J=8.0$ $\mathrm{Hz}, 1 \mathrm{H}, 6-\mathrm{H}), 8.73$ (s, br, 1H, 3-OH ), 10.27 (s, br, 2H, 1-OH und 8-OH).

${ }^{13}$ C-NMR (150.8 MHz, Aceton-d ${ }_{6}$ ): $\delta=100.9$ (t, C-8), 101.3 (d, C-6b), 105.8 (d, C-7), 109.9 (s, C-6a), 118.0 (s, C-3a), 127.0 (d, C-11), 138.3 (s, C-9a), 154.3 (d, C-2), 155.7 (d, C-12b), $156.4(\mathrm{~d}, \mathrm{C}-5)$.

\section{2-Hydroxyjuglon ${ }^{102}$ (69)}

$\mathrm{C}_{10} \mathrm{H}_{6} \mathrm{O}_{4}(190.16)$

leicht gelbes Öl

UV-löschend (254 nm)<smiles>O=C1C=C(O)C(=O)c2c(O)cccc21</smiles>

$\mathbf{R}_{\mathbf{f}}$-Wert: 0.01 (Chloroform/Methanol 9:1)

Anfärbeverhalten: gelbbraun (Anisaldehyd)

Smp.: $>200^{\circ} \mathrm{C}$

EI-MS: $\mathrm{m} / \mathrm{z}(\%)=190(100)[\mathrm{M}]^{+}, 162(20), 121(60), 44(30)$.

HREI-MS: 190.0266 gefunden wie berechnet für $\mathrm{C}_{10} \mathrm{H}_{6} \mathrm{O}_{4}$.

IR(KBr): $\widetilde{v}=3432,2927,1617,1466,1384,1264,1021 \mathrm{~cm}^{-1}$.

UV: $(\mathrm{MeOH}): \quad\left[\lambda_{\max }(\lg \varepsilon)\right]=226(4.00), 260(3.88), 370(3.26), 462(3.15) \mathrm{nm}$;

$(\mathrm{MeOH} / \mathrm{HCl}): \quad\left[\lambda_{\max }(\lg \varepsilon)\right]=220(3.86), 242(\mathrm{sh}, 3.61) 283(3.66), 407(3.09) \mathrm{nm}$;

$(\mathrm{MeOH} / \mathrm{NaOH}):\left[\lambda_{\max }(\lg \varepsilon)\right]=228$ (3.94), $262(3.82), 375(3.23), 451(3.03) \mathrm{nm}$.

${ }^{1}$ H-NMR (600 MHz, CD $\left.{ }_{3} \mathrm{OD}\right): \delta=5.65(\mathrm{~s}, 1 \mathrm{H}, 3-\mathrm{H}), 7.10(\mathrm{dd}, J=1.0,8.0 \mathrm{~Hz}, 1 \mathrm{H}, 7-\mathrm{H})$, 7.40 (dd, $J=8.0 \mathrm{~Hz}, 1 \mathrm{H}, 6-\mathrm{H}), 7.47$ (dd, $J=1.0,8.0 \mathrm{~Hz}, 1 \mathrm{H}, 5-\mathrm{H})$.

${ }^{13}$ C-NMR (150.8 MHz, CD ${ }_{3}$ OD)*: $\delta=108$ (d, C-3), 117.1 (s, C-3a), 118.6 (d, C-6), 125.4 (d, C-7), 133.2 (d, C-5), 138(s, C-8a), 161.7 (s, C-8), 175 (s, C-2), 187.7 (s, C-1 und C-4).

* Die ${ }^{13} \mathrm{C}-\mathrm{NMR}-\mathrm{Daten}$ wurden aus Kopplungen im HSQC und HMBC abgeleitet. 
Sphaerolon $^{103}(70)$

$\mathrm{C}_{20} \mathrm{H}_{12} \mathrm{O}_{6}(348.32)$

leicht gelbes Öl

UV-löschend (254 nm)

$\mathbf{R}_{\mathbf{f}}$-Wert: 0.80 (Chloroform/Methanol 9:1)

Anfärbeverhalten: blaugrau (Anisaldehyd)

Smp.: $>200^{\circ} \mathrm{C}$

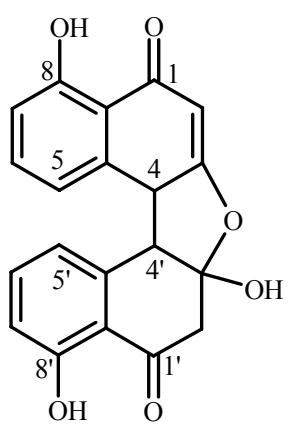

EI-MS: $\mathrm{m} / \mathrm{z}(\%)=348(100)[\mathrm{M}]^{+}, 176(30), 134(20)$.

HREI-MS: 348.0633 gefunden wie berechnet für $\mathrm{C}_{20} \mathrm{H}_{12} \mathrm{O}_{6}$.

IR(KBr): $\widetilde{v}=3434,1643,1606,1586,1475,1400,1236 . \mathrm{cm}^{-1}$.

UV: $(\mathrm{MeOH}): \quad\left[\lambda_{\max }(\lg \varepsilon)\right]=257 \quad(3.74), \quad 287 \quad(3.72), \quad 328 \quad(3.87), \quad 370 \quad$ (3.65), $425(3.67) \mathrm{nm}$;

$(\mathrm{MeOH} / \mathrm{HCl}): \quad\left[\lambda_{\max }(\lg \varepsilon)\right]=216(\mathrm{sh}, 4.19), 255$ (3.84), 285 (3.79), 332 (3.83), 370 (3.75), $424(3.77) \mathrm{nm}$;

$(\mathrm{MeOH} / \mathrm{NaOH}):\left[\lambda_{\max }(\lg \varepsilon)\right]=218$ (sh, 4.24), 235 (4.24), 349 (3.84), 373 (3.69), $584(3.58) \mathrm{nm}$.

${ }^{1}$ H-NMR (600 MHz, Aceton-d d $\left._{6}\right): \delta=3.54$ (s, 2H, 2'- $\mathrm{H}_{2}$ ), 5.96 (s, 1H, 2-H), 7.06 (dd, $J=1.0$, $8.0 \mathrm{~Hz}, 1 \mathrm{H}, 7-\mathrm{H}), 7.24$ (d, $\left.J=8.0 \mathrm{~Hz}, 1 \mathrm{H}, 5^{\prime}-\mathrm{H}\right), 7.51$ (dd, $\left.J=8.0 \mathrm{~Hz}, 1 \mathrm{H}, 6-\mathrm{H}\right), 7.84$ (d, $\left.J=8.0 \mathrm{~Hz}, 1 \mathrm{H}, 7^{\prime}-\mathrm{H}\right), 7.84\left(\mathrm{dd}, J=8.0 \mathrm{~Hz}, 1 \mathrm{H}, 6^{\prime}-\mathrm{H}\right), 8.10(\mathrm{dd}, J=1.0,8.0 \mathrm{~Hz}, 1 \mathrm{H}, 5-\mathrm{H})$, 12.36 (s, br, 1H, 8'-OH), 13,69 (s, 1H, 8-OH).

${ }^{13}$ C-NMR (150.8 MHz, Aceton-d 6 ): $\delta=51.1$ (t, C-2'), 101.5 (d, C-2), 113.7 (s, C-3'), 116.1 (s, C-8a), 117.5 (s, C-8a'), 117.7 (d, C-5), 121.3 (d, C-7), 121.5 (d, C-5'), 121.7 (d, C-7'), 123.9 (s, C-4), 131.2 (d, C-4a), 135.0 (s, C-4'a), 135.4 (d, C-6), 138.2 (d, C-6'), 147.2 (s, C-4'), 164.0 (s, C-8), 164.5 (s, C-8'), 174.5 (s, C-3), 192.9 (s, C-1), 202.3 (s, C-1').

Scytalone $^{104}(64)$

$\mathrm{C}_{10} \mathrm{H}_{10} \mathrm{O}_{4}(194.19)$

leicht gelbes Ö1<smiles>O=C1C[C@H](O)Cc2cc(O)cc(O)c21</smiles>

UV-löschend (254 nm)

gelb fluoreszierend $(366 \mathrm{~nm})$

$\mathbf{R}_{\mathbf{f}}$-Wert: 0.29 (Chloroform/Methanol 9:1)

Anfärbeverhalten: violett (Anisaldehyd) 
IR(KBr): $\widetilde{v}=3400,2930,1629,1497,1461,1366,1311,1266,1165,1070,1039 \mathrm{~cm}^{-1}$.

UV: $(\mathrm{MeOH}): \quad\left[\lambda_{\max }(\lg \varepsilon)\right]=221(4.24), 282(4.23), 315(\mathrm{sh}, 3.88) \mathrm{nm}$;

$(\mathrm{MeOH} / \mathrm{HCl}): \quad\left[\lambda_{\max }(\lg \varepsilon)\right]=221(4.24), 282(4.24), 309(\mathrm{sh}, 3.85) \mathrm{nm}$;

$(\mathrm{MeOH} / \mathrm{NaOH}):\left[\lambda_{\max }(\lg \varepsilon)\right]=210(4.25), 251(3.85), 330(4.51) \mathrm{nm}$.

${ }^{1}$ H-NMR (600 MHz, CD CD $_{3}$ ): $\delta=2.60\left(\mathrm{ddd}, J=7.5,17.0 \mathrm{~Hz}, 1 \mathrm{H}, 2-\mathrm{H}_{\mathrm{a}}\right), 2.83(\mathrm{dd}, J=4.0$, $\left.17.0 \mathrm{~Hz}, 1 \mathrm{H}, 2-\mathrm{H}_{\mathrm{a}}\right), 2.84\left(\mathrm{~d}, J=16.5 \mathrm{~Hz}, 1 \mathrm{H}, 4-\mathrm{H}_{\mathrm{a}}\right), 3.07\left(\mathrm{dd}, J=4.0,16.5 \mathrm{~Hz}, 1 \mathrm{H}, 4-\mathrm{H}_{\mathrm{b}}\right)$, 4.24 (ddd, $J=4.0,7.5 \mathrm{~Hz}, 1 \mathrm{H}, 3-\mathrm{H}), 6.09$ (d, $J=2.5 \mathrm{~Hz}, 1 \mathrm{H}, 7-\mathrm{H}), 6.21$ (d, $J=2.5 \mathrm{~Hz}, 1 \mathrm{H}$, 5-H).

${ }^{13}$ C-NMR (150.8 MHz, CD 3 OD): $\delta=39.1$ (t, C-4), 47.4 (t, C-2), 66.9 (d, C-3), 101.7 (d, C-7), 109.5 (d, C-5), 111.6 (s, C-8a), 146.0 (s, C-4a), 166.5 (s, C-8), 166.8 (s, C-6), 202.4 (s, C-1). 


\section{Aspergillus flavus Stamm Gö 100/4}

\subsection{Kultivierung und Isolierung der Metabolite}

Der Stamm Aspergillus flavus Gö 100/4 wurde wie unter B.II.3.1. beschrieben in Schüttelkolben (4 L, 40 x 100 mL) mit SGG als Nährmedium kultiviert und aufgearbeitet. Aus diesem Fermentationsansatz konnten insgesamt fünf Metabolite (91, 92, 93, 96 und 98) isoliert werden. Die Isolierung der Metabolite ist in Abbildung 69 dargestellt.

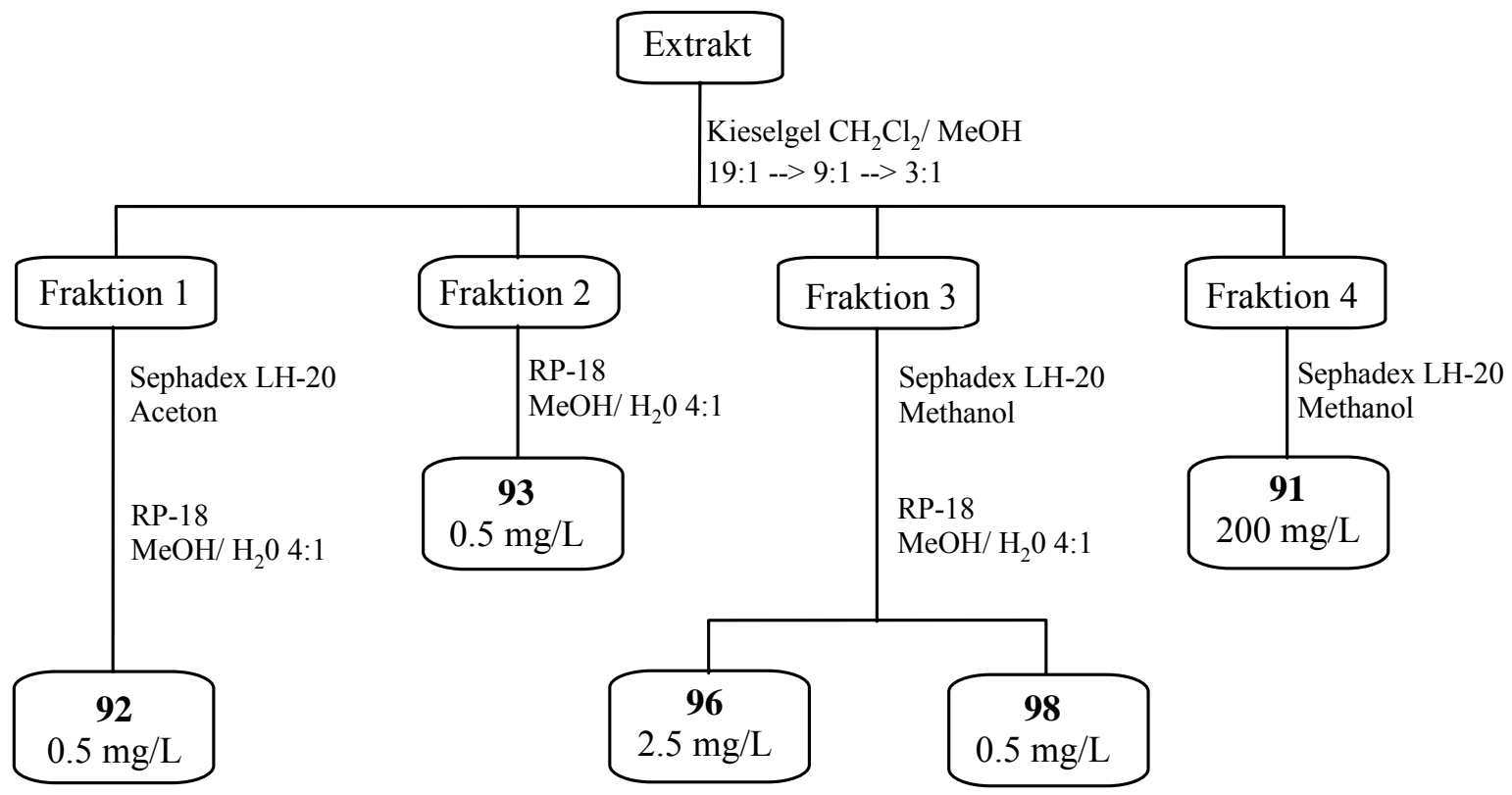

Abbildung 69: Aufreinigung des Extraktes aus der Fermentation von Aspergillus flavus Stamm Gö 100/4 in Schüttelkultur (Medium: SGG).

\subsection{Aufnahme einer Wachstumskurve}

Zur Aufnahme des zeitabhängigen Produktionsverlaufes von Gö 100/9 wurde der Stamm wie unter B.II.3.1. beschrieben kultiviert. Von der 6. bis zur 114. Stunde im Abstand von $6 \mathrm{~h}$, sowie nach 125, 134, 149 und 158 Stunden wurden zwei Kolben entnommen, mit derselben Menge Ethylacetat versetzt und 5 min mit dem Ultraturax behandelt. Anschließend wurde das Mycel abzentrifugiert und verworfen. Nach Abtrennen der organischen Phase wurde ein weiteres Mal mit Ethylacetat extrahiert und die organischen Phase vereinigt. Die nach dem Entfernen des Lösungsmittels erhaltenen Extrakte wurden dünnschichtchromatographisch und per HPLC (Säule 4, Programm D) analysiert. 


\subsection{Durchführung der Fütterungsexperimente}

Für die Fütterung von $\left[1-{ }^{13} \mathrm{C}\right]$ Acetat $(1.0 \mathrm{~g} / \mathrm{L}, 12.2 \mathrm{mmol})$ und $\left[1,2-{ }^{13} \mathrm{C}\right]$ Acetat $(0.5 \mathrm{~g} / 500 \mathrm{~mL}$, $6.1 \mathrm{mmol}$ ) wurde der Stamm wie unter B.II.3.1 beschrieben kultiviert. Die markierten Vorläufer wurden in sterilem Wasser gelöst und die Lösung auf einen pH-Wert von 5.0 eingestellt. Nach der 90. 96. 102. und 108. Stunde wurden die markierten Substanzen im Pulse-Feeding-Verfahren zu den Kulturen des Stammes gegeben.

Die Kulturen wurden nach weiteren zwölf Stunden (120 Stunden Fermentationsdauer) geerntet und wie unter B.II.3.1. beschrieben aufgearbeitet. Die Isolierung der Substanzen erfolgte wie unter B.II.6.1 beschrieben, wobei man sich auf die Arthrogrphol (96) enthaltende Fraktion beschränkte.

\subsection{Charakterisierung der Metabolite}

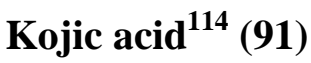

$\mathrm{C}_{6} \mathrm{H}_{6} \mathrm{O}_{4}(142.11)$

farbloser Feststoff

UV-löschend (254 nm)

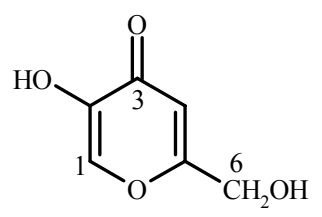

$\mathbf{R}_{\mathbf{f}}$-Wert: 0.1 (Chloroform/Methanol 9:1)

Smp.: $147^{\circ} \mathrm{C}$

EI-MS: m/z (\%) = $142(90)[\mathrm{M}]^{+}, 113$ (48), 69 (100), 57 (62), 55(44), 42(24).

HREI-MS: 142.0266 gefunden wie berechnet für $\mathrm{C}_{6} \mathrm{H}_{6} \mathrm{O}_{4}$.

IR(KBr): $\widetilde{v}=3422,1616,1469,1348,1226,1140,1072 \mathrm{~cm}^{-1}$.

UV: $(\mathrm{MeOH}): \quad\left[\lambda_{\max }(\lg \varepsilon)\right]=217(4.05), 270(3.84) \mathrm{nm}$;

$\mathrm{MeOH} / \mathrm{HCl}): \quad\left[\lambda_{\max }(\lg \varepsilon)\right]=217(4.10), 270(3.90) \mathrm{nm}$;

$(\mathrm{MeOH} / \mathrm{NaOH}):\left[\lambda_{\max }(\lg \varepsilon)\right]=227(4.27), 314$ (3.68) $\mathrm{nm}$.

${ }^{1}$ H-NMR (600 MHz, Pyridin-d $\left.{ }_{5}\right): \delta=4.62\left(\mathrm{~s}, 2 \mathrm{H}, 6-\mathrm{H}_{2}\right), 6.92$ (s, 1H, 4-H), 8.09 (s, 1H, $1-\mathrm{H})$.

${ }^{13}$ C-NMR (150.8 MHz, Pyridin-d $\left.{ }_{5}\right): \delta=60.5$ (t, C-6), 110.5 (d, C-4), 139.4 (d, C-1), 147.6 (s, C-2), 169.0 (s, C-5), 175.4 (s, C-3). 
Miyakamide ${A_{2}}^{116}(92)$

$\mathrm{C}_{31} \mathrm{H}_{32} \mathrm{~N}_{4} \mathrm{O}_{3}(508.62)$

leicht gelbes Öl

UV-löschend (254 nm)

$\mathbf{R}_{\mathbf{f}}$-Wert: 0.78 (Chloroform/Methanol 9:1)

Anfärbeverhalten: hellbraun (Anisaldehyd)

Smp.: $100^{\circ} \mathrm{C}$ (Zersetzung)

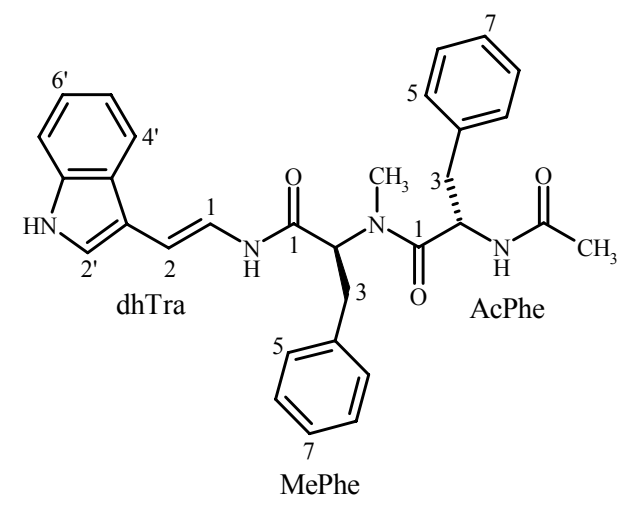

ESI-MS (positive Ionen): $\mathrm{m} / \mathrm{z}(\%)=531(10)[\mathrm{M}+\mathrm{Na}]^{+}, 1039(100)[2 \mathrm{M}+\mathrm{Na}]^{+}$;

(negative Ionen): $\mathrm{m} / \mathrm{z}(\%)=507(100)[\mathrm{M}-\mathrm{H}]^{-}$.

HRESI-MS: 531.23679 gefunden für $\left[\mathrm{C}_{31} \mathrm{H}_{32} \mathrm{~N}_{4} \mathrm{O}_{3} \mathrm{Na}\right]^{+}$.

IR(KBr): $\widetilde{v}=3445,2922,1654,1636,1560,1458,1106 \mathrm{~cm}^{-1}$.

UV: $(\mathrm{MeOH}): \quad\left[\lambda_{\max }(\lg \varepsilon)\right]=307(4.12) \mathrm{nm}$.

CD (MeOH): $\lambda_{\max }([\Theta])=208$ (20912), 234 (-13628), 267 (-3997).

${ }^{1}$ H-NMR (600 MHz, $\left.\left.\mathbf{C D}_{2} \mathbf{C l}_{2}\right): \delta=1.87\left(\mathrm{~s}, 3 \mathrm{H}, \mathrm{Ac}-\mathrm{CH}_{3}\right), 1.87(\mathrm{~m}, 1 \mathrm{H}, \text { AcPhe 3-H })_{\mathrm{a}}\right), 2.64$ $\left(\mathrm{dd}, J=10.0,14.0 \mathrm{~Hz}, 1 \mathrm{H}\right.$, AcPhe 3- $\left.\mathrm{H}_{\mathrm{b}}\right), 2.76\left(\mathrm{dd}, J=9.5,14.0 \mathrm{~Hz}, 1 \mathrm{H}\right.$, MePhe 3- $\left.\mathrm{H}_{\mathrm{a}}\right), 2.99$ (s, 3H, MePhe $\left.\mathrm{NCH}_{3}\right), 3.30\left(\mathrm{dd}, J=5.0,14.0 \mathrm{~Hz}, 1 \mathrm{H}\right.$, MePhe 3- $\left.\mathrm{H}_{\mathrm{b}}\right), 4.81(\mathrm{~m}, 1 \mathrm{H}$, AcPhe 2-H), 5.04 (dd, $J=5.0,9.5 \mathrm{~Hz}, 1 \mathrm{H}$, MePhe 2-H), 6.51 (d, $J=15.0 \mathrm{~Hz}, 1 \mathrm{H}$, dhTra 2-H), 7.07 (d, $J=7.5 \mathrm{~Hz}, 2 \mathrm{H}$, AcPhe 5-H und 9-H), 7.11 (m, 1H, dhTra 5'-H), 7.14 (m, 1H, dhTra 2'-H), 7.18 (m, 1H, dhTra 6'-H), 7.19 (m, 1H, AcPhe 7-H), 7.23 (m, 3H, MePhe 5-H, 7-H und 9-H), 7.24 (s, br., 1H, AcPhe NH), 7.29 (m, 4H, MePhe 6-H und 8-H und AcPhe 6-H und 8-H), $7.35(\mathrm{~d}, J=8.0 \mathrm{~Hz}, 1 \mathrm{H}$, dhTra 7'-H), 7.48 (dd, $J=10.0,15.0 \mathrm{~Hz}, 1 \mathrm{H}$, dhTra 1-H), 7.75 (d, $J=8.0 \mathrm{~Hz}, 1 \mathrm{H}$, dhTra 4'-H), 8.69 (s, 1H, dhTra 1'-NH), 10.02 (d, $J=10.0 \mathrm{~Hz}, 1 \mathrm{H}$, dhTra $\mathrm{NH})$.

${ }^{13}$ C-NMR (150.8 MHz, $\mathbf{C D}_{2} \mathbf{C l}_{2}$ ): $\delta=22.7$ (q, Ac-CH 3 ), 29.6 (q, MePhe NMe), 34.4 (t, MePhe C-3), 36.8 (t, AcPhe C-3), 50.3 (d, AcPhe C-2), 63.0 (d, MePhe C-2), 107.6 (d, dhTra C-2), 111.7 (d, dhTra C-7'), 113.1 (s, dhTra C-3'), 120.0 (d, dhTra C-4'), 120.2 (d, dhTra C-5'), 120.5 (d, dhTra C-1), 122.5 (d, dhTra C-6'), 122.8 (d, dhTra C-2'), 125.5 (s, dhTra C-3a'), 127.2 (d, MePhe C-7 und AcPhe C-7), 128.8 (d, AcPhe C-6 und C-8), 128.9 (d, MePhe C-6 und C-8), 129.3 (d, AcPhe C-5 und C-9), 129.7 (d, MePhe C-5 und C-9), 137.0 (s, dhTra C-7a' und AcPhe C-4), 138.0 (s, MePhe C-4), 165.7 (s, MePhe C-1), 172.2 (s, Ac-CO), 172.9 (s, AcPhe C-1). 
Miyakamide $B_{1}{ }^{116}(93)$

$\mathrm{C}_{31} \mathrm{H}_{32} \mathrm{~N}_{4} \mathrm{O}_{4}(524.62)$

leicht gelbes Öl

UV-löschend (254 nm)

Rf-Wert: 0.45 (Chloroform/Methanol 9:1)

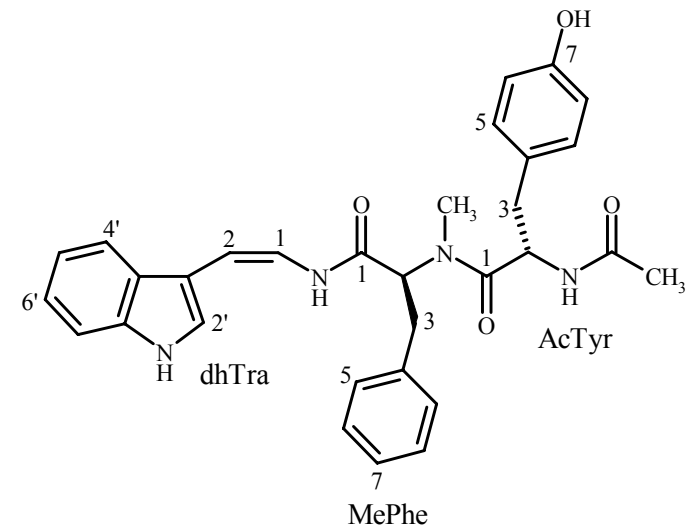

Anfärbeverhalten: hellbraun (Anisaldehyd)

Smp.: $104^{\circ} \mathrm{C}$ (Zersetzung)

ESI-MS (positive Ionen): $\mathrm{m} / \mathrm{z}(\%)=547(90)[\mathrm{M}+\mathrm{Na}]^{+}, 1071(100)[2 \mathrm{M}+\mathrm{Na}]^{+}$;

(negative Ionen): $\mathrm{m} / \mathrm{z}(\%)=523(100)[\mathrm{M}-\mathrm{H}]^{-}, 1047$ (40) [2M-H] .

HRESI-MS: 525.24949 gefunden für $\left[\mathrm{C}_{31} \mathrm{H}_{33} \mathrm{~N}_{4} \mathrm{O}_{3}\right]^{+}$und 547.23140 gefunden für

$$
\left[\mathrm{C}_{31} \mathrm{H}_{32} \mathrm{~N}_{4} \mathrm{O}_{3} \mathrm{Na}\right]^{+} \text {. }
$$

IR(KBr): $\widetilde{v}=3435,2925,1635,1055 \mathrm{~cm}^{-1}$.

UV: $(\mathrm{MeOH}): \quad\left[\lambda_{\max }(\lg \varepsilon)\right]=225(4.29), 285(3.74) \mathrm{nm}$.

CD (MeOH): $\lambda_{\max }([\Theta])=207$ (11617), 234 (-13353), 312 (3451).

${ }^{1}$ H-NMR (600 MHz, $\left.\mathbf{C D}_{2} \mathbf{C l}_{2}\right): \delta=1.85$ (s, 3H, Ac-CH $), 2.68$ (s, 3H, MePhe $\mathrm{NCH}_{3}$ ), 2.70

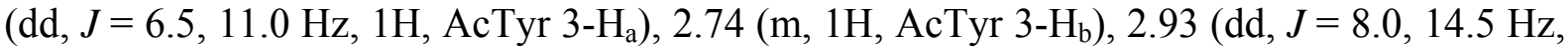
1H, MePhe 3- $\mathrm{H}_{\mathrm{a}}$ ), 3.32 (dd, $J=8.0,14.5 \mathrm{~Hz}, 1 \mathrm{H}$, MePhe 3- $\mathrm{H}_{\mathrm{b}}$ ), 4.97 (ddd, $J=6.0,8.0,14.0$ Hz, 1H, AcTyr 2-H), 5.16 (m, 1H, MePhe 2-H), 6.02 (d, J=9.0 Hz, 1H, dhTra 2-H), 6.10 (s, br., 1H, AcTyr NH), 6.54 (d, $J=8.5 \mathrm{~Hz}, 2 \mathrm{H}$, AcTyr 6-H und 8-H), 6.79 (d, J = 8.5 Hz, 2H, AcTyr 5-H und 9-H), 6.88 (dd, $J=9.0,11.5 \mathrm{~Hz}, 1 \mathrm{H}$, dhTra 1-H), 7.13 (m, 1H, dhTra 5'-H), $7.20(\mathrm{~m}, 1 \mathrm{H}$, dhTra 6'-H), $7.26(\mathrm{~m}, 1 \mathrm{H}$, MePhe 7-H), 7.17 (d, J=7.0 Hz, 1H, MePhe 5-H und 9-H), 7.20 (m, 2H, MePhe 6-H und 8-H), 7.31 (d, $J=2.5 \mathrm{~Hz}, 1 \mathrm{H}$, dhTra 2'-H), 7.40 (d, $\left.J=8.0 \mathrm{~Hz}, 1 \mathrm{H}, \mathrm{dhTra} 7^{\prime}-\mathrm{H}\right), 7.60$ (d, $J=8.0 \mathrm{~Hz}, 1 \mathrm{H}$, dhTra $\left.4^{\prime}-\mathrm{H}\right), 8.12$ (d, $J=11.5 \mathrm{~Hz}, 1 \mathrm{H}$, dhTra NH), 8.58 (s, br., 1H, dhTra 1'-NH).

${ }^{13}$ C-NMR (150.8 MHz, $\mathbf{C D}_{2} \mathbf{C l}_{2}$ )*: $\delta=22.3$ (q, Ac- $\mathrm{CH}_{3}$ ), 29.7 (q, MePhe NMe), 33.3 (t, MePhe C-3), 38.0 (t, AcTyr C-3), 50.7 (d, AcTyr C-2), 62.1 (d, MePhe C-2), 102.7 (d, dhTra C-2), 111.1 (s, dhTra C-3'), 111.4 (d, dhTra C-7'), 115.4 (d, AcTyr C-6 und C-8), 118.8 (d, dhTra C-4'), 118.9 (d, dhTra C-1), 120.0 (d, dhTra C-5'), 122.2 (d, dhTra C-2'),122.9 (d, dhTra C-6'), 126.9 ( dhTra C-3a' und MePhe C-7), 127.4 (s, AcTyr C-4), 129.0 (d, MePhe C-5 und C-9), 129.6 (d, MePhe C-6 und C-8), 130.3 (d, AcTyr C-5 und C-9), 135.8 (s, dhTra C-7a'), 137.0 (s, MePhe C-4), 155.1 (s, AcTyr C-7), 166.3 (s, MePhe C-1), 169.3 (s, Ac-CO), 173.0 (s, AcTyr C-1).

* Die ${ }^{13} \mathrm{C}$-NMR-Daten wurden aus Kopplungen im HSQC und HMBC abgeleitet. 
Arthrographol $^{113,117}(96)$

$\mathrm{C}_{13} \mathrm{H}_{14} \mathrm{O}_{3}(218.25)$

leicht gelbes Öl

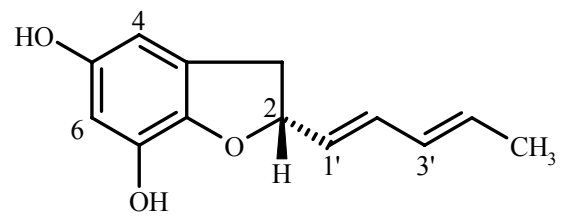

UV-löschend (254 nm)

$\mathbf{R}_{\mathbf{f}}$-Wert: 0.28 (Chloroform/Methanol 9:1)

Anfärbeverhalten: rot-braun (Anisaldehyd)

Smp.: $121^{\circ} \mathrm{C}$

EI-MS: $\mathrm{m} / \mathrm{z}(\%)=218(100)[\mathrm{M}]^{+}, 203(24), 163(60), 138(60), 110(20)$.

HREI-MS: 218.0944 gefunden wie berechnet für $\mathrm{C}_{13} \mathrm{H}_{14} \mathrm{O}_{3}$.

IR(KBr): $\widetilde{v}=3446,1653,1696,1560,1384 \mathrm{~cm}^{-1}$.

UV: $(\mathrm{MeOH}): \quad\left[\lambda_{\max }(\lg \varepsilon)\right]=224(3.61), 287(3.03) \mathrm{nm}$.

CD (MeOH): $\lambda_{\max }([\Theta])=207$ (1317), 234 (-1951).

${ }^{1}$ H-NMR (600 MHz, $\left.\mathbf{C D C l}_{3}\right): \delta=1.80\left(\mathrm{~d}, J=7.0 \mathrm{~Hz}, 3 \mathrm{H}, 5^{\prime}-\mathrm{H}_{3}\right), 3.00(\mathrm{dd}, J=8.5,15.5 \mathrm{~Hz}$, $\left.1 \mathrm{H}, 3-\mathrm{H}_{\mathrm{a}}\right), 3.32\left(\mathrm{dd}, J=8.5,15.5 \mathrm{~Hz}, 1 \mathrm{H}, 3-\mathrm{H}_{\mathrm{b}}\right), 5.22(\mathrm{ddd}, J=8.0,8.5 \mathrm{~Hz} 1 \mathrm{H}, 2-\mathrm{H}), 5.74$ (dd, $J=8.0,15.5 \mathrm{~Hz}, 1 \mathrm{H}, 1$ '-H), 5.82 (dq, $\left.J=7.0,15.0 \mathrm{~Hz}, 1 \mathrm{H}, 4^{\prime}-\mathrm{H}\right), 6.09$ (dd, $J=10.5,15.0$ $\mathrm{Hz}, 1 \mathrm{H}, 3$ '-H), 6.28 (d, J=2.0 Hz, 1H, 4-H), 6.30 (d, J=2.0 Hz, 1H, 6-H), 6.32 (dd, $J=10.5$, $15.5 \mathrm{~Hz}, 1 \mathrm{H}, 2$ '-H).

${ }^{13}$ C-NMR (150.8 MHz, $\mathbf{C D C l}_{3}$ ): $\delta=18.2$ (q, C-5'), 37.3 (t, C-3), 84.6 (d, C-2), 102.5 (d, C-6), 103.6 (d, C-4), 128.1 (d, C-3a), 128.5 (d, C-1'), 130.3 (d, C-3'), 131.8 (d, C-4'), 133.4 (d, C-2'), 140.1 (d, C-7), 140.2 (s, C-7a), 150.4 (s, C-5).

\section{5,7-Di-O-acetylarthrographol (96b)}

$2.0 \mathrm{mg}$ Arthrographol (96) wurden gemäß AAV 1 acetyliert. Nach Chromatographie an

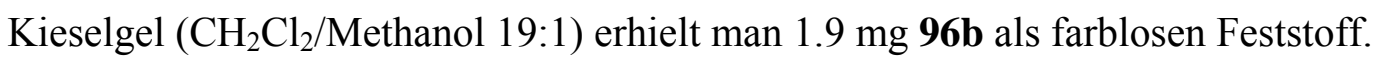

${ }^{1}$ H-NMR (600 MHz, $\mathbf{C D C l}_{3}$ ): $\delta=1.75$ (d, $\left.J=7.0 \mathrm{~Hz}, 3 \mathrm{H}, 5^{\prime}-\mathrm{H}_{3}\right), 2.23$ (s, 3H, Acetyl-CH ${ }_{3}$ ), $2.26\left(\mathrm{~s}, 3 \mathrm{H}\right.$, Acetyl- $\left.\mathrm{CH}_{3}\right), 3.01\left(\mathrm{dd}, J=8.0,16.0 \mathrm{~Hz}, 1 \mathrm{H}, 3-\mathrm{H}_{\mathrm{a}}\right), 3.36(\mathrm{dd}, J=8.5,16.0 \mathrm{~Hz}$, $\left.1 \mathrm{H}, 3-\mathrm{H}_{\mathrm{b}}\right), 5.26$ (ddd, $\left.J=8.0,8.5 \mathrm{~Hz} 1 \mathrm{H}, 2-\mathrm{H}\right), 5.67$ (dd, $J=8.0,15.5 \mathrm{~Hz}, 1 \mathrm{H}, 1$ '-H), 5.76 (dq, $\left.J=7.0,15.0 \mathrm{~Hz}, 1 \mathrm{H}, 4^{\prime}-\mathrm{H}\right), 6.03$ (dd, $\left.J=10.0,15.0 \mathrm{~Hz}, 1 \mathrm{H}, 3^{\prime}-\mathrm{H}\right), 6.26$ (dd, $J=10.0$, $\left.15.5 \mathrm{~Hz}, 1 \mathrm{H}, 2^{\prime}-\mathrm{H}\right), 6.65$ (d, $\left.J=2.5 \mathrm{~Hz}, 1 \mathrm{H}, 4-\mathrm{H}\right), 6.77$ (d, $\left.J=2.5 \mathrm{~Hz}, 1 \mathrm{H}, 6-\mathrm{H}\right)$.

${ }^{13}$ C-NMR (150.8 MHz, $\mathbf{C D C l}_{3}$ ): $\delta=18.2$ (q, C-5'), 20.7 (q, Acetyl-CH ${ }_{3}$ ), 21.0 (q, Acetyl$\mathrm{CH}_{3}$ ), 36.6 (t, C-3), 85.3 (d, C-2), 115.0 (d, C-4 oder C-6), 115.8 (d, C-4 oder C-6), 128.1 (d, 
C-1'), 129.5 (d, C-3a), 130.3 (d, C-3'), 131.9 (d, C-4'), 133.4 (s, C-7), 133.5 (d, C-2'), 143.7 (s, C-5), 148.2 (s, C-7a), 168.3 (s, Acetyl-CO), 169.7 (s, Acetyl-CO).

\section{Arthrolacton (98)}

$\mathrm{C}_{14} \mathrm{H}_{14} \mathrm{O}_{4}(246.26)$

leicht gelbes Öl

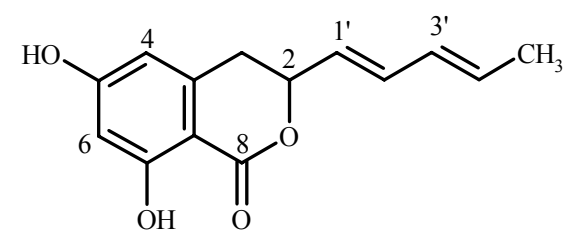

UV-löschend (254 nm)

$\mathbf{R}_{\mathbf{f}}$-Wert: 0.29 (Chloroform/Methanol 9:1)

Anfärbeverhalten: braun (Anisaldehyd)

ESI-MS (negative Ionen): $\mathrm{m} / \mathrm{z}(\%)=245(100)[\mathrm{M}-\mathrm{H}]^{-}, 491(15)[2 \mathrm{M}-\mathrm{H}]^{-}$.

HRESI-MS: 247.09639 gefunden für $\left[\mathrm{C}_{14} \mathrm{H}_{15} \mathrm{O}_{4}\right]^{+}$.

${ }^{1}$ H-NMR (600 MHz, $\mathbf{C D}_{2} \mathbf{C l}_{2}$ ): $\delta=1.77$ (d, $J=6.5 \mathrm{~Hz}, 3 \mathrm{H}, 5^{\prime}-\mathrm{H}_{3}$ ), 2.94 (m, 2H, 4- $\mathrm{H}_{2}$ ), 5.01 (m, 1H, 3-H), 5.66 (dd, $J=7.0,15.0 \mathrm{~Hz}, 1 \mathrm{H}, 1$ '-H), 5.83 (dq, $\left.J=6.5,15.0 \mathrm{~Hz}, 1 \mathrm{H}, 4^{\prime}-\mathrm{H}\right)$, 6.07 (dd, $\left.J=10.5,15.0 \mathrm{~Hz}, 1 \mathrm{H}, 3^{\prime}-\mathrm{H}\right), 6.23$ (d, $\left.J=2.0 \mathrm{~Hz}, 1 \mathrm{H}, 5-\mathrm{H}\right), 6.28$ (d, $J=2.0 \mathrm{~Hz}, 1 \mathrm{H}$, 7-H), 6.32 (dd, $\left.J=10.5,15.0 \mathrm{~Hz}, 1 \mathrm{H}, 2^{\prime}-\mathrm{H}\right)$.

${ }^{13}$ C-NMR (150.8 MHz, $\mathbf{C D}_{2} \mathbf{C l}_{2}$ ): $\delta=18.2$ (q, C-5'), 33.7 (t, C-4), 79.5 (d, C-3), 102.0 (d, C-7), 102.3 (s, C-8a), 106.8 (d, C-5), 126.2 (d, C-1'), 130.3 (d, C-3'), 133.0 (d, C-4'), 134.6 (d, C-2'), 142.0 (d, C-4a), 162.9 (s, C-6), 164.6 (d, C-8), 169.8 (s, C-1).

\section{5,7-Di-O-acetylarthrolacton (98b)}

$2.0 \mathrm{mg}$ Arthrolacton (98) wurden gemäß AAV 1 acetyliert. Nach Chromatographie an Kieselgel $\left(\mathrm{CH}_{2} \mathrm{Cl}_{2} /\right.$ Methanol 19:1) erhielt man $1.9 \mathrm{mg}$ 98b als farblosen Feststoff.

${ }^{1}$ H-NMR (600 MHz, $\mathbf{C D}_{2} \mathbf{C l}_{2}$ ): $\delta=1.77$ (d, $\left.J=7.0 \mathrm{~Hz}, 3 \mathrm{H}, 5^{\prime}-\mathrm{H}_{3}\right), 2.29$ (s, 3H, Acetyl-CH ${ }_{3}$ ), $2.31\left(\mathrm{~s}, 3 \mathrm{H}\right.$, Acetyl- $\left.\mathrm{CH}_{3}\right), 3.00\left(\mathrm{dd}, J=3.5,16.0 \mathrm{~Hz}, 1 \mathrm{H}, 4-\mathrm{H}_{\mathrm{a}}\right), 3.06(\mathrm{dd}, J=10.0,16.0 \mathrm{~Hz}$, $1 \mathrm{H}, 4-\mathrm{H}_{\mathrm{b}}$ ), 4.97 (ddd, $\left.J=3.5,7.0,10.0 \mathrm{~Hz} 1 \mathrm{H}, 3-\mathrm{H}\right), 5.64$ (dd, $J=7.0,15.0 \mathrm{~Hz}, 1 \mathrm{H}, 1$ '- $\mathrm{H}$ ), $5.83\left(\mathrm{dq}, J=6.5,15.0 \mathrm{~Hz}, 1 \mathrm{H}, 4^{\prime}-\mathrm{H}\right), 6.07$ (dd, $\left.J=10.5,15.0 \mathrm{~Hz}, 1 \mathrm{H}, 3{ }^{\prime}-\mathrm{H}\right), 6.33$ (dd, $\left.J=10.5,15.0 \mathrm{~Hz}, 1 \mathrm{H}, 2^{\prime}-\mathrm{H}\right), 6.86$ (d, $\left.J=2.0 \mathrm{~Hz}, 1 \mathrm{H}, 5-\mathrm{H}\right), 6.96$ (d, $\left.J=2.0 \mathrm{~Hz}, 1 \mathrm{H}, 7-\mathrm{H}\right)$. 


\section{Chaetomium sp. Stamm Gö 100/9}

\subsection{Kultivierung und Isolierung der Metabolite}

Der Stamm Aspergillus flavus Gö 100/9 wurde wie unter B.II.3.1. beschrieben in Schüttelkolben (4 L, 40 x 100 mL) mit SGG als Nährmedium kultiviert und aufgearbeitet. Aus diesem Fermentationsansatz konnten neun Metabolite (100, 101, 102, 104, 105, 106, 107, 108, 119) isoliert werden. Die Isolierung der Metabolite ist in Abbildung 43 (Seite 80) dargestellt. Durch Fütterung von Acetat (siehe B.II.7.3.) konnte die Ausbeute an 119 auf $135 \mathrm{mg} / \mathrm{L}$ gesteigert werden, daneben konnten mit Hilfe der präparativen HPLC (Säule 2, Programm B) die Metabolite 120, 121 und 125 in Ausbeuten von 10.5, 7.8 und $3.5 \mathrm{mg} / \mathrm{L}$ erhalten werden.

\subsection{Aufnahme einer Wachstumskurve}

Zur Aufnahme des zeitabhängigen Produktionsverlaufes von Gö 100/9 wurde der Stamm wie unter B.II.3.1. beschrieben kultiviert. Ab der 24. Stunde wurden alle $6 \mathrm{~h}$ zwei Kolben entnommen, mit derselben Menge Ethylacetat versetzt und 5 min mit dem Ultraturax behandelt. Anschließend wurde das Mycel abzentrifugiert und verworfen. Nach Abtrennen der organischen Phase wurde ein weiteres Mal mit Ethylacetat extrahiert und die organischen Phase vereinigt. Die nach dem Entfernen des Lösungsmittels erhaltenen Extrakte wurden dünnschichtchromatographisch und per HPLC (Säule 3, Programm F) analysiert.

\subsection{Durchführung der Fütterungsexperimente}

Für die Fütterung von $\left[1-{ }^{13} \mathrm{C}\right]$ Acetat $(1.0 \mathrm{~g} / \mathrm{L}, 12.2 \mathrm{mmol})$ und $\left[1-{ }^{13} \mathrm{C}\right]$ Glucose $(0.5 \mathrm{~g} / \mathrm{L}$, $2.8 \mathrm{mmol}$ ) wurde der Stamm wie unter B.II.3.1 beschrieben kultiviert. Die markierten Vorläufer wurden in sterilem Wasser gelöst und die Lösung auf einen pH-Wert von 7.0 eingestellt. Nach der 78. 84. 90. und 96. Stunde wurden die markierten Substanzen im PulseFeeding Verfahren $\mathrm{zu}$ den Kulturen des Stammes gegeben. Bei der Fütterung mit $\left[1-{ }^{13} \mathrm{C}\right]$ Glucose wurde zusätzlich unmarkiertes Acetat $(1 \mathrm{~g} / \mathrm{L}, 12.2 \mathrm{mmol})$ gefüttert.

Die Kulturen wurden nach weiteren zwölf Stunden (108 Stunden Fermentationsdauer) geerntet und wie unter B.II.3.1. beschrieben aufgearbeitet. Die Isolierung der Substanzen erfolgte wie unter B.II.7.1 beschrieben mit dem Unterschied, dass die Fraktion, welche die 
Substanzen 101, 102 und 105 enthielt, nach AAV1 acetyliert und dann mittels der präparativen HPLC (Säule 2, Programm C) aufgereinigt wurde.

\subsection{Charakterisierung der Metabolite}

Orsellinsäure $^{120}(100)$

$\mathrm{C}_{8} \mathrm{H}_{8} \mathrm{O}_{4}(168.15)$

weißer Feststoff

UV-löschend (254 nm)

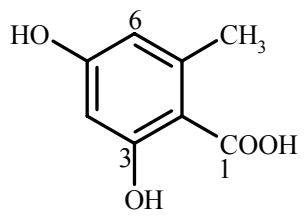

$\mathbf{R}_{\mathbf{f}}$-Wert: 0.05 (Chloroform/Methanol 9:1)

Anfärbeverhalten: rot (Anisaldehyd)

Smp.: $139^{\circ} \mathrm{C}$

EI-MS: m/z (\%) = $168(70)[\mathrm{M}]^{+}, 150(100), 122(50), 94(20), 66(10)$.

HREI-MS: 168.0423 gefunden wie berechnet für $\mathrm{C}_{8} \mathrm{H}_{8} \mathrm{O}_{4}$.

IR(KBr): $\widetilde{v}=3173,2922,2851,1659,1612,1581,1469,1393,1348,1280,1141 \mathrm{~cm}^{-1}$.

UV: $(\mathrm{MeOH}): \quad\left[\lambda_{\max }(\lg \varepsilon)\right]=217(4.15), 270(3.95) \mathrm{nm}$;

$\mathrm{MeOH} / \mathrm{HCl}): \quad\left[\lambda_{\max }(\lg \varepsilon)\right]=217(4.11), 271(3.91) \mathrm{nm} ;$

$(\mathrm{MeOH} / \mathrm{NaOH}):\left[\lambda_{\max }(\lg \varepsilon)\right]=227(4.34), 314(3.76) \mathrm{nm}$.

${ }^{1}$ H-NMR (600 MHz, CD MD): $\delta_{3}=2.45$ (s, 3H, 8- $\mathrm{H}_{3}$ ), 6.14 (d, $\left.J=2.0 \mathrm{~Hz}, 1 \mathrm{H}, 4-\mathrm{H}\right), 6.17$ (d, $J=2.0 \mathrm{~Hz}, 1 \mathrm{H}, 6-\mathrm{H})$.

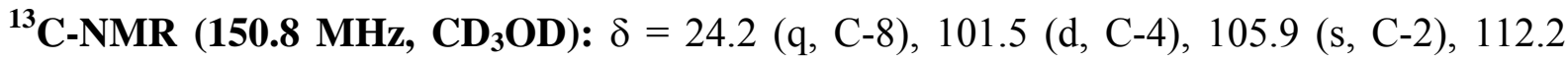
(d, C-6), 145.3 (s, C-7), 163.2 (s, C-5), 166.6 (s, C-3), 175.2 (s, C-1).

Orsellide A (101) und B (102)

(Charakterisierung des $3: 2$ Gemisches)

$\mathrm{C}_{15} \mathrm{H}_{18} \mathrm{O}_{8}$ (326.30)

farbloses Öl

UV-löschend (254 nm)

$\mathbf{R}_{\mathbf{f}}$-Wert: 0.64 (Chloroform/Methanol 9:1)

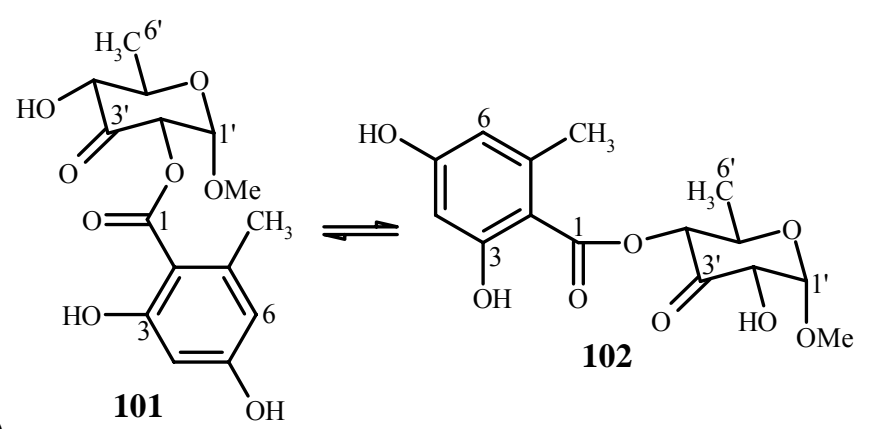

Anfärbeverhalten: rotbraun (Anisaldehyd)

ESI-MS (positive Ionen): $\mathrm{m} / \mathrm{z}(\%)=349(30)[\mathrm{M}+\mathrm{Na}]^{+}, 675(100)[2 \mathrm{M}+\mathrm{Na}]^{+}$;

(negative Ionen): $\mathrm{m} / \mathrm{z}(\%)=325(95)[\mathrm{M}-\mathrm{H}]^{-}, 651(100)[2 \mathrm{M}-\mathrm{H}]^{-}$. 
HRESI-MS: 349.08939 gefunden für $\left[\mathrm{C}_{15} \mathrm{H}_{18} \mathrm{O}_{8} \mathrm{Na}\right]^{+}$.

IR(KBr): $\widetilde{v}=3429,2928,2864$ (sh), 1719, 1631, 1457, 1341, 1241, 1169, $1097 \mathrm{~cm}^{-1}$.

UV: $(\mathrm{MeOH}): \quad\left[\lambda_{\max }(\lg \varepsilon)\right]=216(4.48), 261(4.17), 299(3.83) \mathrm{nm}$;

$\mathrm{MeOH} / \mathrm{HCl}): \quad\left[\lambda_{\max }(\lg \varepsilon)\right]=216(4.52), 262(4.20), 299(3.85) \mathrm{nm}$;

$(\mathrm{MeOH} / \mathrm{NaOH}):\left[\lambda_{\max }(\lg \varepsilon)\right]=210(4.44), 232(\mathrm{sh}, 4.19), 304(4.29) \mathrm{nm}$.

$[\alpha]_{D}^{20}=+58^{\circ}(\mathrm{c}=0.1$, Methanol $)$.

CD (MeOH): $\lambda_{\max }([\Theta])=268$ (2089).

Orsellide A (101):

${ }^{1}$ H-NMR (600 MHz, $\left.\mathbf{C D C l}_{3}\right): \delta=1.48\left(\mathrm{~d}, J=6.5 \mathrm{~Hz}, 3 \mathrm{H}, 6{ }^{\prime}-\mathrm{H}_{3}\right), 2.51$ (s, 3H, 8- $\left.\mathrm{H}_{3}\right), 3.41$ (s, $3 \mathrm{H}, 1$ ' $\left.-\mathrm{OCH}_{3}\right), 3.81\left(\mathrm{dq}, J=6.5,9.5 \mathrm{~Hz}, 1 \mathrm{H}, 5^{\prime}-\mathrm{H}\right), 3.99$ (dd, $\left.J=1.5,9.5 \mathrm{~Hz}, 1 \mathrm{H}, 4^{\prime}-\mathrm{H}\right), 5.22$ (d, $\left.J=4.5 \mathrm{~Hz}, 1 \mathrm{H}, 1^{\prime}-\mathrm{H}\right), 5.65$ (dd, $\left.J=1.5,4.5 \mathrm{~Hz} 1 \mathrm{H}, 2^{\prime}-\mathrm{H}\right), 6.22(\mathrm{~d}, J=2.0 \mathrm{~Hz}, 1 \mathrm{H}, 4-\mathrm{H})$, $6.24(\mathrm{~d}, J=2.0 \mathrm{~Hz}, 1 \mathrm{H}, 6-\mathrm{H}), 11.02(\mathrm{~s}, 1 \mathrm{H}, 3-\mathrm{OH})$.

${ }^{13}$ C-NMR (150.8 MHz, $\mathbf{C D C l}_{3}$ ): $\delta=18.5$ (q, C-6'), 24.2 (q, C-8), 55.5 (q, 1'-OCH $), 71.2$ (d, C-5'), 74.9 (d, C-2'), 77.9 (d, C-4'), 100.0 (d, C-1'), 101.3 (d, C-4), 104.6 (s, C-2), 111.9 (d, C-6), 145.2 (s, C-7), 161.2 (d, C-5), 165.2 (s, C-3), 169.4 (s, C-1), 200.0 (s, C-3').

Orsellide B (102):

${ }^{1}$ H-NMR (600 MHz, $\mathbf{C D C l}_{3}$ ): $\delta=1.44\left(\mathrm{~d}, J=6.5 \mathrm{~Hz}, 3 \mathrm{H}, 6{ }^{\prime}-\mathrm{H}_{3}\right), 2.45$ (s, 3H, 8- $\mathrm{H}_{3}$ ), 3.44 (s, $\left.3 \mathrm{H}, 1^{\prime}-\mathrm{OCH}_{3}\right), 4.14$ (dq, $J=6.5,10.0 \mathrm{~Hz}, 1 \mathrm{H}, 5$ '-H), 4.50 (dd, $\left.J=0.5,4.5 \mathrm{~Hz} 1 \mathrm{H}, 2^{\prime}-\mathrm{H}\right), 5.10$ (d, $\left.J=4.5 \mathrm{~Hz}, 1 \mathrm{H}, 1^{\prime}-\mathrm{H}\right), 5.25$ (dd, $\left.J=0.5,10.0 \mathrm{~Hz}, 1 \mathrm{H}, 4^{\prime}-\mathrm{H}\right), 6.20$ (d, $\left.J=2.0 \mathrm{~Hz}, 1 \mathrm{H}, 4-\mathrm{H}\right)$, $6.24(\mathrm{~d}, J=2.0 \mathrm{~Hz}, 1 \mathrm{H}, 6-\mathrm{H}), 11.17(\mathrm{~s}, 1 \mathrm{H}, 3-\mathrm{OH})$.

${ }^{13}$ C-NMR (150.8 MHz, $\mathbf{C D C l}_{3}$ ): $\delta=18.7$ (q, C-6'), 24.4 (q, C-8), 56.0 (q, 1'-OCH $), 68.4$ (d, C-5'), 75.3 (d, C-2'), 78.0 (d, C-4'), 101.4 (d, C-4), 101.8 (d, C-1'), 104.6 (s, C-2), 111.8 (d, C-6), 144.2 (s, C-7), 161.2 (s, C-5), 165.5 (s, C-3), 169.7 (s, C-1), 199.6 (s, C-3’).

\section{Orsellid C (104)}

$\mathrm{C}_{15} \mathrm{H}_{18} \mathrm{O}_{7}(310.30)$

farbloses Ö1

UV-löschend (254 nm)

$\mathbf{R}_{\mathbf{f}}$-Wert: 0.81 (Chloroform/Methanol 9:1)

Anfärbeverhalten: rotbraun (Anisaldehyd)

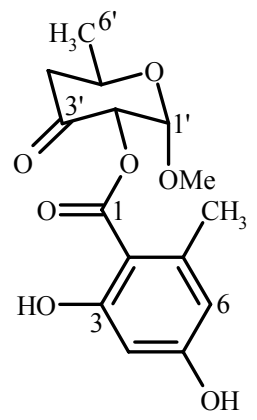

ESI-MS (positive Ionen): $\mathrm{m} / \mathrm{z}(\%)=333(100)[\mathrm{M}+\mathrm{Na}]^{+}, 643(50)[2 \mathrm{M}+\mathrm{Na}]^{+}$;

(negative Ionen): $\mathrm{m} / \mathrm{z}(\%)=309(100)[\mathrm{M}-\mathrm{H}]^{-}$.

HRESI-MS: 333.09447 gefunden für $\left[\mathrm{C}_{15} \mathrm{H}_{18} \mathrm{O}_{7} \mathrm{Na}\right]^{+}$. 
IR(KBr): $\widetilde{v}=3415,2977,2934,2840$ (sh), 1739, 1650, 1621, 1451, 1326, 1258, 1167, 1114, $1049 \mathrm{~cm}^{-1}$.

UV: $(\mathrm{MeOH}): \quad\left[\lambda_{\max }(\lg \varepsilon)\right]=216(4.39), 265(4.19), 302(3.76) \mathrm{nm}$;

$\mathrm{MeOH} / \mathrm{HCl}): \quad\left[\lambda_{\max }(\lg \varepsilon)\right]=216(4.37), 265(4.19), 302(3.76) \mathrm{nm}$;

$(\mathrm{MeOH} / \mathrm{NaOH}):\left[\lambda_{\max }(\lg \varepsilon)\right]=210(4.30), 242(3.98), 306(4.37) \mathrm{nm}$.

$[\alpha]_{D}^{20}=+45^{\circ}(\mathrm{c}=0.1$, Methanol $)$.

CD (MeOH): $\lambda_{\max }([\Theta])=259$ (-5176), 278 (5666).

${ }^{1}$ H-NMR (600 MHz, $\left.\mathbf{C D C l}_{3}\right): \delta=1.37\left(\mathrm{~d}, J=6.0 \mathrm{~Hz}, 3 \mathrm{H}, 6{ }^{\prime}-\mathrm{H}_{3}\right), 2.50$ (s, 3H, 8- $\left.\mathrm{H}_{3}\right), 2.50$ (ddd, $\left.J=0.5,11.0,14.0 \mathrm{~Hz}, 1 \mathrm{H}, 4^{\prime}-\mathrm{H}_{\mathrm{ax}}\right), 2.59$ (dd, $\left.J=3.0,14.0 \mathrm{~Hz}, 1 \mathrm{H}, 4^{\prime}-\mathrm{H}_{\mathrm{äq}}\right), 3.42$ (s, 3H, 1' $-\mathrm{OCH}_{3}$ ), 4.24 (ddq, $J=3.0,6.0,11.0 \mathrm{~Hz}, 1 \mathrm{H}, 5$ '-H), 5.19 (d, $J=4.0 \mathrm{~Hz}, 1 \mathrm{H}, 1$ '-H), 5.56 $\left(\mathrm{dd}, J=0.5,4.0 \mathrm{~Hz} 1 \mathrm{H}, 2^{\prime}-\mathrm{H}\right), 6.20$ (d, $\left.J=2.0 \mathrm{~Hz}, 1 \mathrm{H}, 4-\mathrm{H}\right), 6.24$ (d, $\left.J=2.0 \mathrm{~Hz}, 1 \mathrm{H}, 6-\mathrm{H}\right)$, $11.02(\mathrm{~s}, 1 \mathrm{H}, 3-\mathrm{OH})$.

${ }^{13}$ C-NMR (150.8 MHz, $\mathbf{C D C l}_{3}$ ): $\delta=21.2$ (q, C-6'), 24.1 (q, C-8), 48.5 (t, C-4'), 55.4 (q, 1'-OCH 3 ), 66.0 (d, C-5'), 76.0 (d, C-2'), 100.3 (d, C-1'), 101.2 (d, C-4), 103.8 (s, C-2), 111.7 (d, C-6), 145.0 (s, C-7), 161.1 (s, C-5), 165.0 (s, C-3), 169.5 (s, C-1), 198.2 (s, C-3').

\section{Orsellid D (105)}

$\mathrm{C}_{14} \mathrm{H}_{14} \mathrm{O}_{7}(294.26)$

farbloser Feststoff

UV-löschend (254 nm)

$\mathbf{R}_{\mathbf{f}}$-Wert: 0.63 (Chloroform/Methanol 9:1)

Anfärbeverhalten: rotbrau (Anisaldehyd)

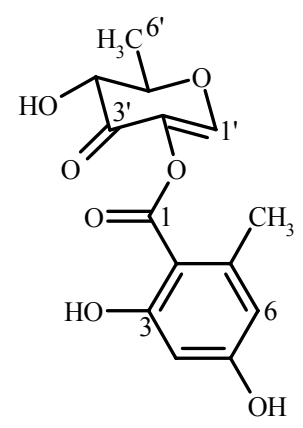

Smp.: $81^{\circ} \mathrm{C}$

ESI-MS (negative Ionen): $\mathrm{m} / \mathrm{z}(\%)=293(100)[\mathrm{M}-\mathrm{H}]^{-}, 587(20)[2 \mathrm{M}-\mathrm{H}]^{-}$.

HRESI-MS: 317.06317 gefunden für $\left[\mathrm{C}_{14} \mathrm{H}_{14} \mathrm{O}_{7} \mathrm{Na}\right]^{+}$.

IR(KBr): $\widetilde{v}=3431,2930,2858$ (sh), 1743 (sh), 1691 (sh), 1655 (sh), 1625, 1505, 1453, $1391,1319,1256,1178 \mathrm{~cm}^{-1}$.

UV: $(\mathrm{MeOH}): \quad\left[\lambda_{\max }(\lg \varepsilon)\right]=215(4.11), 269(3.97), 299(\mathrm{sh}, 3.49) \mathrm{nm}$;

$\mathrm{MeOH} / \mathrm{HCl}): \quad\left[\lambda_{\max }(\lg \varepsilon)\right]=215(4.08), 267(3.97), 299(\mathrm{sh}, 3.48) \mathrm{nm}$;

$(\mathrm{MeOH} / \mathrm{NaOH}):\left[\lambda_{\max }(\lg \varepsilon)\right]=216(4.00), 234$ (sh, 3.70), 276 (sh, 3.71), $302(3.85) \mathrm{nm}$. $[\alpha]_{D}^{20}=+58^{\circ}(\mathrm{c}=0.1$, Methanol $)$.

CD (MeOH): $\lambda_{\max }([\Theta])=205$ (-8254), 264 (6777). 
${ }^{1}$ H-NMR (600 MHz, $\left.\mathbf{C D C l}_{3}\right): \delta=1.60\left(\mathrm{~d}, J=6.5 \mathrm{~Hz}, 3 \mathrm{H}, 6^{\prime}-\mathrm{H}_{3}\right), 2.50\left(\mathrm{~s}, 3 \mathrm{H}, 8-\mathrm{H}_{3}\right), 4.36$ (dq, $\left.J=6.5,13.0 \mathrm{~Hz}, 1 \mathrm{H}, 5^{\prime}-\mathrm{H}\right), 4.19$ (d, $\left.J=13.0 \mathrm{~Hz}, 1 \mathrm{H}, 4^{\prime}-\mathrm{H}\right), 6.22$ (d, $J=2.0 \mathrm{~Hz}, 1 \mathrm{H}$, 4-H), 6.25 (d, J=2.0 Hz, 1H, 6-H), 7.55 (s, 1H, 1'-H), 10.96 (s, 1H, 3-OH).

${ }^{13}$ C-NMR (150.8 MHz, CDCl $_{3}$ ): $\delta=17.8$ (q, C-6'), 24.3 (q, C-8), 72.5 (d, C-5'), 80.8 (d, C-4'), 101.4 (d, C-4), 104.6 (s, C-2), 111.9 (d, C-6), 128.7 (d, C-2'), 144.6 (s, C-7), 157.8 (d, C-1'), 161.4 (s, C-5), 165.9 (s, C-3), 169.2 (s, C-1), 188.1 (s, C-3').

\section{Orsellid E (106)}

$\mathrm{C}_{14} \mathrm{H}_{14} \mathrm{O}_{6}(278.26)$

farbloses Ö1

UV-löschend (254 nm)

$\mathbf{R}_{\mathbf{f}}$-Wert: 0.73 (Chloroform/Methanol 9:1)

Anfärbeverhalten: rotbraun (Anisaldehyd)

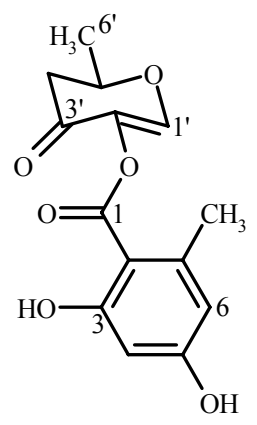

ESI-MS (positive Ionen): $\mathrm{m} / \mathrm{z}(\%)=301(100)[\mathrm{M}+\mathrm{Na}]^{+}$;

(negative Ionen): $\mathrm{m} / \mathrm{z}(\%)=277(100)[\mathrm{M}-\mathrm{H}]^{-}$.

HRESI-MS: 301.06856 gefunden für $\left[\mathrm{C}_{14} \mathrm{H}_{14} \mathrm{O}_{6} \mathrm{Na}\right]^{+}$.

IR(KBr): $\widetilde{v}=3386,2980,2936,1661,1622,1503,1448,1385,1315,1258,1181 \mathrm{~cm}^{-1}$.

UV: $(\mathrm{MeOH}): \quad\left[\lambda_{\max }(\lg \varepsilon)\right]=215(4.35), 269(4.33), 301(\mathrm{sh}, 3.86) \mathrm{nm}$;

$\mathrm{MeOH} / \mathrm{HCl}): \quad\left[\lambda_{\max }(\lg \varepsilon)\right]=215(4.35), 269(4.32), 301(\mathrm{sh}, 3.79) \mathrm{nm}$;

$(\mathrm{MeOH} / \mathrm{NaOH}):\left[\lambda_{\max }(\lg \varepsilon)\right]=210(4.30), 242(3.99), 306(4.38) \mathrm{nm}$.

$[\alpha]_{D}^{20}=+88^{\circ}(\mathrm{c}=0.1$, Methanol $)$.

CD (MeOH): $\lambda_{\max }([\Theta])=273$ (12497).

${ }^{1}$ H-NMR (600 MHz, $\left.\mathbf{C D C l}_{3}\right): \delta=1.52\left(\mathrm{~d}, J=6.5 \mathrm{~Hz}, 3 \mathrm{H}, 6{ }^{\prime}-\mathrm{H}_{3}\right), 2.44$ (s, 3H, 8- $\left.\mathrm{H}_{3}\right), 2.63$

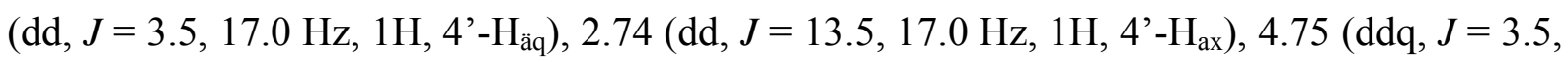
6.5, 13.5 Hz, 1H, 5'-H), 6.13 (d, J=2.0 Hz, 1H, 4-H), 6.18 (d, J=2.0 Hz, 1H, 6-H), 6.95 (s, br, 1H, 5-OH), 7.48 (s, 1H, 1'-H), 10.99 (s, 1H, 3-OH).

${ }^{13}$ C-NMR (150.8 MHz, CDCl 3 ): $\delta=20.1$ (q, C-6'), 24.3 (q, C-8), 42.5 (t, C-4'), 66.0 (d, C-5'), 101.3 (d, C-4), 103.9 (s, C-2), 112.0 (d, C-6), 131.0 (d, C-2'), 144.4 (s, C-7), 157.1 (d, C-1'), 161.8 (s, C-5), 165.7 (s, C-3), 169.4 (s, C-1), 186.3 (s, C-3’). 
(4-Hydroxy-pent-2-on-1-yl)-orsellinat (107)

$\mathrm{C}_{13} \mathrm{H}_{16} \mathrm{O}_{6}(268.27)$

farbloses Öl

UV-löschend (254 nm)<smiles>Cc1cc(O)cc(O)c1C(=O)OCC(=O)CC(C)O</smiles>

$\mathbf{R}_{\mathbf{f}}$-Wert: 0.47 (Chloroform/Methanol 9:1)

Anfärbeverhalten: rotbraun (Anisaldehyd)

ESI-MS (positive Ionen): $\mathrm{m} / \mathrm{z}(\%)=291(100)[\mathrm{M}+\mathrm{Na}]^{+}$;

(negative Ionen): $\mathrm{m} / \mathrm{z}(\%)=267(90)[\mathrm{M}-\mathrm{H}]^{-}, 535(100)[2 \mathrm{M}-\mathrm{H}]^{-}$.

HRESI-MS: 291.08391 gefunden für $\left[\mathrm{C}_{13} \mathrm{H}_{16} \mathrm{O}_{6} \mathrm{Na}\right]^{+}$.

IR(KBr): $\widetilde{v}=3432,2924,1623,1384 \mathrm{~cm}^{-1}$.

UV: $(\mathrm{MeOH}): \quad\left[\lambda_{\max }(\lg \varepsilon)\right]=212(3.60), 267(3.42), 301(2.90) \mathrm{nm}$;

$\mathrm{MeOH} / \mathrm{HCl}): \quad\left[\lambda_{\max }(\lg \varepsilon)\right]=213(3.33), 265(3.00), 301(2.87) \mathrm{nm}$;

$(\mathrm{MeOH} / \mathrm{NaOH}):\left[\lambda_{\max }(\lg \varepsilon)\right]=238(2.89), 304(3.28) \mathrm{nm}$.

$[\alpha]_{D}^{20}=+11^{\circ}(\mathrm{c}=0.1$, Methanol $)$.

${ }^{1}$ H-NMR (600 MHz, CD $\left.\mathbf{C D}_{3} \mathrm{OD}\right): \delta=1.21$ (d, $\left.J=6.5 \mathrm{~Hz}, 3 \mathrm{H}, 5^{\prime}-\mathrm{H}_{3}\right), 2.48$ (s, 3H, 8- $\mathrm{H}_{3}$ ), 2.57 $\left(\mathrm{dd}, J=4.5,15.5 \mathrm{~Hz}, 1 \mathrm{H}, 3^{\prime}-\mathrm{H}_{\mathrm{a}}\right), 2.66\left(\mathrm{dd}, J=8.0,15.5 \mathrm{~Hz}, 1 \mathrm{H}, 3^{\prime}-\mathrm{H}_{\mathrm{b}}\right), 4.23$ (ddq, $J=4.5$, 6.5, 8.0 Hz, 1H, 4'-H), 5.00 (d, $\left.J=17.5 \mathrm{~Hz}, 1 \mathrm{H}, 1^{\prime}-\mathrm{H}_{\mathrm{a}}\right), 5.04$ (d, $\left.J=17.5 \mathrm{~Hz}, 1 \mathrm{H}, 1^{\prime}-\mathrm{H}_{\mathrm{b}}\right), 6.16$ (d, $J=2.0 \mathrm{~Hz}, 1 \mathrm{H}, 4-\mathrm{H}), 6.22$ (d, $J=2.0 \mathrm{~Hz}, 1 \mathrm{H}, 6-\mathrm{H})$.

${ }^{13}$ C-NMR (150.8 MHz, CD 3 OD): $\delta=23.6$ (q, C-5'), 24.2 (q, C-8), 49.9 (t, C-3'), 64.8 (d, C-4'), 69.8 (t, C-1'), 101.7 (d, C-4), 105.4 (s, C-2), 112.6 (d, C-6), 145.0 (s, C-7), 164.1 (s, C5), 166.1 (s, C-3), 171.7 (s, C-1), 204.3 (s, C-2'). 
(Pent-3-en-2-on-1-yl)-orsellinat (108)

$\mathrm{C}_{13} \mathrm{H}_{14} \mathrm{O}_{5}(250.25)$

farbloser Feststoff

UV-löschend (254 nm)

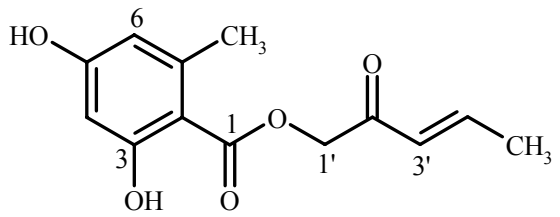

$\mathbf{R}_{\mathbf{f}}$-Wert: 0.73 (Chloroform/Methanol 9:1)

Anfärbeverhalten: rotbraun (Anisaldehyd)

Smp.: $134^{\circ} \mathrm{C}$

ESI-MS (negative Ionen): $\mathrm{m} / \mathrm{z}(\%)=249(100)[\mathrm{M}-\mathrm{H}]^{-}, 499(40)[2 \mathrm{M}-\mathrm{H}]^{-}$.

HRESI-MS: 273.07335 gefunden für $\left[\mathrm{C}_{13} \mathrm{H}_{14} \mathrm{O}_{5} \mathrm{Na}\right]^{+}$.

IR(KBr): $\widetilde{v}=3349,2925,2870$ (sh), 1719, 1684, 1622, 1464 (sh), 1432, 1354, 1249, 1198, $1156,1106 \mathrm{~cm}^{-1}$.

UV: $(\mathrm{MeOH}): \quad\left[\lambda_{\max }(\lg \varepsilon)\right]=217(4.36), 265(4.04), 303(3.66) \mathrm{nm}$;

$\mathrm{MeOH} / \mathrm{HCl}): \quad\left[\lambda_{\max }(\lg \varepsilon)\right]=217(4.33), 265$ (4.00), $301(3.56) \mathrm{nm}$;

$(\mathrm{MeOH} / \mathrm{NaOH}):\left[\lambda_{\max }(\lg \varepsilon)\right]=218(4.14), 236(\mathrm{sh}, 3.91), 305(4.20) \mathrm{nm}$.

CD (MeOH): $\lambda_{\max }([\Theta])=205(-5370)$.

${ }^{1}$ H-NMR (600 MHz, $\left.\mathbf{C D C l}_{3}\right): \delta=1.95\left(\mathrm{dd}, J=2.0,6.5 \mathrm{~Hz}, 3 \mathrm{H}, 5^{\prime}-\mathrm{H}_{3}\right), 2.52\left(\mathrm{~s}, 3 \mathrm{H}, 8-\mathrm{H}_{3}\right)$, 5.08 (s, 2H, 1'- $\left.\mathrm{H}_{2}\right), 5.61$ (s, br, 1H, 5-OH), 6.22 (d, $\left.J=2.0 \mathrm{~Hz}, 1 \mathrm{H}, 4-\mathrm{H}\right), 6.23$ (dd, $J=2.0$, $\left.16.0 \mathrm{~Hz}, 1 \mathrm{H}, 3^{\prime}-\mathrm{H}\right), 6.25$ (d, $\left.J=2.0 \mathrm{~Hz}, 1 \mathrm{H}, 6-\mathrm{H}\right), 7.03$ (dq, $\left.J=6.5,16.0 \mathrm{~Hz}, 1 \mathrm{H}, 4^{\prime}-\mathrm{H}\right), 11.21$ (s, 1H, 3-OH).

${ }^{13}$ C-NMR (150.8 MHz, CDCl 3 ): $\delta=18.7$ (q, C-5'), 24.2 (q, C-8), 67.0 (t, C-1'), 101.4 (d, C-4), 105.2 (s, C-2), 111.5 (d, C-6), 127.5 (d, C-3'), 144.4 (s, C-7), 145.4 (d, C-4'), 160.8 (s, C-5), 165.2 (s, C-3), 170.4 (s, C-1), 191.9 (s, C-2'). 


\section{Acetylierung der Orsellide A - C (101, 102 und 105):}

Gemäß AAV 1 wurde ein Gemisch aus 101, 102 und 105 (5.0 mg) acetyliert. Nach Trennung des erhaltenen Gemisches mittels präparativer HPLC (Säule 2, Programm C) konnten 101b (3.2 mg), 102b (1.1 mg) und 103b (2.4 mg) als Reinsubstanzen erhalten werden.

\section{3,5,4'-Tri-O-acetyl-orsellid A (101b)}

$\mathrm{C}_{21} \mathrm{H}_{24} \mathrm{O}_{11}(452.41)$

farbloser Feststoff

UV-löschend (254 nm)

$\mathbf{R}_{\mathbf{f}}$-Wert: 0.81 (Chloroform/Methanol 9:1)

Anfärbeverhalten: rotbraun (Anisaldehyd)

Smp.: $48-52^{\circ} \mathrm{C}$

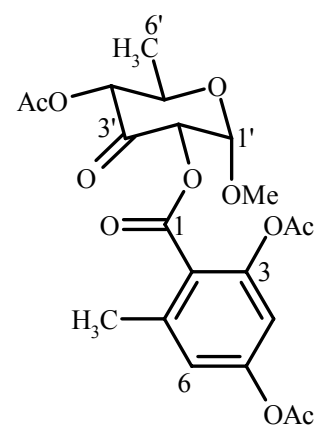

IR(KBr): $\widetilde{v}=3432,2936,1771,1745,1616,1448,1371,1260,1195,1135,1075 \mathrm{~cm}^{-1}$. $[\alpha]_{D}^{20}=+79^{\circ}(\mathrm{c}=0.1$, Methanol).

CD (MeOH): $\lambda_{\max }([\Theta])=205$ (19616), 249 (-6958).

${ }^{1}$ H-NMR (600 MHz, $\mathbf{C D C l}_{3}$ ): $\delta=1.38$ (d, $\left.J=6.0 \mathrm{~Hz}, 3 \mathrm{H}, 6{ }^{\prime}-\mathrm{H}_{3}\right), 2.16$ (s, 3H, Ac-CH $), 2.22$ (s, 3H, Ac- $\left.\mathrm{CH}_{3}\right), 2.26$ (s, 3H, Ac- $\left.\mathrm{CH}_{3}\right), 2.48$ (s, 3H, 8- $\left.\mathrm{H}_{3}\right), 3.40$ (s, 3H, 1'- $\left.\mathrm{OCH}_{3}\right), 4.11$ (dq, $\left.J=6.0,10.0 \mathrm{~Hz}, 1 \mathrm{H}, 5^{\prime}-\mathrm{H}\right), 5.05$ (dd, $\left.J=1.0,10.0 \mathrm{~Hz}, 1 \mathrm{H}, 4^{\prime}-\mathrm{H}\right), 5.15$ (d, $J=4.0 \mathrm{~Hz}, 1 \mathrm{H}$, 1'-H), 5.53 (dd, $J=1.0,4.0 \mathrm{~Hz} 1 \mathrm{H}, 2$ '-H), 6.79 (d, $J=2.0 \mathrm{~Hz}, 1 \mathrm{H}, 4-\mathrm{H}), 6.88$ (d, $J=2.0 \mathrm{~Hz}$, 1H, 6-H).

${ }^{13}$ C-NMR (150.8 MHz, $\mathbf{C D C l}_{3}$ ): $\delta=18.4$ (q, C-6'), 20.4 (q, Ac-CH $\mathrm{CH}_{3}$ oder C-8), 20.8 (q, $\mathrm{Ac}-\mathrm{CH}_{3}$ oder C-8), 20.9 (q, Ac- $\mathrm{CH}_{3}$ oder C-8), 21.1 (q, $\mathrm{Ac}-\mathrm{CH}_{3}$ oder C-8), 55.6 (q, 1'-OCH 3 ), 67.9 (d, C-5'), 75.7 (d, C-2'), 77.6 (d, C-4'), 99.7 (d, C-1'), 114.4 (d, C-4), 121.4 (d, C-6), 122.1 (s, C-2), 140.9 (s, C-7), 150.0 (d, C-3), 152.2 (s, C-5), 164.1 (s, C-1), 168.5 (s, Ac-CO), 169.0 (s, Ac-CO), 169.2 (s, Ac-CO), 192.5 (s, C-3').

\section{3,5,2'-Tri-O-acetyl-orsellid B (102b)}

$\mathrm{C}_{21} \mathrm{H}_{24} \mathrm{O}_{11}(452.41)$

farbloser Feststoff

UV-löschend (254 nm)

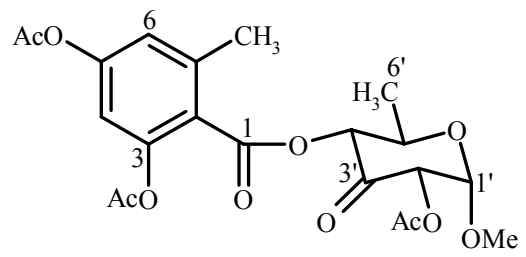

$\mathbf{R}_{\mathbf{f}}$-Wert: 0.81 (Chloroform/Methanol 9:1) 
Anfärbeverhalten: rotbraun (Anisaldehyd)

Smp.: $49^{\circ} \mathrm{C}$

IR(KBr): $\widetilde{v}=3432,2931,2870(\mathrm{sh}), 1771,1750,1619,1369,1196,1136 \mathrm{~cm}^{-1}$.

CD (MeOH): $\lambda_{\max }([\Theta])=210$ (-28891), 247 (14369).

${ }^{1}$ H-NMR (600 MHz, $\left.\mathbf{C D C l}_{3}\right): \delta=1.41\left(\mathrm{~d}, J=6.0 \mathrm{~Hz}, 3 \mathrm{H}, 6^{\prime}-\mathrm{H}_{3}\right), 2.20$ (s, 3H, Ac-CH $), 2.26$ (s, 3H, Ac- $\left.\mathrm{CH}_{3}\right), 2.27$ (s, 3H, Ac- $\left.\mathrm{CH}_{3}\right), 2.47$ (s, 3H, 8- $\left.\mathrm{H}_{3}\right), 3.42$ (s, 3H, 1'- $\left.\mathrm{OCH}_{3}\right), 4.11$ (dq, $\left.J=6.0,10.0 \mathrm{~Hz}, 1 \mathrm{H}, 5^{\prime}-\mathrm{H}\right), 5.13$ (d, $\left.J=4.0 \mathrm{~Hz}, 1 \mathrm{H}, 1^{\prime}-\mathrm{H}\right), 5.18$ (dd, $J=1.0,10.0 \mathrm{~Hz}, 1 \mathrm{H}$, 4'-H), 5.46 (dd, $\left.J=1.0,4.0 \mathrm{~Hz} 1 \mathrm{H}, 2^{\prime}-\mathrm{H}\right), 6.82$ (d, $\left.J=2.0 \mathrm{~Hz}, 1 \mathrm{H}, 4-\mathrm{H}\right), 6.88$ (d, $J=2.0 \mathrm{~Hz}$, $1 \mathrm{H}, 6-\mathrm{H})$.

${ }^{13}$ C-NMR (150.8 MHz, $\mathbf{C D C l}_{3}$ ): $\delta=18.4$ (q, C-6'), 20.4 (q, Ac-CH $\mathrm{CH}_{3}$ oder C-8), 20.5 (q, Ac- $\mathrm{CH}_{3}$ oder C-8), 21.0 (q, Ac- $\mathrm{CH}_{3}$ oder C-8), 21.1 (q, $\mathrm{Ac}-\mathrm{CH}_{3}$ oder C-8), 55.6 (q, 1'- $\mathrm{OCH}_{3}$ ), 67.8 (d, C-5'), 74.9 (d, C-2'), 78.3 (d, C-4'), 99.7 (d, C-1'), 114.2 (d, C-4), 121.2 (d, C-6), 123.0 (s, C-2), 139.7 (s, C-7), 149.1 (d, C-3), 151.9 (s, C-5), 164.2 (s, C-1), 168.6 (s, Ac-CO), 169.0 (s, Ac-CO), 169.4 (s, Ac-CO), 192.5 (s, C-3’).

\section{3,5,4'-Tri-O-acetyl-orsellid D (105b)}

$\mathrm{C}_{20} \mathrm{H}_{20} \mathrm{O}_{10}(420.37)$

farbloses Ö1

UV-löschend (254 nm)

$\mathbf{R}_{\mathbf{f}}$-Wert: 0.81 (Chloroform/Methanol 9:1)

Anfärbeverhalten: rotbraun (Anisaldehyd)

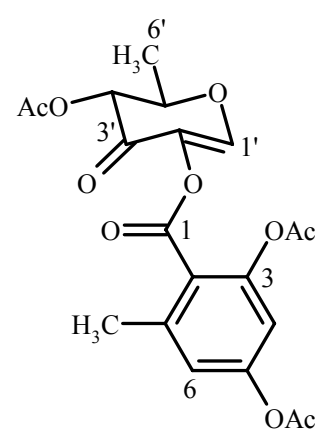

IR(KBr): $\widetilde{v}=3448,2935,1765,1702,1626,1372,1188,1133,1059,1017 \mathrm{~cm}^{-1}$.

$[\alpha]_{D}^{20}=+91^{\circ}(\mathrm{c}=0.1$, Methanol).

CD (MeOH): $\lambda_{\max }([\Theta])=270$ (38234).

${ }^{1}$ H-NMR (600 MHz, $\mathbf{C D C l}_{3}$ ): $\delta=1.49$ (d, $\left.J=6.0 \mathrm{~Hz}, 3 \mathrm{H}, 6{ }^{\prime}-\mathrm{H}_{3}\right), 2.19$ (s, 3H, Ac-CH $), 2.26$ (s, 3H, Ac- $\left.\mathrm{CH}_{3}\right), 2.27$ (s, 3H, Ac- $\left.\mathrm{CH}_{3}\right), 2.49$ (s, 3H, 8- $\left.\mathrm{H}_{3}\right), 4.64$ (dq, J = 6.0, $12.5 \mathrm{~Hz}, 1 \mathrm{H}$, 5'-H), 5.41 (d, $J=12.5 \mathrm{~Hz}, 1 \mathrm{H}, 4$ '-H), 6.82 (d, $J=2.0 \mathrm{~Hz}, 1 \mathrm{H}, 4-\mathrm{H}), 6.90$ (d, $J=2.0 \mathrm{~Hz}, 1 \mathrm{H}$, 6-H), 7.46 (s, 1H, 1'-H).

${ }^{13}$ C-NMR (150.8 MHz, $\mathbf{C D C l}_{3}$ ): $\delta=17.3$ (q, C-6'), 20.5 (q, Ac-CH $\mathrm{Cder}_{3} \mathrm{C}-8$ ), 20.7 (q, Ac- $\mathrm{CH}_{3}$ oder C-8), 20.9 (q, Ac- $\mathrm{CH}_{3}$ oder C-8), 21.1 (q, Ac- $\mathrm{CH}_{3}$ oder C-8), 72.6 (d, C-5'), 78.3 (d, C-4'), 114.2 (d, C-4), 121.3 (d, C-6), 122.2 (s, C-2), 130.3 (s, C-2'), 140.5 (s, C-7), 149.6 (d, C-3), 152.3 (s, C-5), 156.1 (d, C-1'), 163.5 (s, C-1), 168.5 (s, Ac-CO), 169.0 (s, Ac-CO), 169.3 (s, Ac-CO), 181.7 (s, C-3’). 
Chetomin $^{130}$ (119)

$\mathrm{C}_{31} \mathrm{H}_{30} \mathrm{~N}_{6} \mathrm{O}_{6} \mathrm{~S}_{4}(710.88)$

weißer Feststoff

UV-löschend (254 nm)

$\mathbf{R}_{\mathbf{f}}$-Wert: 0.82 (Chloroform/Methanol 9:1)

Anfärbeverhalten: braun (Anisaldehyd)

Smp.: $>200^{\circ} \mathrm{C}$

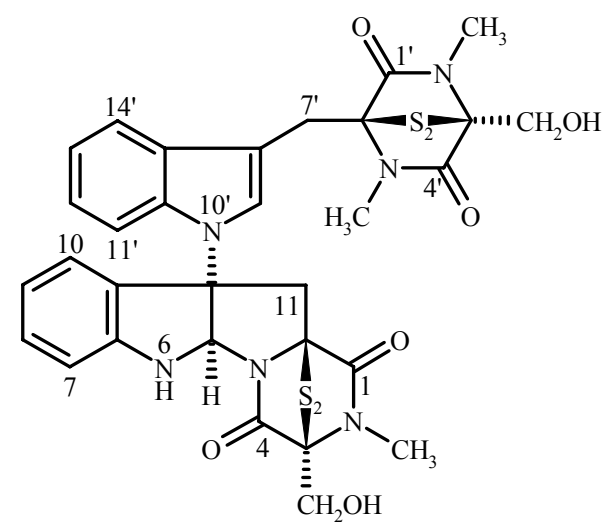

ESI-MS (positive Ionen): $\mathrm{m} / \mathrm{z}(\%)=733(100)[\mathrm{M}+\mathrm{Na}]^{+}, 1443(30)[2 \mathrm{M}+\mathrm{Na}]^{+}$;

(negative Ionen): $\mathrm{m} / \mathrm{z}(\%)=709(100)[\mathrm{M}-\mathrm{H}]^{-}$.

HRESI-MS: 711.11824 gefunden für $\left[\mathrm{C}_{31} \mathrm{H}_{31} \mathrm{~N}_{6} \mathrm{O}_{6} \mathrm{~S}_{4}\right]^{+}$.

IR(KBr): $\widetilde{v}=3421,2930,1682,1612$ (sh), 1457, 1411, 1349, 1233, 1065, $746 \mathrm{~cm}^{-1}$.

UV: $(\mathrm{MeOH}): \quad\left[\lambda_{\max }(\lg \varepsilon)\right]=219(\mathrm{sh}, 4.60), 287(3.92) \mathrm{nm}$.

$[\alpha]_{D}^{20}=+342^{\circ}(\mathrm{c}=0.1$, Methanol $)$.

CD (MeOH): $\lambda_{\max }([\Theta])=238$ (150186), 268 (-38113), 302 (47425).

${ }^{1}$ H-NMR (600 MHz, $\left.\mathbf{C D C l}_{3}\right): \delta=2.93\left(\mathrm{~s}, 3 \mathrm{H}, 5{ }^{\prime}-\mathrm{CH}_{3}\right), 3.12\left(\mathrm{~m}, 1 \mathrm{H}, 11-\mathrm{H}_{\mathrm{a}}\right), 3.15(\mathrm{~s}, 3 \mathrm{H}$, 2- $\mathrm{CH}_{3}$ oder 2' $\left.-\mathrm{CH}_{3}\right), 3.15$ (s, 3H, 2- $\mathrm{CH}_{3}$ oder 2' $\left.-\mathrm{CH}_{3}\right), 3.68$ (d, $J=15.5 \mathrm{~Hz}, 1 \mathrm{H}, 7$ '- $\left.-\mathrm{H}_{\mathrm{a}}\right), 3.83$ $\left(\mathrm{d}, J=15.5 \mathrm{~Hz}, 1 \mathrm{H}, 7^{\prime}-\mathrm{H}_{\mathrm{b}}\right), 4.21\left(\mathrm{~d}, J=12.5 \mathrm{~Hz}, 1 \mathrm{H}, 12-\mathrm{H}_{\mathrm{a}}\right), 4,23(\mathrm{~d}, J=12.5 \mathrm{~Hz}, 1 \mathrm{H}$, $\left.15^{\prime}-\mathrm{H}_{\mathrm{a}}\right), 4.32\left(\mathrm{~d}, J=12.5 \mathrm{~Hz}, 1 \mathrm{H}, 12-\mathrm{H}_{\mathrm{b}}\right), 4.34\left(\mathrm{~d}, J=12.5 \mathrm{~Hz}, 1 \mathrm{H}, 15^{\prime}-\mathrm{H}_{\mathrm{b}}\right), 4.38(\mathrm{~d}, J=15.5$ $\left.\mathrm{Hz}, 1 \mathrm{H}, 11-\mathrm{H}_{\mathrm{b}}\right), 5.65$ (s, br, 1H, 6-NH), 6.19 (s, 1H, 5-H), 6.73 (d, J=8.0 Hz, 1H, 7-H), 6.89 (dd, $J=7.5 \mathrm{~Hz}, 1 \mathrm{H}, 9-\mathrm{H}), 7.19$ (m, 2H, 12'-H und 13'-H), 7.24 (s, 1H, 9'-H), 7.24 (m, 1H, 10-H), 7.29 (m, 1H, 11'-H), 7.31 (m, 1H, 8-H), 7.64 (d, J = 7.0 Hz, 1H, 14'-H).

${ }^{13}$ C-NMR (150.8 MHz, $\mathbf{C D C l}_{3}$ ): $\delta=26.9$ (t, C-7'), 27.4 (q, 2- $\mathrm{CH}_{3}$ oder 2'- $\mathrm{CH}_{3}$ ), 27.5 (q, 2- $\mathrm{CH}_{3}$ oder 2'- $\mathrm{CH}_{3}$ ), 28.1 (q, 5'- $\mathrm{CH}_{3}$ ), 42.6 (t, C-11), 60.0 (t, C-12), 60.8 (t, C-15'), 73.5 (s, C-10b), 73.6 (s, C-11a), 74.8 (s, C-6’), 76.2 (s, C-3), 76.5 (s, C-3'), 80.1 (d, C-5), 107.6 (s, C-8'), 110.9 (d, C-7), 111.4 (d, C-11'), 119.0 (d, C-14'), 120.1 (d, C-9), 120.4 (d, C-13'), 122.7 (d, C-12'), 124.7 (d, C-8), 126.5 (s, C-10a), 127.1 (d, C-9'), 130.2 (s, C-14'a), 131.2 (d, C-10), 133.9 (s, C-10'a), 148.3 (s, C-6a), 162.8 (s, C-4), 165.4 (s, C-1 und C-1'), 166.5 (s, C-4'). 
Chaetoseminudin ${ }^{131}(120)$

$\mathrm{C}_{31} \mathrm{H}_{30} \mathrm{~N}_{6} \mathrm{O}_{6} \mathrm{~S}_{5}(742.95)$

weißer Feststoff

UV-löschend (254 nm)

$\mathbf{R}_{\mathbf{f}}$-Wert: 0.82 (Chloroform/Methanol 9:1)

Anfärbeverhalten: braun (Anisaldehyd)

Smp.: $>200^{\circ} \mathrm{C}$

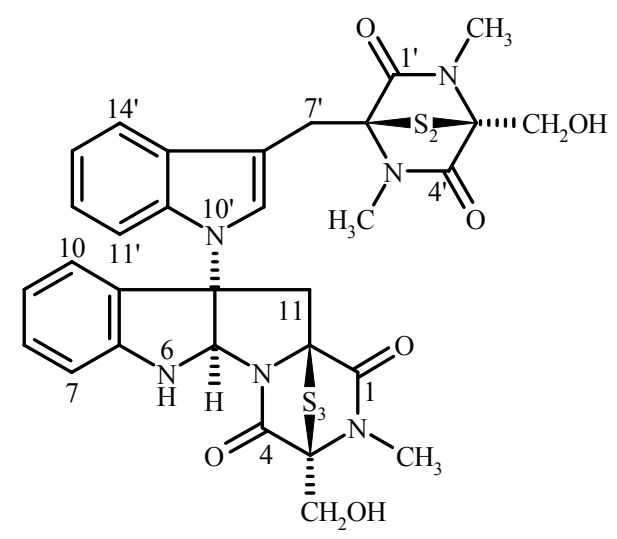

ESI-MS (positive Ionen): $\mathrm{m} / \mathrm{z}(\%)=765(100)[\mathrm{M}+\mathrm{Na}]^{+}, 1507(20)[2 \mathrm{M}+\mathrm{Na}]^{+}$;

(negative Ionen): $\mathrm{m} / \mathrm{z}(\%)=740(40)[\mathrm{M}-\mathrm{H}]^{-}, 786(100)\left[\mathrm{M}+\mathrm{HCO}_{2}\right]^{-}$

HRESI-MS: 743.09002 gefunden für $\left[\mathrm{C}_{31} \mathrm{H}_{31} \mathrm{~N}_{6} \mathrm{O}_{6} \mathrm{~S}_{5}\right]^{+}$.

IR(KBr): $\widetilde{v}=3441,2930,1676,1610$ (sh), 1457, 1354, 1233, 1065, $743 \mathrm{~cm}^{-1}$.

UV: $(\mathrm{MeOH}): \quad\left[\lambda_{\max }(\lg \varepsilon)\right]=207(\mathrm{sh}, 4.60), 294(3.82) \mathrm{nm}$.

$[\alpha]_{D}^{20}=+371^{\circ}(\mathrm{c}=0.1$, Methanol $)$.

CD (MeOH): $\lambda_{\max }([\Theta])=238$ (71478), 303 (29546).

${ }^{1}$ H-NMR (600 MHz, $\left.\mathbf{C D C l}_{3}\right): \delta=3.02$ (s, 3H, 2'- $\mathrm{CH}_{3}$ ), 3.03 (s, 3H, 5'-CH $\left.\mathrm{CH}_{3}\right), 3.05$ (d, $\left.J=15.0 \mathrm{~Hz}, 1 \mathrm{H}, 11-\mathrm{H}_{\mathrm{a}}\right), 3.15\left(\mathrm{~s}, 3 \mathrm{H}, 2-\mathrm{CH}_{3}\right), 3.25\left(\mathrm{~d}, J=15.5 \mathrm{~Hz}, 1 \mathrm{H}, 7^{\prime}-\mathrm{H}_{\mathrm{a}}\right), 3.73(\mathrm{~d}$, $\left.J=12.5 \mathrm{~Hz}, 1 \mathrm{H}, 15^{\prime}-\mathrm{H}_{\mathrm{a}}\right), 4.03\left(\mathrm{~d}, J=15.5 \mathrm{~Hz}, 1 \mathrm{H}, 7^{\prime}-\mathrm{H}_{\mathrm{b}}\right), 4.23\left(\mathrm{~d}, J=12.5 \mathrm{~Hz}, 1 \mathrm{H}, 12-\mathrm{H}_{\mathrm{a}}\right)$, $4.31\left(\mathrm{~d}, J=15.0 \mathrm{~Hz}, 1 \mathrm{H}, 11-\mathrm{H}_{\mathrm{b}}\right), 4.37\left(\mathrm{~d}, J=12.5 \mathrm{~Hz}, 1 \mathrm{H}, 12-\mathrm{H}_{\mathrm{b}}\right), 4.61(\mathrm{~d}, J=12.5 \mathrm{~Hz}, 1 \mathrm{H}$, $\left.15^{\prime}-\mathrm{H}_{\mathrm{b}}\right), 6.07$ (s, 1H, 5-H), 6.68 (s, 1H, 9'-H), 6.73 (m, 1H, 10-H), 6.89 (m, 1H, 9-H), 7.16 (m, 1H, 12'-H), 7.20 (m, 1H, 13'-H), 7.24 (s, 1H, 8-H), 7.26 (d, J = 7.5 Hz, 1H, 7-H), 7.26 (d, $\left.J=8.0 \mathrm{~Hz}, 1 \mathrm{H}, 11^{\prime}-\mathrm{H}\right), 7.56$ (dd, $\left.J=5.0,8.5 \mathrm{~Hz}, 1 \mathrm{H}, 14^{\prime}-\mathrm{H}\right)$.

${ }^{13}$ C-NMR (150.8 MHz, $\left.\mathbf{C D C l}_{3}\right): \delta=27.0$ (t, C-7'), 27.5 (q, 2-CH $\left.\mathrm{CH}_{3}\right), 27.7$ (q, 2'- $\left.\mathrm{CH}_{3}\right), 28.2$ (q, 5'- $\mathrm{CH}_{3}$ ), 49.1 (t, C-11), 61.2 (t, C-15'), 62.1 (t, C-12), 71.9 (s, C-10b), 74.7 (s, C-3'), 75.4 (s, C-3), 76.6 (s, C-6'), 78.2 (s, C-11a), 79.4 (d, C-5), 108.0 (s, C-8'), 111.1 (d, C-10), 111.6 (d, C-11'), 118.8 (d, C-14'), 120.3 (d, C-9), 120.5 (d, C-12'), 122.8 (d, C-13'), 124.8 (d, C-8), 125.7 (s, C-10a), 126.6 (d, C-9'), 130.1 (s, C-14'a), 131.8 (d, C-8), 134.5 (s, C-10'a), 149.7 (s, C-6a), 165.3 (s, C-4), 165.6 (s, C-1') 166.8 (s, C-4'), 168.3 (s, C-1). 


\section{Chetomin B (121)}

$\mathrm{C}_{31} \mathrm{H}_{30} \mathrm{~N}_{6} \mathrm{O}_{6} \mathrm{~S}_{5}$ (742.95)

weißer Feststoff

UV-löschend (254 nm)

$\mathbf{R}_{\mathbf{f}}$-Wert: 0.82 (Chloroform/Methanol 9:1)

Anfärbeverhalten: braun (Anisaldehyd)

Smp.: $>200^{\circ} \mathrm{C}$

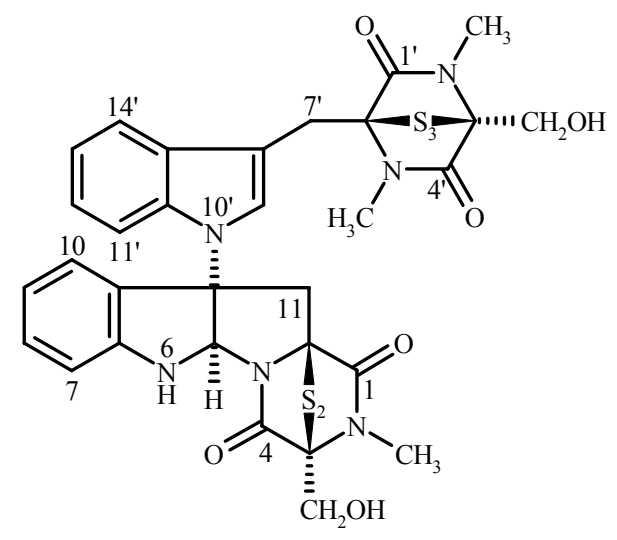

ESI-MS (positive Ionen): $\mathrm{m} / \mathrm{z}(\%)=765(100)[\mathrm{M}+\mathrm{Na}]^{+}, 1507(30)[2 \mathrm{M}+\mathrm{Na}]^{+}$;

(negative Ionen): $\mathrm{m} / \mathrm{z}(\%)=740(40)[\mathrm{M}-\mathrm{H}]^{-}, 786(100)\left[\mathrm{M}+\mathrm{HCO}_{2}\right]^{-}$

HRESI-MS: 765.07283 gefunden für $\left[\mathrm{C}_{31} \mathrm{H}_{31} \mathrm{~N}_{6} \mathrm{O}_{6} \mathrm{~S}_{5} \mathrm{Na}\right]^{+}$.

IR(KBr): $\widetilde{v}=3451,2930,1676,1612$ (sh), 1457, 1419, 1350, 1234, 1065, $744 \mathrm{~cm}^{-1}$.

UV: $(\mathrm{MeOH}): \quad\left[\lambda_{\max }(\lg \varepsilon)\right]=205(\mathrm{sh}, 4.18), 290(3.53) \mathrm{nm}$.

$[\alpha]_{D}^{20}=+270^{\circ}(\mathrm{c}=0.1$, Chloroform $)$.

CD (MeOH): $\lambda_{\max }([\Theta])=238$ (101905), 303 (44132).

Hauptkonformer:

${ }^{1} \mathbf{H}-\mathrm{NMR}\left(600 \mathrm{MHz}, \mathbf{C D C l}_{3}\right): \delta=3.02\left(\mathrm{~s}, 3 \mathrm{H}, 2^{\prime}-\mathrm{CH}_{3}\right), 3.03\left(\mathrm{~s}, 3 \mathrm{H}, 5^{\prime}-\mathrm{CH}_{3}\right), 3.05(\mathrm{~m}, 1 \mathrm{H}$, $\left.11-\mathrm{H}_{\mathrm{a}}\right), 3.15\left(\mathrm{~s}, 3 \mathrm{H}, 2-\mathrm{CH}_{3}\right), 3.25\left(\mathrm{~d}, J=16.0 \mathrm{~Hz}, 1 \mathrm{H}, 7^{\prime}-\mathrm{H}_{\mathrm{a}}\right), 3.73(\mathrm{~d}, J=12.5 \mathrm{~Hz}, 1 \mathrm{H}$, $\left.15^{\prime}-\mathrm{H}_{\mathrm{a}}\right), 4.03\left(\mathrm{~d}, J=16.0 \mathrm{~Hz}, 1 \mathrm{H}, 7^{\prime}-\mathrm{H}_{\mathrm{b}}\right), 4.24\left(\mathrm{~d}, J=12.5 \mathrm{~Hz}, 1 \mathrm{H}, 12-\mathrm{H}_{\mathrm{a}}\right), 4.31(\mathrm{~d}, J=12.5$ $\left.\mathrm{Hz}, 1 \mathrm{H}, 12-\mathrm{H}_{\mathrm{b}}\right), 4.37$ (d, $\left.J=15.5 \mathrm{~Hz}, 1 \mathrm{H}, 11-\mathrm{H}_{\mathrm{b}}\right), 4.60$ (d, J=12.5 Hz, 1H, 15'- $\mathrm{H}_{\mathrm{b}}$ ), 6.07 (s, 1H, 5-H), 6.68 (s, 1H, 9'-H), 6.74 (d, $J=8.0 \mathrm{~Hz}, 1 \mathrm{H}, 10-\mathrm{H}), 6.89$ (dd, $J=8.0 \mathrm{~Hz}, 1 \mathrm{H}, 9-\mathrm{H})$, 7.16 (m, 1H, 12'-H), 7.20 (m, 1H, 13'-H), 7.24 (m, 1H, 8-H), 7.26 (m, 2H, 7-H und 11'-H), $7.56\left(\mathrm{~d}, J=7.0 \mathrm{~Hz}, 1 \mathrm{H}, 14^{\prime}-\mathrm{H}\right)$.

${ }^{13}$ C-NMR (150.8 MHz, $\mathbf{C D C l}_{3}$ ): $\delta=27.5$ (q, 2- $\mathrm{CH}_{3}$ ), 29.0 (q, 5'-CH 3 ), 29.3 (q, 2'- $-\mathrm{CH}_{3}$ ), 30.6 (t, C-7'), 42.2 (t, C-11), 60.5 (t, C-12), 62.5 (s, C-15'), 73.4 (s, C-11a), 73.6 (s, C-10b), 75.7 (s, C-6'), 76.0 (s, C-3), 79.1 (s, C-3'), 80.1 (d, C-5), 108.0 (s, C-8'), 111.2 (d, C-10), 111.3 (d, C-11'), 119.2 (d, C-14'), 120.2 (d, C-9), 120.5 (d, C-12'), 123.0 (d, C-13'), 124.5 (d, C-9'), 125.0 (d, C-7), 126.5 (s, C-10a), 129.6 (s, C-14a'), 131.4 (d, C-8), 134.0 (s, C-10a'), 148.2 (s, C-6a), 163.2 (s, C-4), 165.6 (d, C-1), 166.0 (s, C-4'), 166.6 (d, C-1').

Minderkonformer:

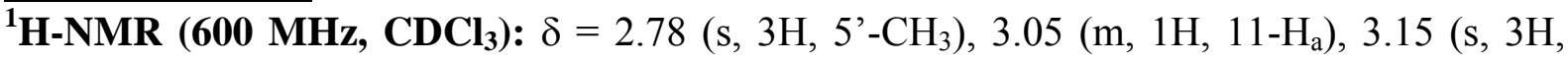
2- $\left.\mathrm{CH}_{3}\right), 3.22\left(\mathrm{~s}, 3 \mathrm{H}, 2^{\prime}-\mathrm{CH}_{3}\right), 3.26\left(\mathrm{~d}, J=16.0 \mathrm{~Hz}, 1 \mathrm{H}, 7^{\prime}-\mathrm{H}_{\mathrm{a}}\right), 3.89(\mathrm{~d}, J=12.5 \mathrm{~Hz}, 1 \mathrm{H}$, $\left.15^{\prime}-\mathrm{H}_{\mathrm{a}}\right), 4.17$ (d, $\left.J=16.0 \mathrm{~Hz}, 1 \mathrm{H}, 7^{\prime}-\mathrm{H}_{\mathrm{b}}\right), 4.23$ (d, $\left.J=12.5 \mathrm{~Hz}, 1 \mathrm{H}, 15^{\prime}-\mathrm{H}_{\mathrm{b}}\right), 4.24$ (d, $J=12.5$ $\left.\mathrm{Hz}, 1 \mathrm{H}, 12-\mathrm{H}_{\mathrm{a}}\right), 4.31\left(\mathrm{~d}, J=12.5 \mathrm{~Hz}, 1 \mathrm{H}, 12-\mathrm{H}_{\mathrm{b}}\right), 4.37$ (d, $\left.J=15.5 \mathrm{~Hz}, 1 \mathrm{H}, 11-\mathrm{H}_{\mathrm{b}}\right), 6.07$ (s, 1H, 5-H), 6.64 (s, 1H, 9’-H), 6.74 (d, $J=8.0 \mathrm{~Hz}, 1 \mathrm{H}, 10-\mathrm{H}), 6.89$ (dd, $J=8.0 \mathrm{~Hz}, 1 \mathrm{H}, 9-\mathrm{H})$, 
$7.16\left(\mathrm{~m}, 1 \mathrm{H}, 12^{\prime}-\mathrm{H}\right), 7.20\left(\mathrm{~m}, 1 \mathrm{H}, 13^{\prime}-\mathrm{H}\right), 7.24(\mathrm{~m}, 1 \mathrm{H}, 8-\mathrm{H}), 7.26(\mathrm{~m}, 2 \mathrm{H}, 7-\mathrm{H}$ und 11'-H), $7.56\left(\mathrm{~d}, J=7.0 \mathrm{~Hz}, 1 \mathrm{H}, 14^{\prime}-\mathrm{H}\right)$.

${ }^{13}$ C-NMR (150.8 MHz, $\left.\mathbf{C D C l}_{3}\right): \delta=27.5$ (q, 2-CH $\mathrm{CH}_{3}$ ), 28.3 (q, 2'- $\mathrm{CH}_{3}$ ), 29.9 (q, 5'-CH ), 32.0 (t, C-7'), 42.0 (t, C-11), 60.5 (t, C-12), 62.2 (s, C-15'), 73.4 (s, C-11a), 73.6 (s, C-10b), 75.8 (s, C-3'), 76.0 (s, C-3), 79.3 (s, C-6'), 80.1 (d, C-5), 108.0 (s, C-8'), 111.2 (d, C-10), 111.3 (d, C-11'), 119.2 (d, C-14'), 120.2 (d, C-9), 120.5 (d, C-12'), 123.0 (d, C-13'), 124.1 (d, C-9'), 125.0 (d, C-7), 126.5 (s, C-10a), 129.6 (s, C-14a'), 131.4 (d, C-8), 134.0 (s, C-10a'), 148.2 (s, C-6a), 163.2 (s, C-4), 165.6 (d, C-1), 165.6 (s, C-4'), 166.8 (d, C-1').

\section{Fumitremorgin $C^{146}(125)$}

$\mathrm{C}_{22} \mathrm{H}_{25} \mathrm{~N}_{3} \mathrm{O}_{3}(379.46)$

leicht gelber Feststoff

UV-löschend (254 nm)

$\mathbf{R}_{\mathbf{f}}$-Wert: 0.62 (Chloroform/Methanol 9:1)

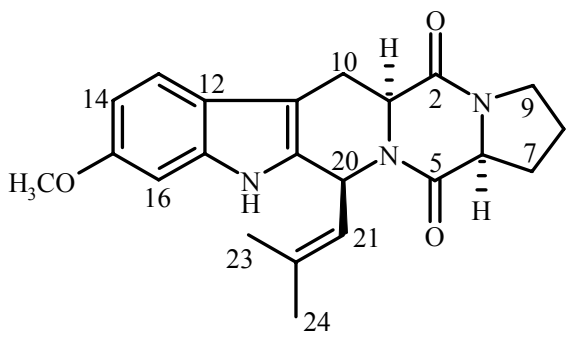

Anfärbeverhalten: grün (Anisaldehyd)

EI-MS: $\mathrm{m} / \mathrm{z}(\%)=379(100)[\mathrm{M}]^{+}, 281(50)$.

IR(KBr): $\widetilde{v}=3451,2930,1676,1612$ (sh), 1457, 1419, 1350, 1234, 1065, $744 \mathrm{~cm}^{-1}$.

UV: $(\mathrm{MeOH}): \quad\left[\lambda_{\max }(\lg \varepsilon)\right]=206(4.42), 223(4.47), 272(3.77), 297(3.83) \mathrm{nm}$.

$[\alpha]_{D}^{20}=-19^{\circ}(\mathrm{c}=0.1$, Methanol).

CD (MeOH): $\lambda_{\max }([\Theta])=203$ (894), 206 (-41405), 216 (-8360), 226 (-25119), 240 (762), 246 (-430), 272 (21078), 284 (8051), 298 (13086) nm.

${ }^{1}$ H-NMR (600 MHz, $\left.\mathbf{C D C l}_{3}\right): \delta_{\mathrm{H}}=1.63\left(\mathrm{~s}, 3 \mathrm{H}, 23-\mathrm{H}_{3}\right), 1.93\left(\mathrm{~m}, 1 \mathrm{H}, 8-\mathrm{H}_{\mathrm{a}}\right), 1.97(\mathrm{~d}, J=1.0$ $\left.\mathrm{Hz}, 3 \mathrm{H}, 24-\mathrm{H}_{3}\right), 2.05\left(\mathrm{~m}, 1 \mathrm{H}, 8-\mathrm{H}_{\mathrm{b}}\right), 2.19\left(\mathrm{~m}, 1 \mathrm{H}, 7-\mathrm{H}_{\mathrm{a}}\right), 2.39\left(\mathrm{~m}, 1 \mathrm{H}, 7-\mathrm{H}_{\mathrm{b}}\right), 3.08(\mathrm{dd}$, $\left.J=11.5,16.0 \mathrm{~Hz}, 1 \mathrm{H}, 10-\mathrm{H}_{\mathrm{a}}\right), 3.51\left(\mathrm{dd}, J=5.0,16.0 \mathrm{~Hz}, 1 \mathrm{H}, 10-\mathrm{H}_{\mathrm{b}}\right), 3.63(\mathrm{dd}, J=5.0$, $\left.8.5 \mathrm{~Hz}, 2 \mathrm{H}, 9-\mathrm{H}_{2}\right), 3.81\left(\mathrm{~s}, 3 \mathrm{H}, 15-\mathrm{OCH}_{3}\right), 4.11(\mathrm{dd}, J=8.5 \mathrm{~Hz}, 1 \mathrm{H}, 6-\mathrm{H}), 4.18(\mathrm{dd}, J=4.5$, $11.5 \mathrm{~Hz}, 1 \mathrm{H}, 3-\mathrm{H}), 4.88$ (dquint, $J=1.0,9.5 \mathrm{~Hz}, 1 \mathrm{H}, 21-\mathrm{H}), 5.97$ (d, $J=9.5 \mathrm{~Hz}, 1 \mathrm{H}, 20-\mathrm{H}$ ), $6.80(\mathrm{dd}, J=2.0,8.5 \mathrm{~Hz}, 1 \mathrm{H}, 14-\mathrm{H}), 6.84(\mathrm{~d}, J=2.0 \mathrm{~Hz}, 1 \mathrm{H}, 16-\mathrm{H}), 7.41(\mathrm{~d}, J=8.5 \mathrm{~Hz}, 1 \mathrm{H}$, 13-H), 7.64 (s, br, 1H, 18-NH).

${ }^{13}$ C-NMR (150.8 MHz, CDCl 3 ): $\delta_{\mathrm{C}}=18.1$ (q, C-24), 21.9 (t, C-10), 23.0 (t, C-8), 25.7 (q, C-23), 28.6 (t, C-7), 45.4 (t, C-9), 51.0 (d, C-20), 55.7 (q, 15- $\left.-\mathrm{OCH}_{3}\right), 56.7$ (d, C-3), 59.2 (d, C-6), 95.2 (d, C-16), 106.1 (s, C-11), 109.5 (d, C-14), 118.8 (d, C-13), 120.6 (s, C-12), 124.1 (d, C-21), 132.1 (s, C-19), 134.0 (s, C-22), 137.0 (s, C-17), 156.4 (s, C-15), 165.7 (s, C-2), 169.5 (s, C-5). 


\section{Trichoderma sp. Stamm Gö 101/19}

\subsection{Kultivierung und Isolierung der Metabolite}

Der Stamm Trichoderma sp. Gö 101/19 wurde wie unter B.II.3.1. beschrieben in Schüttelkolben (2 L, 20 x $100 \mathrm{~mL}$ ) mit 1158 als Nährmedium kultiviert und aufgearbeitet. Durch Chromatographie an Kieselgel $\left(\mathrm{CH}_{2} \mathrm{Cl}_{2} / \mathrm{MeOH} 19: 1 \rightarrow 9: 1 \rightarrow 3: 1\right)$ und Sephadex ${ }^{\circledR}$ LH-20 (Aceton) konnte Sorbicillin (126) in einer Ausbeute von $1.2 \mathrm{mg} / \mathrm{L}$ erhalten werden.

\subsection{Charakterisierung der Metabolite}

\section{Sorbicillin $^{147}(126)$}

$\mathrm{C}_{14} \mathrm{H}_{16} \mathrm{O}_{3}(232.28)$

gelber Feststoff

UV-löschend (254 nm)

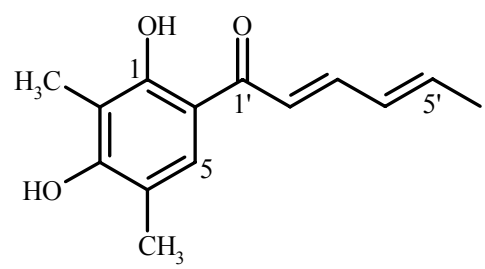

$\mathbf{R}_{\mathbf{f}}$-Wert: 0.5 (Chloroform/Methanol 9:1)

Anfärbeverhalten: gelb (Anisaldehyd)

EI-MS: $\mathrm{m} / \mathrm{z}(\%)=232(100)[\mathrm{M}]^{+}, 217(90), 191(40), 165(60), 136(36)$.

HRESI-MS: 255.09926 gefunden wie berechnet für $\left[\mathrm{C}_{14} \mathrm{H}_{16} \mathrm{O}_{3} \mathrm{Na}\right]^{+}$.

IR(KBr): $\widetilde{v}=3419,2925,1622,1564,1484,1366,1290,1232,1152 \mathrm{~cm}^{-1}$.

UV: $(\mathrm{MeOH}): \quad\left[\lambda_{\max }(\lg \varepsilon)\right]=320(3.97) \mathrm{nm}$;

$\mathrm{MeOH} / \mathrm{HCl}): \quad\left[\lambda_{\max }(\lg \varepsilon)\right]=321(3.96) \mathrm{nm}$;

$(\mathrm{MeOH} / \mathrm{NaOH}):\left[\lambda_{\max }(\lg \varepsilon)\right]=221$ (3.80), 274 (3.88), $298(3.79), 408(4.05) \mathrm{nm}$.

${ }^{1}$ H-NMR (600 MHz, $\mathbf{C D C l}_{3}$ ): $\delta=1.89$ (d, $\left.J=7.0 \mathrm{~Hz}, 3 \mathrm{H}, 6{ }^{\prime}-\mathrm{H}_{3}\right), 2.13$ (s, 3H, 4- $\left.\mathrm{H}_{3}\right), 2.20$ (s, $\left.3 \mathrm{H}, 2-\mathrm{H}_{3}\right), 6.26\left(\mathrm{dq}, J=7.0,15.0 \mathrm{~Hz}, 1 \mathrm{H}, 5^{\prime}-\mathrm{H}\right), 6.33$ (dd, $\left.J=11.0,15.0 \mathrm{~Hz}, 1 \mathrm{H}, 4^{\prime}-\mathrm{H}\right), 6.92$ (d, $\left.J=14.5 \mathrm{~Hz}, 1 \mathrm{H}, 2^{\prime}-\mathrm{H}\right), 7.42$ (s, 1H, 3-OH), 7.44 (dd, $J=11.0,14.5 \mathrm{~Hz}, 1 \mathrm{H}, 3$ '-H), 13.58 (s, 1H, 1-OH).

${ }^{13}$ C-NMR (150.8 MHz, $\left.\mathbf{C D C l}_{3}\right): \delta=7.5$ (q, C-), 15.6 (q, C-), 18.9 (q, C-), 110.4 (s, C-), 113.5 (s, C-), 114.4 (s, C-), 121.8 (d, C-), 128.7 (d, C-), 130.5 (d, C-), 141.2 (d, C-), 144.5 (d, C-), 158.7 (s, C-), 162.5 (s, C-), 192.5 (s, C-1'). 


\section{Humicola grisea Stamm Gö 101/26}

\subsection{Kultivierung und Isolierung der Metabolite}

Der Stamm Humicola grisea Gö 101/26 wurde wie unter B.II.3.1. beschrieben in Schüttelkolben (2 L, 20 x 100 mL) mit SGG als Nährmedium kultiviert und aufgearbeitet. Aus diesem Fermentationsansatz konnten durch Chromatographie an Kieselgel $\left(\mathrm{CH}_{2} \mathrm{Cl}_{2} / \mathrm{MeOH}\right.$ 19:1 $\left.\rightarrow 9: 1 \rightarrow 3: 1\right)$, Sephadex ${ }^{\circledR}$ LH-20 (Aceton) und erneut an Kieselgel $\left(\mathrm{CH}_{2} \mathrm{Cl}_{2} / \mathrm{MeOH}\right.$ 9:1) 5-Methoxysterigmatocystin (127) sowie Fuscoatrosid (128) in Ausbeuten von 1.0 bzw. $2.5 \mathrm{mg} / \mathrm{L}$ isoliert werden.

\subsection{Isolierung und Charakterisierung der Metabolite}

\section{6-Methoxysterigmatocystin ${ }^{148}(127)$}

$\mathrm{C}_{19} \mathrm{H}_{14} \mathrm{O}_{7}(354.32)$

leicht gelber Feststoff

UV-löschend (254 nm)

$\mathbf{R}_{\mathbf{f}}$-Wert: 0.88 (Chloroform/Methanol 9:1)

Anfärbeverhalten: hell braun (Anisaldehyd)

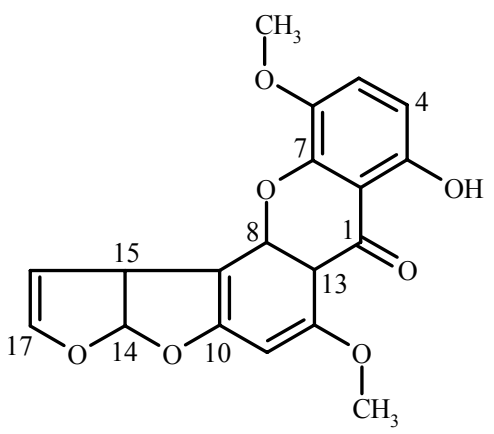

Smp.: $147^{\circ} \mathrm{C}$

EI-MS: m/z (\%) = 354 (100) [M] , 339 (20), 325 (32), 311 (44), 296 (20), 267 (24).

HRESI-MS: 355.08113 gefunden wie berechnet für $\left[\mathrm{C}_{19} \mathrm{H}_{15} \mathrm{O}_{7}\right]^{+}$.

IR(KBr): $\widetilde{v}=3421,2925,1625,1582,1455,1274,1228,1132,1096 \mathrm{~cm}^{-1}$.

UV: $(\mathrm{MeOH}): \quad\left[\lambda_{\max }(\lg \varepsilon)\right]=253(4.18), 328(3.90), 353(\mathrm{sh}, 3.51) \mathrm{nm}$;

$\mathrm{MeOH} / \mathrm{HCl}): \quad\left[\lambda_{\max }(\lg \varepsilon)\right]=254(4.17), 327(3.83), 354(\mathrm{sh}, 3.48) \mathrm{nm}$;

$(\mathrm{MeOH} / \mathrm{NaOH}):\left[\lambda_{\max }(\lg \varepsilon)\right]=243$ (4.19), 325 (3.81), 351 (sh, 3.47) nm.

$[\alpha]_{D}^{20}=-12^{\circ}(\mathrm{c}=0.1$, Methanol $)$.

CD (MeOH): $\lambda_{\max }([\Theta])=225$ (-24270), $337(-3522)$.

${ }^{1}$ H-NMR (600 MHz, $\left.\mathbf{C D C l}_{3}\right): \delta=3.91\left(\mathrm{~s}, 3 \mathrm{H}, 6-\mathrm{OCH}_{3}\right.$ oder 12- $\left.\mathrm{OCH}_{3}\right), 3.98\left(\mathrm{~s}, 3 \mathrm{H}, 6-\mathrm{OCH}_{3}\right.$ oder 12- $\mathrm{OCH}_{3}$ ), 4.78 (ddd, $\left.J=2.5,7.0 \mathrm{~Hz}, 1 \mathrm{H}, 15-\mathrm{H}\right), 5.43$ (dd, $\left.J=2.5 \mathrm{~Hz}, 1 \mathrm{H}, 16-\mathrm{H}\right), 6.40$ (s, 1H, 11-H), 6.48 (dd, $J=2.5 \mathrm{~Hz}, 1 \mathrm{H}, 17-\mathrm{H}), 6.79$ (d, $J=9.0 \mathrm{~Hz}, 1 \mathrm{H}, 4-\mathrm{H}), 6.81$ (d, $J=7.0$ $\mathrm{Hz}, 1 \mathrm{H}, 14-\mathrm{H}), 7.18$ (d, J = 9.0 Hz, 1H, 5-H), 13.45 (s, 1H, 3-OH). 
${ }^{13}$ C-NMR (150.8 MHz, $\mathbf{C D C l}_{3}$ ): $\delta=48.1$ (d, C-15), 56.8 (q, 6- $-\mathrm{OCH}_{3}$ oder 12-OCH $), 56.9$ (q, 6- $-\mathrm{OCH}_{3}$ oder 12- $\mathrm{OCH}_{3}$ ), 90.2 (d, C-11), 102.5 (s, C-16), 104.3 (d, C-4), 105.5 (s, C-13), 106.4 (d, C-9), 109.1 (d, C-2), 113.2 (d, C-14), 118.8 (d, C-5), 143.2 (s, C-6), 145.3 (d, C-17), 148.5 (s, C-3), 151.3 (s, C-8), 154.4 (s, C-7), 163.4 (s, C-12), 164.6 (s, C-10), 181.9 (s, C-1).

\section{Fuscoatrosid $^{149}(128)$}

$\mathrm{C}_{38} \mathrm{H}_{62} \mathrm{O}_{10}(678.91)$

farbloser Feststoff

$\mathbf{R}_{\mathbf{f}}$-Wert: 0.15 (Chloroform/Methanol 9:1)

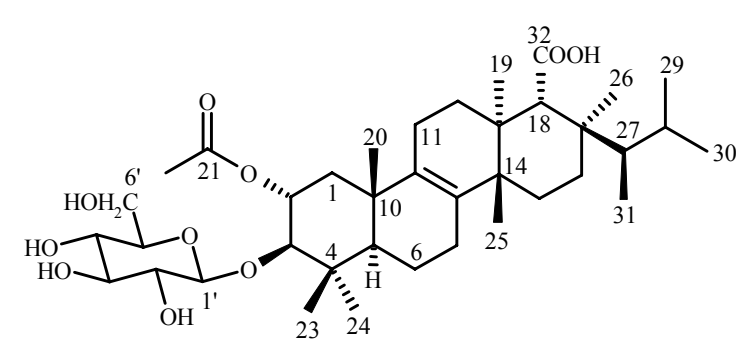

Anfärbeverhalten: violett (Anisaldehyd)

ESI-MS (positive Ionen): $\mathrm{m} / \mathrm{z}(\%)=701(90)[\mathrm{M}+\mathrm{Na}]^{+}, 1379(100)[2 \mathrm{M}+\mathrm{Na}]^{+}$.

HRESI-MS: 701.42352 gefunden für $\left[\mathrm{C}_{38} \mathrm{H}_{62} \mathrm{O}_{10} \mathrm{Na}\right]^{+}$.

${ }^{1}$ H-NMR (600 MHz, d $\mathbf{d}_{\mathbf{6}}$-DMSO): $\delta=0.73$ (d, $\left.J=7.0 \mathrm{~Hz}, 3 \mathrm{H}, 31-\mathrm{H}_{3}\right), 0.82(\mathrm{~d}, J=7.0 \mathrm{~Hz}$, $\left.3 \mathrm{H}, 30-\mathrm{H}_{3}\right), 0.86\left(\mathrm{~s}, 3 \mathrm{H}, 23-\mathrm{H}_{3}\right), 0.86\left(\mathrm{~d}, J=7.0 \mathrm{~Hz}, 3 \mathrm{H}, 29-\mathrm{H}_{3}\right), 0.95$ (s, 3H, 25- $\left.\mathrm{H}_{3}\right), 1.00$ (s, $\left.3 \mathrm{H}, 20-\mathrm{H}_{3}\right), 1.04\left(\mathrm{~s}, 3 \mathrm{H}, 19-\mathrm{H}_{3}\right), 1.08\left(\mathrm{~s}, 3 \mathrm{H}, 24-\mathrm{H}_{3}\right), 1.12\left(\mathrm{~m}, 2 \mathrm{H}, 5-\mathrm{H}\right.$ und 11- $\left.\mathrm{H}_{\mathrm{b}}\right), 1.15-1.20$ $\left(\mathrm{m}, 4 \mathrm{H}, 1-\mathrm{H}_{\mathrm{a}}, 12-\mathrm{H}_{\mathrm{a}}\right.$ und 27- $\left.\mathrm{H}_{2}\right), 1.22\left(\mathrm{~m}, 1 \mathrm{H}, 16-\mathrm{H}_{\mathrm{a}}\right), 1.24\left(\mathrm{~s}, 3 \mathrm{H}, 26-\mathrm{H}_{3}\right), 1.30\left(\mathrm{~m}, 1 \mathrm{H}, 6-\mathrm{H}_{\mathrm{a}}\right)$, $1.37\left(\mathrm{~m}, 1 \mathrm{H}, 15-\mathrm{H}_{\mathrm{a}}\right), 1.58\left(\mathrm{~m}, 2 \mathrm{H}, 12-\mathrm{H}_{\mathrm{b}}\right.$ und 16- $\left.\mathrm{H}_{\mathrm{b}}\right), 1.66-1.73\left(\mathrm{~m}, 2 \mathrm{H}, 6-\mathrm{H}_{\mathrm{b}}\right.$ und 16- $\left.\mathrm{H}_{\mathrm{b}}\right)$, $1.89-1.94\left(\mathrm{~m}, 3 \mathrm{H}, 1-\mathrm{H}_{\mathrm{b}} 7-\mathrm{H}_{\mathrm{a}}\right.$ und 11- $\left.\mathrm{H}_{\mathrm{b}}\right), 2.00\left(\mathrm{~s}, 3 \mathrm{H}, 22-\mathrm{H}_{3}\right), 2.14\left(\mathrm{~m}, 1 \mathrm{H}, 7-\mathrm{H}_{\mathrm{b}}\right), 2.17(\mathrm{~m}$, 1H, 28-H), 2.61 (s, 1H, 18-H), 2.93 (m, 1H, 2'-H), $3.05-3.08$ (m, 2H, 4'-H und 5'-H), 3.13 (dd, br, $J=7.5 \mathrm{~Hz}, 1 \mathrm{H}, 3$ '-H), 3.22 (d, $J=10.0 \mathrm{~Hz}, 1 \mathrm{H}, 3-\mathrm{H}), 3.49$ (d, br, $J=11.5 \mathrm{~Hz}, 1 \mathrm{H}$, 6'- $-\mathrm{H}_{\mathrm{a}}$ ), 3.65 (d, br, J=11.5 Hz, 1H, 6'- $\left.-\mathrm{H}_{\mathrm{b}}\right), 4.19$ (s, br, 1H, 6'-OH), 4.21 (d, J = $7.5 \mathrm{~Hz}, 1 \mathrm{H}$, 1'-H), 4.76 (s, br, 1H, 4'-OH), 4.81 (s, br, 1H, 3'-OH), 4.91 (d, br, J = 5.0 Hz, 1H, 2'-OH), 4.98 (ddd, $J=4.0,10.0,12.0 \mathrm{~Hz}, 1 \mathrm{H}, 2-\mathrm{H})$.

${ }^{13}$ C-NMR (150.8 MHz, d6-DMSO): $\delta=8.0$ (q, C-31), 16.5 (q, C-19), 17.4 (q, C-23), 18.2 (q, C-29), 18.3 (t, C-11), 18.9 (t, C-6), 20.5 (q, C-26), 20.7 (q, C-20), 21.0 (q, C-22), 21.7 (q, C-25), 24.7 (q, C-30), 25.2 (t, C-15), 25.9 (t, C-28), 26.3 (t, C-7), 27.6 (q, C-24), 28.9 (t, C-16), 30.8 (t, C-12), 37.1 (s, C-13), 37.7 (s, C-10), 39.8 (s, C-17), 40.0 (s, C-14), 40.3 (s, C-4), 41.0 (t, C-1), 47.7 (d, C-27), 49.4 (d, C-5), 52.4 (d, C-18), 61.1 (t, C-6'), 69.4 (d, C-2), 70.1 (d, C-4'), 74.3 (d, C-2'), 76.5 (d, C-5'), 77.0 (d, C-3'), 87.7 (d, C-3), 104.7 (d, C-1'), 133.4 (s, C-8), 133.6 (s, C-9), 170.0 (s, C-21), 175.1 (s, C-32). 


\section{Stamm A6651}

\subsection{Kultivierung und Aufarbeitung}

Der Stamm A6651 wurde wie unter B.II.3.1. beschrieben in Schüttelkolben $(2 \mathrm{~L}, 20 \mathrm{x}$ $100 \mathrm{~mL}$ ) in M2-Medium kultiviert und aufgearbeitet. Die erste Aufreinigung der Extrakte aus Kulturfiltrat und Mycel erfolgte durch Säulenchromatographie an Kieselgel $\left(\mathrm{CH}_{2} \mathrm{Cl}_{2} / \mathrm{MeOH}\right.$ $19: 1 \rightarrow 9: 1 \rightarrow 3: 1)$.

\subsection{Variation der Fermentationsbedingungen}

\section{Variation A: Zufütterung von Carbonsäuren}

Um Eremophilane mit anderen Carbonsäuren in der Seitenkette zu erhalten wurden sieben verschiedene Carbonsäuren (Ascorbinsäure, Essigsäure, Benzoesäure, Hexensäure, Nicotinsäure, Sorbinsäure und Zimtsäure) an den Stamm verfüttert. Dazu wurde der Stamm A6651 wie unter B.II.3.1. beschrieben kultiviert. Nach 24 Stunden wurden die obigen Carbonsäuren, die vorher in sterilem Wasser gelöst und auf einen pH-Wert von 5.0 gebracht wurden, in Konzentrationen von 0.1 und $1.0 \mathrm{~g} / \mathrm{L} \mathrm{zu}$ den Fermentationsansätzen gegeben. Nach 96 Stunden Fermentationsdauer wurde der Stamm geerntet. Die verschiedenen Ansätze wurden auf einen pH-Wert von 4-5 gebracht, mit derselben Menge Ethylacetat versetzt und 5 min mit dem Ultraturax behandelt. Anschließend wurde das Mycel abzentrifugiert und verworfen. Nach Abtrennen der organischen Phase wurde ein weiteres Mal mit Ethylacetat extrahiert und die organischen Phasen vereinigt. Die nach dem Entfernen des Lösungsmittels erhaltenen Extrakte wurden dünnschichtchromatographisch und per HPLC-MS analysiert. Dabei wurde selektiv nach den bei einem Einbau erwarteten Molmassen gesucht.

\section{Variation B: Kultivierung in P-Kolben}

Die Ruhekultivierung in P-Kolben erfolgte bei Raumtemperatur in den Medien M2, SGG, G20, 1158, 1549 und E2 (jeweils 2 x 1 L). Die P-Kolben wurden mit je 2 x 1/8 einer gut bewachsenen Agarplatte des Stammes beimpft und direkt nach dem Animpfen und nach 7 Tagen kräftig geschüttelt, um ein gleichmäßiges Wachstum zu ermöglichen. Die P-Kolben wurden nach 28 Tagen nach dem unter B.II.3.2. beschriebenen Verfahren aufgearbeitet. 


\section{Variation C: Kultivierung unter Beleuchtung}

Je zwei Agarplatten wurden mit dem Stamm A6651 beimpft und im Dunkeln, unter Einfluss von Tageslicht und unter permanenter Beleuchtung kultiviert. Nach zwei Wochen wurden die Agarplatten mit Aceton versetzt und fünf Minuten mit dem Ultraturax zerkleinert. Die so erhaltenen Extrakte wurden mit der HPLC (Säule 4, Programm G) analysiert.

\subsection{Isolierung und Charakterisierung der Metabolite}

\section{Dihydrobipolaroxin $^{151}$ (129)}

129 konnte sowohl aus dem Mycel- als auch aus dem Kulturfiltratextrakt durch weitere Chromatographie an Sephadex ${ }^{\circledR}$ LH-20 (MeOH) und Kieselgel $\left(\mathrm{CH}_{2} \mathrm{Cl}_{2} / \mathrm{MeOH}\right.$ 9:1) in Ausbeuten von $3.0 \mathrm{mg} / \mathrm{L}$ erhalten werden.

$\mathrm{C}_{15} \mathrm{H}_{20} \mathrm{O}_{4}(264.32)$

leicht gelbes Öl

UV-löschend (254 nm)

$\mathbf{R}_{\mathbf{f}}$-Wert: 0.30 (Chloroform/Methanol 9:1)

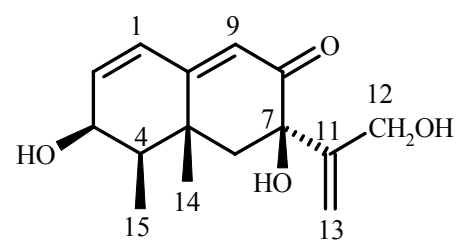

Anfärbeverhalten: blau (Anisaldehyd)

ESI-MS (positive Ionen): $\mathrm{m} / \mathrm{z}(\%)=287(17)[\mathrm{M}+\mathrm{Na}]^{+}, 551(100)[2 \mathrm{M}+\mathrm{Na}]^{+}$.

HRESI-MS: 287.12596 gefunden wie berechnet für $\left[\mathrm{C}_{15} \mathrm{H}_{20} \mathrm{O}_{4} \mathrm{Na}\right]^{+}$.

UV: $(\mathrm{MeOH}): \quad\left[\lambda_{\max }(\lg \varepsilon)\right]=225(3.55), 281(3.71) \mathrm{nm}$;

$\mathrm{MeOH} / \mathrm{HCl}): \quad\left[\lambda_{\max }(\lg \varepsilon)\right]=231(3.77), 281(3.31) \mathrm{nm} ;$

$(\mathrm{MeOH} / \mathrm{NaOH}):\left[\lambda_{\max }(\lg \varepsilon)\right]=225(3.55), 281(3.71) \mathrm{nm}$.

${ }^{1}$ H-NMR (500 MHz, $\left.\mathbf{C D C l}_{3}\right): 1.17$ (d, $\left.J=7.0 \mathrm{~Hz}, 3 \mathrm{H}, 15-\mathrm{H}_{3}\right), 1.46$ (s, 3H, 14- $\mathrm{H}_{3}$ ), 1.76 (dq, $J=4.5,7.0 \mathrm{~Hz}, 1 \mathrm{H}, 4-\mathrm{H}), 1.95\left(\mathrm{~d}, J=14.5 \mathrm{~Hz}, 1 \mathrm{H}, 6-\mathrm{H}_{\mathrm{a}}\right), 2.00\left(\mathrm{~d}, J=14.5 \mathrm{~Hz}, 1 \mathrm{H}, 6-\mathrm{H}_{\mathrm{b}}\right)$, $4.13\left(\mathrm{~d}, J=13.5 \mathrm{~Hz}, 1 \mathrm{H}, 12-\mathrm{H}_{\mathrm{a}}\right), 4.17(\mathrm{dd}, J=4.5 \mathrm{~Hz}, 1 \mathrm{H}, 3-\mathrm{H}), 4.23$ (d, $J=13.5 \mathrm{~Hz}, 1 \mathrm{H}$, $\left.12-\mathrm{H}_{\mathrm{b}}\right), 5.26\left(\mathrm{~s}, 1 \mathrm{H}, 13-\mathrm{H}_{\mathrm{a}}\right), 5.28\left(\mathrm{~s}, 1 \mathrm{H}, 13-\mathrm{H}_{\mathrm{a}}\right), 5.92(\mathrm{~s}, 1 \mathrm{H}, 9-\mathrm{H}), 6.32(\mathrm{~s}, 1 \mathrm{H}, 1-\mathrm{H}), 6.33(\mathrm{~d}$, $J=4.5 \mathrm{~Hz}, 1 \mathrm{H}, 2-\mathrm{H})$.

${ }^{13}$ C-NMR (150.8 MHz, CDCL 3 ): $\delta=10.6$ (q, C-15), 23.0 (q, C-14), 36.5 (s, C-5), 42.0 (d, C-4), 45.5 (t, C-6), 64.1 (s, C-12), 68.2 (d, C-3), 78.2 (s, C-7), 123.5 (d, C-9), 129.2 (d, C-2), 137.2 (d, C-1), 113.5 (t, C-13), 151.8 (s, C-11), 164.1 (s, C-10), 199.5 (s, C-8). 


\section{Bipolaroxin $^{151}$ (130)}

130 konnte aus dem Kulturfiltratextrakt durch weitere Chromatographie an Sephadex ${ }^{\circledR}$ LH-20 (Aceton) und Kieselgel $\left(\mathrm{CH}_{2} \mathrm{Cl}_{2} / \mathrm{MeOH} 9: 1\right)$ in Ausbeuten von $5.8 \mathrm{mg} / \mathrm{L}$ erhalten werden.

$\mathrm{C}_{15} \mathrm{H}_{18} \mathrm{O}_{4}(262.31)$

leicht gelbes Öl

UV-löschend (254 nm)

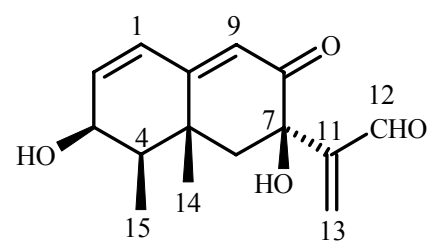

$\mathbf{R}_{\mathbf{f}}$-Wert: 0.45 (Chloroform/Methanol 9:1)

Anfärbeverhalten: blau (Anisaldehyd)

Smp.: $164^{\circ} \mathrm{C}$

EI-MS: $\mathrm{m} / \mathrm{z}(\%)=262(2)[\mathrm{M}]^{+}, 164(10), 154$ (100), $86(20), 70$ (48), 41(10).

ESI-MS (positive Ionen): $\mathrm{m} / \mathrm{z}(\%)=285(100)[\mathrm{M}+\mathrm{Na}]^{+}, 547(45)[2 \mathrm{M}+\mathrm{Na}]^{+}$.

HRESI-MS: 285.11031 gefunden wie berechnet für $\left[\mathrm{C}_{15} \mathrm{H}_{18} \mathrm{O}_{4} \mathrm{Na}\right]^{+}$.

IR(KBr): $\widetilde{v}=3425,3269,2957,2930,2873,1668,1651,1636,1558,1542,1470,1453$, $1440,1385,1340,1302,1272,1238,1117 \mathrm{~cm}^{-1}$.

UV: $(\mathrm{MeOH}): \quad\left[\lambda_{\max }(\lg \varepsilon)\right]=279(3.60) \mathrm{nm}$;

$\mathrm{MeOH} / \mathrm{HCl}): \quad\left[\lambda_{\max }(\lg \varepsilon)\right]=279(3.54) \mathrm{nm}$;

$(\mathrm{MeOH} / \mathrm{NaOH}):\left[\lambda_{\max }(\lg \varepsilon)\right]=249(3.58), 278(3.38) \mathrm{nm}$.

CD (MeOH): $\lambda_{\max }([\Theta])=210$ (-7309), 240 (3517), 251 (776), 260 (3248), 334 (4051).

${ }^{1}$ H-NMR (500 MHz, $\left.\mathbf{C D C l}_{3}\right): \delta=1.03\left(\mathrm{~d}, J=7.0 \mathrm{~Hz}, 3 \mathrm{H}, 15-\mathrm{H}_{3}\right), 1.45$ (s, 3H, 14-H $\left.{ }_{3}\right), 1.74$ $(\mathrm{dq}, J=5.0,7.0 \mathrm{~Hz}, 1 \mathrm{H}, 4-\mathrm{H}), 1.93$ (d, $\left.J=14.5 \mathrm{~Hz}, 1 \mathrm{H}, 6-\mathrm{H}_{\mathrm{a}}\right), 2.04$ (d, $J=14.5 \mathrm{~Hz}, 1 \mathrm{H}$, $\left.6-\mathrm{H}_{\mathrm{b}}\right), 4.13(\mathrm{dd}, J=5.0 \mathrm{~Hz}, 1 \mathrm{H}, 3-\mathrm{H}), 5.93(\mathrm{~s}, 1 \mathrm{H}, 9-\mathrm{H}), 6.27\left(\mathrm{~s}, 1 \mathrm{H}, 13-\mathrm{H}_{\mathrm{a}}\right), 6.30(\mathrm{~d}, J=5.0$ $\mathrm{Hz}, 1 \mathrm{H}, 2-\mathrm{H}), 6.30$ (s, 1H, 1-H), 6.82 (s, 1H, 13- $\left.\mathrm{H}_{\mathrm{b}}\right), 9.48$ (s, 1H, 12-H).

${ }^{13}$ C-NMR (125.7 MHz, CDCl 3 ): $\delta=10.5$ (q, C-15), 23.0 (q, C-14), 36.4 (s, C-5), 41.9 (d, C-4), 44.5 (t, C-6), 68.2 (d, C-3), 74.8 (s, C-7), 123.5 (d, C-9), 128.6 (d, C-2), 136.8 (d, C-1), 135.9 (t, C-13), 154.7 (s, C-11), 163.0 (s, C-10), 192.8 (s, C-12), 197.3 (s, C-8). 


\section{Dendryphiellin $E^{152}(131)$}

131 konnte aus dem Mycelextrakt durch weitere Chromatographie an Sephadex ${ }^{\circledR}$ LH-20 (Aceton) und Kieselgel $\left(\mathrm{CH}_{2} \mathrm{Cl}_{2} / \mathrm{MeOH} 19: 1\right)$ in Ausbeuten von $6.0 \mathrm{mg} / \mathrm{L}$ erhalten werden.

$\mathrm{C}_{24} \mathrm{H}_{32} \mathrm{O}_{5}(400.51)$

leicht gelbes Ö1

UV-löschend (254 nm)

Rf-Wert: 0.62 (Chloroform/Methanol 9:1)

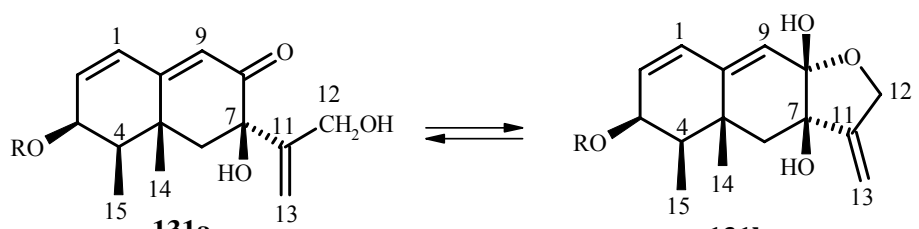

131b

Anfärbeverhalten: blau (Anisaldehyd)

ESI-MS (positive Ionen): $\mathrm{m} / \mathrm{z}(\%)=423(15)[\mathrm{M}+\mathrm{Na}]^{+}, 823(100)[2 \mathrm{M}+\mathrm{Na}]^{+}$;

(negative Ionen): $\mathrm{m} / \mathrm{z}(\%)=399(40)[\mathrm{M}-\mathrm{H}]^{-}, 799(100)[2 \mathrm{M}-\mathrm{H}]^{-}$.

HRESI-MS: 423.21478 gefunden wie berechnet für $\left[\mathrm{C}_{24} \mathrm{H}_{32} \mathrm{O}_{5} \mathrm{Na}\right]^{+}$.

IR(KBr): $\widetilde{v}=3441,2962,2927,2867,1709,1662,1636,1594,1458,1421,1380,1340$, $1303,1259,1241,1187,1139,1110,1081,1066,1029,996,936,912,882 \mathrm{~cm}^{-1}$.

UV: $(\mathrm{MeOH}): \quad\left[\lambda_{\max }(\lg \varepsilon)\right]=268(4.52) \mathrm{nm}$;

$\mathrm{MeOH} / \mathrm{HCl}): \quad\left[\lambda_{\max }(\lg \varepsilon)\right]=242(\mathrm{sh}, 4.38), 261(4.46) \mathrm{nm}$;

$(\mathrm{MeOH} / \mathrm{NaOH}):\left[\lambda_{\max }(\lg \varepsilon)\right]=258(4.50) \mathrm{nm}$.

CD (MeOH): $\lambda_{\max }([\Theta])=214$ (-15784), 220 (-11274), 232 (-32157), 243 (-11805), 254 (-19172), 278 (92810).

Ketoform (131a):

${ }^{1}$ H-NMR (500 MHz, $\left.\mathbf{C D C l}_{3}\right): \delta=0.84\left(\mathrm{t}, J=7.5 \mathrm{~Hz}, 3 \mathrm{H}, 8^{\prime}-\mathrm{H}_{3}\right), 1.00(\mathrm{~d}, J=7.0 \mathrm{~Hz}, 3 \mathrm{H}$, 9'- $\left.\mathrm{H}_{3}\right), 1.04$ (d, $\left.J=6.5 \mathrm{~Hz}, 3 \mathrm{H}, 15-\mathrm{H}_{3}\right), 1.35$ (m, 2H, 7'-H), 1.48 (s, 3H, 14- $\mathrm{H}_{3}$ ), 1.50 (d, $\left.J=14.5 \mathrm{~Hz}, 1 \mathrm{H}, 6-\mathrm{H}_{\mathrm{a}}\right), 1.96(\mathrm{dq}, J=5.0,7.0 \mathrm{~Hz}, 1 \mathrm{H}, 4-\mathrm{H}), 1.98$ (d, $\left.J=14.0 \mathrm{~Hz}, 1 \mathrm{H}, 6-\mathrm{H}_{\mathrm{b}}\right)$, $2.15\left(\mathrm{~m}, 1 \mathrm{H}, 6\right.$ '-H), 4.14 (d, $\left.J=13.5 \mathrm{~Hz}, 1 \mathrm{H}, 12-\mathrm{H}_{\mathrm{a}}\right), 4.24$ (d, $\left.J=13.5 \mathrm{~Hz}, 1 \mathrm{H}, 12-\mathrm{H}_{\mathrm{b}}\right), 5.25$ $\left(\mathrm{s}, 1 \mathrm{H}, 13-\mathrm{H}_{\mathrm{a}}\right), 5.28\left(\mathrm{~s}, 1 \mathrm{H}, 13-\mathrm{H}_{\mathrm{b}}\right), 5.42(\mathrm{dd}, J=5.0 \mathrm{~Hz}, 1 \mathrm{H}, 3-\mathrm{H}), 5.79(\mathrm{~d}, J=15.5 \mathrm{~Hz}, 1 \mathrm{H}$, 2'-H), 5.93 (s, 1H, 9-H), 6.00 (dd, $J=7.5,15.5 \mathrm{~Hz}, 1 \mathrm{H}, 5$ '-H), 6.13 (dd, $J=10.5,15.5 \mathrm{~Hz}$, 1H, 4'-H), 6.28 (dd, $J=5.0,10.0 \mathrm{~Hz}, 1 \mathrm{H}, 2-\mathrm{H}), 6.37$ (d, $J=10.0 \mathrm{~Hz}, 1 \mathrm{H}, 1-\mathrm{H}), 7.22$ (dd, $\left.J=10.5,15.5 \mathrm{~Hz}, 1 \mathrm{H}, 3^{\prime}-\mathrm{H}\right)$.

${ }^{13}$ C-NMR (125.7 MHz, CDCl 3 ): $\delta=10.3$ (q, C-15), 11.7 (q, C-8'), 19.5 (q, C-9'), 22.5 (q, C-14), 29.3 (t, C-7'), 36.4 (s, C-5), 38.8 (d, C-6'), 41.1 (d, C-4), 45.5 (t, C-6), 64.0 (s, C-12), 68.8 (d, C-3), 78.1 (s, C-7), 113.5 (t, C-13), 118.7 (d, C-2'), 123.9 (C-9), 126.6 (d, C-4'), 
130.8 (d, C-1), 133.7 (d, C-2), 146.1 (d, C-3'), 151.0 (d, C-5'), 151.8 (s, C-11), 163.4 (s, C-10), 166.8 (s, C-1'), 199.5 (s, C-8).

Halbacetalform (131b):

$\mathbf{R}_{\mathbf{f}}$-Wert: 0.89 (Chloroform/Methanol 9:1)

Anfärbeverhalten: blau (Anisaldehyd)

${ }^{1}$ H-NMR (500 MHz, $\left.\mathbf{C D C l}_{3}\right): \delta=0.84\left(\mathrm{t}, J=7.5 \mathrm{~Hz}, 3 \mathrm{H}, 8^{\prime}-\mathrm{H}_{3}\right), 0.97$ (d, $J=6.5 \mathrm{~Hz}, 3 \mathrm{H}$,

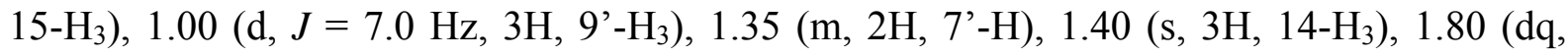
$J=5.0,7.0 \mathrm{~Hz}, 1 \mathrm{H}, 4-\mathrm{H}), 1.99\left(\mathrm{~d}, J=14.5 \mathrm{~Hz}, 1 \mathrm{H}, 6-\mathrm{H}_{\mathrm{a}}\right), 2.15\left(\mathrm{~m}, 1 \mathrm{H}, 6{ }^{\prime}-\mathrm{H}\right), 2.26$ (d, $\left.J=14.0 \mathrm{~Hz}, 1 \mathrm{H}, 6-\mathrm{H}_{\mathrm{b}}\right), 4.46\left(\mathrm{dd}, J=2.0,13.5 \mathrm{~Hz}, 1 \mathrm{H}, 12-\mathrm{H}_{\mathrm{a}}\right), 4.56(\mathrm{dd}, J=2.0,13.5 \mathrm{~Hz}, 1 \mathrm{H}$, $\left.12-\mathrm{H}_{\mathrm{b}}\right), 5.05\left(\mathrm{dd}, J=2.0 \mathrm{~Hz}, 1 \mathrm{H}, 13-\mathrm{H}_{\mathrm{a}}\right), 5.28\left(\mathrm{dd}, J=2.0 \mathrm{~Hz}, 1 \mathrm{H}, 13-\mathrm{H}_{\mathrm{b}}\right), 5.36(\mathrm{dd}, J=5.0$ $\mathrm{Hz}, 1 \mathrm{H}, 3-\mathrm{H}), 5.54$ (s, 1H, 9-H), 5.79 (d, $\left.J=15.5 \mathrm{~Hz}, 1 \mathrm{H}, 2^{\prime}-\mathrm{H}\right), 6.00$ (dd, $J=7.5,15.5 \mathrm{~Hz}$, 1H, 5'-H), 6.13 (dd, $\left.J=10.5,15.5 \mathrm{~Hz}, 1 \mathrm{H}, 4^{\prime}-\mathrm{H}\right), 5.90$ (dd, $\left.J=5.0,10.0 \mathrm{~Hz}, 1 \mathrm{H}, 2-\mathrm{H}\right), 6.19$ (d, $J=10.0 \mathrm{~Hz}, 1 \mathrm{H}, 1-\mathrm{H}), 7.22$ (dd, $\left.J=10.5,15.5 \mathrm{~Hz}, 1 \mathrm{H}, 3^{\prime}-\mathrm{H}\right)$.

${ }^{13}$ C-NMR (125.7 MHz, CDCl 3 ): $\delta=10.1$ (q, C-15), 11.7 (q, C-8'), 19.5 (q, C-9'), 20.6 (q, C-14), 29.3 (t, C-7'), 36.1 (s, C-5), 38.8 (d, C-6'), 42.1 (d, C-4), 43.4 (t, C-6), 67.6 (s, C-12), 69.9 (d, C-3), 79.0 (s, C-7), 99.5 (s, C-8), 106.1 (t, C-13), 119.2 (d, C-2'), 122.5 (C-9), 126.7 (d, C-4'), 127.1 (d, C-2), 131.1 (d, C-1), 144.5 (s, C-11), 145.5 (d, C-3'), 150.4 (d, C-5'), 153.4 (s, C-10), 167.0 (s, C-1').

\section{$\mathrm{KM}^{101}{ }^{153}(132)$}

132 konnte aus dem Kulturfiltratextrakt durch weitere Chromatographie an Sephadex ${ }^{\circledR}$ LH-20 (Aceton) und Kieselgel $\left(\mathrm{CH}_{2} \mathrm{Cl}_{2} / \mathrm{MeOH} 19: 1\right)$ in Ausbeuten von $5.5 \mathrm{mg} / \mathrm{L}$ erhalten werden.

$\mathrm{C}_{24} \mathrm{H}_{30} \mathrm{O}_{5}(398.50)$

leicht gelbes Öl

UV-löschend (254 nm)

$\mathbf{R}_{\mathbf{f}}$-Wert: 0.77 (Chloroform/Methanol 9:1)

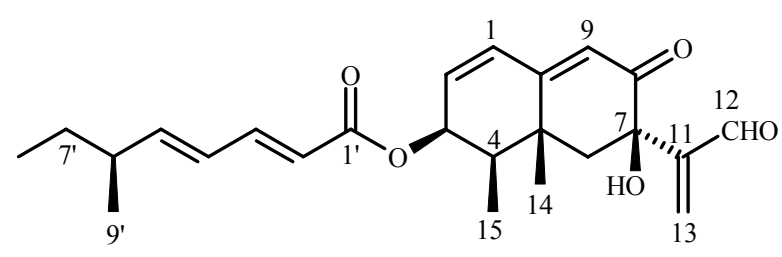

Anfärbeverhalten: blau (Anisaldehyd)

ESI-MS (positive Ionen): $\mathrm{m} / \mathrm{z}(\%)=421(50)[\mathrm{M}+\mathrm{Na}]^{+}, 819(100)[2 \mathrm{M}+\mathrm{Na}]^{+}$.

HRESI-MS: 421.19913 gefunden wie berechnet für $\left[\mathrm{C}_{24} \mathrm{H}_{30} \mathrm{O}_{5} \mathrm{Na}\right]^{+}$.

IR(KBr): $\widetilde{v}=3427,2958,2923,2852,1711,1664,1636,1459,1379,1342,1300,1258$, $1241,1185,1139,1110,1038,997,915,884 \mathrm{~cm}^{-1}$. 
UV: $(\mathrm{MeOH}): \quad\left[\lambda_{\max }(\lg \varepsilon)\right]=273(4.39) \mathrm{nm} ;$

$\mathrm{MeOH} / \mathrm{HCl}): \quad\left[\lambda_{\max }(\lg \varepsilon)\right]=271(4.36) \mathrm{nm}$;

$(\mathrm{MeOH} / \mathrm{NaOH}):\left[\lambda_{\max }(\lg \varepsilon)\right]=258(4.37) \mathrm{nm}$.

CD (MeOH): $\lambda_{\max }([\Theta])=210$ (-15940), 253 (-33500), 278 (82227), 324(3489), 352(6344).

${ }^{1}$ H-NMR (500 MHz, $\mathbf{C D C l}_{3}$ ): $\delta=0.83\left(\mathrm{t}, J=7.0 \mathrm{~Hz}, 3 \mathrm{H}, 8^{\prime}-\mathrm{H}_{3}\right), 0.98$ (d, $J=7.0 \mathrm{~Hz}, 3 \mathrm{H}$, 9'- $\left.\mathrm{H}_{3}\right), 1.00$ (d, $\left.J=6.5 \mathrm{~Hz}, 3 \mathrm{H}, 15-\mathrm{H}_{3}\right), 1.34$ (m, 2H, 7'-H), 1.48 (s, 3H, 14- $\left.\mathrm{H}_{3}\right), 1.96$ (d, $\left.J=14.0 \mathrm{~Hz}, 1 \mathrm{H}, 6-\mathrm{H}_{\mathrm{a}}\right), 1.96(\mathrm{dq}, J=5.0,6.5 \mathrm{~Hz}, 1 \mathrm{H}, 4-\mathrm{H}), 2.05$ (d, $\left.J=14.0 \mathrm{~Hz}, 1 \mathrm{H}, 6-\mathrm{H}_{\mathrm{b}}\right)$, 2.14 (m, 1H, 6'-H), 2.80 (s, br, 1H, 7-OH), 5.40 (dd, $J=5.0 \mathrm{~Hz}, 1 \mathrm{H}, 3-\mathrm{H}), 5.78$ (d, $J=15.5$ Hz, 1H, 2'-H), 5.97 (s, 1H, 9-H), 5.99 (dd, $J=8.0,15.5$ Hz, 1H, 5'-H), 6.13 (dd, $J=11.0$, $15.5 \mathrm{~Hz}, 1 \mathrm{H}, 4$ '-H), 6.25 (dd, $J=5.0,10.0 \mathrm{~Hz}, 1 \mathrm{H}, 2-\mathrm{H}), 6.39$ (d, $J=10.0 \mathrm{~Hz}, 1 \mathrm{H}, 1-\mathrm{H}), 6.82$ (s, 2H, 13- $\left.\mathrm{H}_{2}\right), 7.22$ (dd, $\left.J=11.0,15.5 \mathrm{~Hz}, 1 \mathrm{H}, 3^{\prime}-\mathrm{H}\right), 9.50$ (s, 1H, 12-H).

${ }^{13}$ C-NMR (125.7 MHz, CDCl $)$ ): $\delta=10.2$ (q, C-15), 11.7 (q, C-8'), 19.5 (q, C-9'), 22.5 (q, C-14), 29.3 (t, C-7'), 36.2 (s, C-5), 38.8 (d, C-6'), 40.9 (d, C-4), 44.4 (t, C-6), 68.9 (d, C-3), 74.8 (s, C-7), 118.7 (d, C-2'), 124.0 (C-9), 126.6 (d, C-4'), 131.1 (d, C-1), 133.2 (d, C-2), 135.9 (t, C-13), 146.0 (d, C-3'), 150.9 (d, C-5'), 154.6 (s, C-11), 162.2 (s, C-10), 166.8 (s, C-1'), 192.7 (s, C-12), 197.4 (s, C-8).

\section{Metabolite A6651-E (133)}

133 konnte sowohl aus dem Mycel- als auch aus dem Kulturfiltratextrakt durch weitere Chromatographie an Sephadex ${ }^{\circledR}$ LH-20 $(\mathrm{MeOH})$ und Kieselgel $\left(\mathrm{CH}_{2} \mathrm{Cl}_{2} / \mathrm{MeOH}\right.$ 9:1 $+0.1 \%$ $\mathrm{AcOH}$ ) in Ausbeuten von $4.0 \mathrm{mg} / \mathrm{L}$ erhalten werden.

$\mathrm{C}_{24} \mathrm{H}_{30} \mathrm{O}_{6}(414.50)$

leicht gelbes Öl

UV-löschend (254 nm)

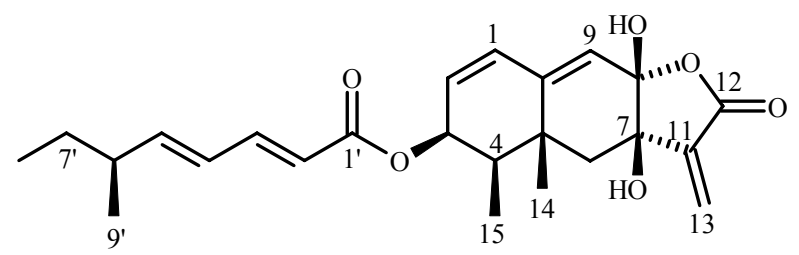

$\mathbf{R}_{\mathbf{f}}$-Wert: 0.19 (Chloroform/Methanol 9:1)

Anfärbeverhalten: blau (Anisaldehyd)

ESI-MS (positive Ionen): $\mathrm{m} / \mathrm{z}(\%)=437(20)[\mathrm{M}+\mathrm{Na}]^{+}, 851(100)[2 \mathrm{M}+\mathrm{Na}]^{+}$.

(negative Ionen): $\mathrm{m} / \mathrm{z}(\%)=413(100)[\mathrm{M}-\mathrm{H}]^{-}$.

HRESI-MS: 437.19404 gefunden wie berechnet für $\left[\mathrm{C}_{24} \mathrm{H}_{30} \mathrm{O}_{6} \mathrm{Na}\right]^{+}$.

IR(KBr): $\widetilde{v}=3425,2963,2929,2877,1707,1663,1536,1458,1380,1343,1304,1259$, $1242,1204,1187,1141,1107,1035,996,915,887,667,575 \mathrm{~cm}^{-1}$. 
UV: $(\mathrm{MeOH}): \quad\left[\lambda_{\max }(\lg \varepsilon)\right]=275(4,37) \mathrm{nm}$.

CD (MeOH): $\lambda_{\max }([\Theta])=226$ (3906), 253 (-53981), 279 (105962), 334 (7591), 350 (6683).

${ }^{1}$ H-NMR (500 MHz, $\left.\mathbf{C D C l}_{3}\right): \delta=0.84\left(\mathrm{t}, J=7.5 \mathrm{~Hz}, 3 \mathrm{H}, 8{ }^{\prime}-\mathrm{H}_{3}\right), 0.99$ (d, $J=7.0 \mathrm{~Hz}, 3 \mathrm{H}$,

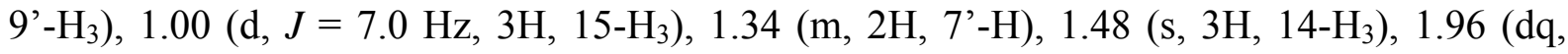
$J=5.0,7.0 \mathrm{~Hz}, 1 \mathrm{H}, 4-\mathrm{H}), 2.05\left(\mathrm{~d}, J=14.0 \mathrm{~Hz}, 1 \mathrm{H}, 6-\mathrm{H}_{\mathrm{a}}\right), 2.13\left(\mathrm{~d}, J=14.0 \mathrm{~Hz}, 1 \mathrm{H}, 6-\mathrm{H}_{\mathrm{b}}\right)$, 2.16 (m, 1H, 6'-H), 5.39 (dd, $J=5.0 \mathrm{~Hz}, 1 \mathrm{H}, 3-\mathrm{H}), 5.78$ (d, J = $\left.15.5 \mathrm{~Hz}, 1 \mathrm{H}, 2^{\prime}-\mathrm{H}\right), 5.91$ (s, 1H, 9-H), 5.99 (dd, $\left.J=8.0,15.0 \mathrm{~Hz}, 1 \mathrm{H}, 5^{\prime}-\mathrm{H}\right), 6.13$ (dd, $\left.J=11.0,15.0 \mathrm{~Hz}, 1 \mathrm{H}, 4^{\prime}-\mathrm{H}\right), 6.23$ $(\mathrm{dd}, J=5.0,10.0 \mathrm{~Hz}, 1 \mathrm{H}, 2-\mathrm{H}), 6.24\left(\mathrm{~s}, 2 \mathrm{H}, 13-\mathrm{H}_{2}\right), 6.36$ (d, $\left.J=10.0 \mathrm{~Hz}, 1 \mathrm{H}, 1-\mathrm{H}\right), 7.22$ (dd, $\left.J=11.0,15.5 \mathrm{~Hz}, 1 \mathrm{H}, 3^{\prime}-\mathrm{H}\right)$.

${ }^{13}$ C-NMR (125.7 MHz, $\mathbf{C D C l}_{3}$ ): $\delta=10.2$ (q, C-15), 11.7 (q, C-8'), 19.5 (q, C-9'), 22.3 (q, C-14), 29.3 (t, C-7'), 36.3 (s, C-5), 38.8 (d, C-6'), 41.1 (d, C-4), 45.0 (t, C-6), 68.9 (d, C-3), 75.3 (s, C-7), 118.8 (d, C-2'), 123.9 (C-9), 126.6 (d, C-4'), 127.7 (s, C-,13), 131.1 (d, C-1), 133.0 (d, C-2), 145.1 (s, C-11), 146.0 (d, C-3'), 150.9 (d, C-5'), 161.8 (s, C-10), 166.9 (s, C-1'), 168.9 (s, C-12).

Die chemische Verschiebung von C-8 kann aufgrund zu geringer Intensität nicht bestimmt werden.

\section{Dendryphiellinsäure $A^{152}$ (139)}

139 konnte aus dem Mycelextrakt durch weitere Chromatographie an Sephadex ${ }^{\circledR}$ LH-20 $(\mathrm{MeOH})$ und Kieselgel $\left(\mathrm{CH}_{2} \mathrm{Cl}_{2} / \mathrm{MeOH} 9: 1+0.1 \% \mathrm{AcOH}\right)$ in Ausbeuten von $4.5 \mathrm{mg} / \mathrm{L}$ erhalten werden.

$\mathrm{C}_{9} \mathrm{H}_{14} \mathrm{O}_{2}(154.21)$

farbloses Ö1

UV-löschend (254 nm)

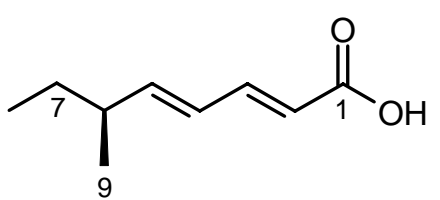

$\mathbf{R}_{\mathbf{f}}$-Wert: 0.19 (Chloroform/Methanol 9:1)

Anfärbeverhalten: blau (Anisaldehyd)

EI-MS: $\mathrm{m} / \mathrm{z}(\%)=154(56)[\mathrm{M}]^{+}, 139(28)\left[\mathrm{M}-\mathrm{CH}_{3}\right]^{+}, 125(40), 97(100), 79(42), 56(40)$.

IR(KBr): $\widetilde{v}=3425,2987,2963,2929,2871,2362,1692,1636,1616,1558,1458,1418$, $1383,1302,1276,1197,1157,1110,1002,671 \mathrm{~cm}^{-1}$.

UV: $(\mathrm{MeOH}): \quad\left[\lambda_{\max }(\lg \varepsilon)\right]=257(4.30) \mathrm{nm}$;

$\mathrm{MeOH} / \mathrm{HCl}): \quad\left[\lambda_{\max }(\lg \varepsilon)\right]=259(4.32) \mathrm{nm}$;

$(\mathrm{MeOH} / \mathrm{NaOH}):\left[\lambda_{\max }(\lg \varepsilon)\right]=251(4.29) \mathrm{nm}$. 
CD (MeOH): $\lambda_{\max }([\Theta])=235$ (2732), 244 (-6029), 253 (5486), 260(-2450), 269 (2847), 277 (sh, 1428), 331(1101).

${ }^{1}$ H-NMR (500 MHz, $\mathbf{C D C l}_{3}$ ): $\delta=0.89\left(\mathrm{t}, J=7.5 \mathrm{~Hz}, 3 \mathrm{H}, 8-\mathrm{H}_{3}\right), 1.05(\mathrm{~d}, J=7.0 \mathrm{~Hz}, 3 \mathrm{H}$, 9- $\left.\mathrm{H}_{3}\right), 1.40\left(\mathrm{dq}, J=7.0,7.5 \mathrm{~Hz}, 2 \mathrm{H}, 7-\mathrm{H}_{2}\right), 2.21(\mathrm{dtq}, J=7.0,7.5 \mathrm{~Hz}, 1 \mathrm{H}, 6-\mathrm{H}), 5.82(\mathrm{~d}$, $J=15.0 \mathrm{~Hz}, 1 \mathrm{H}, 2-\mathrm{H}), 6.09$ (dd, $J=7.5,15.0 \mathrm{~Hz}, 1 \mathrm{H}, 5$ '-H), 6.19 (dd, $J=11.0,15.0 \mathrm{~Hz}, 1 \mathrm{H}$, 4-H), 7.37 (dd, $J=11.0,15.0 \mathrm{~Hz}, 1 \mathrm{H}, 3-\mathrm{H})$.

${ }^{13}$ C-NMR (125.7 MHz, $\mathbf{C D C l}_{3}$ ): $\delta=11.7$ (q, C-8), 19.4 (q, C-9), 29.2 (t, C-7), 38.8 (d, C-6), 118.4 (d, C-2), 126.6 (d, C-4), 147.7 (d, C-3), 151.6 (d, C-5), 172.8 (s, C-1).

\section{Cynodontin $^{160}$ (140)}

1,4,5,8-Tetrahydroxy-2-methylanthrachinon

140 konnte sowohl aus dem Mycel- als auch aus dem Kulturfiltratextrakt durch weitere Chromatographie an Sephadex ${ }^{\circledR}$ LH-20 (Aceton) in Ausbeuten von $0.75 \mathrm{mg} / \mathrm{L}$ erhalten werden.

$\mathrm{C}_{15} \mathrm{H}_{10} \mathrm{O}_{6}(286.24)$

roter Feststoff

UV-löschend (254 nm)

rötlich fluoreszierend $(366 \mathrm{~nm})$

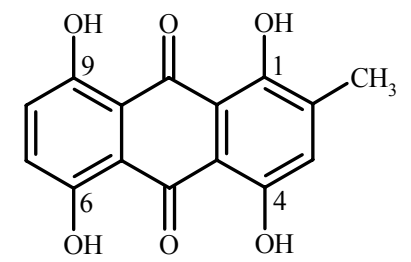

$\mathbf{R}_{\mathbf{f}}$-Wert: 0.82 (Chloroform/Methanol 9:1)

Eigenfarbe: rot

Smp.: $258^{\circ} \mathrm{C}$

ESI-MS (positive Ionen): $\mathrm{m} / \mathrm{z}(\%)=285(100)[\mathrm{M}+\mathrm{H}]^{+}, 593(8)[2 \mathrm{M}-2 \mathrm{H}+\mathrm{Na}]^{-}$.

HRESI-MS: 287.05556 gefunden wie berechnet für $\left[\mathrm{C}_{15} \mathrm{H}_{11} \mathrm{O}_{6}\right]^{+}$.

IR(KBr): $\widetilde{v}=3443,2956,2923,2856,1734,1719,1701,1650,1635,1620,1577,1542$, $1450,1431,1394,1311,1272,1254,1173,1078,1022,973,787,761,735 \mathrm{~cm}^{-1}$.

UV: $(\mathrm{MeOH}): \quad\left[\lambda_{\max }(\lg \varepsilon)\right]=237(4.16), 293(3.38), 485$ (3.52), 506 (3.68), 518 (3.74), $543(3.71), 556(3.75) \mathrm{nm}$;

$\mathrm{MeOH} / \mathrm{HCl}): \quad\left[\lambda_{\max }(\lg \varepsilon)\right]=237$ (4.20), 293 (3.52), 487 (3.57), 507 (3.73), 517 (3.78), 543 (3.74), $556(3.77) \mathrm{nm}$;

$(\mathrm{MeOH} / \mathrm{NaOH}):\left[\lambda_{\max }(\lg \varepsilon)\right]=240(4.24), 294$ (3.44), $573(3.80), 614(3.81) \mathrm{nm}$.

${ }^{1}$ H-NMR (500 MHz, $\left.\mathbf{C D C l}_{3}\right): \delta=2.40\left(\mathrm{~s}, 3 \mathrm{H}, 11-\mathrm{H}_{3}\right), 7.20(\mathrm{~s}, 1 \mathrm{H}, 3-\mathrm{H}), 7.31$ (s, 2H, 7-H und 8-H), 12.40 (s, 1H, OH), $12.43(\mathrm{~s}, 1 \mathrm{H}, \mathrm{OH}), 12.45(\mathrm{~s}, 1 \mathrm{H}, \mathrm{OH}), 12.83(\mathrm{~s}, 1 \mathrm{H}, \mathrm{OH})$. 


\section{Cerebrosid $C^{163}(141)$}

141 konnte aus dem Mycelextrakt durch weitere Chromatographie an Sephadex ${ }^{\circledR}$ LH-20 $(\mathrm{MeOH})$ in Ausbeuten von $2.0 \mathrm{mg} / \mathrm{L}$ erhalten werden.

$\mathrm{C}_{43} \mathrm{H}_{79} \mathrm{NO}_{9}(754.10)$

farbloser Feststoff

$\mathbf{R}_{\mathbf{f}}$-Wert: 0.06 (Chloroform/Methanol 9:1)

Anfärbeverhalten: braun (Anisaldehyd)

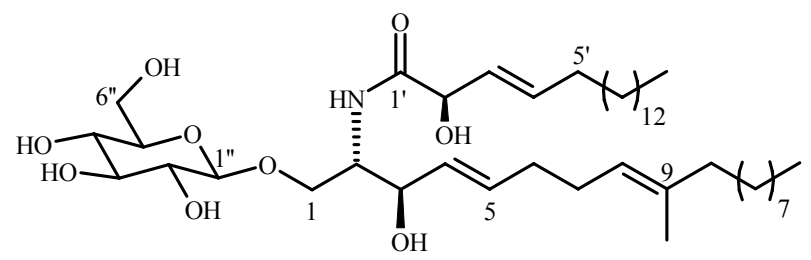

Smp.: $162^{\circ} \mathrm{C}$

ESI-MS (positive Ionen): $\mathrm{m} / \mathrm{z}(\%)=777(20)[\mathrm{M}+\mathrm{Na}]^{+}, 1531(100)[2 \mathrm{M}+\mathrm{Na}]^{+}$;

(negative Ionen): $\mathrm{m} / \mathrm{z}(\%)=753(100)[\mathrm{M}-\mathrm{H}]^{-}, 1507$ (35) $[2 \mathrm{M}-\mathrm{H}]^{-}$.

HRESI-MS: 776.56529 gefunden wie berechnet für $\left[\mathrm{C}_{43} \mathrm{H}_{79} \mathrm{NO}_{9} \mathrm{Na}\right]^{+}$.

IR(KBr): $\widetilde{v}=3424,2955,2923,2852,1773(\mathrm{sh}), 1752(\mathrm{sh}), 1735(\mathrm{sh}), 1719(\mathrm{sh}), 1698(\mathrm{sh})$, 1651, 1559(sh), 1542, 1522(sh), 1474, 1458, 1404, 1382, 1260, 1164, 1106, 1241, 1187, $1139,1110,1081,1066,1029,996,936,912,882 \mathrm{~cm}^{-1}$.

${ }^{1}$ H-NMR (500 MHz, $\left.\mathbf{C D C l}_{3}\right): \delta=0.89\left(\mathrm{t}, \mathrm{J}=7.0 \mathrm{~Hz}, 6 \mathrm{H}, 16{ }^{\prime}-\mathrm{H}_{3}\right.$ und 18- $\left.\mathrm{H}_{3}\right), 1.28(\mathrm{~m}, 38 \mathrm{H}$, $6^{\prime}-\mathrm{H}_{2}-15^{\prime}-\mathrm{H}_{2}$ und 11- $\left.\mathrm{H}_{2}-17-\mathrm{H}_{2}\right), 1.59$ (s, 3H, 19- $\left.\mathrm{H}_{3}\right), 1.97$ (t, $\left.J=7.5 \mathrm{~Hz}, 2 \mathrm{H}, 10-\mathrm{H}\right)$, $2.01-2.07$ (m, 6H, 6- $\mathrm{H}_{2}, 7-\mathrm{H}_{2}$ und 5' $\left.-\mathrm{H}_{2}\right), 3.19$ (dd, $J=8.0,9.5 \mathrm{~Hz}, 1 \mathrm{H}, 2$ ' '-H), 3,27 (m, 2H, 4' '-H und 5' '-H), 3.35 (dd, $J=9.5 \mathrm{~Hz}, 1 \mathrm{H}, 3$ ''-H), 3.66 (dd, $J=5.0,12.0 \mathrm{~Hz}, 1 \mathrm{H}, 6$ ' $\left.-\mathrm{H}_{\mathrm{a}}\right)$, $3.70\left(\mathrm{dd}, J=3.5,10.0 \mathrm{~Hz}, 1 \mathrm{H}, 1-\mathrm{H}_{\mathrm{a}}\right), 3.86\left(\mathrm{dd}, J=2.0,12.0 \mathrm{~Hz}, 1 \mathrm{H}, 6\right.$ ' '- $\left.\mathrm{H}_{\mathrm{b}}\right), 3.96$ (ddd, $J=3.5,5.0,8.0 \mathrm{~Hz}, 1 \mathrm{H}, 2-\mathrm{H}), 4.12$ (dd, $J=7.5,8.0 \mathrm{~Hz}, 1 \mathrm{H}, 3-\mathrm{H}), 4.13$ (dd, $J=5.0,10.0 \mathrm{~Hz}$, $\left.1 \mathrm{H}, 1-\mathrm{H}_{\mathrm{b}}\right), 4.27$ (d, $J=8.0 \mathrm{~Hz}, 1 \mathrm{H}, 1$ '’-H), 4.43 (dd, $J=1.0,6.0 \mathrm{~Hz}, 1 \mathrm{H}, 2$ '-H), 5.14 (dd, $J=6.5,7.5 \mathrm{~Hz}, 1 \mathrm{H}, 8-\mathrm{H}), 5.44$ (dd, $J=7.5,15.0 \mathrm{~Hz}, 1 \mathrm{H}, 4-\mathrm{H}), 5.48$ (dd, $J=6.0,15.0 \mathrm{~Hz}, 1 \mathrm{H}$, 3'-H), 5.71 (ddd, $J=6.0,7.0,15.0 \mathrm{~Hz}, 1 \mathrm{H}, 5-\mathrm{H}), 5.83$ (ddd, $\left.J=6.0,8.0,15.0 \mathrm{~Hz}, 1 \mathrm{H}, 4^{\prime}-\mathrm{H}\right)$.

${ }^{13}$ C-NMR (125.7 MHz, CDCl 3 ): $\delta=14.5$ (q, C-16'), 14.5 (q, C-18), 16.2 (q, C-19), 23.8 (t, C-17 und C-15'), 28.8 - 33.8 (t, C-6, C-7, C11-C16 und C-5'-C14'), 40.8 (t, C-10), 54.6 (d, C-2), 62.7 (t, C-6'’), 69.7 (t, C-1), 71.6 (d, C-3 oder C-4'’), 72.9 (d, C-3 oder C-4'’), 74.1 (d, C-2' oder C-2'), 77.9 (d, C-3'”), 77.9 (t, C-5'’), 104.7 (d, C-1'’), 124.9 (d, C-8), 129.0 (d, C-4'), 131.0 (d, C-5), 134.5 (d, C-4 oder C-3'), 134.7 (d, C-4 oder C-3'), 136.7 (s, C-9), 175.4 (s, C-1'). 


\section{LITERATURVERZEICHNIS}

1 D. J. Newman, G. M. Cragg, K. M. Snader, J. Nat. Prod. 2003, 66, 1022-1037.

2 W. Tang, G. Eisenbrand, Chinese Drugs of Plant Origin, Chemistry, Pharmacology and Use in Traditional and Modern Medicine, Springer Verlag, Berlin, Heidelberg, 1992.

3 1) A. Fleming, Brit. J. Pat. 1929, 10, 226-236.

2) E. P. Abraham, W. Baker, E. Chain, H. W. Florey, E. R. Holiday, R. Robinson, Nature 1942, 149, 356.

4 H. Umezawa, I. R. Hooper, Aminoglycoside Antibiotics, Springer Verlag, New York, Heidelberg, Berlin, Tokyo, 1982.

5 A. L. Demain, Nature Biotechnology 2002, 20, 331.

6 W. Bergmann, R. Feeney, Journal of Organic Chemistry 1951, 16, 981-987.

7 A. Kijjoa, P. Sawangwong, Mar. Drugs 2004, 2, 73-82.

8 D. J. Newman, G. M. Cragg, J. Nat. Prod. 2004, 67, 1216-1238.

9 www.finanznachrichten.de/nachrichten-2004-12/artikel-4247901.asp

10 1) K. Rinehart, T. G. Holt, N. L. Fregeau, J. G. Stroh, P. A. Kiefer, F. Sun, L. H. Li, D. G. Martin, J. Org. Chem. 1990, 55, 4512-4515.

2) A. E. Wright, D. A. Forleo, G. P. Gunwardana, S. P. Gunasekera, F. E. Koehn, O. J. McConnell, J. Org. Chem. 1990, 55, 4508-4512.

11 M. Zewail-Foote, L. H. Hurley, J. Med. Chem. 1999, 42, 2493-2497.

12 R. Mutter, M. Wills, Bioorg. Med. Chem. 2000, 8, 1841-1860.

13 J. Kortmansky, G. K. Schwartz, Cancer Investigation 2003, 21, 924-936.

14 1) P. A. Wender, B. Lippa, Tetrahedron Lett. 2000, 41, 1007-1011.

2) P. A. Wender, J. L. Baryza, S. E. Brenner, M. O. Clarke, C. G. Gamber, J. C. Horan, T. C. Jessop, C. Kan, K. Pattabiraman, T. J. Williams, Pure and Appl. Chem. 2003, 75, 143 155.

15 M. G. Haygood, S. K. Davidson, Appl. Env. Microbiol. 1997, 63, 4612-4616.

16 M. Hildebrand, L. E. Waggoner, H. Liu, S. Sudek, S. Allen, C. Anderson, D. H. Sherman, M. Haygood, Chemistry and Biology 2004, 11, 1543-1552.

17 P. Proksch, R. A. Edrada, R. Ebel, Applied Microbiology and Biotechnology 2002, 59, 125-134.

18 C. Cuevas, M. Perez, M. J. Martin, J. L. Chicharro, C. Fernandez-Rivas, M. Flores, A. Francesch, P. Gallego, M. Zarzuelo, F. de la Calle, J. Garcia, C. Polanco, I. Rodruiguez, I. Manzanares, Org. Lett. 2000, 2, 993-996. 
19 1) C. A. Bewley, N. D. Holland, D. J. Faulkner, Experientia 1996, 52, 716-722.

2) C. A. Bewley, D. J. Faulkner, Angewandte Chemie Internationale Edition 1998, 37, 2162- 2178 .

20 E. W. Schmid, A. Y. Obraztsova, S. K. Davidson, D. J. Faulkner, M. G. Haygood, Marine Biology 2000, 136, 969-977.

21 R. L. L. Kellner, K. Dettner, Oecologia 1996, 107, 293-300.

22 1) J. Piel, Proc. Natl. Acad. Sci. USA 2002, 99, 14002-14007.

2) J. Piel, G. Wen, M. Platzer, D. Hui, ChemBioChem 2004, 5, 93-98.

23 N. B. Perry, J. W. Blunt, M. H. G. Munro, A. M. Thompson, J. Org. Chem. 1990, 55, $223-$ 227.

24 S. Sakemi, T. Ichiba, S. Kohmoto, G. Saucy, T. Higa, J. Am. Chem. Soc. 1988, 110, 48514853.

25 J. K. Stone, C. W. Bacon, J. F. White, An Overview of Endophytic Microbes: Endophytism Defined, in Microbial Endophytes, edited by C. W. Bacon, J. F. White, Marcel Dekker Inc., New York, Basel, 2000, 3-31.

26 G. A. Strobel, D. M. Long, ASM News 1998, 64, 263-268.

27 A. E. Arnold, Z. Maynard, G. S. Gilbert, P. D. Coley, T. A. Kursar, Ecol. Lett. 2000, 3, 267-274.

28 1) M. Arachevaleta, C. W. Bacon, C. S. Hoveland, D. E. Radcliffe, Agron. J. 1989, 81, 8390.

2) F. Ravel, C. Courty, A. Coudret, G. Charmet, Agronomie 1997, 17, 173-181.

29 1) J. Hallmann, R. A. Sikora, Eur. J. Plant Pathol. 1996, 102, 155-162.

2) H. H. Wilkinson, M. R. Siegel, J. D. Blankenship, A. C. Mallory, L. P. Bush, C. L. Shardl, Mol. Plant-Microbe Interact. 2000, 13, 1027-1033.

3) R. W. Preszler, E. S. Gaylord, W. J. Boecklen, Oecologia 1996, 108, 159-166, 336-342.

30 M. J. Christensen, Aust. Plant Pathol. 1996, 25, 186-191.

31 A. V. Sturz, B. R. Christie, B. G. Matheson, W. J. Arsenault, N. A. Buchanan, Plant Pathol. 1999, 48, 360-369.

32 1) G. Strobel, B. Daisy, Microbiol. Mol. Biol. Rev. 2003, 67, 491-502.

2) G. Strobel, B. Daisy, U. Castillo, J. Harper, J. Nat. Prod. 2004, 67, 257-268.

33 J. Y. Li, J. K. Harper, D. M. Grant, B. O. Tombe, B. Bashyal, W. M. Hess, G. A. Strobel, Phytochemistry 2001, 56, 463-468.

34 J. Y. Li, G. A. Strobel, J. K. Harper, E. Lokovsky, J. Clardy, Org. Lett. 2000, 2, 767-770. 

Microbiology 2000, 146, 2079-2089. 1998, 20, 259-264.

40

2) H. B. Bode, B. Bethe, R. Höfs, A. Zeeck, ChemBioChem 2002, 3, 619-627. Verlag, Berlin, Heidelberg, New York, 1992.

Eds.: J. D. Bullock, L. J. Nisbet, D. J. Winstley, Academic Press, London, New York 1982, 51-70. from Nature, edited by S. Grabley, R. Thierecke, Springer Verlag, Berlin Heidelberg New York, 1999, 124-149. $187-195$ G. Bringmann, K. Messer, M. Wohlfarth, J. Kraus, K. Dumbuya, M. Rückert, Anal. Chem. 1999, $71,2678-2686$.

51 K. Ingkaninan, A. Hazekamp, C. M. de Best, H. Irth, U. R. Tjaden, R. van der Heijden. J. van der Greef, R. Verpoorte, J. Nat. Prod. 2000, 63, 803-806.

L. Krasu, A. Kocj, S. Hoffstetter-Kuhn, Dünnschichtchromatographie, Springer-Verlag, Berlin, Heidelberg, New York, 1995.

53 Autorenkollektiv, Chapman\&Hall/CRC Dictionary of Natural Products on CD-ROM, Version 9:1, Chapmann\& Hall, CRC., 2000. 

Trans. I 1994, 13, 1865-1869. 2565. 4528.

60 1054.

2) J. A. Findlay, S. Buthelezi, R. Lavoie, L. Pena-Rodriguez, J. D. Miller, J. Nat. Prod. 1995, 58, 1759-1766.

1) L. L. Woods, P. A. Dix, J. Org. Chem. 1961, 26, 1028-1030.

2) J. Fried, R. C. Elderfield, J. Org. Chem. 1941, 6, 577.

1) H. Sato, K. Konoma, S. Sakamura, A. Furusaki, T. Matsumoto, T. Matsuzaki, Agric. Biol. Chem. 1981, 45, 795-797.

2) S. A. Sparace, J. B. Mudd, B.A. Burke, A. J. Aasen, Phytochemistry 1984, 23, 2693 2694.

3) T. Amagata, K. Minoura, A. Numata, J. Antibiot. 1998, 51, 432-434. Rojas, J. F. Reyes, Tetrahedron 1993, 49, 141-151.

G. Schlingmann, L. Milne, G. T. Carter, Tetrahedron 1998, 4, 13013-13022.

L. J. Mulheirn, R. B. Beechey D. P. Leworthy, M. D. Osselton, J. Chem. Soc. Chem. Comm. 1974, 21, 874-876.

W. Kohl, H. Irschik, H. Reichenbach, G. Höfle, Liebigs Ann. Chem. 1983, 10, 1656-1667. A. F. Barrero, J. E. Oltra, M. M. Herrador, E. Cabrera, J. F. Sanchez, J. F. Quilez, F. J.

Y. Shizuri, M. Niwa, H. Furukawa, S. Yamamura, Tetrahedron Lett. 1983, 24, 1053-1054.

T. Nakada, S. Sudo, S. Kosemura, S. Yamamura, Tetrahedron Lett. 1999, 40, 6831-6834. 
73 A. Ichihara, H. Oikawa, K. Hayashi, S. Sakamura, J. Am. Chem. Soc. 1983, 105, $2907-$ 2908.

74 I. Barash, S. Manulis, Y. Kashman, J. P. Springer, M. H. M. Chen, J. Clardy, G. A. Stroebel, Science 1983, 220, 1065-1066.

S. Manulis, Y. Kashman, D. Netzer, I. Barash, Phytochemistry 1984, 10, 2193-2198.

76 1) A. Ichihara, H. Oikawa, M. Hashimoto, S. Sakamura, T. Haraguchi, H. Nagano, Agric. Biol. Chem. 1983, 47, 2965-2967.

2) H. Oikawa, A. Ichihara, S. Sakamura, J. Chem. Soc. Chem. Comm. 1988, 35, 600-602.

3) G. Brauers, R. A. Edrada, R. Ebel, P. Proksch, V. Wray, A. Berg, U. Gräfe, C. Schächtele, F. Totzke, G Finkenzeller, D. Marme, J. Kraus, M. Münchbach, M. Michel, G. Bringmann, K. Schaumann, J. Nat. Prod. 2000, 63, 739-745.

S. Manulis, D. Netzer, I. Barash, J. Phytopath. 1986, 115, 283-287.

H. Oikawa, A. Ichihara, S. Sakamura, J. Chem. Soc. Chem. Comm. 1984, 31, 814-815. M. Svensson, L. N. Lundgren, C. Woods, J. Fatehi, J. Stenlid, Phytochemistry 2001, 56, 747-751.

81 A. Ichihara, H. Tazaki, S. Sakamura, Tetrahedron Lett. 1983, 24, 5373-5376.

82 A. Ichihara, H. Oikawa, Biosci. Biotech. Biochem. 1997, 61, 12-18.

83 S. Laschat, Angew. Chem. 1996, 108, 313-315.

84 1) G. Pohnert, ChemBioChem 2001, 2, 873-875.

2) E. M. Stocking, R. M. Williams, Angew. Chem. 2003, 115, 3186-3223

3) H. Oikawa, T. Tokiwano, Nat. Prod. Rep. 2004, 21, 321-352.

R. J. Cole, R. H. Cox, Handbook of Toxic Fungal Metabolites 1981, Academic Press INC., 615.

R. J. Cole, R. H. Cox, Handbook of Toxic Fungal Metabolites 1981, Academic Press INC., 623.

1) L. M. Seitz, H. E. Mohr, D. B. Sauer, R. Burrogh, J. V. Paukstelis, J. Agric. Food Chem. 1975, 23, 1-4.

2) S. da Motta, L. M. V. Soares, Food Addit. Contam. 2001, 18, 630-634.

E. E. Stinson, J. Food Prot. 1985, 48, 80-91. 530.

91 M. E. Stack, M. J. Prival, Appl. Environ. Microbiol. 1986, 52, 718-722. 
92 T. Okuno, I. Natsume, K. Sawai, K. Sawamura, A. Furusaki, T. Matsumoto, Tetrahedron Lett. 1983, 24, 5653-5656.

1) A. Okubo, S. Yamazaki, K. Fuwa, Agr. Biol. Chem. 1975, 39, 1173-1175.

2) D. G. Panaccione, R. D. Johnson, J. B. Rasmussen, T.L. Friesen, Fungal Phytotoxins in Mycota 2002, Springer, Berlin, 311-340.

D. C. Allport, J. D. Bullock, J. Chem. Soc. 1958, 4090-4094.

G. San-Blas, O. Guanipa, B. Moreno, S. Pekerar, F. San-Blas, Curr. Microbiol. 1996, 32, 11-16.

96 A. A. Brakhage, K. Langfelder, G. Wanner, A. Schmidt, B. Jahn, Contrib. Microbiol. 1999, 2, 205-215.

H.-F. Tsai, M. H. Wheeler, Y. C. Chang, K. J. Kwon-Chung, J. Bacteriol. 1999, 181, 6469-6477.

R. G. Washburn, Opportunistic mold infections; in The Mycota VI, L. Esser, P. A. Lembke (eds.), 1996, Springer Verlag, Heidelberg, 147-158.

D. P. Woloshuk, H. D. Sisler, M. C. Tokousbalides, S. R. Dutky, Pest. Biochem. Physiol. 1980, $14,256-264$.

100 M. H. Wheeler, M. A. Klich, Pest. Biochem. Physiol. 1995, 52, 125-136.

101 T. J. Simpson, J. Chem. Soc. Perkin Trans. I 2000, 19, 2771-2775.

102 G. Barre, M. Hocquaux, B. Jacquet, M. De Min, M. T. Maurette, E. Oliveros, Tetrahedron Lett. 1986, 27, 6197-6200.

103 H. B. Bode, A. Zeeck, Phytochemistry 2000, 54, 597-601.

104 J. A. Findlay, D. Kwan, Can. J. Chem. 1973, 51, 3299-3301.

${ }^{105}$ Aldrich Library of ${ }^{13} \mathrm{C}$ and ${ }^{1} \mathrm{H}$ FT NMR Spectra 1992, 3, 570B.

106 M. Radzom, Diplomarbeit, Universität Göttingen 2003.

107 M. Speitling, Dissertation, Universität Göttingen 1998.

${ }^{108}$ Aldrich Library of ${ }^{13} \mathrm{C}$ and ${ }^{1} \mathrm{H}$ FT NMR Spectra 1992, 2, 385A, 1203A.

${ }^{109}$ Aldrich Library of ${ }^{13} \mathrm{C}$ and ${ }^{1} \mathrm{H}$ FT NMR Spectra 1992, 3, $130 \mathrm{~B}$.

${ }^{110}$ S. Lösgen, Diplomarbeit, Universität Göttingen 2004.

${ }^{111}$ K. I. Fujita, Y. Nagamine, X. Ping, M. Taniguchi, J. Antibiot. 1999, 52, 628-634.

${ }^{112}$ M. Aoki, Y. Itezono, H. Spirai, N. Nakayama, A. Sakai, Y. Tanaka, A. Yamaguchi, N. Shimma, K. Yokose, H. Seto, Tetrahedron Lett. 1991, 36, 4737-4740.

113 W. Pfeferle, H. Anke, M. Bross, B. Stefan, R. Vianden, W. Steglich, J. Antibiot. 1990, 43, 648-654 
114 R. J. Cole, R. H. Cox, Handbook of Toxic Fungal Metabolites 1981, Academic Press INC., 759.

115 H. R. V. Arnstein, R. Bentley, Biochem. J. 1953, 54, 493-508, 508-516, 517-522.

${ }^{116}$ K. Shiomi, K. Hatae, Y. Yamaguchi, R. Masuma, H. Tomoda, S. Kobayashi, S. Omura, J. Antibiot. 2002, 55, 952-961.

117 W. A. Ayer, K. Nozawa, Can. J. Microbiol. 1990, 36, 83-85.

118 1) R. Siebertz, P. Proksch, V. Wray, L. Witte, Phytochemistry 1989, 28, 789-793.

2) J. D. Bu'Lock, A. T. Hudson, B. Kaye, J. Chem. Soc. Chem. Comm. 1967, 814-815.

3) J. D. Bu'Lock, B. Kaye, A. T. Hudson, Phytochemistry 1971, 10, 1037-1046.

119 W. B. Turner, D. C. Aldridge, Fungal Metabolites, Academic Press, London 1983, 115 117.

${ }^{120}$ Aldrich Library of ${ }^{13} \mathrm{C}$ and ${ }^{1} \mathrm{H}$ FT NMR Spectra 1992, 2, $1258 \mathrm{~B}$.

${ }^{121}$ I. Tvaroska, F. Taravel, Adv. Carbohyd. Chem. Biochem. 1995, 51, 15-61.

122 T. Müller, R. R. Schmidt, Liebigs Ann. 1997, 1907-1914.

123 J. S. Brimacombe, C. W. Smith, J. Minshall, Tetrahedron Lett. 1974, 35, 2997-3000.

124 1) T. Müller, R. R. Schmidt, Liebigs Ann. 1997, 1907-1914.

2) H. Chen, S-M. Yeung, N. L. S. Que, T. Müller, R. R. Schmidt, H. Liu, J. Am. Chem. Soc. 1999, 121, 7166-7167.

125 A. Nakagawa, Y. Konda, A. Hatano, Y. Harigaya, M. Onda, J. Org. Chem. 1988, 53, 2660-2661.

${ }^{126}$ H. Sasamori, K. S. Reddy, M. P. Kirkup, J. Shabanowitz, D. G. Lynn, S. M. Hecht, K. A. Woode, R. F. Bryan, J. Campell, W. S. Lynn, E. Eggert, G. M. Sheldrick, J. Chem. Soc. Perkin Trans. I 1983, 31, 1333-1347.

127 1) W. D. Celmer, W. P. Cullen, H. Maeda, J. Tone, U.S. Patent 454334, 24.09.1985.

2) M. Morioka, I. Takahashi, K. Suzuki, K. Nagai, H. Yamaguchi, T. Sasaki, M. Shibazaki, M. Hiramoto, Y. Takebayashi, PCT Int. Appl. WO 9212988, 06.08.1992.

128 T. Nitoda, H. Usuki, H. Kanzaki, Z. Naturforsch. 2003, 58c, 891-894.

129 A. Kirschning, A. F.-W. Bechthold, J. Rohr, Topics in Current Chemistry 1997, 188, 1-84, Springer-Verlag, Berlin, Heidelberg, New York.

${ }^{130}$ R. J. Cole, R. H. Cox, Handbook of Toxic Fungal Metabolites 1981, Academic Press INC., 601-605.

${ }^{131}$ H. Fujimoto, M. Sumino, E. Okuyama, M. Ishibashi, J. Nat. Prod. 2004, 67, 98-102.

132 T. Saito, Y. Suzuki, K. Koyama, S. Natori, Y. Iitaka, T. Kinoshita, Chem. Pharm. Bull. 1988, 36, 1942-1956. 
${ }^{133}$ N. Kawahara, K. Nozawa, M. Yamazaki, S. Nakajima, K. Kawai, Chem. Pharm. Bull. 1990, 38, 73-78.

134 1) J. R. Johnson, W. F. Bruce, J. D. Dutcher, J. Am. Chem. Soc. 1943, 65, 2005-2009.

2) A. F. Beecham, J. Fridrichsons, A. M. Mathieson, Tetrahedron Lett. 1966, 27, 31313138.

135 1) R. J. Cole, R. H. Cox, Handbook of Toxic Fungal Metabolites 1981, Academic Press INC., 571 .

2) F. Seigle-Murandi, S. Krivobok, R. Steiman, D. Marzin, J. Agricult. Food Chem. 1990, $38,1854-1856$.

${ }^{136}$ G. M. Strunz, M. Kakushima, M. A. Stillwell, C. Heissner, J. Chem. Soc. Perkin I 1973, 21, 2600-2602.

137 T. Rouxel, Y. Chupeau, R. Fritz, A. Kollmann, J. F. Bousquet, Plant Sci. 1988, 57, 45-53.

138 D. Hauser, H. P. Weber, H. P. Sigg, Helv. Chim. Acta 1970, 53, 1061-1073.

${ }^{139}$ K. Katagiri, K. Sato, S. Hayakawa, T. Matsushima, H. Minato, J. Antibiot. 1970, 23, 420422.

140 1) F. R. Ahmed, M. Przybylska, Acta Crystallographica 1977, B33, 168-170.

2) R. L. De Vault, W. Rosenbrook, J. Antibiotics 1973, 26, 532-534.

3) G. W. Kirby, G. V. Rao, D. J. Robins, J. Chem. Soc. Perkin I 1988, 36, 301-304.

141 1) T. Saito, Y. Suzuki, K. Koyama, S. Natori, Y. Iitaka, T. Kinoshita, Chem. Pharm. Bull. 1988, 36, 1942-1956.

2) Y. Feng, J. W. Blunt, A. L. J. Cole, M. H. G. Munroe, J. Nat. Prod. 2004, 67, 20902092.

3) H. Fujimoto, M. Sumino, E. Okuyama, M. Ishibashi, J. Nat. Prod. 2004, 67, 98-102.

142 P. Waring, J. Beaver, Gen. Pharmacol. 1996, 27, 1311-1316.

143 J. D. Bu'Lock, C. Leigh, J. Chem. Soc. Chem. Comm. 1975, 628-629.

144 M. I. Pita Boente, G. W. Kirby, G. L. Patrick, D. J. Robins, J. Chem. Soc. Perkin I 1991, 39, 1283-1290.

145 D. M. Gardiner, A. J. Cozijnsen, L. M. Wilson, M. S. C. Pedras, B. J. Howlett, Mol. Microbiol. 2004, 53, 1307, 1318.

146 R. J. Cole, R. H. Cox, Handbook of Toxic Fungal Metabolites 1981, Academic Press INC., 364-367.

147 R. Andrade, W. A. Ayer, P. P. Mebe, Can. J. Chem. 1992, 70, 2526-2535.

148 R. J. Cole, R. H. Cox, Handbook of Toxic Fungal Metabolites 1981, Academic Press INC., 89. 
149 B. K. Joshi, J. B. Gloer, D. T. Wicklow, J. Nat. Prod. 2002, 65, 1734-1737.

${ }^{150}$ H. B. Bode, B. Bethe, R. Höfs, A. Zeeck, ChemBioChem 2002, 3, 619-627.

${ }^{151}$ F. Sugawara, G. Strobel, L. E. Fisher, G. D. van Duyne, J. Clardy, Proc. Natl. Acad. Sci. USA 1985, 82, 8291-8294.

152 A. Guerriero, M. D’Ambrosio, V. Cuomo, F. Vanzanella, F. Pietra, Helv. Chim. Acta 1989, $72,438-446$

153 S-K. Kim, K. Mizuno, M. Hatori, S. Marumo, Tetrahedron Lett. 1994, 35, 1731-1734.

154 1) B. M. Fraga, Nat. Prod. Rep. 2004, 21, 669-693.

2) B. M. Fraga, Nat. Prod. Rep. 2003, 20, 392-413.

3) B. M. Fraga, Nat. Prod. Rep. 2002, 19, 650-672.

4) B. M. Fraga, Nat. Prod. Rep. 2001, 18, 650-673.

155 S. Kim, T. Asano, S. Marumo, Biosci. Biotech. Biochem. 1995, 59, 1394-1397.

${ }^{156}$ D. C. Ridderbusch, R. W. S. Weber, T. Anke, O. Sterner, Z. Naturforsch. 2004, 59c, 379383.

${ }^{157}$ K. Harimaya, E. Magome, Y. Tabata, T. Sasaki, T. Tsuruoka, Meiji Seika Kaisha 1997, $36,19-27$.

158 D. E. Cane, P. C. Prabhakaran, J. S. Oliver, D. B. McIlwaine, J. Am. Chem. Soc. 1990, 112, 3209-3210.

${ }^{159}$ K. A. Rising, C. M. Starks, J. P. Noel, J. Chappell, J. Am. Chem. Soc. 2000, 122, 18611866.

${ }^{160}$ H. Raistrick R. Robinson, A. R. Todd, Biochem. J. 1933, 27, 1170-1175.

${ }^{161}$ M. Chrysayi-Tokousbalides, M. A. Kastanias, J. Agric. Food Chem. 2003, 51, 4920-4923.

162 S. A. Mabadeje, W. E. Jefferson, J. D. Wander, Exp. Mycology 1978, 2, 359-365.

163 R. D. Sitrin, G. Chan, J. Dingerdissen, C. DeBrosse, R. Mehta, G. Roberts, S. Rottschaefer, D. Staiger, J. Valenta, K. M. Snader, R. J. Stedman, J. R. E. Hoover, J. Antibiot. 1988, 41, 469-480.

164 R. X. Tan, J. H. Chen, Nat. Prod. Rep. 2003, 20, 509-534.

165 A. G. M. Barett, J. C. Beall, D. C. Bradock, K. Flack, V. C. Gibson, M. M. Salter, J. Org. Chem. 2000, 65, 6508-6514.

166 1) G. Kawai, Y Ikeda, Biochim. Biophys. Acta 1982, 719, 612-618.

2) G. Kawai, Y Ikeda, Biochim. Biophys. Acta 1983, 754, 243-248.

3) G. Kawai, Y Ikeda, J. Lipid Res. 1985, 26, 338-343.

167 G. Kawai, Biochim. Biophys. Acta 1989, 1001, 185-190. 
168 J. Koga, T. Yamauchi, M. Shimura, N. Ogawa, K. Oshima, K. Umemura, M. Kikuchi, N. Ogasawara, J. Biol. Chem. 1998, 273, 31985-31991.

169 Merck, Anfärbereagenzien für die Dünnschichtchromatographie, Darmstadt, 1980. 


\section{DANKSAGUNG}

Mein besonderer Dank gilt Kerstin Vogel, die durch ihre engagierte experimentelle Mitarbeit maßgeblich zum Gelingen dieser Arbeit beigetragen hat.

Allen Mitgliedern der NMR-Abteilung und den Abteilungsoperatoren danke ich für die gute Zusammenarbeit. Besonders Herrn R. Machinek sei für die Bereitstellung von reichlich Meßzeit und seine Hilfs- und Diskussionsbereitschaft gedankt. Für die Einarbeitung und gute Zusammenarbeit am 500er möchte ich Sven Meyer danken sowie Carola Zolke, Christiane Siebert und Martin Weitemeyer, die mit Tips und Tricks häufig weiterhelfen konnte.

Für die Aufnahme der Massenspektren gilt mein Dank Frau G. Udvarnoki und Herrn Dr. Frauendorf. Aus unserer Abteilung sei Luise Hoffmann und Nadine Czempinski für die LCMS-Messungen gedankt.

Für die gute Kooperation bei der Bearbeitung der endophytischen Pilze danke ich Frau Dr. Schulz und ihren Mitarbeitern, besonder Herrn Dr. Draeger für die Bestimmung der Pilze.

Michaela Klingebiel danke ich für die Aufnahme von Drehwerten, IR-, UV- und CDSpektren, Jutta Gerber-Nolte für die Bereitstellung von Testplatten und Hans-Peter Kroll für seine Hilfe und Unterstützung im Umgang mit der HPLC.

Ein ganz herzliches Dankeschön an Luise Hoffmann und Markus Radzom für das sorgfältige und konstruktive Korrekturlesen dieser Arbeit.

Der Besetzung von Z-2 Kerstin, Lui und Tim danke ich für die gute Atmosphäre im Labor und das meist klaglose Ertragen meiner Musik.

Allen Mitgliedern der Arbeitsgruppe danke ich für die freundschaftliche Atmosphäre, viele schöne Stunden bei Kuchen, Feierabendbierchen oder anderen Anlässen.

Vielen herzlichen Dank auch an alle meine Freunde für die schöne Zeit.

Dies gilt im Besonderen für Kerstin, die auch in schwierigen Zeiten für mich da war und es versteht mich immer wieder aufzumuntern.

Mein ganz besonderer Dank gilt meiner Familie dafür, dass sie mein Handeln stets unterstützt haben und immer für mich da waren. 


\section{LEBENSLAUF}

Am 13. Februar 1977 wurde ich als Kind von Annemarie Schlörke, geb. Nebe und Manfred Schlörke in Berlin-Wilmersdorf geboren.

Von August 1983 bis Juli 1987 besuchte ich die Grundschule Bad Pyrmont und anschließend bis Juli 1989 die Orientierungsstufe Bad Pyrmont. Ab August 1989 war ich Schüler des Humboldt Gymnasiums Bad Pyrmont, das ich im Juni 1996 mit der Allgemeinen Hochschulreife verließ.

Von Juli 1996 bis April 1997 absolvierte ich meinen Wehrdienst beim Transportbataillion 11 in Dörverden.

Zum Sommersemester 1997 begann ich mit dem Studium der Chemie an der Georg-AugustUniversität in Göttingen, wo ich im April 1999 die Vordiplomprüfung bestand. In der Zeit von Januar 2001 bis Oktober 2001 fertigte ich im Arbeitskreis von Prof. Dr. A. Zeeck meine Diplomarbeit mit dem Titel „Strukturaufklärung und Biosynthese der Cetoniacytone sowie Identifizierung von Sekundärmetaboliten aus Actinomyceten“ an und absolvierte im November 2001 die Diplom-Chemiker Hauptprüfung.

Seit Januar 2002 arbeite ich unter Anleitung von Prof. Dr. A. Zeeck an der vorliegenden Dissertation. 